\title{
Somatoforme Störungen in der Hausarztpraxis \\ Konstruktion und Evaluation eines psychosozialen Gruppenprogramms
}

\author{
Inauguraldissertation \\ zur Erlangung des Doktorgrades \\ der Humanwissenschaftlichen Fakultät \\ der Universität zu Köln \\ nach der Promotionsordnung vom 10.05.2010 \\ vorgelegt von
}

\section{Sarah Magdalena Elisabeth Rembold}

aus

Bergisch Gladbach

Gutachter: Prof. Dr. Jörg Fengler

Prof. Dr. Bodo Januszewski

Tag der Disputation: 27. Oktober 2010

Diese Dissertation wurde von der Humanwissenschaftlichen Fakultät der Universität zu Köln im Oktober 2010 angenommen. 


\section{Danksagungen}

\section{- Dank an meinen Doktorvater Prof. Dr. Jörg Fengler}

Prof. Jörg Fengler durfte ich bereits im 3 . Semester kennen lernen und bin dankbar für alles, was ich in den letzten 6 Jahren von inm lernen durfte und konnte. Seine Begleitung durch alle persönlichen und fachlichen Tiefen und Höhen, sein unerschütterlicher Glaube an mich und seine Leichtigkeit gegenüber vielen meiner Ängste und Sorgen, haben mir durch die Zeit der Promotion sehr geholfen. Ich danke inm für die Möglichkeit, dass ich meine eigenen Ideen und Interessen als seine Doktorandin sehr frei und eigenständig verwirklichen durfte.

\section{- Probandinnen und Probanden sowie Hausärztinnen und Hausärzte}

Ich möchte mich von ganzem Herzen für das Vertrauen und die zuverlässige Teilnahme an meinem Gruppenprogramm bei allen Probanden und Probandinnen, allen Kontrollprobanden sowie allen teilnehmenden Hausärztinnen und Hausärzten bedanken. Ohne sie hätte ich diese Doktorarbeit nicht realisieren können. Danke!!

\section{- Thorsten Franzen}

Meinem lieben Thorsten danke ich für jeden Tag auf dem Spielplatz, für die „Eimaschine“ und für jeden Tag mit inm in meiner „Gegenwelt“. Sein ungebrochener Humor beförderte mich zu jeder Zeit, selbst aus den tiefsten Tiefen und Löchern, unweigerlich wieder hinaus. Ich habe mich noch niemals so bedingungslos begleitet und angenommen gefühlt und bin dafür mehr als dankbar. Aber nicht nur für das (Er-)Leben mit inm in meiner "Gegenwelt" bin ich glücklich. Thorstens außerordentlicher künstlerischer Begabung verdanke ich die kreative Beratung der Bilder in diesem Buch!

\section{Außerdem danke ich von Herzen:}

Meiner Mutter für die unerschütterliche Unterstützung meines Weges und ihrem fürsorglichen Ehemann Michael, meinem lustig-langsam-schlauen Bruder Mario, Meinem Vater Bruno und seiner Lebenspartnerin Bettina für Geduld in tabellarischen Herausforderungen sowie alltäglicher Verpflegung, dem Doktorandenkolloquium, der Benedikt und Helene-Schmittmann-Wahlen Stiftung für die Unterstützung meiner Promotion durch ein Stipendium, Dr. Adina Bunea, Dr. Ursula Tönnes, Frank Dobers, Dr. Heribert Wiemer, Anette Muckenhaupt und Carsten Birkenberger der AOK Bergisch Gladbach, Dr. Thomas Hennemann, Sören Weyer, Prof. Dieter Lotz, Prof. Bodo Januszewski als langjährigen Wegbegleiter, Regina Hömberg, Prof. Ulrich Schultz-Venrath, Rainer sowie meinen wunderbaren Freunden und Freundinnen Simone, Katja, Laura, Mona, Maria, Anja, Inga, Sandro und Till. Danke Thomas für das, was in Worten nicht zu fassen ist... 


\section{Vorwort}

Die vorliegende Forschungsarbeit entstand von Oktober 2007 bis Oktober $2010 \mathrm{im}$ Rahmen einer Promotion an der Humanwissenschaftlichen Fakultät der Universität zu Köln. Das Thema der Arbeit ergab sich aus meiner Beobachtung, dass sich organisch nicht begründbare körperliche Beschwerden in der Gegenwartsgesellschaft häufen. Ich erlebte dies insbesondere bei Menschen, die ein hohes eigenes Ideal verfolgen und in ihrem Berufsleben sehr erfolgreich sind. Das seelische Klagen über den Körper erscheint sehr viel legitimer als über Bedürttigkeiten und eigene Emotionen zu sprechen. Menschen mit somatoformen Störungen machen heute den Großteil aller Patienten in hausärztlichen Praxen aus und können häufig nicht vor Eintritt einer Krankheitschronifizierung angemessen psychosozial erreicht und versorgt werden. Dies hat für betroffene Patienten massive negative Folgen. Dazu zählen Jahre lange Odysseen durch das Gesundheitssystem sowie viele unnötige aufwendige medizinische Untersuchungen bis hin zu invasiven Eingriffen. Andererseits wird auch das Gesundheitssystem durch diese Fehlbehandlungen finanziell stark strapaziert. Es war mir daher ein besonderes Anliegen ein Konzept zu entwickeln, das Patienten unmittelbar in der Primärversorgung erreichen kann, bevor die somatoforme Störung chronifiziert oder voranschreitet und häufig ein Leben lang unverändert den Alltag der Menschen zu einem andauernden Leidenszustand verwandelt. Des Weiteren ist mein Anliegen mit dieser Studie deutlich werden zu lassen, dass die Investition in eine psychosoziale Behandlungsmaßnahme sich langfristig nicht nur für den Leidensweg der Patienten sondern auch für das Gesundheitssystem weitaus ökonomischer gestaltet als die alleinige medizinische Betreuung.

Bergisch Gladbach, Oktober 2010 
1 EINLEITUNG

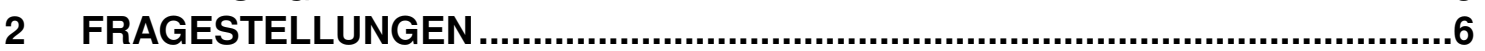

3 STAND DER FORSCHUNG ................................................................

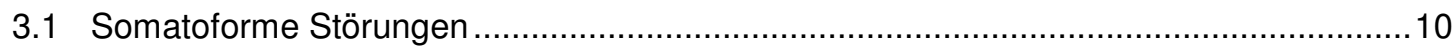

3.2 Der Prozess der depressiven Somatisierung und das narzisstisch-altruistische Bewältigungsverhalten ...................................................................................23

3.3 Prävalenz in der Hausarztpraxis und Ökonomieaspekte für das Gesundheitssystem ....43

3.4 Die Arzt-Patient-Beziehung und die Notwendigkeit interdisziplinärer Kooperation .........47

3.5 Ergebnisse deutschsprachiger Studien in der psychosozialen Gruppenarbeit mit Somatisierungspatienten .........................................................................................

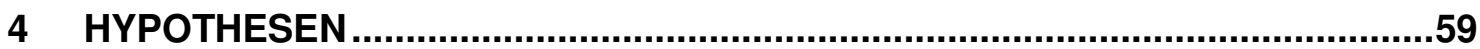

5 UNTERSUCHUNGSMETHODIK UND UNTERSUCHUNGSVERLAUF................79

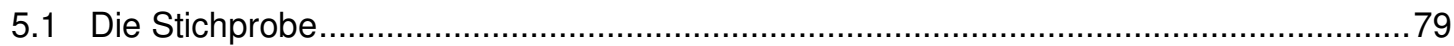

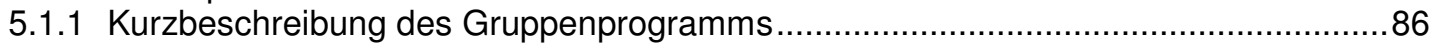

5.1.2 Das Untersuchungsdesign ............................................................................ 90

5.2 Screening für somatoforme Störungen (SOMS), Gesundheitsfragebogen für Patienten (PHQ-D), Whiteley-Index (WI7) und eine Beschwerdeskala ………………………......91

5.3 Skalen zum Erleben von Emotionen (SEE) und eine Emotionswahrnehmungsskala......99

5.4 Veränderungsfragebogen des Erlebens und Verhaltens (VEV) ...................................103

5.5 Arzt-Patient-Fragen und Psychotherapiebereitschaft...................................................107

5.6 Frage zu einem narzisstischen Verhaltensmuster ....................................................109

5.7 Evaluationen des Resonanzerlebens sowie des Beschwerdeerlebens .........................110

5.8 Statistische Untersuchungsverfahren ……………………………………………....112

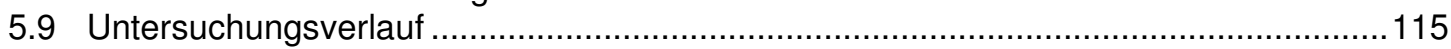

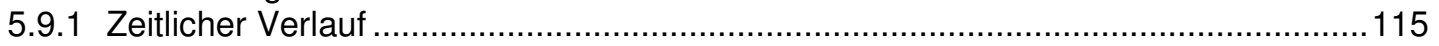

5.9.2 Entwicklung des Studienkonzepts nach Abschluss des Programms ........................121

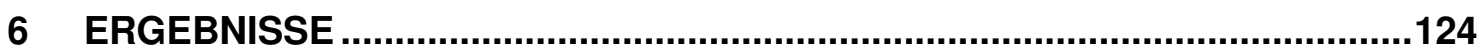

6.1 Vortestunterschiede der Versuchs- und Kontrollgruppe ..........................................125

6.2 Entwicklung der Symptomatik .........................................................................129

6.3 Akzeptanz, Mangel und körperbezogene Symbolisierung der Emotionen .....................137

6.4 Veränderungsfragen des Erlebens und Verhaltens ................................................... 142

6.5 Erlebte Resonanz und ihre Korrelation zu der erlebten Stärke der Beschwerden ..........145

6.6 Arzt-Patienten-Angaben und Psychotherapiemotivation .............................................148

6.7 Narzisstisches Verhalten in Altag und Gruppe ……………..................................155

6.8 Nicht hypothesengebundene Ergebnisse ...........................................................157

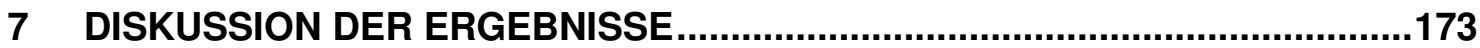

7.1 Zusammenfassung der Ergebnisse und Schlussfolgerungen ...................................208

7.2 Eigene Stellungnahme zum Promotionsprojekt: Persönliche Erfahrungen und Entwicklungsprozesse.

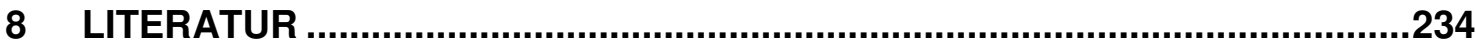

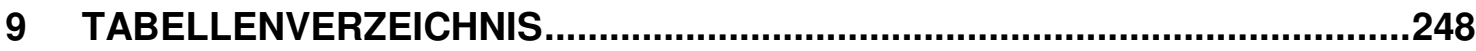

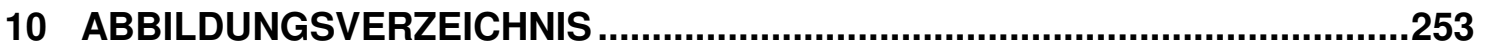

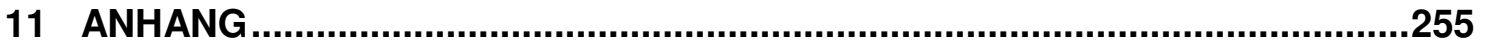





\section{$1 \quad$ Einleitung}

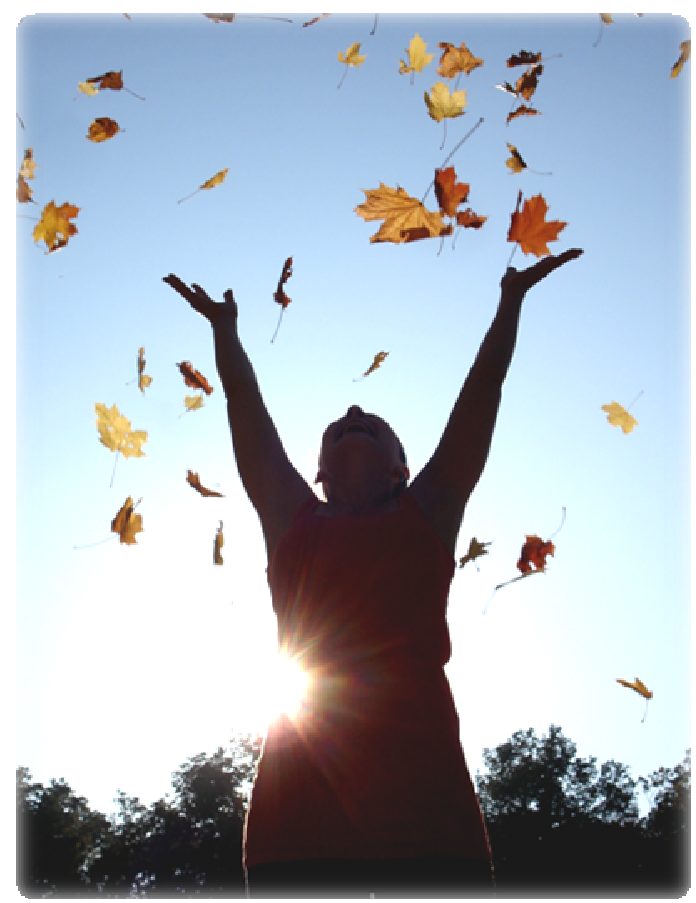

\section{„Der Körper ist der Übersetzer der Seele ins Sichtbare.“ (Christian Morgenstern 1871-1914)}

Somatoforme Störungen beziehungsweise medizinisch unklare Körperbeschwerden sind für das Gesundheitssystem, sowohl wegen ihrer hohen Prävalenz als auch wegen des intensiven Inanspruchnahmeverhaltens medizinisch kostspieliger Leistungen, von großer Bedeutung.

Die somatoformen Störungen gehören zu den psychischen Störungsbildern, bei denen Patienten ihren Hausarzt vorrangig als erste Anlaufstelle konsultieren. Noch heute besteht von Seiten der behandelnden Ärzte und Psychologen eine reservierte Haltung gegenüber dieser Patientengruppe (Nanke \& Rief, 2003).

Dies beruht vor allem darauf, dass diese Patienten nur schwer zu einer psychotherapeutischen Behandlung zu motivieren sind, da die Betroffenen befürchten, als Simulanten oder eingebildete Kranke stigmatisiert zu werden (Hiller \& Rief, 1998).

Der schwierige Umgang mit dieser Klientel mag ein Grund dafür sein, dass den somatoformen Störungen sowohl von klinischer als auch von wissenschaftlicher Seite bisher eher geringes Interesse entgegengebracht wurde und daher verhältnismäßig wenige Forschungsarbeiten zum Gesamtspektrum dieser Krankheit vorliegen. 
Im Bereich der psychosozialen gruppenspezifischen Interventionen, die nicht in stationärem Setting, sondern in direkter Kooperation mit den behandelnden Hausärzten stattfinden, existieren im deutschsprachigen Raum bisher keine veröffentlichten Studien.

Das Forschungsinteresse dieser Arbeit setzt genau an diesem bisher vernachlässigten Bereich der Kooperation an. Sowohl, um den Patienten lange, quälende, Reisen durch Krankenhäuser und verschiedene Facharztpraxen zu ersparen und eine direkte Hilfsmöglichkeit anzubieten als auch dafür, die Ökonomie des Gesundheitssystems zu unterstützen. Dafür soll das in dieser Arbeit vorgestellte psychosoziale Gruppenkonzept, in direkter Kooperation mit den behandelnden Hausärzten als eine neue Möglichkeit für den Umgang mit Somatisierungspatienten vorgestellt werden.

Um auch einen präventiven Beitrag zur Vermeidung von Chronifizierungen somatoformer Störungen zu leisten werden nicht nur langjährig betroffene Patienten, sondern auch Personen, die (noch) weniger massive Symptome aufweisen und am möglichen Anfang einer Somatisierungsstörung stehen in die Studie einbezogen.

Da die somatoformen Störungen als Störungsklasse in der ICD-10 erst seit 1993 bestehen (Dilling et al., 1994) und die Kriterien sich von DSM-II-R zu DSM-IV nochmals verändert haben, ist der Forschungsstand für erfolgreiche gruppenspezifische psychosoziale Interventionskonzepte für diese Patientengruppe in Deutschland noch sehr gering.

Diesem Stand der Forschung folgt die in dieser Arbeit dargestellte Studie. Es wurde ein gruppenorientiertes Kurzzeitinterventionsprogramm von acht Sitzungen konzipiert und an 20 somatoformen Patienten mit einer Pretest-, Posttest-, und Follow Up-Messung getestet. Als Kontrollgruppe dienten 20 Patienten mit einer somatoformen Störung, die nicht an dem Gruppenprogramm teilnahmen und nur von ihrem Hausarzt alleine behandelt wurden.

Das Gruppenkonzept orientiert sich an einer psychodynamischen Theorie für die Entstehung somatoformer Störungen nach Gerd Rudolf. Rudolf beschreibt die Entwicklung eines depressiven Grundkonflikts und stützt sich dabei auf die strukturbezogene Psychotherapie. Diese Form des psychodynamischen Ansatzes beschäftigt sich vor allem mit der Arbeit an defizitär entwickelten strukturellen Dimensionen einer Persönlichkeit.

Für eine Kurzzeitintervention liegt der besondere Fokus auf der Förderung defizitär entwickelter struktureller Dimensionen, die sich bei somatoformen Störungen als relevant erwiesen haben. 
Das Programm beachtet in der Konzeption aber auch den zugrundeliegenden depressiven Grundkonflikt und beinhaltet zudem Ressourcen- und Lösungsorientierung.

Bevor der Stand der Forschung mit relevanten bisher bekannten Ergebnissen und bedeutsamen Aspekten zur Studienthematik erläutert wird, werden die diese Arbeit betreffenden Fragestellungen benannt.

Anschließend werden die Forschungshypothesen sowie die dazu ausgewählten Untersuchungsinstrumente dargestellt, bevor eine Präsentation der Ergebnisse und deren Diskussion und Interpretation erfolgt. 


\section{$2 \quad$ Fragestellungen}

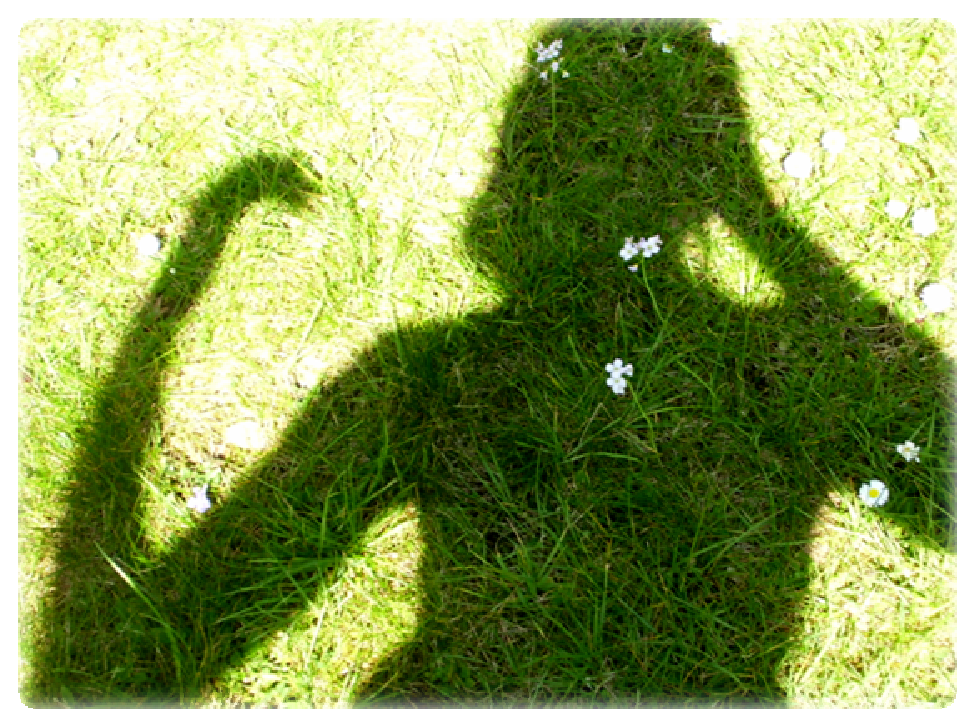

Wohin „die Reise“ führen soll....

Patienten mit somatoformen Störungen wurde in der Forschungslandschaft bisher nur wenig Aufmerksamkeit gewidmet. Die Betroffenen stehen unter einem massiven Leidensdruck und die behandelnden Ärzte, nach abgeschlossener organischer Diagnostik, häufig selbst vor der Hilflosigkeit.

Zwar existieren einzelne wenige Untersuchungen zu gruppentherapeutischen Maßnahmen bei chronifizierten somatoformen Patienten (Nickel \& Egle, 1999, Rief et al., 2002, Martin et al., 2007, Weber \& Tschuschke, 2007), jedoch bisher keine Studien zu einer direkten Kooperation mit behandelnden Hausärzten. Das bedeutet, es fehlen ambulante, niedrigschwellige Maßnahmen, die Patienten angeboten werden können und sie möglicherweise erreichen, bevor sie Jahre lang, ohne symptomatische Verbesserung, das gesamte Gesundheitssystem duchwandert haben.

Es interessiert daher sehr, ob Patienten mit einer somatoformen Störung, auf die Empfehlung ihres Hausarztes hin, und unter parallel weiter bestehender medizinischer Betreuung seinerseits, von der Teilnahme an einem niedrigschwelligen psychosozialen Kurzgruppeninterventionsprogramm, bezogen auf ihre somatoforme Symptomatik, profitieren können. 
Aber nicht nur die körperliche Symptomatik und deren Verbesserung sind bedeutsam. Für Menschen mit somatoformen Störungen gilt als relevant, dass sie körperliche Beschwerden nicht mehr oder nur noch wenig mit ihrem affektiven Erleben in Zusammenhang bringen können (Rudolf, 2006a). Daher stellt sich die Frage, ob oder inwiefern Betroffene diesen Zusammenhang erleben und ob sich diesbezüglich eine Veränderung nach der Teilnahme am Gruppenprogramm ergibt.

Das gesamte Erleben und Wahrnehmen eigener Emotionen, stellt sich als relevant für die Verbesserung und Heilung dieses Störungsbildes dar (Rudolf, 2006b).

Aus speziell heilpädagogischer Sicht interessiert zudem ganz besonders auch das Erleben und Verhalten der Betroffenen in ihrem Alltag. Die physiologischen Beschwerden haben einen großen negativen Einfluss auf den gesamten Alltag und dessen Bewältigung. Vor allem stellt sich die Frage, ob eine zunehmende Hoffnungslosigkeit, Anspannung und ein grundlegend negativ geprägtes Denken und Erleben den Teufelskreis körperlicher Symptome zusätzlich verstärkt. Es soll herausgefunden werden, ob die Probanden nach der Teilnahme am konzipierten Gruppenprogramm ihr Erleben und Verhalten im Alltag positiver, gelassener und optimistischer einschätzen als vor der Teilnahme.

Es existiert aktuell zudem ein großes Beklagen über ständig wachsende Kosten in unserem Gesundheitssystem, die u. a. auch massiv durch dieses Patientenklientel mit verursacht werden sollen (Nanke \& Rief, 2003). Dies betrifft ursächlich vor allem überdurchschnittlich häufige Arztkontakte und viele unnötige medizinische Untersuchungen.

Außerdem soll evaluiert werden, ob die Teilnahme an einem kooperativen psychosozialen Angebot möglicherweise in einen Zusammenhang mit der Reduktion von unnnötigen Arztkontakten und massiven medizinischen Untersuchungsforderungen gebracht werden kann.

Ein weiteres Interesse richtet sich auf die psychodynamische Theorie zur Entstehung somatoformer Störungen nach Gerd Rudolf. Innerhalb des Prozesses der depressiven Somatisierung beschreibt er anschaulich, was Betroffenen in der frühen Entwicklung fehlte und welche Verhaltens- und vor allem Beziehungsmuster, sich bis ins Erwachsenenalter entwickelt und verfestigt haben. 
Durch stetig fehlende Resonanz auf seelisches Klagen verlieren Betroffene die positive Erfahrung in ihren Bedürfnissen wirklich gesehen und beantwortet zu werden. Als Form der Bewältigung dieser unbefriedigten Bedürfnisse entwickeln sie ein narzsisstischalturistisch geprägtes Verhalten. Bricht dieses Muster aus diversen Gründen zusammen, kommt es nach Rudolf zum Symptomausbruch (Rudolf, 2007c).

Es wird daher erhoben, ob die erfahrene Resonanz in einer psychosozialen Gruppe, in der mehr Raum um zu klagen angeboten wird als die Menschen dies bisher in ihrem Alltag erfahren konnten, einen Zusammenhang aufweisen kann zur erlebten Stärke der Beschwerden. Außerdem ist fraglich, ob das narzisstisch geprägte Verhalten, durch den geschützten Gruppenraum, ggf. in der Gruppe weniger stark zum eigenen Schutz benötigt beziehungsweise gezeigt wird als außerhalb.

Es wird zudem fortwährend davon berichtet, dass Patienten mit einem Somatisierungssyndrom nur sehr schwer zu einer psychosozialen Hilfsmaßnahme zu motivieren sind, weil sie sich ausschließlich auf das somatische Ursachenmodell fixieren (Henningsen et al., 2002). Es soll überprüft werden, ob dies der Wahrheit entspricht oder ob möglicherweise auch andere Faktoren, wie die negative Arzt-Patient-Beziehung oder ein zu geringer Einsatz für den Patienten den Zugang zu dieser Hilfsmöglichkeit versperren.

Die nun folgenden Forschungsfragestellungen sowie die in Kapitel 4 aufgeführten Hypothesen beziehen eine parallel mitlaufende Kontrollgruppe somatoformer Patienten als Vergleich in allen Variablen ein. 
Im Einzelnen lauten die Fragestellungen, die dieser Forschungsarbeit zugrunde gelegt werden:

1. Zeigen Patienten der Versuchsgruppe im Posttest und Follow Up signifikant bessere Ergebnisse in ihren Kontrollvariablen (Erlebte Beschwerdeintensität/Beeinträchtigungsempfinden/Krankheitsängste/Erleben und Verhalten/Emotionserleben/Anzahl der Arztwechsel/Beziehungsqualität zum behandelnden Hausarzt) als im Pretest?

2. Zeigen die behandelnden Hausärzte der Patienten der Versuchsgruppe im Posttest und Follow Up eine signifikant besser eingeschätzte Beziehungsqualität zu ihren Patienten als im Pretest?

3. Zeigen Patienten der Versuchsgruppe im Posttest und Follow Up eine signifikant geringere Anzahl an Arztkonsultationen als im Pretest?

4. Zeigen Patienten der Versuchsgruppe im Posttest und Follow Up ein signifikant geringeres Ausmaß an Forderungen nach weiteren medizinischen Untersuchungen als im Pretest?

5. Besteht ein signifikant negativer Zusammenhang zwischen den selbst eingeschätzten Resonanzerfahrungen der Patienten der Versuchsgruppe und der subjektiv erlebten Stärke der körperlichen Beschwerden während den Gruppenstunden?

6. Erleben Patienten der Versuchsgruppe ihre Beschwerden während den Gruppensitzungen signifikant weniger stark als im Verlauf einer Woche in ihrem Alltagsleben?

7. Zeigt sich bei Patienten der Versuchsgruppe ein signifikanter Unterschied zwischen dem narzisstisch geprägten (Bewältgungs)-Verhalten im Altag und dem narzisstisch geprägten (Bewältigungs)-Verhalten in den Gruppenstunden?

8. Zeigen Patienten der Versuchsgruppe im Posttest und Follow Up eine signifikant höhere Bereitschaft zu einer Psychotherapie als im Pretest? 


\section{$3 \quad$ Stand der Forschung}

\subsection{Somatoforme Störungen}

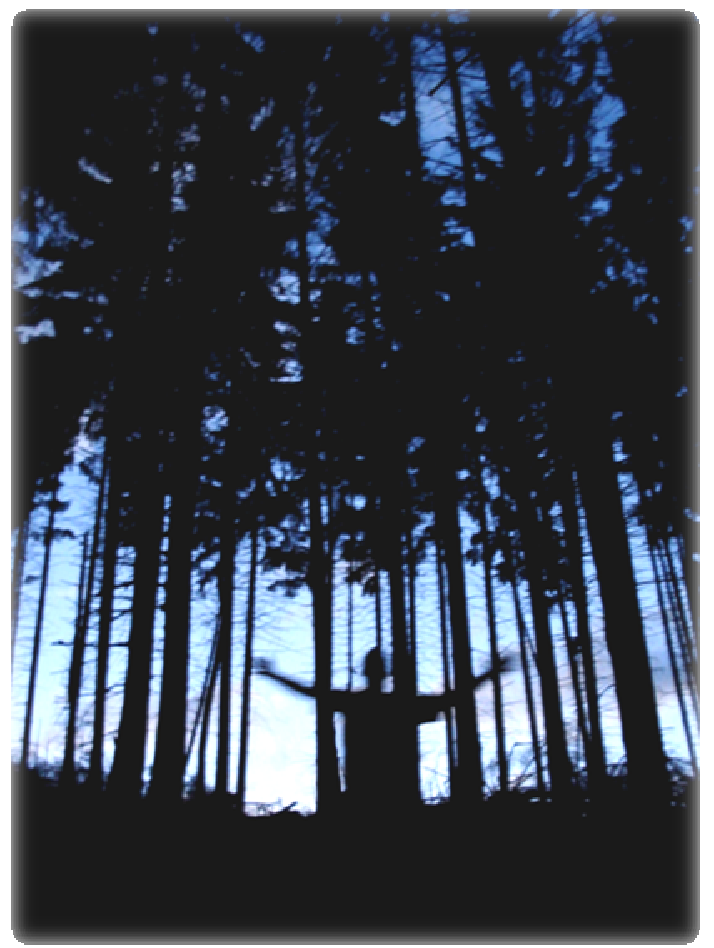

Worum es in dieser Arbeit eigentlich geht...

Somatoforme Störungen verweisen seit der Einführung von DSM-III (Diagnostik and Statistical Manual, 3. Auflage) im Jahre 1980 auf eine Klasse von psychiatrischen Störungen, deren wesentliche Charakteristika körperliche Symptome darstellen. Diese haben den Anschein, eine somatische Krankheit anzuzeigen, ohne, dass hierfür jedoch ausreichende organische Ursachen oder bekannte pathophysiologische Mechanismen vorliegen (Kapfhammer, 2001a).

Die Somatisierung als Oberbegriff für die körperliche, nicht auf organische Ursachen zurückzuführende Symptomatik ist eines der häufigsten Phänomene im psychischen und psychosomatischen Kontext. 
Je nach fachlichem Schwerpunkt einer Arztpraxis, des Krankenhauses oder anderer Einrichtungen des psychosozialen Gesundheitssystems wird davon ausgegangen, dass zwischen $15 \%$ und $50 \%$ der hilfesuchenden Personen unter einem Somatisierungssyndrom leiden (Rief \& Hiller, 1992).

Eindeutige somatische Erklärungen für körperliche Beschwerden sind eher die Ausnahme als die Regel (Rudolf, 2008).

Es besteht die Annahme, dass psychologische Einflüsse oder Konflikte und psychosoziale Stressoren in der Symptombildung bedeutungsvoll sind. Im Unterschied zu vorgetäuschten (artifiziellen) Störungen oder einer Simulation liegt bei den betroffenen Patienten keine willentliche Kontrolle über die körperliche Symptomatik vor.

Somatisierungssymptome treten meist nicht als singuläres Phänomen auf, sondern kombiniert oder in zeitlicher Anknüpfung mit weiteren körperlichen Symptomen. Aus diesem Grund wurde in früheren Arbeiten der Begriff multiples somatoformes Syndrom vorgeschlagen (Rief, 1995).

Das Konzept der psychosomatischen Krankheiten steht den somatoformen Störungen nahe, ebenso wie weiterhin ein Zusammenhang mit den historischen Konzepten der Hysterie, Hypochondrie, Konversion und Dissoziation besteht (Rief, 1998).

Bereits in der Antike beschrieb der Begriff der Hysterie ein Krankheitsbild, das auf Frauen beschränkt war und durch einen wandernden Uterus im Unterleib entstehen sollte, vor allem bei Frauen mit unerfülltem Kinderwunsch. Paul Briquet widmete sich im 19. Jahrhundert diesem Krankheitsbild und untersuchte über 400 Patienten, die an multiplen körperlichen Beschwerden ohne organische Erklärung litten. Schon in Briquets Standardwerk 1859 wurden erste Vorschläge für eine deskriptive Diagnostik vorgenommen, wie sie dann 1980 durch das DSM-III erstmals in einem offiziellen Diagnosesystem realisiert wurden (Rief \& Hiller, 2001).

In seinem Buch schrieb Briquet auch über belastende Lebensereignisse und erkannte eine Häufigkeit dieser Störung im Zusammenhang mit sozial schwachen Schichten. Heute haben sich diese Faktoren als Risikofaktoren zur Entstehung somatoformer Störungen bestätigt (Rief \& Hiller, 1992).

Erst in den 1960er Jahren wurde durch eine amerikanische Arbeitsgruppe von Samuel Guze et. al das Konzept der Briquet-Störung erneut aufgenommen. 
Dies führte dazu, dass im amerikanischen Diagnosesystem DSM-III 1980 die „Somatisierungsstörung" aufgenommen wurde, die eine Weiterentwicklung des BriquetSyndroms darstellt (Rief \& Hiller, 2001).

In Europa, und damit auch in Deutschland, wurde erst 13 Jahre nach der Einführung im DSM-III ein ebenfalls deskriptiv-operationalisierter Klassifikationsansatz dieser Störung gewählt und in die ICD-10 (International Statistical Classifikation of Diseases) eingeführt (Dilling et al., 1994).

Wenngleich die Logik der Klassifikation in beiden Systemen ähnlich ist, so ergeben sich im Detail doch Unterschiede, die zu gravierenden Diskrepanzen führen können. Beide Systeme verwenden Symptomlisten, allerdings zum Teil mit unterschiedlichen Kriterien und Symptomen. Im DSM-IV ist, gegenüber der ICD-10, beispielsweise ein Höchstalter von 30 Jahren bei Auftreten der ersten Symptome gefordert.

Einen weiteren Unterschied in der Klassifikation der Somatisierungsstörung nach den Klassifikationssystemen ICD-10 und DSM-IV stellt die Einführung der somatoformen autonomen Funktionsstörung in der ICD-10 dar. Die Kriterien einer SAD (somatoformen autonomen Funktionsstörung) sind in Tabelle 4 aufgelistet.

Die vollen Kriterien einer Somatisierungsstörung erfüllen bei epidemiologischen Studien nur wenige Personen. Wittchen et al. beschreibt, dass maximal $0,2 \%$ bis $2 \%$ aller Frauen an diesem Störungsbild leiden, bei Männern ist der Prozentsatz noch geringer (Wittchen et al, 1989).

Weder die Veränderungen von DSM-III-R nach DSM-IV noch der Ansatz in der ICD-10 konnten zu einer Erhöhung des Auftretens einer Somatisierungsstörung führen (Rief et al, 1996).

Eine solch niedrige Prävalenzrate steht jedoch in krassem Gegensatz zur derzeitigen gesundheitspolitischen Relevanz des Problems mit dieser Klientel.

Aus diesem Grund war eine Lockerung der Diagnosekriterien der Somatisierungsstörung wichtig. Dafür wurde in die Klassifikationssysteme die Untergruppe undifferenzierte somatoforme Störung eingeführt. Werden nicht alle Kriterien einer vollen Somatisierungsstörung erfüllt, reicht für die abgeschwächte Form bereits ein körperliches Symptom aus der Liste der Symptome für eine Diagnose aus, wobei auch andere Beschwerden möglich sind. (z. B: chronische Müdigkeit oder Erschöpfung, Appetitverlust oder andere). 
Die Mindestdauer der Beschwerden wird hier auf einen Zeitraum von sechs Monaten festgelegt.

Die Beschwerden dürfen nicht ausschließlich Symptome einer affektiven Störung, einer Angststörung, einer sexuellen Störung, einer Schlafstörung oder einer psychotischen Störung sein (Rief \& Hiller, 2001).

Es ist diesbezüglich festzustellen, dass es über die somatoformen Störungen im engeren Sinne hinaus keine psychischen Störungen ohne erhebliche Körperbeteiligung gibt. Man denke dabei beispielsweise an eine Depression mit ihrer oft körperlichen Erschöpfung und häufigen Schlafstörungen oder an eine Angststörung, bei der während einer Panikattacke Atemnot, Zittern und Schwitzen erlebt wird und die Essstörung mit ihrer Deformierung des Körpers sowie diverse Persönlichkeitsstörungen, bei der Betroffene sich häufig körperlich selbst verletzen (Rudolf, 2008).

Schmerzen (Kopf-, Rücken-, Brust- und Gliederschmerzen) sind weltweit die häufigsten somatoformen Körperbeschwerden, gefolgt von funktionellen Beschwerden wie Übelkeit, Herzrasen, Schwindel, Blähungen (Isaac et al., 1995, Rief et al., 2001).

Daher erhalten auch Schmerzsymptome sowohl in der ICD-10 als auch im DSM-IV eine eigene somatoforme Diagnosekategorie.

Das DSM-IV unterscheidet zwischen Schmerzstörungen in Verbindung mit psychischen Faktoren und Schmerzstörungen in Verbindung mit sowohl medizinischen als auch psychischen Krankheitsfaktoren.

In der ICD-10 findet sich die anhaltende somatoforme Schmerzstörung, die, abgegrenzt von den anderen somatoformen Störungen, nicht im Rahmen einer Somatisierungsstörung auftreten, andererseits aber wiederum auch seit sechs Monaten bestehen muss.

Vor allem wenn pseudoneurologische Symptome auftreten, wäre des Weiteren an eine Konversionsstörung zu denken. Diese manifestiert sich allerdings meist relativ akut und kurz, während eine somatoforme Störung eine sehr viel stärkere Tendenz zur Chronizität aufweist. Angesichts des häufig chronischen Verlaufs stellt sich zusätzlich die Frage, ob es sich nach dem DSM-IV bei der Somatisierungsstörung oder der Hypochondrie weniger um eine Achse-I Diagnose als vielmehr um eine Persönlichkeitsstörung, also eine Achse-II Diagnose handelt (Tyrer et al., 1990, Bass et al., 1995). 
Nach dem DSM-IV wird schließlich noch die körperdysmorphe Störung klassifiziert, über die jedoch bisher wenig bekannt ist. Hierbei hält ein Mensch einen bestimmten Körperteil von sich für missgestaltet und muss ständig an dieses denken.

In der ICD-10 wird diese Form der Störung als Untergruppe unter dem Begriff der Hypochondrie aufgeführt.

In beiden Diagnosesystemen wird die Hypochondrie unter den somatoformen Störungen aufgeführt.

Es wird und wurde immer hinterfragt, ob diese Zuteilung sinnvoll ist, da Personen, die unter Hypochondrie leiden, in erster Linie Ängste (Gesundheitsängste) und weniger körperliche Beschwerden zeigen.

Daher kommt es vor, dass hypochondrische Patienten keinerlei körperliche Beschwerden, aber z. B. große Angst zeigen mit einer ansteckenden Krankheit infiziert zu sein (Rief \& Hiller, 2001).

\section{Komorbidität}

Somatoforme Störungen weisen jedoch in der Tat eine hohe Komorbidität zu anderen psychischen Störungen auf. Die Komorbidität zwischen depressiven und Angststörungen ist konsistent hoch. Zwischen der Somatisierungsstörung und einer depressiven Störung wird von einer Komorbiditätsrate von $75 \%$ bis $90 \%$, mit einer Panikstörung von $10 \%$ bis $50 \%$, mit phobischen Störungen von $17 \%$ bis $70 \%$ berichtet (Ebel \& Podoll, 1998).

Neben der hohen Verbindung somatoformer mit Angst- und depressiven Störungen ist vor allem die Komorbidität mit Persönlichkeitsstörungen beachtenswert. Es werden Raten zwischen einer Somatisierungsstörung und mindestens einer Persönlichkeitsstörung von $28 \%$ bis $80 \%$ angegeben, wobei sich frühere Annahmen zur besonderen Häufung antisozialer oder histrionischer Persönlichkeitsstörungen nicht bestätigen konnten (Ebel \& Podoll, 1998).

Jenseits der Bezeichnungen der ICD-Diagnosen, finden sich außerdem zahlreiche alternative Bezeichnungen für psychosomatische Störungen in allen medizinischen Disziplinen (z. B. Fibromyalgie, orofazialer Schmerz, Weichteilrheumatismus, Pelvipathie, funktionelles Oberbauchsyndrom etc.), die zum Teil zu Verwirrung und uneinheitlicher Kommunikation unter den unterschiedlichen Helfern führen können (Rudolf, 2008.) 
In Deutschland sollte daher eine einheitliche Orientierung an den Kriterien der ICD-10 stattfinden.

\section{Somatoforme Störungen: Definition und Klassifikation nach ICD-10}

Nicht nur das Vorhandensein somatoformer Körperbeschwerden gehört zu der allgemeinen Charakterisierung somatoformer Störungen, die der diagnostischen Gruppe F45 in den klinisch-diagnostischen Leitlinien der ICD-10 vorangestellt sind.

Folgende fünf Punkte sind Bestanteil der Definition (Henningsen et al, 2002):

- wiederholte Darbietung körperlicher Symptome

- hartnäckige Forderung nach medizinischen Untersuchungen trotz wiederholter negativer Ergebnisse und Versicherung durch die Ärzte, dass die vorhandenen Symptome nicht körperlich begründbar sind

- Der Patient widersetzt sich den Versuchen, die Möglichkeit einer psychischen Ursache zu diskutieren. Der Patient ist von einer körperlichen Ursache überzeugt.

- Das zu erreichende Verständnis für die Verursachung der Symptome ist häufig für Patienten und Arzt enttäuschend. Die Schwierigkeiten des Patienten zu akzeptieren, dass keine ausreichende organmedizinische Ursache für die Beschwerden vorliegt, führt häufig zu einer Belastung in der Arzt-PatientBeziehung. Oft wechselt der Patient dann enttäuscht den Arzt, der nicht der erhoffte Helfer sein konnte.

- Die Beschwerden werden deutlich von körperlich ausreichend begründbaren Krankheiten abgegrenzt. 
Auch wenn in Zukunft damit zu rechnen ist, dass weitere Änderungen in der Klassifikation der somatoformen Störungen in der ICD-10 durchgeführt werden, ist für das Verständnis dieses Störungsbildes innerhalb dieser Studie die derzeitig gültige Version der ICD-10 maßgeblich (Dilling et al., 2010).

Als Ausschlussdiagnosen bei somatoformen Störungen gelten: (Dilling et al., 2010)

- Ausreißen der Haare (F98.4)

- Daumenlutschen (F98.8)

- Dissoziative Störungen (F44)

- Haarezupfen (F98.4, stereotype Bewegungsstörungen)

- Lallen (F80.0)

- Lispeln (F80.8)

- Nägelkauen (F98.8)

- Psychologische Faktoren oder Verhaltensfaktoren bei andernorts klassifizierten Krankheiten (F54)

- Sexuelle Funktionsstörungen, nicht organisch bedingt (F52)

- Ticstörungen im Kindes- und Jugendalter (F95)

- Tourette-Syndrom (F95.2)

- Trichotillomanie (F63.3)

- Simulation (Z76.5)

- Auf die körperlichen Funktionen oder Körperform fixierte Wahnphänomene (F22.0)

- Wahnhafte Dysmorphophobie (F22.8) 
Die nachfolgenden Tabellen 1-7 geben in übersichtlicher Form Auskunft über die somatoformen Störungen nach der ICD-10. Außerdem werden verwandte Störungen, die in der psychosomatischen Praxis ebenfalls bedeutsam sind und in der ICD-10 nicht explizit unter die somatoformen Störungen fallen, aufgeführt.

Aufgrund der Schwierigkeit kooperierende Hausärzte sowie bereitwillige Probanden zu gewinnen, konnte sich in dieser Studie nicht nur auf eine der folgenden somatoformen Störungen bezogen werden. Teilnehmen konnten alle Patienten, die an einer der in Tabellen 1-6 aufgeführten somatoformen Störungen litten. 
Tabelle 1: Diagnostische Kriterien der Somatisierungsstörung nach ICD-10 (Dilling et.al., 2010)

\section{Somatisierungsstörung (F.45)}

A. Eine Vorgeschichte von mindestens zwei Jahre anhaltenden Klagen über multiple und wechselnde körperliche Symptome, die durch keine diagnostizierbare körperliche Erkrankung erklärt werden können. Die eventuell vorliegende bekannte körperliche Erkrankung erklärt nicht die Schwere, das Ausmaß, die Vielfalt und die Dauer der körperlichen Beschwerden oder die damit verbundene soziale Behinderung. Wenn einige vegetative Symptome vorliegen, bilden sie nicht das Hauptmerkmal der Störung, d. h. sie sind nicht besonders anhaltend oder belastend.

B. Die ständige Sorge um die Symptome führt zu andauerndem Leiden und dazu, dass die Patienten mehrfach (drei- oder mehrmals) um Konsultationen oder

Zusatzuntersuchungen in der Primärversorgung oder beim Spezialisten nachsuchen. Wenn aus finanziellen oder geographischen Gründen medizinische Einrichtungen nicht erreichbar sind, kommt es zu andauernder Selbstmedikation oder mehrfachen Konsultationen bei örtlichen Laienheilern.

C. Hartnäckige Weigerung, die medizinische Feststellung zu akzeptieren, dass keine ausreichende körperliche Ursache für die körperlichen Symptome vorliegt. Akzeptanz der ärztlichen Mitteilung allenfalls für kurze Zeiträume bis zu einigen Wochen oder unmittelbar nach einer medizinischen Untersuchung.

D. Insgesamt sechs oder mehr Symptome aus der folgenden Liste, mit Symptomen aus mindestens zwei verschiedenen Gruppen.

\section{Gastrointestinale Beschwerden:}

(1) Bauchschmerzen

(2) Übelkeit

(3) Gefühl von Überblähung

(4) Schlechter Geschmack im Mund oder extrem belegte Zunge

(5) Klagen über Erbrechen oder Regurgitation von Speisen

(6) Klagen über häufigen Durchfall oder Austreten von Flüssigkeit aus dem Anus

\section{Kardiovaskuläre Symptome:}

(7) Atemlosigkeit ohne Anstrengung

(8) Brustschmerzen

\section{Urogenitale Symptome:}

(9) Dysurie oder Klagen über Miktionshäufigkeit

(10) Unangenehme Empfindungen in oder um den Genitalbereich

(11) Klagen über ungewöhnlichen oder verstärkten vaginalen Ausfluß

Haut - und Schmerzsymptome:

(12) Klagen über Fleckigkeit oder Farbveränderungen der Haut

(13) Schmerzen in den Gliedern, Extremitäten oder Gelenken

(14) Unangenehme Taubheit oder Kribbelgefühl

E. Häufigstes Ausschlusskriterium: Die Störung tritt nicht ausschließlich während einer Schizophrenie oder einer verwandten Störung (F2), einer affektiven Störung (F3) oder einer Panikstörung (F41.0) auf. 
Tabelle 2: Diagnostische Kriterien der undifferenzierten Somatisierungsstörung (UDS) nach ICD-10 (Henningsen et al., 2002, Dilling et al., 2010)

\section{Undifferenzierte Somatisierungsstörung (F45.1)}

A. Mindestens sechs Monate anhaltende multiple und körperliche Symptome, für die keine ausreichende somatische Erklärung gefunden wurde.

B. Die symptomatischen Diagnosekriterien für eine Somatisierungsstörung sind nicht vollständig erfüllt.

$\rightarrow$ Die Entwicklung somatoformer Körperbeschwerden ist als Kontinuum anzusehen: Je mehr somatoforme Symptome vorliegen, desto schwerer ist die Störung. Entsprechend stellt die undifferenzierte Somatisierungsstörung eine mildere Verlaufsform der Somatisierungsstörung mit geringerer Schwere, ansonsten aber gleichen Charakteristika dar (Henningsen et al., 2002).

Tabelle 3: Diagnostische Kriterien der hypochondrischen Störung (HS) nach ICD-10 (Henningsen et al., 2002, Dilling et al., 2010)

\section{Hypochondrische Störung (F45.2)}

A. Eine mindestens sechs Monate anhaltende Überzeugung vom Vorhandensein wenigstens einer ernsthaften, vom Patienten benennbaren körperlichen Krankheit, als Ursache für vorhandene Symptome, auch wenn wiederholte Untersuchungen keine ausreichende körperliche Erklärung erbracht haben.

B. Ständige Weigerung, den Rat und die Versicherung mehrer Ärzte zu akzeptieren, dass den Symptomen keine körperliche Krankheit zugrunde liegt.

C. Als Ausschlusskriterium gilt das Auftreten einer hypochondrischen Störung während einer Schizophrenie oder einer verwandten Störung (F2, insbesondere F22) oder während einer affektiven Störung (F3). 
Tabelle 4: Diagnostische Kriterien der somatoform autonomen Funktionsstörung (SAD) nach ICD-10 (Rief \& Hiller, 2002, Dillling et al., 2010)

\section{Somatoform autonome Funktionsstörung (F45.3)}

A. Symptome der autonomen (vegetativen) Erregung, die von den Patienten einer körperlichen Krankheit in einem oder mehreren der folgenden Systeme oder Organe zugeordnet werden:

1. Herz und kardiovaskuläres System

2. oberer Gastrointestinaltrakt (Oesophagus und Magen)

3. unterer Gastrointestinaltrakt

4. respiratorisches System

5. Urogenitalsystem

B. Zwei oder mehrere der folgenden vegetativen Symptome:

1. Palpitationen

2. Schweißausbrüche (heiß oder kalt)

3. Mundtrockenheit

4. Hitzewallungen oder Erröten

5. Druckgefühl im Epigastrium, Kribbeln oder Unruhe im Bauch

C. Eines oder mehrere der folgenden Symptome:

1. Brustschmerzen oder Druckgefühl in der Herzgegend

2. Dyspnoe oder Hyperventilation

3. außergewöhnliche Ermüdbarkeit bei leichter Anstrengung

4. Aerophagie, Singultus oder brennendes Gefühl im Brustkorb oder im Epogastrium

5. Bericht über häufigen Stuhlgang

6. erhöhte Miktionsfrequenz oder Dysurie

7. Gefühl der Überblähung oder Völlegefühl

D. Kein Nachweis einer Störung von Struktur oder Funktion der Organe oder Systeme, über die die Patienten klagen.

E. Häufiges Ausschlusskriterium: Die Symptome treten nicht ausschließlich im Zusammenhang mit einer phobischen (F40.0-F40.3) oder einer Panikstörung (F41.0) auf. 
Tabelle 5: Diagnostische Kriterien der anhaltenden somatoformen Schmerzstörung (ASS) nach ICD-10 (Henningsen et al.,2002, Dilling et al., 2010)

\section{Anhaltende somatoforme Schmerzstörung (F45.4)}

A. Die vorherrschende Beschwerde ist ein andauernder, schwerer und quälender Schmerz, der durch einen physiologischen Prozess oder eine körperliche Störung nicht hinreichend erklärt werden kann. Er tritt in Verbindung mit emotionalen Konflikten oder psychosozialen Belastungen auf, denen die Hauptrolle für Beginn, Schweregrad, Exazerbation oder Aufrechterhaltung der Schmerzen zukommt. Die Folge ist meist eine beträchtlich gesteigerte persönliche oder medizinische Hilfe und Unterstützung.

B. Die Schmerzsymptomatik muss länger als sechs Monate bestehen.

Tabelle 6: Diagnostische Kriterien sonstiger somatoformer Störungen nach ICD-10 (Henningsen et al., 2002, Dilling et al., 2010)

\section{Sonstige somatoforme Störungen (F45.8)}

Hier sollten alle anderen Störungen der Wahrnehmung, der Körperfunktion und des Krankheitsverhaltens klassifiziert werden, die nicht durch das vegetative Nervensystem vermittelt werden, die auf spezifische Teile oder Systeme des Körpers begrenzt sind und mit belastenden Ereignissen oder Problemen eng in Verbindung stehen.

- Psychogener Globus hystericus

- Psychogener Pruritus

- Psychogene Dysmenorrhö

- Psychogenes Zähneknirschen

- Psychogener Tortikollis 
Tabelle 7: Verwandte Störungen in der ICD-10 (Dilling et al., 2010)

Dissoziative Störung der Bewegung und Empfindung bzw. Konversionsstörung (F44.4-7)

Das allgemeine Kennzeichen der dissoziativen oder Konversionsstörungen besteht in teilweisem oder völligem Verlust der normalen Integration der Erinnerung an die Vergangenheit, des Identitätsbewusstseins, der Wahrnehmung unmittelbarer Empfindungen sowie der Kontrolle von Körperbewegungen. Alle dissoziativen Störungen neigen nach einigen Wochen oder Monaten zur Remission, besonders, wenn der Beginn mit einem traumatisierenden Lebensereignis verbunden ist. Eher chronische Störungen, besonders Lähmungen und Gefühlsstörungen, entwickeln sich, wenn der Beginn mit unlösbaren Problemen oder interpersonalen Schwierigkeiten verbunden ist. Diese Störungen wurden früher als verschiedene Formen der "Konversionsneurose oder Hysterie" klassifiziert. Sie werden als ursächlich psychogen angesehen, in enger zeitlicher Verbindung mit traumatisierenden Ereignissen, unlösbaren oder unerträglichen Konflikten oder gestörten Beziehungen. Die Symptome verkörpern häufig das Konzept der betroffenen Person, wie sich eine körperliche Krankheit manifestieren müsste. Körperliche Untersuchung und Befragungen geben keinen Hinweis auf eine bekannte somatische oder neurologische Krankheit. Zusätzlich ist der Funktionsverlust offensichtlich Ausdruck emotionaler Konflikte oder Bedürfnisse. Die Symptome können sich in enger Beziehung zu psychischer Belastung entwickeln und erscheinen oft plötzlich. Nur Störungen der körperlichen Funktionen, die normalerweise unter willentlicher Kontrolle stehen, und Verlust der sinnlichen Wahrnehmung sind hier eingeschlossen. Störungen mit Schmerz und anderen komplexen körperlichen Empfindungen, die durch das vegetative Nervensystem vermittelt werden, sind unter Somatisierungsstörungen ( $\underline{F 45.0}$ ) zu klassifizieren. Die Möglichkeit eines späteren Auftretens ernsthafter körperlicher oder psychiatrischer Störungen muss immer mitbedacht werden.

Inklusive:

- Hysterie

- Hysterische Psychose

- Konversionshysterie

- Konversionsreaktion

Neurasthenie und chronic fatigue syndrome (F48.0)

Im Erscheinungsbild zeigen sich beträchtliche kulturelle Unterschiede. Zwei Hauptformen überschneiden sich beträchtlich. Bei einer Form ist das Hauptcharakteristikum die Klage über vermehrte Müdigkeit nach geistigen Anstrengungen, häufig verbunden mit abnehmender Arbeitsleistung oder Effektivität bei der Bewältigung täglicher Aufgaben. Die geistige Ermüdbarkeit wird typischerweise als unangenehmes Eindringen ablenkender Assoziationen oder Erinnerungen beschrieben, als Konzentrationsschwäche und allgemein ineffektives Denken. Bei der anderen Form liegt das Schwergewicht auf Gefühlen körperlicher Schwäche und Erschöpfung nach nur geringer Anstrengung, begleitet von muskulären und anderen Schmerzen und der Unfähigkeit, sich zu entspannen. Bei beiden Formen finden sich eine ganze Reihe von anderen unangenehmen körperlichen Empfindungen wie Schwindelgefühl, Spannungskopfschmerz und allgemeine Unsicherheit. Sorge über abnehmendes geistiges und körperliches Wohlbefinden, Reizbarkeit, Freudlosigkeit, Depression und Angst sind häufig. Der Schlaf ist oft in der ersten und mittleren Phase gestört, es kann aber auch Hypersomnie im Vordergrund stehen.

Inklusive: Ermüdungssyndrom 


\subsection{Der Prozess der depressiven Somatisierung und das narzisstisch- altruistische Bewältigungsverhalten}

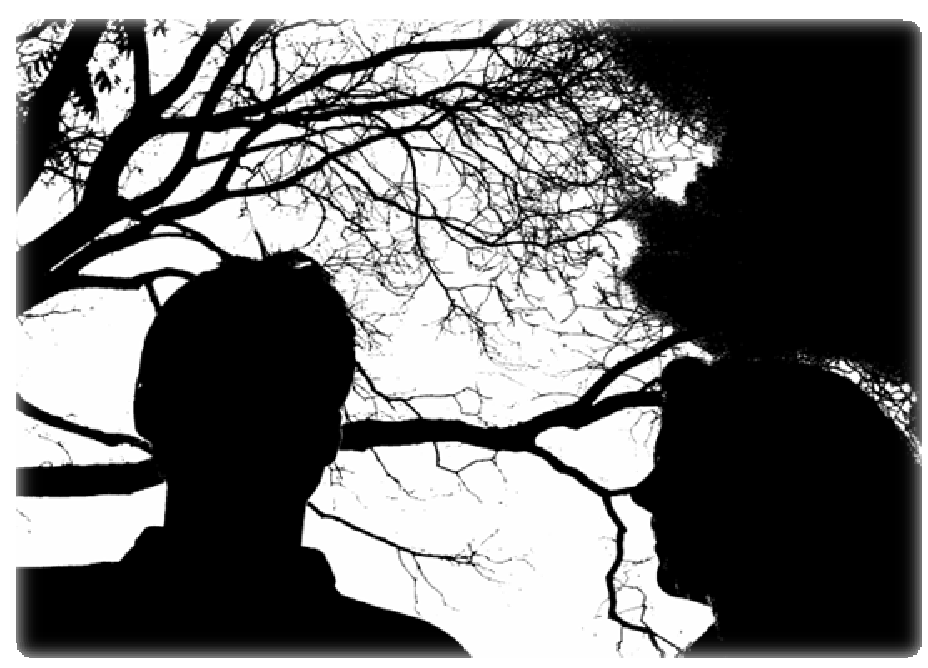

„Das Symptom erlaubt, endlich zu klagen, anzuklagen und zu fordern“ (Rudolf, 2007b).

Die Grundfragen, die sich insgesamt stellen, lauten: Wie kann es geschehen, dass ein Patient überhaupt somatoforme Beschwerden entwickelt, warum erlebt er diese und nicht vorrangig eine andere psychische Störung? Wieso sind die somatischen Ursachenüberzeugungen so wichtig?

Diese und das Thema betreffende Fragen rund um die Entstehung somatoformer Störungen sollen nun behandelt und geklärt werden. 


\section{Der ätiologische Ansatz}

Für die Erklärung zur Entstehung somatoformer Störungen wird in dieser Arbeit ein tiefenpsychologischer Ansatz nach Gerd Rudolf verfolgt. Dieser Ansatz bildet die Basis der Programmkonzeption sowie für einige ausgewählte Forschungshypothesen.

Es ist bereits an dieser Stelle anzumerken, dass es selbstverständlich weitere Ätiologien und Theorien für die Erklärung zur Entstehung somatoformer Störungen gibt. Diese werden in dieser Arbeit jedoch nicht weiter verfolgt. Für ein über diese Studie hinausragendes Interesse an weiteren Theorien und Konzepten, vor allem in der kognitivverhaltenstherapeutisch orientierten Arbeit, seien zur Vertiefung u. a. genannt: Bleichhardt und Weck "Kognitive Verhaltenstherapie bei Hypochondrie und Krankheitsangst“ (2007), Studien von Rief, Bleichhardt und Timmer zur Gruppentherapie für somatoforme Störungen - Behandlungsleitfaden, Akzeptanz und Prozessqualität (2002) sowie „One Session Treatment for patients suffering from medically unexplained Symptoms in primary care: A randomized clinical trail“ von Martin, Rauh, Fichter und Rief (2007).

Um der Begrifflichkeit innerhalb der Arbeit gut folgen zu können und die theoretische Basis einzelner Fragestellungen sowie die Konzeption des Programms zu verstehen, erfolgt eine Darstellung des von Rudolf (2008b) genannten narzisstisch-altruistischen Bewältigungsverhaltens sowie des zugrundeliegenden depressiven Grundkonflikts und dessen Entstehungsprozess.

Gerd Rudolf ist Vertreter der strukturbezogenen Psychotherapie, die er stetig versucht, mehr in die allägliche psychosoziale Praxis zu integrieren und sie vor allem in einem großen Arbeitskreis in eine operationalisierbare psychodynamisch-therapeutische Methode verwandelt. Er ist Mitbegründer der OPD (Operationalisierte Psychodynamische Diagnostik), die sich um eine besondere Objektivität bemüht.

Innerhalb der OPD kann die Stärke einer strukturellen Störung anhand von vier unterschiedlichen Stufen des Strukturniveaus unterschieden werden. Es soll an dieser Stelle nicht näher auf die OPD eingegangen werden. Für tieferes Interesse an der OPD sei jedoch das aktuelle Werk "Operationalisierte Psychodynamische Diagnostik OPD-2. Das Manual zur Diagnostik und Therapieplanung vom Arbeitskreis OPD (Hrsg.) (2009)“ empfohlen. 


\section{Die Arbeit mit strukturbezogener Psychotherapie}

Die strukturbezogene Psychotherapie ist eine Modifikation tiefenpsychologischen und analytischen Vorgehens bei psychischen Störungen, die nicht in erster Linie durch das Wirken unbewusster Konfliktspannung erklärt werden können, sondern bei denen entwicklungsbedingt die Verfügbarkeit über regulative psychische Funktionen eingeschränkt ist (Rudolf, 2006a).

Dabei ist Struktur definiert als die Verfügbarkeit über psychische Funktionen, die für die Organisation des Selbst und seine Beziehungen zu den inneren und äußeren Objekten erforderlich sind.

Rudolf nennt dafür vier Strukturdimensionen beziehungsweise Ebenen, die sich jeweils auf das Selbst und auf andere Objekte, also auf die Beziehung, übertragen lassen. Diese Funktionen werden demnach in den ersten Lebensjahren entwickelt und nachhaltig angelegt. Seelische Struktur meint die Art und Weise, wie wir uns selbst und andere Menschen wahrnehmen, mit uns und innen emotional kommunizieren, unsere Impulse und Gefühle regulieren und sie in Beziehung bringen. Außerdem beinhaltet seelische Struktur unsere Bindungsfähigkeit. Dabei geht es erstrangig um die Frage, ob wir gute Modelle von Beziehungen verinnerlichen konnten und diese zur Selbststeuerung nutzen können und ob wir uns an andere Menschen binden und uns ggf. auch wieder lösen können.

Im Einzelnen benennt Rudolf die vier Ebenen unserer seelischen Struktur wie folgt:

1. Kognitive Ebene (Das Selbst und andere Objekte wahrnehmen können)

2. Regulative Ebene (Das Selbst und den Bezug zu anderen Objekten regulieren zu können)

3. Emotionale Ebene (Eigene emotionale Kommunikation nach innen und die emotionale Kommunikation mit anderen gestalten können)

4. Bindungsebene (An eigene innere Objekte sowie an äußere Objekte gebunden sein zu können).

Besteht eine strukturelle Störung, beinhaltet dies die unzureichende Verfügbarkeit über diese psychischen Funktionen beziehungsweise ihre Vulnerabilität. 


\section{Strukturelle Aspekte bei somatoformen Störungen}

Für Menschen mit somatoformen Störungen haben sich die Bereiche der Affektdifferenzierung bezüglich körperlicher Aspekte von Emotionen und die Mitteilung eigener Affekte in der Kommunikation nach außen als deifizitär erwiesen. Außerdem besteht häufig eine Schwierigkeit in Umgang und Mitteilung von Ärger- und Wutaffekten im Kontakt zu anderen Personen. Fehlende Selbstinternalisierung guter Objekte und eine erschwerte Loslösung von idealisierten Objekten werden als weitere strukturell defizitär entwickelte Bereiche benannt (Rudolf, 2006a).

Durch frühe verfehlte Beziehungen fehlt den Patienten eine Differenzierung von Körperzuständen und affektiven Verfassungen, um eine eigene innerpsychische Vorstellung von den beiden Aspekten eines Selbst, dem körperlichen und dem psychischen, zu erlangen.

So können Betroffene im Erwachsenenalter zwischen den affektiven Aspekten ihres Körpererlebens (z. B. Hunger, Krankheit, Müdigkeit) und den körperlichen Anteilen ihrer affektiv-psychischen Verfassung nicht gut unterscheiden. Sie übersehen, dass der Körper Affekte ausdrückt und halten dann alles, was sich körperlich ereignet für den Ausdruck einer Körperkrankheit.

Aber nicht nur die im Körperlichen spürbaren affektiven Vorgänge können nicht gut wahrgenommen und differenziert werden, sondern auch das Körperselbst als Ganzes ist nur schwer realistisch spürbar. Dies bedeutet, dass sich der Mensch zwar als psychisches und auch als körperliches Ich begreifen kann, aber doch beide Teilaspekte zu einer Identität einmünden sollten. Es besteht eine Tendenz zur Spaltung zwischen psychischem und körperlichem Ich, wobei das psychische Ich den Anspruch erhebt, das körperliche Ich jederzeit kontrollieren und beherrschen zu können und zu wollen.

Die Mitteilung und das in Ausdruck bringen eigener Affekte, z. B. Ärger und Enttäuschung, fällt schwer. Ebenso schwer ist die Mitteilung von weichen Gefühlsregungen, die mit Bedürttigkeit im Zusammenhang stehen. Hier steht das geübte narzisstische Unabhängigkeitsverhalten im Wege. Diese Menschen haben selten von anderen etwas gebraucht, dafür aber um so mehr zu geben gehabt. Auf das narzisstisch-altruistische Verhalten somatoformer Patienten wird im Späteren noch eingegangen. Tabelle 10 zeigt die strukturellen Aspekte bei somatoformen Störungen noch einmal im Überblick. 
Tabelle 8: Strukturelle Aspekte der Somatisierung (Rudolf, 2006a, S. 173).

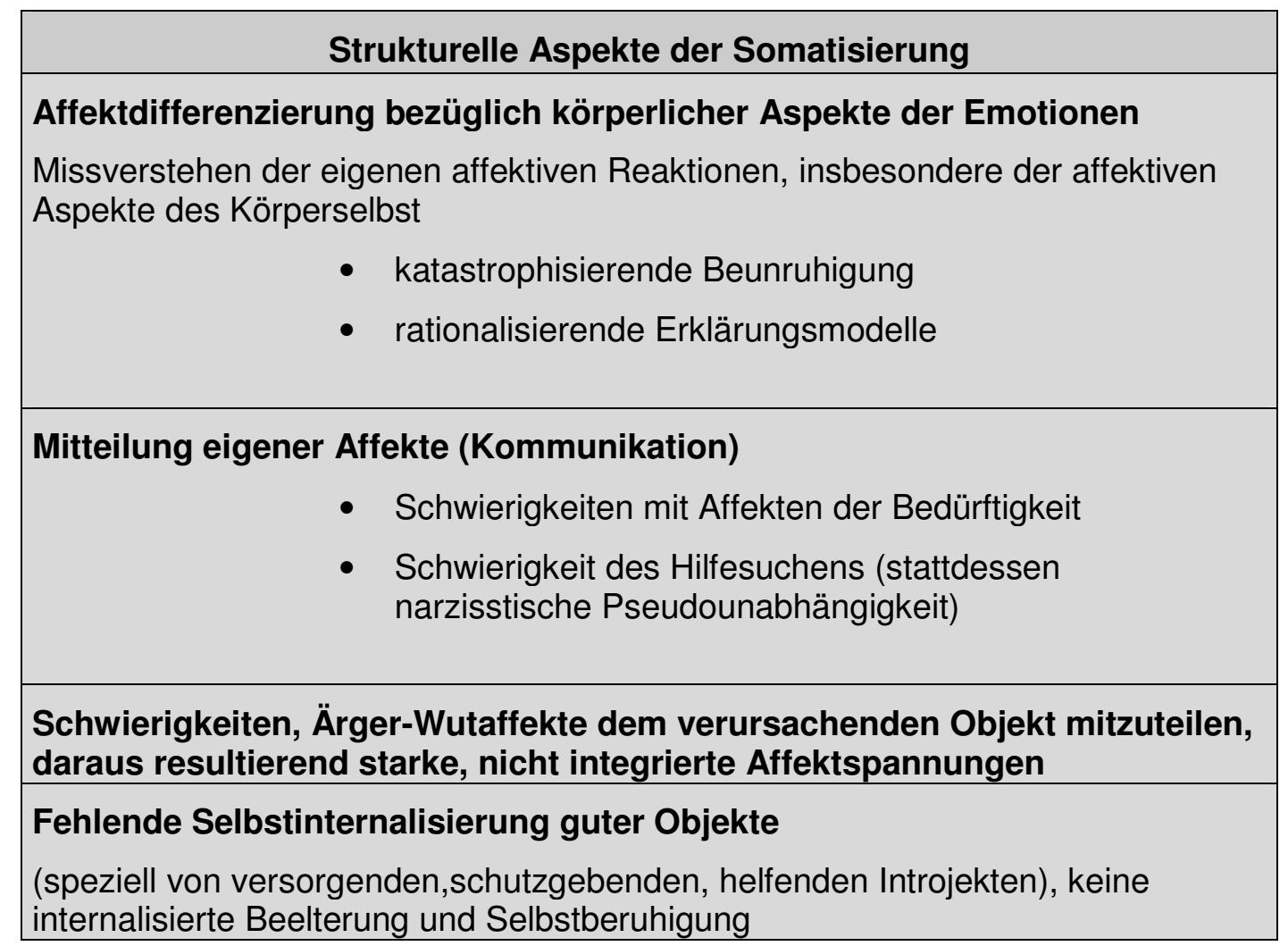

\section{Die Entstehung struktureller Störungen und des zugrundeliegenden depressiven}

\section{Grundkonflikts}

Strukturelle Störungen sind nach diesem Konzept die Folge frühkindlicher Beziehungsstörungen. Die Ursache frühkindlicher Beziehungsstörungen liegt meist darin, dass Eltern oder andere wichtige Bezugspersonen kein gutes Vorbild sein können. Sie sind entweder gar nicht vorhanden oder mit eigenen Problemen überfordert und verhalten sich extrem unsicher und uneinfühlsam. Durch einen Mangel an hilfreicher Rückmeldung von der Umwelt kann sich ein junger Mensch selbst nur unzureichend kennenlernen und erlebt nicht, wie er modellhaft psychisch gesund mit sich und anderen umgehen kann. Nur sehr wenige Kinder können unter solchen Bedingungen ein sicheres Bindungsmuster entwickeln und gehen fortan als bindungsunsichere Menschen, sichtbar vor allem in der Gestaltung von Beziehungen, durchs Leben.

Bei Personen mit diesen frühkindlichen Beziehungsstörungen rufen körperliche Symptome Irritation und Besorgnis hervor und nicht selten reagieren sie darauf mit Angst, Depression oder selbstschädigendem Verhalten. 
Gerade bei zwischenmenschlichen Problemen und Schwierigkeiten sind die Betroffenen schnell überfordert und reagieren, aufgrund keiner anderen vorhandenen psychosozialen Handlungsalternativen, mit psychischen Krankheitsbildern beziehungsweise körperlicher Symptomatik (Mück, 2005). Tabelle 8 zeigt überblickend die Konfliktaspekte der Somatisierung.

Tabelle 9: Konflikt-Aspekte der Somatisierung (Rudolf, 2006a, S. 172)

\begin{tabular}{|l|}
\hline \multicolumn{1}{|c|}{ Konflikt-Aspekte der Somatisierung } \\
\hline Depressiver Grundkonflikt (verlassen, unversorgt sein, Objektidealisierung, \\
Objektenttäuschung, Objektverlustangst) \\
(im OPD-Sinne vorrangig Autarkie-Versorgungs-Konflikt) \\
\hline Bewältigungsform: narzisstisch-altruistische Bewältigung als forciertes \\
Angebot \\
(Im OPD-Sinne vorrangig Selbstwertkonflikt). \\
\hline
\end{tabular}

Die fehlende Erfahrung von zuverlässiger Beelterung findet sich bei den meisten Betroffenen. Es besteht ein Mangel an positiven, wohlwollenden und versorgenden Introjekten, die es mit der Person gut meinen und sie fördern möchten (Rudolf, 2006a).

Rudolf spricht zudem davon, dass somatoforme Patienten ihre bedeutsamen frühen Objekte nicht gänzlich entbehren mussten, sondern eher vorzeitig verloren haben und daher zumindest eine innere Vorstellung von einem Idealobjekt besitzen.

In der Folge wenden sie sich oft an Beziehungspartner, die ihrem idealisierten Objekt nahe kommen und versuchen diese mit allen Mitteln zu erreichen und zu behalten. Wird ein Idealobjekt gefunden, gibt es häufig große Schwierigkeiten, sich von diesem Objekt auch wieder lösen zu können, sich zu verabschieden und den Abschied ggf. betrauern zu können. Tabelle 9 zeigt die Psychodynamik des zugrundeliegenden depressiven Grundkonflikts in der Übersicht. 
Tabelle 10: Psychodynamik des depressiven Grundkonflikts (Rudolf, 2008).

\begin{tabular}{|c|}
\hline Psychodynamik des depressiven Grundkonflikts \\
\hline - Verlassenheitserfahrung \\
- Bindungssehnsucht \\
- Objektidealisierung \\
- Anpassungsbereitschaft \\
- Objektverlustangst \\
- Objektenttäuschung \\
- Enttäuschungsabwehr \\
\hline
\end{tabular}

Natürlich ist dies keine Typologie und nicht alles ist gleichermaßen auf alle betroffenen Patienten anzuwenden. Jeder Mensch bleibt auch in seiner Störungspräsentation ein Individuum und hat seine ganz eigene Geschichte. Die aufgeführten strukturellen Bereiche sind jedoch in gehäuftem Maße bei Patienten mit somatoformen Störungen beobachtet worden und bedürfen daher einer besonderen Aufmerksamkeit in der störungsspezfischen Behandlung und Begleitung (Rudolf, 2006a).

Dass strukturbezogene Arbeit in Anbetracht all dieser Informationen vermutlich die effektivste und wirtschaftlichste Form der Prävention überhaupt ist, dürfte anhand der Bedeutung dieser früh entwickelten psychischen Funktionen wenig zweifelhaft sein.

\section{Die Bewältigungsfunktionen des frühen depressiven Grundkonflikts}

Für Personen mit somatoformen Störungen haben Henningsen (1998), Henningsen et al. (2002) und Rudolf $(1992,1998,2004)$ die typischen biografischen Hintergründe und die Dynamik der Persönlichkeitsentwicklung herausgearbeitet und an größeren Patientenstichproben überprüft.

Sie fanden bei Menschen, die eine somatoforme Symptomatik entwickelten, bevorzugt frühe Verlusterfahrungen und soziale Notlagen der Familien. Besonders wichtig erscheint die bereits von Kindheit an bestehende Depressionsabwehr in Form überhöhter Leistungsangebote und Anpassungsbereitschaft, die bei den erwachsenen somatoformen Patienten in narzisstischen Stolz auf die eigene Belastbarkeit mündet. 
In einer Studie von Steinhausen (1997) wurden Gutachtentexte von 45 somatoformen Patienten qualitativ textanalytisch untersucht. Eine massive frühe biographische Belastung hat dabei bei allen Patienten bestanden.

Gekennzeichnet war sie im einzelnen vor allem durch materielle Not (58\%), Nichtverfügbarkeit der elterlichen Bezugsperson (86\%), massive Zurücksetzungserlebnisse (23\%), belastende und traumatisierende Erlebnisse (21\%), zeitliche Einschränkung durch Pflichten (23\%), geringe Möglichkeit der Schulbildung (23\%) und keine selbst bestimmte Berufswahl (16\%).

Als zweite Konstellation ließ sich bei $60 \%$ im Kindesalter eine frühe Verantwortungsübernahme und bei $95 \%$ eine starke Pflicht- und Leistungsorientierung im Erwachsenenalter nachweisen.

Rudolf beschreibt dieses leistungs- und pflichtorientierte Verhalten als eine Bewältigungsfunktion des frühen, von inm bezeichneten, depressiven Grundkonflikts, der somatoformen Störungen zugrunde liegt.

Wie bereits oben erwähnt resultiert die Störung aus einer massiven Diskrepanz zwischen den angeborenen starken Bindungs- und Beziehungsbedürfnissen eines Kindes und den fehlenden oder widersprüchlichen Beziehungsangeboten der Bezugspersonen.

Der depressive Grundkonflikt entnimmt seine motivationale Kraft also nicht aus einer Triebregung, sondern aus einem interpersonellen Bedürfnis: Dem Wunsch nach Geborgenheit bei einem starken und sicheren Objekt.

Wichtig für das Verständnis des Krankheitsentstehens ist das Modell der Verarbeitung des frühen Grundkonflikts. Klinisch bedeutsam ist nach Rudolf die narzisstisch-altruistische Verarbeitung des depressiven Grundkonflikts bei somatoformen Patienten. Eine Form, die er mit Abwehr- aber auch mit schützendem Bewältigungsverhalten betitelt.

Die vom Ich ausgehenden Verarbeitungs- beziehungsweise Abwehrweisen bestimmen die Form des klinischen Bildes, während der zugrundeliegende Konflikt die Ursache darstellt. 


\section{Die altruistische Verarbeitung}

Um die enttäuschten Bedürfnisse abzuwehren, opfern sich Betroffene später häufig selbst auf. Aggressionen können nicht ausgelebt werden. Es besteht ein großes Harmoniebedürfnis und ein zu strenges Über-Ich. Durch ein fürsorgliches und harmoniebetontes Verhalten erreichen sie konstante Objektbeziehungen, jedoch nur unter großer Anstrengung und dem Verzichten auf Aggression und Wut. Die vorhandene Wut wird durch den Abwehrmechanismus der Reaktionsbildung in Liebe umgewandelt.

Dem altruistischen Verhalten könnte man das Motto zusprechen: "Wenn sich schon niemand um mich kümmert, dann kümmere ich mich um andere, um überhaupt Beziehungen zu erleben“.

\section{Die narzisstische Verarbeitung}

In der narzisstischen Verarbeitung will eine Person sich vor allem von der Masse abheben, etwas Besonderes, Besseres sein und entwertet andere Objekte, die als "weniger" wahrgenommen werden. Ständig wird eine Atmosphäre von Rivalität erzeugt, um sich selbst aufzuwerten und dem permanenten Verlangen nach Anerkennung nach zu kommen.

Dabei leiden Betroffene stark. Sie stehen unter ständiger Anstrengung etwas Besonderes erschaffen zu wollen, zu müssen und haben die große Sorge, nicht (mehr) beachtet zu werden.

Dieser Bewältigungsstil der narzisstischen Selbstidealisierung bedarf allerdings einer Grundbegabung für irgendeine Fähigkeit und der frühen Förderung dieser durch die Eltern.

Dem narzisstischen Bewältigungsverhalten könnte man das Motto zusprechen: "Ich brauche niemanden, ich schaffe alles alleine und ohne Hilfe, weil die Enttäuschung, zurückgewiesen zu werden, beim Zeigen von Bedürftigkeit, nicht (mehr) aushaltbar ist."

Insgesamt entwickelt das Selbst eines Menschen einen eigenen Stil der Selbstorganisation und des Umgangs mit anderen Objekten, der lediglich einen Selbstheilungsversuch darstellt, um sich selbst zu stabilisieren. Die Folge dessen ist entweder eine forcierte Unabhängigkeit durch Verzicht auf wirkliche Objektnähe oder im Gegenteil eine zu enge Objektbindung zu Lasten der Selbstständigkeit. 


\section{Symptomausbruch beim Zusammenbruch der Bewältigungsfunktionen}

Wenn aus einer Situation zusätzlicher Überforderung irgendwann diese narzisstischaltruistischen Bewältigungsfunktionen zusammenbrechen, kommt es zum Ausbruch der Symptomatik.

Dies kann z. B. eine Mutter zu dem Zeiptunkt treffen, wenn ihre Kinder das Elternhaus verlassen und sie ihre altruistische Rolle gegenüber ihren Kindern nicht mehr ausüben kann.

Vor allem die narzisstische Seite des Bewältigungsverhaltens erscheint wichtig und dominant. Die Symptombildung kann beispielsweise durch den Alterungsprozess einsetzen ein, wenn Bewunderungen für Schönheit oder besondere Erfolge ausbleiben.

Darauf folgen Affektausbrüche, dramatische Verzweiflung, eine klagsame Depression und oft sogar selbstzerstörerische Kränkungswut, weil der eigene Selbstwert ausschließlich über die Anerkennung anderer aufrechterhalten wird.

So schreibt Rudolf in Sandweg (2004, S.93-94 \& S.99.): „Das ganze belastete Leben zuvor und die Anstrengungen, es zu meistern, haben die meisten Patienten als etwas Persönliches angenommen. Das krankheitsauslösende Ereignis jedoch erscheint ihnen als etwas Schicksalhaftes, von außen Kommendes, für das sie keine Verantwortung übernehmen können und zu dessen Bewältigung und Wiedergutmachung sie sich an die Mächtigen der Gesellschaft wenden müssen. Die Gesellschaft aber weist sie zurück und zeigt sich verständnislos. Jetzt beginnt ein quälerischer Kampf um Recht und Genugtuung......

So gelingt es der Medizin nicht, die gesellschaftlich Verletzten zu versorgen. Zwischen diesem Typus der Patienten und unserem Typus von Medizin arrangiert sich eine Reinszenierung von jener Zurückweisung, Nichtwahrnehmung, jenem Nichternstnehmen, unter Umständen quälend und schädigend, welche der Patient aus seinem bisherigen Leben, vor allem aus seinen frühen Erfahrungen nur zu gut kennt."

Die seit frühester Kindheit bestehende Lücke an befriedigter Fürsorge, Beelterung und Schutz kann bei Zusammenbruch der Bewältigungsfunktionen nicht mehr ausreichend abgewehrt werden. Der Symptomausbruch ist der letzte Weg, um das eigentlich schon lange, aber mit Angst vor Ablehnung besetzte, ersehnte seelische Klagen zu umgehen. 


\section{Der gesamte Prozess der depressiven Somatisierung}

Den gesamten Prozess der depressiven Somatisierung beschreibt Rudolf (1998) anschaulich über drei wesentliche Bereiche.

1. Intrapsychische Aspekte

2. Interpersonelle Aspekte

3. Psychophysiologische Aspekte

\section{Depressive Somatisierung: Intrapsychische Dynamik}

Die Somatisierung ist eine Folgeerscheinung des Zusammenbruchs der Bewältigungsfunktionen beziehungsweise Abwehrstrukturen.

Das psychodynamische Grundmuster des frühen Objektverlustes hinterlässt eine schmerzliche Spur der Enttäuschung am Objekt, ebenso wie die Sehnsucht nach dem guten Objekt. Dem Selbst fehlt damit insbesondere die stützende Kraft positiver Introjekte.

Diese Enttäuschungserfahrung hinterlässt Selbstzweifel, Selbstverachtung und Selbsthass. Aggressive Impulse wenden sich in strafender Weise gegen das eigene Selbst und werden zugleich auf Objekte projeziert.

Als Bewältigung dieser depressiven Kernproblematik finden sich altruistischüberfürsorgliche Züge oder/und narzisstische Elemente.

„Der Symptomauslösende Konflikt trifft die Abwehrstruktur zu einem bereits sehr erschöpften Zeitpunkt. Oft genügt eine geringfügige zwischenmenschliche Enttäuschung, eine zusätzliche narzisstische Kränkung oder ein Entmächtigungserlebnis eines Unfalltraumas, um die Symptomatik auszulösen. Daraufhin folgen häufige Chronifizierungstendenzen." (Rudolf, 1998, S.192).

Diese Eigendynamik, insbesondere die Erfahrung von Enttäuschungen, charakterisiert die Arzt-Patienten-Beziehung. Hier erhofft der Patient sich immer wieder das gute und ideale Objekt zu finden, wird aber, durch keine Diagnose und damit abgewiesen werden, immer wieder neu enttäuscht. Dies betonen vor allem die interpersonellen Aspekte (s. u.).

Ein häufiges Erscheinungsbild der depressiven Somatisierung ist die somatoforme Schmerzstörung. Abbildung 1 zeigt die intrapsychischen Aspekte der depressiven Somatisierung in einer Übersicht. 


\section{Die depressive Somatisierung \\ am Beispiel der somatoformen Schmerzstörung \\ Intrapsychische Aspekte}

Frühe Biographie:

Fehlende Unterstützung

Depressiver Grundkonflikt
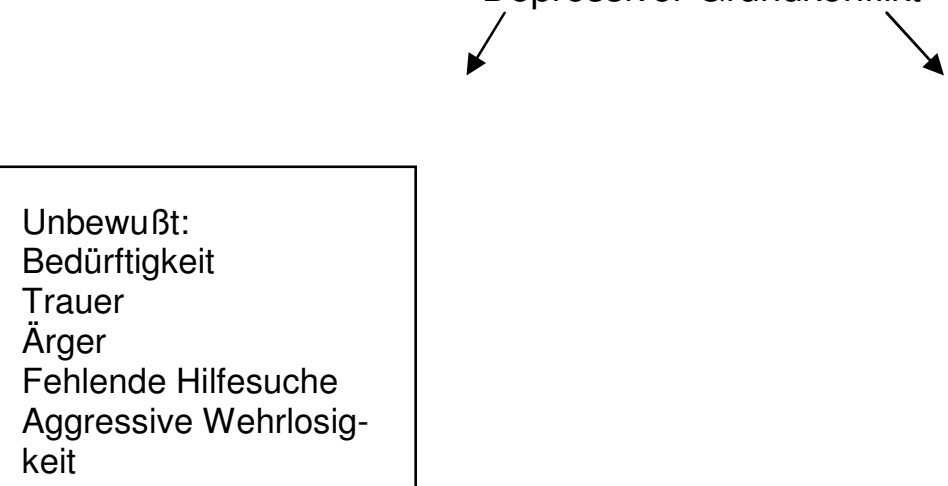

\section{Emotionale}

Dauerspannung

Fehlende emotionale

Entlastung

Fehlende kommunika-

tive Entlastung

Fehlende regressive

Entlastung

Aufmerksamkeit

Ängstliche Bewertung

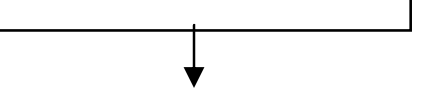

Chronische

Selbstverleugnung

Chronische

$\rightarrow$ Selbstüberforderung

Daueranspannung

Abwehr der Bedürftigkeit und Modus der

Verarbeitung:

Durchhalten

Forciertes Angebot
Erschöpfung

Burn-Out

Verzweiflung

\section{Schonhaltung}

Soziale Vermeidung

Symptomchroni-

fizierung

Abbildung 1: Intrapsychische Aspekte der depressiven Somatisierung (Rudolf \& Henningsen, 1998, S. 193). 


\section{Interpersonelle Dynamik und Arzt-Patient Beziehung}

Das Klageverhalten ist als Teil der Symptomatik zu sehen und dient dem primären Krankheitsgewinn.

Der Klage geht eine lange Entwicklung voraus, die auf dem depressiven Grundkonflikt basiert, verstärkt durch die Tatsache, dass das Appellieren und Einklagen immer hoffnungslos oder verboten war.

Dem voran geht der Bewältigungsversuch des forcierten autonomen Verhaltens und der Fürsorge für andere. Hier wird dem Objekt, im Gegensatz zum Einfordern, nichts abverlangt, sondern der Betroffene stellt sich in den Dienst des anderen, um das Objekt so auf eine andere Art und Weise für sich zu gewinnen. Er nimmt die Last auf sich, sich zusammenzureißen, klaglos Dinge zu ertragen und dies stets in der Hoffnung, dass damit irgendwann einmal alles gut werden wird.

Nach Jahren der Selbstüberforderung erfolgt eine Erschöpfungsreaktion. Die damit eigentlich empfundene Enttäuschung und Verzweiflung werden aber nicht gefühlt, sondern das Symptom erlaubt endlich zu klagen, anzuklagen und zu fordern.

Es entsteht eine Dynamik zwischen regressiven Bedürfnissen und aggressivkämpferischen Seiten. In der Arzt-Patient-Beziehung bedeutet das in etwa: „Helfen Sie mir, kümmern Sie sich um mich, los, sofort, helfen Sie mir"! Eine Mischung aus regressivem Verhalten und aggressivem Einfordern. In dieser Erwartungshaltung droht jedoch bereits die erneute Enttäuschung.

Das Klageverhalten und die jeweilige Klage werden vom Patienten nur durch das Körpersymptom gerechtfertigt. Wenn der Arzt nun mit einem alleinigen psychologischen Krankheitsmodell argumentiert, nimmt er dem Patienten damit etwas Wichtiges weg. Er nimmt ihm sein einziges Mittel weg, endlich zu klagen.

Seelischer Schmerz hat in der Lebenserfahrung des Patienten keine kommunikative Realität und gilt in seiner Erfahrung nicht als Mitteilung, die ein Objekt erreichen könnte.

Körperbeschwerden sind für den Patienten, der jede psychische Belastung und Bedürftigkeit strikt abzuwehren gelernt hat, das adäquate Ausdrucksmittel.

Das Beziehungsangebot an den Arzt ist als Idealobjekt zu betrachten. Dieser versucht in der Regel auch alles, um seinem klagenden Patienten zu helfen und seine medizinische Kunst so gut es geht durchzuführen; die organische Ursachenforschung beginnt. 
Irgendwann beginnt der Arzt aus Verzweiflung, mehr Medikamente zu verschreiben, mehr operative Eingriffe vorzunehmen bis er sich, am Ende seiner medizinischen Möglichkeiten, für den somatisierenden Patient vom idealen zum enttäuschenden Objekt wandelt und der Patient schließlich den Arzt wechselt. Dort beginnt der gesamte Prozess der Idealisierung und Enttäuschung erneut, denn ein Arzt mit seinem medizinischen Krankheitsmodell kann inm nicht hinreichend helfen.

Abbildung 2 zeigt diese interpersonellen Aspekte der depressiven Somatisierung.

\begin{tabular}{|c|c|c|c|c|c|}
\hline \multicolumn{6}{|c|}{$\begin{array}{c}\text { Die depressive Somatierung } \\
\text { Am Beispiel der somatoformen Schmerzstörung } \\
\text { Interaktionelle Aspekte }\end{array}$} \\
\hline & $\begin{array}{c}\text { Intrapsychisches } \\
\text { Erleben }\end{array}$ & & $\begin{array}{c}\text { Interpersonelles } \\
\text { Angebot }\end{array}$ & & $\begin{array}{c}\text { Erlebte } \\
\text { Objektresonanz }\end{array}$ \\
\hline $\begin{array}{l}\text { Frühe } \\
\text { Biographie: } \\
\text { Fehlende } \\
\text { Unterstützung } \\
\downarrow\end{array}$ & Bedürftigkeit & $\rightarrow$ & Appell & $\leftarrow$ & $\begin{array}{l}\text { Fehlende } \\
\text { Resonanz }\end{array}$ \\
\hline $\begin{array}{l}\text { Depressiver } \\
\text { Grundkonflikt } \\
\downarrow\end{array}$ & $\begin{array}{l}\text { Enttäuschung, } \\
\text { Schmerz }\end{array}$ & $\rightarrow$ & Appell & $\leftarrow$ & $\begin{array}{l}\text { Verbot des } \\
\text { Appellierens }\end{array}$ \\
\hline $\begin{array}{l}\text { Verarbeitung } \\
\downarrow\end{array}$ & $\begin{array}{l}\text { Überkomp. } \\
\text { Bemühen }\end{array}$ & $\rightarrow$ & $\begin{array}{c}\text { Forciertes } \\
\text { Angebot }\end{array}$ & $\leftarrow$ & $\begin{array}{l}\text { Fehlende } \\
\text { Resonanz }\end{array}$ \\
\hline $\begin{array}{l}\text { Körpersymp- } \\
\text { tombildung } \\
\downarrow\end{array}$ & $\begin{array}{l}\text { Selbstüber- } \\
\text { forderung, } \\
\text { Erschöpfung }\end{array}$ & $\rightarrow$ & Symptomklage & $\leftarrow$ & $\begin{array}{l}\text { Unverständnis } \\
\text { Durchhalte- } \\
\text { appelle }\end{array}$ \\
\hline $\begin{array}{l}\text { Symptom- } \\
\text { chronifizierung }\end{array}$ & Enttäuschung & $\rightarrow$ & Aggravation & $\epsilon$ & Ablehnung \\
\hline
\end{tabular}

Abbildung 2: Interaktionelle Aspekte der depressiven Somatisierung (Rudolf \& Henningsen, 1998, S. 195). 


\section{Psychophysiologie der depressiven Somatisierung}

Als dritte Komponente der depressiven Somatisierung ist natürlich die Psychophysiologie zu nennen.

Menschen reagieren auf emotionale Konflikte immer auch physiologisch. Dies ist bereits bei einjährigen Kindern im Rahmen der Bindungsforschung beobachtet worden. Unsichervermeidend gebundene Kinder zeigen in der Fremde-Situation, einem diagnostischen Verfahren zur Bestimmung des Bindungsstils kleiner Kinder, kein Signalverhalten, weinen nicht, zeigen keinen Kummer über den Verlust der Mutter oder des angespannten Gefühls für eine Weile allein oder mit einer fremden Person im Raum zu bleiben. Sie wirken ruhig. Physiologisch konnte man jedoch messen, dass der Kortisolspiegel bei diesen Kindern in der Stresssituation ansteigt (Spangler in: Friedlmeyer \& Holodynksi, 1999). Kortisol ist ein Stresshormon, das vor allem in anstrengenden und auch emotional belastenden Situationen produziert wird, um die Stressreaktion wieder zu bremsen.

Findet eine Daueranspannung und eine dauernde Kortisolausschüttung statt, können die Speicher sich erschöpfen. Dann ist der Körper nicht mehr resistent gegen Angriffe von außen. Die Entzündungsbereitschaft kann ansteigen, weil kein Kortisol mehr zur Ausschüttung zur Verfügung steht (Hypokortisolismus). Die Stressreaktion kann nicht mehr oder nur suboptimal gebremst werden.

Die Disposition zur somatoformen Symptombildung entsteht vor allem durch dysfunktionale Bewältigung von emotionalem Stress und Daueranspannung mit altruistischen oder narzisstischen Zügen. Daraus resultiert entweder ein Beziehungsangebot mit durchhaltewilligen, leistungsbereiten und nach Anerkennung suchenden Seiten oder eines, das pflichtorientiert, aufopferungsvoll und anhänglich ist.

Beide münden in einer ausgeprägten emotionalen Daueranspannung, die sich auch körperlich auswirkt. Letztlich führt dies zu Selbstüberforderung und Erschöpfung. Angesichts der ständigen emotionalen und psychischen Überforderungen besteht das Risiko, dass das psychophysische Gleichgewichtssystem des Körpers irgendwann in einem Burn-Out zusammenbricht. 
Die zugrundeliegende physiologische Situation lässt sich dann aus der emotionalen Daueranspannung als anhaltende sympathikotone Erregung mit ständig erhöhtem Muskeltonus und Minderdurchblutung, Entzündungsbereitschaft sowie der oben bereits erwähnten irgendwann einsetzenden Erschöpfung der Kortisolspeicher verstehen. Abbildung 3 zeigt die psychophysiologischen Aspekte der depressiven Somatisierung (Rudolf, 1998, S.192). 


\section{Die depressive Somatisierung \\ am Beispiel der somatoformen Schmerzstörung \\ Psychophysiologische Aspekte}

\begin{tabular}{|c|}
\hline Frühe Biographie: \\
Fehlende \\
Unterstützung \\
\hline
\end{tabular}

\begin{tabular}{c|}
\hline Depressiver \\
Grundkonflikt \\
\hline
\end{tabular}

\begin{tabular}{|c|}
\hline Fehlende Entlastung \\
Emotionale \\
Dauerspannung \\
Fehlende Hilfesuche
\end{tabular}

Noradrenerges System

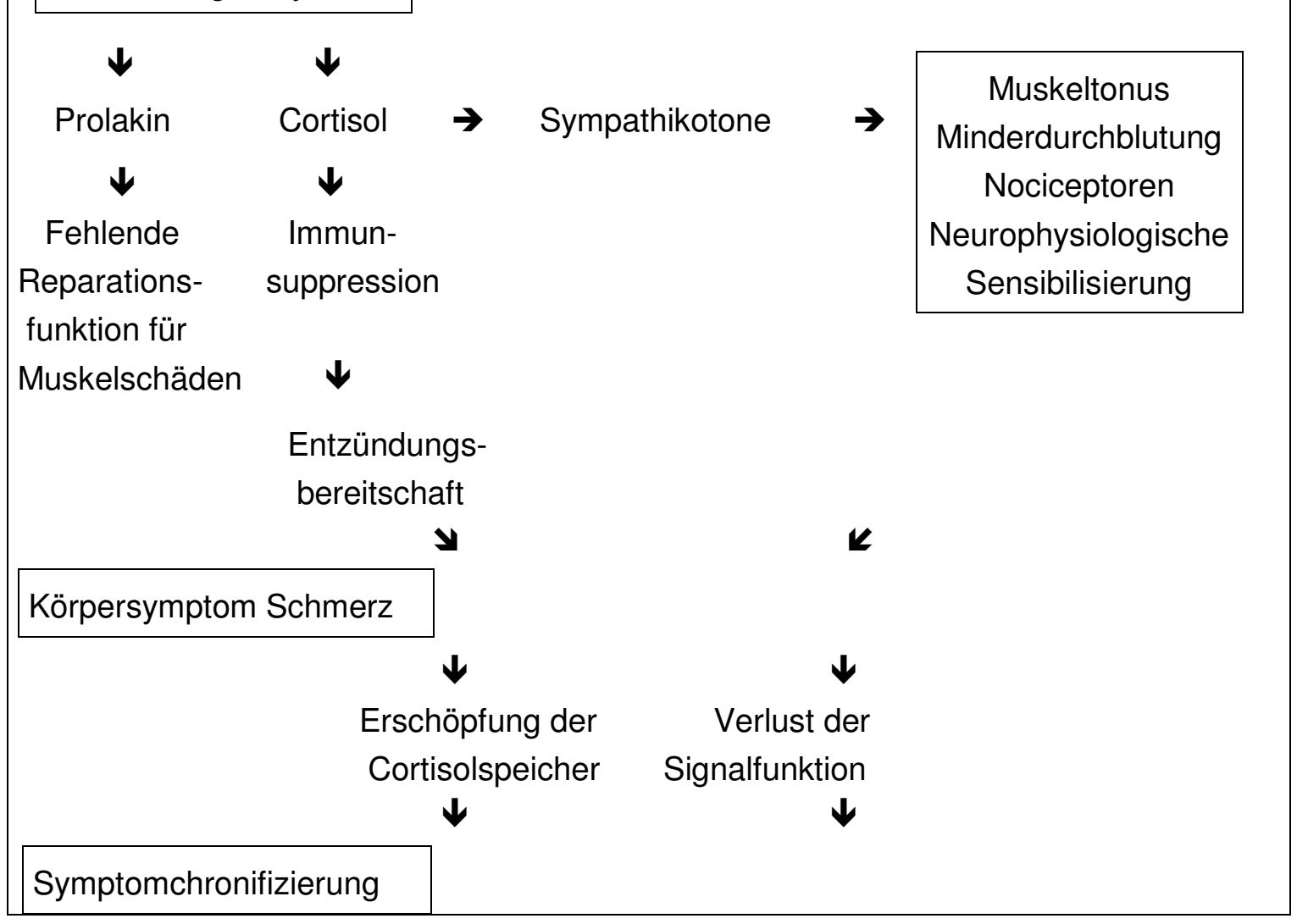

Abbildung 3: Psychophysiologische Aspekte der depressiven Somatisierung (Rudolf \& Henningsen, 1998, S.196). 
Selbst wenn bei somatoformen Störungen der depressive Grundkonflikt zugrunde liegt, bestehen, wie oben erläutert, auch bei diesen Patienten strukturelle Defizite. Es ist aufgrund der häufigen Behandlungsschwierigkeit dieser Patienten wichtig, an den strukturellen Dimensionen anzusetzen.

Denn selbst wenn aktuelle und biografische Konflikte für die Betroffenen unübersehbar sind, scheinen Selbstreflexion und Einsicht strikt abgelehnt zu werden. Hinweise auf eine depressive und ängstliche Verfassung empfinden Patienten mit somatoformen Symptomatiken zu Beginn einer Behandlung häufig als beleidigend (Rudolf, 2008).

Die klassischen therapeutischen Interventionen eines Psychoanalytikers können einen Patienten mit diesem Störungsbild nicht erreichen. Unter der Berücksichtigung der strukturell defizitären Gesichtspunkte, wird die Situation jedoch besser verständlich und therapeutisch handhabbar.

Es ist, entgegen vieler Ansichten klassischer Psychoanalytiker, nicht immer vorrangig möglich und notwendig einen Konflikt zu behandeln und zu bearbeiten. Im Gegensatz dazu gilt es heute als weitgehend gesichert, dass eine erfolgreiche Arbeit an Konflikten erst dann möglich ist, wenn die dafür erforderlichen strukturellen Grundlagen geschaffen sind (Mück, 2005).

Insbesondere die Arbeit an strukturellen Dimensionen ist Ziel der in dieser Studie durchgeführten Gruppenintervention.

Es gilt zunächst, die wichtigen Bewältigungsfunktionen nicht gänzlich wegzunehmen, sondern eher nach dem „Was-Du-Brauchst-Schema“, nach Hanne Seemann eine Lösungsorientierung mit einzubringen. Sie stellt die These auf, dass betroffenen Menschen nicht einfach alles weggenommen werden sollte, über das sie sich selbst stark definieren. Beispielsweise liegt es bei einem narzisstisch betonten Berufserfolg eines Managers nahe, ihm zu raten, er solle seine Arbeit zurückschrauben oder verändern. Hier sollte aber dem Gegenüber eher die Frage gestellt werden: Was fehlt auf der anderen Seite der Waage? Was können wir dazu geben, nicht wegnehmen, damit ein Ausgleich stattfinden kann? (Seemann, 1998). 


\section{Haltung des Helfers gegenüber einem somatisierenden Patienten}

Zum Ende des Kapitels soll noch erwähnt werden, dass die Haltung des Helfers gegenüber einem somatisierenden Patienten elementar ist, um einen guten gemeinsamen Arbeitsprozess in Gang bringen zu können.

Gegenüber somatoformen Patienten ist nicht die Einstellung eines alles durchblickenden Experten empfehlenswert, denn dafür sind die Zusammenhänge viel zu kompliziert, sondern die eines anteilnehmenden und wohlwollend interessierten Gegenübers.

Die therapeutischen Mittel sind nicht die eines konfliktaufdeckenden Therapeuten, sondern eher die eines wohlwollend-loyalen Mitmenschen, der Verständnis und Interesse für die Beschwerden, Enttäuschungen und Verzweiflungen seines Patienten aufbringt und inm seine Begleitung und Unterstützung anbietet (Rudolf, 2008).

Das therapeutische Handeln ist gerade bei somatoformen Patienten nicht auf die intime Zweierbeziehung zurückgezogen, sondern einzig und allein durch die Erschaffung von psychosozialen Netzwerken zwischen unterschiedlichen Helfern und Helferinnen zu ermöglichen. So müssen Ärzte, Psychotherapeuten, Heilpädagogen und Berater vernetzt miteinander arbeiten und so kooperieren, dass sie einen Patienten, trotz aller Beziehungsschwierigkeiten, vorsichtig und anhaltend begleiten können.

\section{Behandlungsziele}

Das, was mit Patienten erreicht werden kann, ist meist leider keine gänzliche Heilung. Im günstigsten Fall lernen sie, mit eigenen Defiziten fürsorglich, selbstverantwortlich und annehmend umzugehen und dabei die krankmachenden und selbstüberfordernden, selbstschädigenden Verhaltensweisen, insbesondere in der Konstruktion von Beziehungen, zu vermeiden.

Ziel ist der Erwerb von Selbstkompetenz und Selbstvertrauen im Umgang mit eigenen Problemen sowie ein größeres Maß an Selbstverantwortung und Selbstfürsorglichkeit.

Befragt man einen somatoformen Patienten nach einem erfolgreichen Therapieverlauf zu seiner Symptomatik, ist ein typischer Satz: „Ich habe die Beschwerden immer noch, aber das spielt für mich jetzt keine so große Rolle mehr" (Mück, 2005).

Dies bedeutet, dass sich für den Betroffenen neue Lebensziele, neue Sichtweisen, vor allem eine neue Sichtweise auf sich selbst eröffnet haben, die ein lebenswertes Leben, mit oder trotz somatischer Einschränkung, ermöglichen (Rudolf, 2008). 


\section{Studiendesign Gruppe - warum?}

Besonders die Arbeit in einer Gruppe soll viele Aspekte für den Umgang mit somatoformen Patienten berücksichtigen und ihnen ermöglichen, ihre Situation zu verbessern.

Die vielen Möglichkeiten für Resonanz, den mehrperspektivischen interpersonellen Kontakt und die Erlaubnis, endlich in einem gemeinschaftlichen Raum klagen zu dürfen, sollen zu seelischer und körperlicher Erleichterung verhelfen.

Außerdem kann, durch die Vielzahl der Teilnehmer in einer Gruppe, die Idealisierung nur eines Objekts möglicherweise vermieden werden, indem sich viele Menschen als hilfreich und einfühlsam erweisen. 


\subsection{Prävalenz in der Hausarztpraxis und Ökonomieaspekte für das Gesundheitssystem}

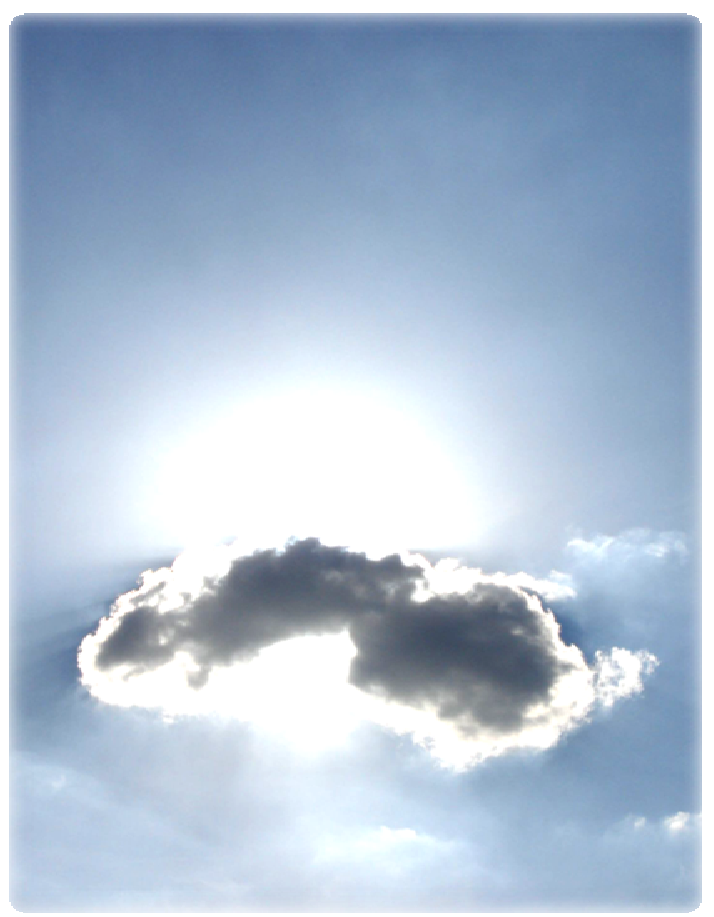

„Eindeutige somatische Erklärungen für körperliche Beschwerden sind eher die Ausnahme als die Regel“" (Rudolf, 2008).

Somatoforme Störungen sind für das Gesundheitssystem sowohl aufgrund ihrer hohen Prävalenz als auch wegen der intensiven Inanspruchnahme medizinischer Leistungen von großer Bedeutung.

Patienten mit somatoformen Störungen stellen in vielen Arztpraxen, insbesondere in der hausärztlichen Versorgung, bei Internisten, Gynäkologen, Orthopäden und Allgemeinkrankenhäusern mit $20 \%$ bis $40 \%$ die größte Patientengruppe dar (Nanke \& Rief, 2003, Kruse et al., 2004).

Befunde aus dem in Deutschland durchgeführten Zusatzsurvey „psychische Störungen“ des Bundesgesundheitssurveys berichten bei somatoformen Störungen bei einer zwölfMonats-Prävalenz von 11\%. Damit stellen die somatoformen Störungen neben affektiven und Angststörungen die größte psychische Störungsgruppe dar (Jacobi et al., 2004). 
Eine in Lübeck und Umgebung durchgeführte Studie zur Häufigkeit von psychischen Krankheitssymptomen an 4075 Probanden zeigte eine Lebenszeitprävalenz von 19,6\% für die undifferenzierte Somatisierungsstörung (Grabe et al., 2003a; Grabe et al., 2003b).

Zusätzlich litten 12,3\% während ihres bisherigen Lebens unter einer schweren somatoformen Schmerzstörung. Weniger stringente diagnostische Kriterien ergaben sogar Lebenszeitprävalenzen der somatoformen Schmerzstörung bis zu 33,7\%.

Die in Greifswald und Stralsund durchgeführte SHIP-Studie (Study of Health in Pomerania) erfasste 4310 Probanden aus der Allgemeinbevölkerung und zeigte eine Prävalenz somatoformer Störungen von 16,1\%.

$18,2 \%$ der Patienten aus Allgemeinarztpraxen litten an einem somatoformen Syndrom, in internistischen waren es $25,1 \%$, in chirurgischen $21,3 \%$ und in orthopädischen Praxen $27,6 \%$. Somatisierungspatienten beanspruchten $32,5 \%$ aller Termine in Allgemeinarztpraxen, $41 \%$ in internistischen Praxen, $49,5 \%$ in chirurgischen und $51,7 \%$ in orthopädischen Praxen (John et al., 2001).

Die Relevanz der somatoformen Störungen für das Gesundheitssystem lässt sich am Inanspruchnahmeverhalten medizinischer Dienste bemessen. Gerade gegenwärtige Diskussionen thematisieren, dass unser Gesundheitssystem durch stetig steigende Behandlungskosten gekennzeichnet ist. Daher erhalten gesundheitsökonomische Fragestellungen eine besondere Bedeutung (Nanke \& Rief, 2003).

Von 218,4 Mrd. Euro an Gesamtausgaben im Jahr 2000 entfielen ca. 60\% allein auf Leistungen der Primärversorgung (Statstisches Bundesamt, 2002).

Durchschnittlich suchen Deutsche elf Mal im Jahr einen niedergelassenen Arzt auf, wobei die Inanspruchnahme bei Frauen im Vergleich zu Männern höher ist (12,8 zu 9,1 Kontakten) und mit zunehmendem Alter ansteigt (Nanke \& Rief, 2003).

Mehrere Studien zeigen, dass die Inanspruchnahme medizinischer Leistungen bei Patienten mit somatoformen Störungen im Vergleich zu anderen klinischen Gruppen besonders hoch ist (Fink et al., 1999). 
Im Vergleich zu psychisch gesunden Personen, die im Durchschnitt einmal pro Quartal einen Arzt aufsuchen, berichten Smith et al. (1986) davon, dass die Behandlungskosten bei somatoformen Patienten um das ca. neunfache erhöht sind.

Außerdem werden von dieser Patientengruppe signifikant häufiger nichtpsychiatrische Gesundheitsleistungen in Anspruch genommen im Vergleich zu Patienten ohne eine somatoforme Störung (Fink et al., 1999). Dies hängt damit zusammen, dass Betroffene überwiegend auf ein somatisches Erklärungsmodell fixiert sind und weiterhin den Arzt dazu auffordern, die somatische Ursache zu finden.

Daher werden diese Patienten häufiger untersucht, stärker invasiv behandelt und laboranalytisch unter größerem Kostenaufwand vermessen als der durchschnittliche Patient einer Hausarztpraxis (Zimmermann \& Kaduszkiewicz, 2003). Dabei ist anzumerken, dass jede erneute Diagnostik für den Patienten nur eine weitere Verunsicherung bedeutet, denn wenn der Arzt weitere Untersuchungen durchführt, dann heißt das für den Patienten, dass auch der Arzt weiterhin auf der Suche nach einer organischen Ursache ist, wobei er eigentlich bereits weiß, dass er sie nicht finden wird (Cuntz, 1998).

Arbeitsausfälle durch Krankschreibungen und sogar vorzeitige Berentung finden sich bei kaum einer Krankheitsgruppe in einer solchen Häufigkeit wie bei den somatoformen Störungen (Rief \& Fichter, 1998).

Während dieses Klientel medizinisch überversorgt ist, berichtet Katon et. al (1990) davon, dass die Mehrheit dieser Personen (67\%) psychiatrisch jedoch als unterversorgt eingeschätzt wird. Hier stellt sich die berechtigte Frage, ob vielleicht zu wenig geeignete Hilfsmaßnahmen und Ressourcen für die Betroffenen zugänglich sind.

Auch das Ausmaß an Krankheitsängsten lassen die Tendenz zum Aufsuchen medizinischer Hilfe ansteigen (Conroy et al., 1999).

Die Studie von Nanke und Rief (2003) ergab bei 295 teilnehmenden Probanden, die ihren Hausarzt mit organisch unklaren körperlichen Beschwerden aufsuchten, dass insgesamt bei $82 \%$ (242) der Patienten eine somatoforme Störung vorlag. Neben dem Kontakt zum behandelnden Hausarzt wurden fünf weitere Fachärzte häufig aufgesucht: Zahnärzte, Orthopäden, Gynäkologen, Internisten und Augenärzte. 
Insgesamt gaben $68 \%$ der befragten Patienten an, zu keinem Zeitpunkt psychotherapeutische oder psychiatrische Behandlung in Anspruch genommen zu haben.

Die niedrige Rate psychotherapeutischer Vorerfahrung und der Aspekt, dass der Hausarzt für die Gesamtbevölkerung in der Regel der erste und wichtigste Ansprechpartner ist, verweisen auf die Bedeutung und Verantwortung der praktizierenden Ärzte. Zwischen $40 \%$ und $70 \%$ der psychischen Störungen werden jedoch von den Hausärzten übersehen oder nicht erkannt (Hiller \& Rief, 1998).

Ergebnisse einer Studie von Rossa \& Breull (2004) zeigen zudem, dass fast jeder zweite Patient in der allgemeinmedizinischen Praxis seit durchschnittlich sieben Jahren an einer somatoformen Störung in Kombination mit ein bis zehn anderen Erkrankungen, litt.

Obwohl 92\% über psychosoziale Probleme, besonders im Beruf, $67 \%$ über psychische Beschwerden und $72 \%$ über Verlustängste klagten, wurde dieser deutliche psychosoziale Zusammenhang nur bei einem Viertel der Patienten berücksichtigt. Auch Kruse et al. (1998) berichten, dass nur $50 \%$ der psychogenen Erkrankungen vom Hausarzt ausreichend diagnostiziert werden.

Dies betont einmal mehr die Notwendigkeit zu einer interdisziplinären Kooperation zwischen Ärzten und anderen Helferberufen. Auch die in dieser Arbeit dargestellte Studie setzt den besonderen Fokus auf eine direkte Kooperation mit den behandelnden Hausärzten. Denn nicht nur die Kosten gilt es damit zu kontrollieren, sondern auch frühzeitig das Beschwerdebild des Patienten zu erfassen, zu erkennen und eine Chronifizierung der Krankheit im Sinne des Patienten zu verhindern.

Es ist daher von großem interdisziplinärem Interesse, effektive Interventionen zu finden und $\mathrm{zu}$ entwickeln, die einerseits den Betroffenen helfen und andererseits gesundheitsökonomische Gesichtspunkte berücksichtigen können (Yates, 1994). 


\subsection{Die Arzt-Patient-Beziehung und die Notwendigkeit interdisziplinärer Kooperation}

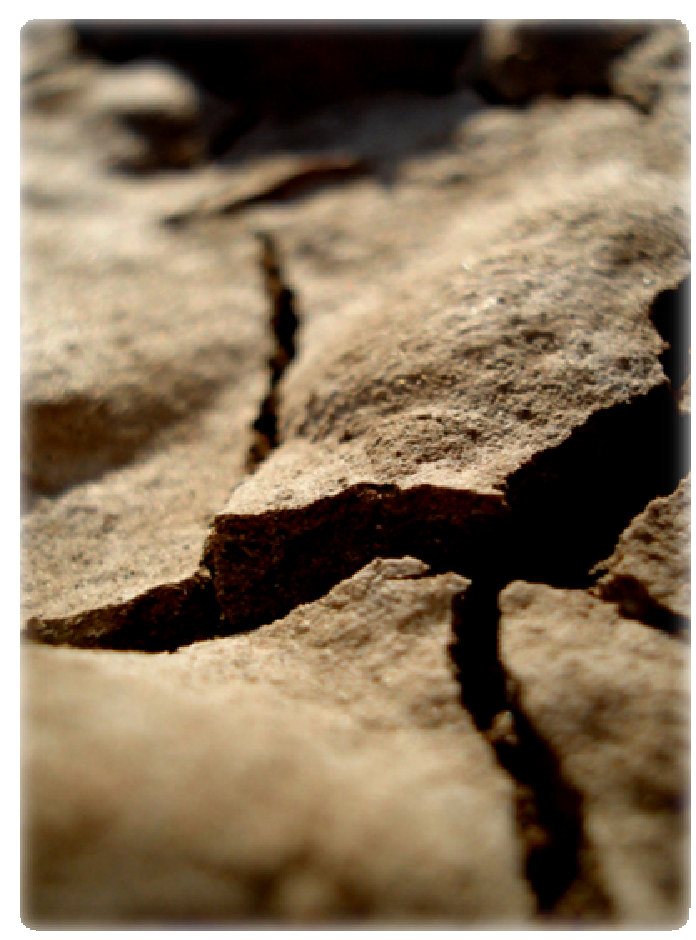

Ich war mein ganzes Leben lang noch nie depressiv, ich habe nie geklagt oder gejammert, ich habe meine Pflichten alle getan, habe immer funktioniert. Und jetzt kommen Sie daher und sagen ich sei „psycho“?

Das ärztliche Handeln ist sehr stark von einem medizinischen Krankheitsverständnis, durch das körperlichen Beschwerden eine einheitliche Ursache zugewiesen und daraus eine Behandlungsstrategie abgeleitet wird, geprägt.

Jedoch sind es auch die Rollenerwartungen an den Arzt, die wesentlich durch die Popularität des ätiologischen Krankheitsverständnisses geprägt sind. Der Patient erwartet von seinem Arzt die Aufklärung seiner Leiden. Er soll sie ursächlich benennen und medizinisch therapieren. Diese Art der Beziehung bedeutet, dass wir dem Arzt die Verantwortung für das Heilen unserer Beschwerden abgeben und von inm erhoffen, dass er uns die richtige Behandlung gibt.

Wichtig ist zu Beginn jeder Behandlung von Seiten des Arztes sowohl das medizinischsomatische als auch das psychophysiologische Krankheitsmodell als Möglichkeit der Ursache mit darzustellen. Es sollte normaler werden, beide Modelle als mögliche Ursache anzuerkennen. 
Es wäre förderlich, dem Patienten immer die grundsätzliche Beziehung zwischen körperlichen Beschwerden und psychischem Befinden zu erklären.

Körperliche Beschwerden beeinträchtigen immer, ob sie körperlich erklärbar sind oder nicht, die Lebensqualität und damit auch das psychische Befinden. Umgekehrt können emotionale Konflikte, Niedergeschlagenheit, Antriebsarmut und Gesundheitsängste eine Vielzahl körperlicher Symptome hervorrufen.

Was letztlich „Ei und was Henne ist“, lässt sich manchmal kaum entscheiden. Doch die Besserung einer Seite bessert immer auch das Befinden und Erleben der Anderen (Zimmermann \& Kaduszekiewicz, 2003).

Rudolf beschreibt (2008a) als Arzt und Psychotherapeut treffend:

„Viktor von Weizäcker ist einer der bekanntesten Väter der Psychosomatik. Er formulierte zum ersten Mal aus einer anderen Perspektive: „Es geht nicht um Krankheiten, sondern um kranke MENSCHEN - nichts Psychisches hat keinen Leib, nichts Leibliches hat keinen Sinn, der Ausbruch von Krankheiten erfolgt in biographisch sinnhaften Situationen. ““

Besonders wichtig ist es, den Patienten zu motivieren und inm nicht das Gefühl zu geben, inn abschieben zu wollen. Der Arzt sollte bestenfalls den bisherigen Kontakt zu ihm aufrechterhalten und inm ein Vertrauensverhältnis anbieten (Grabe, 2003a).

Das dominierende medizinische Krankheitsmodell bietet für die Ärzte jedoch eine weitaus bessere zeitliche Ökonomie in ihrer täglichen, von permanentem Zeitmangel gekennzeichneten Praxis. Eine organbezogene Diagnose macht den Hausarzt (schnell) therapeutisch handlungsfähig. Meist erfolgt dies durch Medikamentenverschreibungen und führt dazu, dass der Patient, fast immer, erfolgreich behandelt wird. Dies wiederum sichert dem behandeInden Arzt die Zufriedenheit und Anerkennung seines Patienten, was für eine gute Arzt-Patient-Beziehung sorgt.

Auch der Patient ist über eine Diagnosestellung, die auf körperlichen Ursachen beruht zufriedener als mit einer psychophysiologischen Diagnose. Die klare Nennung einer einfachen Ursache wirkt angstreduzierend und die angesetzte Therapie verspricht kurzfristige Heilung, ohne dem Patienten selbst einen aktiven Beitrag zur Heilung abzuverlangen (Cuntz, 1998). 
Ein Leiden unter einer organisch definierten Krankheit ist sozial eher akzeptiert als das Vorliegen einer somatoformen Erkrankung und das, bei den oben genannten hohen Prävalenzen von psychischen Störungen in der Allgemeinarztpraxis.

Benennt der behandelnde Arzt irgendeine körperliche Krankheit, ergibt sich für den Patienten, dass inm nun endlich die Krankenrolle zusteht.

Wie in Kapitel 3.2 erläutert, geht es für Somatisierungspatienten darum, auf körperlichem Wege zu klagen, was auf psychischem nicht (mehr) möglich ist. Eine vielleicht jahrelange Arbeitsbelastung, die immer tapfer gemeistert wurde, das narzisstisch-alturistische Verhalten, was lediglich der Abwehr der eigentlichen Bedürfnisse diente, bricht zusammen. Dann gibt es für diese Menschen meist „nur noch“ den Weg, über den Körper zu kommunizieren.

Wie in Kapitel 3.2 bereits erläutert, holen die Patienten nach, was innen lange versagt blieb: Zu klagen, zu fordern und endlich auch einmal krank, nicht funktionstüchtig und hilfebedürttig zu sein (Rudolf, 2008).

Das Dilemma ist, dass sie die Hilfe am falschen Ort suchen und durch ständige neue negative organische Befunde für ihre körperlichen Beschwerden auch permanente neue Beziehungsenttäuschungen zu ihren idealisierten Ärzten als Helfern erleben. „Da brauche ich einmal Hilfe und keiner kann mir helfen....und dann erklären die Ärzte mich noch für verrückt, ich bilde mir das doch alles nicht ein" sind typische und sicher auch nachvollziehbare Gedanken betroffener Patienten.

So schreibt Rudolf (2006a, S.171-172): „Das meist schmerzgetönte und ängstlich gefärbte Körpererleben der Patienten ist eingebettet in ein typisches Beziehungsmuster mit großen Heilserwartungen gegenüber einem mächtigen Helfer - einem Arzt, der die Körpersymptome kraft seiner medizinischen Kompetenz verstehen und beseitigen soll. Da diese riesigen Erwartungen regelmäßig unerfüllt bleiben - der Arzt findet „nichts“ - schlägt die idealisierende Hoffnung um in resignierte Enttäuschung, bis dann irgendwann ein neuer Hoffnungsträger am Horizont auftaucht. Das für die Arzt-Patient-Beziehung typische Muster findet sich auch in anderen Beziehungen wieder: Der Patient bekommt nie, was er dringend bräuchte, er sucht ständig und kommt stets zu kurz."

Nicht selten vermutet der behandelnde Arzt irgendwann hinter dem Klagen seines Patienten sogar Simulation oder, dass der Patient inn belästigen will. 
Ein wichtiger, häufig von den Ärzten übersehener Aspekt beim Stellen der Verdachtsdiagnose sind die Gegenübertragungsgefühle der Praxishelferinnen, der Ärztinnen und Ärzte oder des Pflegepersonals: Desinteresse, Müdigkeit, Ärger, und Aggressivität gegenüber den Patienten (Zimmermann \& Kaduszkiewicz, 2003).

Der Patient nimmt jedoch, trotz negativen Befunds, weiterhin organische Ursachen an, weil nur diese eine Legitimation für inn und seine Beschwerden darstellen können. Er ist enttäuscht, fühlt sich emotional zurückgewiesen, mit seinen Beschwerden nicht ernst genommen und es kommt zum Abbruch der Arzt-Patient-Beziehung und dem typischen, häufigen Ärztewechsel (Ärztehopping) (Henningsen et al., 2002b).

An dieser Stelle setzt die in Kapitel 3.2 beschriebene typische Enttäuschungsspirale ein. Der Patient klagt, etwas, was er zuvor in seinem Leben nur wenig oder gar nicht getan hat. Sein Klagen hat aber größere Erwartungen als das bloße Heilen eines körperlichen Leidens, denn dahinter steckt die tiefe Bedürftigkeit nach zwischenmenschlicher Annahme, Wertschätzung und dem Gesehenwerden in der absoluten Erschöpfung (Rudolf, 2007b).

Im schlechtesten Fall erfährt der Patient hier dann jedoch erneut, was er immer schon auf sein Klagen erfahren hat: Ablehnung und fehlende Resonanz. Der dahinterliegende Wunsch nach einem tröstenden idealen Objekt wird von den Betroffenen nicht wahrgenommen und kann daher nicht emotional, sondern nur über die Symptomklage kommuniziert werden.

Andererseits kann jedoch auch eine anhaltende Übereinstimmung für organische Ursachenüberzeugungen Probleme erzeugen, beispielsweise, wenn selbst ernannte Wunderheiler irgendwelche Wunderpillen gegen die „organische Krankheit“ oder sonstige esoterische Heilversprechen abgeben.

Der Verlauf einer somatoformen Störung wird im Wesentlichen vom Verhalten der Ärzte, insbesondere der Hausärzte in der Primärversorgung, mitbestimmt.

Andauernde weitere organische Diagnostik trägt zu einer Chronifizierung bei, während regelmäßige Einbestellung des Patienten, unabhängig von aktuellen Beschwerden, das inadäquate Krankheitsverhalten verringert (Henningsen et al., 2002b). Die zum Teil sehr aufwändigen apparativen Untersuchungen werden fast garantiert irgendwelche Diagnosen liefern, die sich dann im Verständnis des Patienten fest etablieren, selbst wenn sie die gegenwärtigen Beschwerden eigentlich gar nicht erklären können. 
Medizinische Bagatelldiagnosen verstärken das organische Krankheitskonzept und die entsprechenden Behandlungserwartungen bei den Patienten.

Morriss et al. (1999) untersuchten den Effekt eines Trainingsprogramms für Hausärzte, welches speziell auf die Vermittlung von Kommunikationsstrategien mit somatisierenden Patienten ausgerichtet war. Als Kontrollgruppe dienten Patienten, die von den gleichen Ärzten auch vor Absolvierung des Trainings behandelt wurden.

In der Nachuntersuchung nach drei Monaten schätzte die Kontrollgruppe die Kommunikation zu ihrem Arzt signifikant besser ein, während bei Patienten mit einer Somatisierungssymptomatik keine Verbesserung der erlebten Behandlungsqualität erzielt werden konnte.

Die Betroffenen fühlten sich durch die trainierten Ärzte mit ihren Symptomen sogar weniger gut verstanden. Es besteht demnach weiterhin dringender Bedarf, den Umgang mit dieser Klientel durch neue Interventionsstrategien zu verbessern.

In einer Studie von Nanke und Rief (2003) konnte hingegen gezeigt werden, dass entgegen der Erwartungen betroffene Patienten ihre Beziehung zu ihrem behandelnden Arzt eher positiv einschätzten, wobei die wenige Zeit, die der Hausarzt innen widmete, den am schlechtesten eingeschätzten Faktor darstellte.

Insgesamt gelten somatoforme Patienten jedoch bei den Ärzten als schwieriges Klientel und die Beziehung zu innen wird von den Ärzten als problematisch dargestellt. Andererseits empfinden auch die Patienten oft negative Gefühle gegenüber ihren Ärzten, aufgrund der ausbleibenden effektiven Hilfeleistung und der zum späteren Zeitpunkt unterschiedlichen Ursachenüberzeugung.

Allerdings stecken die Behandler in einem aktuell kaum auflösbaren Systemzwang. Trotz, dass sie sich der psychosozialen Einflüsse auf die Beschwerden bewusst sind, haben sie nicht viele Möglichkeiten, diese dem Patienten angemessen verständlich zu machen.

Selbst wenn Hausärzte sich Zeit nehmen mit den betroffenen Patienten zu reden, wird diese Leistung über das Abrechnungssystem nur gering honoriert. Das System favorisiert körpermedizinische Diagnostik und Therapie und lässt dabei nur sehr wenig Raum für Gespräche mit dem Patienten (Zimmermann \& Kaduszkiewicz, 2003). 
Dem Hausarzt bleibt dabei nur die Wahl zwischen zwei jeweils unerfreulichen Optionen: Entweder sucht er das Gespräch mit dem Patienten und verdient dabei wenig Geld, oder er behelligt inn mit unnötigerweise wiederholter körperlicher Diagnostik und verschreibt Medikamente, um bestenfalls die aktuellen Beschwerden zu lindern. Damit kann sich der Arzt zwar ökonomisch absichern, aber seinen Patienten keineswegs optimal versorgen, denn die Ursache der Beschwerden bleibt weiter verborgen und unbehandelt.

Obwohl den ausgebildeten Ärzten psychosoziale Einflüsse sicher bewusst sind, stellt die Handhabung einer nicht somatisch erklärbaren Krankheit dennoch ein Hindernis für sie dar. Ist es doch immer noch so, dass Gesprächstechniken noch nicht zu den Grundlagen des Medizinstudiums gehören (Zimmermann \& Kaduszkiewicz, 2003).

Nach heutigen Erkenntnissen verwundert bei all diesen Informationen nicht, dass sich wirkliche Behandlungserfolge nur interdisziplinär erzielen lassen.

In der Praxis ist man davon jedoch leider noch weit entfernt. Nach wie vor sind Allgemeinmediziner häufig die erste und auch dauerhaft längste Anlaufstelle für Betroffene.

Psychosoziale Hilfsmaßnahmen treten meistens erst nach langem Störungsverlauf und fehlgeschlagenen Behandlungen hinzu. Es ist dringend notwendig, ein Umdenken anzuregen und generell für eine viel größere Kooperation direkt vor Ort mit den Hausärzten zu sorgen (Sonnenmoser, 2005).

Dies erfordert beidseitige Achtung vor den mitgebrachten Kompetenzen der unterschiedlichen Helfer. Es Bedarf des Austauschs und nicht der Konkurrenz.

Somatoforme Störungen sind sehr stabil und weder einfach zu behandeln noch zu diagnostizieren. Daher setzen viele Experten die Therapieziele sehr niedrig an.

Schon eine leichte Verminderung von Symptomen, Arztbesuchen und Schmerzen sowie einer positiven Veränderung der Einstellung und des Altagslebens, werden als Erfolg gewertet (Sonnenmoser, 2005). 
Es ist festzuhalten, dass wir in Deutschland erst am Anfang der Entwicklung adäquater Therapie- und Versorgungsmaßnahmen für Personen mit somatoformen Störungen stehen. Eine Übersicht über die wenigen bisher evaluierten Gruppenprogramme für Somatisierungspatienten in Kapitel 3.5. machen deutlich, wie groß und notwendig der gegenwärtige Forschungsbedarf auf diesem Feld noch ist.

Es erscheint als ganz besonders wichtig, (Minimal-)Interventionen zu entwickeln und zu evaluieren, die an vorderster Front, z. B. im Rahmen der hausärztlichen Versorgung beziehungsweise in direkter Kooperation mit dem Hausarzt angeboten und durchgeführt werden können (Rief \& Fichter, 1998). 


\subsection{Ergebnisse deutschsprachiger Studien in der psychosozialen Gruppenarbeit mit Somatisierungspatienten}

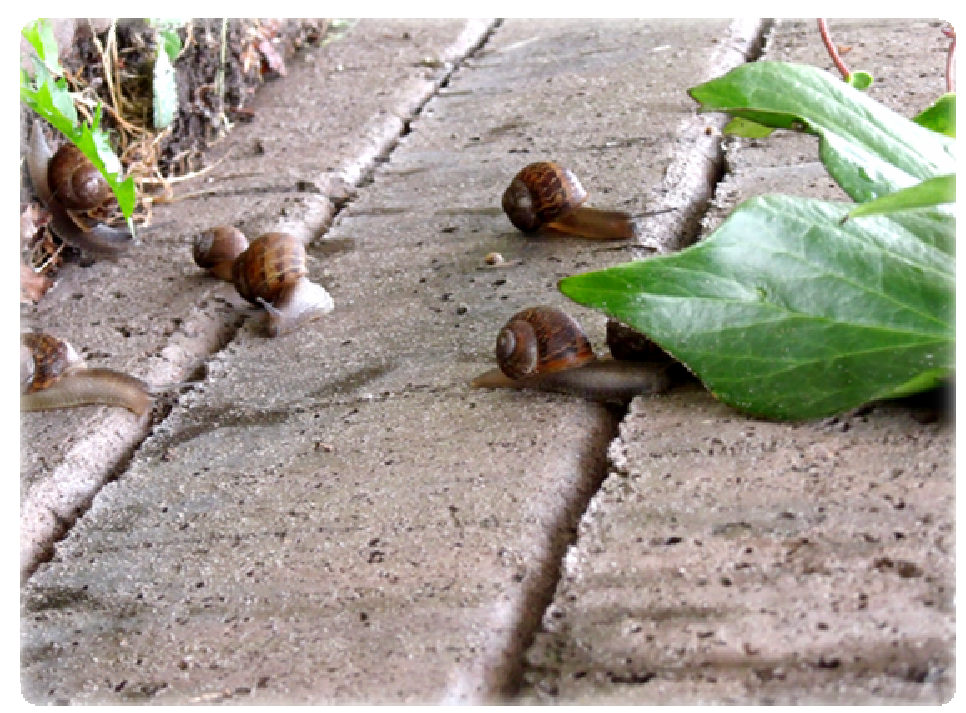

Es gibt noch viel zu tun...

Die Anzahl der bislang vorliegenden Studien mit Kontrollbedingung über die Therapie somatoformer Störungen, das heißt Einbezug einer nicht oder andersartig behandelten Kontrollgruppe, ist noch sehr klein. Rief und Hiller (1998) referieren über nur zehn Studien, wobei sich die Zahl nochmals relativiert, wenn man berücksichtigt, dass vier Publikationen von derselben Arbeitsgruppe um Smith, Rock und Arkansas aus den USA stammen. Es liegt in den Veröffentlichungen zum Gesamtthemenspektrum somatoformer Störungen ein deutlicher Überhang zur kognitiv-behavioralen Therapie vor, wobei sich bei dem geringen Anteil der gruppentherapeutischen Studien die theoretischen Konzepte zwischen Psychodynamisch und Kognitiv-behavioral 2:2 aufteilen (Weber, 2004).

Nähere Informationen zu Studien für das gesamte therapeutische Spektrum somatoformer Störungen liefern die Therapiestudien zur Behandlung von Patienten mit somatoformen Störungen von Rief und Hiller (1998).

Das besondere Interesse und der Hauptfokus dieser Arbeit liegt explizit auf gruppenspezifischen Interventionsformen für dieses Klientel.

Alle Studien befassen sich mit Patienten, die bereits eine chronifizierte Störung aufweisen und überwiegend erst in einem psychiatrisch stationären Setting angetroffen werden konnten. 
Lediglich vier Forschergruppen haben sich bisher im deutschen Sprachraum mit der Entwicklung und Evaluation gruppentherapeutischer Interventionen, speziell für somatoforme Patienten, beschäftigt. Diese sind Nickel und Egle (1999), Rief, Bleihardt und Timmer (2002), Martin, Rauh, Fichter und Rief (2007) und Weber und Tschuschke (2007).

Nickel und Egle konzipierten ein Programm von 40 ambulanten Sitzungen, das zwei Mal pro Woche mit sieben bis neun Patienten pro Gruppe stattfand, wobei die letzten zehn Sitzungen nur noch einmal in der Woche durchgeführt wurden. Nach sechs Monaten wurden Auffrischungssitzungen durchgeführt. Die Patientengruppe schloss Betroffene mit einer somatoformen Schmerzstörung ein. Sie waren in ihrer Symptomatik chronifiziert und sprachen nur schwer auf psychologische Interventionen an. Das Manual ist das erste störungsspezifische und manualisierte psychodynamische Gruppentherapieverfahren für dieses Klientel. Den theoretischen Kern bildet ein psychodynamisch-interaktioneller Ansatz.

Nickel und Egle (1999) berichten jedoch lediglich von der Konzeption des Programms sowie von einer Durchführung an sieben somatisierenden Schmerzpatientinnen, wobei nur eine Einzelfalldarstellung erfolgt. Statistische Ergebnisse werden nicht benannt.

Das Programm ist mit 40 Sitzungen und möglicher Auffrischung zudem recht umfangreich und spricht nur die auf Schmerzen eingegrenzte Gruppe somatisierender Patienten an.

Weber und Tschuschke entwickelten eine ambulante psychodynamische Kurzgruppenpsychotherapie für Patienten mit somatoformen Störungen, womit sie die Wirksamkeit tiefenpsychologischer Kurzgruppentherapie für dieses Störungsbild beweisen konnten.

Kurzgruppentherapie wird bei innen mit 20 Sitzungen à 90 Minuten definiert. Innerhalb einer Studie wurden sechs ambulante Gruppen mit insgesamt 50 Patienten untersucht.

Es gab keine Kontrollgruppe.

Doch auch hier wurden nur selektiv ausgewählte somatoforme Störungsbilder eingeschlossen (F.45 Somatisierungsstörung, F45.1 undifferenzierte Somatisierungsstörung und F45.3 somatoforme autonome Funktionsstörung). Patienten mit Schmerz-, Konversions- oder hypochondrischen Störungen wurden nicht berücksichtigt. 
Es ergaben sich sehr gute Effektstärken für eine signifikante Reduktion der Beeinträchtigungsschwere sowie ein signifikanter Anstieg in der Einschätzung des globalen Funktionsniveaus. Die größte Abnahme der psychischen und körperlichen Belastung wurde erst zwölf Monate nach Abschluss der Gruppentherapie erreicht.

Die Bedeutung und Entwicklung von Krankheitsängsten fehlt hier in der Evaluation aufgrund des Ausschlusses hypochondrischer Patienten.

Rief, Bleichhardt und Timmer (2002) entwickelten ein kognitiv-behaviorales Gruppentherapieprogramm über acht Sitzungen à 100 Minuten für Personen mit chronifizierten und multiplen somatoformen Beschwerden, die in psychosomatischen Kliniken behandelt werden. Pro Gruppe galt eine Stärke von 8 Probanden.

Eingeschlossen wurden 107 Patienten mit einem Somatisierungssyndrom mit mindestens acht Symptomen.

Die Forscher hatten besonderes Interesse an der Frage, ob dieses Gruppenangebot von dieser Patientengruppe gut angenommen und akzeptiert wird. Über $90 \%$ der Betroffenen nahmen die Behandlung in der Klinik wahr. Stundenevaluationsbögen zeigen hohe positive Bewertungen bereits ab der zweiten Therapiesitzung.

Das „One-Session treatment for patients suffering from medically unexplainend symptoms in primary care" von Martin, Rauh, Fichter und Rief (2007) stellt ein einstündiges kognitivbehaviorales Programm dar, dass an 140 Somatisierungspatienten anstatt einer standardallgemeinmedizinischen, einmaligen Behandlung getestet wurde. Jeweils 70 Patienten bildeten die Versuchs- und die Kontrollgruppe.

Diese eine Behandlungsepisode erstreckte sich über drei bis vier Stunden in einer kleinen Gruppe von zwei bis vier Patienten und einem klinischen Psychologen sowie einem Arzt für psychotherapeutische Medizin als Leitung. Vor allem beinhaltet diese Sitzung Psychoedukation, kognitive Beeinflussbarkeit der Beschwerden und Entspannungsübungen.

Besonderer Fokus der Studie lag auf der Betrachtung der Inanspruchnahme medizinischer Dienste und deren Verringerung.

Bei der Anzahl der Arztkontakte zum Facharzt und der zu Allgemeinmedizinern konnte sich nach sechs Monaten ein signifikanter Unterschied zwischen Versuchs- und Kontrollgruppe zeigen. Probanden der Versuchsgruppe verringerten die Arztbesuche mehr als Probanden der Kontrollgruppe. 
Zwar konnten nach vier Wochen in der Versuchsgruppe auch die Anzahl somatoformer Symptome sowie die Krankheitsangst geringfügig absinken, jedoch zeigten sich beide Ausgangswerte in der Kontrollgruppe niedriger und sanken ebenfalls nach vier Wochen ab.

Keine der hier aufgeführten Studien fokussierte sich auf einen direkten Kontakt zu behandelnden Hausärzten und holte sich demnach auch von diesen keine weiteren Informationen über die Patienten ein.

Alle aufgeführten Konzepte werden als gruppen-therapeutisch betitelt und setzen damit nicht niedrigschwellig an. Bis auf die Einstundenintervention gestalten sich die Konzepte insgesamt, besonders im ambulanten Setting, eher zeitlich aufwendig und daher wenig ökonomisch.

Für kurzzeitige, niedrigschwellige gruppenspezifische psychosoziale Interventionen, die in direkter Kooperation mit dem Hausarzt durchgeführt werden, liegen bislang keine Studien in Deutschland vor.

Eine Übersicht über die bisherigen gruppentherapeutischen Evaluationen zeigt Tabelle 11.

Die Heidelberger Forschungsgruppe um Herzog, Schäfert, Kaufmann und Gadow befindet sich mit inrem großen Forschungsprojekt „SpeziALL“ an der Universitätsklinik derzeit in intensiven Prozessen zu weiteren gruppenspezifischen Evaluationen.

Bisher sind zu diesem Projekt jedoch noch keine veröffentlichten Ergebnisse bekannt geworden. Schäfert et al. befassen sich in einem professionellen Team bereits seit Jahren mit somatoformen Störungen und stellen damit einen wesentlichen Anker bezüglich dieser Thematik in der Deutschen Forschungslandschaft dar. 
Tabelle 11: Übersicht über bisherige gruppentherapeutische Programme für Menschen mit somatoformen Störungen im deutschsprachigen Raum

\begin{tabular}{|c|c|c|}
\hline $\begin{array}{l}\text { Autoren und } \\
\text { Universität/Ort }\end{array}$ & Gruppendesign & Mögliche Kritikpunkte \\
\hline $\begin{array}{l}\text { Nickel und Egle } \\
(1999) \\
\text { Klinik der } \\
\text { Johannes } \\
\text { Gutenberg - } \\
\text { Universität } \\
\text { Mainz }\end{array}$ & $\begin{array}{l}40 \text { Sitzungen, zwei mal pro Woche, die } \\
\text { letzten } 10 \text { : Ein Mal die Woche, nach } \\
\text { sechs Monaten } \\
\text { Auffrischungssitzungen, sieben bis } \\
\text { neun Personen in einer Gruppe, } \\
\text { chronifizierte Patienten, die nur schwer } \\
\text { ansprechbar sind auf psychologische } \\
\text { Interventionen, psychodynamisch- } \\
\text { interaktionelles Konzept. }\end{array}$ & $\begin{array}{l}\text { > Keine statistischen Ergebnisse } \\
\text { vorhanden } \\
\text { > Geringe Probandenanzahl } \\
>\text { Eingegrenzt auf die } \\
\text { somatoforme Schmerzströung } \\
>\text { Keine Kontrollgruppe } \\
>40 \text { Sitzungen sind aufwendig } \\
\text { und lang } \\
>\text { Keine Hausarztbeteiligung }\end{array}$ \\
\hline $\begin{array}{l}\text { Rief, } \\
\text { Bleichhardt } \\
\text { und Timmer } \\
(2002) \\
\text { Klinik Roseneck, } \\
\text { Prien am } \\
\text { Chiemsee }\end{array}$ & $\begin{array}{l}\text { Acht Sitzungen für Personen mit } \\
\text { multiplen somatoformen Beschwerden, } \\
\text { die in einer psychosomatischen Klinik } \\
\text { behandelt werden, zusätzlich erhalten } \\
\text { sie parallel breites stationäres } \\
\text { Programm, kognitiv-behaviorales } \\
\text { Konzept. }\end{array}$ & $\begin{array}{l}\text { > Nur für Patienten in einer } \\
\text { psychosomatischen Klinik } \\
\text { konzipiert } \\
\text { > Mit acht Symptomen und dem } \\
\text { vollen klinischen Bild der } \\
\text { Somatisierungsstörung sehr } \\
\text { eingerenzte Probandenauswahl } \\
\text { > Keine } \\
\text { Symptomentwicklungsevaluation } \\
\text { > Kein explizit gestalteter } \\
\text { Kontakt zum weiter } \\
\text { behandelnden Hausarzt }\end{array}$ \\
\hline $\begin{array}{l}\text { Tschuschke } \\
\text { und Weber } \\
\text { (2007) } \\
\text { Universität Köln, } \\
\text { Köln- } \\
\text { Düsseldorfer } \\
\text { Studie }\end{array}$ & $\begin{array}{l}20 \text { Sitzungen für Patienten mit mittlerer } \\
\text { Erkrankungsdauer von zehn Jahren in } \\
\text { ambulantem Setting, } \\
\text { psychodynamisches Konzept. }\end{array}$ & $\begin{array}{l}\text { > Keine Kontrollgruppe } \\
\text { > Selektion der somatoformen } \\
\text { Störungsbilder } \\
\text { > Fehlende Evaluation von } \\
\text { Krankheitsängsten und deren } \\
\text { Entwicklung } \\
\text { > Keine Hausarztbeteiligung }\end{array}$ \\
\hline $\begin{array}{l}\text { Martin, Rauh, } \\
\text { Fichter und } \\
\text { Rief } \\
(2007) \\
\text { Philipps } \\
\text { Universität } \\
\text { Marburg }\end{array}$ & $\begin{array}{l}\text { Eine Treatment-Sitzung von drei bis } \\
\text { vier Stunden, pro Gruppe zwei bis vier } \\
\text { Patienten mit zwei Therapeuten, } \\
\text { kognitiv-behaviorales Konzept }\end{array}$ & $\begin{array}{l}\text { > Möglicherweise sind vier } \\
\text { Stunden für eine Frequenz zu } \\
\text { lang } \\
>\text { Eine Sitzung ist ggf. zu wenig } \\
\text { > Wenig Tiefgang und Raum für } \\
\text { Gespräche und Entwicklung von } \\
\text { Gruppendynamik } \\
>\text { Keine Hausarztbeteiligung }\end{array}$ \\
\hline
\end{tabular}




\section{$4 \quad$ Hypothesen}

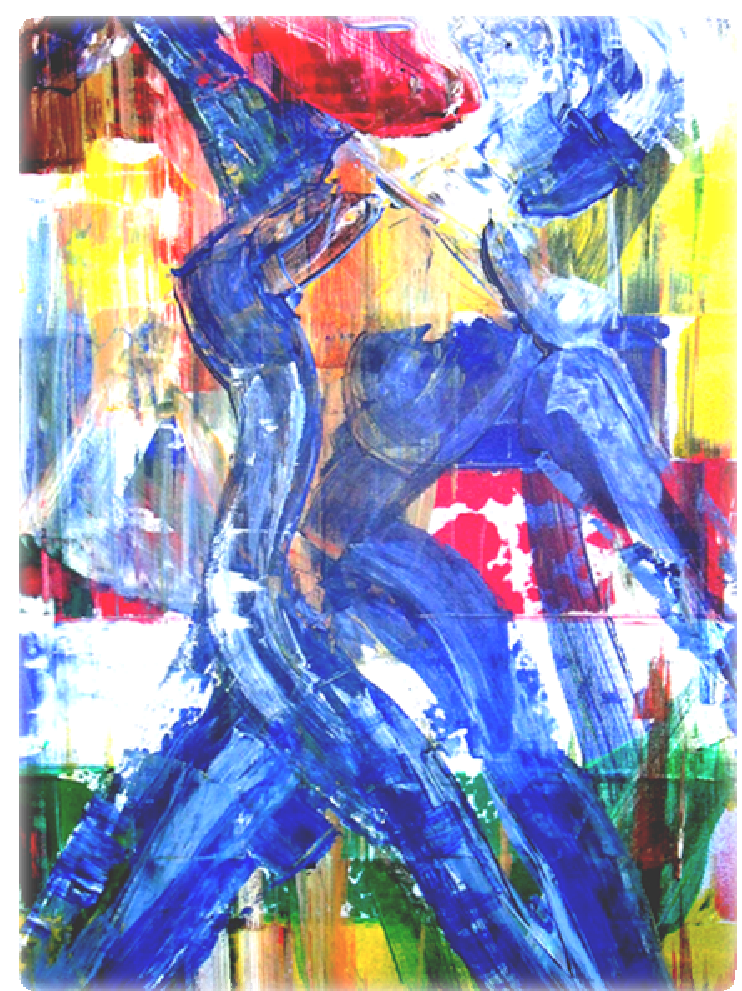

„Das Projekt ist die Inkarnation der Idee, und das Design des Projektes ist ihre Gestalt. Bei diesem Design stellt sich der Betrachter in die Mitte des Blickfeldes, es gibt keinen anderen Platz für ihn.

Der Forscher IST IN seinem Projekt, und er liebt die Anordnung der noch ungeöffneten und bestiegenen Pyramiden der Erkenntnis, die er zu erobern hofft, eine nach der andern, beraten vom weisen Ibis am Ufer seiner Wüste, und er denkt, dass er nur zwei Farben entdecken wird: Schwarz und Weiß“" (Mertens, 1992, S.20).

Auf der Grundlage des in Kapitel 3 dargestellten Stands der Forschung sowie den aufgestellten Fragestellungen wurden insgesamt 51 Forschungshypothesen aufgestellt.

Der Übersichtlichkeit halber werden die Hypothesen, angelehnt an den Fragestellungen, in acht Gruppen unterteilt, wobei aufgrund der Vielseitigkeit der Fragestellung 1 weitere Hypothesen-Subgruppen für diese gebildet werden. 


\section{Hypothesen zur Fragestellung 1}

Für die wissenschaftliche Überprüfung der Fragestellung 1 wurden unterschiedliche Kontrollvariablen gewählt. Die Hypothesensubgruppen wurden dementsprechend zusammengefasst:

1. Hypothesen zur Entwicklung der körperlichen Symptomatik

2. Hypothesen zum Erleben und Verhalten im Alltag

3. Hypothesen zum emotionalen Erleben

4. Hypothesen zur erlebten Beziehung zum Hausarzt sowie zum ÄrztehoppingVerhalten

\section{Hypothesen zur Fragestellung 2, 3 und 4}

> Hypothesen zu den Einschätzungen und Angaben der behandelnden Hausärzte

\section{Hypothesen zur Fragestellung 5}

Hypothesen zum Erleben von Resonanz in der Gruppe und der Korrelation zu den erlebten Beschwerden

\section{Hypothesen zur Fragestellung 6}

Hypothesen zum Vergleich der erlebten Symptomatik in Alltag und Gruppe

\section{Hypothesen zur Fragestellung 7}

> Hypothesen zu einem narzisstischen Verhaltensmuster

\section{Hypothesen zur Fragestellung 8}

Hypothesen zur Psychotherapiebereitschaft

Die Hypothesen werden übersichtlich in tabellarischer Form dargestellt. In der Tabelle befinden sich Hypothese, die dazugehörigen Untersuchungsinstrumente und, wenn möglich, das konkrete Einzel-Item beziehungsweise der Verweis auf die Gesamtskala, die der Prüfung dieser Hypothese dienen sowie die dazugehörige Fragestellung.

Eine genaue Beschreibung der herangezogenen Untersuchungsmethodik erfolgt in Kapitel 5.

Für alle Hypothesentabellen gilt die Abkürzung VG = Versuchsgruppe. 


\section{Hauptfokus der Studie: Hypothesengruppe „Entwicklung der Symptomatik“}

Das Hautpmerkmal somtatoformer Störungen sind körperliche Beschwerden ohne ausreichenden medizinischen Befund. Daher ist es nachvollziehbar, dass der Hauptfokus in den Hypothesen auf die Entwicklung der Symptomatik gelegt wird. Gerade weil die Veränderung der Symptome als sehr schwer eingestuft wird, und auch eine geringfügige Verbesserung bereits als Erfolg gilt, gebührt der Evaluation des körperlichen Empfindens die Hauptaufmerksamkeit innerhalb dieser Studie. Tabelle 12 zeigt Hypothesen 1-15 auf den Seiten 57-60, die der ersten Subgruppe „Hypothesen zur Entwicklung der somatoformen körperlichen Symptomatik" angehören.

Tabelle 12: Hypothesen zur Entwicklung der körperlichen Symptomatik

\begin{tabular}{|c|c|c|c|c|}
\hline & Hypothese & $\begin{array}{l}\text { Untersuchungs- } \\
\text { instrument }\end{array}$ & Konkrete/s Item/s & $\begin{array}{l}\text { Frage- } \\
\text { stellung }\end{array}$ \\
\hline & $\begin{array}{l}\text { Patienten der VG } \\
\text { zeigen im } \\
\text { Posttest eine } \\
\text { signifikant } \\
\text { geringer erlebte } \\
\text { Beein- } \\
\text { trächtigung } \\
\text { durch ihre } \\
\text { Beschwerden } \\
\text { als im Pretest. }\end{array}$ & $\begin{array}{l}\text { PHQ } 15 \text { - Patient } \\
\text { Health } \\
\text { Questionnaire } \\
\text { (Löwe) } \\
\text { Pretest und } \\
\text { Posttest }\end{array}$ & $\begin{array}{l}\text { Wie stark fühlten Sie sich } \\
\text { in den letzten } 8 \\
\text { Wochen durch die } \\
\text { folgenden Beschwerden } \\
\text { beeinträchtigt? } \\
\text { a) Bauchschmerzen } \\
\text { b) } \\
\text { Rückenschmerzen....usw. } \\
\rightarrow \text { Nicht, Wenig, Stark } \\
\text { beeinträchtigt }\end{array}$ & 1 \\
\hline 2. & $\begin{array}{l}\text { Patienten der VG } \\
\text { zeigen im Follow } \\
\text { Up eine } \\
\text { signifikant } \\
\text { geringer erlebte } \\
\text { Beein- } \\
\text { trächtigung } \\
\text { durch ihre } \\
\text { Beschwerden } \\
\text { als im Pretetst. }\end{array}$ & $\begin{array}{l}\text { PHQ 15 - Patient } \\
\text { Health } \\
\text { Questionnaire } \\
\text { (Löwe) } \\
\text { Pretest und } \\
\text { Follow Up }\end{array}$ & II & 1 \\
\hline 3. & $\begin{array}{l}\text { Patienten der VG } \\
\text { zeigen im Follow } \\
\text { Up eine } \\
\text { signifikant } \\
\text { geringer erlebte } \\
\text { Beein- } \\
\text { trächtigung } \\
\text { durch ihre } \\
\text { Beschwerden } \\
\text { als im Posttest. }\end{array}$ & $\begin{array}{l}\text { PHQ } 15 \text { - Patient } \\
\text { Health } \\
\text { Questionnaire } \\
\text { (Löwe) } \\
\text { Posttest und } \\
\text { Follow Up }\end{array}$ & II & 1 \\
\hline
\end{tabular}




\begin{tabular}{|c|c|c|c|}
\hline Fortsetzung Tab. 12 & & & \\
\hline Hypothese & $\begin{array}{l}\text { Untersuchungs- } \\
\text { instrument }\end{array}$ & Konkrete/s Item/s & $\begin{array}{l}\text { Frage- } \\
\text { stellung }\end{array}$ \\
\hline $\begin{array}{l}\text { 4. Patienten der VG } \\
\text { zeigen im } \\
\text { Posttest eine } \\
\text { signifikant } \\
\text { geringer erlebte } \\
\text { Intensität ihrer } \\
\text { Beschwerden } \\
\text { als im Pretest. }\end{array}$ & $\begin{array}{l}\text { SOMS - Screening } \\
\text { für somatoforme } \\
\text { Störungen (Rief und } \\
\text { Hiller) } \\
\text { Pretest und } \\
\text { Posttest }\end{array}$ & $\begin{array}{l}\text { Alle } 53 \text { Items des } \\
\text { SOMS } 7 \\
\text { (standardisiert) }\end{array}$ & 1 \\
\hline $\begin{array}{l}\text { 5. Patienten der VG } \\
\text { zeigen im Follow } \\
\text { Up eine } \\
\text { signifikant } \\
\text { geringer erlebte } \\
\text { Intensität ihrer } \\
\text { Beschwerden } \\
\text { als im Pretest. }\end{array}$ & $\begin{array}{l}\text { soms - Screening } \\
\text { für somatoforme } \\
\text { Störungen (Rief und } \\
\text { Hiller) } \\
\text { Pretest und } \\
\text { Follow Up }\end{array}$ & II & 1 \\
\hline $\begin{array}{l}\text { 6. Patienten der VG } \\
\text { zeigen im Follow } \\
\text { Up eine } \\
\text { signifikant } \\
\text { geringer erlebte } \\
\text { Intensität ihrer } \\
\text { Beschwerden } \\
\text { als im Posttest. }\end{array}$ & $\begin{array}{l}\text { SOMS - Screening } \\
\text { für somatoforme } \\
\text { Störungen (Rief und } \\
\text { Hiller) } \\
\text { Posttest und } \\
\text { Follow Up }\end{array}$ & II & 1 \\
\hline $\begin{array}{l}\text { 7. Patienten der VG } \\
\text { zeigen im } \\
\text { Posttest eine } \\
\text { signifikant } \\
\text { geringere } \\
\text { Anzahl an } \\
\text { Beschwerden } \\
\text { als im Pretest. }\end{array}$ & $\begin{array}{l}\text { SOMS - Screening } \\
\text { für somatoforme } \\
\text { Störungen (Rief und } \\
\text { Hiller) } \\
\text { Pretest und } \\
\text { Posttest }\end{array}$ & II & 1 \\
\hline $\begin{array}{l}\text { 8. Patienten der VG } \\
\text { zeigen im Follow } \\
\text { Up eine } \\
\text { signifikant } \\
\text { geringere } \\
\text { Anzahl an } \\
\text { Beschwerden } \\
\text { als im Pretest. }\end{array}$ & $\begin{array}{l}\text { SOMS - Screening } \\
\text { für somatoforme } \\
\text { Störungen (Rief und } \\
\text { Hiller) } \\
\text { Pretest und } \\
\text { Follow Up }\end{array}$ & II & 1 \\
\hline
\end{tabular}




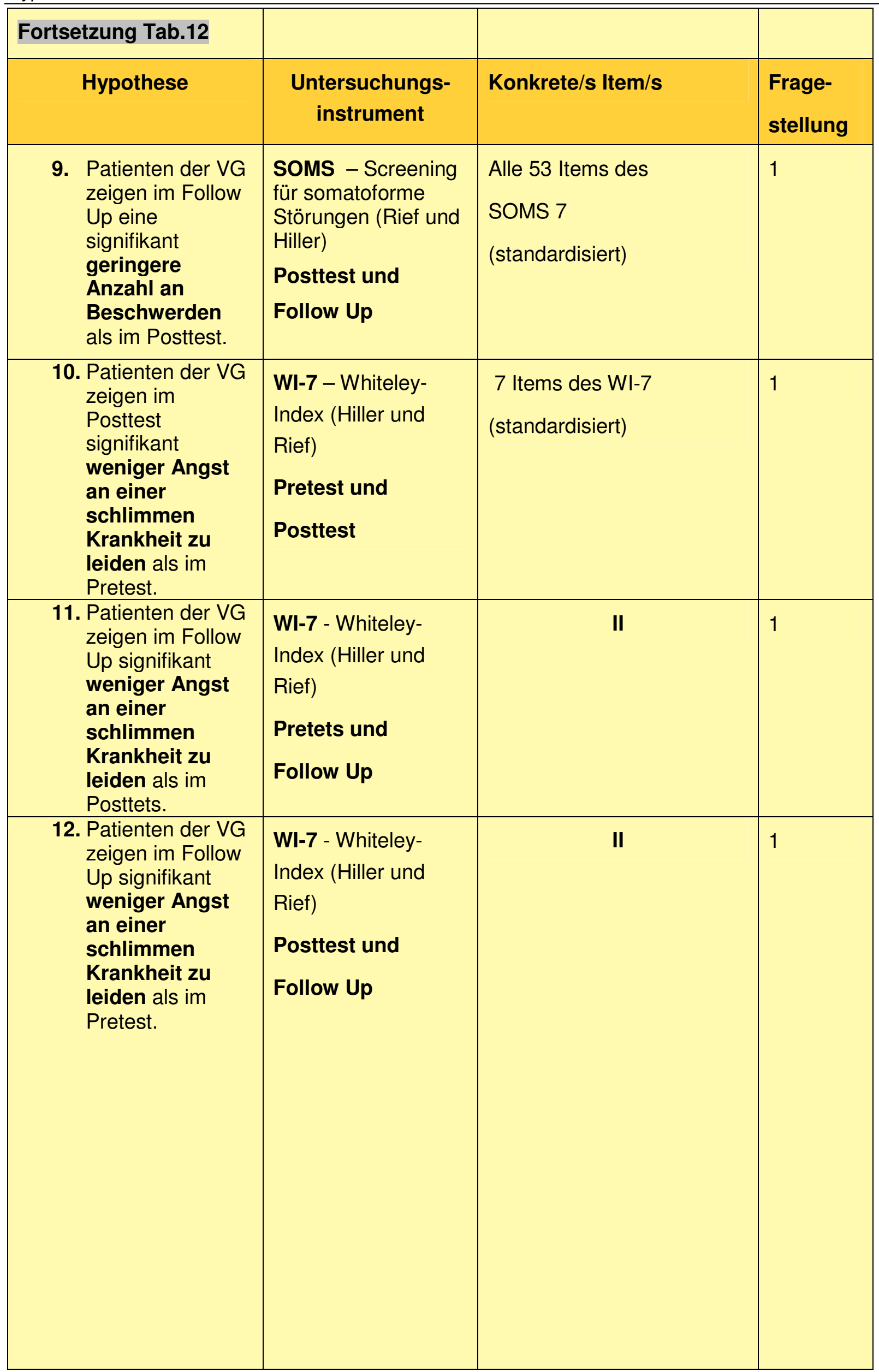




\begin{tabular}{|c|c|c|c|}
\hline \multicolumn{4}{|l|}{ Fortsetzung Tab.12 } \\
\hline Hypothese & $\begin{array}{l}\text { Untersuchungs- } \\
\text { instrument }\end{array}$ & Konkrete/s Item/s & $\begin{array}{l}\text { Frage- } \\
\text { stellung }\end{array}$ \\
\hline $\begin{array}{l}\text { 13. Im Posttest } \\
\text { geben Probanden } \\
\text { der VG eine } \\
\text { signifikant } \\
\text { geringer erlebte } \\
\text { Stärke der } \\
\text { körperlichen } \\
\text { Beschwerden an } \\
\text { als im Pretest. }\end{array}$ & $\begin{array}{l}\text { Ratingskala } \\
\text { Pretest und } \\
\text { Posttest }\end{array}$ & $\begin{array}{l}\text { Wie stark erleben Sie } \\
\text { derzeit Ihre körperlichen } \\
\text { Beschwerden auf einer } \\
\text { Skala von } 0 \text { bis } 10 \text {, bitte } \\
\text { kreuzen Sie an: } \\
0=\text { keine Beschwerden } \\
10=\text { extrem starke } \\
\text { Beschwerden }\end{array}$ & 1 \\
\hline $\begin{array}{l}\text { 14. Im Follow Up } \\
\text { geben Probanden } \\
\text { der VG eine } \\
\text { signifikant } \\
\text { geringer erlebte } \\
\text { Stärke der } \\
\text { körperlichen } \\
\text { Beschwerden an } \\
\text { als im Pretest. }\end{array}$ & $\begin{array}{l}\text { Ratingskala } \\
\text { Pretest und } \\
\text { Follow Up }\end{array}$ & II & 1 \\
\hline $\begin{array}{l}\text { 15. Im Follow Up } \\
\text { geben Probanden } \\
\text { der VG eine } \\
\text { signifikant } \\
\text { geringer erlebte } \\
\text { Stärke der } \\
\text { körperlichen } \\
\text { Beschwerden an } \\
\text { als im Posttest. }\end{array}$ & $\begin{array}{l}\text { Ratingskala } \\
\text { Posttest und } \\
\text { Follow Up }\end{array}$ & II & 1 \\
\hline
\end{tabular}

Aufbauend auf den Fokushypothesen zur Entwicklung der körperlichen Symptomatik sind die folgenden weiteren Bereiche von großem Interesse. 


\section{Das Erleben und Verhalten}

Wie im Stand der Forschung deutlich wurde, ist das Erleben und Verhalten der Patienten, gerade nach Ausbruch der Symptomatik, häufig durch Enttäuschung und misslingende Beziehungsversuche geprägt. Die Betroffenen geraten in einen Kreislauf von negativen Erfahrungen und zunehmender Hoffnungslosigkeit und Pessimissmuss. Die Hypothesen 16-18 zum Erleben und Verhalten erscheinen vor dem Bewusstsein dieser Aspekte als besonders interessant. Tabelle 13 zeigt die Hypothesen zum Erleben und Verhalten.

Tabelle 13: Hypothesen zum Erleben und Verhalten

\begin{tabular}{|c|l|l|l|}
\hline Hypothese & $\begin{array}{l}\text { Untersuchungs- } \\
\text { instrument }\end{array}$ & Konkrete/s Item/s & Frage- \\
stellung
\end{tabular}




\section{Das emotionale Erleben}

Durch das frühe Versagen einer Reaktion auf das Äußern emotionaler Bedürftigkeit und das später aufgebaute narzisstisch-altruistische Schutzverhaltensmuster, ist es vielen Betroffenen nicht möglich, angemessen eigene Emotionen zu erleben, wahrzunehmen und sie in Beziehung zu körperlichen Symptomen zu bringen. Ihnen fehlen häufig die „Übung“ und der Erfolg im emotionalen Klagen sowie ein angemessener Umgang mit eigenen Gefühlen. Hypothesen 19-30 in Tabelle 14 auf den Seiten 62-65 befassen sich mit der Untersuchung dieser Thematik.

Tabelle 14: Hypothesen zum emotionalen Erleben

\begin{tabular}{|l|l|l|l|}
\hline $\begin{array}{l}\text { Hypothese } \\
\text { instrument }\end{array}$ & Konkrete/s Item/s & Frage- \\
stellung
\end{tabular}




\begin{tabular}{|c|c|c|c|}
\hline Fortsetzung Tab.14 & & & \\
\hline Hypothese & $\begin{array}{l}\text { Untersuchungs- } \\
\text { instrument }\end{array}$ & Konkrete/s Item/s & $\begin{array}{l}\text { Frage- } \\
\text { stellung }\end{array}$ \\
\hline $\begin{array}{l}\text { 22. Patienten der VG } \\
\text { zeigen im } \\
\text { Posttest ein } \\
\text { signifikant } \\
\text { weniger großes } \\
\text { Erleben von } \\
\text { Emotions- } \\
\text { mangel als im } \\
\text { Pretest. }\end{array}$ & $\begin{array}{l}\text { SEE - Skalen zum } \\
\text { Erleben von } \\
\text { Emotionen (von M. } \\
\text { Behr und M. Becker) } \\
\text { Pretest und } \\
\text { Posttest }\end{array}$ & $\begin{array}{l}\text { Siehe standardisierte } \\
\text { Skala }\end{array}$ & 1 \\
\hline $\begin{array}{l}\text { 23. Patienten der VG } \\
\text { zeigen im Follow } \\
\text { Up ein signifikant } \\
\text { weniger großes } \\
\text { Erleben von } \\
\text { Emotions- } \\
\text { mangel als im } \\
\text { Pretest. }\end{array}$ & $\begin{array}{l}\text { SEE - Skalen zum } \\
\text { Erleben von } \\
\text { Emotionen (von M. } \\
\text { Behr und M. Becker) } \\
\text { Pretest und } \\
\text { Follow Up }\end{array}$ & II & 1 \\
\hline $\begin{array}{l}\text { 24. Patienten der VG } \\
\text { zeigen im Follow } \\
\text { Up ein signifikant } \\
\text { weniger großes } \\
\text { Erleben von } \\
\text { Emotions- } \\
\text { mangel als im } \\
\text { Posttest. }\end{array}$ & $\begin{array}{l}\text { SEE - Skalen zum } \\
\text { Erleben von } \\
\text { Emotionen (von M. } \\
\text { Behr und M. Becker) } \\
\text { Posttest und } \\
\text { Follow Up }\end{array}$ & II & 1 \\
\hline $\begin{array}{l}\text { 25. Patienten der VG } \\
\text { (A) zeigen im } \\
\text { Posttest ein } \\
\text { signifikant } \\
\text { größeres } \\
\text { Verständnis für } \\
\text { die Verbindung } \\
\text { zwischen } \\
\text { körperlichen } \\
\text { Beschwerden } \\
\text { und ihrem } \\
\text { seelischen } \\
\text { Befinden als im } \\
\text { Pretest. }\end{array}$ & $\begin{array}{l}\text { SEE - Skalen zum } \\
\text { Erleben von } \\
\text { Emotionen (von M. } \\
\text { Behr und M. Becker) } \\
\text { Pretest und } \\
\text { Posttest }\end{array}$ & $\begin{array}{l}\text { Siehe standardisierte } \\
\text { Skala }\end{array}$ & 1 \\
\hline
\end{tabular}




\begin{tabular}{|c|c|c|c|}
\hline \multicolumn{4}{|l|}{ Fortsetzung Tab.14 } \\
\hline Hypothese & $\begin{array}{l}\text { Untersuchungs- } \\
\text { instrument }\end{array}$ & Konkrete/s Item/s & $\begin{array}{l}\text { Frage- } \\
\text { stellung }\end{array}$ \\
\hline $\begin{array}{l}\text { 26. Patienten der VG } \\
\text { zeigen im Follow } \\
\text { Up ein signifikant } \\
\text { größeres } \\
\text { Verständnis für } \\
\text { die Verbindung } \\
\text { zwischen } \\
\text { körperlichen } \\
\text { Beschwerden } \\
\text { und ihrem } \\
\text { seelischen } \\
\text { Befinden als im } \\
\text { Pretest. }\end{array}$ & $\begin{array}{l}\text { SEE - Skalen zum } \\
\text { Erleben von } \\
\text { Emotionen (von M. } \\
\text { Behr und M. Becker) } \\
\text { Pretest und } \\
\text { Follow Up }\end{array}$ & $\begin{array}{l}\text { Siehe standardisierte } \\
\text { Skala }\end{array}$ & 1 \\
\hline $\begin{array}{l}\text { 27. Patienten der VG } \\
\text { zeigen im Follow } \\
\text { Up ein signifikant } \\
\text { größeres } \\
\text { Verständnis für } \\
\text { die Verbindung } \\
\text { zwischen } \\
\text { körperlichen } \\
\text { Beschwerden } \\
\text { und ihrem } \\
\text { seelischen } \\
\text { Befinden als im } \\
\text { Posttest. }\end{array}$ & $\begin{array}{l}\text { SEE - Skalen zum } \\
\text { Erleben von } \\
\text { Emotionen (von M. } \\
\text { Behr und M. Becker) } \\
\text { Posttest und } \\
\text { Follow Up }\end{array}$ & II & 1 \\
\hline $\begin{array}{l}\text { 28. Patienten der VG } \\
\text { zeigen im } \\
\text { Posttest eine } \\
\text { signifikant } \\
\text { bessere selbst } \\
\text { eingeschätzte } \\
\text { Fähigkeit zur } \\
\text { Wahrnehmung } \\
\text { ihrer eigenen } \\
\text { Gefühle als im } \\
\text { Pretest. }\end{array}$ & $\begin{array}{l}\text { Ratingskala } \\
\text { Pretest und } \\
\text { Posttest }\end{array}$ & $\begin{array}{l}\text { Wie gut können Sie nach } \\
\text { Ihrer eigenen Ein- } \\
\text { schätzung auf einer } \\
\text { Skala von } 0 \text { bis } 10 \\
\text { derzeit Ihre eigenen } \\
\text { Gefühle wahrnehmen? } \\
\text { Bitte kreuzen Sie an: } \\
0=\text { überhaupt nicht } \\
10=\text { sehr gut }\end{array}$ & 1 \\
\hline $\begin{array}{l}\text { 29. Patienten der VG } \\
\text { zeigen im } \\
\text { Posttest eine } \\
\text { signifikant } \\
\text { bessere selbst } \\
\text { eingeschätzte } \\
\text { Fähigkeit zur } \\
\text { Wahrnehmung } \\
\text { ihrer eigenen } \\
\text { Gefühle als im } \\
\text { Pretest. }\end{array}$ & $\begin{array}{l}\text { Ratingskala } \\
\text { Pretest und } \\
\text { Follow Up }\end{array}$ & II & 1 \\
\hline
\end{tabular}




\begin{tabular}{|c|c|c|c|}
\hline \multicolumn{4}{|l|}{ Fortsetzung Tab.14 } \\
\hline Hypothese & $\begin{array}{l}\text { Untersuchungs- } \\
\text { instrument }\end{array}$ & Konkrete/s Item/s & $\begin{array}{l}\text { Frage- } \\
\text { stellung }\end{array}$ \\
\hline $\begin{array}{l}\text { 30. Patienten der VG } \\
\text { zeigen im Follow } \\
\text { Up eine } \\
\text { signifikant } \\
\text { bessere selbst } \\
\text { eingeschätzte } \\
\text { Fähigkeit zur } \\
\text { Wahrnehmung } \\
\text { ihrer eigenen } \\
\text { Gefühle als im } \\
\text { Posttest. }\end{array}$ & $\begin{array}{l}\text { Ratingskala } \\
\text { Posttest und } \\
\text { Follow Up }\end{array}$ & $\begin{array}{l}\text { Wie gut können Sie nach } \\
\text { Ihrer eigenen Ein- } \\
\text { schätzung auf einer } \\
\text { Skala von } 0 \text { bis } 10 \\
\text { derzeit Ihre eigenen } \\
\text { Gefühle wahrnehmen? } \\
\text { Bitte kreuzen Sie an: } \\
0=\text { überhaupt nicht } \\
10=\text { sehr gut }\end{array}$ & 1 \\
\hline
\end{tabular}

\section{Die Beziehung zum Hausarzt und das Ärztehopping-Verhalten}

In Kapitel 3.4 wurde über die Problematiken in der Arzt-Patient-Beziehung berichtet. Es wäre sinnvoll und, ein wichtiges Ziel im Rahmen einer Maßnahme, das ständige Ärztehopping zu vermeiden und eine konstante und vertrauensvolle Beziehung zum behandelnden Arzt zu erschaffen. Hypothesen 31-36 in Tabelle 15 auf den Seiten 65-67 konzentrieren sich auf diese Thematik.

Tabelle 15: Hypothesen zur Arztbeziehung und dem Ärztehopping

\begin{tabular}{|c|c|c|c|}
\hline Hypothese & $\begin{array}{l}\text { Untersuchungs- } \\
\text { instrument }\end{array}$ & Konkrete/s Item/s & $\begin{array}{l}\text { Frage- } \\
\text { stellung }\end{array}$ \\
\hline $\begin{array}{l}\text { 31. Im Posttest } \\
\text { bewerten die } \\
\text { Probanden der } \\
\text { VG die } \\
\text { Beziehung zu } \\
\text { ihrem Hausarzt } \\
\text { signifikant besser } \\
\text { als im Pretest. }\end{array}$ & $\begin{array}{l}\text { Ratingskala } \\
\text { Pretest und } \\
\text { Posttest }\end{array}$ & $\begin{array}{l}\text { Wie bewerten Sie mit } \\
\text { einer Note von 1-5 die } \\
\text { derzeitige Beziehung zu } \\
\text { Ihrem behandelnden } \\
\text { Hausarzt? Bitte kreuzen } \\
\text { Sie an: } \\
\text { Bewertung: } \\
\text { O Sehr gut (1) } \\
\text { O Gut (2) } \\
\text { O Befriedigend (3) } \\
\text { O Ausreichend (4) } \\
\text { O Mangelhaft (5) }\end{array}$ & 1 \\
\hline
\end{tabular}




\begin{tabular}{|c|c|c|c|}
\hline \multicolumn{4}{|l|}{ Fortsetzung Tab.15 } \\
\hline Hypothese & $\begin{array}{l}\text { Untersuchungs- } \\
\text { instrument }\end{array}$ & Konkrete/s Item/s & $\begin{array}{l}\text { Frage- } \\
\text { stellung }\end{array}$ \\
\hline $\begin{array}{l}\text { 32. Im Follow Up } \\
\text { bewerten die } \\
\text { Probanden der } \\
\text { VG die } \\
\text { Beziehung zu } \\
\text { ihrem Hausarzt } \\
\text { signifikant besser } \\
\text { als im Pretest. }\end{array}$ & $\begin{array}{l}\text { Ratingskala } \\
\text { Pretest und } \\
\text { Follow Up }\end{array}$ & $\begin{array}{l}\text { Wie bewerten Sie mit } \\
\text { einer Note von 1-5 die } \\
\text { derzeitige Beziehung zu } \\
\text { lhrem behandelnden } \\
\text { Hausarzt? Bitte kreuzen } \\
\text { Sie an: } \\
\text { Bewertung: } \\
\text { O Sehr gut (1) } \\
\text { O Gut (2) } \\
\text { O Befriedigend (3) } \\
\text { O Ausreichend (4) } \\
\text { O Mangelhaft (5) }\end{array}$ & 1 \\
\hline $\begin{array}{l}\text { 33. Im Follow Up } \\
\text { bewerten die } \\
\text { Probanden der } \\
\text { VG die } \\
\text { Beziehung zu } \\
\text { ihrem Hausarzt } \\
\text { signifikant besser } \\
\text { als im Posttest. }\end{array}$ & $\begin{array}{l}\text { Ratingskala } \\
\text { Posttest und } \\
\text { Follow Up }\end{array}$ & II & 1 \\
\hline $\begin{array}{l}\text { 34. Im Posttest } \\
\text { geben Probanden } \\
\text { der VG eine } \\
\text { signifikant } \\
\text { geringere Anzahl } \\
\text { der Arztwechsel } \\
\text { an als im Pretest. }\end{array}$ & $\begin{array}{l}\text { Einzelitem } \\
\text { Pretest und } \\
\text { Posttest }\end{array}$ & $\begin{array}{l}\text { Wie oft haben Sie in den } \\
\text { letzten } 2 \text { Monaten den } \\
\text { Hausarzt gewechselt? } \\
\text { Bitte kreuzen Sie an: } \\
\text {...................x(mal) }\end{array}$ & 1 \\
\hline $\begin{array}{l}\text { 35. Im Follow Up } \\
\text { geben Probanden } \\
\text { der VG eine } \\
\text { signifikant } \\
\text { geringere Anzahl } \\
\text { der Arztwechsel } \\
\text { an als im Pretest. }\end{array}$ & $\begin{array}{l}\text { Einzelitem } \\
\text { Pretest und } \\
\text { Follow Up }\end{array}$ & II & 1 \\
\hline
\end{tabular}




\begin{tabular}{|c|c|c|c|}
\hline \multicolumn{4}{|l|}{ Fortsetzung Tab.15 } \\
\hline Hypothese & $\begin{array}{l}\text { Untersuchungs- } \\
\text { instrument }\end{array}$ & Konkrete/s Item/s & $\begin{array}{l}\text { Frage- } \\
\text { stellung }\end{array}$ \\
\hline $\begin{array}{l}\text { 36. Im Follow Up } \\
\text { geben Probanden } \\
\text { der VG eine } \\
\text { signifikant } \\
\text { geringere Anzahl } \\
\text { der Arztwechsel } \\
\text { an als im } \\
\text { Posttest. }\end{array}$ & $\begin{array}{l}\text { Einzelitem } \\
\text { Posttest und } \\
\text { Follow Up }\end{array}$ & $\begin{array}{l}\text { Wie oft haben Sie in den } \\
\text { letzten } 2 \text { Monaten den } \\
\text { Hausarzt gewechselt? } \\
\text { Bitte kreuzen Sie an: } \\
\text {..................x(mal) }\end{array}$ & 1 \\
\hline
\end{tabular}

\section{Einschätzungen und Angaben der behandelnden Hausärzte}

Die Anzahl der Arztkontakte und das Fordern nach medizinischen Untersuchungen stellen sich bei somatoformen Patienten, wie in Kapitel 3.3 aufgezeigt, als problematisch hoch für das Gesundheitssystem dar. Aber auch der Leidensweg für die Patienten wird dadurch nicht verkürzt. Die Enttäuschung einer erneuten nicht medizinischen Diagnose ist vorprogrammiert. Es ist daher von Interesse, wie sich die Anzahl der Arztkontakte über den Studienverlauf hinweg entwickeln als auch, inwiefern die behandelnden Ärzte sich weiterhin dazu aufgefordert fühlen, zusätzliche medizinische Untersuchungen durchzuführen.

Zudem ist von Seiten der Ärzte die erlebte Beziehungsqualität von großer Bedeutung. Ausgelebte negative Gegenübertragungsgefühle tragen nicht zu einer konstanten und vertrauten Beziehung bei. Hypothesen 37-45 in Tabelle 16 auf den Seiten 68 -70 widmen sich diesem Forschungsinteresse. 
Tabelle 16: Hypothesen zu den Einschätzungen und Angaben der behandelnden Hausärzte

\begin{tabular}{|c|c|c|c|}
\hline Hypothese & $\begin{array}{l}\text { Untersuchungs- } \\
\text { instrument }\end{array}$ & Konkrete/s Item/s & $\begin{array}{l}\text { Frage- } \\
\text { stellung }\end{array}$ \\
\hline $\begin{array}{l}\text { 37. Im Posttest } \\
\text { bewerten die } \\
\text { behandelnden } \\
\text { Ärzte der } \\
\text { Probanden der } \\
\text { Versuchsgruppe } \\
\text { die Beziehung } \\
\text { zu ihren } \\
\text { Patienten } \\
\text { signifikant besser } \\
\text { als im Pretest. }\end{array}$ & $\begin{array}{l}\text { Arztfragebogen } \\
\text { (Frage1) } \\
\text { Ratingskala } \\
\text { entsprechende } \\
\text { Frage wie Patienten } \\
\text { Pretest und } \\
\text { Follow Up }\end{array}$ & $\begin{array}{l}\text { Wie bewerten Sie mit } \\
\text { einer Note von 1-5 die } \\
\text { derzeitige Beziehung zu } \\
\text { Ihrem Patienten? Bitte } \\
\text { kreuzen Sie an: } \\
\text { Bewertung: } \\
\text { O Sehr gut (1) } \\
\text { O Gut (2) } \\
\text { O Befriedigend (3) } \\
\text { O Ausreichend (4) } \\
\text { O Mangelhaft (5) }\end{array}$ & 2 \\
\hline $\begin{array}{l}\text { 38. Im Follow Up } \\
\text { bewerten die } \\
\text { behandelnden } \\
\text { Ärzte der } \\
\text { Probanden der } \\
\text { Versuchsgruppe } \\
\text { die Beziehung } \\
\text { zu ihren } \\
\text { Patienten } \\
\text { signifikant besser } \\
\text { als im Pretest. }\end{array}$ & $\begin{array}{l}\text { Arztfragebogen } \\
\text { (Frage 1) } \\
\text { Ratingskala } \\
\text { entsprechende } \\
\text { Frage wie Patienten } \\
\text { Pretest und } \\
\text { Follow Up }\end{array}$ & II & 2 \\
\hline $\begin{array}{l}\text { 39. Im Follow Up } \\
\text { bewerten die } \\
\text { behandelnden } \\
\text { Ärzte der } \\
\text { Probanden der } \\
\text { Versuchsgruppe } \\
\text { die Beziehung } \\
\text { zu ihren } \\
\text { Patienten } \\
\text { signifikant besser } \\
\text { als im Posttest. }\end{array}$ & $\begin{array}{l}\text { Arztfragebogen } \\
\text { (Frage 1) } \\
\text { Ratingskala } \\
\text { entsprechende } \\
\text { Frage wie Patienten } \\
\text { Posttest und } \\
\text { Follow Up }\end{array}$ & II & 2 \\
\hline
\end{tabular}




\begin{tabular}{|c|c|c|c|}
\hline Fortsetzung Tab.16 & & & \\
\hline Hypothese & $\begin{array}{l}\text { Untersuchungs- } \\
\text { instrument }\end{array}$ & Konkrete/s Item/s & $\begin{array}{l}\text { Frage- } \\
\text { stellung }\end{array}$ \\
\hline $\begin{array}{l}\text { 40. Im Posttest } \\
\text { geben die } \\
\text { behandelnden } \\
\text { Ärzte bei } \\
\text { Probanden der } \\
\text { VG im Hinblick } \\
\text { auf die letzten } 2 \\
\text { Monate eine } \\
\text { signifikant } \\
\text { geringe Anzahl } \\
\text { von } \\
\text { Arztkonsultation } \\
\text { en an als im } \\
\text { Pretest. }\end{array}$ & $\begin{array}{l}\text { Arztfragebogen } \\
\text { (Frage 2) } \\
\text { Einzelitem } \\
\text { Pretest und } \\
\text { Posttest }\end{array}$ & $\begin{array}{l}\text { Wie oft hat der Patient } \\
\text { Mr. X Sie in den letzten } 2 \\
\text { Monaten konsultiert? } \\
\text {............... } \text { (mal) }\end{array}$ & 3 \\
\hline $\begin{array}{l}\text { 41. Im Follow Up } \\
\text { geben die } \\
\text { behandelnden } \\
\text { Ärzte bei } \\
\text { Probanden der } \\
\text { VG im Hinblick } \\
\text { auf die letzten } 2 \\
\text { Monate eine } \\
\text { signifikant } \\
\text { geringe Anzahl } \\
\text { von } \\
\text { Arztkonsultation } \\
\text { en an als im } \\
\text { Pretest. }\end{array}$ & $\begin{array}{l}\text { Arztfragebogen } \\
\text { (Frage 2) } \\
\text { Einzelitem } \\
\text { Pretest und } \\
\text { Follow Up }\end{array}$ & II & 3 \\
\hline $\begin{array}{l}\text { 42. Im Follow Up } \\
\text { geben die } \\
\text { behandelnden } \\
\text { Ärzte bei } \\
\text { Probanden der } \\
\text { VG im Hinblick } \\
\text { auf die letzten } 2 \\
\text { Monate eine } \\
\text { signifikant } \\
\text { geringe Anzahl } \\
\text { von } \\
\text { Arztkonsultation } \\
\text { en an als im } \\
\text { Posttest. }\end{array}$ & $\begin{array}{l}\text { Arztfragebogen } \\
\text { (Frage 2) } \\
\text { Einzelitem } \\
\text { Posttest und } \\
\text { Follow Up }\end{array}$ & II & 3 \\
\hline
\end{tabular}




\begin{tabular}{|c|c|c|c|}
\hline \multicolumn{4}{|l|}{ Fortsetzung Tab.16 } \\
\hline Hypothese & $\begin{array}{l}\text { Untersuchungs- } \\
\text { instrument }\end{array}$ & Konkrete/s Item/s & $\begin{array}{l}\text { Frage- } \\
\text { stellung }\end{array}$ \\
\hline $\begin{array}{l}\text { 43. Im Posttest } \\
\text { geben die } \\
\text { behandelnden } \\
\text { Ärzte bei } \\
\text { Probanden der } \\
\text { VG im Hinblick } \\
\text { auf die letzten } 2 \\
\text { Monate ein } \\
\text { signifikant } \\
\text { geringeres } \\
\text { Ausmaß an } \\
\text { Forderungen } \\
\text { nach weiteren } \\
\text { medizinischen } \\
\text { Untersuchungen } \\
\text { an als im Pretest. }\end{array}$ & $\begin{array}{l}\text { Arztfragebogen } \\
\text { (Frage 3) } \\
\text { Ratingskala } \\
\text { Pretest und } \\
\text { Posttest }\end{array}$ & $\begin{array}{l}\text { Wie ausgeprägt ist } \\
\text { derzeit das Ausmaß der } \\
\text { Forderungen des } \\
\text { Patienten nach weiteren } \\
\text { medizinischen } \\
\text { Untersuchungen? Bitte } \\
\text { kreuzen Sie an: } \\
\begin{array}{ll}0 \ldots . . .1 . . .2 \ldots 3 . . . .4 \\
\text { gar nicht } & \text { (0) } \\
\text { mittel } & \text { (2) } \\
\text { sehr stark (4) }\end{array}\end{array}$ & 4 \\
\hline $\begin{array}{l}\text { 44. Im Follow Up } \\
\text { geben die } \\
\text { behandelnden } \\
\text { Ärzte bei } \\
\text { Probanden der } \\
\text { VG im Hinblick } \\
\text { auf die letzten } 2 \\
\text { Monate ein } \\
\text { signifikant } \\
\text { geringeres } \\
\text { Ausmaß an } \\
\text { Forderungen } \\
\text { nach weiteren } \\
\text { medizinischen } \\
\text { Untersuchungen } \\
\text { an als im Pretest. }\end{array}$ & $\begin{array}{l}\text { Arztfragebogen } \\
\text { (Frage 3) } \\
\text { Ratingskala } \\
\text { Pretest und } \\
\text { Follow Up }\end{array}$ & II & 4 \\
\hline $\begin{array}{l}\text { 45. Im Follow Up } \\
\text { geben die } \\
\text { behandelnden } \\
\text { Ärzte bei } \\
\text { Probanden der } \\
\text { VG im Hinblick } \\
\text { auf die letzten } 2 \\
\text { Monate ein } \\
\text { signifikant } \\
\text { geringeres } \\
\text { Ausmaß an } \\
\text { Forderungen } \\
\text { nach weiteren } \\
\text { medizinischen } \\
\text { Untersuchungen } \\
\text { an als im } \\
\text { Posttest. }\end{array}$ & $\begin{array}{l}\text { Arztfragebogen } \\
\text { (Frage 3) } \\
\text { Ratingskala } \\
\text { Posttest und } \\
\text { Follow Up }\end{array}$ & II & 4 \\
\hline
\end{tabular}




\section{Resonanz- und Beschwerdeerleben in der Gruppe}

Rudolf beschreibt, vor allem im interaktionellen Aspekt der depressiven Somatisierung, die Bedeutung von immer währender fehlender Resonanz auf seelisches Klagen während des gesamten Entwicklungsverlaufs. Daher ist von Interesse, ob ein hohes Resonanzerleben während den Gruppensitzungen signifikant negativ mit einer Abnahme des Erlebens der körperlichen Beschwerden korreliert, weil den Patienten hier nun endlich Raum zum körperlichen und seelischen Klagen geboten wird. Hypothese 46 in Tabelle 17 bildet die Grundlage dieses Forschungsinteresses.

Tabelle 17: Hypothese zur Korrelation zwischen Resonanz - und Beschwerdeerleben in den Gruppensitzungen

\begin{tabular}{|c|c|c|c|}
\hline Hypothese & $\begin{array}{l}\text { Untersuchungs- } \\
\text { instrument }\end{array}$ & Konkrete/s Item/s & $\begin{array}{l}\text { Frage- } \\
\text { stellung }\end{array}$ \\
\hline $\begin{array}{l}\text { 46. Die Höhe der } \\
\text { selbst } \\
\text { eingeschätzten } \\
\text { Resonanzer- } \\
\text { fahrung, der } \\
\text { einzelnen } \\
\text { Teilnehmer der } \\
\text { VG, die sie in } \\
\text { den einzelnen } \\
\text { Gruppensitz- } \\
\text { ungen erhalten, } \\
\text { korreliert } \\
\text { negativ mit der } \\
\text { subjektiv } \\
\text { erlebten Stärke } \\
\text { der } \\
\text { Beschwerden } \\
\text { während der } \\
\text { Gruppenstunde. }\end{array}$ & $\begin{array}{l}3 \text { Selbstein- } \\
\text { schätzungsfragen } \\
\text { nach jeder Sitzung }\end{array}$ & 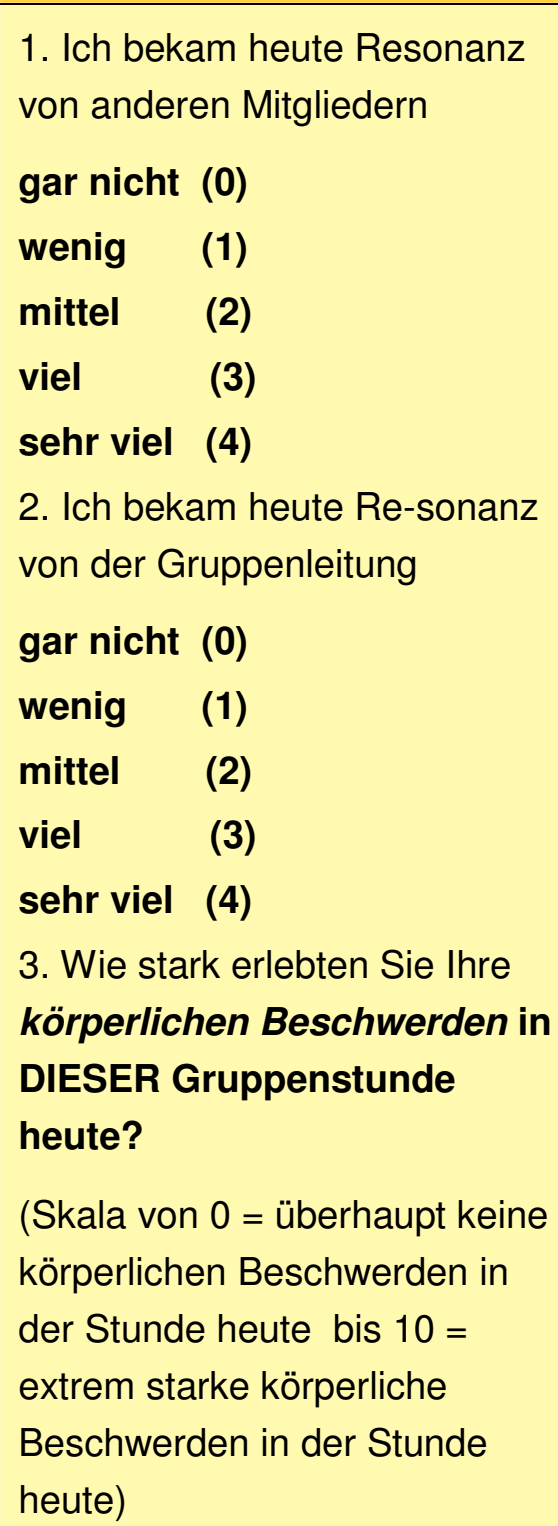 & 5 \\
\hline
\end{tabular}




\section{Symptomatikerleben in Alltag und Gruppe}

Aufbauend auf der These, dass Klagen und Gesehenwerden in der Gruppe statt finden kann, ist von Interesse, ob die Betroffenen ihre Beschwerden in den Gruppensitzungen signifikant weniger stark erleben als im Verlauf einer Woche in ihrem Alltagsleben.

Erleben die Patienten in der Gruppe weniger Beschwerden, ist ggf. eine Übertragung des Verhaltens in den Alltag möglich. Hypothese 47 in Tabelle 18 dient der wissenschaftlichen Formulierung dieses Interesses.

Tabelle 18: Hypothese zum Symptomerleben in Alltag und Gruppe

\begin{tabular}{|c|c|c|c|}
\hline Hypothese & $\begin{array}{l}\text { Untersuchungs- } \\
\text { instrument }\end{array}$ & Konkrete/s Item/s & $\begin{array}{l}\text { Frage- } \\
\text { stellung }\end{array}$ \\
\hline $\begin{array}{l}\text { 47. Während den } \\
\text { Gruppen- } \\
\text { stunden erleben } \\
\text { Patienten der VG } \\
\text { signifikant } \\
\text { weniger } \\
\text { körperliche } \\
\text { Beschwerden } \\
\text { als im Verlauf } \\
\text { einer ganzen } \\
\text { Woche in ihrem } \\
\text { Alltagsleben. }\end{array}$ & $\begin{array}{l}\text { Ratingskala nach } \\
\text { JEDER Sitzung } \\
\text { Ratingskala nach } \\
\text { JEDER Sitzung }\end{array}$ & $\begin{array}{l}\text { 1. Wie stark erlebten Sie } \\
\text { Ihre körperlichen } \\
\text { Beschwerden im } \\
\text { Verlauf der gesamten } \\
\text { letzten Woche? } \\
\text { 2. Wie stark erlebten Sie } \\
\text { Ihre körperlichen } \\
\text { Beschwerden in } \\
\text { DIESER } \\
\text { Gruppenstunde heute? } \\
\text { Jeweils: } \\
\text { (Skala von } 0=\text { überhaupt } \\
\text { keine körperlichen } \\
\text { Beschwerden in der } \\
\text { Stunde heute bis } 10= \\
\text { extrem starke körperliche } \\
\text { Beschwerden in der } \\
\text { Stunde heute) }\end{array}$ & 6 \\
\hline
\end{tabular}




\section{Narzisstisches Verhaltensmuster}

Rudolf erläutert, dass der frühe depressive Grundkonflikt durch ein häufig narzisstisch geprägtes Bewältigungsverhalten abgewehrt und gehandhabt wird. Die Patienten erfahren, dass sie konstante Beziehungen gewinnen können, in dem sie sich stark und sicher zeigen und sich zusätzlich um andere sorgen. Sie verhindern, selbst Hilfe in Anspruch zu nehmen, um keine erneuten Enttäuschungserfahrungen zu machen und zeigen sich forciert autonom und unabhängig.

Wiederum aufbauend auf der These, dass den Patienten in der Gruppe ermöglicht wird, sich neu und anders zu verhalten als in ihrem Alltagsleben, wurde Hypothese 48 aufgestellt, um zu überprüfen, ob ein narzisstisches Verhaltensmuster in der Gruppe signifikant weniger ausgelebt wird, als im Alltagsleben der Patienten. Tabelle 19 zeigt Hypothese 48.

Tabelle 19: Hypothese zum narzisstischen Verhalten in Alltag und Gruppe

\begin{tabular}{|c|c|c|c|}
\hline Hypothese & $\begin{array}{l}\text { Untersuchungs- } \\
\text { instrument }\end{array}$ & Konkrete/s Item/s & $\begin{array}{l}\text { Frage- } \\
\text { stellung }\end{array}$ \\
\hline $\begin{array}{l}\text { 48. Probanden der } \\
\text { VG }(\mathrm{A}) \text { erleben } \\
\text { sich in der } \\
\text { Interventions- } \\
\text { Gruppe weniger } \\
\text { forciert autonom } \\
\text { und stark als in } \\
\text { ihrem } \\
\text { Alltagsleben. }\end{array}$ & $\begin{array}{l}\text { Einzeltitems zum } \\
\text { narzisstisch- } \\
\text { altruistischen } \\
\text { Bewältigungsverhalt } \\
\text { en (angelehnt an } \\
\text { das Modell der } \\
\text { depressiven } \\
\text { Somatisierung von } \\
\text { Gerd Rudolf) } \\
\text { Posttest }\end{array}$ & 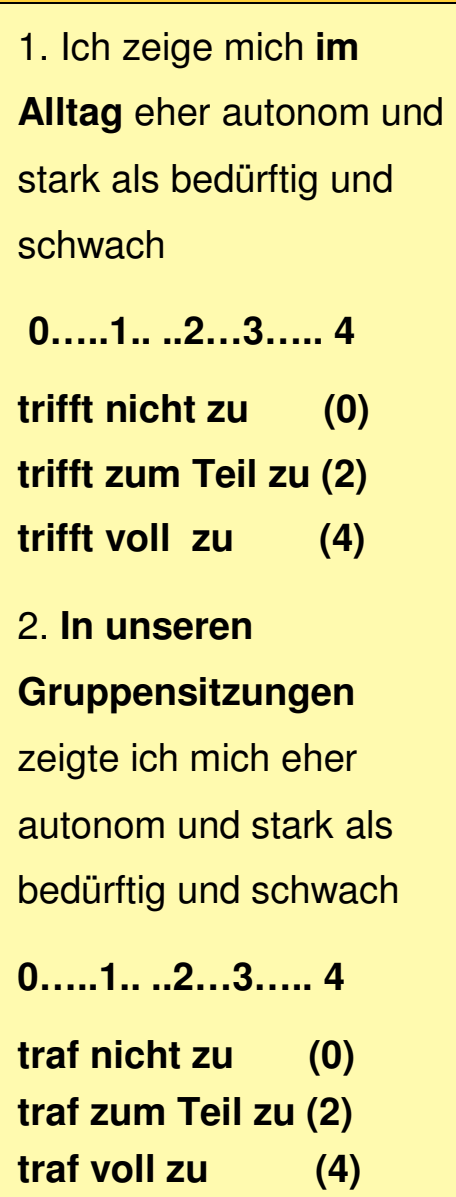 & 7 \\
\hline
\end{tabular}




\section{Psychotherapiebereitschaft}

Laut bisher veröffentlichter Literatur (siehe Kapitel 3), gelten somatoforme Patienten als schwer motivierbar für die Teilnahme an psychosozialen Maßnahmen. Sie werden als nicht kooperationsbereit und als ausschließlich fixiert auf die somatische Behandlung dargestellt. Ein Angebot für psychosoziale Hilfe sollen diese Patienten als beleidigend empfinden. Anhand einer Frage zur Psychotherapiebereitschaft, sollen diese Aussagen überprüft werden. Dazu wurden die letzten Hypothesen 49-51 in Tabelle 20 aufgestellt.

Tabelle 20: Hypothesen zur Psychotherapiebereitschaft

\begin{tabular}{|c|c|c|c|}
\hline Hypothese & $\begin{array}{l}\text { Untersuchungs- } \\
\text { instrument }\end{array}$ & Konkrete/s Item/s & $\begin{array}{l}\text { Frage- } \\
\text { stellung }\end{array}$ \\
\hline $\begin{array}{l}\text { 49. Patienten der VG } \\
\text { zeigen im } \\
\text { Posttest eine } \\
\text { signifikant } \\
\text { höhere } \\
\text { Bereitschaft } \\
\text { eine } \\
\text { Psychotherapie } \\
\text { zu machen als } \\
\text { im Pretest. }\end{array}$ & $\begin{array}{l}\text { Ratingskala zur } \\
\text { Psychotherapie- } \\
\text { motivation } \\
\text { Pretest und } \\
\text { Posttest }\end{array}$ & $\begin{array}{l}\text { Wie hoch ist derzeit auf } \\
\text { einer Skala von } \mathbf{0} \text { bis } 10 \\
\text { Ihre Bereitschaft eine } \\
\text { Psychotherapie zu } \\
\text { machen? Bitte kreuzen Sie } \\
\text { EINE Zahl an: } \\
0=\text { überhaupt keine } \\
\text { Bereitschaft } \\
10=\text { sehr hohe Bereitschaft }\end{array}$ & 8 \\
\hline $\begin{array}{l}\text { 50. Patienten der VG } \\
\text { zeigen im Follow } \\
\text { Up eine } \\
\text { signifikant } \\
\text { höhere } \\
\text { Bereitschaft } \\
\text { eine } \\
\text { Psychotherapie } \\
\text { zu machen als } \\
\text { im Pretest. }\end{array}$ & $\begin{array}{l}\text { Ratingskala zur } \\
\text { Psychotherapie- } \\
\text { motivation } \\
\text { Pretest und } \\
\text { Follow Up }\end{array}$ & II & 8 \\
\hline $\begin{array}{l}\text { 51. Patienten der VG } \\
\text { zeigen im Follow } \\
\text { Up eine } \\
\text { signifikant } \\
\text { höhere } \\
\text { Bereitschaft } \\
\text { eine } \\
\text { Psychotherapie } \\
\text { zu machen als } \\
\text { im Posttest. }\end{array}$ & $\begin{array}{l}\text { Ratingskala zur } \\
\text { Psychotherapie- } \\
\text { motivation } \\
\text { Posttest und } \\
\text { Follow Up }\end{array}$ & II & 8 \\
\hline
\end{tabular}




\section{Untersuchungsmethodik und Untersuchungsverlauf}

\subsection{Die Stichprobe}

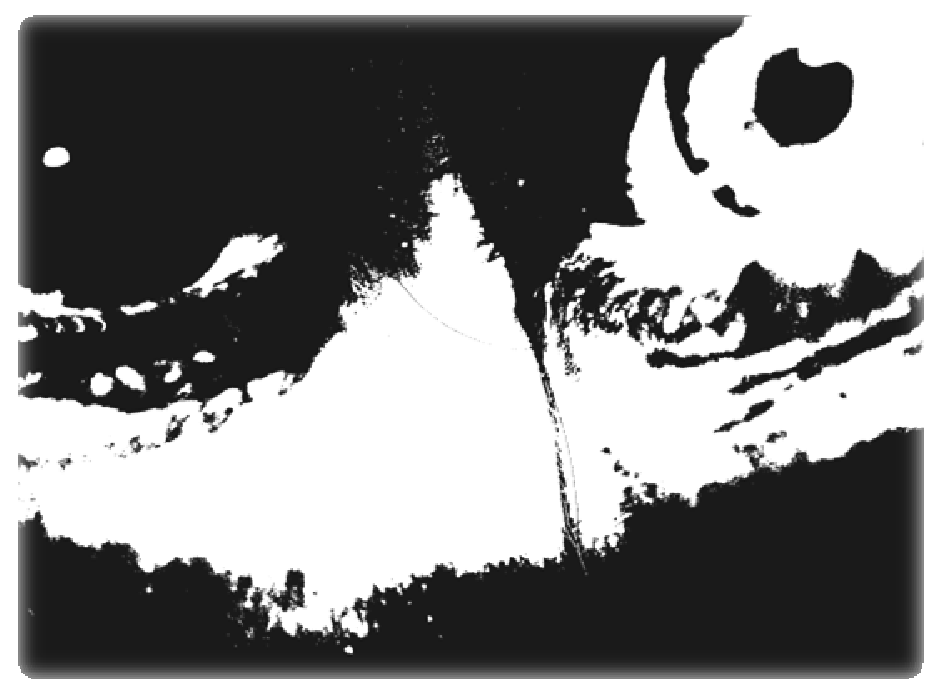

\section{Proband}

„Er/Sie ist unschuldig, namen- und gesichtslos. Eine Person steht für viele, welche von den suchenden Vogel-Kundschaftern investigierend in der Welt der Wirklichkeit vorgefunden werden.

Sie bietet sich dar, doch spricht sie eine Sprache, die der Forscher nicht versteht. Man wird sie übersetzen und interpretieren.

Der Proband wird davon nichts erfahren. Er schaut bei diesem Vorgang in den Mond“ (Mertens, 1992, S.24).

Zur Stichprobengewinnung gehörte das Aufstellen von Einschluss- und Ausschlusskriterien. Die Diagnose und Einschätzung des behandelnden Hausarztes war dafür ein wichtiges Kriterium. Wichtig für den Einschluss in die Versuchsgruppe war dabei besonders die Einschätzung, ob sie ihren Patienten für die Teilnahme an einer Gruppenintervention als geeignet befanden.

Akut suizidgefährdete Patienten wurden, ebenso wie Menschen mit schweren organischen oder psychiatrischen Diagnosen wie z. B. einer Psychose, von der Teilnahme der Studie ausgeschlossen. 
Alle Teilnehmer der Versuchsgruppe unterschrieben vor Beginn des Gruppenprogramms eine Einverständniserklärung dafür, dass sie die volle Selbstverantwortung für die Teilnahme an der Gruppe übernehmen.

Diese lautete im Wortlaut:

„Hiermit erkläre ich mich einverstanden an dem psychosozialen Gruppeninterventionsprogramm unter der Leitung von Frau Dipl. Heilpäd. Sarah Rembold im Rahmen einer Studie des Fachbereichs Psychologie und Psychotherapie der Universität zu Köln, die von Herrn Prof. Dr. Jörg Fengler begleitet wird, teilzunehmen.

Ich verantworte die Teilnahme selbst.“

Die behandelnden Hausärzte der Patienten erhielten eine kurze Einschätzungstabelle, die Kriterien zum Ein- und Ausschluss für die Studienteilnahme enthielten. Abgesehen von der Einschätzung des Arztes, ob er seinen Patienten für eine Gruppenbehandlung als geeignet befindet, galten alle Kriterien für die Versuchs- und Kontrollgruppe gleich. Eingeschlossen wurden nur erwachsene Personen im Alter zwischen 18 und 70 Jahren.

Tabelle 21 zeigt die Einschluss- und Ausschlusskriterien für die Teilnahme an der Studie. Wenn der Arzt Punkt 7 mit nein beantwortete, die Patienten aber von sich aus gerne teilnehmen wollten, war dies kein Ausschlusskriterium. 
Tabelle 21: Einschlusskriterien für die Teilnehmer der Studie (nach Einschätzung der behandelnden Hausärzte)

\begin{tabular}{|l|l|l|}
\hline Einschlusskriterien & \multicolumn{2}{|l|}{ Liegt vor } \\
\hline 1. Alter: 18 - 70 Jahre & Ja & Nein \\
\hline $\begin{array}{l}\text { 2. Ausreichende deutsche Sprach- und } \\
\text { Schriftkenntnisse }\end{array}$ & Ja & Nein \\
\hline 3. Ausreichend gute Hör- und Sehfähigkeiten & Ja & Nein \\
\hline $\begin{array}{l}\text { 5.Keine akute Suizidialität oder schwere organisch } \\
\text { begründete oder psychiatrische Erkrankung, z. B. } \\
\text { Psychose }\end{array}$ & Ja & Nein \\
\hline $\begin{array}{l}\text { 3. Körperliche Beschwerden, die organisch nicht } \\
\text { ausreichend erklärbar sind und das Wohlbefinden } \\
\text { stark beeinträchtigen }\end{array}$ & Ja & Nein \\
\hline $\begin{array}{l}\text { 5. Nach meiner Einschätzung hat der Patient } \\
\text { somatoforme/funktionelle Beschwerden }\end{array}$ & Ja & Nein \\
\hline $\begin{array}{l}\text { 6. Generelle Bereitschaft des Patienten zur } \\
\text { Teilnahme an einer Studie und einer kooperativen } \\
\text { Behandlung zwischen Medizin und psychosozialer } \\
\text { Arbeit }\end{array}$ & Ja & Nein \\
\hline $\begin{array}{l}\text { 7. Ich kann mir für diesen Patienten eine } \\
\text { Gruppenbehandlung gut vorstellen }\end{array}$ & Ja Nein \\
\hline
\end{tabular}

Im Folgenden liefern Tabellen 22 bis 31 eine Gesamtübersicht über die Stichprobe der Versuchs- und der Kontrollprobanden. Die deskriptiven Daten dienen, vor der Darstellung der statistischen Ergebnisse, vor allem dazu, ein allgemeines Verständnis und Gefühl für die behandelte Stichprobe zu erlangen.

Die Anzahl der Probanden von $\mathrm{N}=20$ als auch der Geschlechter von N=5 männlich und $\mathrm{N}=15$ weiblich sind in der Versuchsgruppe wie in der Kontrollgruppe gleich verteilt.

Aufgrund einer kleinen Stichprobe $(\mathrm{N}=40)$ wird in der Auswertung jedoch auf eine Geschlechter getrennte Darstellung verzichtet, da allgemein aussagekräftige Informationen bei jeweils $\mathrm{N}=5$ männliche Teilnehmer und $\mathrm{N}=$ jeweils 15 weibliche Teilnehmer pro Gruppe nicht zu treffen sind. 
Der Altersdurchschnitt in der Versuchsgruppe beträgt 49,35, in der Kontrollgruppe 40,9 Jahre. Bei acht möglichen Sitzungen beträgt die durchschnittliche Teilnahme der Probanden der Versuchsgruppe 6,8 Sitzungen.

Die mittlere Anzahl der Kinder pro Person liegt in der Versuchsgruppe bei 1, in der Kontrollgruppe bei 1,15.

11 der 20 Probanden der Versuchsgruppe sind verheiratet, 4 leben in einer festen Partnerschaft und 5 Personen haben derzeit keinen Lebenspartner.

In der Kontrollgruppe sind 7 Probanden verheiratet, 7 Personen leben in einer festen Partnerschaft, 6 Teilnehmer haben derzeit keinen Lebenspartner.

14 Probanden der Versuchsgruppe sind in einem festen Beschäftigungsverhältnis, während 6 derzeit ohne Arbeit sind. In der Kontrollgruppe befinden sich 13 Probanden derzeit in einem festen Beschäftigungsverhältnis. 7 Teilnehmer sind derzeit ohne Arbeit.

Die höchsten Schulabschlüsse verteilen sich bei 20 Probanden in der Versuchsgruppe auf 4x Abitur, 5x Fachhochschulreife, $8 x$ Realschulabschluss und $3 x$ Hauptschulabschluss. Keiner der Teilnehmer hat keinen Schulabschluss.

In der Kontrollgruppe haben 7 Personen das Abitur, 5 verfügen über Fachhochschulreife, 3 über einen Realschulabschluss und 4 besitzen den Hauptschulabschluss. Einer der Teilnehmer hat keinen Schulabschluss. 
Tabelle 22: Gesamtübersicht deskriptive Daten der Versuchsgruppe (FP = Feste Partnerschaft, $\mathrm{V}=$ Veheiratet, $\mathrm{KP}=$ Keine Partnerschaft, $\mathrm{MR}=$ Mittlere Reife, HS = Hauptschulabschluß, A = Abitur, FHR = Fachhochschulreife, $\mathrm{w}=$ weiblich, $\mathrm{m}=$ männlich)

\begin{tabular}{|l|c|c|c|c|c|c|c|}
\hline Proband & Geschlecht & $\begin{array}{c}\text { Alter } \\
\text { in } \\
\text { Jahren }\end{array}$ & $\begin{array}{c}\text { Anwesen- } \\
\text { heit von 8 } \\
\text { Sitzungen }\end{array}$ & $\begin{array}{c}\text { Anzahl } \\
\text { der } \\
\text { Kinder }\end{array}$ & $\begin{array}{c}\text { Feste } \\
\text { Arbeitsstelle } \\
\text { (Ja/Nein) }\end{array}$ & $\begin{array}{c}\text { Beziehungs- } \\
\text { status }\end{array}$ & $\begin{array}{c}\text { Höchster } \\
\text { Schul- } \\
\text { abschluss }\end{array}$ \\
\hline P1 & w & 44 & 8 & 2 & ja & FP & MR \\
\hline P2 & m & 65 & 7 & 1 & nein & V & MR \\
\hline P3 & w & 54 & 6 & 2 & ja & V & HS \\
\hline P4 & w & 27 & 6 & 0 & ja & FP & A \\
\hline P5 & m & 33 & 5 & 0 & ja & KP & FHR \\
\hline P6 & m & 45 & 6 & 1 & ja & V & HS \\
\hline P7 & w & 51 & 8 & 2 & nein & KP & MR \\
\hline P8 & w & 53 & 7 & 2 & ja & V & HS \\
\hline P9 & w & 56 & 8 & 0 & ja & V & A \\
\hline P10 & w & 57 & 8 & 0 & ja & FP & MR \\
\hline P11 & m & 38 & 7 & 0 & ja & V & FHR \\
\hline P12 & w & 54 & 7 & 2 & ja & FP & A \\
\hline P13 & w & 58 & 7 & 0 & nein & V & MR \\
\hline P14 & m & 52 & 8 & 2 & nein & KP & MR \\
\hline P15 & w & 53 & 7 & 2 & ja & KP & FHR \\
\hline P16 & w & 60 & 8 & 1 (tod) & nein & V & FHR \\
\hline P17 & w & 60 & 7 & 0 & nein & V & FHR \\
\hline P18 & w & 40 & 8 & 0 & ja & KP & A \\
\hline P19 & w & 40 & 6 & 0 & ja & V & MR \\
\hline P20 & w & 47 & 8 & 3 & ja & V & MR \\
\hline
\end{tabular}


Tabelle 23: Gesamtübersicht deskriptive Daten der Kontrollgruppe (FP = Feste Partnerschaft, $V=$ Veheiratet, $\mathrm{KP}=$ Keine Partnerschaft, $\mathrm{MR}=$ Mittlere Reife, $\mathrm{HS}=$ Hauptschulabschlu $\mathrm{B}, \mathrm{A}=\mathrm{Abitur}$, $\mathrm{FHR}=$ Fachhochschulreife, $\mathrm{w}=$ weiblich, $\mathrm{m}=$ männlich)

\begin{tabular}{|l|c|c|c|c|c|c|}
\hline Proband & Geschlecht & $\begin{array}{c}\text { Alter } \\
\text { in } \\
\text { Jahren }\end{array}$ & $\begin{array}{c}\text { Anzahl } \\
\text { der } \\
\text { Kinder }\end{array}$ & $\begin{array}{c}\text { Feste } \\
\text { Arbeitsstelle } \\
\text { (Ja/Nein) }\end{array}$ & $\begin{array}{c}\text { Beziehungs- } \\
\text { status }\end{array}$ & $\begin{array}{c}\text { Höchster } \\
\text { Schul- } \\
\text { abschluss }\end{array}$ \\
\hline P1 & w & 69 & 3 & nein & V & HS \\
\hline P2 & w & 37 & 1 & ja & KP & MR \\
\hline P3 & w & 46 & 2 & ja & V & FHR \\
\hline P4 & w & 51 & 2 & ja & V & A \\
\hline P5 & m & 59 & 2 & nein & V & FHR \\
\hline P6 & m & 61 & 3 & ja & KP & HS \\
\hline P7 & w & 30 & 1 & ja & KP & MR \\
\hline P8 & m & 44 & 0 & ja & V & A \\
\hline P9 & w & 44 & 1 & nein & KP & FHR \\
\hline P10 & w & 18 & 0 & nein & FP & KA \\
\hline P11 & w & 65 & 3 & nein & FP & HS \\
\hline P12 & w & 32 & 0 & ja & FP & A \\
\hline P13 & m & 35 & 1 & ja & V & A \\
\hline P14 & w & 30 & 0 & ja & FP & A \\
\hline P15 & m & 43 & 0 & ja & FP & A \\
\hline P16 & w & 22 & 1 & ja & FP & MR \\
\hline P17 & w & 27 & 1 & nein & KP & FHR \\
\hline P18 & w & 37 & 2 & ja & FP & A \\
\hline P19 & w & 49 & 0 & ja & V & HS \\
\hline P20 & w & 19 & 0 & nein & KP & FHR \\
\hline
\end{tabular}

Tabelle 24: Geschlechterverteilung in der Versuchsgruppe

\begin{tabular}{|l|c|}
\hline Geschlecht & Häufigkeiten \\
\hline männlich & 5 \\
\hline weiblich & 15 \\
\hline
\end{tabular}

Tabelle 25: Geschlechterverteilung in der Kontrollgruppe

\begin{tabular}{|l|c|}
\hline Geschlecht & Häufigkeiten \\
\hline männlich & 5 \\
\hline weiblich & 15 \\
\hline
\end{tabular}

Tabelle 26: Beziehungsstatus: Häufigkeiten in der Versuchsgruppe

\begin{tabular}{|l|c|}
\hline Beziehungsstatus & Häufigkeiten \\
\hline verheiratet & 11 \\
\hline feste Partnerschaft & 4 \\
\hline keinen Partner & 5 \\
\hline
\end{tabular}


Tabelle 27: Beziehungsstatus: Häufigkeiten in der Kontrollgruppe

\begin{tabular}{|l|c|}
\hline Beziehungsstatus & Häufigkeiten \\
\hline verheiratet & 7 \\
\hline feste Partnerschaft & 7 \\
\hline keinen Partner & 6 \\
\hline
\end{tabular}

Tabelle 28: Arbeitssituation: Häufigkeiten in der Versuchsgruppe

\begin{tabular}{|l|c|}
\hline Arbeitssituation & Häufigkeiten \\
\hline Feste Arbeitsstelle & 14 \\
\hline Keine Arbeitsstelle & 6 \\
\hline
\end{tabular}

Tabelle 29: Arbeitssituation: Häufigkeiten in der Kontrollgruppe

\begin{tabular}{|l|c|}
\hline Arbeitssituation & Häufigkeiten \\
\hline Feste Arbeitsstelle & 13 \\
\hline Keine Arbeitsstelle & 7 \\
\hline
\end{tabular}

Tabelle 30: Höchster Schulabschluss: Häufigkeiten in der Versuchsgruppe

\begin{tabular}{|l|c|}
\hline Höchster Schulabschluss & Häufigkeiten \\
\hline Keinen Schulabschluss & 0 \\
\hline Hauptschulabschluss & 3 \\
\hline Realschulabschluss & 8 \\
\hline Fachhochschulreife & 5 \\
\hline Abitur & 4 \\
\hline
\end{tabular}

Tabelle 31: Höchster Schulabschluss: Häufigkeiten in der Kontrollgruppe

\begin{tabular}{|l|c|}
\hline Höchster Schulabschluss & Häufigkeiten \\
\hline Keinen Schulabschluss & 1 \\
\hline Hauptschulabschluss & 4 \\
\hline Realschulabschluss & 3 \\
\hline Fachhochschulreife & 5 \\
\hline Abitur & 7 \\
\hline
\end{tabular}




\subsubsection{Kurzbeschreibung des Gruppenprogramms}

Das Programm ist über acht Sitzungen a ein einhalb Stunden einmal die Woche konzipiert. Die Teilnehmeranzahl sollte zehn Personen dabei nicht überschreiten.

Die Inhalte der einzelnen Sitzungen, das heißt Interventionen, Übungen, Rituale und Foki der Leitung, wurden überwiegend auf dem in Kapitel 3.2 dargestellten Modell der depressiven Somatisierung nach Gerd Rudolf und den sich als defizitär entwickelten strukturellen Bereichen bei somatoformen Störungen aufgebaut.

Eine kurze Gesamtübersicht über die Themen, Inhalte sowie Haupt- und allgemeine Ziele der Sitzungen eins bis acht liefert Tabelle 32.

Die darauf folgende Tabelle 33 fokussiert im Anschluss die besonderen emotionalen Förderbereiche des Programms. Innerhalb der Anfangs- und Endrituale, die in allen Sitzungen gleich ablaufen, findet in jeder Stunde die Förderung der emotionalen Selbstwahrnehmung sowie der Affektdifferenzierung statt.

Es geht dabei um das klare Äußern der eigenen gegenwärtigen Emotionen und dient der Übung, diese nach außen klar zu kommunizieren. Zudem liegt in jeder Stunde zusätzlich ein Fokus auf den neu gefundenen Ressourcen der Patienten, die ihnen im Umgang mit ihrer Symptomatik und Lebenssituation helfen können. Auch das, in der ersten Stunde formulierte, Alltagsziel wird im Anfangsritual in jeder Stunde beachtet und dessen Status erfragt. 
Tabelle 32: Übersicht über Themen, Inhalte, Hauptziele und allgemeine Ziele der Stunden

\begin{tabular}{|c|c|c|}
\hline Themen/Inhalt & Hauptziele der Stunde & Allgemeine Ziele \\
\hline $\begin{array}{l}\text { Stunde 1: } \\
\text { Kennen lernen } \\
\text { /Beziehungsaufbau/ } \\
\text { Gruppengefühl } \\
\text { entwickeln/Klagen }\end{array}$ & $\begin{array}{l}\text { Beziehungsaufbau/Vertrauen und } \\
\text { Sicherheit gewinnen/Verbundenheit } \\
\text { mit anderen erleben/Beschwerdeklage } \\
\text { annehmen/ Persönlichen Zielfokus } \\
\text { entwickeln }\end{array}$ & $\begin{array}{l}\text { Resonanz erleben/ } \\
\text { Selbstwahrnehmung }\end{array}$ \\
\hline $\begin{array}{l}\text { Stunde 2: } \\
\text { Selbstwahrnehmung/,,Der } \\
\text { Körper erzählt“‘ } \\
\text { /Klagen/Ressourcen }\end{array}$ & $\begin{array}{l}\text { Bewussteres (affektives) Selbst- } \\
\text { wahrnehmen/Zielstatus überprüfen u. } \\
\text { Ressourcen anhören/Körper und } \\
\text { Seele als Einheit erfahren/ } \\
\text { Körperselbst erfahren }\end{array}$ & $\begin{array}{l}\text { Resonanz erleben/ } \\
\text { Selbstwahrnehmung }\end{array}$ \\
\hline $\begin{array}{l}\text { Stunde 3: } \\
\text { Selbstwahrnehmung } \\
\text { /,,Was kann ich gut - Was } \\
\text { fehlt mir"? } \\
\text { /Wertschätzung/Ausgleich }\end{array}$ & $\begin{array}{l}\text { (Affektive) Selbstwahrnehmung/ } \\
\text { Zielstatus prüfen und Ressourcen } \\
\text { anhören/ Stärken erkennen und } \\
\text { Ausgleiche dazu finden/ Motivation zur } \\
\text { Veränderung entfachen/ Positives } \\
\text { Feedback erfahren }\end{array}$ & $\begin{array}{l}\text { Resonanz erleben/ } \\
\text { Selbstwahrnehmung } \\
\text { /Selbstsicherheit }\end{array}$ \\
\hline $\begin{array}{l}\text { Stunde 4: } \\
\text { Selbstwahrnehmung/ } \\
\text {,Tabuemotionen"/ } \\
\text { Kognition ,Emotion und } \\
\text { Körper }\end{array}$ & $\begin{array}{l}\text { Erkennen abgelehnter Gefühle/ } \\
\text { Erschaffung neuer Selbstakzeptanz/ } \\
\text { Gruppe als Hilfe zum verstehen der } \\
\text { Verbindung Kognition,Emotion u. } \\
\text { Körper }\end{array}$ & $\begin{array}{l}\text { Resonanz erleben/ } \\
\text { Selbstwahrnehmung } \\
\text { /Emotionen erfahren/ } \\
\text { Selbstakzeptanz }\end{array}$ \\
\hline $\begin{array}{l}\text { Stunde 5: } \\
\text { Selbstwahrnehmung/Ress } \\
\text { ourcen+Zielkontrolle/ } \\
\text { "Personen als } \\
\text { Ressourcen"/Modelle und } \\
\text { Fremdwahrnehmung }\end{array}$ & $\begin{array}{l}\text { Selbstwahrnehmung/ Zielstatus prüfen } \\
\text { und Ressourcen anhören/Erfahrung } \\
\text { von Wertschätzung und positiver } \\
\text { Fremdwahrnehmung aus der Gruppe/ } \\
\text { Mitglieder als positive Modelle/Trotz } \\
\text { Symptom Vorbild sein zu können }\end{array}$ & $\begin{array}{l}\text { Resonanz erleben/ } \\
\text { Selbstwahrnehmung } \\
\text { /Selbstsicherheit }\end{array}$ \\
\hline $\begin{array}{l}\text { Stunde 6: } \\
\text { Selbstwahrnehmung/“"Mein } \\
\text { Symptomsinn"/Selbst- } \\
\text { akzeptanz- u.verständnis }\end{array}$ & $\begin{array}{l}\text { Selbstwahrnehmung/Zielstatus prüfen } \\
\text { und Ressourcen anhören/ Individuelle } \\
\text { Sinnhaftigkeit der Symptomatik } \\
\text { erkennen } \rightarrow \text { Negatives in Positives } \\
\text { Umkehren }\end{array}$ & $\begin{array}{l}\text { Resonanz erleben/ } \\
\text { Selbstwahrnehmung } \\
\text { /Akzeptanz erleben }\end{array}$ \\
\hline $\begin{array}{l}\text { Stunde 7: } \\
\text { Selbstwahrnehmung/ Die } \\
\text { Gruppe und mein } \\
\text { Alltag"/Übertragen/ } \\
\text { Veränderungen/ } \\
\text { Erkenntnisse }\end{array}$ & $\begin{array}{l}\text { Zielstatus prüfen und Ressourcen } \\
\text { anhören/ Veränderungsprozesse } \\
\text { wahrnehmen/ Differenzen zwischen } \\
\text { Alltag und Gruppe erkennen und } \\
\text { versuchen, Positives mit in den Alltag } \\
\text { zu nehmen/ Gruppenbindung } \\
\text { (Selbsthilfeteilnahme) stärken/ } \\
\text { Bessere Selbstwahrnehmung }\end{array}$ & $\begin{array}{l}\text { Resonanz erleben/ } \\
\text { Selbstwahrnehmung } \\
\text { /Ressourcen } \\
\text { wahrnehmen/ } \\
\text { Selbstsicherheit }\end{array}$ \\
\hline $\begin{array}{l}\text { Stunde 8: } \\
\text { Selbstwahrnehmung/ } \\
\text { Abschied/Beschwerden } \\
\text { heute u. } \\
\text { Ressourcen/Selbsthilfe }\end{array}$ & $\begin{array}{l}\text { Fokussierung auf positiv Erreichtes/ } \\
\text { Veränderte Wahrnehmung der } \\
\text { Beschwerden/ Motivation zur } \\
\text { Selbsthilfegruppe }\end{array}$ & $\begin{array}{l}\text { Resonanz erleben/ } \\
\text { Selbstwahrnehmung }\end{array}$ \\
\hline
\end{tabular}


Tabelle 33: Gesamtübersicht über die geförderten Bereiche innerhalb des gesamten Programms und den einzelnen Sitzungen

In ALLEN Stunden geförderte Bereiche

$\rightarrow$ Anfangsritual fördert immer: (An Gerd Rudolf orientierte strukturelle Bereiche, die sich bei somatoformen Störungen als defizitär erwiesen haben)

1. Emotionale Ebene: Emotionale Kommunikation nach außen/Affekte gegenüber anderen ausdrücken

2. Kognitive Ebene: Das Selbst wahrnehmen (Die eigene emotionale Situation klären: Affektdifferenzierung sowie

Alltags- und Ressourcenorientierung (Zielstatusüberprüfung und Ressourcentagebuch)

Die Leitung achtet insgesamt darauf, dass ein Bezug zwischen Körper und

psychischem Erleben hergestellt wird und fungiert dabei als Hilfs-Ich.

$\rightarrow$ Die „Offene Runde“ fördert: (Nach Gerd Rudolf orientiert an dem zugrundeliegenden depressiven Grundkonflikt)

1. Eine in geringem Maße korrigierende Erfahrung von Resonanz in der Gruppe

$\rightarrow$ Das Abschlussritual fördert immer:

1.Die bereits im Anfangsritual aufgeführten strukturellen Bereiche

2.Die Wahrnehmungsschärfung auf positive Erlebnisse und neu erworbene

Ressourcen für den Umgang mit den Beschwerden innerhalb der Sitzung

\footnotetext{
Stunde

$1 \rightarrow$ Kennen

lernen/Beziehungsaufbau/Gruppengefühl entwickeln/Klagen
}

$2 \rightarrow$ Selbstwahrnehmung $/$, Der Körper erzählt" /Klagen/Ressourcen

$3 \rightarrow$ Selbstwahrnehmung /,, Was kann ich
gut - Was fehlt mir“"?
/Wertschätzung/Ausgleich

$4 \rightarrow$ Selbstwahrnehmung/

"Tabuemotionen"/

Kognition und Emotion und Körper
$5 \rightarrow$ Selbstwahrnehmung/Ressourcen+
Zielkontrolle/" Personen als
Ressourcen"/Modelle und
Fremdwahrnehmung

$6 \rightarrow$ Selbstwahrnehmung/" Mein

Symptomsinn"/Selbstakzeptanz-

u.verständnis

$7 \rightarrow$ Selbstwahrnehmung/ Die Gruppe

und mein

Alltag "Übertragen/Veränderungen/Er-

kenntnisse

$8 \rightarrow$ Selbstwahrnehmung/Abschied/

Beschwerden heute $u$.

Ressourcen/Selbsthilfe
Weitere berücksichtigte Bereiche und Foki in den offenen Runden

$\rightarrow$ Vertrauensvolle Basis erschaffen

$>$ Gruppenkohäsion erleben

$>$ Klagen

$>$ Beziehungen aufbauen/Vertrauen erleben

$\rightarrow$ Die „Offene Runde“: (strukturelle Bereiche)

$>$ Hier: „Der Körper erzählt“: Körperselbst stärken + Affektdifferenzierung

$>$ Resonanz erleben

Resonanz erleben

$>$ Evt. wahrnehmen bestimmter Bewältigungsmuster $\rightarrow$ Lösungsorientierung: Finden von Ausgleichsaktivitäten

$>$ Seelisch klagen dürfen und dafür Resonanz erfahren - versus nur für körperliches Klagen

Aufmerksamkeit zu bekommen

$>$ Andere als Modelle erleben/ Feedback geben+erhalten/ Wertschätzung erfahren

$>$ Resonanz erleben

$>$ Blick auf die „positiven Seiten“ der Krankheit zu lenken

$>$ Differenzen erkennen

$>$ Reflektieren und Gelerntes in den Alltag übertragen

$>$ Differenzen erkennen/Resonanz erleben

$>$ Sich verabschieden lernen/Gruppe als positive Objekte wahrnehmen

$>$ Animation zur Selbsthilfegruppe 
Das gesamte Manual befindet sich mit jeder Sitzung ausformuliert im Anhang dieser Arbeit. Darin werden die einzelnen Themen der Sitzungen noch einmal detailliert erläutert.

In diesem Manual werden exakte Anweisungen für den Leiter der Gruppe als Möglichkeit zur Orientierung der Strukturierung der Sitzungen gegeben. Dies ermöglicht, dass das Programm auch von anderen Helfern problemlos durchgeführt werden kann.

Ergänzend zum ausformulierten Manual befinden sich im Anhang zu jeder Stunde einseitige Stundenblätter zu jeder Sitzung. Sie geben der leitenden Person des Programms eine hilfreiche Unterstützung und Orientierung für eine sichere Durchführung. Dabei beinhalten sie acht Bereiche:

1. Inhalt/Ablauf der Sitzung

2. Zeit (genaue Zeitangaben für die einzelnen Übungen, Interventionen etc.)

3. Störungsmodell (welches Störungsmodell hier besondere Berücksichtung erhält.)

4. Ziele (Welche Ziele diese Sitzung verfolgt.)

5. Gruppenwirkfaktoren

6. Leiter Verhalten

7. Innerer Forkus der Leitung

8. Material. 


\subsubsection{Das Untersuchungsdesign}

Das hier angewandte Untersuchungsdesign entspricht einem zwei-Gruppen-Plan mit Pretest, Intervention, Posttest und Follow Up. Damit erfüllt die Untersuchung eigentlich die Kriterien eines starken experimentellen Designs. Allerdings gibt es Einschränkungen, die im Bereich der vollständigen Randomisierung zu nennen sind. Die Patienten für die Versuchs- und Kontrollgruppe wurden durch die Ärzte zwar zufällig zusammengestellt, jedoch existierten zum Zeitpunkt des Gruppenstarts der Versuchsgruppe noch nicht zeitgleich alle Kontrollprobanden. Das bedeutet, dass ein Teil der Kontrollprobanden erst im Laufe der Studie hinzukamen und deshalb nicht mehr zufällig der Versuchsgruppe zugeordnet werden konnten. Die Kontrollprobanden wurden über das parallel laufende Gruppeninterventionsprogramms nicht informiert. Daher ist das Design als nicht randomisierter zwei-Gruppen-Plan mit Pretest, Intervention, Posttest und Follow Up und damit als quasi-experimentell zu bezeichnen.

Abbildung 4 zeigt diesen Versuchsplan mit Versuchs- und Kontrollgruppe.

\begin{tabular}{|ccccc|}
\hline VG: & U1 & $X$ & U2 & U3 \\
KG: & U1 & - & U2 & U3 \\
\hline
\end{tabular}

U1 = Untersuchungszeitpunkt 1 (Pretest)
U2 = Untersuchungszeitpunkt 2 (Posttest)
X = Intervention
U3 = Untersuchungszeitpunkt 3 (Follow Up)

Abbildung 4: Nicht randomisierter zwei-Gruppen-Plan mit Pretest, Intervention, Posttest und Follow Up (quasi-experimentell).

Für den Untersuchungsabstand zwischen Pretest, Posttest und Follow Up wurde jeweils ein Zeitintervall von zwei Monaten gewählt. Sowohl die Patienten der Versuchsgruppe als auch diese der Kontrollgruppe wurden zu diesen drei Messzeitpunkten mit denselben diagnostischen Instrumenten untersucht.

Das gewählte Intervall von zwei Monaten begründet sich vor allem auf die ebenfalls zweimonatige Dauer des Gruppenprogramms. Dieser gleichbleibende zeitliche Abstand für die Untersuchungen ermöglicht eine einheitliche und präzise Interpretation und Bewertung der Ergebnisse. Dennoch ist bereits an dieser Stelle einschränkend zu sagen, dass bei einer Versuchspersonenanzahl von $\mathrm{N}=40$ eine gewisse Vorsicht und Zurückhaltung für Aussagen zur Allgemeingültigkeit der Ergebnisse gewahrt werden sollte.

Das Untersuchungsintervall hätte selbstverständlich auch länger oder kürzer gewählt werden können. 


\subsection{Screening für somatoforme Störungen (SOMS), Gesundheitsfragebogen für Patienten (PHQ-D), Whiteley-Index (WI7) und eine Beschwerdeskala}

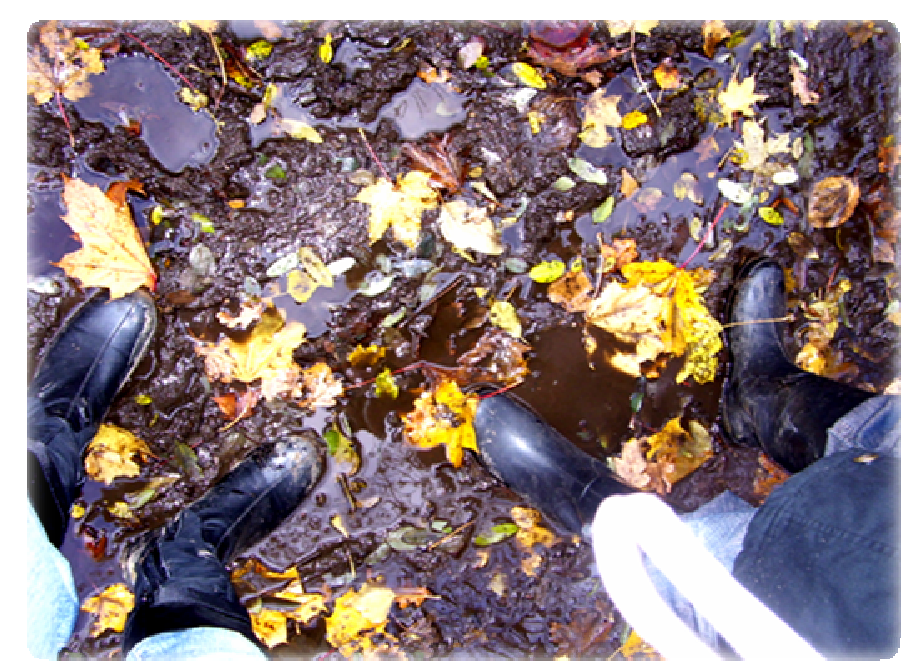

Recherche

„Nun macht sich der Forscher ans Werk, baut sich eine Wohnstatt und entsendet Kundschafter verschiedener Couleur in den Wind, auf dass er sie davontrage in die Wirklichkeit der weiten Welt, und auf dass sie heimkehren mögen mit der Beute der Fakten, die der Forscher zur Speise benötigt, um sich zu bestätigen - oder zu widerlegen (aber letzteres nur ausnahmsweise, wie wir wissen)“

( Mertens, 1992 S. 22.).

\section{PHQ- D : Gesundheitsfragebogen für Patienten}

Der "Gesundheitsfragebogen für Patienten“ (PHQ-D) ist ein Screeninginstrument, welches eine valide und zeitökonomische Diagnostik psychischer Störungen ermöglicht. Der PHQ(D) ist eine autorisierte deutsche Version des „PRIME MD Patient Health Questionnaire (PHQ), der von der Heidelberger Forschungsgruppe Löwe et al. (2002b) übersetzt wurde. $\mathrm{Zu}$ diesem liegen mittlerweile mehrere Validierungsstudien vor, welche die guten diagnostischen Eigenschaften dieses Diagnostikinstruments belegen (Spitzer et al., 1994; Kroenke et al., 2001).

Der PHQ-D lässt sich flexibel an die jeweilige inhaltliche Fragestellung anpassen und besteht, je nach Anzahl der gewählten Module, aus mindestens einer bis maximal vier Seiten. Die Module ermöglichen die Diagnostik somatoformer Störungen, depressiven Störungen, Angststörungen, Esstörungen und von Alkoholmissbrauch. 
Für die in dieser Arbeit dargestellte Studie wurde in der Diagnostik das Modul für die Erfassung somatoformer Störungen ausgewählt.

Der Skalensummenwert "somatische Symptome“ umfasst 15 somatische Symptome, die sowohl den häufigsten Beschwerden von ambulanten Patienten als auch den wichtigsten DSM-IV Kriterien für die Somatisierungsstörung entsprechen. Dieser Skalensummenwert wird PHQ-15 genannt. Gebildet wird der Skalensummenwert aus diesen 15 Items des somatoformen Moduls, die jeweils mit 0 („Nicht beeinträchtigt“), 1 („Wenig beeinträchtigt“) oder 2 („Stark beeinträchtigt“) bewertet werden. Der Skalensummenwert liegt somit im Bereich 0 bis 30 .

Diese drei Einschätzungsmöglichkeiten gelten für folgende 15 Items „, Wie stark fühlten Sie sich im Verlauf der letzten acht Wochen durch die folgenden Beschwerden beeinträchtigt?"
a. Bauschmerzen
b. Rückenschmerzen
c. Schmerzen in Armen, Beinen und Gelenken (Knie, Hüfte etc.)
d. Menstruationsbeschwerden oder andere Probleme mit der Menstruation
e. Schmerzen oder Probleme beim Geschlechtsverkehr
f. Kopfschmerzen
g. Schmerzen im Brustbereich
h. Schwindel
i. Ohnmachtsanfälle
j. Herzklopfen oder Herzrasen
k. Kurzatmigkeit
I. Verstopfung, nervöser Darm oder Durchfall
m. Übelkeit, Blähungen oder Verdauungsbeschwerden
n. Schwierigkeiten ein oder durchzuschlafen oder vermehrter Schlaf
o. Müdigkeit oder Gefühle keine Energie zu haben 
Im PHQ-15 ist die rückblickende Zeit auf vier Wochen datiert und wurde für diese Studie auf acht Wochen erweitert.

Ab einem Wert von 6 ist laut dem Gesundheitsfragebogen von einem somatoformen Syndrom auszugehen (Löwe et al, 2002b). Innerhalb der hier vorliegenden Studie wurden jedoch auch Patienten mit einem Wert ab 3 einbezogen, um möglicherweise auch einen sekundär präventiven Effekt ausüben zu können.

Es sollten nicht nur schwer chronifizierte Patienten, sondern auch Personen, die (noch) weniger massive Symptome aufweisen und am Anfang einer Somatisierungsstörung stehen, behandelt und einbezogen werden.

Im Rahmen der Validierung des PHQ-15 wurde ein enger Zusammenhang mit dem Funktionsstatus und der gesundheitsbezogenen Lebensqualität belegt (Kroenke et al., 2001).

Die Validierung des deutschen PHQ-D wurde an einer Stichprobe von ambulanten Klinikpatienten und Hausarztpatienten durchgeführt. Diagnostischer Goldstandard war dabei das "Strukturierte Klinische Interview für das DSM-IV (SKID-I)“ (Wittchen et al.,1997). Insgesamt wurde eine Stichprobe von 1619 Patienten mit dem PHQ-D untersucht. Innerhalb dieser wurde bei 528 Patienten das SKID-I durchgeführt.

Erste Ergebnisse zeigen eine gute Übereinstimmunhg von PHQ-D und SKID, so dass von einer guten Validität des Instruments ausgegangen werden kann (Gräfe et al, 2001b; Löwe et al, 2002).

Da die amerikanische Orginalversion des PHQ-D auf die in den USA angewandten diagnostischen Kriterien des DSM-IV konstruiert wurde, entsprechen weitgehend auch die diagnostischen Kriterien in der deutschen Version diesem System. Da in Deutschland im Wesentlichen die ICD-10 als diagnostischer Schlüssel eingesetzt wird, ist zusätzlich zu einer Diagnose über den PHQ-D ein ärztliches Gespräch anzuraten. Niedergelassene Ärzte können dann ergänzend die Kriterien der ICD-10 berücksichtigen.

Innerhalb der Patientenrekrutierung wurde deshalb durchgehend eng mit den niedergelassenen Hausärzten der teilnehmenden Patienten kooperiert.

Der PHQ-15 diente der Überprüfung der Hypothesen 1, 2 und 3. 


\section{SOMS: Screening für somatoforme Störungen}

Das Screening für somatoforme Störungen (SOMS) dient der Erfassung von körperlichen Beschwerden, die nicht auf eine organische Erkrankung zurückzuführen sind und wurde von Rief und Hiller entwickelt. 2008 haben sie das SOMS vollständig überarbeitet und neu normiert.

Es werden darin sowohl die Kriterien nach ICD-10 als auch nach DSM-IV berücksichtigt.

Es liegen zwei Formen des SOMS für die Diagnostik somatoformer Störungen vor.

Der SOMS-2 ermöglicht mit 68 Items eine Statusdiagnostik im Rückblick auf das Erleben körperlicher Beschwerden innerhalb der letzten 2 Jahre.

Der SOMS-2 wird hier als Statusdiagnostik im Pre/Post und Follow Up-Test benutzt, um eine mögliche Veränderung in der Stärke der Somatisierungsstörung innerhalb des Studienzeitraums dokumentieren zu können.

Die 68 Fragen werden ausschließlich mit "Ja“ oder „Nein“ beantwortet und bestätigen oder verneinen damit das Auftreten eines Symptoms während des untersuchten Zeitraums.

Das SOMS 7T ermöglicht mit 53 Items eine Veränderungsmessung der körperlichen Beschwerden und ihrer Intensität im Rückblick auf die letzten sieben Tage. Innerhalb dieser Studie wurde das SOMS 7T als Veränderungsmessungsinstrument für die Pre/Post und Follow Up-Messung im Rückblick auf die letzten zwei Monate verwendet.

Da hier der klassifikationssystemübergreifende SAD-Index (BeschwerdeindexSomatisierung) benutzt wurde, galten für die Beantwortung der ja/nein Fragen ebenfalls nur die 53 Items des SOMS 7T für die Auswertung.

Die Beschwerdeitems konnten mit 0 (gar nicht), 1 (leicht), 2 (mittelmäßig), 3 (stark) oder 4 (sehr stark) beantwortet werden.

In der Testanleitung fanden die Patienten folgende Anweisungen:

„Bitte geben Sie an, ob und wie sehr Sie im Laufe der vergangenen 8 Wochen unter diesen Beschwerden gelitten haben.

Geben Sie nur solche Beschwerden an, für die von den Ärzten keine genauen Ursachen gefunden wurden und die Ihr Wohlbefinden stark beeinträchtig haben."

Ich habe in den vergangenen 8 Wochen unter folgenden Beschwerden gelitten: (Ausmaßbeeinträchtigung $0=$ gar nicht, $1=$ leicht, 2= mittelgradig, 3= stark, $4=$ sehr stark) 
1. Kopf-oder Gesichtsschmerzen

2. Schmerzen im Bauch oder in der Magengegend

3. Rückenschmerzen

4. Gelenkschmerzen

5. Schmerzen in den Armen oder Beinen

6. Brustschmerzen

7. Schmerzen im Enddarm

8. Schmerzen beim Geschlechtsverkehr

9. Schmerzen beim Wasserlassen

10. Übelkeit

11. Völlegefühl (sich aufgebläht fühlen)

12. Druckgefühl, Kribbeln oder Unruhe im Bauch

13. Erbrechen (außerhalb einer Schwangerschaft)

14. Vermehrtes Aufstoßen (in der Speiseröhre)

15. „Luftschlucken“, Schluckauf oder Brennen im Brust - oder Magenbereich

16. Unverträglichkeit von verschiedenen Speisen

17. Appetitverlust

18. Schlechter Geschmack im Mund oder stark belegte Zunge

19. Mundtrockenheit

20. Häufiger Durchfall

21. Flüssigkeitsaustritt aus dem Darm

22. Häufiges Wasserlassen

23. Häufiger Stuhldrang

24. Herzrasen oder Herzstolpern

25. Druckgefühl in der Herzgegend

26. Schweißausbrüche (heiß oder kalt)

27. Hitzewallungen oder Erröten

28. Atemnot (außer bei Anstrengungen)

29. Übermäßig schnelles Ein- und Ausatmen

30. außergewöhnliche Müdigkeit bei leichter Anstrengung

31. Flecken oder Farbveränderungen der Haut

32. Sexuelle Gleichgültigkeit

33. Unangenehme Empfindungen im oder am Genitalbereich

34. Koordinations- oder Gleichgewichtsstörungen 
35. Lähmung oder Muskelschwäche

36. Schwierigkeiten beim Schlucken oder Kloßgefühl

37. Flüsterstimme oder Stimmverlust

38. Harnverhalten oder Schwierigkeiten beim Wasserlassen

39. Sinnestäuschungen

40. Verlust von Berührungs- oder Schmerzempfindungen

41. Unangenehme Kribbelempfindungen

42. Sehen von Doppelbildern

43. Blindheit

44. Verlust des Hörvermögens

45. Krampfanfälle

46. Gedächtnisverlust

47. Bewusstlosigkeit

Für Frauen:

48. Schmerzhafte Regelblutungen

49. Unregelmäßige Regelblutungen

50. Übermäßige Regelblutungen

51. Erbrechen während der Schwangerschaft

52. ungewöhnlicher oder verstärkter Ausfluß aus der Scheide

Für Männer:

53, Impotenz oder Störungen des Samenergusses

Im SOMS 7T lassen sich auch leichte Veränderungen darstellen. Das ist deshalb vorteilhaft, weil sich aus Erfahrung sagen lässt, dass es unwahrscheinlich ist, dass langjährig chronifizierte Beschwerden in kurzer Zeit vollständig verschwinden. Häufiger lässt nach einer Intervention lediglich die Intensität der Beschwerden nach. Deshalb wurde neben dem SOMS 2 das SOMS 7T neu entwickelt. Es erfasst nicht nur das Vorhandensein, sondern auch die Intensität und die Anzahl der körperlichen Beschwerden und deren Veränderungen (Rief und Hiller, 2008).

Das SOMS wurde an Stichproben zwischen 100 und 2500 Personen normiert. Es liegen Normdaten für Gesunde und für psychosomatisch Kranke vor. Die RetestReliabilitätswerte ( $\mathrm{rtt}=, 70$ bis $\mathrm{rtt}=, 87)$ und internen Konsistenzwerte $(\mathrm{a}=, 80$ bis $\mathrm{a}=, 95)$ bestätigen die Reliabilität. 
Die Validität des Verfahrens wird durch Korrelationen der Fragebogenergebnisse aus dem SOMS-2 mit der Anzahl somatoformer Symptome laut Interview zwischen $r=, 72$ und $r=, 75$ sowie einer deutlichen Differenzierung verschiedener klinischer Gruppen und Schweregrade belegt (Rief \& Hiller, 2008).

Das SOMS bietet einen klassifikationssystemübergreifenden (DSM-IV und ICD 10) „Beschwerdeindex Somatisierung (SAD)“, der für die Darstellung mit Prozentrangwerten hier benutzt wird.

Aufgrund der übergreifenden Eigenschaft des SAD-Index gehen in ihn die meisten Informationen ein.

Das SOMS diente der Überprüfung der Hypothesen 4, 5, 6, 7, 8 und 9.

\section{WI: Der Whiteley-Index zur Erfassung hypochondrischer Ängste}

Auf internationaler Ebene hat sich der Whiteley-Index als ökonomisches und valides Instrument zur Erfassung hypochondrischer Ängste durchgesetzt. Das ursprünglich von Pilkowsky (1967) entwickelte Instrument mit 14 Items wurde von Rief und Hiller (2004) ins Deutsche übertragen und für den deutschen Sprachraum auf seine Gütekriterien überprüft (Hiller \& Rief, 2004).

Es stehen erstmals Normwerte für klinische und nicht-klinische Gruppen zur Verfügung. Der WI wurde an 2050 Personen aus der Allgemeinbevölkerung und 743 Patienten einer Medizinisch-Psychosomatischen-Klinik normiert.

Die Test-Retest-Reliabilitäten für den WI liegen zwischen ,73 und ,94.

Für die Faktorenstruktur des Whiteley-Index ergab sich ähnlich wie bei der englischen Originalfassung eine dreifaktorielle Lösung.

Faktor 1: „Krankheitsängste“, also primär Ängste und Sorgen, eine schwere Erkrankung zu bekommen.

Faktor 2: „Somatische Beschwerden“ beschreibt die Selbsteinschätzung der Patienten an einer Vielzahl von körperlichen Beschwerden zu leiden.

Faktor 3: „Krankheitsüberzeugung“ gibt im Gegensatz zum ersten Faktor die feste Überzeugung wieder, an einer ernsthaften Erkrankung zu leiden. 
Angelehnt an die Forschungsgruppe Schäfert et al. der Medizinischen Fakultät Heidelberg wurden auch in dieser Studie sieben Items zusammengestellt, die eine gute Einschätzung über hypochondrische Ängste zulassen.

Die sieben unten aufgeführten Items konnten mit „gar nicht“, „wenig“, „mittelgradig“, „relativ stark“ oder „sehr stark“ bewertet werden. Ab der Wahl „mittelgradig“ wird ein Punktwert gegeben, der für Hypochondrie spricht. Innerhalb dieser Version des Tests können die Probanden also einen Wert von 0 bis 7 erreichen.

Auch hier wurde die ursprüngliche rückblickende Zeitangabe von vier auf acht Wochen erweitert.

„Wie sehr fühlten Sie sich im Verlauf der letzten 8 Wochen beeinträchtigt durch...."

1. Sorgen, dass mit Ihrem Körper ernsthaft etwas nicht in Ordnung ist?

2. Sorgen, dass Sie an einer Krankheit leiden, von der Sie gelesen oder gehört haben?

3. Eine Vielzahl von Schmerzen?

4. Sorgen, möglicherweise eine ernsthafte Krankheit zu haben?

5. Einer Vielzahl unterschiedlicher Symptome

6. Gedanken, der Arzt könnte sich irren, wenn er Ihnen sagt, dass kein Grund zur Besorgnis besteht?

7. Viele Sorgen über Ihre Gesundheit?

Der WI-7 wurde nicht als Einschlusskriteriumsinstrument, sondern lediglich zur Evaluation des Therapieverlaufs verwendet.

Der WI-7 diente der Überprüfung der Hypothesen 10, 11 und 12. 


\section{Ratingskala zur Stärke der derzeit erlebten körperlichen Beschwerden}

Zur zusätzlichen Erfassung der erlebten Stärke der körperlichen Beschwerden an den unterschiedlichen Messzeitpunkten (Pre/Post und Follow Up) diente eine Ratingskala, die folgende Frage vorranstellte:

„Wie stark erleben Sie auf einer Skala von 0-10 derzeit Ihre körperlichen Beschwerden? Bitte kreuzen Sie EINE Zahl an“

$$
0=\text { keine Beschwerden }
$$

$10=$ sehr starke Beschwerden

\begin{tabular}{|l|l|l|r|r|r|r|r|r|r|r|}
\hline 0 & $=$ sehr starke Beschwerden \\
\hline 0 & 1 & 2 & 3 & 4 & 5 & 6 & 7 & 8 & 9 & 10 \\
\hline
\end{tabular}

Die Ratingskala diente der Überprüfung der Hypothesen 13, 14 und 15.

\subsection{Skalen zum Erleben von Emotionen (SEE) und eine Emotionswahrnehmungsskala}

Die Skalen zum Erleben von Emotionen (SEE) wurden von Behr und Becker (2004) entwickelt.

Sie dienen der Persönlichkeits- und Störungsdiagnostik und stellen einen voll standardisierten kurzen und praktisch gut einsetzbaren Selbstauskunft-Fragebogen dar, der das individuelle Gefühlserleben sowie die Bewertung und die Regulation von Gefühlen erfasst.

In der Forschung kann der SEE vor allem auch für eine Prozessmessung gut angewendet werden. 
Die sieben faktorenanalytisch gewonnenen und voneinander unabhängigen Skalen gliedern sich insgesamt in 42 Items.

Die unabhängigen Skalen messen:

1. Akzeptanz eigener Emotionen

2. Erleben von Emotionsüberflutung

3. Erleben von Emotionsmangel

4. Körperbezogene Symbolisierung von Emotionen

5. Imaginative Symbolisierung von Emotionen

6. Erleben von Emotionsregulation

7. Erleben von Selbstkontrolle.

Da in den psychodynamischen Konzepten zur Entstehung somatoformer Störungen vor allem auf die Verdrängung eigener Emotionen, narzisstisches Abwehren eigener Bedürftigkeit und einem Mangel an der Koppelung zwischen seelischem Erleben und körperlichem Empfinden geachtet wird, wurden innerhalb der hier dargestellten Studie die Skalen 1, 3 und 4 für die Verlaufsmessung verwendet (Rudolf, 2008).

Tabelle 34 zeigt eine Übersicht über die Bedeutung hoher oder niedriger Werte dieser Skalen. 
Tabelle 34: Übersicht der Bedeutungen hoher und niedriger Werte in den Skalen der SEE (Behr \& Becker, 2004).

\begin{tabular}{|c|c|}
\hline \multicolumn{2}{|c|}{ Akzeptanz eigener Emotionen } \\
\hline hohe Werte & niedrige Werte \\
\hline $\begin{array}{l}\text { - } \quad \text { kann Empfindungen so } \\
\text { annehmen wie sie sind } \\
\text { - } \quad \text { steht zu den eigenen Gefühlen } \\
\text { - } \\
\text { schämt sich nicht für die } \\
\text { eigenen Gefühle }\end{array}$ & $\begin{array}{l}\text { - } \text { schämt sich für die eigenen } \\
\text { Gefühle } \\
\text { - } \quad \text { kann die eigenen Gefühle nicht } \\
\text { annehmen oder nur zum Teil } \\
\text { annehmen }\end{array}$ \\
\hline \multicolumn{2}{|c|}{ Erleben von Emotionsmangel } \\
\hline hohe Werte & niedrige Werte \\
\hline $\begin{array}{l}\text { - } \quad \text { spürt die inneren Empfindungen } \\
\text { oft nicht } \\
\text { - } \quad \text { würde die eigenen Gefühle } \\
\text { gerne besser wahrnehmen } \\
\text { - } \quad \text { nimmt die eigenen } \\
\quad \text { Körpersignale schlecht wahr }\end{array}$ & $\begin{array}{ll}\text { - } & \text { erlebt innerlich genug } \\
\text { - } & \text { nimmt die eigenen Gefühle wahr } \\
\text { - } & \text { spürt die eigenen Körpersignale }\end{array}$ \\
\hline \multicolumn{2}{|c|}{ Körperbezogene Symbolisierung von Emotionen } \\
\hline Hohe Werte & Niedrige Werte \\
\hline $\begin{array}{ll}\text { - } & \text { nutzt Körpersignale beim } \\
\text { Treffen von Entscheidungen } \\
\text { - } \quad \text { erlebt Intuition aus dem } \\
\text { Körperempfinden heraus } \\
\text { - } \quad \text { empfindet die eigenen } \\
\text { Körperwahrnehmungen als } \\
\text { Spiegel des seelischen } \\
\text { Befindens } \\
\end{array}$ & $\begin{array}{l}\text { - } \quad \text { nutzt die eigenen Körpersignale } \\
\text { nicht zur Entscheidungsfindung } \\
\text { oder zur Orientierung } \\
\text { - } \quad \text { sieht keinen Zusammenhang } \\
\text { zwischen dem körperlichen und } \\
\text { seelischen Erleben }\end{array}$ \\
\hline
\end{tabular}

Die Probanden bewerteten alle unten aufgeführten Items jeweils mit „stimmt gar nicht“, „stimmt kaum, „stimmt mittelmäßig“, „stimmt ziemlich“ oder „stimmt völlig“.

\section{Für die Skala „Akzeptanz eigener Emotionen“ gelten folgende 6 Items:}

1. Ich stehe zu all meinen Empfindungen.

2. Ich fühle, was ich fühle, und das ist ok.

3. Es ist für mich keine Frage, dass ich ein Recht auf alle meine Gefühle habe.

4. Für meine Gefühle schäme ich mich nicht.

5. Alle meine Gefühle dürfen genauso sein, wie sie sind.

6. Was bei mir an Gefühlen hochkommt, kann ich immer zunächst auch annehmen. 
Für die Skala „Erleben von Emotionsmangel“ gelten folgende 5 Items:

1. Meine Körpersignale bemerke ich schnell.

2. Mein inneres Erleben fühle ich oft nicht.

3. Oft wünsche ich, ich könnte meine Gefühle besser wahrnehmen.

4. Ich würde gerne innerlich mehr erleben.

5. Die Signale meines Körpers spüre ich oft nicht.

Für die Skala „Körperbezogene Symbolisierung“ gelten folgende 8 Items:

1. Meine Gefühle spiegeln sich oft auch in Körperempfindungen wider.

2. Die Wahrnehmung von inneren Verkrampfungen, Entspannungen usw. hilft mir bei Entscheidungen.

3. Mein körperliches Befinden entspricht meist meinem seelischen Befinden.

4. Bei Entscheidungen verlasse ich mich meist auf meine leiblichen Empfindungen.

5. Empfindungen wie Herzklopfen, Magendrücken, Herzkribbeln sind für mich eine gute Orientierung für das, was ich will.

6. Wenn ich mich in einer Situation unwohl fühle, dann merke ich dies z. B. im Magen, an der Haut, an Verspannungen o.ä.

7. Körperliche Beschwerden empfinde ich oft als Ausdruck seelischen Unbehagens.

8. Meine Intuition hat für mich viel mit der Wahrnehmung meines Leibes zu tun.

Für die inneren Konsistenzen der Skalen konnten sehr gute bis gute valide Werte $(a=, 70$ bis a =,86) ermittelt werden. Die Retest-Reliabilität liegt über Intervalle von 2, $3,4,10$ und 14 Wochen hinweg, für alle Skalen konstant, im Bereich von ,60 bis ,90.

Zudem liegen Untersuchungen mit anderen Verfahren zur Emotionswahrnehmung, mit Selbstkonzeptinventaren, klinischen Tests und Verfahren zur Einschätzung von interpersonellen Beziehungen vor.

Die Skalen korrelieren jeweils theorie- und hypothesenkonform. Eine klinische Stichprobe unterscheidet sich von einer Zufallsstichprobe ebenso hypothesenkonform wie die Skalenwerte von Männern und Frauen.

Für die Normierung liegen, getrennt für Alters- und Geschlechtergruppen sowie Vergleichswerte für Psychotherapie-Patienten, z-, T-. Prozentrang- und Statinenwerte $(\mathrm{N}=1047)$ vor. Außerdem liegen eine italienische und eine türkische Version vor (Behr \& Becker, 2004). 
Für die Darstellung werden hier die ermittelten T-Werte verwendet. Testwerte werden als auffällig bewertet, wenn sich Abweichungen von einer Standardabweichung über oder unter dem Mittel der Vergleichsstichprobe ergeben, das heißt T-Werte $>60$ oder $<40$.

Dies bedeutet, dass diese Skala so normiert wurde, dass sie einen Mittelwert von $M=50$ und eine Standardabweichung von $\mathrm{S}=10$ (T-Wert-Skala) besitzt. Damit liegt ein einheitlicher Interpretationsmaßstab vor (Rost, 2005).

Die SEE dienten der Überprüfung der Hypothesen 19 bis 27.

Ratingskala zur Einschätzung der Wahrnehmung eigener Gefühle

Zur zusätzlichen Erfassung der erlebten Fähigkeit eigene Gefühle wahrzunehmen diente eine Ratingskala, der folgende Frage voran ging:

„Wie gut können Sie nach Ihrer eigenen Einschätzung auf einer Skala von 0 bis 10 derzeit Ihre eigenen Gefühle wahrnehmen?"Bitte kreuzen Sie EINE Zahl an

O = überhaupt nicht
$10=$ sehr intensiv
\begin{tabular}{|l|l|l|l|l|l|l|l|l|l|l|}
\hline 0 & 1 & 2 & 3 & 4 & 5 & 6 & 7 & 8 & 9 & 10 \\
\hline
\end{tabular}

Die Ratingskala diente der Überprüfung der Hypothesen 28, 29 und 30.

\subsection{Veränderungsfragebogen des Erlebens und Verhaltens (VEV)}

Der Veränderungsfragebogen des Erlebens und Verhaltens (VEV) wurde von Zielke und Mehnert 1978 entwickelt.

Der Fragebogen dient der quantitativen Einschätzung der subjektiv wahrgenommenen Veränderungen im Erleben und Verhalten und eignet sich für Verlaufsuntersuchungen an einzelnen Probanden oder Gruppen von Probanden. Ursprünglich wurde er für Klienten nach Beendigung einer klientenzentrierten Psychotherapie angewandt, wird aber umfassender auch im Rahmen der Erfolgskontrolle von Psychotherapie anderer Ausrichtungen benutzt. 
Der VEV erfasst einen bipolaren Veränderungsfaktor des Erlebens und Verhaltens mit den Polen Entspannung/Gelassenheit/Optimismus und Spannung/Unsicherheit/Pessimismus. Dabei wird für 42 Erlebens- und Verhaltensweisen die Veränderung, vor allem durch eine Behandlung, direkt eingeschätzt. Die Items, von denen 28 positiv und 14 negativ formuliert sind, sind als Veränderungen (besser/schlechter geworden) direkt ausformuliert.

Die Beurteilung erfolgt anhand einer siebenstufigen Skala, die die Veränderung in der formulierten Richtung und in der Gegenrichtung je dreistufig umfasst und als Indifferenzpunkt „keine Änderung“ als Auswahlmöglichkeit bietet.

Da der Bogen eine gewisse Komplexität beinhaltet und ein genaues Verständnis des Systems erforderlich ist, finden Probanden auf der ersten Seite eine detaillierte Einleitung, die folgende Erklärungen beinhaltet:

„Bitte stellen Sie sich den Zeitpunkt vor 8 Wochen vor. Das war der-----(Datum). Überlegen Sie, was Sie zu diesem Zeitpunkt machten und wie Sie sich fühlten. Überblicken Sie bitte nunmehr immer den Zeitraum vom obigen Datum bis heute. Bitte prüfen Sie bei den nachfolgenden Fragen immer, ob sich bei Ihnen innerhalb dieses Zeitraums eine Änderung in die eine oder andere Richtung vollzogen hat. Das Ausmaß der Änderung geben Sie bitte durch Ankreuzen einer Zahl an.

Hier ein Beispiel:

\begin{tabular}{|c|c|c|c|}
\hline & \multicolumn{3}{|c|}{ Änderung } \\
\hline & $\begin{array}{l}\text { In } \\
\text { gleicher } \\
\text { Richtung }\end{array}$ & keine & $\begin{array}{l}\text { In } \\
\text { entgegengesetzter } \\
\text { Richtung }\end{array}$ \\
\hline & $\begin{array}{lll}+3 & +2 & +1 \\
\text { Stark } & \text { mittel } & \text { schwac }\end{array}$ & h & $\begin{array}{ccc}-1 & -2 & -3 \\
\text { schwach } & \text { mittel stark }\end{array}$ \\
\hline Ich bin ruhiger geworden & & & \\
\hline
\end{tabular}

Abbildung 5: Beispielabbildung zur Erfassung der erlebten Veränderung des Erlebens und Verhaltens im Veränderungsfragebogen (VEV). 
Wenn Sie innerhalb des Zeitraums keine Änderung erlebt haben, wenn Sie also weder ruhiger noch unruhiger geworden sind, durchkreuzen Sie bitte 0 .

Wenn Sie eine starke Änderung in entgegengesetzter Richtung erlebt haben, wenn Sie also während des Zeitraums unruhiger geworden sind, durchkreuzen Sie bitte die - 3 .

Wenn Sie eine starke Änderung in die gleiche Richtung erlebt haben, wenn Sie also während des Zeitraums ruhiger geworden sind, durchkreuzen Sie bitte die +3 .

Nun beantworten Sie bitte zügig und spontan alle Fragen!

Alle 42 Items sind ebenso aufgebaut, wie das Beispiel oben. Die einzuschätzenden Aussagen lauten:

1. Ich fühle mich weniger gehetzt.

2. Ich rege mich über viele Dinge nicht mehr auf.

3. Ich weiß jetzt eher, was ich tun will und tun kann.

4. Ich habe jetzt das Gefühl, in einer Sackgasse zu stecken, aus der ich nicht herauskomme.

5. Das Leben hat für mich keinen rechten Inhalt mehr.

6. Ich bin mit mir zufrieden und mache mir weniger Gedanken über mich selbst.

7. Ich sehe Schwierigkeiten gelassener entgegen.

8. Ich bin innerlich ruhiger geworden.

9. Alle Dinge haben jetzt Bedeutung und Leben für mich.

10. Ich habe weniger Ausdauer und gebe schneller auf.

11. Ich bin heiterer geworden.

12. Meine Stimmungen schwanken jetzt stärker als früher.

13. Ich fühle mich unabhängiger von anderen.

14. Ich leide nicht mehr unter meiner Unsicherheit.

15. Im Umgang mit anderen Menschen bin ich ruhiger geworden.

16. Ich fühle mich weniger beunruhigt, wenn ich an die Zukunft denke.

17. Diese quälende Ungewissheit ist stärker geworden.

18. Unerwartete Ereignisse nehme ich gelassener hin.

19. Ich fühle mich stärker isoliert als früher.

20. Ich weiß jetzt manchmal nicht mehr, wie es weitergehen soll.

21. Ich kann es noch nicht verstehen, aber es geht mir seit einiger Zeit besser.

22. Ich kann jetzt freier sprechen.

23. Ich habe weniger Selbstvertrauen.

24. Irgendwie sehe ich wieder mehr Sinn in meinem Leben. 
25. Ich fühle mich freier.

26. Mit Personen des anderen Geschlechts kann ich schlechter Kontakt aufnehmen.

27. Meine Schwierigkeiten im Umgang mit anderen Menschen haben zugenommen.

28. Ich bin häufiger niedergestimmt.

29. Ich fühle mich meinen Aufgaben nicht mehr gewachsen.

30. Ich bin entspannter.

31. Ich fühle mich ruhiger und ausgeglichener.

32. Ich habe ein klareres Bild von mir und meiner Zukunft.

33. Irgendwie bin ich froh und sehe die Dinge optimistischer.

34. Ich glaube, dass ich jetzt eher weiß, was für mich wichtig ist.

35. Ich kann mich jetzt mit meinen Problemen besser abfinden.

36. Im Gespräch mit anderen fühle ich mich nicht mehr so unsicher.

37. Ich komme mit mir selbst schlechter aus.

38. Ich kann das Ungemach des alltäglichen Lebens besser ertragen.

39. Ich habe keine Angst mehr, in einer Sache zu versagen, die mir gelingen soll.

40. Es macht mich nicht mehr so unsicher, wenn sich ein anderer mir gegenüber selbstbewusst gibt.

41. Ich liege nicht mehr im Kriegszustand mit mir selbst.

42. Ich habe immer größere Schwierigkeiten, mich mit anderen Menschen zu unterhalten.

Der Bogen ermöglicht die Berechnung eines Gesamtwerts aus den in einer Komparativform formulierten Items, die in einer retrospektiven Selbsteinschätzung angekreuzt werden.

Für diesen Gesatmwert liegen Veränderungsnormen aus den Testparametern einer nichtbehandelten klinischen Population vor. Es liegen Veränderungswerte vor, die erreicht werden müssen, um eine Änderung als signifikant ansehen zu können.

168 beschreibt dabei den Wert, bei dem sich weder zum Positiven noch zum Negativen etwas verändert hat.

Die Werte im Positiven liegen bei $187(p<=5 \%), 193(p<=1 \%)$ und ab $200(p<=0,1 \%)$. Im Negativen liegen die Veränderungswerte bei 149 ( $p<=5 \%), 143(p<=1 \%)$ und ab 136 $(p<=0,1 \%)$.

Der Gesamtscore reicht von 42 bis 294. 
Dieser Wert sagt etwas darüber aus, in welcher Richtung und mit welcher Stärke sich ein Klient nach einer abgeschlossenen Therapie beziehungsweise psychosozialen Intervention in seinem Verhalten und Erleben verändert hat.

Er hat die Funktion, eine Veränderung des subjektiven Befindens durch einen rückschauenden Schätzungsproze $B$ anhand einzelner Veränderungsaussagen zu objektivieren und zu quantifizieren.

Es wurden Reliabilitätskoeffizienten für eine Therapie- und eine Wartegruppe ( $\mathrm{N}$ jeweils 45) berechnet. Diese Werte liegen für die Gesamtgruppe um ,98. Für Normalpersonen erreichte die Retestreliabilität bei einem Intervall von acht Wochen einen Wert von ,61.

Die Werte im VEV korrelieren mit anderen konstruktnahen Veränderungsskalen (Symptombelastung, $r=, 63$, neurotische Tendez, $r=, 61$, innere Fehlanpassung, $r=, 57$, Psychasthenie, $r=, 56$, Angst, $r=, 55$, jeweils Differenzwerte zwischen Pre- und Postwerten) (Zielke und Mehnert, 1978).

Der VEV diente der Überprüfung der Hypothesen 16, 17 und 18.

\subsection{Arzt-Patient-Fragen und Psychotherapiebereitschaft}

Den Patienten wurden zu den drei Messzeitpunkten Pretest, Posttest und Follow Up zwei Fragen bezüglich des Kontakts zu ihrem behandelnden Hausarzt gestellt.

Die erste Frage bezieht sich auf die Beziehungsqualität, die sie zu ihrem Arzt erleben.

„Wie bewerten Sie mit einer Note von 1-5 die derzeitige Beziehung zu Ihrem behandelnden Hausarzt? - Bitte kreuzen Sie EINE Zahl an“"

sehr gut (1), gut (2), befriedigend (3), ausreichend (4) mangelhaft (5)

Diese Frage diente der Überprüfung der Hypothesen 31, 32 und 33.

Die zweite Frage bezog sich auf das Ärztehopping-Verhalten.

Wie oft haben Sie in den letzten 2 Monaten Ihren Hausarzt gewechselt?

Diese Frage diente der Überprüfung der Hypothesen 34, 35 und 36. 
Eine weitere Frage bezog sich auf die derzeitige Bereitschaft zur Durchführung einer Psychotherapie.

Wie hoch ist derzeit auf einer Skala von 0 bis 10 Ihre Bereitschaft eine Psychotherapie zu machen? Bitte kreuzen Sie EINE Zahl an.

\begin{tabular}{|c|c|c|c|c|c|c|c|c|c|c|}
\hline \multicolumn{11}{|c|}{$\begin{array}{c}0=\text { überhaupt keine Bereitschaft } \\
10=\text { sehr hohe Bereitschaft }\end{array}$} \\
\hline 0 & 1 & 2 & 3 & 4 & 5 & 6 & 7 & 8 & 9 & 10 \\
\hline
\end{tabular}

\section{Die Ratingsskala diente der Überprüfung der Hypothesen 49, 50 und 51.}

Ein besonderes Interesse im Rahmen dieser Studie war es, die behandelnden Hausärzte einzubeziehen. Einerseits aufgrund ihrer professionellen Einschätzung und Diagnose über die teilnehmenden Patienten, andererseits aber auch, weil der Kontakt und die Beziehung zu innen als äußerst wichtig und relevant für Patienten mit einer somatoformen Störung gelten. Sie sind meist die erste und häufigste Anlaufstelle und erfüllen damit eine bedeutsame Funktion für den Verlauf und die Behandlung dieser Menschen (Henningsen et al., 2002b).

Somatoforme Patienten gelten als besonders schwierig und fordernd, möchten immer wieder neue medizinische Untersuchungen bekommen, auch wenn oder gerade weil diese schon einige Male zu negativen pathophysiologischen Ergebnissen geführt haben. Diese Untersuchungen sind nicht nur kostenintensiv, sondern führen den Betroffenen auch immer weiter in die falsche Richtung.

Es interessierte daher, ob nach dem Gruppenprogramm eine andere Sicht auf die Krankheit entsteht und die Forderungen zur Durchführung weiterer medizinischer Untersuchungen sowie die Anzahl der Arztkonsultationen im Untersuchungszeitraum sinken.

Neben diesen Aspekten war auch von Interesse, die erlebte Beziehung des Arztes zu seinem Patienten $z u$ erfassen. Aufgrund der oft massiven Forderungen und der permanenten Unzufriedenheit mit der medizinischen Behandlung, erleben Ärzte die Beziehung zu ihren Patienten häufig als anstrengend und wenig erfüllend. 
Folgende Fragen beantworteten die behandelnden Hausärzte der teilnehmenden Patienten im Pretest, Posttest und Follow:

Wie oft hat Ihr Patient Mr. X Sie in den letzten 2 Monaten konsultiert?

Diese Frage diente der Überprüfung der Hypothesen 40, 41 und 42.

Wie ausgeprägt ist derzeit das Ausmaß der Forderungen des Patienten nach weiteren medizinischen Untersuchungen? Bitte kreuzen Sie EINE Zahl an:

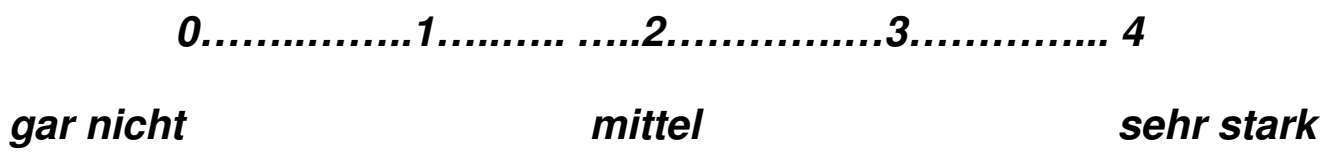

Diese Frage diente der Überprüfung der Hypothesen 43, 44 und 45.

Wie bewerten Sie mit einer Note von 1-5 die derzeitige Beziehung zu Ihrem Patienten? Bitte durchkreuzen Sie Ihre Bewertung:

sehrgut(1) gut(2) befriedigend(3) ausreichend(4) mangelhaft(5)

Diese Frage diente der Überprüfung der Hypothesen 37, 38 und 39.

\subsection{Frage zu einem narzisstischen Verhaltensmuster}

Bisher gibt es kein Instrument, welches das, nach Gerd Rudolfs Theorie beschriebene, narzisstische Verhaltensmuster somatoformer Patienten erfasst.

Es ist jedoch interessant zu erfahren, ob die Probanden der Versuchsgruppe sich in der Interventionsgruppe weniger pseudoautonom verhalten, das heißt ihr Bewältigungsverhalten ggf. weniger benötigen als im Alltag. Dafür wurde den Probanden der Versuchsgruppe im Posttest zusätzlich eine Frage bezogen auf das erlebte eigene Verhalten in der Gruppe gestellt. 
Die narzisstische Pseudoautonomie wird bei Rudolf als ein typisches Abwehrverhalten des zugrundeliegenden depressiven Grundkonflikts bei Patienten mit somatoformen Störungen genannt und findet daher in der Untersuchung Beachtung (Rudolf, 2008). Es wurde den Probanden eine einzelne, einfache Frage gestellt, die mit einer 5 stufigen Skala, 0 (trifft nicht zu), 2 (trifft zum Teil zu) und 4 (trifft voll zu) sowie 1 und 3 als offene Möglichkeiten, bewertet werden konnte.

1. „ICh zeige mich im Alltag eher autonom und stark als bedürftig und schwach.“

0 . 1.

2 .

3. 4

2. "Ich zeigte mich in der Gruppe eher autonom und stark als bedürftig und schwach."

0 . 1. .2 3 4

Diese Items dienten der Überprüfung der Hypothese 48.

\subsection{Evaluationen des Resonanzerlebens sowie des Beschwerdeerlebens}

Die Versuchsgruppenprobanden schätzten die erlebte Resonanz nach jeder Sitzung selbst ein. Die Bedeutung von Resonanz wurde in der ersten Sitzung erklärt und auf jedem Bogen noch einmal erläutert.

Durch Ankreuzen war dies ökonomisch und einfach möglich.

Wie viel Resonanz * konnten Sie heute in der Gruppe erleben? Bitte kreuzen Sie an:

\begin{tabular}{|l|l|l|l|l|l|}
\hline & Gar nicht & Wenig & Mittel & Viel & Sehr viel \\
\hline Ich bekam heute & & & & & \\
Resonanz von & & & & & \\
anderen & & & & & \\
Mitgliedern & & & & & \\
\hline $\begin{array}{l}\text { Ich habe heute } \\
\text { von der }\end{array}$ & & & & & \\
Gruppenleiterin & & & & & \\
Resonanz & & & & & \\
bekommen & & & & & \\
\hline
\end{tabular}

*Resonanz hier: Das Wort Resonanz stammt vom lateinischen resonare ab und bedeutet soviel wie Widerhall. Hier ist Ihr unmittelbares Gefühl von konstruktiven Reaktionen auf Ihre Aussagen, Bitten, Fragen in dieser Stunde gemeint. Konstruktive Reaktionen müssen nicht zwangsläufig Zuspruch darstellen, sondern können auch Kritik und hilfreiche Anstöße in einem respektvollen Rahmen beinhalten. 
Um dieses Resonanzerleben nach dem Gruppenprogramm mit der erlebten Beschwerdeintensität während den Gruppensitzungen korrelieren zu können, wurde zusätzlich nach jeder Sitzung die erlebte Beschwerdestärke während der heutigen Gruppensitzung sowie als weitere Vergleichsmöglichkeit im Verlauf der gesamten letzten Woche im Alltagsleben erfragt. Dazu verhalfen zwei Ratingskalen.

1. Wie stark erlebten Sie auf einer Skala von 0 bis 10 Ihre körperlichen Beschwerden im Verlauf der gesamten letzten Woche?

$0=$ keine körperlichen Beschwerden bis $10=$ sehr starke körperliche Beschwerden

\begin{tabular}{|l|l|l|l|l|l|l|l|l|l|l|}
\hline 0 & 1 & 2 & 3 & 4 & 5 & 6 & 7 & 8 & 9 & 10 \\
\hline
\end{tabular}

2. Wie stark erlebten Sie auf einer Skala von 0 bis 10 Ihre körperlichen Beschwerden in DIESER Gruppensitzung heute?

$0=$ keine körperlichen Beschwerden bis $10=$ sehr starke körperliche Beschwerden

\begin{tabular}{|l|l|l|l|l|l|l|l|l|l|l|}
\hline 0 & 1 & 2 & 3 & 4 & 5 & 6 & 7 & 8 & 9 & 10 \\
\hline
\end{tabular}

Die Fragen nach dem Resonanzerleben und der erlebten Beschwerdestärke in den Gruppensitzungen dienten der Überprüfung der Hypothesen 46 und 47. 


\subsection{Statistische Untersuchungsverfahren}

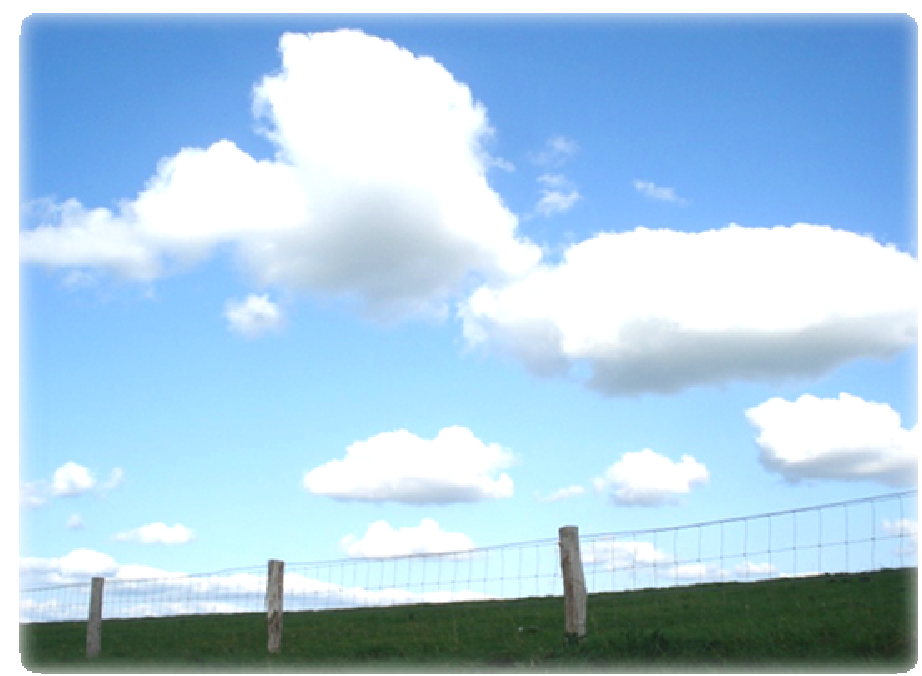

Die statistischen Helfer für den Weg ans Ziel..

Für die Berechnung der Ergebnisse wurden unterschiedliche statistische Verfahren benutzt, die in Tabelle 35 in einer Übersicht dargestellt sind.

Die nonparametrischen Verfahren wurden primär zur nochmaligen Überprüfung der Ergebnisse des fast ausschließlich verwendeten t-Tests benutzt.

Nach vorheriger Disukssion mit Forscherkollegen und dem Vergleich anderer pädagogisch-psychologischer Studien, wurden die hier angewandten Ratingskalen für die Auswertungsmethodik als Intervallskalen behandelt. Auch andere Kollegen und Kolleginnen schlossen sich dieser Meinung an.

Um dennoch die Genauigkeit der ermittelten Ratingskalen-Ergebnisse über den t-Test zu überprüfen, wurden alle Ergebnisse der Ratingskalen zusätzlich mit einem equivalenten nonparametrischen Verfahren kontrolliert. Die Ergebnisse unterschieden sich nicht zu denen des t-Tests.

Als Berechnungshilfe wurde das Programm SPSS Statistics Version 17 herangezogen.

Tabelle 35 zeigt eine Auflistung der Verfahren und ihrer individuellen Nutzen im Einzelnen in der Gesamtübersicht. 
Tabelle 35: Auflistung der verwendeten statistischen Verfahren für die Auswertung der Ergebnisse

\begin{tabular}{|c|c|}
\hline $\begin{array}{l}\text { Statistisches } \\
\text { Verfahren }\end{array}$ & Konkreter Einsatz in dieser Studie \\
\hline $\begin{array}{l}\text { Einfaktorielle } \\
\text { ANOVA mit den } \\
\text { Zusatzoptionen: } \\
\text { Test auf } \\
\text { Homogenität } \\
\text { der Varianzen } \\
\text { und dem } \\
\text { Welch-Test }\end{array}$ & $\begin{array}{l}\rightarrow \text { zur Ermittlung der Vortest-Unterschiede zwischen } \\
\text { Versuchsgruppe und Kontrollgruppe. Der Test auf Homogenität der } \\
\text { Varianzen prüft, ob die Vorraussetzung der Varianzgleichheit erfült } \\
\text { ist. Wird dieser Test signifikant, so sind die Varianzen ungleich. } \\
\text { Das bedeutet, dass die Vorraussetzung verletzt ist. Ob diese } \\
\text { Verletzung aber dazu führt, dass die Ergebnisse nicht interpretiert } \\
\text { werden können, überprüft nun der Welch-Test. Dieser ist nicht an } \\
\text { die Vorraussetzung der Varianzgleichheit gebunden. Ist der Wert } \\
\text { dieses Tests ähnlich dem Ergebnis der ANOVA, dann fällt die } \\
\text { Verletzung der Varianzhomogenität nicht ins Gewicht und man } \\
\text { kann das Ergebnis der ANOVA getrost verwenden. Bei einem 2- } \\
\text { Gruppen Design ist das Ergebnis der ANOVA gleich mit dem des t- } \\
\text { Tests, der ebenfalls die Mittelwerte zu Messzeitpunkt } 2 \text { und } 3 \\
\text { vergleicht (Bortz \& Döring, 2005). }\end{array}$ \\
\hline $\begin{array}{l}\text { Kolmogorow- } \\
\text { Smirnov-Test }\end{array}$ & $\begin{array}{l}\rightarrow \text { zur Überprüfung der Normalverteilung der Stichprobe, die u. a. } \\
\text { Vorraussetzung für die Anwendung des t-Tests ist. }\end{array}$ \\
\hline $\begin{array}{l}\text { t-Test bei } \\
\text { unabhängigen } \\
\text { Stichproben }\end{array}$ & $\begin{array}{l}\rightarrow \text { zur Ermittlung der Mittelwertunterschiede im Posttest und Follow } \\
\text { Up zwischen Versuchsgruppe und Kontrollgruppe (nach vorheriger } \\
\text { Prüfung aller nötigen Bedingungen zur Durchführung des t-Tests.) } \\
\text { Der t-Test liefert somit eine Entscheidungshilfe dafür, ob ein } \\
\text { gefundener Mittelwertsunterschied rein zufällig entstanden ist, oder } \\
\text { ob es wirklich bedeutsame d. h. signifikante Unterschiede } \\
\text { zwischen zwei untersuchten bzw. innerhalb einer untersuchten } \\
\text { Gruppe gibt (s. u. t-Test bei verbundenen Stichproben). }\end{array}$ \\
\hline $\begin{array}{l}\text { t-Test bei } \\
\text { verbundenen } \\
\text { Stichproben }\end{array}$ & $\begin{array}{l}\rightarrow \text { zur Ermittlung der Pretest/Posttest-, Pretest/Follow Up- und } \\
\text { Posttest/Follow Up-Veränderungen innerhalb der einzelnen } \\
\text { Gruppen (Versuchsgruppe und Kontrollgruppe) isoliert betrachtet. }\end{array}$ \\
\hline $\begin{array}{l}\text { Bivariate } \\
\text { Korrelationen }\end{array}$ & $\rightarrow$ zur Ermittlung des Korrelationskoeffizienten nach Pearson. \\
\hline
\end{tabular}




\begin{tabular}{|c|c|}
\hline & Fortsetzung Tab. 35 \\
\hline $\begin{array}{l}\text { Statistisches } \\
\text { Verfahren }\end{array}$ & Konkreter Einsatz in dieser Studie \\
\hline Cohen`s d & $\begin{array}{l}\rightarrow \text { zur Ermittlung der Effektstärken der einzelnen Ergebnisse } \\
\rightarrow \text { Einige Kurzinformationen zur Effektstärke d: } \\
\text { In der empirischen Forschung interessiert nicht nur, ob ein Effekt da ist } \\
\text { (Ablehnung der Nullhypothese) oder nicht (Annahme der Nullhypothese), } \\
\text { sondern auch wie groß der Effekt ist. Effektstärke bezeichnet ein } \\
\text { statistisches Maß, das die (relative) Größe eines Effekts angibt. Ist ein } \\
\text { Unterschied zwischen Gruppen statistisch signifikant, muss noch nach } \\
\text { seiner praktischen Bedeutsamkeit gefragt werden. Ob eine Differenz } \\
\text { signifikant wird, hängt vor allem auch von der Stichprobengröße N ab. Die } \\
\text { Versuchspersonenzahl bestimmt den Standardfehler einer Teststatistik } \\
\text { und je größer die Probandenzahl, desto kleiner der SD. Das führt dazu, } \\
\text { dass bei sehr großen Stichproben auch sehr kleine, praktisch belanglose } \\
\text { Effekte, die pädagogisch-psychologisch trivial sind, noch statistisch } \\
\text { signifikant werden. Eine signifikante Differenz zwischen Gruppen muss } \\
\text { also noch auf die praktische Bedeutsamkeit hin analysiert werden. Dabei } \\
\text { sollten wir uns fragen, ob ein Effekt wirklich bedeutsam genug ist, um } \\
\text { sinnvolle Umsetzungen in die Praxis zu gestatten. Andererseits kann } \\
\text { es passieren, dass ein theoretisch wie praktisch bedeutsamer Effekt nicht } \\
\text { statistisch (mit einem signifikanten Ergebnis) abgesichert werden kann, } \\
\text { einfach weil die Stichprobe zu klein gewählt worden ist (Rost, 2005). } \\
\text { Es gibt mehrere Maße zur Berechnung der Effektstärke. Für } \\
\text { Mittelwertsunterschiede, wie beim t-Test, wird die Effektstärke d (Cohen`s } \\
\text { d) berechnet. Die Formel für Cohen`s d lautet: } \\
\text { d = (M1-M2):((SD1+SD2):2) } \\
\text { Die Effektstärke kann im Gegensatz zu den Werten einer Korrelation } \\
\text { Werte annehmen, die größer als 1 sind. Dadurch, dass dies eine } \\
\text { Interpretation willkürlicher macht, gibt es für die Effekte Konventionen, die } \\
\text { in den Sozialwissenschaften wie folgt klassifiziert werden: } \\
\text { d = 0,20 entspricht einem kleinen Effekt } \\
\text { d = 0,50 entspricht einem mittleren Effekt } \\
\text { d = 0,80 entspricht einem großen Effekt }\end{array}$ \\
\hline $\begin{array}{l}\text { Mann-Whitney- } \\
\text { U-Test }\end{array}$ & $\begin{array}{l}\rightarrow \text { Ein nonparametrisches Alternativverfahren zum t-Test bei } \\
\text { unabhängigen Stichproben, der dann angewendet wird, wenn die } \\
\text { Vorrausetzungen des t-Tests für unabhängige Stichproben stark } \\
\text { verletzt sind, d. h. wenn die Messwerte nicht normalverteilt oder } \\
\text { die Vortestunterschiede zwischen den beiden Gruppen hoch } \\
\text { signifikant sind oder sogar beides gegeben ist. Im Allgemeinen } \\
\text { reagiert der t-Test auf Vorraussetzungsverletzungen jedoch } \\
\text { relativ robust, } d \text {. h. um die Ergebnisse verwerfen zu müssen, } \\
\text { müssen die Vorraussetzungen eklatant verletzt werden. Um zu } \\
\text { überprüfen, wie sehr sich mögliche Verletzungen auf das Ergebnis } \\
\text { auswirken, kann deshalb zusätzlich zum t-Test zur Absicherung } \\
\text { der Mann-Whitney-U-Test berechnet werden. (Bortz, 2005) }\end{array}$ \\
\hline Wilcoxon Test & $\begin{array}{l}\rightarrow \text { Ein nonparametrisches Alternativverfahren zum t-Test bei } \\
\text { verbundenen Stichproben, der dann angewendet wird, wenn die } \\
\text { Vorrausetzungen des t-Tests für abhängige Stichproben stark } \\
\text { verletzt sind. Auch dieser Test kann zur Absicherung der } \\
\text { Ergebnisse zusätzlich zum t-Test durchgeführt werden. }\end{array}$ \\
\hline
\end{tabular}




\subsection{Untersuchungsverlauf}

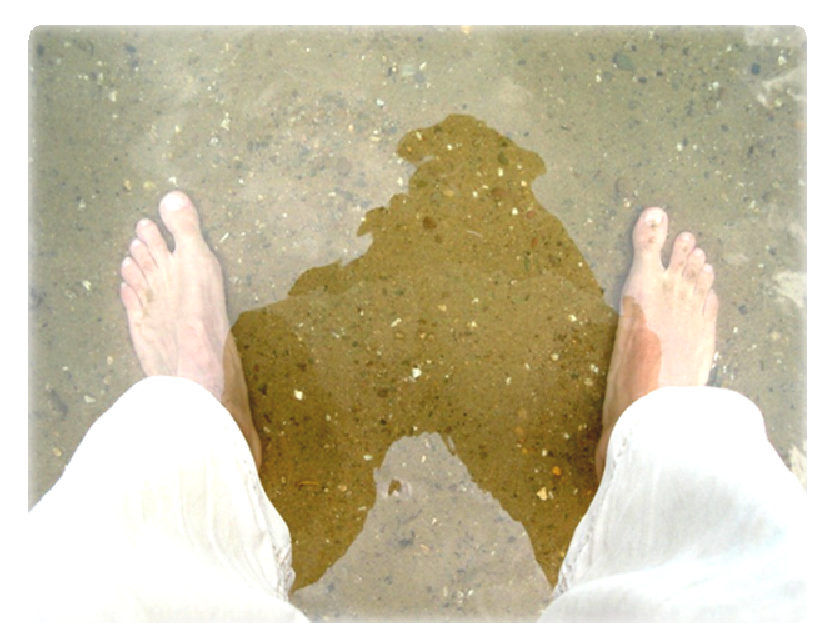

Planung ist die halbe Arbeit...oder?

\subsubsection{Zeitlicher Verlauf}

Der Verlauf der gesamten Studie, inklusive Spezifizierung des Themas und schriftliche Zusammenfassung der Ergebnisse, verlief über einen Zeitraum von zweieinhalb Jahren hinweg, von Oktober 2007 bis Mai 2010. Oktober 2007 bis Februar 2008 dienten vor allem der Themeneingrenzung und der Recherche über den gegenwärtigen Forschungsstand in der Gruppenarbeit mit Patienten, die unter somatoformen Störungen leiden. Zudem fand ein reger und sehr inspirierender Austausch mit Professoren, Kollegen und Forschungsteams anderer Universitäten in Deutschland statt. An der Universität zu Köln gab es schriftlichen Kontakt und persönliche Gespräche mit Prof. Klosterkötter, Prof. Albus und Dr. Weber. An der Universität Freiburg konnte Prof. Fritzsche und Prof. Scheidt für ein Gespräch und einen Erfahrungsaustausch gewonnen werden. Prof. Fritzsche verwies anschließend auf Prof. Henningsen, der an Universität München forscht. Über den freundlichen und ermutigenden Kontakt zu inm gelang schließlich die Verbindung zu Dr. Schäfert, dem für diese Arbeit wichtigsten und intensivsten Kontakt an der Universität Heidelberg.

Das Forschungsteam Schäfert et.al befasst sich auch derzeit weiterhin in einem großen interdisziplinären Forschungsteam mit dem Thema somatoforme Störungen, Gruppentherapie und der Kooperation mit Hausärzten. Bisher ist jedoch noch keine Veröffentlichung ihrer Ergebnisse bekannt geworden. 
Nach der intensiven Auseinandersetzung mit anderen deutschen Forschern auf dem Gebiet der somatoformen Störungen und dem Herausarbeiten des derzeitigen eher geringen Forschungsstandes in der Gruppenarbeit mit diesem Klientel, konnte zwischen März und Oktober 2008 das Konzept des dieser Arbeit zugrundeliegenden Gruppenprogramms entwickelt und fertiggestellt werden. Das entwickelte Programm basiert hauptsächlich auf dem im Stand der Forschung dargestellten tiefenpsychologischen Ansatz zur Entstehung und Dynamik somatoformer Störungen nach Gerd Rudolf.

Während dieser Zeitspanne konnte zudem der Vorsitzende der Kassenärztlichen Vereinigung für den Bereich Rheinisch-Bergischer Kreis, Herr Dr. Wiemer, als Unterstützung für die Realisierung der Studie gewonnen werden. Über den vierteljährlichen Rundbrief, den alle 369 praktizierenden Kollegen und Kolleginnen erhielten, wurde das Projekt für Oktober 2008 angekündigt und alle Hausärzte eingeladen, betroffene Patienten zu überweisen. Um einen persönlichen Kontakt sowie die Möglichkeit für Rückfragen zu ermöglichen, fand zudem mit 70 der 396 umliegenden Hausärzten ein direktes Gespräch über die zugrundeliegende Motivation des Projekts, mögliche Patienten und dem Wunsch nach besserer Kooperation zwischen Ärzten und anderen Helferberufen statt.

Die Resonanz war zunächst beinah durchweg positiv, dennoch zeigte sich im Anschluss eine große Schwere in der Zuverlässigkeit des Einsatzes für betroffene Patienten von ärztlicher Seite. Ein wichtiges Kriterium für die Notwendigkeit des ständig erneuten Nachhörens lag sicherlich an der massiven Zeitnot, unter der Hausärzte aufgrund einiger neuer zuätzlicher bürokratischer Hürden derzeit leiden.

Größtenteils wurde dabei jedoch eine Desillusionierung der optimistischen Hoffnungen und zuvor etwas überschwänglich positiven Einstellung gegenüber der Bereitschaft von ärztlicher Seite, sich für die Studie und das Patientenklientel einzusetzen, erlebt.

Für eine besonders gute, sehr unterstützende, motivierende und hilfreiche Kooperation soll an dieser Stelle insbesondere Frau Dr. Tönnes, Frau Dr. Bunea, Herrn Dr. Dobers und Herrn Dr. Thiel gedankt werden. 
Ohne sie wäre die Verwirklichung dieses Projekts beinah unmöglich geworden. Insgesamt kooperierten trotz anfänglicher Schwierigkeiten dennoch insgesamt 27 Ärzte innerhalb der Studie und ermöglichten damit die Verwirklichung einer Versuchs- und einer Kontrollgruppe mit jeweils 20 Patienten.

Der Begriff der „Kooperation“ ist wohl extrem dehnungsfähig und diesbezüglich mußten Einbußen gemacht werden. Der Kontakt, der zunächst erhofft und erwünscht war, beschränkte sich auf ein Minimalmaß. Dieses Thema findet in Kapitel 7.2, in einer persönlichen Stellungnahme, noch einmal Raum.

Für die Durchführung der Gruppensitzungen war es das Bestreben einen, einerseits professionellen aber dennoch nicht psychiatrisch besetzten Raum zu finden. Da viele der Patienten erst einmal Ängste und Vorurteile über die psychosoziale Arbeit mitbrachten und teilweise zum ersten Mal in Berührung mit dieser Art der Auseinandersetzung mit ihrer somatoformen Thematik kamen, war es ein besonderes Anliegen, sie nicht direkt durch einen Raum in einer psychiatrischen Einrichtung abzuschrecken.

Nach einigen Kontakten und Gesprächen gelang ein Kontakt mit Frau Muckenhaupt, der zuständigen Betreuerin bei der AOK Bergisch Gladbach für den Bereich Selbsthilfegruppen. Die Darstellung der geplanten Studie begeisterte die Mitarbeiterin und sie vermittelte zu Herrn Birkenberger, der die Raumkoordination im Bereich Rehabilitation, Fitness und sonstiger Gruppen im Haus übernahm. Die AOK als Gesundheitskasse stellte mir für die Gruppensitzungen kostenlos einen großen Raum zur Verfügung. Die Räumlichkeit in der Gesundheitskasse bot einen guten Mittelweg zwischen einem ggf. eher beängstigenden Sitzungsraum in der Psychiatrie und einer ggf. privat organisierten eher unprofessionell wirkenden Räumlichkeit.

Von Oktober bis Ende Dezember 2008 konnte das Gruppenprogramm mit drei Gruppen jeweils einmal in der Woche mit einer Zeitfrequenz von ein einhalb Stunden pro Sitzung in den Räumen der AOK Bergisch Gladbach durchgeführt werden. Der Zeitraum wurde gewählt, weil er alle acht Sitzungen des Konzepts in acht ferienfreien Wochen am Stück ermöglichte. Unmittelbar vor Beginn wurde an allen Patienten der Versuchsgruppe der Pretest durchgeführt. 
Für die Versuchsgruppe waren insgesamt 30 Patienten angemeldet. Davon haben insgesamt fünf Personen die Teilnahme am Programm frühzeitig abgebrochen und fünf weitere der 30 angemeldeten Patienten sind nicht erschienen. Diese zehn Probanden konnten daher nicht mehr in die Versuchsgruppenauswertung einbezogen werden.

Gruppe 1 startete mit sechs festen Patienten in der Gruppe, wobei bis zum Ende drei ausstiegen. Vier von zehn angemeldeten Personen erschienen nicht zum Gruppenstart.

Gruppe 2 startete mit neun Personen und blieb über den Verlauf hinweg konstant. Eine angemeldete Person erschien nicht.

Gruppe 3 begann mit einer Gruppenstärke von zehn Patienten, wobei zwei bis zum Ende hin ausstiegen.

Mit allen 30 angemeldeten Personen wurde im Vorfeld ein ausführliches Telefonat über Ihre Beschwerden sowie über das geplante Gruppenprogramm geführt.

Frühzeitig ausgeschieden oder nicht erschienen sind drei Männer und sieben Frauen. Dies ergibt eine Drop Out Quote von 1/3 bzw. 33,3\%.

Über den Zeitraum von Oktober 2008 bis Oktober 2009 wurden, ein wenig auseinander gezogen, die ebenfalls 20 Patienten der Kontrollgruppe im Pretest, Posttest und Follow Up untersucht.

Die Posttestphase der Versuchsgruppe lag unmittelbar nach Abschluss des Gruppenprogramms im Dezember 2008. Das Follow Up dieser Gruppe fand im März 2009 statt. Nach Eingang aller Untersuchungsbögen der Versuchs- und Kontrollgruppe im Oktober 2009, konnte die Auswertung aller Daten bis Januar 2010 erfolgen.

Von Januar bis Juni 2010 wurden alle Ergebnisse schriftlich zusammengefasst und jeder Kontrollpatient erhielt als Dank für die Teilnahme eine individuelle schriftliche Auswertung seiner Angaben. Einer der unerwarteten aber sehr erfreulichen Ergebnisse und Verläufe ist die Tatsache, dass 14 der 20 Personen der Versuchsgruppe sich im Anschluss an das offizielle Gruppenprogramm das Weiterführen der Gruppe wünschten. Dazu gibt Kapitel 5.9.2 noch einmal ausführlich Auskunft. 
Zudem schicken bis heute einzelne Hausärzte neue Patienten in die Gruppen, die von den Studienteilnehmern herzlich und gut aufgenommen und integriert werden.

Einen kompakten Überblick des gesamten Untersuchungsverlaufs liefert Tabelle 36. 
Tabelle 36: Zeitlicher Verlauf der Untersuchung

\begin{tabular}{|c|c|}
\hline $\begin{array}{l}\text { Oktober } 2007 \text { - } \\
\text { Februar } 2008\end{array}$ & $\begin{array}{l}\text { - Recherche zum gegenwärtigen Stand der Forschung in der } \\
\text { Gruppenarbeit mit Somatisierungspatienten. } \\
\text { - Kontaktaufnahme und Austausch mit Professoren und } \\
\text { Forschungsteams der Universität München, Köln, Freiburg } \\
\text { und Heidelberg. }\end{array}$ \\
\hline $\begin{array}{l}\text { März 2008 - } \\
\text { Oktober } 2008\end{array}$ & $\begin{array}{l}\text { - Entwicklung und Fertigstellung des Gruppenprogramms } \\
\text { - Über den zuständigen Vorsitzenden der Kassenärztlichen } \\
\text { Vereinigung für den Rheinisch Bergischen Kreis. Dr. } \\
\text { Wiemer, Ankündigung der Studie über einen Rundbrief an } \\
\text { alle } 369 \text { praktizierenden Ärzte im Umkreis. } \\
\text { - Persönliches Gespräch mit } 70 \text { der } 369 \text { niedergelassenen } \\
\text { Hausärzte } \\
\text { - Suche eines geeigneten Ortes für die Durchführung der } \\
\text { Sitzungen. Ergebnis: AOK Bergisch Gladbach. } \\
\text { - Patienten- und Ärzterekrutierung/ Vorgespräche }\end{array}$ \\
\hline $\begin{array}{l}\text { Oktober } 2008 \text { - } \\
\text { Dezember } 2008\end{array}$ & $\begin{array}{l}\text { - } \text { Pretest - Phase Versuchsgruppe } \\
\text { - Pretest - Phase von zehn Kontrollprobanden } \\
\text { - Durchführung des Gruppenprogramms an drei Gruppen in } \\
\text { der AOK Bergisch Gladbach }\end{array}$ \\
\hline $\begin{array}{l}\text { Dezember } 2008- \\
\text { Januar } 2009\end{array}$ & $\begin{array}{l}\text { - Posttest-Phase Versuchsgruppe } \\
\text { - Posttest-Phase von zehn Kontrollprobanden } \\
\text { - Pretest-Phase fünf weiterer Kontrollprobanden }\end{array}$ \\
\hline März 2009 & $\begin{array}{l}\text { - } \text { Follow Up Messzeitpunkt Versuchsgruppe } \\
\text { - } \text { Follow Up Messzeitpunkt von zehn Kontrollprobanden } \\
\text { - }\end{array}$ \\
\hline Mai 2009 & $\begin{array}{l}\text { - Follow Up Messzeitpunkt von fünf Kontrollprobanden } \\
\text { - Pretest-Phase fünf weiterer Kontrollprobanden }\end{array}$ \\
\hline Juli 2009 & - Posttest-Phase der letzten fünf Kontrollprobanden \\
\hline September 2009 & - Follow Up Messzeitpunkt der letzten fünf Kontrollprobanden \\
\hline Oktober 2009 & $\begin{array}{l}\text { - Letzte Rückläufe der Fragebögen der } \\
\text { Kontrollgruppenpatienten }\end{array}$ \\
\hline $\begin{array}{c}\text { Oktober } 2009- \\
\text { Januar } 2010 \\
\end{array}$ & $\begin{array}{l}\text { - Auswertung der Daten der Versuchs- und Kontrollgruppe } \\
\text { - Ärzte schicken neue Patienten }\end{array}$ \\
\hline $\begin{array}{l}\text { Januar } 2010- \\
\quad \text { Mai } 2010\end{array}$ & $\begin{array}{l}\text { - Schriftliche Zusammenfassung der Arbeit } \\
\text { - Individuelle Auswertungen für Kontrollpatienten erstellen }\end{array}$ \\
\hline $\begin{array}{c}\text { Januar } 2009 \text { - } \\
\text { Heute (Mai 2010) }\end{array}$ & $\begin{array}{l}\text { - } 14 \text { der } 20 \text { Patienten äußerten den Wunsch die Gruppe } \\
\text { weiter zu führen. Seit einem Jahr und drei Monaten erfolgt } \\
\text { die Teilnahme konstant und erfolgreich. }\end{array}$ \\
\hline
\end{tabular}




\subsubsection{Entwicklung des Studienkonzepts nach Abschluss des Programms}

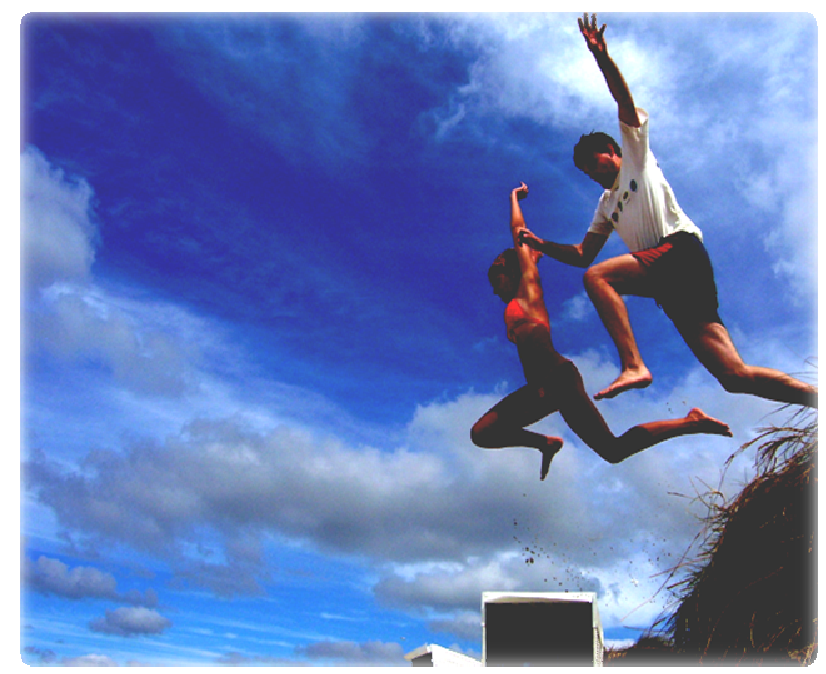

„Theorie ist, wenn man alles weiß und nichts klappt; Praxis ist, wenn alles klappt, und keiner weiß warum" (volkstümlicher Aphorismus).

Dieser Aphorismus steht zu Beginn dieses Kapitels, weil er auch eine vielleicht etwas provokante aber doch existente Wahrheit psychosozialer Interventionen anspricht. Zwar ist es das Bestreben aller Wissenschaftler und Forscher, möglichst alle erfolgreichen psychosozialen Prozesse zu erklären, zu beweisen und damit replizierbar zu machen, dennoch soll die These in den Raum gestellt werden, dass jede Intervention, auch wenn sie genau anhand eines Konzepts entlang verläuft, einzigartig und damit auch einzigartig wirksam ist.

Die in Kapitel 6 dargestellten Ergebnisse sind zum Teil sehr interessant und auch erfolgreich, andere wiederum sind wenig erfolgreich und aus Sicht der Forscherin nicht zufriedenstellend.

Alle Ergebnisse beruhen jedoch auf quantitativen Evaluationsmethoden, die wissenschaftlich zwar sehr anerkannt und hilfreich aber qualitativ doch wenig aussagekräftig sind. Nicht an die statistischen Ergebnisse gebunden ist jedoch die Entwicklung der Gruppen nach Beendigung des offiziellen Programms.

Von 20 teilnehmenden Patienten haben sich 14 direkt und begeistert dafür ausgesprochen, dass sie ihre Gruppen weiterführen möchten. 
Betrachtet man das häufig vorliegende Problem des Ärztehoppings und der schweren Erreichbarkeit für psychosoziale Maßnahmen bei somatoformen Patienten (Zimmermann \& Kaduszkiewicz, 2003), ist die konstante Teilnahme an einer weiterführenden Gruppe seit Januar 2008 bis fortwährend, also nun über ein Jahr, wohl eines der schönsten und erfolgreichsten Ergebnisse der gesamten Studie.

Wenn auch das wirtschaftliche hier vor dem humanen Interesse genannt wird und dies im ersten Moment eher unmenschlich wirkt, ist objektiv dennoch als wichtiges Kriterium anzumerken, dass auch die für das Gesundheitssystem anfallenden, oft massiv hohen Kosten, durch immer wiederholende, ergebnislose medizinische Untersuchungen an diesen Patienten, mit der Gruppenfortführung eingegrenzt werden.

Die Patienten zeigen einen anderen Umgang mit ihrer Krankheit, sie verstehen ihren Körper nicht mehr als isoliert, sondern als einen Teil ihrer ganzen Persönlichkeit. Zudem hält die Leitung weiterhin Kontakt zu den behandelnden Hausärzten und versucht das vertraute Verhältnis zu innen als medizinischen Ansprechpartner für die Patienten zu unterstützen und zu erhalten. Das Ärztehopping wird unterbunden, wenn die Patienten in interdisziplinären fachkompetenten Händen sind.

Meist sprechen die Betroffenen bei Rückfällen oder einer erneuten Verstärkung der Symptomatik erst einmal in der Gruppe über das Geschehen und versuchen ein Verständnis für die derzeitige Situation zu erlangen.

Dass immer auch die medizinische Abklärung zu dieser Arbeit gehört, ist selbstverständlich. Oft erfahren die Betroffenen jedoch in der Gruppensituation oder in den Tagen danach bereits Besserung und bemerken für sich, dass sie auch einen Einfluss und eine Veränderungsmöglichkeit auf und für ihre Beschwerden selbst in sich tragen.

Alle Gruppenteilnehmer haben zudem die Möglichkeit, die sie aber kaum nutzen, in akuten Situationen auch ein Einzelgespräch mit der Gruppenleitung zu führen. Der wenige Gebrauch dieser Option zeigt, dass die Gruppe als eine Form der Selbsthilfe für sie der Raum des Vertrauens und der Hilfe darstellt. Insgesamt wird die vorher ungeplante und unerwartete Entwicklung der Gruppen als eines der bedeutsamsten Ergebnisse der Studie bewertet.

Ausführlicher werden diese und weitere Erfahrungen, die im Rahmen des Projekts gesammelt wurden, in Kapitel 7.2 beschrieben. 
Zum Ende diese Kapitels zum Anfangsaphorismus zurück. Er ist provokant und sicher nicht gänzlich wörtlich zu verstehen, aber vielleicht trifft es diese vorgenommene Veränderung ein wenig besser:

Theorie ist, wenn man sich aus wissenschaftlichen Ergebnissen alles erhofft und nie alles erreicht; Praxis ist, wenn all das passiert, womit niemand vorher gerechnet hat. 


\section{6}

\section{Ergebnisse}

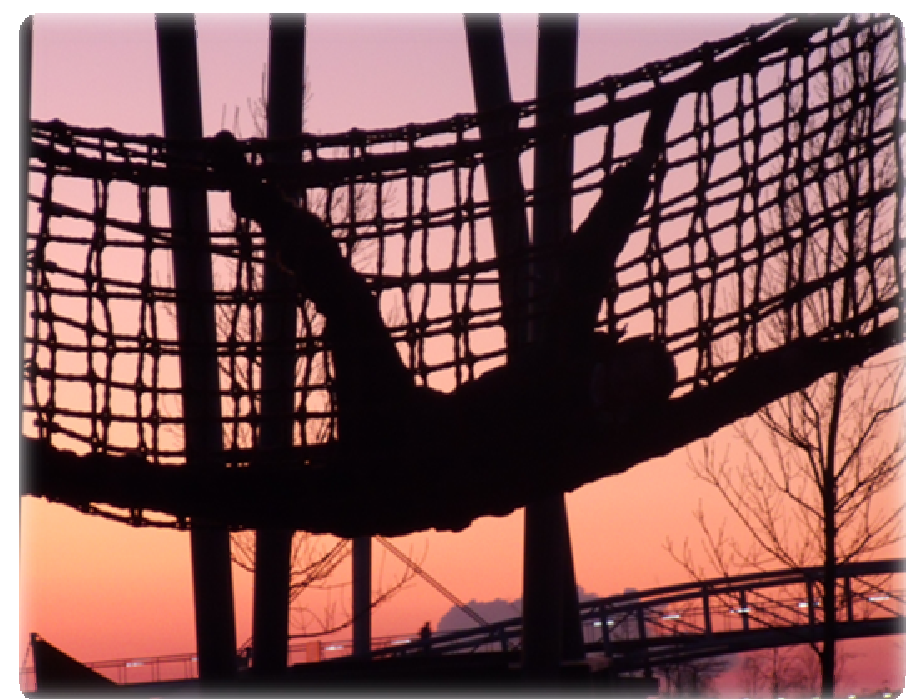

\section{Befund}

„Da ist er, ein prächtiges Engels- oder Teufelswerk (wer will das entscheiden?), noch schwer entwirr-, entziffer- und enträtselbar, doch alle Möglichkeiten der

Erkenntnis lustvoll versprechend, über der Wüste der Ignoranz und ihren fruchtbaren Hervorbringungen. Schon zeigen wir das Ding den wichtigsten Kollegen, den Batz, das Knäul, - unsicher noch, aber auch ein wenig stolz schon: Dies hier haben wir entdeckt, das hier ist es, seht nur, Hoppla, Ecce, Heureka!

Aber: Es wirft Schatten in verschiedene Richtungen?

Und: Was fangen wir nun damit an?

Es ist ja alles andere, nur nicht schwarz und weiß (wie erhofft)“

(Mertens, 1992, S.34).

Nach einem kurzen Ausflug zu eher qualitativen Gesichtspunkten in Kapitel 5.9.2, erfolgt nun die Darstellung der quantitativen Untersuchungsergebnisse.

Diese nun folgenden statistischen Ergebnisse der Evaluation der Studie dienen der Überprüfung der in Kapitel 4 aufgestellten Hypothesen. Es erfolgt zunächst eine deutungsarme Darstellung der Ergebnisse, die in Kapitel 7 im Anschluss diskutiert und interpretiert werden. 
Einzelne ausgewählte Ergebnisse werden zusätzlich durch die Abbildung eines Diagramms hervorgehoben. Dies ermöglicht, durch die bildliche Darstellung, bei besonders interessanten Ergebnissen eine intensivere Veranschaulichung.

\subsection{Vortestunterschiede der Versuchs- und Kontrollgruppe „Homogenität der Gruppen? - Freigabe für den t-Test?“}

Bevor eine Entscheidung für ein statistisches Rechenverfahren getroffen werden konnte, wurden die Vortestunterschiede zwischen Versuchs- und Kontrollgruppe ermittelt. Da sich keine signifikanten Unterschiede zwischen Versuchsgruppe und Kontrollgruppe zeigten, das heißt kein Ergebnis ist <,05, war es möglich im Folgenden die Mittelwerte der beiden Gruppen auch im Posttest und Follow Up miteinander zu vergleichen und auf signifikante Unterschiede hin zu untersuchen. Tabelle 37 zeigt die Überprüfung der Vortestunterschiede und die ermittelten Werte.

Tabelle 37: Überprüfung der Vortest-Unterschiede zwischen Versuchsgruppe und Kontrollgruppe nach den Ergebnissen des Pretests.

\begin{tabular}{|l|c|}
\hline \multicolumn{1}{|c|}{ Variablen } & Signifikanz \\
\hline Psychotherapiebereitschaft &, 923 \\
\hline Beschwerdeindex Somatisierung &, 932 \\
\hline Beschwerdeintensitätsmittelwert &, 065 \\
\hline Beschwerdeanzahl &, 180 \\
\hline PHQ 15 &, 626 \\
\hline WI 7 &, 309 \\
\hline VEV &, 899 \\
\hline SEE Emotionsmangel &, 514 \\
\hline SEE körperbezogene Symbolisierung &, 224 \\
\hline SEE Akzeptanz der Emotionen &, 375 \\
\hline Beschwerdeskala 0-10 &, 471 \\
\hline Gefühle wahrnehmen Skala 0-10 &, 947 \\
\hline Beziehung zum Arzt &, 134 \\
\hline Anzahl Arztwechsel &, 172 \\
\hline Beziehung zum Patient &, 284 \\
\hline Anzahl Arztkonsultationen &, 177 \\
\hline Forderungen nach Untersuchungen &, 727 \\
\hline Narzisstisches Verhaltensmuster &, 563 \\
\hline
\end{tabular}

Erläuterungen Tabelle 37:

PHQ 15: Gesundheitsfragebogen

WI 7: Whtieley Index

VEV: Veränderungsfragebogen des Erlebens und Verhaltens

SEE: Skalen zum Erleben von Emotionen

${ }^{*} p<.05,{ }^{* *} p<.01,{ }^{* * *} p<.001$ 
Aus Tabelle 2 ergibt sich eine Normalverteilung der Ergebnisse, ohne signifikante Unterschiede von dieser. Ab hier sind alle Bedingungen für den T-test für unabhängige Stichproben beziehungsweise die Wiederholung der einfaktoriellen ANOVA erfüllt. Es durften Mittelwertsvergleiche zwischen Kontroll- und Versuchsgruppe im Posttest und Follow Up durchgeführt werden, deren Ergebnisse im Hinblick auf Signifikanz eine wahre Interpretation zulassen und welche zusätzlich auf die Effektstärke d getestet wurden, um die praktische Bedeutsamkeit des Ergebnisses beziehungsweise das Ausmaß der Wirkung eines experimentellen Faktors herauszufinden.

Tabelle 38: Überprüfung der Normalverteilung der gesamten Stichprobe im Pretest.

\begin{tabular}{|l|c|c|}
\hline \multicolumn{1}{|c|}{ Variablen } & N & $\begin{array}{c}\text { Asymptotische Signifikanz } \\
\text { (2-seitig) }\end{array}$ \\
\hline Psychotherapiebereitschaft & 40 &, 153 \\
\hline Beschwerdeindex Somatisierung & 40 &, 708 \\
\hline Beschwerdeintensitätsmittelwert & 40 &, 582 \\
\hline Beschwerdeanzahl & 40 &, 099 \\
\hline PHQ 15 & 40 &, 254 \\
\hline WI 7 & 40 &, 255 \\
\hline VEV & 40 &, 159 \\
\hline SEE Emotionsmangel & 40 &, 156 \\
\hline SEE Körperbezogene & 40 &, 923 \\
Symbolisierung & & \\
\hline SEE Akzeptanz der Emotionen & 40 &, 990 \\
\hline Beschwerdeskala 0-10 & 40 &, 273 \\
\hline Gefühle wahrnehmen Skala 0-10 & 40 &, 053 \\
\hline Beziehung zum Arzt & 40 &, 132 \\
\hline Anzahl Arztwechsel & 40 &, 076 \\
\hline Beziehung zum Patient & 40 &, 315 \\
\hline Anzahl Arztkonsultationen & 40 &, 403 \\
\hline Forderungen nach Untersuchungen & 40 &, 056 \\
\hline
\end{tabular}

\section{Erläuterungen Tabelle 38:}

PHQ 15: Gesundheitsfragebogen

WI 7: Whtieley Index

VEV: Veränderungsfragebogen des Erlebens und Verhaltens

SEE: Skalen zum Erleben von Emotionen

$\mathrm{N}=$ Probandenanzahl

${ }^{*} \mathrm{p}<.05,{ }^{* *} \mathrm{p}<.01,{ }^{* * *} \mathrm{p}<.001$ 
Im gesamten Ergebnisteil gilt:

$\mathbf{N}$ = Probandenanzahl

VG $=$ Versuchsgruppe

KG $=$ Kontrollgruppe

Um ständige Wiederholungen zu vermeiden, werden als Synonyme für die Versuchs- und Kontrollgruppe des Weiteren verwendet:

Gruppe $(\mathbf{A})=$ Versuchsgruppe

Gruppe (B) = Kontrollgruppe

In den folgenden Tabellen befindet sich unter „Vergleichende Statistik Teil I“ der Vergleich der Mittelwerte im Hinblick auf Signifikanz und Effektstärke im Posttest und Follow Up zwischen den beiden Gruppen.

Unter „Vergleichende Statistik Teil II“ befinden sich die Signifikanzen und Effektstärken der Versuchs- und Kontrollgruppe in der isolierten Betrachtung voneinander im Pretest/Posttest-, Pretest/Follow Up- und Posttest/Follow Up-Vergleich der ermittelten Mittelwerte.

Die „Deskriptive Statistik“ zeigt die Mittelwerte (M), die Standardabweichungen (SD) sowie die Probandenanzahl $(\mathrm{N})$ im Pretest, Posttest und Follow Up in den einzelnen Gruppen.

Für alle Tabellen gelten folgende Abkürzungen:

Erläuterungen der Abkürzungen:

$\mathbf{P}=$ Pretest, $\mathbf{P O}=$ Posttest, $\mathbf{F}=$ Follow Up, $\mathbf{M}=$ Mittelwert, $\mathbf{S D}=$ Standardabweichung,

$\mathbf{N}=$ Probandenanzahl

$\mathbf{V G}=$ Versuchsgruppe, $\mathbf{K G}=$ Kontrollgruppe

POvG-POKG $=$ Postest Vergleich der Mittelwerte zwischen VG und KG.

FvG-FKG = Follow Up Vergleich der Mittelwerte zwischen VG und KG.

d PO = Effektstärke(praktische Bedeutsamkeit) der statistischen Ergebnisse im Posttest Vergleich zwischen VG und KG.

$\mathbf{d} \mathbf{F}=$ Effektstärke (praktische Bedeutsamkeit) der statistischen Ergebnisse im Follow Up Vergleich zwischen VG und KG. 
P-PO = Pretest-Posttest Vergleich zwischen den Mittelwerten der einzelnen Gruppen (VG u. KG) isoliert betrachtet

P-F = Pretest-Follow Up Vergleich zwischen den Mittelwerten der einzelnen Gruppen (VG. u. KG) isoliert betrachtet.

PO-F = Posttest-Follow Up Vergleich zwischen den Mittelwerten der einzelnen Gruppen (VG und $\mathrm{KG}$ ) isoliert betrachtet.

d P-PO = Effektstärke des Pre/Post Vergleichergebnisses in den einzelnen Gruppen (VG und $K G)$.

d P-F = Effektstärke des Pre/Follow Up Vergleichergebnisses in den einzelnen Gruppen (VG und $K G)$.

d PO-F = Effektstärke des Post/Follow Up Vergleichergebnisses in den einzelnen Gruppen (VG und $K G)$.

${ }^{*} p<.05,{ }^{\star *} p<.01,{ }^{* \star *} p<.001$

$\mathrm{d}=$ kleiner Effekt ab 0.20, $\mathrm{d}=$ mittlerer Effekt ab 0.50, $\mathrm{d}=$ großer Effekt ab 0.80 


\subsection{Entwicklung der Symptomatik}

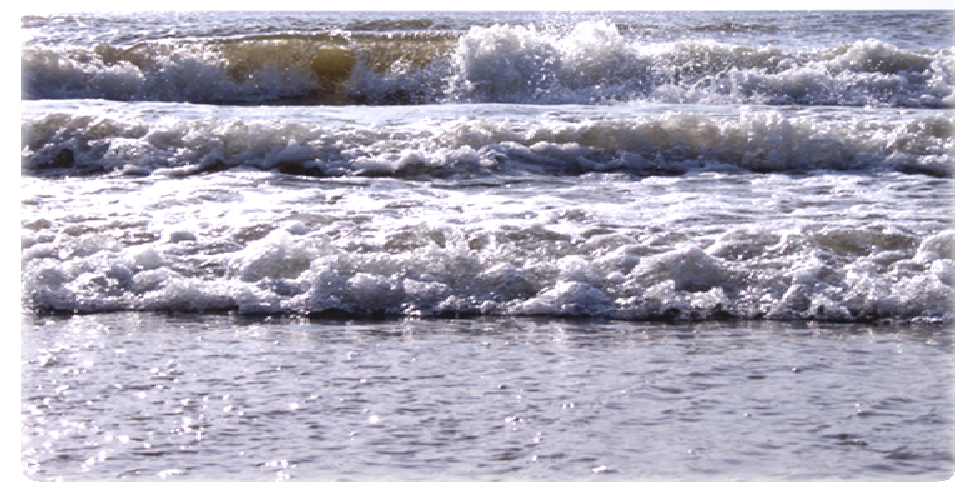

\section{Wo sind die Symptome? - Überraschende Ergebnisse}

In Kapitel 6.2 wurden die Hypothesen 1 bis 15 untersucht. Sie fokussierten sich auf das Hauptmerkmal somatoformer Störungen: die körperliche Symptomatik und damit auch auf den Hauptfokus der untersuchten Hypothesen sowie dem Ziel des Gruppenprogramms. Es war erwünscht, dass die erlebte Symptomatik sich verringert. Es war jedoch nur ein kleiner Effekt zu erhoffen und zu erwarten. Umso erstaunlicher sind die Ergebnisse.

Wichtig ist hier die Beobachtung der Entwicklung der erlebten Beeinträchtigung durch die Beschwerden, die erlebte Beschwerdeintensität, die Anzahl der körperlichen Beschwerden, die Angst, an einer schrecklichen Krankheit erkrankt zu sein und die erlebte Stärke der augenblicklichen Beschwerden.

Tabelle 39 zeigt keinen signifikanten Unterschied der erlebten Beeinträchtigung der Beschwerden zwischen VG und KG im Mittelwertsvergleich im Posttest als auch im Follow Up. Dennoch ist im Posttestvergleich der Gruppen der ermittelte Wert von ,059 als grenzwertig signifikant zu erwähnen. Interessant ist hier die Effektstärke, die trotz nicht vorhandener Signifikanz, zu beiden Messzeitpunkten einen mittleren Effekt aufweist. Sichtbar ist, dass die Mittelwerte der erlebten Beeinträchtigung durch die Beschwerden in der Versuchsgruppe stetig absinken. Wenngleich dies auch in der Kontrollgruppe geschieht, jedoch in einem viel geringerem Ausmaß. 
Unter Vergleichender Statistik Teil II ergeben sich dagegen in der Versuchsgruppe isoliert betrachtet, sowohl der Pretest/Postest- als auch der Pretest/Follow Up-Vergleich als signifikant. Besonders hervorzuheben ist hier, dass sich die erlebte Beeinträchtigung im Follow Up, also 2 Monate nach dem Posttest, nochmals verringert hat.

In der Kontrollgruppe ist von Posttest zu Follow Up eine signifikante Veränderung zu betrachten.

In Gruppe (A) ist zu jedem Vergleichszeitpunkt ein Effekt sichtbar, der zwischen Pretest und Follow Up mit ,76 an einen großen Effekt heranreicht.

In der Kontrollgruppe sind hingegen nur kleine Effekte zwischen Pretest und Follow Up und Posttest und Follow Up zu beobachten.

Insgesamt konnten sich die Hypothesen 1 und 2 zwar für die Versuchsgruppe isoliert betrachtet erfüllen, jedoch zeigte sich kein signifikanter Unterschied im Vergleich zur Kontrollgruppe. Hypothese 3 konnte sich nicht bestätigen. Wichtig ist aber, dass auch im Follow Up die erlebte Beeinträchtigungsschwere noch einmal gesunken ist, wenn auch nicht signifikant.

Tabelle 39: Ergebnisse der Versuchsgruppe und der Kontrollgruppe in der erlebten Beeinträchtigung durch die Beschwerden (0-30)

\begin{tabular}{|c|c|c|c|c|c|c|c|c|c|c|c|c|c|}
\hline \multicolumn{8}{|c|}{ Deskriptive Statistik } & \multicolumn{6}{|c|}{ Vergleichende Statistik Teil I } \\
\hline \multirow{2}{*}{$\begin{array}{c}\text { Unter- } \\
\text { suchungs- } \\
\text { Instrument }\end{array}$} & & \multicolumn{3}{|c|}{ Versuchsgruppe } & \multicolumn{3}{|c|}{ Kontrollgruppe } & \multicolumn{3}{|c|}{ Signifikanzen } & \multicolumn{3}{|c|}{ Effektstärken d } \\
\hline & & $P$ & $\mathrm{PO}$ & $\mathrm{F}$ & $P$ & $\mathrm{PO}$ & $\mathrm{F}$ & \multicolumn{2}{|c|}{ POvg-POKG } & $F_{V G}-F_{K G}$ & $d P O$ & \multicolumn{2}{|c|}{$d F$} \\
\hline \multirow{3}{*}{ PHQ 15} & M & 10,55 & 8,43 & 7,45 & 11,28 & 11,30 & 10,05 & \multirow{3}{*}{\multicolumn{2}{|c|}{059}} & \multirow[t]{3}{*}{,095 } & \multirow[t]{3}{*}{,63 } & \multirow{3}{*}{\multicolumn{2}{|c|}{, 55}} \\
\hline & SD & 4,0 & 3,23 & 4,17 & 5,26 & 5,77 & 5,37 & & & & & & \\
\hline & $\mathrm{N}$ & 20 & 20 & 20 & 20 & 20 & 20 & & & & & & \\
\hline \multirow{4}{*}{ PHQ 15} & & \multicolumn{12}{|c|}{ Vergleichende Statistik Teil II } \\
\hline & & \multicolumn{3}{|c|}{$\begin{array}{c}\text { Versuchsgruppe } \\
\text { Signifikanzen } \\
\end{array}$} & \multicolumn{3}{|c|}{$\begin{array}{l}\text { Kontrollgruppe } \\
\text { Signifikanzen }\end{array}$} & \multicolumn{3}{|c|}{$\begin{array}{c}\text { Versuchsgruppe } \\
\text { Effektstärken d }\end{array}$} & \multicolumn{3}{|c|}{$\begin{array}{l}\text { Kontrollgruppe } \\
\text { Effektstärken d }\end{array}$} \\
\hline & & P-PO & P-F & PO-F & P-PO & P-F & $\mathrm{PO}-\mathrm{F}$ & d P-PO & d P-F & d PO-F & d P-PO & d P-F & d PO-F \\
\hline & &, $032^{*}$ &, $003^{\star *}$ & ,139 & ,966 & ,100 &, $013^{*}$ &, 59 & ,76 & ,26 & ,005 & ,23 & ,23 \\
\hline
\end{tabular}

Tabelle 40 zeigt die Entwicklung der erlebten Beschwerdeintensität. Während sich die erlebte Intensität in der Kontrollgruppe nur ,09 Punkte im Mittelwert verringert, kann in der Versuchsgruppe eine konstante Veränderung von ,18 Punkten im Posttest und Follow Up beobachtet werden.

Der Posttestvergleich zwischen Versuchs- und Kontrollgruppe ergibt mit ,04 einen signifikanten Unterschied. Auch die Effektstärken bewegen sich, sowohl im Posttest- als auch im Follow-Up-Vergleich auf einem mittelgroßen Niveau. 
In der isolierten Betrachtung der beiden Gruppen, ergibt sich jedoch weder bei Gruppe (A) noch bei Gruppe (B) eine signifikante Veränderung, weiterhin jedoch in beiden Gruppen kleine praktisch bedeutsame Effekte.

In der Versuchsgruppe isoliert betrachtet konnte sich keine signifikante Veränderung ergeben, das heißt, Hypothesen 3, 4 und 5 konnten sich nicht mit Signifikanz bestätigen. Dafür entstand jedoch im Posttest, zumindest auf Hypothese 4 bezogen, ein signifikanter Unterschied zwischen Versuchs- und Kontrollgruppe.

Tabelle 40: Ergebnisse der Versuchsgruppe und der Kontrollgruppe in der erlebten Beschwerdeintensität (0-4)

\begin{tabular}{|c|c|c|c|c|c|c|c|c|c|c|c|c|c|}
\hline \multicolumn{8}{|c|}{ Deskriptive Statistik } & \multicolumn{6}{|c|}{ Vergleichende Statistik Teil I } \\
\hline \multirow{2}{*}{$\begin{array}{c}\text { Unter- } \\
\text { suchungs- } \\
\text { Instrument }\end{array}$} & & \multicolumn{3}{|c|}{ Versuchsgruppe } & \multicolumn{3}{|c|}{ Kontrollgruppe } & \multicolumn{3}{|c|}{ Signifikanzen } & \multicolumn{3}{|c|}{ Effektstärken d } \\
\hline & & $\mathrm{P}$ & $\mathrm{PO}$ & $\mathrm{F}$ & $\mathrm{P}$ & $\mathrm{PO}$ & $\mathrm{F}$ & POva-PO & & FvG-FkG & $\mathrm{dPO}$ & & $\mathrm{dF}$ \\
\hline \multirow{3}{*}{ SOMS } & M & 1,88 & 1,7 & 1,70 & 2,12 & 2,03 & 2,0 & ,040* & & ,084 & ,67 & & ,58 \\
\hline & SD & 46 & 49 & 62 & ,35 & 49 & 38 & & & & & & \\
\hline & $\mathrm{N}$ & 20 & 20 & 20 & 20 & 20 & 20 & & & & & & \\
\hline \multirow{4}{*}{ SOMS } & \multicolumn{13}{|c|}{ Vergleichende Statistik Teil II } \\
\hline & & \multicolumn{3}{|c|}{$\begin{array}{l}\text { Versuchsgruppe } \\
\text { Signifikanzen }\end{array}$} & \multicolumn{3}{|c|}{$\begin{array}{l}\text { Kontrollgruppe } \\
\text { Signifikanzen }\end{array}$} & \multicolumn{3}{|c|}{$\begin{array}{l}\text { Versuchsgruppe } \\
\text { Effektstärken d }\end{array}$} & \multicolumn{3}{|c|}{$\begin{array}{l}\text { Kontrollgruppe } \\
\text { Effektstärken d }\end{array}$} \\
\hline & & P-PO & P-F & PO-F & P-PO & P-F & PO-F & d P-PO & d P-F & d PO-F & d P-PO & d P-F & d PO-F \\
\hline & & ,102 & ,268 & ,944 & ,275 & 150 & ,682 & ,38 &, 32 &, 01 & ,23 &, 35 &, 08 \\
\hline
\end{tabular}

Die Anzahl der erlebten körperlichen Beschwerden zeigt in der Versuchsgruppe eine deutliche und vor allem konstante Abnahme im Posttest und Follow Up. Auch in der Kontrollgruppe sinkt die Anzahl der Beschwerden, jedoch in viel geringerem Ausmaß.

Der Vergleich der Mittelwerte zwischen Versuchs- und Kontrollgruppe ist sowohl im Posttest als auch im Follow Up signifikant. Es ergeben sich im Gruppenvergleich mit ,82 ein großer Effekt im Posttest und ein guter mittlerer Effekt von ,68 im Follow Up.

Hier sind die hohen Standardabweichungen auch zu beachten, die bei der Kontrollgruppe fast doppelt so groß sind als in der Versuchsgruppe. Im Posttest und Follow Up ergeben die Standardabweichungen in der Versuchsgruppe, dass auch Patienten dabei sind, deren Anzahl der Beschwerden gegen 0 gehen beziehungsweise gegen 12. In der Kontrollgruppe liegt die Standardabweichung durchgehend bei ca. 10. Dies ermöglicht eine massive Erhöhung für die Beschwerdeanzahl einzelner Probanden. 
In der Einzelbetrachtung der Gruppen sind hoch signifikante Ergebnisse in der Versuchsgruppe im Pretest/Posttest- und Pretest/Follow Up-Vergleich zu beobachten, ebenso gute mittlere Effekte in diesen beiden Vergleichsepisoden. Die Kontrollgruppe zeigt ebenfalls eine signifikante Veränderung zwischen Pretest und Follow Up, jedoch kann man hier die jeweilige Effektstärke zwischen den Gruppen vergleichen, die in der Kontrollgruppe nur klein, in der Versuchsgruppe hingegen mittelgroß ausfällt.

Hypothesen 7, 8 und 9 können insgesamt bestätigt werden, wobei sich im Pretest/Follow Up-Vergleich in der Versuchsgruppe keine signifikante Veränderung mehr feststellen lässt, jedoch trotzdem die Anzahl der Beschwerden weiter absinkt. Sowohl in der Versuchsgruppe isoliert betrachtet als auch im Gruppenvergleich zu den Zeitpunkten Posttest und Follow Up ergeben sich signifikante Veränderungen und Unterschiede zwischen den beiden Gruppen sowie innerhalb der Versuchsgruppe isoliert betrachtet.

Tabelle 41: Ergebnisse der Versuchsgruppe und der Kontrollgruppe für die Anzahl der erlebten Beschwerden

\begin{tabular}{|c|c|c|c|c|c|c|c|c|c|c|c|c|c|}
\hline \multicolumn{8}{|c|}{ Deskriptive Statistik } & \multicolumn{6}{|c|}{ Vergleichende Statistik Teil I } \\
\hline \multirow{2}{*}{$\begin{array}{c}\text { Unter- } \\
\text { suchungs- } \\
\text { Instrument }\end{array}$} & & \multicolumn{3}{|c|}{ Versuchsgruppe } & \multicolumn{3}{|c|}{ Kontrollgruppe } & \multicolumn{3}{|c|}{ Signifikanzen } & \multicolumn{3}{|c|}{ Effektstärken d } \\
\hline & & $\mathrm{P}$ & $\mathrm{PO}$ & $\mathrm{F}$ & $\mathrm{P}$ & $\mathrm{PO}$ & $\mathrm{F}$ & POva-PC & & $F_{V G-F K G ~}$ & $\mathrm{~d} P O$ & & $d F$ \\
\hline \multirow{3}{*}{ SOMS } & M & 10,10 & 6,50 & 6,20 & 13,80 & 12,96 & 11,75 & \multirow{3}{*}{\multicolumn{2}{|c|}{, $018^{\star}$}} & \multirow[t]{3}{*}{, $044^{*}$} & \multirow[t]{3}{*}{82} & \multirow{3}{*}{\multicolumn{2}{|c|}{68}} \\
\hline & SD & 6,33 & 5,47 & 6,14 & 10,32 & 10,34 & 10,21 & & & & & & \\
\hline & $\mathrm{N}$ & 20 & 20 & 20 & 20 & 20 & 20 & & & & & & \\
\hline \multirow{4}{*}{ SOMS } & & \multicolumn{12}{|c|}{ Vergleichende Statistik Teil II } \\
\hline & & \multicolumn{3}{|c|}{$\begin{array}{l}\text { Versuchsgruppe } \\
\text { Signifikanzen }\end{array}$} & \multicolumn{3}{|c|}{$\begin{array}{c}\text { Kontrollgruppe } \\
\text { Signifikanzen }\end{array}$} & \multicolumn{3}{|c|}{$\begin{array}{l}\text { Versuchsgruppe } \\
\text { Effektstärken d }\end{array}$} & \multicolumn{3}{|c|}{$\begin{array}{l}\text { Kontrollgruppe } \\
\text { Effektstärken d }\end{array}$} \\
\hline & & P-PO & P-F & PO-F & P-PO & P-F & PO-F & d P-PO & d P-F & d PO-F & d P-PO & $d P-F$ & d PO-F \\
\hline & &, $009^{\star \star}$ &, $005^{\star \star}$ & ,753 &, 462 &, $045^{*}$ & ,279 &, 61 & ,63 &, 05 & ,08 & , 20 &, 12 \\
\hline
\end{tabular}


Pre/Post und Follow Up-Vergleich der Mittelwerte zwischen Versuchs- und Kontrollgruppe bezogen auf die erlebte Anzahl der Beschwerden

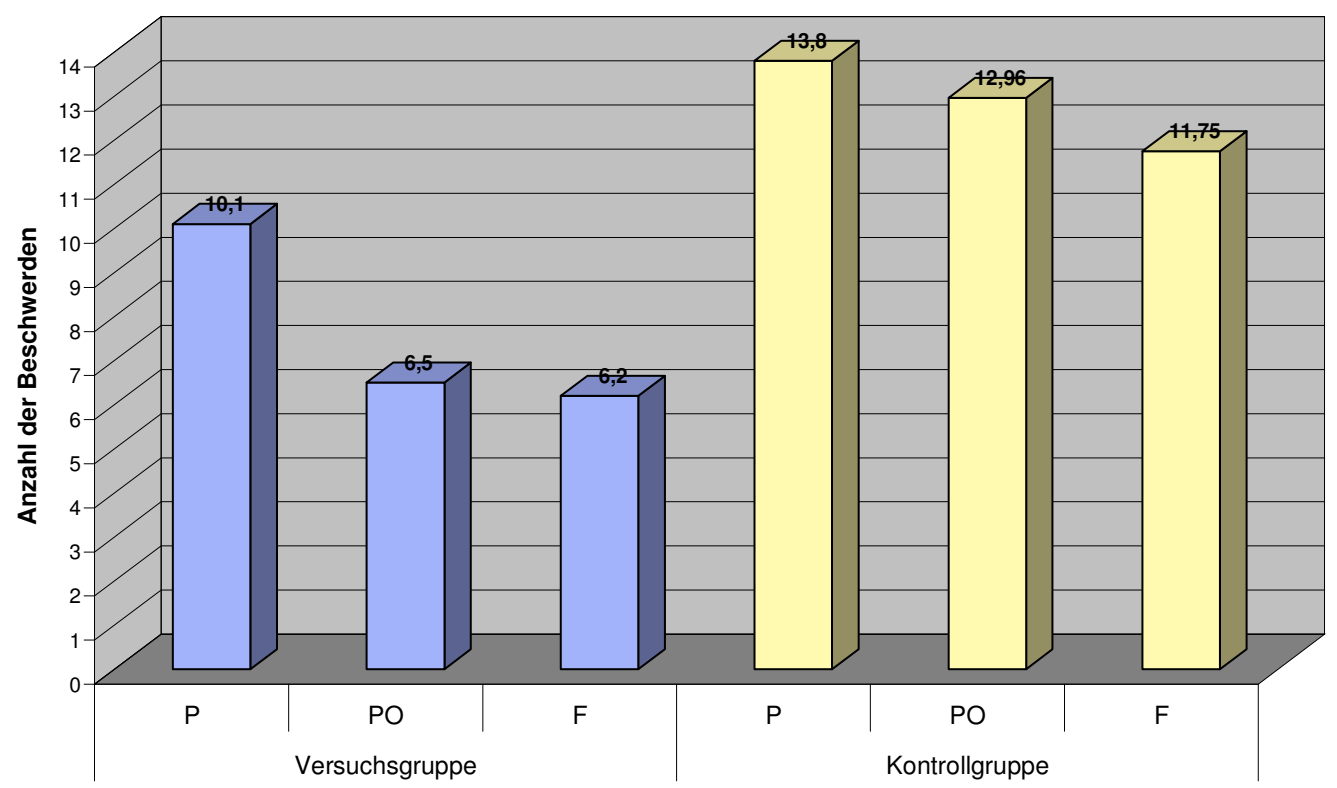

Abbildung 6: Graphische Darstellung: Mittelwertsvergleich zwischen Versuchs- und Kontrollgruppe bezogen auf die erlebte Anzahl der Beschwerden.

Tabelle 6 zeigt die Entwicklung der Krankheitsängste. Hier findet bei der Versuchsgruppe eine konstante Abnahme statt. Auch die Kontrollgruppe zeigt im Mittel eine Abnahme der Ängste, jedoch wiederum in geringerem Ausmaß als in der Versuchsgruppe. Daher ergeben sich die Vergleiche der Gruppen im Posttest und Follow Up mit ,011 und ,025 als signifikant. Auch die Effektstärke fällt zu beiden Zeitpunkten mit ,86 und ,76 groß aus.

Während die Standardabweichung in der Versuchsgruppe konstant sinkt, steigt sie in der Kontrollgruppe stetig an.

Betrachtet man die Versuchsgruppe isoliert, wird die hohe signifikante Veränderung von Pretest zu Posttest und von Pretest zu Follow Up sichtbar. Aber auch die positive Veränderung der Krankheitsängste in der Kontrollgruppe zwischen Pretest und Follow Up ist signifikant.

Besonders hervorzuheben sind die hohen Effektstärken in der Versuchsgruppe, die Werte von ,96 im Pretest/Posttest- und sogar 1,02 im Pretest/Follow Up-Vergleich erreichen.

Kleine bis mittlere Effekte sind aber auch in der Kontrollgruppe zu beobachten. 
Die Hypothesen 10, 11 und 12 konnten sich insgesamt bestätigen. Innerhalb der Versuchsgruppe sinken die Krankheitsängste von Pretest zu Posttest sowie von Pretest zu Follow Up signifikant, wobei von Posttest zu Follow Up zwar ein weiteres Absinken, jedoch ohne Signifikanz zu beobachten ist.

Auch zwischen den Gruppen ergeben sich signifikante Unterschiede zu Gunsten der Versuchsgruppe.

Tabelle 42: Ergebnisse der Versuchsgruppe und der Kontrollgruppe bezogen auf die Entwicklung der Krankheitsängste ( 0 -7)

\begin{tabular}{|c|c|c|c|c|c|c|c|c|c|c|c|c|c|}
\hline & [ & eskr & ptive & Stati & tik & & & Ver & rgleic & ichende & Statis & tik Te & eil I \\
\hline Unter- & & Vers & uchsgr & uppe & Kon & trollgr & uppe & Sign & ifikar & anzen & Effel & tststärk & ken d \\
\hline Instrument & & $\mathrm{P}$ & $\mathrm{PO}$ & $\mathrm{F}$ & $\mathrm{P}$ & PO & $\mathrm{F}$ & $\mathrm{POva}-\mathrm{PO}$ & & FvG-FKG & dPO & & $d F$ \\
\hline & $M$ & 3,75 & 1,6 & 1,55 & 4,55 & 3,6 & 3,35 &, $011^{*}$ & &, $025^{*}$ & ,86 & & ,76 \\
\hline WI 7 & SD & 2,49 & 2,01 & 1,82 & 2,42 & 2,66 & 2,94 & & & & & & \\
\hline & $\mathrm{N}$ & 20 & 20 & 20 & 20 & 20 & 20 & & & & & & \\
\hline & & & & & & gle & her & St & 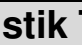 & Teil II & & & \\
\hline WI 7 & & $\begin{array}{r}\text { Vers } \\
\text { Sig }\end{array}$ & $\begin{array}{l}\text { uchsgr } \\
\text { nifikan }\end{array}$ & $\begin{array}{l}\text { uppe } \\
\text { zen }\end{array}$ & $\begin{array}{c}\text { Kon } \\
\text { Sig }\end{array}$ & $\begin{array}{l}\text { trollgr } \\
\text { nifikar }\end{array}$ & $\begin{array}{l}\text { uppe } \\
\text { zen }\end{array}$ & $\begin{array}{l}\text { Versu } \\
\text { Effek }\end{array}$ & $\begin{array}{l}\text { Ichsg } \\
\text { tstärr }\end{array}$ & $\begin{array}{l}\text { gruppe } \\
\text { ken d }\end{array}$ & $\begin{array}{l}\text { Kon } \\
\text { Effel }\end{array}$ & $\begin{array}{l}\text { rollgr } \\
\text { ststärk }\end{array}$ & $\begin{array}{l}\text { uppe } \\
\text { ken d }\end{array}$ \\
\hline & & P-PO & P-F & PO-F & P-PO & P-F & PO-F & d P-PO & d P-F & $=d$ PO-F & d P-PO & $d P-F$ & d PO-F \\
\hline & &, $003^{\star \star}$ & $002^{* *}$ & 934 & 087 & $028^{*}$ & 561 & ,96 & 1,02 & 03 & ,37 & ,45 & ,09, \\
\hline
\end{tabular}

Pre/Post und Follow Up-Vergleich der Mittelwerte zwischen Versuchs- und Kontrollgruppe bezogen auf die erlebten Krankheitsängste

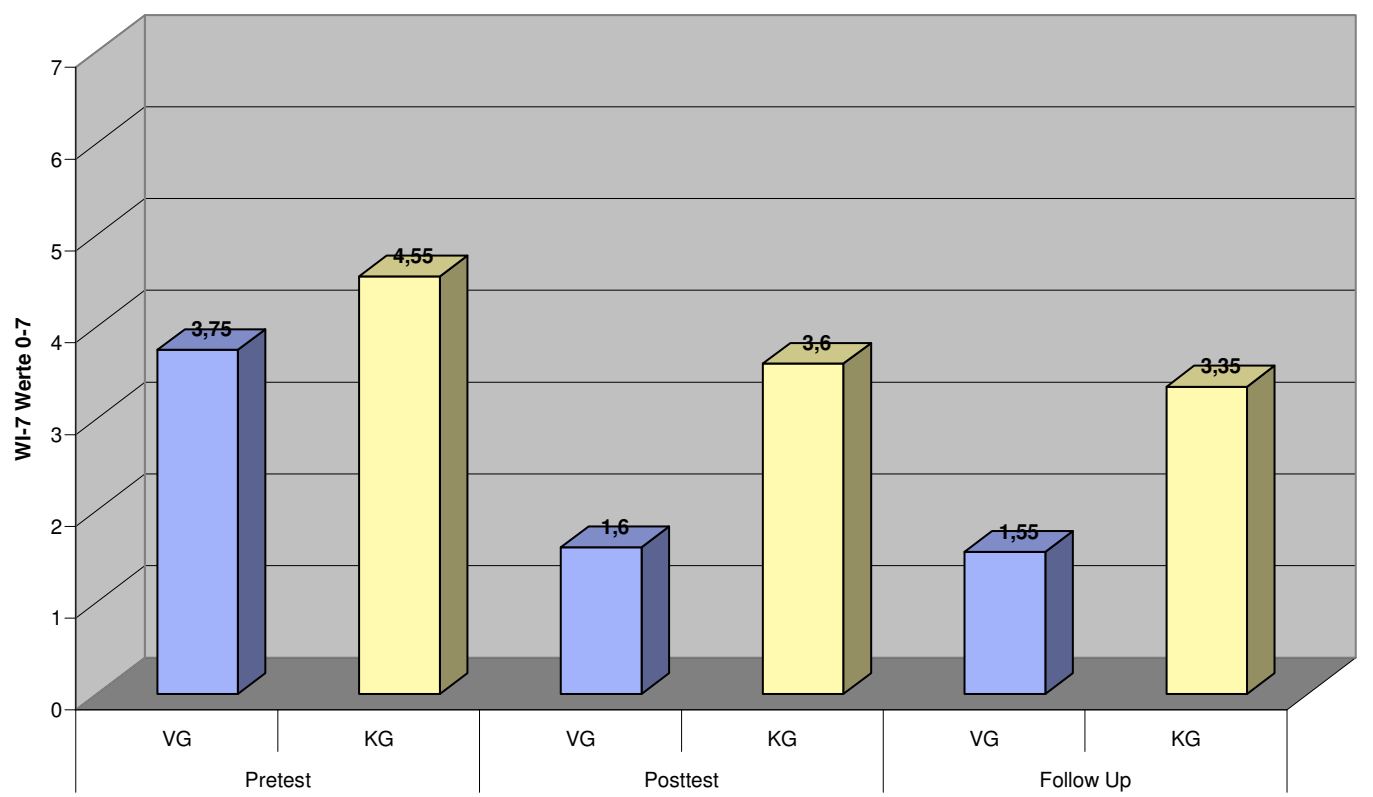

Abbildung 7: Graphische Darstellung: Mittelwertvergleich zwischen Versuchs- und Kontrollgruppe bezogen auf die erlebten Krankheitsängste. 
Tabelle 43 zeigt die Entwicklung der erlebten Stärke der Beschwerden in der Einschätzung auf einer Skala von 0-10. Auch hier fällt die konstant positive Entwicklung der Versuchsgruppe auf, die sich im Mittelwert im Posttest und Follow Up beinah um die Hälfte der erlebten Beschwerdestärke reduzieren kann. Auch die Kontrollgruppe zeigt ein geringes Absinken der erlebten Beschwerdestärke, jedoch wiederum sehr viel geringer als bei den Probanden der Versuchsgruppe.

Die Mittelwertsvergleiche zwischen Versuchs- und Kontrollgruppe zeigen daher sowohl im Posttest als auch im Follow Up einen signifikanten Unterschied und mit ,96 und ,72 große, bedeutsame Effekte.

Während die Berücksichtigung der Standardabweichung im Posttest und Follow Up bei der Versuchsgruppe Werte um 1 ermöglicht, ist dies trotz Beachtung der Standardabweichung in der Kontrollgruppe nicht zu erreichen.

In der isolierten Betrachtung der beiden Gruppen ergibt sich mit ,001 im Pretest/Posttest-. und Pretest/Follow Up-Vergleich eine hoch signifikante Veränderung innerhalb der Versuchsgruppe. Aber auch zwischen den Zeitpunkten Pretest/Follow Up findet statistisch eine signifikante Veränderung in der Kontrollgruppe statt. Wichtig ist hier wieder die Betrachtung der Effektstärken in den einzelnen Gruppen, die in Gruppe (A) mit 1,1 sehr groß und in Gruppe (B) mit ,25 und ,37 klein ausfallen.

Die Hypothesen 13 und 14 konnten sich insgesamt bestätigen. Innerhalb der Versuchsgruppe sinkt die erlebte Stärke der körperlichen Beschwerden von Pretest zu Posttest sowie von Pretest zu Follow Up signifikant.

Hypothese 15 konnte sich nicht bestätigen, da von Posttest zu Follow Up innerhalb der Versuchsgruppe keine Veränderung mehr zu beobachten ist. Wohl aber zeigen sich im Posttest sowie im Follow Up Gruppenvergleich signifikante Unterschiede zu Gunsten der Versuchsgruppe. 
Tabelle 43: Ergebnisse der Versuchsgruppe und der Kontrollgruppe bezogen auf die Entwicklung der erlebten Stärke der Beschwerden auf einer Skala von 0-10

\begin{tabular}{|c|c|c|c|c|c|c|c|c|c|c|c|c|c|}
\hline \multicolumn{8}{|c|}{ Deskriptive Statistik } & \multicolumn{6}{|c|}{ Vergleichende Statistik Teil I } \\
\hline \multirow{2}{*}{$\begin{array}{l}\text { Unter- } \\
\text { suchungs- } \\
\text { Instrument }\end{array}$} & & \multicolumn{3}{|c|}{ Versuchsgruppe } & \multicolumn{3}{|c|}{ Kontrollgruppe } & \multicolumn{3}{|c|}{ Signifikanzen } & \multicolumn{3}{|c|}{ Effektstärken d } \\
\hline & & $P$ & $\mathrm{PO}$ & $\mathrm{F}$ & $\mathrm{P}$ & $\mathrm{PO}$ & $\mathrm{F}$ & POvg-P & & 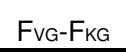 & $\mathrm{d} P O$ & & $d F$ \\
\hline \multirow{3}{*}{$\begin{array}{l}\text { RATING- } \\
\text { SKALA }\end{array}$} & M & 6,25 & 3,85 & 3,85 & 6,8 & 6,15 & 5,75 & \multirow{3}{*}{\multicolumn{2}{|c|}{, $004^{\star \star}$}} & \multirow[t]{3}{*}{, $030^{*}$} & \multirow[t]{3}{*}{,96 } & \multirow{3}{*}{\multicolumn{2}{|c|}{,72 }} \\
\hline & SD & 2,05 & 2,30 & 2,35 & 2,69 & 2,50 & 2,94 & & & & & & \\
\hline & $\mathrm{N}$ & 20 & 20 & 20 & 20 & 20 & 20 & & & & & & \\
\hline \multirow{4}{*}{$\begin{array}{l}\text { RATING- } \\
\text { SKALA }\end{array}$} & & \multicolumn{12}{|c|}{ Vergleichende Statistik Teil II } \\
\hline & & \multicolumn{3}{|c|}{$\begin{array}{l}\text { Versuchsgruppe } \\
\text { Signifikanzen }\end{array}$} & \multicolumn{3}{|c|}{$\begin{array}{l}\text { Kontrollgruppe } \\
\text { Signifikanzen }\end{array}$} & \multicolumn{3}{|c|}{$\begin{array}{l}\text { Versuchsgruppe } \\
\text { Effektstärken d }\end{array}$} & \multicolumn{3}{|c|}{$\begin{array}{l}\text { Kontrollgruppe } \\
\text { Effektstärken d }\end{array}$} \\
\hline & & P-PO & P-F & PO-F & P-PO & P-F & PO-F & d P-PO & d P-F & $=$ d PO-F & d P-PO & d P-F & d PO-F \\
\hline & &, $001^{* \star}$ &, $001^{\star \star}$ & 1,0 & ,142 &, $011^{*}$ & 214 & 1,1 & 1,1 & 0 & ,25 & ,37 & ,15 \\
\hline
\end{tabular}

Pre/Post und Follow Up-Vergleich der Mittelwerte zwischen Versuchs- und Kontrollgruppe bezogen auf die erlebte Beschwerdestärke

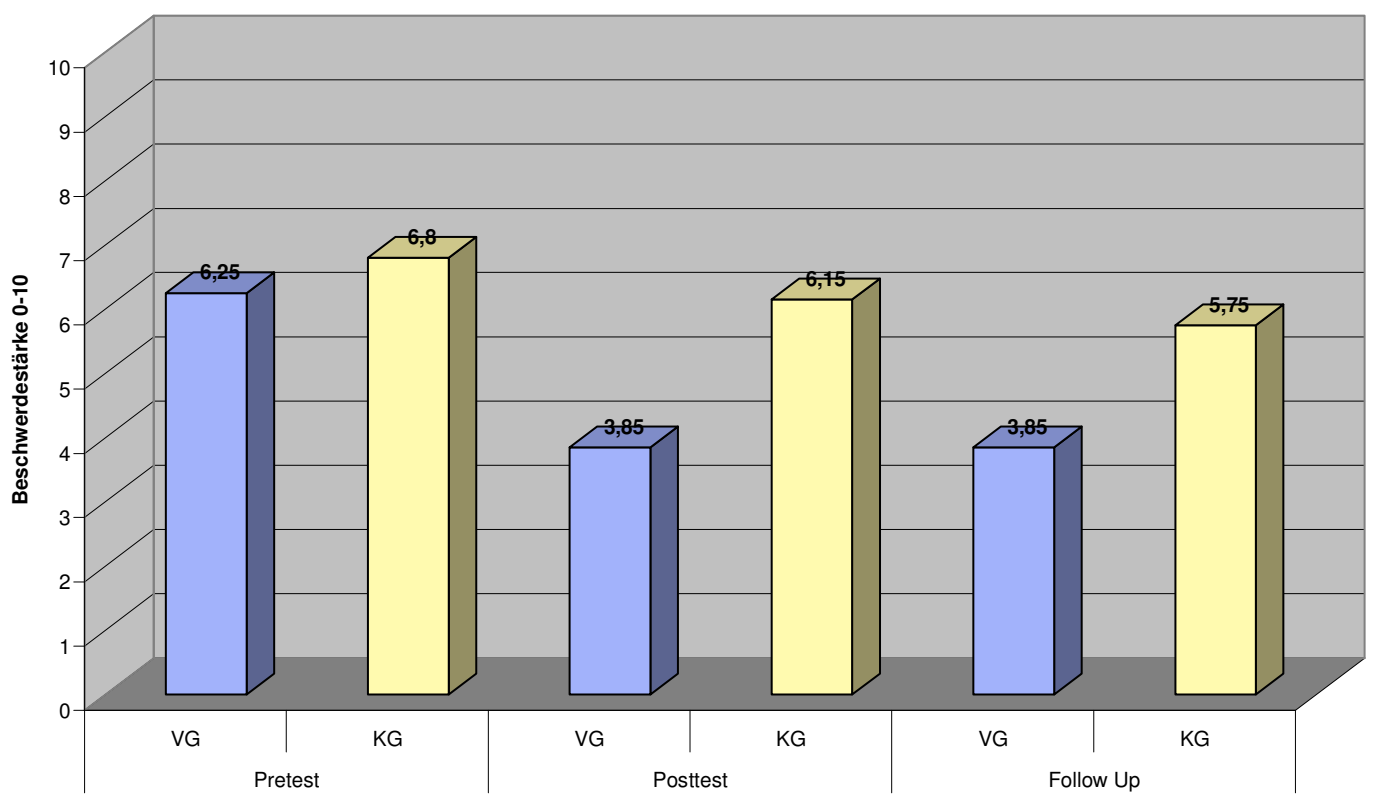

Abbildung 8: Graphische Darstellung: Mittelwertvergleich zwischen Versuchs- und Kontrollgruppe bezogen auf die erlebte Beschwerdestärke 


\subsection{Akzeptanz, Mangel und körperbezogene Symbolisierung der Emotionen}

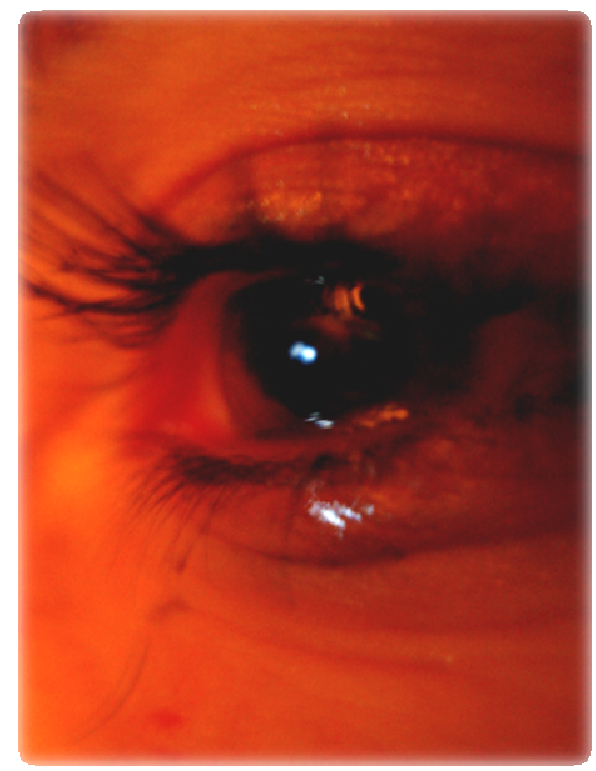

\section{Gefühle zu erleben ist nicht selbstverständlich...}

\section{Die Komplexität des Emotionserlebens}

Kapitel 6.3 prüft die Hypothesen 17-27. Die Ergebnistabellen 44-47 befassen sich mit der Entwicklung der Akzeptanz eigener Emotionen, dem erlebten Mangel an Emotionen, der Koppelung von körperlichen Beschwerden und psychischem Empfinden sowie der Selbsteinschätzung zur Wahrnehmung eigener Gefühle.

Tabelle 8 zeigt in T-Werten, dass eine signifikant positive Verbesserung der Akzeptanz eigener Gefühle in der Versuchsgruppe erst im Pretest/Follow Up-Vergleich stattfindet. Dies gilt zwar auch für die Kontrollgruppe, jedoch erneut in geringfügigem und nicht signifikantem Ausmaß. Die Mittelwertvergleiche der beiden Gruppen ergeben jedoch keine signifikanten Unterschiede, dennoch ist interessant, dass im Follow Up-Vergleich mit ,58 ein mittelgroßer bedeutsamer Effekt im Vergleich entsteht.

Die in beiden Gruppen relativ hohe Standardabweichung über 10 zeigt jeweils eine recht große Spanne für Werte in den oberen und unteren Bereichen.

In der Einzelbetrachtung der beiden Gruppen ist nur in der Versuchsgruppe zwischen Posttest und Follow Up mit ,003 eine sehr signifikante Verbesserung der Akzeptanz der eigenen Emotionen zu beobachten. 
Während sich in der Kontrollgruppe weder signifikante Veränderungen noch bedeutsame Effekte ergeben, weisen der Pretest/Follow Up- und Post/Follow Up-Vergleich in der Versuchsgruppe kleine bis mittelgroße Effekte auf.

Hypothesen 19 und 20 konnten sich nicht bestätigen, da sich die Akzeptanz der eigenen Emotionen weder in der Versuchsgruppe isoliert betrachtet noch im Gruppenvergleich signifikant verändert oder unterscheidet.

Interessant ist jedoch, dass sich Hypothese 21 bezogen auf die VG isoliert betrachtet bestätigte. Von Posttest zu Follow Up fand in der Versuchsgruppe ein signifikanter Anstieg der Akzeptanz eigener Emotionen statt.

Tabelle 44: Ergebnisse der Versuchsgruppe und der Kontrollgruppe bezogen auf die Entwicklung der Akzeptanz eigener Emotionen (T-Wert 12-70)

\begin{tabular}{|c|c|c|c|c|c|c|c|c|c|c|c|c|c|}
\hline \multicolumn{8}{|c|}{ Deskriptive Statistik } & \multicolumn{6}{|c|}{ Vergleichende Statistik Teil I } \\
\hline \multirow{2}{*}{$\begin{array}{c}\text { Unter- } \\
\text { suchungs- } \\
\text { Instrument }\end{array}$} & & \multicolumn{3}{|c|}{ Versuchsgruppe } & \multicolumn{3}{|c|}{ Kontrollgruppe } & \multicolumn{3}{|c|}{ Signifikanzen } & \multicolumn{3}{|c|}{ Effektstärken d } \\
\hline & & $P$ & $\mathrm{PO}$ & $\mathrm{F}$ & $\mathrm{P}$ & $\mathrm{PO}$ & $\mathrm{F}$ & POvG-PC & & 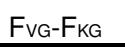 & d PO & & $d F$ \\
\hline \multirow{3}{*}{ SEE } & M & 46,35 & 44,85 & 49,90 & 42,85 & 42,05 & 43,55 & \multirow{3}{*}{\multicolumn{2}{|c|}{,477 }} & \multirow[t]{3}{*}{,077 } & \multirow[t]{3}{*}{,23 } & \multirow{3}{*}{\multicolumn{2}{|c|}{,58 }} \\
\hline & SD & 12,98 & 12,00 & 10,12 & 11,65 & 12,66 & 11,90 & & & & & & \\
\hline & $\mathrm{N}$ & 20 & 20 & 20 & 20 & 20 & 20 & & & & & & \\
\hline \multirow{4}{*}{ SEE } & & \multicolumn{12}{|c|}{ Vergleichende Statistik Teil II } \\
\hline & & \multicolumn{3}{|c|}{$\begin{array}{c}\text { Versuchsgruppe } \\
\text { Signifikanzen } \\
\end{array}$} & \multicolumn{3}{|c|}{$\begin{array}{c}\text { Kontrollgruppe } \\
\text { Signifikanzen }\end{array}$} & \multicolumn{3}{|c|}{$\begin{array}{c}\text { Versuchsgruppe } \\
\text { Effektstärken d }\end{array}$} & \multicolumn{3}{|c|}{$\begin{array}{l}\text { Kontrollgruppe } \\
\text { Effektstärken d }\end{array}$} \\
\hline & & P-PO & P-F & PO-F & P-PO & P-F & PO-F & d P-PO & d P-F & $=d$ PO-F & d P-PO & d P-F & d PO-F \\
\hline & & ,242 & ,127 &, $003^{* *}$ & ,744 & ,743 & ,442 & ,12 & ,31 & ,47 & ,07 &, 06 & ,12 \\
\hline
\end{tabular}

Der erlebte Emotionsmangel kann sich in der Versuchsgruppe minimal verbessern, das heisst, es werden im Posttest und Follow Up mehr Emotionen erlebt als im Pretest. Dies gilt in kleinem Ausmaß auch für die Kontrollgruppe, wobei hier der Emotionsmangel im Follow Up wieder gering ansteigt. Im Posttest- und Follow Up-Vergleich der Mittelwerte zwischen den Gruppen konnte kein statistisch signifikanter Unterschied ermittelt werden, dennoch zeigen die Effektstärken mit ,57 und ,46 zumindest mittelgroße Effekte, die für die Praxis sinnvolle Schlüsse ermöglichen.

In der Einzelbetrachtung der beiden Gruppen ergibt sich kein Messzeitpunktvergleich als signifikant verändert, wobei sich jedoch im Pretest/Posttest- und Pretest/Follow UpVergleich in der Versuchsgruppe ein kleiner bis mittlerer Effekt ermitteln lässt.

Hypothesen 22, 23 und 24 konnten sich nicht bestätigen. Zwar zeigt sich eine Veränderung in Richtung geringer erlebtem Emotionsmangel in der Versuchsgruppe, jedoch sind diese Veränderungen weder signifikant noch zeigen sich im Vergleich zur Kontrollgruppe im Posttest und Follow Up signifikante Unterschiede. 
Tabelle 45: Ergebnisse der Versuchsgruppe und der Kontrollgruppe bezogen auf die Entwicklung des erlebten Mangels an Emotionen (T-Wert 28-91)

\begin{tabular}{|c|c|c|c|c|c|c|c|c|c|c|c|c|c|}
\hline \multicolumn{8}{|c|}{ Deskriptive Statistik } & \multicolumn{6}{|c|}{ Vergleichende Statistik Teil I } \\
\hline \multirow{2}{*}{$\begin{array}{l}\text { Unter- } \\
\text { suchungs- } \\
\text { Instrument }\end{array}$} & & \multicolumn{3}{|c|}{ Versuchsgruppe } & \multicolumn{3}{|c|}{ Kontrollgruppe } & \multicolumn{3}{|c|}{ Signifikanzen } & \multicolumn{3}{|c|}{ Effektstärken d } \\
\hline & & $\mathrm{P}$ & $\mathrm{PO}$ & $\mathrm{F}$ & $\mathrm{P}$ & $\mathrm{PO}$ & $\mathrm{F}$ & POvG-PC & & 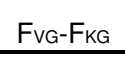 & $\mathrm{dPO}$ & & $\mathrm{dF}$ \\
\hline \multirow{3}{*}{ SEE } & M & 52,9 & 49,35 & 50,35 & 54,4 & 54,0 & 54,2 & \multirow{3}{*}{\multicolumn{2}{|c|}{081}} & \multirow{3}{*}{, 155 } & \multirow[t]{3}{*}{,57 } & \multirow{3}{*}{\multicolumn{2}{|c|}{ 46 }} \\
\hline & SD & 7,14 & 8,94 & 8,44 & 7,27 & 7,39 & 8,33 & & & & & & \\
\hline & $\mathrm{N}$ & 20 & 20 & 20 & 20 & 20 & 20 & & & & & & \\
\hline \multirow{4}{*}{ SEE } & & \multicolumn{12}{|c|}{ Vergleichende Statistik Teil II } \\
\hline & & \multicolumn{3}{|c|}{\begin{tabular}{|c} 
Versuchsgruppe \\
Signifikanzen \\
\end{tabular}} & \multicolumn{3}{|c|}{$\begin{array}{c}\text { Kontrollgruppe } \\
\text { Signifikanzen }\end{array}$} & \multicolumn{3}{|c|}{$\begin{array}{c}\text { Versuchsgruppe } \\
\text { Effektstärken d }\end{array}$} & \multicolumn{3}{|c|}{$\begin{array}{l}\text { Kontrollgruppe } \\
\text { Effektstärken d }\end{array}$} \\
\hline & & P-PO & P-F & PO-F & P-PO & P-F & PO-F & d P-PO & d P-F & $=$ d PO-F & d P-PO & d P-F & d PO-F \\
\hline & &, 063 &, 141 &, 650 &, 770 & ,884 &, 881 &, 44 &, 33 &, 12 &, 05 &, 03 &, 03 \\
\hline
\end{tabular}

Tabelle 46 zeigt, wie sehr die Probanden ihre körperlichen Beschwerden mit ihrem psychischen Empfinden koppeln. In der Versuchsgruppe findet eine geringe, aber konstante Steigerung der Koppelung dieser beiden Variablen statt, während dies in der Kontrollgruppe insgesamt minimal absinkt.

Es ergeben sich in den Messzeitpunktvergleichen weder zwischen den einzelnen Gruppen, noch innerhalb der einzelnen Gruppen signifikante Unterschiede.

Interessant ist, dass jedoch ein kleiner Effekt bezüglich der Verbesserung in Gruppe (A) sichtbar wird, wo hingegen ein kleiner bedeutsamer Effekt zum negativen im Pretest/Posttest-Vergleich innerhalb der Kontrollgruppe zu beobachten ist.

Hypothesen 25, 26 und 27 konnten sich nicht bestätigen. Zwar zeigt sich eine Veränderung in Richtung einer größeren Koppelung zwischen körperlichen Bechwerden und seelischem Befinden in der Versuchsgruppe, jedoch sind diese Veränderungen innerhalb der Gruppe nicht signifikant. Es zeigen sich auch im Vergleich zur Kontrollgruppe im Posttest und Follow Up keine signifikanten Unterschiede. 
Tabelle 46: Ergebnisse der Versuchsgruppe und der Kontrollgruppe bezogen auf die Entwicklung der Koppelung von körperlichen Beschwerden und psychischem Empfinden (T-Wert 14-78)

\begin{tabular}{|c|c|c|c|c|c|c|c|c|c|c|c|c|c|}
\hline \multicolumn{8}{|c|}{ Deskriptive Statistik } & \multicolumn{6}{|c|}{ Vergleichende Statistik Teil I } \\
\hline \multirow{2}{*}{$\begin{array}{c}\text { Unter- } \\
\text { suchungs- } \\
\text { Instrument }\end{array}$} & & \multicolumn{3}{|c|}{ Versuchsgruppe } & \multicolumn{3}{|c|}{ Kontrollgruppe } & \multicolumn{3}{|c|}{ Signifikanzen } & \multicolumn{3}{|c|}{ Effektstärken d } \\
\hline & & $P$ & $\mathrm{PO}$ & $\mathrm{F}$ & $P$ & $\mathrm{PO}$ & $\mathrm{F}$ & POvg-PO & & 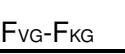 & $\mathrm{dPO}$ & & $\mathrm{F}$ \\
\hline \multirow{3}{*}{ SEE } & M & 46,0 & 48,30 & 49,35 & 49,3 & 46,4 & 48,85 & \multirow{3}{*}{\multicolumn{2}{|c|}{, 582}} & \multirow[t]{3}{*}{,860 } & \multirow[t]{3}{*}{, 18} & \multirow{3}{*}{\multicolumn{2}{|c|}{05}} \\
\hline & SD & 8,58 & 9,45 & 10,87 & 8,31 & 12,05 & 9,99 & & & & & & \\
\hline & $\mathrm{N}$ & 20 & 20 & 20 & 20 & 20 & 20 & & & & & & \\
\hline \multirow{4}{*}{ SEE } & & \multicolumn{12}{|c|}{ Vergleichende Statistik Teil II } \\
\hline & & \multicolumn{3}{|c|}{$\begin{array}{c}\text { Versuchsgruppe } \\
\text { Signifikanzen }\end{array}$} & \multicolumn{3}{|c|}{$\begin{array}{l}\text { Kontrollgruppe } \\
\text { Signifikanzen }\end{array}$} & \multicolumn{3}{|c|}{$\begin{array}{l}\text { Versuchsgruppe } \\
\text { Effektstärken d }\end{array}$} & \multicolumn{3}{|c|}{$\begin{array}{l}\text { Kontrollgruppe } \\
\text { Effektstärken d }\end{array}$} \\
\hline & & P-PO & P-F & PO-F & P-PO & P-F & PO-F & d P-PO & d P-F & d PO-F & d P-PO & d P-F & d PO-F \\
\hline & & 201 & ,301 & ,666 & 247 & 837 & ,148 & ,26 & ,34 & ,10 & ,28 & ,05 & ,22 \\
\hline
\end{tabular}

Tabelle 47 zeigt die Selbsteinschätzung zur Wahrnehmung eigener Gefühle auf einer Skala von 0-10.

Insgesamt schätzen die Probanden beider Gruppen ihre Fähigkeit eigene Emotionen wahrzunehmen mit einem Wert um 7,0 recht hoch ein. Dass der Wert der Versuchsgruppe von 7,05 auf 6,9 absinkt findet in der Diskussion Raum. Die Mittelwerte unterscheiden sich zwischen Gruppe (A) und (B) zu keinem Zeitpunkt signifikant.

Auch in der Einzelbetrachtung beider Gruppen in der vergleichenden Statistik Teil II ergeben sich keine signifikanten Unterschiede zwischen den Messzeitpunktvergleichen sowie keine bedeutsamen Effektstärken.

Hypothesen 28, 29 und 30 konnten sich nicht bestätigen. Die Selbsteinschätzung zur Wahrnehmung eigener Gefühle ist innerhalb der Versuchsgruppe beinah konstant. Im Posttest sinkt die eingeschätzte Fähigkeit der Wahrnehmung eigener Gefühle minimal ab. 
Tabelle 47: Ergebnisse der Versuchsgruppe und der Kontrollgruppe bezogen auf die Entwicklung der Selbsteinschätzung zur Wahrnehmung eigener Gefühle auf einer Skala von $0-10$

\begin{tabular}{|c|c|c|c|c|c|c|c|c|c|c|c|c|c|}
\hline \multicolumn{8}{|c|}{ Deskriptive Statistik } & \multicolumn{6}{|c|}{ Vergleichende Statistik Teil I } \\
\hline \multirow{2}{*}{\begin{tabular}{|c|} 
Unter- \\
suchungs- \\
Instrument
\end{tabular}} & & \multicolumn{3}{|c|}{ Versuchsgruppe } & \multicolumn{3}{|c|}{ Kontrollgruppe } & \multicolumn{3}{|c|}{ Signifikanzen } & \multicolumn{3}{|c|}{ Effektstärken d } \\
\hline & & $\mathrm{P}$ & PO & $\mathrm{F}$ & $\mathrm{P}$ & $\mathrm{PO}$ & $\mathrm{F}$ & POva-PC & & Fva-Fkg & $\mathrm{dPO}$ & & $d F$ \\
\hline \multirow{3}{*}{$\begin{array}{c}\text { RATING- } \\
\text { SKALA }\end{array}$} & $M$ & 7,05 & 6,9 & 7,15 & 7,0 & 6,7 & 6,6 & \multirow{3}{*}{\multicolumn{2}{|c|}{6991}} & \multirow[t]{3}{*}{467} & \multirow[t]{3}{*}{08} & \multirow{3}{*}{\multicolumn{2}{|c|}{, 13}} \\
\hline & SD & 2,19 & 1,94 & 1,95 & 2,49 & 3,18 & 2,27 & & & & & & \\
\hline & $\mathrm{N}$ & 20 & 20 & 20 & 20 & 20 & 20 & & & & & & \\
\hline \multirow{4}{*}{$\begin{array}{l}\text { RATING- } \\
\text { SKALA }\end{array}$} & & \multicolumn{12}{|c|}{ Vergleichende Statistik Teil II } \\
\hline & & \multicolumn{3}{|c|}{\begin{tabular}{|c|} 
Versuchsgruppe \\
Signifikanzen
\end{tabular}} & \multicolumn{3}{|c|}{$\begin{array}{l}\text { Kontrollgruppe } \\
\text { Signifikanzen }\end{array}$} & \multicolumn{3}{|c|}{$\begin{array}{l}\text { Versuchsgruppe } \\
\text { Effektstärken d }\end{array}$} & \multicolumn{3}{|c|}{$\begin{array}{l}\text { Kontrollgruppe } \\
\text { Effektstärken d }\end{array}$} \\
\hline & & P-PO & P-F & PO-F & $\mathrm{P}-\mathrm{PO}$ & P-F & PO-F & d P-PO & $d P-F$ & d PO-F & d P-PO & $d P-F$ & d PO-F \\
\hline & & 679 &, 781 & , 549 & , 474 & ,372 & 854 & 07 &, 07 &, 13 &, 11 &, 15 & .03 \\
\hline
\end{tabular}




\subsection{Veränderungsfragen des Erlebens und Verhaltens}

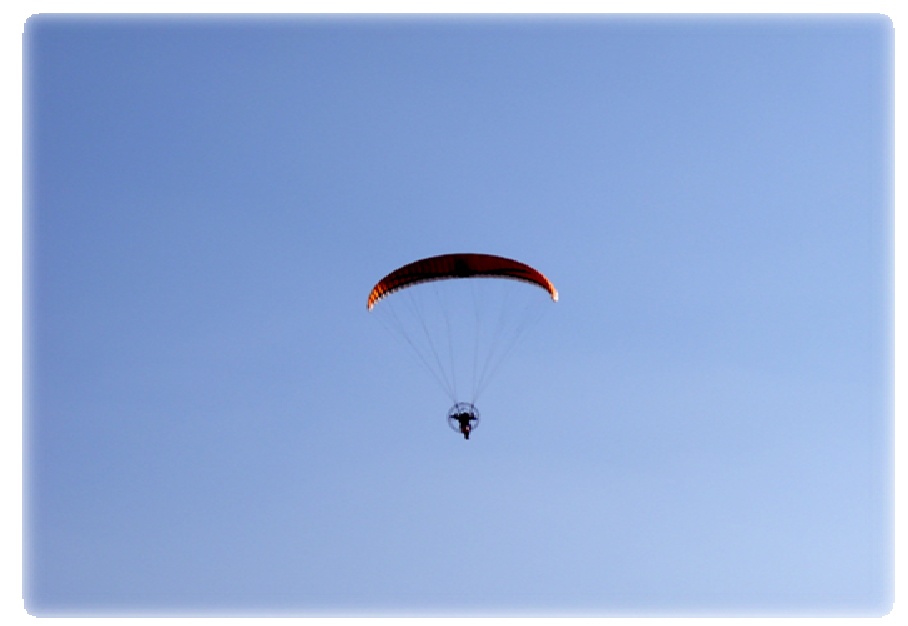

Und plötzlich war da wieder Hoffnung...

Kapitel 6.4 liefert Ergebnisse zur Überprüfung der Hypothesen 16, 17 und 18.

Tabelle 48 zeigt die Entwicklung des eigenen Erlebens und Verhaltens im Alltag der Patienten. Dabei wurde, wie in Kapitel 5.4 beschrieben, die Veränderung des Erlebens und Verhaltens in Richtung der zwei Pole Entspannung/Gelassenheit/Optimismus und Spannung/Unsicherheit/Pessimismus erfasst.

Wie an den Mittelwerten sichtbar wird, verändert sich das Erleben und Verhalten der Versuchsgruppe deutlich und stetig Richtung Entspannung/Gelassenheit und Optimismus. Demgegenüber steht eine gegensätzliche Entwicklung in Richtung Spannung/Unsicherheit und Pessimismus innerhalb der Kontrollgruppe. In beide Gruppen bewegt sich die Standardabweichung im Bereich zwischen 30 und 40, wobei die Kontrollgruppe die höheren Werte aufweist. Berücksichtigt man diese bedeuete dies, dass auch in der Kontrollgruppe einzelne Patienten eine positive Entwicklung ihres Erlebens und Verhaltens im Alltag erleben, dennoch nicht in derselben Stärke wie Patienten der Versuchsgruppe.

Der Mittelwertvergleich der beiden Gruppen im Posttest und Follow Up fällt mit ,000 hoch signifikant aus und auch die Effektstärken erreichen für den Gruppenvergleich Spitzenwerte von 1,31.

Wie in Kapitel 5.4 beschrieben, gilt laut Testmanual für Werte im positiven Bereich Optimismus ab 200 eine Irrtumswahrscheinlichkeit von $p<=0,1 \%$. 
Auffällig sind hier in beiden Gruppen die relativ hohen Standardabweichungen mit Werten zwischen 31 und 40.

Trotz dieser hohen Standardabweichung können sich im niedrigen Bereich in der Versuchsgruppe keine schlechteren Ergebnisse als im Pretest ergeben, während unter Berücksichtigung der Standardabweichung eine Überschreitung des Pretestergebnisses zum Positiven in der Kontrollgruppe nur gering möglich ist.

Auch in der Einzelbetrachtung des Pretest/Posttest- und Pretest/Follow Up-Vergleichs, zeigt die Versuchsgruppe sehr signifikante Unterschiede von ,004 und ,017 und jeweils einen großen Effekt.

In der Kontrollgruppe wird kein Ergebnis im Vergleich der Messzeitpunkte signifikant. Trotzdem zeigen sich für die Verschlechterung in dieser Gruppe im Pretest/Posttest- und im Pretest/Follow Up-Vergleich mit ,37 und ,46 kleine bis mittlere Effekte, die praktisch bedeutsam sind.

Die Hypothesen 16 und 17 konnten sich damit bestätigen. Zwar verändert sich von Posttest zu Follow Up das Erleben und Verhalten in der Versuchsgruppe nicht mehr signifikant, jedoch zeigt der Gruppenvergleich sowohl im Posttest als auch im Follow Up hoch signifikante Unterschiede zu Gunsten der Versuchsgruppe.

Hypothese 18 kann sich demnach zwar innerhalb der Versuchsgruppe isoliert betrachtet nicht bestätigen, jedoch zeigt der Gruppenvergleich eine positive Entwicklung zu Gunsten der Versuchsgruppe. 
Tabelle 48: Ergebnisse der Versuchsgruppe und der Kontrollgruppe bezogen auf die Entwicklung des Erlebens und Verhaltens im Alltag (42-294)

\begin{tabular}{|c|c|c|c|c|c|c|c|c|c|c|c|c|c|}
\hline \multicolumn{8}{|c|}{ Deskriptive Statistik } & \multicolumn{6}{|c|}{ Vergleichende Statistik Teil I } \\
\hline \multirow{2}{*}{$\begin{array}{c}\text { Unter- } \\
\text { suchungs- } \\
\text { Instrument }\end{array}$} & & \multicolumn{3}{|c|}{\begin{tabular}{|l} 
Versuchsgruppe \\
\end{tabular}} & \multicolumn{3}{|c|}{ Kontrollgruppe } & \multicolumn{3}{|c|}{ Signifikanzen } & \multicolumn{3}{|c|}{ Effektstärken d } \\
\hline & & $\mathrm{P}$ & $\mathrm{PO}$ & $\mathrm{F}$ & $\mathrm{P}$ & $\mathrm{PO}$ & $\mathrm{F}$ & POvg-P & & FvG-FKG & $\mathrm{dPO}$ & \multicolumn{2}{|r|}{$d F$} \\
\hline \multirow{3}{*}{ VEV } & M & 174,75 & 204,05 & 200,35 & 173,3 & 159,3 & 155,75 & \multirow{3}{*}{\multicolumn{2}{|c|}{, $000^{* * *}$}} &, $000^{\star \star \star}$ & 1,30 & & 1,31 \\
\hline & SD & 31,52 & 33,52 & 31,8 & 39,78 & 36,59 & 36,45 & & & & & & \\
\hline & $\mathrm{N}$ & 20 & 20 & 20 & 20 & 20 & 20 & & & & & & \\
\hline \multirow{4}{*}{ VEV } & & \multicolumn{12}{|c|}{ Vergleichende Statistik Teil II } \\
\hline & & \multicolumn{3}{|c|}{$\begin{array}{l}\text { Versuchsgruppe } \\
\text { Signifikanzen }\end{array}$} & \multicolumn{3}{|c|}{$\begin{array}{l}\text { Kontrollgruppe } \\
\text { Signifikanzen }\end{array}$} & \multicolumn{3}{|c|}{$\begin{array}{l}\text { Versuchsgruppe } \\
\text { Effektstärken d }\end{array}$} & \multicolumn{3}{|c|}{$\begin{array}{l}\text { Kontrollgruppe } \\
\text { Effektstärken d }\end{array}$} \\
\hline & & P-PO & P-F & PO-F & P-PO & P-F & PO-F & d P-PO & $d P-F$ & d PO-F & d P-PO & d P-F & d PO-F \\
\hline & &, $004^{\star *}$ &, $017^{*}$ & ,596 & ,152 & ,082 & ,371 &, 90 &, 81 &, 11 &, 37 &, 46 &, 10 \\
\hline
\end{tabular}

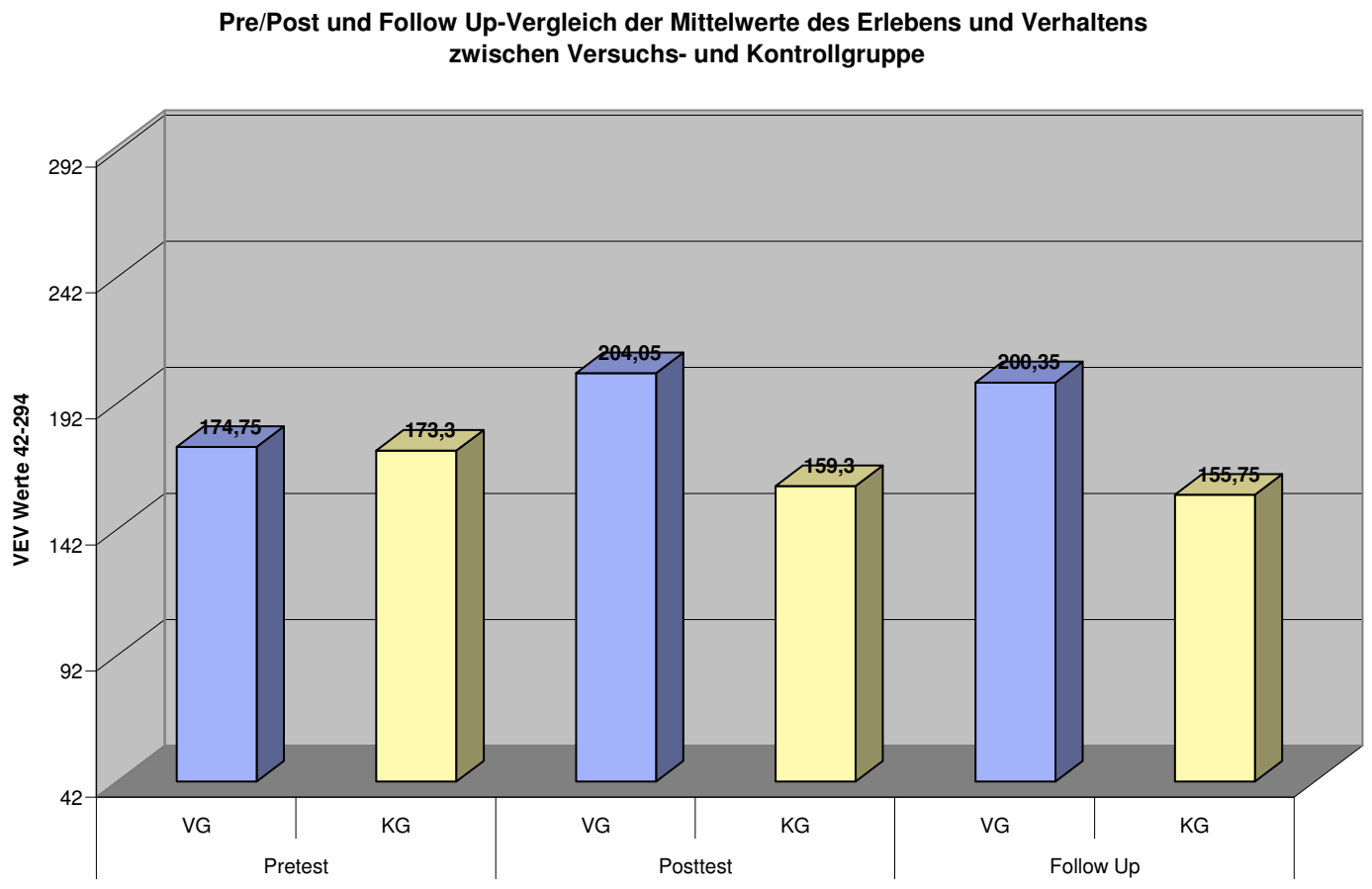

Abbildung 9: Mittelwertvergleich zwischen Versuchs- und Kontrollgruppe bezogen auf die Entwicklung des Erlebens und Verhaltens im Alltag (Richtung: Entspannung/Gelassenheit/Optimismus oder Spannung/UnsicherheitPessimismus) 


\subsection{Erlebte Resonanz und ihre Korrelation zu der erlebten Stärke der Beschwerden}

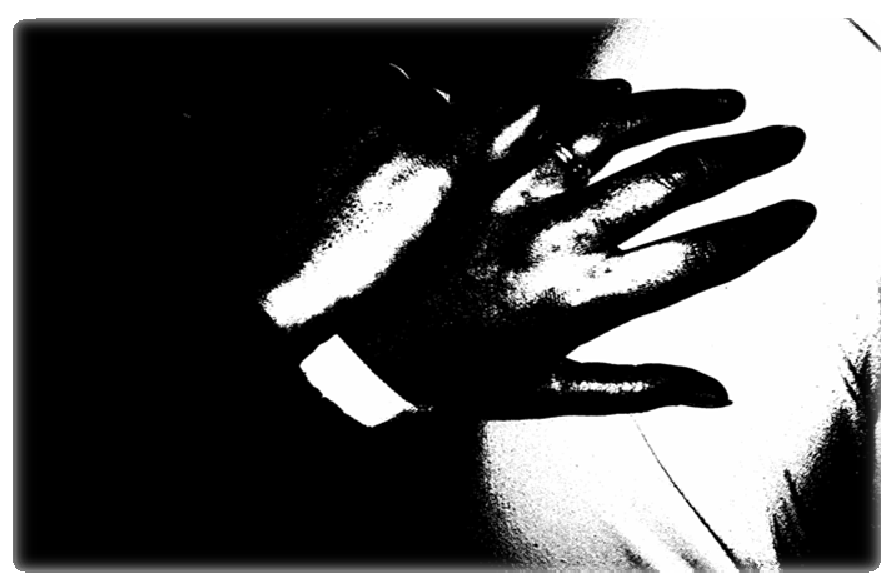

Resonanz - ein wirksames und preiswertes „Medikament“?

Innerhalb dieses Kapitels wurden die Hypothesen 43 und 44 untersucht.

Zunächst wurden Beschwerdemittelwerte für die Sitzungen 1-2, 3-4, 5-6 und 7-8 mit ebenfalls zusammengefassten Mittelwerten der erlebten Resonanz durch die Gruppenleitung und durch andere Gruppenmitglieder für die Sitzungen 1-2, 3-4, 5-6 und 78 ermittelt.

In Tabelle 49 wird die erlebte Beschwerdestärke im Alltag mit der erlebten Beschwerdestärke während den Gruppensitzungen verglichen.

Die Korrelationen in Tabelle 49 ergeben sich nicht, wie erwartet, als signifikant. Auch eine entgegengesetzte positive Korrelation ist nicht zu erkennen.

Dennoch fällt bis Sitzung 7 auf, je größer der ermittelte Resonanzwert desto geringer ist der Gesamtmittelwert der erlebten Beschwerden während den Sitzungen.

Sitzungen 7-8 zeigen erstaunlicherweise den geringsten erlebten Resonanzwert und dennoch gleichzeitig den geringsten erlebten Beschwerdewert.

Hypothese 46 konnte sich statistisch nicht bestätigen, jedoch zeigt sich, trotz nicht vorhandener negativer Korrelationen zwischen Resonanzerleben und Beschwerdeintensität, dass bei viel Resonanz auch weniger Beschwerdeintensität angegeben wird. 
Tabelle 49: Korrelationsuntersuchung im Hinblick auf die erlebte Resonanz und der erlebten Beschwerdeintensität während den einzelnen Sitzungen

(Beschwerdeskala 0-10, möglicher Gesamtresonanzwert in einer Stunde $=8$ (addierte Resonanz von Mitgliedern und Leitung))

\begin{tabular}{|l|c|c|c|c|c|}
\hline & Korrelation & Signifikanz & Stunden & $\begin{array}{c}\text { Gesamt- } \\
\text { Mittelwert } \\
\text { Resonanz } \\
\text { (0-8) }\end{array}$ & $\begin{array}{c}\text { Gesamt- } \\
\text { Mittelwert } \\
\text { Beschwerden } \\
\text { (0-10) }\end{array}$ \\
\hline $\begin{array}{l}\text { Beschwerdenmittelwert Stunden 1-2 } \\
\text { korreliert mit } \\
\text { Resonanzmittelwert Stunden 1-2 }\end{array}$ &, 004 &, 987 & $1+2$ & 4,46 & 4,35 \\
\hline $\begin{array}{l}\text { Beschwerdenmittelwert Stunden 3-4 } \\
\text { korreliert mit } \\
\text { Resonanzmittelwert Stunden 3-4 }\end{array}$ &, 196 &, 408 & $3+4$ & 4,83 & 3,93 \\
\hline $\begin{array}{l}\text { Beschwerdenmittelwert Stunden 5-6 } \\
\text { korreliert mit } \\
\text { Resonanzmittelwert Stunden 5-6 }\end{array}$ &, 254 &, 293 & $5+6$ & 5,13 & 2,79 \\
\hline $\begin{array}{l}\text { Beschwerdenmittelwert Stunden 7-8 } \\
\text { korreliert mit } \\
\text { Resonanzmittelwert Stunden 7-8 }\end{array}$ &, 136 &, 567 & $7+8$ & 4,41 & 2,33 \\
\hline
\end{tabular}

Tabelle 50 zeigt die Mittelwerte der erlebten Beschwerdestärke der Patientengruppe (A) im Verlauf einer Woche im Alltag im Vergleich zum Mittelwert der erlebten Stärke der Beschwerden in den Gruppensitzungen.

Dabei ergibt sich ein sehr signifikanter Unterschied zwischen dem Beschwerdeerleben während den Sitzungen und diesem während des Alltags der Probanden. Im Alltag erleben die Patienten im Durchschnitt eine Beschwerdestärke von 4,57. In den Gruppensitzungen hingegen ergibt sich nur ein Wert von 3,22. Auch die Beachtung der Standardabweichungen verändert das Ergebnis der unterschiedlich wahrgenommenen Beschwerdestärke in Alltag und Gruppe nicht.

Der Unterschied ergibt eine Signifikanz von ,003 und eine mittelgroße Effektstärke von ,58.

Hypothese 47 konnte sich damit bestätigen. Es besteht ein signifikanter Unterschied zwischen der erlebten Beschwerdestärke innerhalb des Alltags und während den Gruppensitzungen, wobei die Beschwerden in den Sitzungen geringer erlebt werden als im Alltagsleben. 
Tabelle 50: Ergebnisse der Versuchsgruppe der erlebten Beschwerdestärke auf einer Skala von 0-10 während einer ganzen Woche im Vergleich zur erlebten Beschwerdestärke während den Sitzungen.

\begin{tabular}{|c|c|c|c|c|c|}
\hline $\begin{array}{l}\text { Untersuchungs- } \\
\text { instrument }\end{array}$ & & \multicolumn{2}{|c|}{$\begin{array}{c}\text { Deskriptive } \\
\text { Statistik }\end{array}$} & \multicolumn{2}{|c|}{$\begin{array}{c}\text { Vergleichende } \\
\text { Statistik }\end{array}$} \\
\hline \multirow[t]{4}{*}{$\begin{array}{l}\text { RATING- } \\
\text { SKALA }\end{array}$} & & $\begin{array}{l}\text { Beschwerde- } \\
\text { wert innerhalb } \\
\text { der Wochen im } \\
\text { Alltag und } \\
\text { Untersuchungs- } \\
\text { zeitraum }\end{array}$ & $\begin{array}{l}\text { Beschwerde- } \\
\text { wert in den } \\
\text { Gruppen- } \\
\text { sitzungen }\end{array}$ & $\begin{array}{l}\text { Signi- } \\
\text { fikanz }\end{array}$ & $\begin{array}{l}\text { Effekt- } \\
\text { stärke d }\end{array}$ \\
\hline & $M$ & 4,57 & 3,22 & \multirow{3}{*}{, $003^{\star *}$} & \multirow{3}{*}{,58 } \\
\hline & SD & 2,49 & 2,26 & & \\
\hline & $\mathrm{N}$ & 20 & 20 & & \\
\hline
\end{tabular}




\subsection{Arzt-Patienten-Angaben und Psychotherapiemotivation}

\section{Die Ergebnisse des bisherigen Forschungsstandes werden in Frage gestellt - Oder fehlt der Mut zur Wahrheit?}

In Kapitel 6.6 wurden die Hypothesen 31-45 sowie 49-51 überprüft.

Die Ergebnisse zeigen die Entwicklung der Bewertung über die erlebte Beziehung der Versuchs- und Kontrollgruppe zu ihren behandelnden Hausärzten, die Anzahl der Arztwechsel, die Entwicklung der Bewertung über die erlebte Beziehung zu den Patienten durch die behandelnden Hausärzte eingeschätzt, die Anzahl der Arztkonsultationen, die Forderungen nach weiteren medizinischen Untersuchungen sowie die derzeitige Bereitschaft der Probanden, eine Psychotherapie zu machen.

Tabelle 51 zeigt in Schulnoten die Bewertung der Beziehung der Patienten zu ihren Hausärzten. Es zeigt sich zwischen Versuchs- und Kontrollgruppe zu keinem Messzeitpunkt ein signifikanter Unterschied in der Bewertung. Die Noten liegen im Durchschnitt zwischen 2,0 und 2,45. Trotzdem verschlechtert sich bei den Patienten der Kontrollgruppe die Beziehung zu den behandelnden Ärzten im Posttest minimal. Dies genügt nicht, um einen statistisch signifikanten Unterschied zu erreichen, dennoch ist die Effektstärke im Posttest mit ,46 und auch im Follow Up mit ,30 noch klein bis mittelgradig hoch.

Die Standardabweichung liegt in der Kontrollgruppe bei maximal 1,08 und ist damit größer als der Maximalwert der Standardabweichung von ,92 in der Versuchsgruppe. 
In den Einzelbetrachtungen der Gruppen ergeben sich keine signifikanten Ergebnisse und keine bedeutsamen Effektstärken.

Insgesamt konnten sich demnach die Hypothesen 31, 32 und 33 statistisch nicht bestätigen.

Tabelle 51: Ergebnisse der Versuchsgruppe und der Kontrollgruppe bezogen auf die Entwicklung der Bewertung der Beziehung zu ihren behandelnden Hausärzten mit Schulnoten von 1-5

\begin{tabular}{|c|c|c|c|c|c|c|c|c|c|c|c|c|c|}
\hline \multicolumn{8}{|c|}{ Deskriptive Statistik } & \multicolumn{6}{|c|}{ Vergleichende Statistik Teil I } \\
\hline \multirow{2}{*}{$\begin{array}{l}\text { Unter- } \\
\text { suchungs- } \\
\text { Instrument }\end{array}$} & & \multicolumn{3}{|c|}{ Versuchsgruppe } & \multicolumn{3}{|c|}{ Kontrollgruppe } & \multicolumn{3}{|c|}{ Signifikanzen } & \multicolumn{3}{|c|}{ Effektstärken d } \\
\hline & & $P$ & $\mathrm{PO}$ & $\mathrm{F}$ & $\mathrm{P}$ & $\mathrm{PO}$ & $\mathrm{F}$ & POvg-P & & $F_{V G-F K G}$ & $\mathrm{dPO}$ & & $d F$ \\
\hline \multirow{3}{*}{$\begin{array}{l}\text { RATING- } \\
\text { SKALA }\end{array}$} & M & 2,05 & 2,05 & 2,0 & 2,45 & 2,5 & 2,3 & \multirow{3}{*}{\multicolumn{2}{|c|}{,350 }} & \multirow[t]{3}{*}{ 152 } & , 46 & \multirow{3}{*}{\multicolumn{2}{|c|}{,30 }} \\
\hline & SD & ,76 & 89 & ,92 & 89 & 1,05 & 1,08 & & & & & & \\
\hline & $\mathrm{N}$ & 20 & 20 & 20 & 20 & 20 & 20 & & & & & & \\
\hline \multirow{4}{*}{$\begin{array}{l}\text { RATING- } \\
\text { SKALA }\end{array}$} & \multicolumn{13}{|c|}{ Vergleichende Statistik Teil II } \\
\hline & & \multicolumn{3}{|c|}{$\begin{array}{l}\text { Versuchsgruppe } \\
\text { Signifikanzen }\end{array}$} & \multicolumn{3}{|c|}{$\begin{array}{l}\text { Kontrollgruppe } \\
\text { Signifikanzen }\end{array}$} & \multicolumn{3}{|c|}{$\begin{array}{l}\text { Versuchsgruppe } \\
\text { Effektstärken d }\end{array}$} & \multicolumn{3}{|c|}{$\begin{array}{l}\text { Kontrollgruppe } \\
\text { Effektstärken d }\end{array}$} \\
\hline & & P-PO & P-F & PO-F & P-PO & P-F & PO-F & d P-PO & $d P-F$ & d PO-F & d P-PO & $d P-F$ & d PO-F \\
\hline & & 1,0 &, 804 & ,789 & ,772 & ,419 & ,163 &, 0 & , 06 &, 06 &, 05 & , 15 &, 19 \\
\hline
\end{tabular}

Die Anzahl der Arztwechsel war, aufgrund des in der Literatur häufig genannten Ärztehoppings somatoformer Patienten, ein weiteres Forschungsinteresse. Wie in Tabelle 52 sichtbar wird, findet in der Kontrollgruppe kein einziger Arztwechsel im Untersuchungszeitraum statt. Auch die Versuchsgruppe liefert unerwartete Ergebnisse und tendiert mit Werten wie ,50 und ,40 ebenfalls Richtung null. Die Standardabweichungen kommen hier lediglich durch einzelne Personen zu Stande. Der mittelgroße Effekt von ,45 im Vergleich zwischen Versuchs- und Kontrollgruppe, ergibt sich aus dem durchgehenden Wert von 0 in Gruppe (B).

Die Hypothesen 34, 35 und 36 konnten sich aufgrund dieser Ergebnisse nicht bestätigen. 
Tabelle 52: Ergebnisse der Versuchsgruppe und der Kontrollgruppe bezogen auf die Anzahl der Arztwechsel

\begin{tabular}{|c|c|c|c|c|c|c|c|c|c|c|c|c|c|}
\hline \multicolumn{8}{|c|}{ Deskriptive Statistik } & \multicolumn{6}{|c|}{ Vergleichende Statistik Teil I } \\
\hline \multirow{2}{*}{$\begin{array}{c}\text { Unter- } \\
\text { suchungs- } \\
\text { Instrument }\end{array}$} & & \multicolumn{3}{|c|}{ Versuchsgruppe } & \multicolumn{3}{|c|}{ Kontrollgruppe } & \multicolumn{3}{|c|}{ Signifikanzen } & \multicolumn{3}{|c|}{ Effektstärken d } \\
\hline & & $P$ & $\mathrm{PO}$ & $\mathrm{F}$ & $P$ & $\mathrm{PO}$ & $\mathrm{F}$ & POvg-PC & OKG & 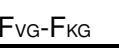 & d PO & & $\mathrm{dF}$ \\
\hline \multirow{3}{*}{$\begin{array}{l}\text { EINZEL- } \\
\text { FRAGE }\end{array}$} & M &, 50 &, 50 & ,40 & 0 & 0 & 0 & \multirow{3}{*}{\multicolumn{2}{|c|}{,324 }} & \multirow[t]{3}{*}{,324 } & \multirow[t]{3}{*}{,45 } & \multirow{3}{*}{\multicolumn{2}{|c|}{45}} \\
\hline & SD & ,34 & ,45 & ,35 & 0 & 0 & 0 & & & & & & \\
\hline & $\mathrm{N}$ & 20 & 20 & 20 & 20 & 20 & 20 & & & & & & \\
\hline \multirow{4}{*}{$\begin{array}{l}\text { EINZEL- } \\
\text { FRAGE }\end{array}$} & & \multicolumn{12}{|c|}{ Vergleichende Statistik Teil II } \\
\hline & & \multicolumn{3}{|c|}{$\begin{array}{c}\text { Versuchsgruppe } \\
\text { Signifikanzen }\end{array}$} & \multicolumn{3}{|c|}{$\begin{array}{c}\text { Kontrollgruppe } \\
\text { Signifikanzen }\end{array}$} & \multicolumn{3}{|c|}{$\begin{array}{c}\text { Versuchsgruppe } \\
\text { Effektstärken d }\end{array}$} & \multicolumn{3}{|c|}{$\begin{array}{l}\text { Kontrollgruppe } \\
\text { Effektstärken d }\end{array}$} \\
\hline & & P-PO & P-F & PO-F & P-PO & P-F & PO-F & d P-PO & d P-F & d PO-F & d P-PO & d P-F & d PO-F \\
\hline & & 1,0 & ,428 & ,330 & 0 & 0 & 0 & 0 & ,06 & ,05 & 0 & 0 & 0 \\
\hline
\end{tabular}

Das in der Literatur genannte schwierige Verhältnis des Arztes zum somatoformen Patienten konnte sich in dieser Studie insgesamt auch nicht bestätigen. Dennoch verbessert sich in der Versuchsgruppe, aus Sicht des Arztes, die Beziehung zum Patienten von 2,20 im Pretest auf 1,65 im Follow Up. Die relativ kleinen Standardabweichungen von maximal ,83 könnten dieses Ergebnis nicht gravierend verändern. In der Kontrollgruppe verschlechtert sich demgegenüber die Beziehung zu den Patienten nach Arzteinschätzung von 1,95 im Pretest auf 2,25 im Follow Up.

Der Follow Up-Vergleich zwischen den Gruppen ergibt mit ,028 einen signifikanten Unterschied. Auch die Effektstärke ergibt sich im Follow Up als mittelmäßig groß.

Hier wird erneut deutlich, dass selbst mit einer geringen Veränderung, trotz fehlender statistischer hoher Signifikanz, ein bedeutsamer praktischer Effekt erzielt werden kann.

In der Versuchsgruppe ergeben sich auch in der Einzelbetrachtung der Messzeitpunktvergleiche zwischen Pretest/Posttest und Pretest/Follow Up signifikante Unterschiede sowie durchweg kleine bis mittelgroße Effektstärken.

Es ergeben sich in der Kontrollgruppe zwar keine signifikanten Ergebnisse bezüglich der ermittelten eingeschätzten Beziehungsqualität der Ärzte zu ihren Patienten, dennoch ergibt sich aus der ermittelten Verschlechterung im Pretest/Follow Up- und im Posttest/Follow Up-Vergleich ein kleiner bis mittelmäßig bedeutsamer Effekt für die Praxis.

Die Hypothesen 37 und 38 konnten sich statistisch bestätigen. Es zeigt sich innerhalb der Vesuchsgruppe eine signifikante Verbesserung in der erlebten Beziehungsqualität im Pretest/Posttest- und Pre/Follow Up-Vergleich. Nicht bestätigen konnte sich Hypothese 39, da sich im Posttest/Follow Up-Vergleich keine signifikante Verbesserung ergibt. Dafür zeigt sich jedoch im Follow Up-Vergleich zwischen den Gruppen ein signifikanter Unterschied zu Gunsten der Versuchsgruppe. 
Tabelle 53: Ergebnisse der Versuchsgruppe und der Kontrollgruppe zur Bewertung der Hausärzte über die Beziehung zu ihren Patienten mit Schulnoten von 1-5

\begin{tabular}{|c|c|c|c|c|c|c|c|c|c|c|c|c|c|}
\hline \multicolumn{8}{|c|}{ Deskriptive Statistik } & \multicolumn{6}{|c|}{ Vergleichende Statistik Teil I } \\
\hline \multirow{2}{*}{$\begin{array}{c}\text { Unter- } \\
\text { suchungs- } \\
\text { Instrument }\end{array}$} & & \multicolumn{3}{|c|}{ Versuchsgruppe } & \multicolumn{3}{|c|}{ Kontrollgruppe } & \multicolumn{3}{|c|}{ Signifikanzen } & \multicolumn{3}{|c|}{ Effektstärken d } \\
\hline & & $\mathrm{P}$ & $\mathrm{PO}$ & $\mathrm{F}$ & $\mathrm{P}$ & $\mathrm{PO}$ & $\mathrm{F}$ & POvg-P & OKG & $F_{V G}-F_{K G}$ & $\mathrm{dPO}$ & & $d F$ \\
\hline \multirow{3}{*}{$\begin{array}{l}\text { RATING- } \\
\text { SKALA }\end{array}$} & M & 2,20 & 1,90 & 1,65 & 1,95 & 2,0 & 2,25 & \multirow{3}{*}{\multicolumn{2}{|c|}{,647 }} & \multirow[t]{3}{*}{, $028^{*}$} & \multirow[t]{3}{*}{,15 } & \multirow{3}{*}{\multicolumn{2}{|c|}{, 72}} \\
\hline & SD & ,83 & 64 & ,81 & 60 & ,73 & ,85 & & & & & & \\
\hline & $\mathrm{N}$ & 20 & 20 & 20 & 20 & 20 & 20 & & & & & & \\
\hline \multirow{4}{*}{$\begin{array}{l}\text { RATING- } \\
\text { SKALA }\end{array}$} & & \multicolumn{12}{|c|}{ Vergleichende Statistik Teil II } \\
\hline & & \multicolumn{3}{|c|}{$\begin{array}{c}\text { Versuchsgruppe } \\
\text { Signifikanzen }\end{array}$} & \multicolumn{3}{|c|}{$\begin{array}{l}\text { Kontrollgruppe } \\
\text { Signifikanzen }\end{array}$} & \multicolumn{3}{|c|}{$\begin{array}{l}\text { Versuchsgruppe } \\
\text { Effektstärken d }\end{array}$} & \multicolumn{3}{|c|}{$\begin{array}{l}\text { Kontrollgruppe } \\
\text { Effektstärken d }\end{array}$} \\
\hline & & P-PO & P-F & $\mathrm{PO}-\mathrm{F}$ & P-PO & P-F & PO-F & d P-PO & $d P-F$ & d PO-F & d P-PO & d P-F & d PO-F \\
\hline & &, $030^{*}$ &, $008^{* *}$ &, 135 &, 716 &, 083 &, 135 & 41 & ,67 & ,34 & ,08 & ,41 & ,32 \\
\hline
\end{tabular}

Tabelle 54 gibt Auskunft über die Anzahl der Arztkonsultationen der beiden Gruppen innerhalb des Untersuchungszeitraums. Die Anzahl der Konsultationen sinkt bei der Versuchsgruppe von 4,4 im Pretest auf 1,45 im Follow Up ab. Patienten der Kontrollgruppe konsultieren demgegenüber ihren Hausarzt im Posttest und Follow Up häufiger als im Pretest.

Auch hier zeigt sich kein signifikanter Unterschied im Posttest und Follow Up zwischen den beiden Gruppen. Dennoch ergibt sich im Follow Up eine mittelgroße Effektstärke.

Die Standardabweichung ist in beiden Gruppen sehr hoch, was jeweils große, meist doppelt so große, Abweichungen in höhere und niedrigere Bereiche ermöglicht. Die hohen Standardabweichungen werden jedoch von Einzelpersonen verursacht.

Die Versuchsgruppe einzeln betrachtet zeigt sowohl im Pretest/Posttest- als auch im Pretest/Follow Up-Vergleich eine signifikante Verringerung der Anzahl der Arztkonsultationen. Die Effektstärke liegt im Pretest/Follow Up-Bereich mit ,81 in einem hohen Bereich.

In der Kontrollgruppe ergeben sich hingegen keine signifikanten Unterschiede bezüglich der Anzahl der Arztkonsultationen sowie keine bedeutsamen Effektstärken.

Insgesamt konnten sich die Hypothesen 40 und 41 innerhalb der Versuchsgruppe statistisch bestätigen. Zwar nimmt die Anzahl der Arztkonsultationen in der Versuchsgruppe auch im Follow Up nochmals ab, führt jedoch nicht mehr zu einer signifikanten Veränderung zum Posttest/Follow Up-Vergleich. Damit kann sich Hypothese 42 statistisch nicht bestätigen. 
Tabelle 54: Ergebnisse der Versuchsgruppe und der Kontrollgruppe über die Anzahl der Arztkonsultationen

\begin{tabular}{|c|c|c|c|c|c|c|c|c|c|c|c|c|c|}
\hline \multicolumn{8}{|c|}{ Deskriptive Statistik } & \multicolumn{6}{|c|}{ Vergleichende Statistik Teil I } \\
\hline \multirow{2}{*}{\begin{tabular}{|c} 
Unter- \\
suchungs- \\
Instrument \\
\end{tabular}} & & \multicolumn{3}{|c|}{ Versuchsgruppe } & \multicolumn{3}{|c|}{ Kontrollgruppe } & \multicolumn{3}{|c|}{ Signifikanzen } & \multicolumn{3}{|c|}{ Effektstärken d } \\
\hline & & $\mathrm{P}$ & $\mathrm{PO}$ & $\mathrm{F}$ & $\mathrm{P}$ & $\mathrm{PO}$ & $\mathrm{F}$ & POvG-POKc & & $F_{V G-F K G}$ & $\mathrm{~d} P O$ & & $d F$ \\
\hline \multirow{3}{*}{$\begin{array}{l}\text { EINZEL- } \\
\text { FRAGE }\end{array}$} & M & 4,4 & 2,25 & 1,45 & 2,7 & 3,15 & 2,9 & \multirow[t]{3}{*}{,322 } & \multirow{3}{*}{\multicolumn{2}{|c|}{,069 }} & \multirow[t]{3}{*}{,33 } & \multirow{3}{*}{\multicolumn{2}{|c|}{, 59}} \\
\hline & SD & 4,4 & 2,1 & 1,28 & 2,41 & 3,01 & 2,61 & & & & & & \\
\hline & $\mathrm{N}$ & 20 & 20 & 20 & 20 & 20 & 20 & & & & & & \\
\hline \multirow{4}{*}{$\begin{array}{l}\text { EINZEL- } \\
\text { FRAGE }\end{array}$} & & \multicolumn{12}{|c|}{ Vergleichende Statistik Teil II } \\
\hline & & \multicolumn{3}{|c|}{\begin{tabular}{|c|} 
Versuchsgruppe \\
Signifikanzen
\end{tabular}} & \multicolumn{3}{|c|}{$\begin{array}{c}\text { Kontrollgruppe } \\
\text { Signifikanzen }\end{array}$} & \multicolumn{3}{|c|}{$\begin{array}{l}\text { Versuchsgruppe } \\
\text { Effektstärken d }\end{array}$} & \multicolumn{3}{|c|}{$\begin{array}{l}\text { Kontrollgruppe } \\
\text { Effektstärken d }\end{array}$} \\
\hline & & P-PO & P-F & PO-F & P-PO & P-F & PO-F & d P-PO & $d P-F$ & d PO-F & d P-PO & d P-F & d PO-F \\
\hline & &, $05^{*}$ &, $01^{*}$ & , 163 & ,251 &, 519 & ,522 & ,61 & ,81 & ,37 &, 15 &, 08 & ,08 \\
\hline
\end{tabular}

Interessant sind zudem die Ergebnisse der Einschätzung der behandelnden Hausärzte über das Ausmaß an Forderungen nach weiteren medizinischen Untersuchungen in Tabelle 55.

In der Versuchsgruppe nehmen, nach Einschätzung der behandelnden Ärzte, die Forderung nach weiteren medizinischen Untersuchungen von 1,68 auf 0,45 ab.

Die Forderungen in der Kontrollgruppe sinken ebenfalls von 1,55 auf 1,2, jedoch in geringerem Maß als in der Versuchsgruppe.

Die Standardabweichungen sind in beiden Gruppen sehr hoch. Berücksichtigt man diese können auch Werte um 0 erreicht werden. Ebenso Werte, die doppelt so hoch sind als die errechneten Mittelwerte.

Der Vergleich der Mittelwerte zwischen Versuchs- und Kontrollgruppe ergibt sowohl im Posttest als auch im Follow Up einen signifikanten Unterschied.

Auch die großen Effektstärken von 1,04 im Posttest und ,89 im Follow Up unterstützen die Bedeutsamkeit der statistisch signifikanten Ergebnisse.

Die Messzeitpunktvergleiche innerhalb der Versuchsgruppe ergeben einen hoch signifikanten Unterschied von ,000 und ,000 im Pretest/Posttest- und Pretest/Follow UpVergleich. Hier werden zudem die höchsten Effektstärken der Studie von 1,5 und 1,7 ermittelt.

Die Messzeitpunktvergleiche innerhalb der Kontrollgruppe ergeben keine signifikant ermittelten Unterschiede. Es ergeben sich im Pretest/Follow Up- und Posttest/Follow UpVergleich jedoch kleine Effekte, die für die Praxis relevant sind. 
Die Hypothesen 43 und 44 können sich innerhalb der Versuchsgruppe demnach statistisch, mit hochsignifikanten Veränderungen bezogen auf die Verringerung des Ausmaßes der Forderungen nach weiteren medizinischen Untersuchungen, bestätigen. Hypothese 45 konnte sich in der Versuchsgruppe isoliert betrachtet nicht bestätigen. Dennoch sinkt das Forderungsausma $\beta$ weiter ab und es zeigt sich im Follow UpGruppenvergleich ein signifikanter Unterschied zu Gunsten der Versuchsgruppe.

Tabelle 55: Ergebnisse der Versuchsgruppe und der Kontrollgruppe über das von den behandelnden Hausärzten angegebene Ausmaß an Forderungen nach weiteren medizinischen Untersuchungen auf einer Skala von 0-4

\begin{tabular}{|c|c|c|c|c|c|c|c|c|c|c|c|c|c|}
\hline \multicolumn{8}{|c|}{ Deskriptive Statistik } & \multicolumn{6}{|c|}{ Vergleichende Statistik Teil I } \\
\hline \multirow{2}{*}{$\begin{array}{l}\text { Unter- } \\
\text { suchungs- } \\
\text { Instrument }\end{array}$} & & \multicolumn{3}{|c|}{ Versuchsgruppe } & \multicolumn{3}{|c|}{ Kontrollgruppe } & \multicolumn{3}{|c|}{ Signifikanzen } & \multicolumn{3}{|c|}{ Effektstärken d } \\
\hline & & $\mathrm{P}$ & $\mathrm{PO}$ & $\mathrm{F}$ & $\mathrm{P}$ & $\mathrm{PO}$ & $\mathrm{F}$ & POvG-PC & & $\mathrm{FvG}_{\mathrm{VG}} \mathrm{F}_{\mathrm{KG}}$ & $\mathrm{dPO}$ & & $d F$ \\
\hline \multirow{3}{*}{$\begin{array}{l}\text { RATING- } \\
\text { SKALA }\end{array}$} & M & 1,68 & ,55 & ,45 & 1,55 & 1,45 & 1,2 & \multirow{3}{*}{\multicolumn{2}{|c|}{, $003^{\star \star}$}} &, $009^{\star \star}$ & 1,04 & \multirow{3}{*}{\multicolumn{2}{|c|}{89}} \\
\hline & SD & 1,05 & ,45 & ,39 & 1,2 & 1,1 & 1,0 & & & & & & \\
\hline & $\mathrm{N}$ & 20 & 20 & 20 & 20 & 20 & 20 & & & & & & \\
\hline \multirow{4}{*}{$\begin{array}{l}\text { RATING- } \\
\text { SKALA }\end{array}$} & \multicolumn{13}{|c|}{ Vergleichende Statistik Teil II } \\
\hline & & \multicolumn{3}{|c|}{$\begin{array}{l}\text { Versuchsgruppe } \\
\text { Signifikanzen }\end{array}$} & \multicolumn{3}{|c|}{$\begin{array}{c}\text { Kontrollgruppe } \\
\text { Signifikanzen }\end{array}$} & \multicolumn{3}{|c|}{$\begin{array}{l}\text { Versuchsgruppe } \\
\text { Effektstärken d }\end{array}$} & \multicolumn{3}{|c|}{$\begin{array}{l}\text { Kontrollgruppe } \\
\text { Effektstärken d }\end{array}$} \\
\hline & & P-PO & P-F & PO-F & P-PO & $\mathrm{P}-\mathrm{F}$ & PO-F & d P-PO & $d P-F$ & d PO-F & d P-PO & $d P-F$ & d PO-F \\
\hline & &, $000^{* \star \star}$ &, $000^{\star \star \star}$ &, 479 & ,629 & , 185 & ,234 & 1,5 & 1,7 &, 24 &, 09 &, 32 &, 24 \\
\hline
\end{tabular}

Pre/Post und Follow Up-Vergleich der Mittelwerte zwischen Versuchs- und Kontrollgruppe bezogen auf das vom Arzt eingeschätzte Ausmaß an Forderungen nach weiteren medizinischen Untersuchungen

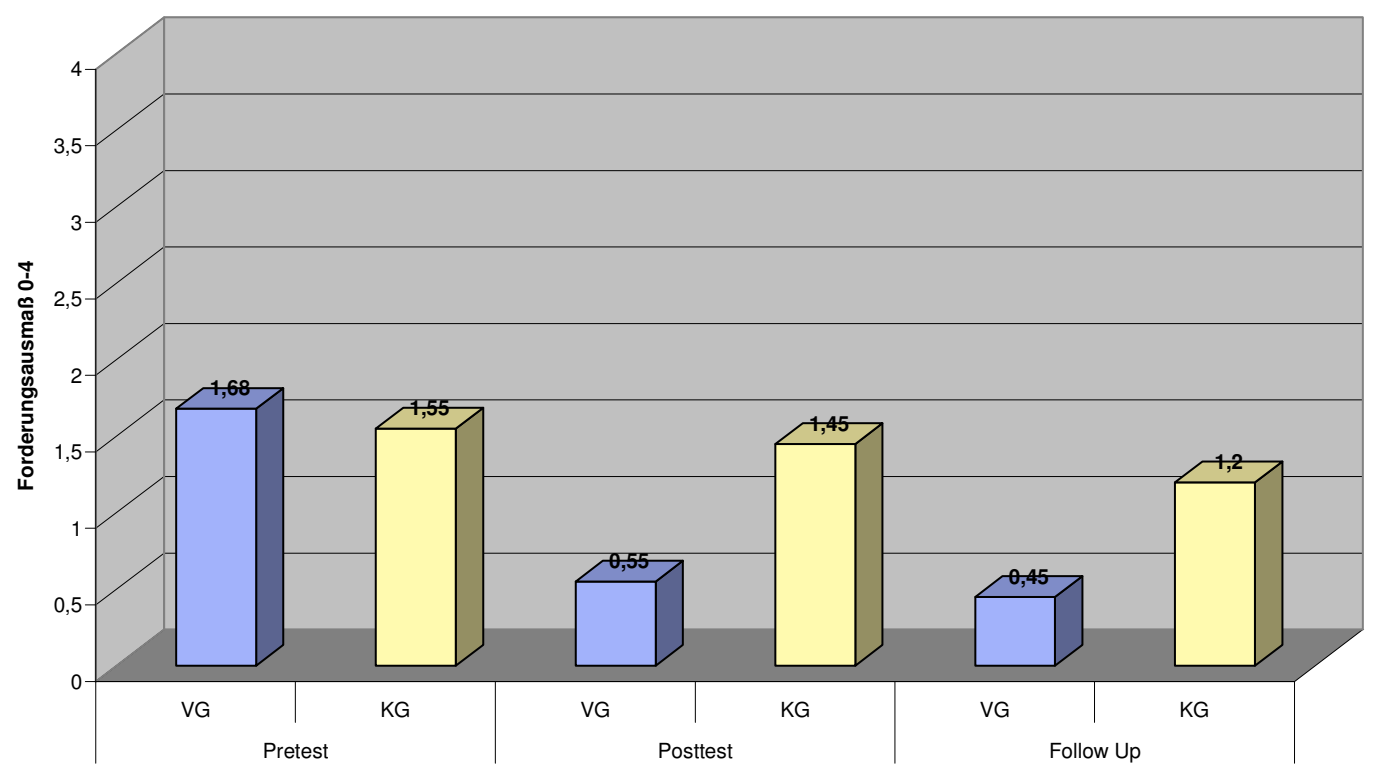

Abbildung 10: Graphische Darstellung: Mittelwertvergleich zwischen Versuchs- und Kontrollgruppe bezogen auf die Entwicklung der Forderungen nach weiteren medizinischen Untersuchungen. 
Die Psychotherapiebereitschaft der Patienten wird in Tabelle 56 dargestellt.

In beiden Gruppen nimmt die Bereitschaft bis zum Follow Up stetig ab, wobei die Kontrollgruppe einen größeren Verlust aufzeigt.

Trotz nicht vorhandenener signifikantener Unterschiede ist die mittelgroße Effektstärke von ,49 im Vergleich der Mittelwerte der beiden Gruppen im Follow Up zu nennen.

Die Standardabweichungen um 3 lassen vor allem für eine höhere Bereitschaft zur Therapie bei einigen Patienten einen Wert von beinahe 10 vermuten.

Innerhalb der Versuchsgruppe ergeben sich in allen Messzeitpunktvergleichen keine signifikanten Veränderungen, während in der Kontrollgruppe die Psychotherapiebereitschaft zwischen Pretest/Follow Up und Posttest/Follow Up signifikant absinkt.

Diese beiden statistisch signifikanten Ergebnisse weisen jeweils eine mittelgroße Effektstärke auf, die praktisch bedeutsam ist.

Die Hypothesen 49, 50 und 51 konnten sich hier nicht bestätigen. Die Psychotherapiebereitschaft steigt in der Versuchsgruppe zu keinem Messzeitpunkt signifikant an. In der Kontrollgruppe nimmt sie jedoch signifikant im Pretest/Follow Up- und Posttest/Follow Up-Vergleich ab.

Tabelle 56: Ergebnisse der Versuchsgruppe und der Kontrollgruppe bezogen auf die Entwicklung der Psychotherapiebereitschaft auf einer Skala von 0-10.

\begin{tabular}{|c|c|c|c|c|c|c|c|c|c|c|c|c|c|}
\hline & & eskr & ptive & Stat & stik & & & Ver & rgleic & chende & Statis & tik Te & eil I \\
\hline Unter- & & Vers & uchsg & uppe & Kon & rollgrt & ippe & Sign & ifikan & nzen & Effel & ktstärk & ken d \\
\hline Instrument & & $P$ & $\mathrm{PO}$ & $\mathrm{F}$ & $P$ & $\mathrm{PO}$ & $\mathrm{F}$ & POva-PO & & $F_{V G-F G G}$ & $\mathrm{dPO}$ & & $\mathrm{dF}$ \\
\hline & $M$ & 7,10 & 7,05 & 6,4 & 7,00 & 6,25 & 4,55 & , 483 & & ,129 & ,25 & & 49 \\
\hline RATING- & SD & 2,83 & 2,92 & 3,35 & 2,99 & 3,45 & 4,15 & & & & & & \\
\hline SKALA & $\mathrm{N}$ & 20 & 20 & 20 & 20 & 20 & 20 & & & & & & \\
\hline & & & & & & argl & chen & Statis & is & Teil II & & & \\
\hline RATING- & & \begin{tabular}{|c|} 
Vers \\
Sig \\
\end{tabular} & $\begin{array}{l}\text { uchs } \\
\text { nifike }\end{array}$ & $\begin{array}{l}\text { ppe } \\
\text { en }\end{array}$ & $\begin{array}{r}\text { Kon } \\
\text { Sig } \\
\end{array}$ & $\begin{array}{l}\text { rollgrt } \\
\text { ifikan }\end{array}$ & $\begin{array}{l}\text { ippe } \\
\text { zen }\end{array}$ & $\begin{array}{l}\text { Versuc } \\
\text { Effekt }\end{array}$ & $\begin{array}{l}\text { Ichsgr } \\
\text { ttstärk }\end{array}$ & $\begin{array}{l}\text { ruppe } \\
\text { ken d }\end{array}$ & $\begin{array}{l}\text { Kont } \\
\text { Effel }\end{array}$ & $\begin{array}{l}\text { trollgrt } \\
\text { ktstärk }\end{array}$ & $\begin{array}{l}\text { uppe } \\
\text { sen d }\end{array}$ \\
\hline SKALA & & P-PO & P-F & PO-F & P-PO & P-F & PO-F & d P-PO & $d P-F$ & d PO-F & d P-PO & d P-F & d PO-F \\
\hline & & ,952 & ,482 & ,333 & ,250 &, $013^{\star \star}$ &, $024^{\star}$ & ,02 & ,23 & , 21 & ,23 & ,69 & ,45 \\
\hline
\end{tabular}




\subsection{Narzisstisches Verhalten in Alltag und Gruppe}

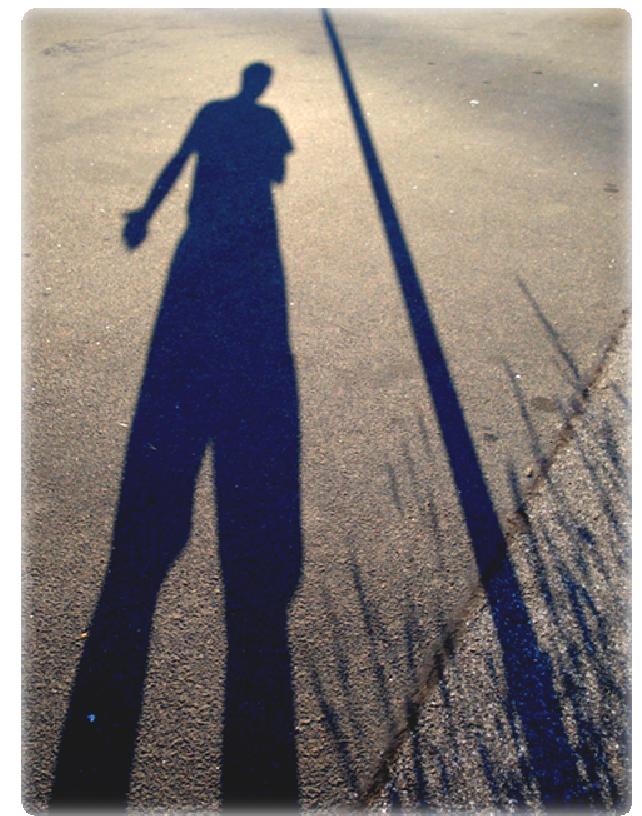

Wer Höhen erreichen möchte, sollte in Tiefen laufen können...

In Punkt 6.7 wurde Hypothese 45 überprüft. Hier interessierte, ob sich die Patienten der Versuchsgruppe in den Gruppensitzungen, im Vergleich zu ihrem Verhalten im Alltag, weniger narzisstisch zeigen konnten. Demgegenüber liegt das offene Zeigen von Schwäche und Bedürftigkeit. Wie in Gerd Rudolfs Theorie unter 3.2 erläutert, ist ein narzisstisch betontes Verhalten bei Menschen mit somatoformen Störungen oft zu beobachten und gilt als ein Bewältigungsversuch des zugrundeliegenden depressiven Grundkonflikts.

In Tabelle 57 wird deutlich, dass sich Patienten in der Gruppe weniger betont autonom und stark als in inrem Alltagsleben verhalten. Dieser Unterschied ist mit ,02 statistisch signifikant und mit einer Effektstärke von ,59 zudem auch praktisch bedeutsam.

Insgesamt konnte sich Hypothese 48 bestätigen. Die Patienten verhalten sich in der Gruppe signifikant weniger narzisstisch als in ihrem Alltagsleben. 
Tabelle 57: Ergebnisse der Versuchsgruppe für ein selbst eingeschätztes narzisstisches Verhaltensmuster in den Gruppensitzungen im Vergleich zum Verhalten im Alltag auf einer Skala von 0-4.

\begin{tabular}{|c|c|c|c|c|c|}
\hline $\begin{array}{l}\text { Untersuchungs- } \\
\text { instrument }\end{array}$ & & $\begin{array}{r}\text { Deskri } \\
\text { Stati }\end{array}$ & & $\begin{array}{r}\text { Vergl } \\
\text { St }\end{array}$ & $\begin{array}{l}\text { chende } \\
\text { istik }\end{array}$ \\
\hline \multirow[t]{4}{*}{$\begin{array}{l}\text { RATING- } \\
\text { SKALA }\end{array}$} & & $\begin{array}{c}\text { Narzisstisches } \\
\text { Verhaltens- } \\
\text { muster im Alltag } \\
\text { (Gesamt- } \\
\text { mittelwert } \\
\text { Pre/Post/Fu) }\end{array}$ & $\begin{array}{c}\text { Narzisstisches } \\
\text { Verhaltens- } \\
\text { muster in der } \\
\text { Gruppe } \\
\text { (Gesamt- } \\
\text { mittelwert) }\end{array}$ & $\begin{array}{l}\text { Signi- } \\
\text { fikanz }\end{array}$ & $\begin{array}{l}\text { Effekt- } \\
\text { stärke d }\end{array}$ \\
\hline & $M$ & 2,83 & 2,2 & \multirow{3}{*}{,02* } & \multirow{3}{*}{,56 } \\
\hline & SD & 1,13 & 1,1 & & \\
\hline & $\mathrm{N}$ & 20 & 20 & & \\
\hline
\end{tabular}




\subsection{Nicht hypothesengebundene Ergebnisse}

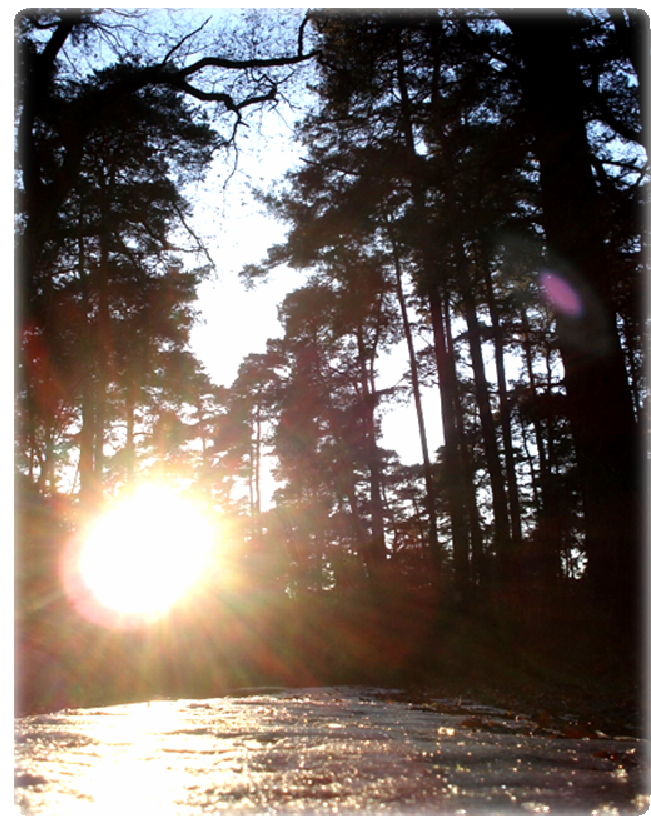

Am schönsten ist immer das, was wir uns nicht gewagt haben zu erhoffen...

In diesem Kapitel sollen alle Ergebnisse dargestellt werden, für deren Ermittlung zuvor keine Hypothesen aufgestellt wurden. Dennoch liefern sie weitere interessante Informationen über die symptomatische Entwicklung der Probanden sowie die Bewertung des konzipierten Gruppenprogramms.

Da eine Wiederholung des Programms durchaus möglich ist und in Erwägung gezogen wird, kann die Bewertung einzelner elementarer Bereiche helfen, das Programm zu verbessern, auszuarbeiten und weiterhin sinnvoll einzusetzen.

Da vorher nicht zu erwarten war, dass sich, in einem Untersuchungszeitraum von insgesamt sechs Monaten, der Gesamtbeschwerdeindex-Somatisierung signifikant verändert, wurde diese Hypothese zuvor nicht aufgestellt. Trotzdem wurde der, über das Screening für somatoforme Störungen ermittelte, Beschwerdeindex mit evaluiert.

Der Beschwerdeindex-Somatisierung ist ein klassifikationssystemübergreifender Somatisierungsindex über ICD-10 und DSM-IV hinaus. Er wurde benutzt, weil sich die Kriterien in der ICD-10 und dem DSM-IV teilweise unterscheiden und sich zudem auch derzeit noch in der Veränderung befinden. 
Zudem erfasst er sämtliche Somatisierungssymptome und grenzt sich nicht auf eine Störung, beispielsweise die Hypochondrie, ein.

Da innerhalb dieser Studie nicht eine spezielle, sondern alle existierenden somatoformen Störungen einbezogen wurden, eignete sich der Beschwerdeindex-Somatisierung sehr gut für die Entwicklungsbeobachtung des Störungsbildes.

Auch die Diskussion um das Klassifizieren von Störungsbildern und ICD-10 Kriterien wird als wichtig markiert. Sicher werden diese Klassifikationen für eine übergreifende, einheitliche Kommunikation, vielleicht auch für die Therapie benötigt, aber wenn sich Kriterien für eine Krankheit immer wieder verändern, dann sollte doch ein gewisser Spielraum für das Hinterfragen und Diskutieren dieses Krankheitsbegriffs offen bleiben.

Tabelle 58 zeigt, dass zwischen der Versuchs- und Kontrollgruppe weder im Posttest noch im Follow Up ein signifikanter Unterschied in der Veränderung besteht.

Im Pretest/Posttest- und Pretest/Follow Up-Vergleich der Versuchsgruppe zeigen sich jedoch jeweils signifikante Abnahmen des Beschwerdeindex Somatisierung, der in Prozentrangwerten berechnet wird.

Im Pretest gibt es nur 41,25\% einer psychosomatischen Vergleichsgruppe, deren Somatisierung stärker ist als der Durchschnitt der Versuchsgruppe. Im Follow Up hingegen sind es bereits 59,95\%, die im Vergleich zur Versuchsgruppe eine stärkere Somatisierungsstörung aufweisen.

Aber auch in der Kontrollgruppe zeigt sich eine signifikante Verbesserung im Pretest/Follow Up-Vergleich von 40,35\% hin zu 50,85\%, die im Vergleich zu Gruppe (B) eine stärkere Somatisierung zeigen.

Während sich für die Kontrollgruppe aber nur kleine Effektstärken zeigen, sind die Effekte in der Versuchsgruppe mittelgroß. 
Tabelle 58: Ergebnisse der Versuchsgruppe und der Kontrollgruppe im Beschwerdeindex Somatisierung nach dem Soms (Angaben in Prozentrangwerten 1100)

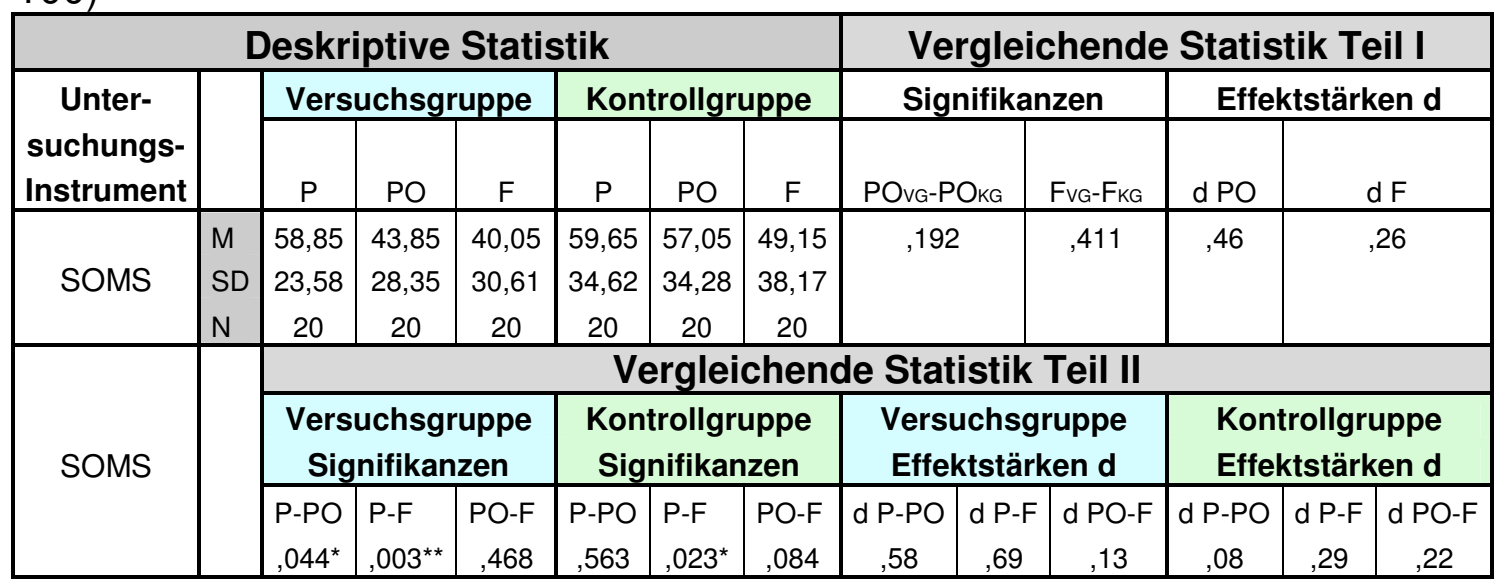

Pre/Post und Follow Up-Vergleich der Mittelwerte des Beschwerdeindex-Somatisierung zwischen Versuchs- und Kontrollgruppe

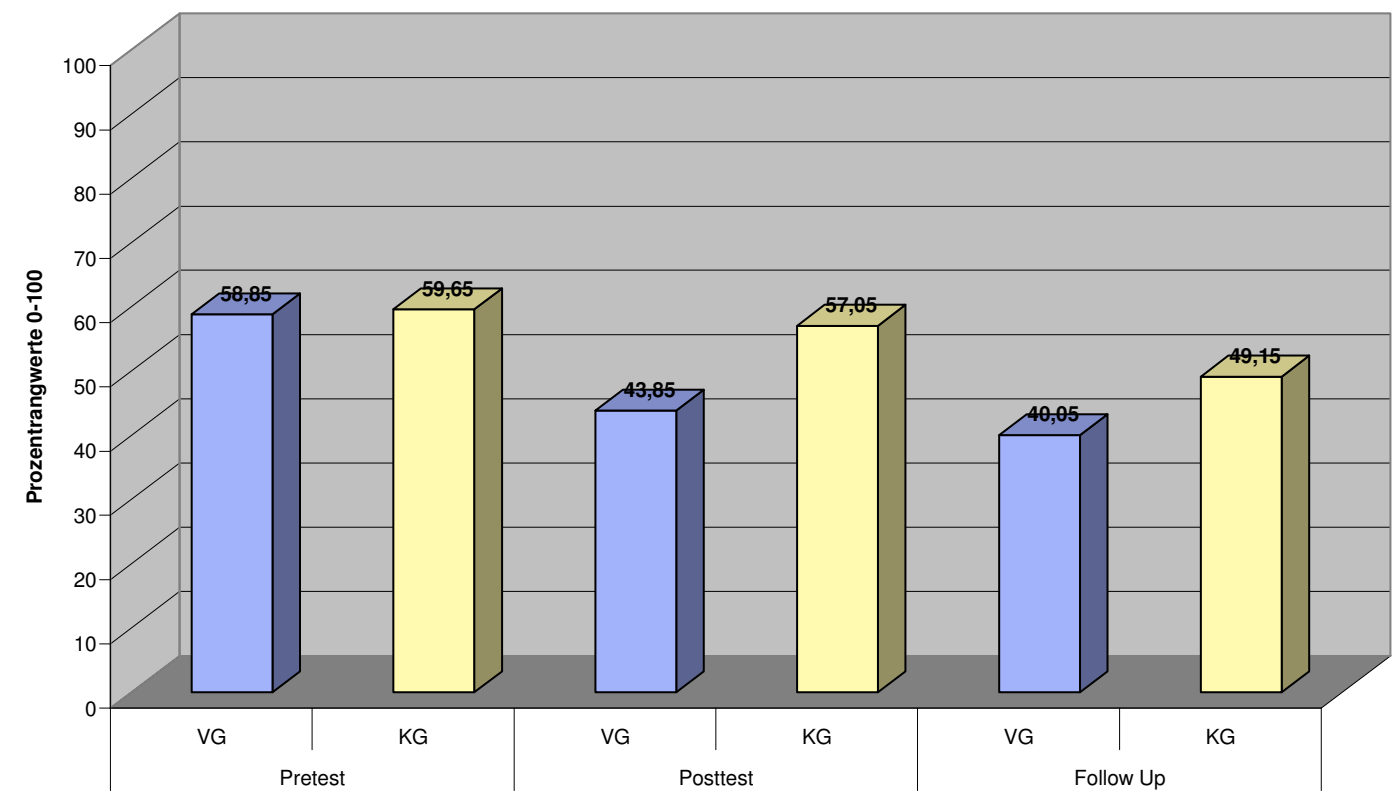

Abbildung 11: Graphische Darstellung: Mittelwertvergleich zwischen Versuchs- und Kontrollgruppe bezogen auf die Entwicklung des Beschwerdeindex-Somatisierung.

Im Folgenden werden nun die deskriptiven Daten über die Bewertung des Programms, die von den Probanden im Anschluss an die Teilnahme abgegeben wurde, aufgezeigt.

Weil sich 14 von 20 Teilnehmern im Anschluss an das Programm in der Gruppe so gut aufgehoben fühlten, und wie im Untersuchungsverlauf erwähnt, weiterhin in der Gruppe arbeiten wollten, soll die Einschätzung der Patienten dargestellt werden. 
Selbst wenn sich nicht alle Forschungshypothesen bestätigen konnten ist das unerwartete Ergebnis, dass 14 Patienten auch ein Jahr später noch in der Gruppe miteinander arbeiten, prägnant und erfreulich.

Außerdem besteht die Möglichkeit, dass dieses Programm wiederholt wird. Insgesamt erhielt das Programm positive Resonanz von den kooperierenden Hausärzten. Für eine mögliche Wiederholung mit neuen Patienten ist es wichtig Feedback von denjenigen zu erhalten, die bereits das Programm durchlaufen haben, um es möglicherweise an schwachen Stellen modifizieren und verbessern zu können und einen Überblick über den Nutzen für die Patienten zu bekommen.

Zunächst werden Ergebnisse dargestellt, die durch Skalenangaben in Tabellen ausgedrückt werden können. Der Mittelwert soll hier lediglich der Anschauung dienen. Danach erfolgt eine Zusammenfassung der wichtigsten und häufigsten Angaben zu offenen Fragen.

14 Probanden nehmen an einer weiterführenden Gruppe teil.

6 Probanden nehmen nach der Beendigung des Gruppenprogramms nicht weiter an einer Gruppe teil.

Tabelle 59: Häufigkeiten der Teilnahme an einer weiterführenden Gruppe nach Abschluss des offiziellen Programms der Patienten der Versuchsgruppe

\begin{tabular}{|l|c|c|}
\hline \multirow{2}{*}{ Geschlecht } & \multicolumn{2}{|c|}{ Teilnahme } \\
\cline { 2 - 3 } & ja & nein \\
\hline männlich & 2 & 3 \\
\hline weiblich & 12 & 3 \\
\hline $\mathbf{N}$ & \multicolumn{2}{|c|}{$\mathbf{2 0}$} \\
\hline
\end{tabular}

7 Probanden haben früher schon einmal eine Therapie wegen anderer Probleme durchgeführt und 2 Probanden sind kurz vor der Gruppe in eine Einzeltherapie eingestiegen.

11 Probanden der Versuchsgruppe hatten vor dem Programm noch keinerlei psychotherapeutische Erfahrung.

6 dieser 11 Personen haben nach dem Programm eine Einzel-Psychotherapie begonnen oder werden bald eine Einzeltherapie beginnen.

5 der 11 Versuchsgruppenprobanden möchten keine Therapie beginnen. Jedoch nehmen 4 der 5 Patienten weiterhin regelmäßig an der weiterführenden Gruppe teil und betrachten die Gruppe als ihre Therapie. 
Tabelle 60: Deskriptive Daten der Versuchsgruppe zur Durchführung einer EinzelPsychotherapie (Fragen im Follow Up)

\begin{tabular}{|l|c|c|}
\hline \multicolumn{1}{|c|}{$\begin{array}{c}\text { Angaben zur Durchführung einer } \\
\text { Einzel-Psychotherapie }\end{array}$} & \multicolumn{2}{c|}{ Häufigkeiten } \\
\cline { 2 - 3 } & männlich & weiblich \\
\hline $\begin{array}{l}\text { Weder vor noch nach der Studienteilnahme eine } \\
\text { Therapie begonnen und möchte auch keine beginnen }\end{array}$ & 1 & 4 \\
\hline Möchte bald eine Therapie beginnen & 0 & 4 \\
\hline $\begin{array}{l}\text { Vor Beginn der Studienteilnahme einmal eine Therapie } \\
\text { gemacht, die aber beendet ist }\end{array}$ & 1 & 6 \\
\hline $\begin{array}{l}\text { Vor Studienteilnahme Therapie begonnen, die noch nicht } \\
\text { beendet ist }\end{array}$ & 2 & 0 \\
\hline Während der Studienteilnahme eine Therapie begonnen & 1 & 1 \\
\hline $\mathbf{N}$ & $\mathbf{2 0}$ \\
\hline
\end{tabular}

11 der 20 Probanden der Kontrollgruppe möchten keine Therapie beginnen und haben auch vor Studienteilnahme noch nie eine Therapie absolviert.

6 der 20 Probanden der Kontrollgruppe haben in ihrem Leben schon einmal eine Therapie gemacht, die aber bereits beendet ist.

Nur 3 der 20 Kontrollprobanden befinden sich derzeit in einer laufenden Psychotherapie.

Tabelle 61: Deskriptive Daten der Kontrollgruppe zur Durchführung einer EinzelPsychotherapie (Fragen im Follow Up)

\begin{tabular}{|l|c|c|}
\multicolumn{1}{|c|}{$\begin{array}{c}\text { Angaben zur Durchführung einer } \\
\text { Einzel-Psychotherapie }\end{array}$} & \multicolumn{2}{c|}{ Häufigkeiten } \\
\cline { 2 - 3 } & männlich & weiblich \\
\hline $\begin{array}{l}\text { Weder vor noch nach der Studienteilnahme eine } \\
\text { Therapie begonnen und möchte auch keine beginnen }\end{array}$ & 1 & 10 \\
\hline Möchte bald eine Therapie beginnen & 0 & 0 \\
\hline $\begin{array}{l}\text { Vor Beginn der Studienteilnahme einmal eine Therapie } \\
\text { gemacht, die aber beendet ist }\end{array}$ & 3 & 3 \\
\hline $\begin{array}{l}\text { Vor Studienteilnahme Therapie begonnen, die noch nicht } \\
\text { beendet ist }\end{array}$ & 1 & 2 \\
\hline Während der Studienteilnahme eine Therapie begonnen & 0 & 0 \\
\hline $\mathbf{N}$ & \multicolumn{2}{|c|}{$\mathbf{2 0}$} \\
\hline
\end{tabular}


Tabellen 62 bis 73 zeigen die Evaluationen des Gruppenprogramms durch Skalenbewertungen von 0-10 für:

- Die Empfehlung für andere betroffene Menschen

- Das Empfinden, ob das Programm eine berechtigte Ergänzung zur hausärztlichen Behandlung ist

- Wie ernst sich die Teilnehmer von der Gruppenleitung und anderen Gruppenmitgliedern genommen gefühlt haben

Außerdem gibt es Angaben dazu, wie es den Patienten körperlich und psychisch nach der Teilnahme geht, wie nah sie ihrem zuvor formulierten Alltagsziel gekommen sind und wie die Dauer des Programms von zwei Monaten sowie die Dauer einer Sitzung von 90 Minuten bewertet wird. Zudem wird die Bewertung einer durchschnittlichen Gruppengröße von acht Personen, die Bedeutung der Raumgestaltung und Atmosphäre, um sich öffnen zu können und zuletzt die Bewertung des Verhältnisses der Anteile von angeleiteten Übungen und freier Gruppensprechzeit, dargestellt. 
Tabelle 62: Wie sehr empfehlen Probanden der Versuchsgruppe auf einer Skala von 0-10 $(0=$ gar nicht bis $10=$ sehr stark) anderen Menschen mit ähnlicher Problematik die Teilnahme an diesem Programm?

\begin{tabular}{|c|c|}
\hline \multicolumn{2}{|c|}{ Deskriptive Ergebnisse } \\
\hline$M$ & 9,3 \\
\hline $\mathrm{N}$ & 20 \\
\hline \multicolumn{2}{|c|}{ Statistische Ergebnisse } \\
\hline Skalenwert & Häufigkeit \\
\hline 0 & 0 \\
\hline 1 & 0 \\
\hline 2 & 0 \\
\hline 3 & 0 \\
\hline 4 & 0 \\
\hline 5 & 1 \\
\hline 6 & 1 \\
\hline 7 & 1 \\
\hline 8 & 0 \\
\hline 9 & 2 \\
\hline 10 & 15 \\
\hline
\end{tabular}

Tabelle 63: Wie sehr empfinden Probanden der Versuchsgruppe auf einer Skala von 0- 10 ( $0=$ gar nicht bis $10=$ sehr stark) die Teilnahme an diesem Programm als eine berechtigte und sinnvolle Ergänzung zu ihrer hausärztlichen Behandlung ihrer somatoformen Beschwerden? (auch stellvertretend für andere betroffene Patienten)

\begin{tabular}{|c|c|}
\hline \multicolumn{2}{|c|}{ Deskriptive Ergebnisse } \\
\hline$M$ & 9,4 \\
\hline $\mathrm{N}$ & 20 \\
\hline \multicolumn{2}{|c|}{ Statistische Ergebnisse } \\
\hline Skalenwert & Häufigkeit \\
\hline 0 & 0 \\
\hline 1 & 0 \\
\hline 2 & 0 \\
\hline 3 & 0 \\
\hline 4 & 0 \\
\hline 5 & 1 \\
\hline 6 & 0 \\
\hline 7 & 1 \\
\hline 8 & 1 \\
\hline 9 & 2 \\
\hline 10 & 15 \\
\hline
\end{tabular}


Tabelle 64: Wie ernst genommen fühlen sich Probanden der Versuchsgruppe auf einer Skala von 0-10 (0=gar nicht bis $10=$ sehr stark) von der Gruppenleitung?

\begin{tabular}{|c|c|}
\hline \multicolumn{2}{|c|}{ Deskriptive Ergebnisse } \\
\hline $\mathrm{M}$ & 9,15 \\
\hline $\mathrm{N}$ & 20 \\
\hline \multicolumn{2}{|c|}{ Statistische Ergebnisse } \\
\hline Skalenwert & Häufigkeit \\
\hline 0 & 0 \\
\hline 1 & 0 \\
\hline 2 & 0 \\
\hline 3 & 0 \\
\hline 4 & 0 \\
\hline 5 & 1 \\
\hline 6 & 1 \\
\hline 7 & 0 \\
\hline 8 & 3 \\
\hline 9 & 2 \\
\hline 10 & 13 \\
\hline
\end{tabular}

Tabelle 65: Wie ernst genommen fühlen sich Probanden der Versuchsgruppeauf einer Skala von 0-10 (0=gar nicht bis 10=sehr stark) von den anderen Gruppenmitgliedern?

\begin{tabular}{|c|c|}
\hline \multicolumn{2}{|c|}{ Deskriptive Ergebnisse } \\
\hline $\bar{M}$ & 9,15 \\
\hline $\mathrm{N}$ & 20 \\
\hline \multicolumn{2}{|c|}{ Statistische Ergebnisse } \\
\hline Skalenwert & Häufigkeit \\
\hline 0 & 0 \\
\hline 1 & 0 \\
\hline 2 & 0 \\
\hline 3 & 0 \\
\hline 4 & 0 \\
\hline 5 & 1 \\
\hline 6 & 1 \\
\hline 7 & 0 \\
\hline 8 & 3 \\
\hline 9 & 2 \\
\hline 10 & 13 \\
\hline
\end{tabular}


Tabelle 66: Wie geht es den Probanden der Versuchsgruppe körperlich nach der Teilnahme am Gruppenprogramm im Vergleich zu vorher?

\begin{tabular}{|l|c|}
\hline \multicolumn{1}{|c|}{ Items } & Häufigkeit \\
\hline Sehr viel besser & 1 \\
\hline besser & 15 \\
\hline Genau wie vorher & 4 \\
\hline schlechter & 0 \\
\hline Sehr viel schlechter & 0 \\
\hline \multicolumn{1}{|c|}{$\mathbf{N}$} & $\mathbf{2 0}$ \\
\hline
\end{tabular}

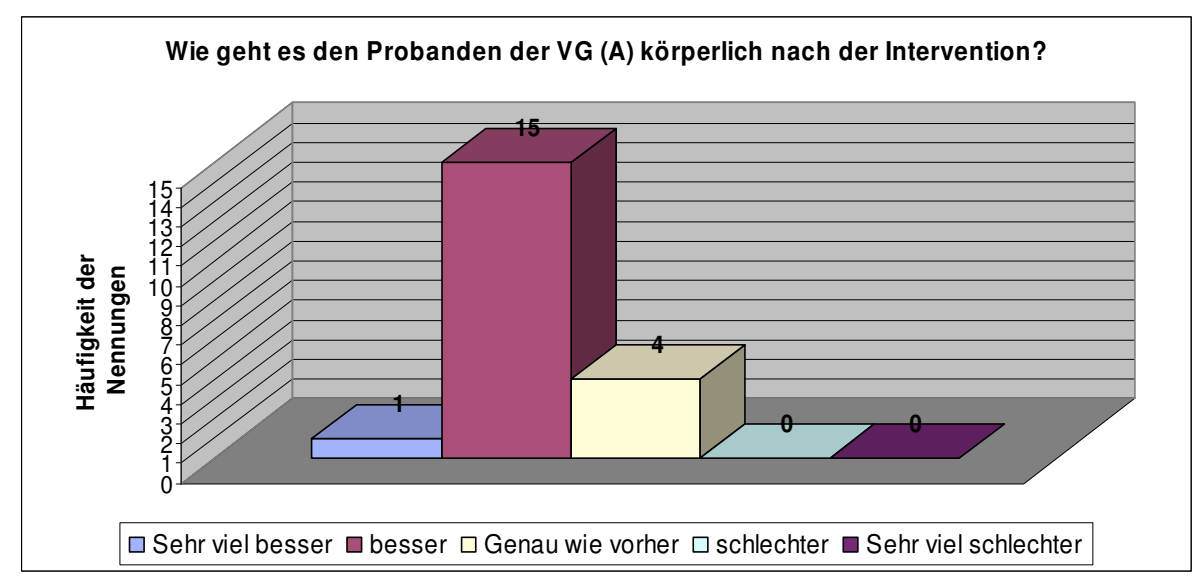

Abbildung 12: Graphische Darstellung: Wie geht es den Probanden der VG (A) körperlich nach der Intervention?

Tabelle 67: Wie geht es den Probanden der Versuchsgruppe psychisch nach der Teilnahme am Gruppenprogramm im Vergleich zu vorher?

\begin{tabular}{|c|c|}
\hline Items & Häufigkeit \\
\hline Sehr viel besser & 2 \\
\hline besser & 15 \\
\hline Genau wie vorher & 2 \\
\hline schlechter & 1 \\
\hline Sehr viel schlechter & 0 \\
\hline $\mathbf{N}$ & 20 \\
\hline
\end{tabular}




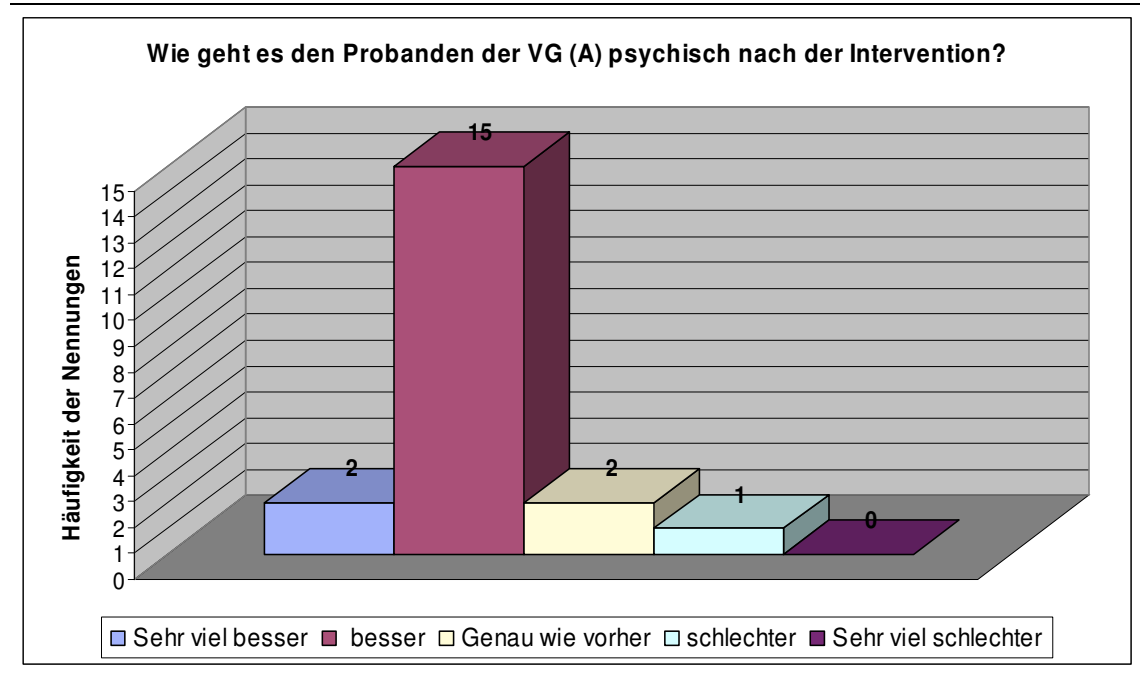

Abbildung 13: Graphische Darstellung: Wie geht es den Probanden der VG (A) psychisch nach der Intervention?

Tabelle 68: Wie nah sind die Probanden der Versuchsgruppe, nach ihrer persönlichen Einschätzung, ihrem vor dem Programm formulierten Alltagsziel gekommen?

\begin{tabular}{|l|c|}
\hline \multicolumn{1}{|c|}{ Items } & Häufigkeit \\
\hline Kaum näher & 4 \\
\hline $\begin{array}{l}\text { Etwas näher als es mir vor der } \\
\text { Gruppe möglich war }\end{array}$ & 9 \\
\hline Sehr nah & 6 \\
\hline Ich habe mein Ziel erreicht & 1 \\
\hline \multicolumn{1}{|c|}{$\mathbf{N}$} & $\mathbf{2 0}$ \\
\hline
\end{tabular}

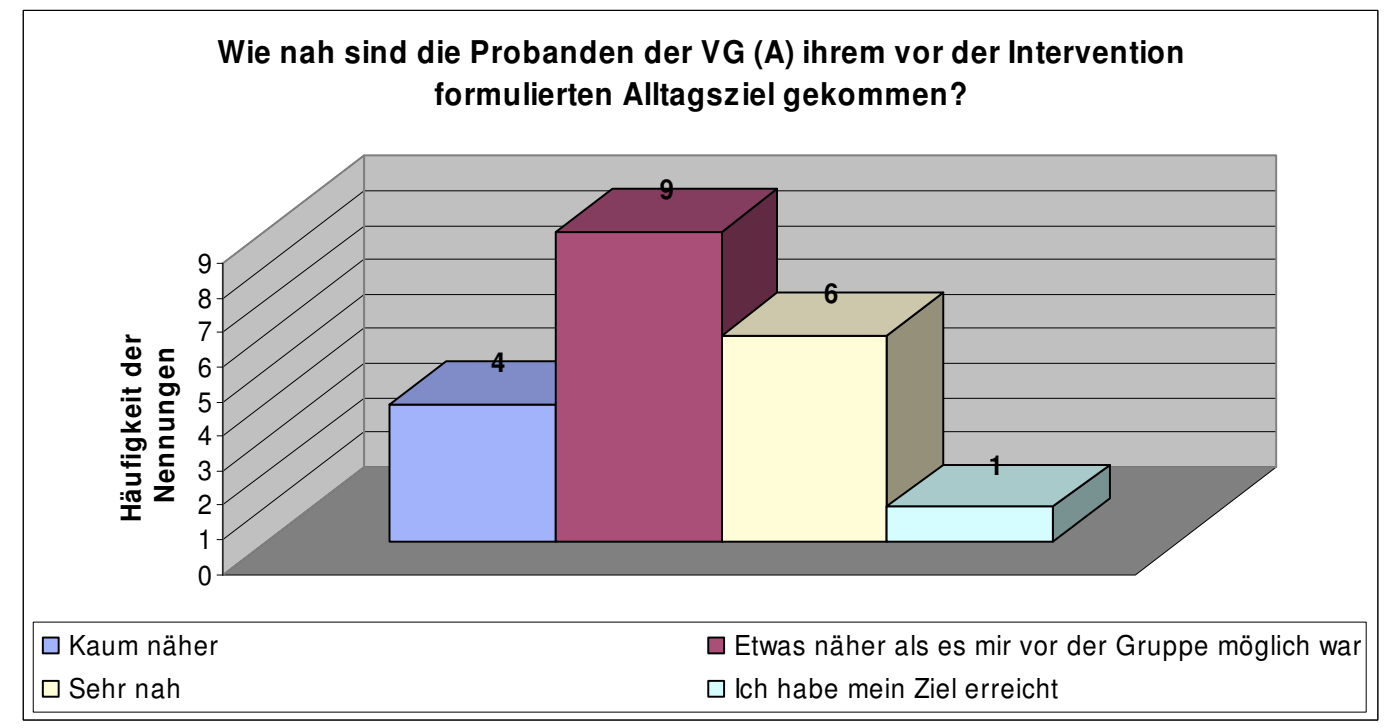

Abbildung 14: Graphische Darstellung: Wie nah sind die Probanden der VG (A) ihrem vor der Intervention formulierten Alltagsziel gekommen? 
Tabelle 69: Wie bewerten Probanden der Versuchsgruppe die Dauer von zwei Monaten (acht Sitzungen) des Programms?

\begin{tabular}{|l|c|}
\hline \multicolumn{1}{|c|}{ Items } & Häufigkeit \\
\hline Zu kurz & 15 \\
\hline Genau richtig & 5 \\
\hline Zu lang & 0 \\
\hline \multicolumn{1}{|c|}{$\mathbf{N}$} & $\mathbf{2 0}$ \\
\hline
\end{tabular}

Tabelle 70: Wie bewerten Probanden der Versuchsgruppe die Dauer einer Sitzung a 90 Minuten?

\begin{tabular}{|l|c|}
\hline \multicolumn{1}{|c|}{ Items } & Häufigkeit \\
\hline Zu kurz & 3 \\
\hline Genau richtig & 17 \\
\hline Zu lang & 0 \\
\hline \multicolumn{1}{|c|}{$\mathbf{N}$} & $\mathbf{2 0}$ \\
\hline
\end{tabular}

Tabelle 71: Wie bewerten Probanden der Versuchsgruppe eine Gruppengröße von durchschnittlich acht Personen?

\begin{tabular}{|l|c|}
\hline \multicolumn{1}{|c|}{ Items } & Häufigkeit \\
\hline Zu klein & 0 \\
\hline Genau richtig & 19 \\
\hline Zu groß & 1 \\
\hline \multicolumn{1}{|c|}{$\mathbf{N}$} & $\mathbf{2 0}$ \\
\hline
\end{tabular}

Tabelle 72: Als wie wichtig bewerten Probanden der Versuchsgruppe die Gestaltung und die Atmosphäre des Gruppenraums, um sich öffnen zu können?

\begin{tabular}{|l|c|}
\hline \multicolumn{1}{|c|}{ Items } & Häufigkeit \\
\hline Sehr wichtig & 9 \\
\hline Eher Neutral & 11 \\
\hline Unwichtig & 0 \\
\hline $\mathbf{N}$ & $\mathbf{2 0}$ \\
\hline
\end{tabular}

Tabelle 73: Wie bewerten Probanden der Versuchsgruppe das Verhältnis der Anteile von angeleiteten Übungen und freier Gruppensprechzeit? (Was wünschen sie sich?)

\begin{tabular}{|l|c|}
\hline \multicolumn{1}{|c|}{ Items } & Häufigkeit \\
\hline Mehr Übungen, weniger freie Sprechzeit & 3 \\
\hline Weniger Übungen, mehr freie Sprechzeit & 1 \\
\hline So wie es war fand ich es genau richtig & 15 \\
\hline Gar keine Übungen - nur freie Sprechzeit & 1 \\
\hline $\mathbf{N}$ & $\mathbf{2 0}$ \\
\hline
\end{tabular}




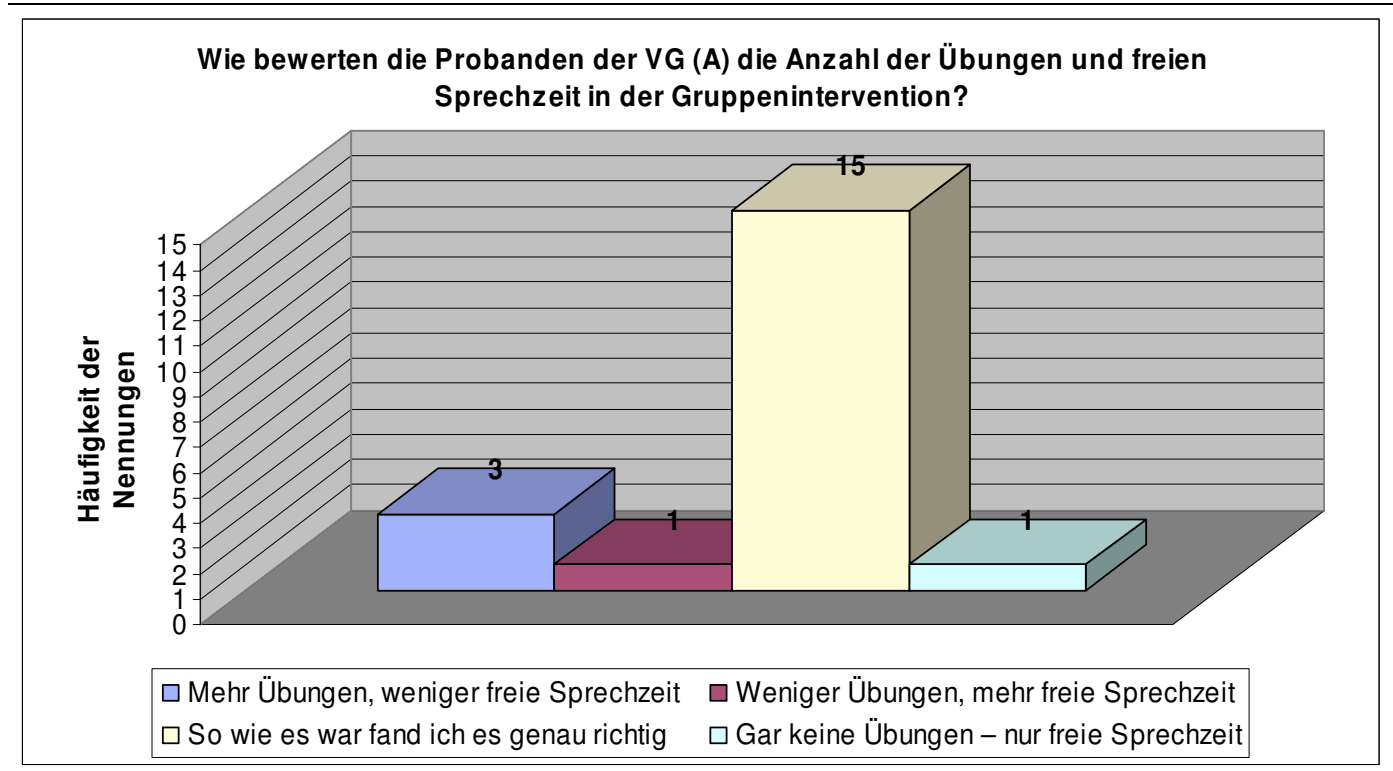

Abbildung 15: Graphische Darstellung der Bewertung der VG (A) des Anteils der angeleiteten Übungen und der freien Sprechzeit im Gruppenprogramm 
Im Anschluss an das Gruppenprogramm wurden den Teilnehmern zusätzlich offene Fragen zur Bewertung der Konzeption gestellt. Diese werden in drei Bereiche unterteilt:

1. Erlebte Auswirkungen im Alltag

2. Erleben in der Gruppe

3. Offen gebliebene Wünsche und Erwartungen.

$\mathrm{Zu}$ jeder Frage werden maximal drei Antworten zitiert, die von den Teilnehmern am häufigsten genannt wurden. Es ist beabsichtigt, dass alle Antworten im Originalwortlaut dargestellt werden, das bedeutet auch in Umgangssprache. Diese Art der Darstellung ermöglicht dem Leser ein authentisches Erleben der Probanden und eine lebensnahe Wiedergabe des Empfindens. Die vollständige Auflistung der Antworten zu jeder Frage befindet sich im Anhang der Arbeit.
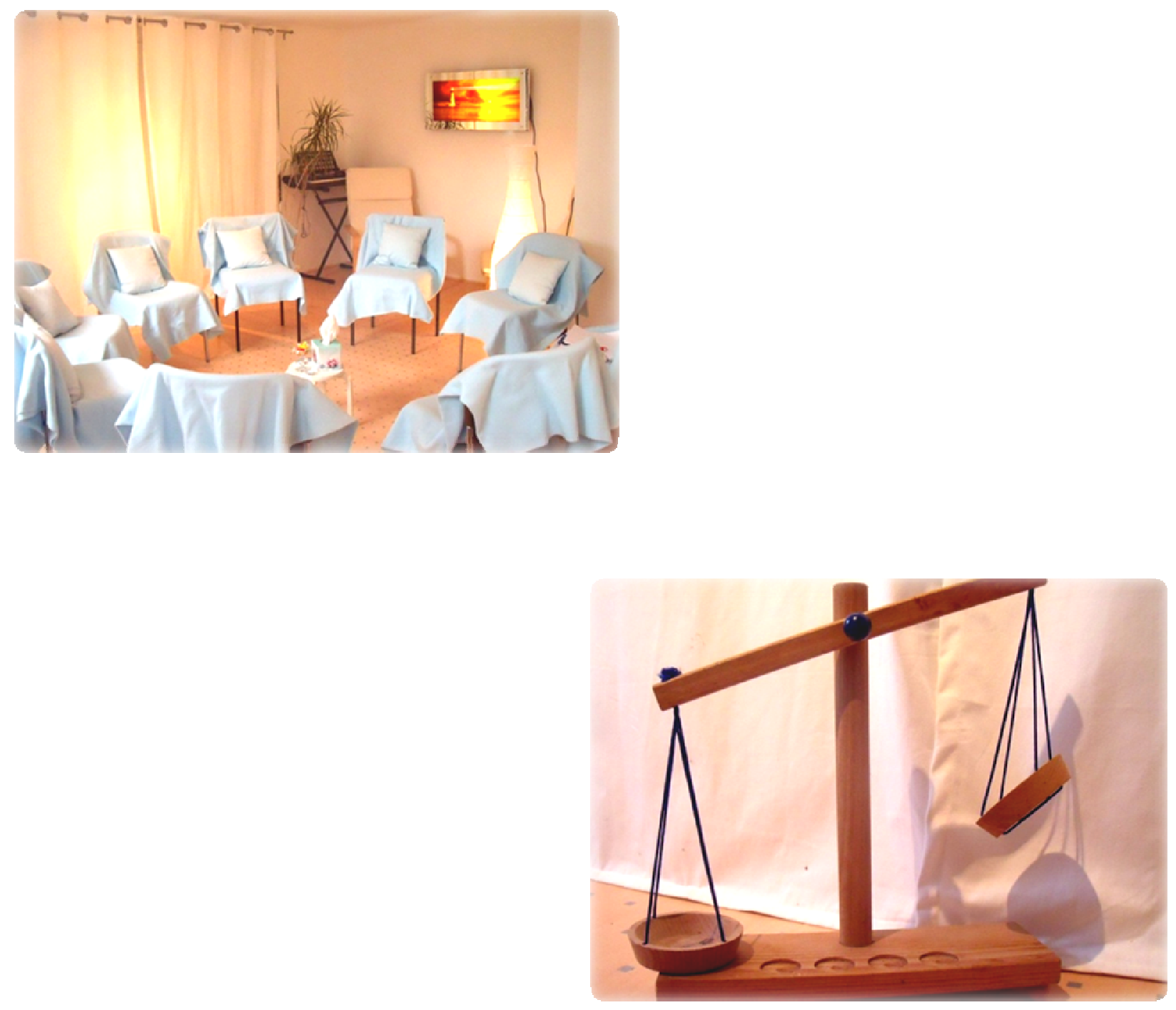
Tabelle 74: Offene Fragen und offene Antworten nach Beendigung des Programms

Erlebte persönliche Auswirkungen und Veränderungen im Alltag durch die Teilnahme an dem Gruppenprogramm

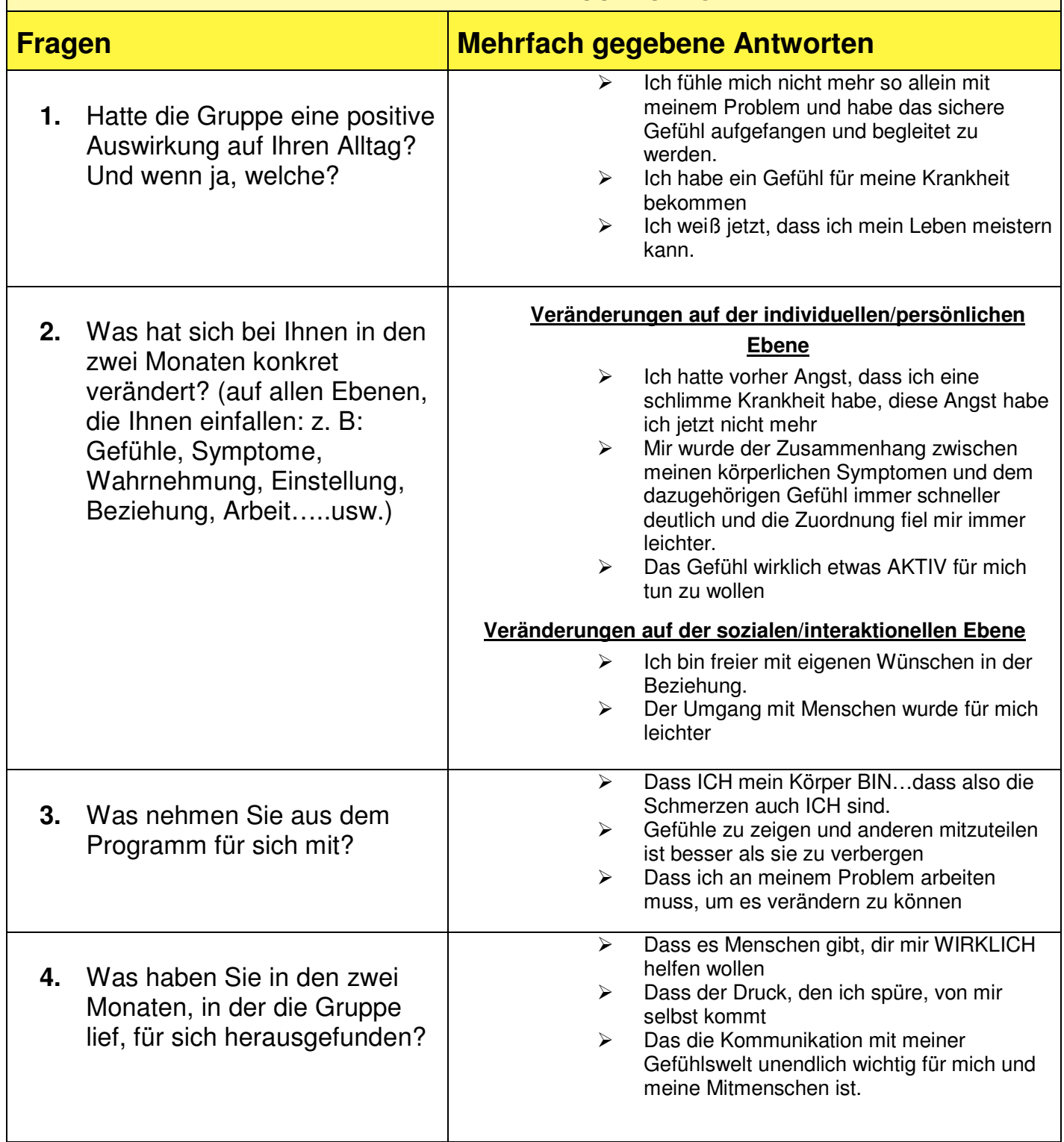


Fortsetzung Tabelle 74

Interventionen und Erleben in der Gruppe, mit den Gruppenmitgliedern und der Gruppenleitung

\begin{tabular}{|l|l|}
\hline Fragen & Mehrfach gegebene Antworten
\end{tabular}

5. Welche Äußerung aus der Gruppe oder von der Gruppenleitung war der entscheidende Wendepunkt innerhalb dieses Programms für Sie?

6. Was war Ihnen bzgl. der Gruppenleitung besonders wichtig?
Äußerungen/Handlungen durch die Gruppenmitglieder

> Die erste Sitzung, als eine Teilnehmerin über Tod und Traurigkeit sprach, da bekam ich Panik und konnte dann mit Hilfe klären, dass es mit dem Tod meines Vaters zu tun hatte, über den ich noch gar nicht richtig traurig sein konnte.

> Die Fähigkeit einer anderen Teilnehmerin, die sich gut abgrenzen konnte: „Was hat das mit mir zu tun? Ist das wirklich relevant für mich?"

$>\quad$ Als wir über die Beziehung zu meiner Mutter gesprochen haben

Äußerungen/Handlungen durch die Gruppenleitung

$>$ Dass $\mathrm{ICH}$ „,mein Gefühl, mein Körper, meine Schmerzen, meine Gedanken" bin...

$>$ „Ich lebe JETZT“.

> Dass „Lachen“ etwas ist, was mir gut tut und was ich viel mehr tun sollte.

$>$ Dass sie das Entscheidende immer zusammenfasste und nachhackte, wenn es nötig war, aber doch die richtige Mischung zwischen Einmischen und Zurückhaltung fand.

Dass sie mich ernst genommen hat.

$>$ Dass sie klar und freundlich war und geleitet und gelenkt hat.
7. Was aus bzw. an der Gruppe war Ihnen besonders wichtig?
Dass jeder an dem anderen interessiert war, zugehört hat und sich wieder gefunden hat.

$>$ Dass ein großer Rückhalt da war, obwohl man sich vorher nicht kannte.

$>$ Verschwiegenheit, Fremdheit, Achtung.
8. Welche Übung/Intervention war für Sie am prägnantesten?
Die Waage (fast durchweg genannt)

Ich muss - ich entscheide mich zu, Ich darf nicht - ich erlaube mir nicht - Ich kann nicht - ich will nicht

$>$ Mein schmerzendes Körperteil schreibt mir einen Brief..
9. Gibt es etwas, bzgl. der Atmosphäre, das dazu beigetragen hat, dass Sie sich öffnen konnten? Wenn ja, was war es?
Dass in der Gruppe das Gefühl vermittelt wurde, dass jeder und jede Einzelne wichtig und gleichberechtigt mit seinen/ihren Problemen ist.

$>$ Dass es ein geschlossener Raum war und nichts nach außen drang.

$>\quad$ Licht, Kerzen, die Offenheit der Teilnehmer und der Leitung. 
Fortsetzung Tabelle 74

Zur Konzeption: Erfüllte und offen gebliebene Erwartungen sowie Anregungen für die Verbesserung des Programms

\begin{tabular}{|c|c|}
\hline Fragen & Mehrfach gegebene Antworten \\
\hline $\begin{array}{l}\text { 10. Was waren im Vorfeld Ihre } \\
\text { Erwartungen? Welche davon } \\
\text { wurden erfüllt, welche blieben } \\
\text { offen? }\end{array}$ & $\begin{array}{c}\text { Erfüllte Erwartungen } \\
>\quad \begin{array}{l}\text { mein Selbstwertgefühl zu stärken und freier sprechen zu } \\
\text { können } \\
\text { Die Frage zu klären, ob Schmerzen auch psychisch } \\
\text { bedingt sein können }\end{array} \\
>\quad \text { Meine Probleme annehmen zu lernen } \\
\quad \text { Offen gebliebene Erwartungen } \\
>\quad \begin{array}{l}\text { Zu lernen wie ich mich bei akuten Beschwerden } \\
\text { verhalten soll }\end{array} \\
>\quad \text { Wie kann ich meine Probleme wirklich verändern? } \\
\text { Dass ich die Ursachen meiner Schmerzen heraus } \\
\text { bekomme und daran arbeite. Einiges ist davon erfüllt, } \\
\text { aber es braucht noch mehr Zeit. }\end{array}$ \\
\hline $\begin{array}{l}\text { 11. Was hat Ihnen besonders } \\
\text { gefallen? }\end{array}$ & $\begin{array}{l}\text { Dass ich so sein konnte, wie ich bin. } \\
\text { Dass ich jede Stunde dazu angeregt wurde neue } \\
\text { persönliche Ressourcen im Alltag für mich zu finden, die } \\
\text { mich stärken. } \\
\text { Dass ich mit meinen Schmerzen ernst genommen } \\
\text { wurde }\end{array}$ \\
\hline 12. Was haben Sie vermisst? & $\begin{array}{l}\text { Mehr männliche Teilnehmer } \\
\text { Mehr Zeit } \\
\text { Ich hätte mir gewünscht, dass sich 2,3 Teilnehmer mehr } \\
\text { geöffnet hätten, aber vielleicht kommt das ja noch }\end{array}$ \\
\hline $\begin{array}{l}\text { 13. Was sollte aus Ihrer Sicht in der } \\
\text { Gruppe noch besprochen } \\
\text { werden? }\end{array}$ & $\begin{array}{l}\text { Die Rolle „Mann“ und die Rolle „Frau“ und das } \\
\text { Zusammenspiel in einer Beziehung } \\
\text { Was man in akuten Situationen tun kann, um Hilfe zu } \\
\text { bekommen. } \\
\text { Dass wir auf jeden fall „da weiter machen, wo wir } \\
\text { aufgehört haben“. }\end{array}$ \\
\hline
\end{tabular}




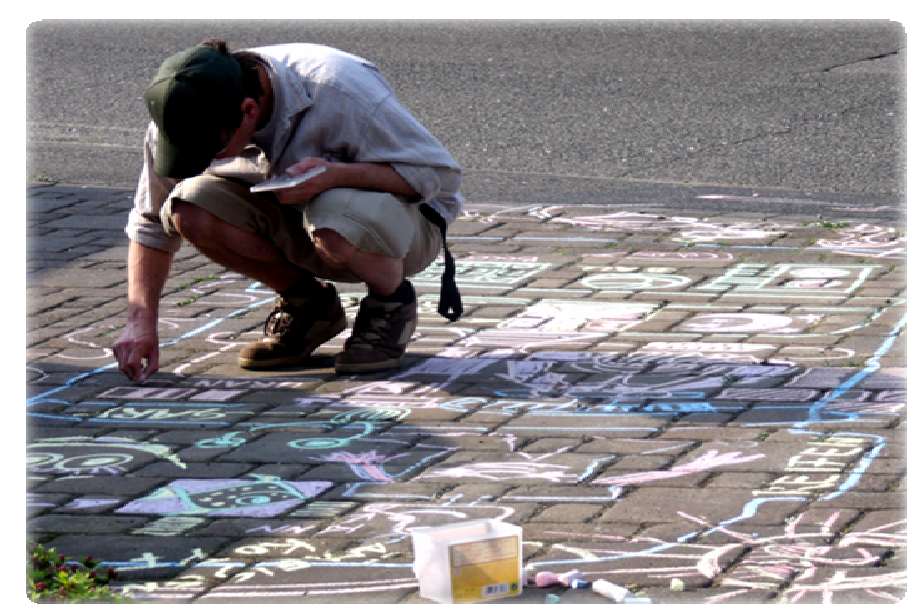

„Wir sind ja nicht umsonst der Herausforderung mit unserer Idee begegnet, haben ein passendes Projektdesign gefunden, haben mehrfach recherchiert, diskutiert, reflektiert, formalisiert, redigiert und reduziert, informiert und diffundiert,

und siehe da:

Nun stehen sie nach der Säuberung wohlgesäubert vor uns, die ehedem so peinlichen Geschöpfe kreatürlicher Vielfalt und Abnormität, cartesianisch geputzt und übersichtlich in ihren Konturen, wie Phönixe (aufgestiegen aus dem Meer der Unwissenheit über die Gebirge der Unerforschtheit bis in den Himmel der Erkenntnis), und in unsere rechtwinkligen Schubladen sollten sie jetzt einigermaßen hineinpassen“" (Mertens, 1992, S. 46).

Ziel der hier vorliegenden Arbeit war es, herauszufinden, ob sich bei Patienten mit somatoformen Störungen nach der Teilnahme an einem niedrigschwelligen Gruppeninterventionsprogramm, das in direkter Kooperation mit den behandelnden Hausärzten durchgeführt wird, positive Effekte, bezogen auf körperliche und psychosoziale Variablen, zeigen können.

Hauptzielfokus war eine positive Abnahmeentwicklung der Beschwerdeintensität. Wobei selbst eine minimale Verbesserung bei den häufig chronisch betroffenen Personen als ein Erfolg anzusehen ist (Sonnenmoser, 2005).

Neben der Symptomreduktion war in der psychosozialen Arbeit zudem die Sensibilisierung für die Wahrnehmung eigener Emotionen und eine bessere Kommunikationsfähigkeit dieser von Bedeutung (Rudolf, 2006a). 
Deshalb war von großem Interesse, ob sich die Akzeptanz eigener Gefühle, die Wahrnehmung dieser und das Erleben einer Koppelung zwischen körperlichen Beschwerden und psychischem Empfinden, verändern können.

Aus heilpädagogischer Sicht wurde auch dem Erleben und Verhalten im Altag der Probanden eine besondere Gewichtung erteilt. Hierbei stand die Frage im Fokus der Beobachtung und Evaluation, ob sich nach der Gruppenteilnahme die Grundeinstellung der Betroffenen zu mehr Optimismuss und Gelassenheit verändern kann.

Aufgrund der häufig konfliktbehafteten und schwierigen Arzt-Patient-Beziehung (Zimmermann \& Kaduszkiewicz, 2003) wurden auch die Entwicklung der erlebten Beziehungsqualität von Seiten der Patienten und des Arztes zueinander als auch die Anzahl der Arztkontakte und Arztwechsel sowie das Ausmaß an Forderungen nach weiteren medizinischen Untersuchungen von Patientenseite evaluiert.

Basierend auf der in Punkt 3.2 vorgestellten tiefenpsychologischen Theorie zur Entstehung somatoformer Störungen galt in der Erhebung ein weiterer Fokus dem bewussten Erleben von Resonanz in der Gruppe und einem möglichen Zusammenhang zwischen der erlebten Resonanz und der wahrgenommenen Beschwerdestärke. Diesbezüglich und bezüglich des narzisstischen Verhaltensmusters galt ein weiterer Fokus dem vergleichenden Blick zwischen Alltags- und Gruppenerleben und Verhalten.

Somatoforme Patienten werden als wenig motivierbar und offen gegenüber den für sie aber dringend notwendigen, psychosozialen Hilfsmaßnahmen dargestellt (Cuntz, 1998). Da für die Patienten die somatische Belastung im Vordergrund steht, stellte sich die Frage, ob eine parallele Weiterbehandlung beim Hausarzt und die von inm unterstützte Teilnahme am Gruppenprogramm dieser Studie möglicherweise in Zusammenhang mit einem positiven Effekt bezogen auf die Psychotherapiebereitschaft interpretiert werden kann.

Die Ergebnisse dieser einzelnen Foki werden nun im Einzelnen sowie aufeinanderbezogen diskutiert und interpretiert.

Es muss an dieser Stelle vorab gesagt werden, dass allgemeingültige und auf alleinige Wahrheit insistierende Aussagen nicht getroffen werden können. Die Arbeit an und mit Menschen lässt immer Raum für viele Variablen, die der Forscher nicht im Blick hat, die aber möglicherweise auch einen Einfluss auf die Entwicklung einer Person haben können. 
Sicher lassen die Ergebnisse einige erfreuliche Entwicklungen beobachten und ermutigen zumindest dazu, in die „richtige Richtung“ gearbeitet zu haben. Für die Population aller Betroffenen bei einer Versuchspersonenanzahl von $\mathrm{N}=20$ verallgemeinernde Aussagen zu treffen, sollte jedoch mit Vorsicht geschehen.

Trotzdem wird in dieser Untersuchung der Blick auf die Gruppenintervention gelegt, die als Fokus für die Diskussion und Interpretation der Ergebnisse dient. Die Teilnahme am konzipierten Gruppenprogramm ist Grundlage der Evaluation und daher Hauptblickpunkt für die Interpretation der Arbeit. 


\section{Symptomentwicklung}

In der Versuchsgruppe konnten sich alle Variablen zur Symptomentwicklung verbessern. Dazu zählten die erlebte Beeinträchtigung durch die Beschwerden, die erlebte Intensität und Stärke der Beschwerden, die Anzahl der körperlichen Symptome sowie die hypochondrische Angst an einer schlimmen Krankheit zu leiden.

Dass sich der gesamte Bereich der körperlichen Symptomatik nach einer niedrigschwelligen Kurzzeitintervention verbessert, war eine stille Hoffnung aber keineswegs ein zu hundert Prozent zu erwartendes Ergebnis. Umso beeindruckender ist die Verbesserung der Symptomvariablen über den Untersuchungszeitraum hinweg.

Hypothesen 1-15 befassten sich mit der Entwicklung der körperlichen Symptomatik.

Hypothesen 1, 2, 7, 8, 10, 11, 13 und 14 konnten sich insofern bestätigen, dass in den Symptomvariablen jeweils eine signfikante Veränderung innerhalb der Versuchsgruppe zwischen Pretest und Posttest sowie zwischen Pretest und Follow Up sichtbar wurde. Für die Hypothesen 3, 9, 12 und 15 ergab sich jedoch auch eine Verbesserung der Symptomatik, die jedoch lediglich als Tendenz bewertet werden kann, da sich keine signifikanten Veränderungen mehr zwischen Posttest und Follow Up ergaben.

Hypothesen 3, 4 und 5 sind die einzigen Hypothesen der Subgruppe Symptomentwicklung, die sich innerhalb der Versuchsgruppe nicht durch statistische Signifikanz bestätigen konnten. Allerdings zeigt sich in der erlebten Beschwerdeintensität im Posttestvergleich der Mittelwerte zwischen den Gruppen, dass dieser signifikant ausfällt. Die Versuchsgruppe verringerte ihre erlebte Beschwerdestärke also signifikant mehr als die Patienten der Kontrollgruppe.

\section{Chronifizierung(sgründe)}

Aufbauend auf den Aussagen von Mück (2005) und Rudolf (2008), dass in der Therapie meist keine gänzliche Heilung, sondern eine Verbesserung des Umgangs mit der Symptomatik erreicht werden kann, sind die Ergebnisse als erfreulich zu bewerten.

Anhand dieser Ergebnisse kann hier gegen die Aussage von Bass und Murphy (1995), welche die Somatisierungsstörung vielmehr als eine Persönlichkeitsstörung bezeichnen, die nach Definiton wesentlich veränderungsresistenter ist als eine neurotische Störung, argumentiert werden. 
Zwar verlaufen Somatisierungsstörungen häufig chronisch, wohl aber auch, weil sie durch die behandelnen Hausärzte über viele Jahre hinweg unerkannt bleiben. Bedenke man dabei Angaben von Rief und Hiller (1998), die über nicht erkannte psychische Störungen in der Hausarztpraxis von $40 \%$ und $70 \%$ berichten.

Es besteht die Annahme, dass der Grund für häufige Chronifizierungen nicht in einer Störung der Persönlichkeit zu suchen ist, sondern in der oft zu spät einsetzenden professionellen Behandlung.

Die in dieser Studie ermittelten hohen Symptomverbesserungen zeigen deutlich, dass Betroffene auf psychosoziale Maßnahmen ansprechen und die Symptome keineswegs veränderungsresistent sind.

Die Studie von Rossa und Breull (2004) zeigte, dass fast jeder zweite Patient in der allgemeinmedizinischen Praxis seit durchschnittlich sieben Jahren an einer somatoformen Störung in Kombination mit ein bis zehn anderen Erkrankungen litt. Obwohl 92\% der Patienten über psychosoziale Probleme klagten, wurde dieser deutliche Zusammenhang nur bei einem Viertel der Patienten berücksichtigt.

Anhand dieser Daten verwundert es wenig, dass ein Großteil somatoformer Patienten eine Chronifzierung der Störung erfährt.

Das in dieser Arbeit angebotene Gruppenkonzept konnte sowohl den Hausärzten als auch den betroffenen Patienten eine direkte Hilfsmöglichkeit anbieten. Ärzte wurden durch das Gruppenangebot zu einer größeren Aufmerksamkeit für die Probleme ihrer Patienten sensibilisiert, während Betroffene auch im Frühstadium der Krankheit unmittelbare zusätzliche psychosoziale Hilfe erhielten.

Nach der zweigleisigen Behandlung zwischen Hausarzt und psychosozialer Gruppenteilnahme konnten sich die erlebte Stärke der Beschwerden, die Anzahl der Beschwerden und die erlebte Beeinträchtigung durch die Beschwerden in der Versuchsgruppe signifikant verringern. 


\section{Krankheitsängste, Arztangaben und gesundheitsökonomische Aspekte}

Auch die untersuchte Entwicklung der Krankheitsängste in den Hypothesen 10, 11 und 12 gibt Anlass zu der Annahme, dass die alleinige Behandlung durch den Hausarzt nicht ausreicht.

Das Ausmaß an Krankheitsängsten erhöht die Tendenz der Betroffenen zum Aufsuchen medizinischer Hilfe (Conroy et al., 1999). Unterbricht man diesen Teufelskreis nicht durch interdisziplinäre Kooperation, ist der Weg zur Störungschronifizierung offen gelegt. Diese Interpretation wird auch durch Yates (1994) unterstützt, der nicht nur über die Kostenkontrolle im Gesundheitssystem schreibt, sondern auch appelliert, das Beschwerdebild frühzeitig zu erfassen, zu erkennen und eine Chronifizierung durch interdisziplinäre Arbeit zu verhindern.

Die Krankheitsängste konnten sich in der Versuchsgruppe sehr signifikant verringern. Auch im Vergleich zur Kontrollgruppe in Posttest und Follow Up zeigt sich ein signifikanter Unterschied der Verringerung der Ängste zu Gunsten der Versuchsgruppe.

Die Tatsache, dass die Versuchsgruppe ihre Krankheitsängste signifikant höher verringern konnte als die Patienten der Kontrollgruppe, lässt die Interpretation zu, dass die zusätzliche psychosoziale Betreuung angstreduzierende Effekte hatte.

An dieser Stelle lassen sich die Hypothesen 38, 39, 40, 41, 42, 43, 44, 45 und 46 über die Angaben der behandelnen Ärzte einbeziehen.

Sowohl die zuvor aufgestellten Hypothesen zur erlebten Beziehungsqualität der Ärzte zu ihren Patienten als auch diese zur Anzahl der Arztkonsultationen und dem Ausmaß an Forderungen nach weiteren medizinischen Untersuchungen von Patientenseite, konnten sich innerhalb der Versuchsgruppe als statstisch signifikant bestätigen.

Zwar zeigt sich im Posttest/Follow Up-Vergleich innerhalb der Versuchsgruppe keine signifikante Veränderung der aufgeführten Variablen mehr, jedoch ergibt sich durchweg eine Tendenz zur weiteren Verbesserung der Werte.

Die im Rahmen des Gruppenprogramms erworbende Angstminderung der Versuchsprobanden konnte sich möglicherweise positiv auf die Beziehung zum behandelnden Arzt, vor allem aber auf die bisherige Anzahl der Arztkonsultationen sowie das Ausmaß an Forderungen weiterer medizinischer Untersuchungen auswirken. 
Smith et al. berichten (1986), dass die Behandlungskosten bei somatoformen Patienten um das ca. neunfache erhöht sind. Gerade aufgrund der hohen Inanspruchnahme medizinischer Leistungen bei diesem Klientel und dem Wissen, dass der Hausarzt die erste Anlaufstelle darstellt, ist interdisziplinäre Arbeit direkt vor Ort unbedingt vonnöten.

Diese Annahme wird durch die Ergebnisse dieser Studie unterstützt, da die alleinige Behandlung der Kontrollprobanden durch den Hausarzt zu überwiegend signifikant schlechteren Ergebnissen führte als bei Patienten, die zusätzlich zur hausärztlichen Behandlung an der Gruppe teilnahmen.

\section{Psychosoziale Versorgung somatoformer Patienten und die Drop-Out-Quote}

Patienten mit somatoformen Störungen gelten in der Praxis in der Mehrheit (67\%) als medizinisch über- und psychiatrisch unterversorgt (Katon et al., 1990).

Wie aber sollen Betroffene psychiatrisch und psychosozial versorgt werden, wenn sie von sich aus diese Hilfen nicht in Anspruch nehmen oder darauf aufmerksam gemacht werden?

Es bedarf einer behutsamen Kooperation vor Ort mit einem psychosozialen Helfer, der um die Störungshintergründe, wie sie in Kapitel 3.2 erläutert wurden, weiß und dementsprechend einfühlsam und verständnisvoll mit den Patienten umgehen kann.

Zu erwähnen ist allerdings auch noch einmal die Drop-Out-Quote von 33,3\% innerhalb dieser Studie. Es ist anzunehmen, dass dies eine Gruppe somatisierender Probanden betrifft, die sowohl über den Arzt als auch über andere Hilfen nur schwer erreichbar sind. Man könnte vermuten, dass sich hinter diesen 33\% die "eigentliche“ Gruppe der Somatisierungspatienten verbirgt, die in der Literatur beschrieben werden.

Zu diskutieren ist dieser Aspekt sicherlich, es gilt hier aber Abstufungen zu machen. Alle an der Studie teilnehmenden Personen litten unter somatoformen Symptomen, verhielten sich aber nicht wie die in vielen Veröffentlichungen dargestellten Patienten. Allerdings liegt die Vermutung nahe, dass es neben hilfesuchenden Patienten auch diese Gruppe gibt, die als „therapieresistent“ oder gar als nicht „therapierbar“ bezeichnet werden muss. 
Nicht jeder Mensch spricht auf psychosoziale Hilfe an und auch nicht jeder möchte diese überhaupt annehmen und das nicht nur aufgrund der Ablehnung eines nichtmedizinischen Krankheitsmodells. Intensive Arbeit an sich selbst ist bei vielen Menschen mit Angst vor Kontrollverlust und Ungewissheit verbunden.

Anhand der Aussage von Hiller und Rief (1998), dass gerade somatoforme Patienten eine sehr niedrige Rate an psychotherapeutischer Vorerfahrung aufweisen, kann die These aufgestellt werden, dass insbesondere Menschen mit einer somatoformen Störungsproblematik ängstlich gegenüber psychosozialen Maßnahmen sind.

Betrachtet man sich die in Kapitel 3.2 dargelegten Hintergründe zur Störungsentstehung, wird die massive Angst vor dem Umgang und Ansprechen eigener Emotionen verstehbar.

Menschen, die wie Rudolf (2008b) darlegt, sich in ihrem Leben ein narzisstischaltruistisches Bewältigungsmuster „erarbeitet“ haben, um sich vor der Ablehnung anderer zu schützen, erleben die Auseinandersetzung mit Gefühlen als Bedrohung dieses Schutzmusters.

Haben sie sich einst durch das Muster gerettet und gelernt eigene Bedürfnisse, Gefühle der Wut und Trauer nicht mehr zu äußern, steigt das Verständnis für die anfängliche Ablehnung von psychosozialer Hilfe.

Gerade an dieser Stelle sind geschulte Helfer, die diesen Hintergrund einbeziehen, akzeptieren und eben genau dort ansetzen und intervenieren können, von großer Bedeutung.

Um zurück zum Ausgangspunkt der Drop-Out-Quote zu kommen, ist zu sagen, dass sich nicht gänzlich klären lässt, warum die Personen sich letztlich gegen die Teilnahme entschieden haben.

Es liegt aber nahe, dass sie eine noch intensivere Betreuung und längere Hinführung benötigt hätten, um sich auf eine solche Maßnahme einlassen zu können. Neben intrapsychischen Widerständen und Ängsten spielt aber auch die intellektuelle Erreichbarkeit eine nicht zu unterschätzende Rolle. Das Programm erfordert eine normale geistige und sprachliche Fähigkeit. Auch das Fehlen dieser Eigenschaften kann auf einige der ausgeschiedenen Teilnehmer zutreffen. 
Die Ergebnisse zur Psychotherapiebereitschaft der behandelten Gruppe untermauern, durch einen konstanten Wert von 7, die Annahme, dass die Personen eine grundlegende Bereitschaft zur psychosozialen Arbeit an sich selbst mitbrachten. Das minimale Absinken auf 6,4 im Follow Up wird hier anhand der Tatsache interpretiert, dass der überwiegende Teil der Probanden die Gruppe weiterhin als Ort des Austauschs und der Hilfe beansprucht und für sich eine Form der Therapie darin gefunden hat. Damit werden weitere Maßnahmen ggf. zunächst nicht mehr so stark in Betracht gezogen.

Die Entwicklung der Kontrollgruppe zeigt eine anfänglich hohe Bereitschaft von ebenfalls 7 auf einer Skala von 0-10, die bis zum Follow Up jedoch auf 4,55 absinkt. Es ergeben sich hier signifikante Verschlechterungen im Posttest und Follow Up sowie mittelgroße Effektstärken, die die praktische Bedeutung dieses Ergebnisses bekräftigen.

Es stellt sich die Frage, warum der behandelnde Hausarzt nicht zu Beginn, bei hoher Motivation, hilfreich zu einer psychosozialen Hilfe vermitteln konnte und die Patienten anscheinend in eine depressive Resignation verfielen. Vielleicht ereignete sich hier zudem die zunehmende negative Gegenübertragung auf Seiten der Ärzte in Gefühlen von steigendem Desinteresse, Müdigkeit oder Ärger gegenüber dem Patienten, die diesen demotivierten (Zimmermann \& Kaduszkiewiscz, 2003).

Die Ergebnisse der Psychotherapiemotivation zeigen deutlich, dass ein möglicher Handlungszeitpunkt überschritten wurde und sich die Symptome derweil festigen konnten. Dadurch stieg möglicherweise die organische Ursachenüberzeugung weiter an.

Bedenkt man an dieser Stelle zudem die Schwierigkeit somatoformer Patienten sich überhaupt Hilfe zu suchen, ist ein solches Ergebnis umso negativer zu bewerten. Zu den strukturell defizitär entwickelten Bereichen zählt laut Rudolf die Kommunikation eigener Affekte der Bedürfigkeit. Selbstständiges Hilfesuchen wird durch eine narzisstische Pseudounabhängigkeit verhindert (Rudolf, 2006a).

Würden die behandelnden Hausärzte den psychodynamischen Hintergrund somatisierender Patienten besser einschätzen und verstehen, würde das Übergehen möglicher (psychosozialer) Bedürftigkeit ggf. weniger häufig vorkommen.

Insgesamt lässt sich doch vermuten, dass auch Menschen mit somatoformen Störungen nach allen ihnen möglichen Hilfsmaßnahmen greifen möchten und auch für psychosoziale Hilfe offen sind. 
Zimmermann und Kaduszkiewicz berichten (2003) über den aktuell kaum auflösbaren Systemzwang, in dem Ärzte stecken. Sie werden für ausführliche Gespräche mit dem Patienten weder honoriert noch fallen gesprächstherapeutische Techniken in das Studium der Mediziner. Auf dem Hintergrund dieser im Stand der Forschung referierten Aspekte stellt sich die Frage, ob betroffene Patienten von den behandelnden Ärzten überhaupt ausreichend auf psychosoziale Maßnahmen aufmerksam gemacht oder darin unterstützt werden. Andernfalls liegt die geringe psychosoziale Behandlungsquote dieses Klientels ggf. auch daran, dass wir über nur unzureichend freie Psychotherapieplätze verfügen.

Verschiedene Patienten nannten Wartezeiten für einen Platz bis zu einem Jahr, was durchaus als normal gilt. Wenn man sich vorstellt, was in diesem einen Jahr alles passieren kann, vor allem bezüglich Chronifizierungsprozessen, dann sollten wir wohl alarmiert sein mehr direkte Hilfsmaßnahmen zur Verfügung zu stellen.

Auch dies war eine grundlegende Motivation für die Konzeption und Durchführung des Gruppenprogramms. Das Gruppendesign ermöglicht bis zu zehn Patienten die gleichzeitge Teilnahme. Da der Hausarzt persönlich informiert wurde, konnte er Patienten ohne langes Zögern in die Gruppe überweisen. Das Programm ist für somatoforme Patienten konzipiert, die zu einem Großteil des Hausarztpatientenklientels gehören. Die Zielgruppe wurde demnach so gewählt, dass sie in Arztpraxen eine hohe und direkte Erreichbarkeit aufweist.

Das Programm könnte jederzeit wiederholt und auch von anderen Leitern durchgeführt werden. Es ist ökonomisch, gut überschaubar und für einen ersten Kontakt mit einer psychosozialen Maßnahme wenig beängstigend. 


\section{Beziehungsaspekte und praktische Bedeutsamkeit der Ergebnisse}

Die von Rudolf (2006a) dargestellte Enttäuschungsspirale in der Arzt-Patient-Beziehung konnte sich anhand der überprüften Hypothesen hier nicht bestätigen.

Es liegt allerdings, zumindest auf Seiten der Ärzte, die Vermutung nahe, dass ihre durchweg positiven Angaben zu ihren Patienten nicht der Wahrheit entsprechen. Mögliche Gründe für Angaben der Schulnoten zwischen 1 und 2 könnten Scham und Angst gewesen sein, dass die Ergebnisse doch in die Hände ihrer Patienten gelangen könnten, was sich selbstverständlich negativ ausgewirkt hätte.

Trotz, dass die Noten sich sowohl in der Versuchs- als auch in der Kontrollgruppe im durchweg guten Bereich befinden, verschlechtert sich die erlebte Beziehungsqualität zu den Kontrollprobanden, während diese zu den Versuchsprobanden sich signifikant verbessert.

Die Anzahl der Arztkonsultationen steigt in der Kontrollgruppe, hingegen einem signifikanten Absinken in der Versuchsgruppe, ebenfalls an. Hier liegt die Interpretation nahe, dass die in Hypothesen 10, 11 und 12 untersuchte Entwicklung der Krankheitsängste, die in der Versuchsgruppe hoch signifikant abgesunken sind, sich ebenfalls positiv auf die Verringerung der Arztkontakte auswirken konnte.

Dennoch ist anzumerken, dass auch in der Kontrollgruppe die Ängste minimal sinken. Betrachtet man die Entwicklung der Anzahl der Arztkonsultationen, kann das minimale Absinken der Krankheitsängste aber nicht zum Absinken weiterer Arztkontakte führten.

Auch die erlebte Stärke der Beschwerden sowie die Anzahl dieser und die erlebte Beeinträchtigung durch sie können in der Kontrollgruppe minimal absinken.

In all diesen Fällen ist jedoch die Beachtung der Effektstärken von besonderem Belang.

Die Versuchsgruppe erreicht in fast allen Variablen zur Symptomentwicklung als auch in den Arzt-Patient-Angaben mitttelgroße bis große Effektstärken. Die Kontrollgruppe hingegen kann, trotz minimaler Verbesserungen, die zum Teil auch signifikant ausfallen, kaum beachtliche Effektstärken erreichen. Anhand dieser Tatsache wird deutlich, dass die ermittelten Ergebnisse, wenn auch nicht durchweg signifikant, für eine praktische Umsetzung sehr bedeutsam sind. 
Das Gruppenprogramm könnte anhand der ermittelten Effektstärken zur Verbesserung der Symptomentwicklung sowie der gesundheitsökonomischen Aspekte, die sich auf die Redukation von Arztkonsultationen und der Durchführung weiterer medizinischer Untersuchungen beziehen, mit gutem Gewissen in die Praxis etabliert werden. Gerade weil die Etablierung von Maßnahmen ebenfalls finanzielle Ressourcen benötigt, ist die Ermittlung von guten Effektstärken unabdingbar.

So ergeben sich für die Verringerung an Forderungen weiterer medizinischer Untersuchungen in der Versuchsgruppe sehr große Effektstärken von 1,5 und 1,7. Ebenso ökonomisch bedeutsam sind die Effektstärken von ,61 und ,81 bezogen auf die Verringerung der Anzahl der Arztkonsultationen innerhalb der Versuchsgruppe.

Aus therapeutischer Sicht können die Effektstärken für die Verringerung der Anzahl der Beschwerden von ,61 und ,63 sowie diese für die Abnahme der Krankheitsängste von ,96 und 1,02 und für die Verminderung der erlebten Stärke der Beschwerden von 1,1 und 1,1 überzeugen.

Effektstärken dieser Größe können in keiner verbesserten Variable der Kontrollgruppe erreicht werden.

\section{Das „typische“ Ärztehopping-Verhalten}

Henningsen et al. (2002b) beschreibt das typische Ärztehopping-Verhalten von Menschen mit somatoformen Störungen. Er begründet es vor allem durch die erlebte emotionale Zurückweisung durch einen negativen organischen Befund. Der Patient fühlt sich nicht ernst genommen und hält weiterhin an seinem Krankheitsmodell fest und wechselt enttäuscht den Arzt, bei dem sich dasselbe erneut ereignen wird.

Die Ergebnisse der überprüften Hypothesen 33, 34 und 35 bezüglich der Anzahl der Arztwechsel im Untersuchungszeitraum widerlegen hingegen dieser Darstellungen das als typisch geltende Arztwechselverhalten gänzlich.

Keiner der 20 Kontrollprobanden hat zu irgendeinem Messzeitpunkt den behandelnen Hausarzt gewechselt. Ähnliches gilt für die Versuchsgruppe, in der ein Mittelwert von ,50 erreicht wird, der sicher nicht einem massiven Ärztehopping-Verhalten entsprechen kann.

Es ist also anzunehmen, dass betroffene Personen sehr wohl den Wunsch haben möglichst lange und konstant die Beziehung zu einem vertrauten Hausarzt zu bewahren. 
Eine andere Erklärung für diese Ergebnisse mag der eher kurzgefasste Untersuchungszeitraum von 6 Monaten sein, wobei anzunehmen ist, dass innerhalb dieser Zeitspanne ein Arztwechsel doch möglich gewesen wäre.

Unterstützt wird das Ergebnis, dass die teilnehmenden Probanden ihren Ärzten vertrauen dadurch, dass sie im Rahmen der Überprüfung der Hypothesen 30, 31 und 32 zur erlebten Beziehungsqualität zu den behandelnden Ärzten konstante Noten von 2,0 gaben.

Dies gilt auch hier sowohl für die Versuchs- als auch für die Kontrollgruppe, wobei die Kontrollgruppe die Beziehung mit 2,5 um eine halbe Note schlechter bewertet als die Versuchsprobanden mit 2,05.

\section{Emotionsentwicklung}

Zu der Verbesserung der körperlichen Symptomatik soll ein Zusammenhang zu den Evaluationen des emotionalen Erlebens hergestellt werden.

Es ist höchst interessant, dass sich zwar unerwartet große positive Veränderungen innerhalb der physiologischen Beschwerden ergeben haben, jedoch nur sehr gering bedeutsame positive Veränderungen für das emotionale Erleben zu benennen sind.

\section{Das Koppeln zwischen körperlichen Symptomen und seelischem Empfinden}

Im Stand der Forschung wurde unter Kapitel 3.2 eine tiefenpsychologische Ätiologie zur Entstehung somatoformer Störungen nach Rudolf (2006a, 2008a) dargestellt. Darin wurden strukturelle Bereiche erläutert, die sich bei Menschen mit einer solchen Problematik als defizitär erwiesen haben. Hierzu zählt die Affektdifferenzierung bezüglich körperlicher Aspekte der Emotionen. Dabei fällt es Betroffenen schwer, zwischen affektiven Aspekten ihres Körpererlebens bei Hunger, Müdigkeit oder Krankheit und den körperlichen Anteilen ihrer affektiv-psychischen Verfassung zu unterscheiden.

Körperliche Signale werden überwiegend als Ausdruck physischer Schädigung interpretiert, selbst wenn psychische Gegebenheiten wie Eheprobleme oder andere psychosoziale Belastungen eindeutig im Zusammenhang stehen. Patienten mit somatoformen Störungen spalten ihr körperliches- und ihr psychisches-lch in zwei unterschiedliche Instanzen auf, wobei das psychische-Ich den Anspruch erhebt das körperliche jederzeit kontrollieren zu können (Rudolf, 2006a). 
Es bestand daher das Interesse in den Hypothesen 25, 26 und 27 zu evaulieren, wie sehr die Probanden ihr körperliches Empfinden mit ihrem seelischen Erleben koppeln. Hingegen der Annahme, dass dieser Prozess erheblich gestört sein sollte, ergab sich weder in der Versuchs- noch in der Kontrollgruppe zu keinem Messzeitpunkt ein wahrhaftig schlechtes Ergebnis dieser Variable. Mit einem T-Wert um 48, bei möglichen 78, erreichen sie sogar recht hohe Werte.

Zwar findet in der Versuchsgruppe eine geringfügig stetige Verbesserung zwischen Pretest, Posttest und Follow Up der Koppelung zwischen körperlichen Beschwerden und psychischem Empfinden statt, die aber zu keinem Zeitpunkt signifikant ist.

In der Kontrollgruppe findet demgegenüber eine stetige minimale Verschlechterung dieser Variable statt. Die kleine Effektstärke von ,34 im Pretest/Follow Up-Vergleich innerhalb der Versuchsgruppe kann dennoch für die therapeutische Praxis als bedeutsam interpretiert werden.

\section{Akzeptanz eigener Emotionen}

Ein weiterer strukturueller Aspekt der Somatisierung betrifft die Mitteilung eigener Affekte, insbesondere die Kommunikation von Affekten der Bedürttigkeit und Wut nach außen. Diesbezüglich ist aufgrund des an dieser Stelle entwickelten pseudoautonomischnarzisstisch geprägten Verhaltens davon auzugehen, dass die Akzeptanz eigener Emotionen durch die frühe Entscheidung gegen das Äußern von Bedürttigkeit oder Wut gering ist. (Rudolf 2006a).

Es interessierte daher, ob sich die Akzeptanz eigener Emotionen nach der Interventionsteilnahme verbessern kann. Zudem war es von Interesse zu erfahren, ob Emotionen nicht nur nicht gezeigt, geäussert oder akzeptiert werden, sondern, ob die Patienten auch innerlich wenige Emotionen erleben. Zur Erhebung dieser Informationen dienten die Hypothesen 19, 20, 21, 22, 23, 24, 28, 29 und 30.

Bis auf Hypothese 21, die die Entwicklung und Erhebung der Akzeptanz eigener Emotionen zwischen Posttest und Follow Up beinhaltet, konnte sich keine der aufgeführten Hypothesen bestätigen.

Dass sich die Akzeptanz eigener Emotionen in der Versuchsgruppe von Posttest zu Follow Up mit ,003 sogar sehr signifkant zum Positiven verändert, könnte in Zusammenhang mit der Gruppenfortführung gebracht werden. 
Dies würde darauf hindeuten, dass sich erst eine längerfristige psychosoziale Begleitung signifkant positiv auf die gewünschte Veränderung emotionaler Prozesse auswirken kann. Wohingegen die positive Veränderung der körperlichen Symptomatiken nach der Teilnahme an einem zwei-monatigen Konzept gelungen sind.

Aus tiefenpsychologischer Sicht liegt hier die Vermutung nahe, dass die Patienten ihren Fokus von der physiologischen- auf die psychische Ebene verlagert haben.

\section{Therapie vor der Therapie - vom Körpersymtpom zur Emotion(swahrnehmung)}

Die These der Verschiebung von Symptom-auf Emotionsebene erlaubt es, das durchgeführte Gruppenprogramm als eine „Therapie vor der Therapie“ zu betiteln. Damit soll ausgedrückt werden, dass es bei diesem Klientel notwendig ist, zunächst eine behutsame Sensibilisierung für psychosoziale Maßnahmen vorzunehmen. Erst im Anschluss sind das Befassen mit eigenen emotionalen Aspekten sowie eine eigentliche Arbeit an diesen möglich. Das Erschaffen struktureller Grundlagen wurde auch im Stand der Forschung als Vorraussetzung für eine erfolgreiche therapeutische Konfliktarbeit benannt (Mück, 2005). Die Arbeit an der Kommunikation eigener Affekte nach außen sowie diese der Affektdifferenzieung, d. h. dem permanenten Fokus auf das Verbinden von psychosozial erlebter Belastung und körperlicfher Symptomatik, war struktureller Hauptfokus innerhalb der Programmdurchführung.

In einem Vortrag über strukturbezogene Psychotherapie an der Universität Köln 2008, konnte auch Prof. Rudolf diese Frage, ob die Arbeit an strukturellen Dimensionen bei Somatisierungspatienten als „Therapie vor der Therapie“ gesehen werden kann, mit seiner Zustimmung beantworten.

Die frühe Verfehlung angemessener Versorgung und Fürsorge führte nach Rudolfs Theorie letztlich dazu, dass der erste Weg des eigentlich seelischen Klagens über den Körper gesucht wird. Erst wenn dieser „ausgeklagt“ hat und Raum in der Kommunikation finden durfte, ist die Arbeit an emotionalen Themen möglich.

Hätten sich die Symptome lediglich insofern verändert, dass beispielsweise statt Rückenschmerzen nun Kopfschmerzen aufgetreten wären, könnte man dies nicht als erfolgreich, sondern nur als Symptomverschiebung bezeichnen. 
Im Verlauf der Untersuchung konnten sich in der Evaluation des erlebten Emotionsmangels in der Versuchsgruppe zwar geringe Verbesserungen ergeben, die aber zu keinem Zeitpunkt signfikant ausfielen. Vielleicht können die Emotionen noch nicht genau benannt werden, die Ergebnisse legen jedoch eine Sensibilisierung für das eigene innere emotionale Erleben bei den Versuchsgruppenprobanden nahe.

In der Kontrollgruppe bleibt der erlebte Emotionsmangel über alle Zeipunkte beinah konstant.

Für die Angabe der Wahrnehmung eigener Gefühle ergibt sich in der Versuchsgruppe sogar eine Verschlechterung zwischen Pretest und Posttest über eine Selbsteinschätzung auf einer zehnstufigen Skala von $M=7,05$ auf $M=6,9$.

Diese Veränderung führt aber weder zu einem signfikanten Ergebnis noch zu bedeutsamen Effektstärken.

Gefühle wahrzunehmen gilt wohl als selbstverständlich und wird wenig hinterfragt. Die Selbsteinschätzung der eigenen Fähigkeit Gefühle wahrzunehmen mag bereits eine Selbstreflektionskompetenz vorraussetzen, dies überhaupt wahrheitsgemäß einschätzen zu können. Insofern ist die eingesetzte Ratingskala insofern anzuzweifeln als dass sie von den Probanden eine intrapsychische Reflektionsfähigkeit verlangte, die möglicherweise nicht alle Teilnehmer von vornherein mitbrachten.

Dennoch ist das Ergebnis als richtig anzunehmen, da es in der Frage eindeutig um die selbst eingeschätzte Fähigkeit zur eigenen Emotionswahrnehmung und nicht um die objektiv nachweisbare Kompetenz ging.

Wenn es „nur“, mit Hilfe des Programms, gelungen ist die Patienten ansprechbarer für die psychosoziale Arbeit an sich selbst zu machen, kann dies als immenser Erfolg genannt werden. 
Mehr Gelassenheit und Optimismus für die Versuchsgruppenpatienten

Aufbauend auf den im Stand der Forschung referierten Aussagen des zunehmend durch Hoffnungslosigkeit und Enttäuschung geprägten Erlebens und Verhaltens somatisierender Patienten, gerade in der Beschreibung der interpersonellen Dynamik, (Rudolf, 1998) wurde anhand der Hypothesen 16, 17 und 18 die Entwicklung des Erlebens und Verhaltens über den Untersuchungszeitraum hinweg evaluiert.

Neben der positiven Symptomentwicklung ist das statistisch beste Ergebnis der ganzen Studie die hochsignifikant positive Veränderung des Erlebens und Verhaltens der Versuchsgruppenprobanden.

Der Unterschied zwischen Versuchs- und Kontrollgruppe bezogen auf das Erleben von Entspannung, Gelassenheit und Optimismus ist mit ,000 sowohl im Posttest als auch im Follow Up hoch signifikant. Es werden hier große Effektstärken von 1,30 und 1,31 erreicht. Gemeinsam mit den Effektstärken zur Verminderung des Ausmaßes an Forderungen nach weiteren medizinischen Untersuchen konnten hier die höchsten Effekte ermittelt werden.

Während sich das Erleben und Verhalten im Alltag der Gruppenteilnehmer nach der Interventionsteilnahme massiv verbessert und auch im Follow Up auf gleich konstantem Niveau bleibt, verschlechtert sich das Erleben und Verhalten der Patienten, die ausschließlich von ihrem Arzt behandelt wurden, im Posttest und im Follow Up nochmals deutlich. Dies bedeutet, dass Patienten der Kontrollgruppe eine negative Entwicklung in Richtung weitere Anspannung, Unsicherheit und Pessimismus während der gesamten Studienteilnahme durchlebten.

Hier ist erneut an die Ergebnisse der Kontrollgruppe zur Entwicklung der Abnahme der Psychotherapiemotivation zu denken, die sich durch mittelgroße Effekte praktisch bedeutsam und alarmierend darstellten.

Auch für die Verschlechterung des Erlebens und Verhaltens bei den Patienten der Kontrollgruppe zeigen sich kleine bis mittelgroße Effektstärken von ,37 und ,46.

Die Annahme, dass Grundoptimismuss und ein inneres positives und gelassenes Erleben der eigenen Person zur therapeutischen Ansprechbarkeit und Symptomverbesserung beitragen, liegt nahe. 
Möglicherweise ist demnach die deutlich positive Veränderung innerhalb der Versuchsgruppe auch ein weiterer Grund dafür, dass die Patienten ihre Symptomatiken im Posttest und Follow Up weniger massiv beeinträchtigend bewerten. Außerdem wurde in der Durchführung des Programms in jeder Sitzung auf das Benennen und Finden neuer Ressourcen bei jedem Einzelnen geachtet, die inn in der Bewältigung seiner Beschwerden unterstützen können.

Die Teilnehmer erlangten dadurch einen positiveren Blick auf sich selbst und ihr Alltagsleben. Sie wurden ermutigt, nicht mehr einzig das Negative, Schmerzvolle und Leidbetonte zu fokussieren, sondern auch ihre individuellen Möglichkeiten der Selbststärkung zu entdecken und diese aktiv zu nutzen.

Man könnte hier anmerken, dass alle Patienten zum Endpunkt des offiziellen Gruppenprogramms hätten weiterhin an einer Gruppe teilnehmen müssen, um nun intensiver an ihrem emotionalen Leben arbeiten zu können. Allerdings muss an dieser Stelle klar gesagt werden, dass es einigen Personen sicher vollends genügte ihre körperliche Symptomatik verbessert zu haben und nicht jeder weiterführendes Interesse an tiefergehender Auseinandersetzung mit sich selbst mitbrachte. 
Erfahrungen, Erleben und Verhalten in den Gruppensitzungen

Der theoretischen Konzeption des Gruppenprogramms wurde die tiefenpsychologische Theorie nach Gerd Rudolf zur Entstehung somatoformer Störungen zu Grunde gelegt. Diese orientiert sich an defizitär entwickelten strukturellen Dimensionen einer Persönlichkeit als auch dem zugrundeliegenden depressiven Grundkonflikt, der durch ein verfrühtes Verlassenwerden gekennzeichnet ist. Dieser Grundkonflikt wird durch ein narzisstisch-altruistisches Bewältigungsverhalten abgewehrt und so für die Betroffenen erträglich (Rudolf, 2008b). Die Theorie wurde in Kapitel 3.2 ausführlich erläutert.

Innerhalb der interaktionellen Aspekte der depressiven Somatisierung betont Rudolf über die gesamte Entwicklung hinweg fehlende Resonanz auf Appelle der Bedürftigkeit, was letztlich zu Enttäuschung und einem Gefühl der emotionalen Ablehnung führt. Diese frühen Erfahrungen reinszenieren sich im Erwachsenenleben dann in der Arzt-PatientBeziehung oder dem Helfer-Patient-Kontakt. Abbildung 2 in Kapitel 3.2 verdeutlicht diesen Prozess bildhaft.

Es wurde daher innerhalb der Programmkonzeption und Durchführung besonders auf das Erleben und Geben von Resonanz auf jegliches Klagen geachtet. Besonders sollten die Teilnehmer Resonanz auf seelisches Klagen erleben, was sie zunächst durch ihre körperlichen Beschwerden ausdrückten. Es war hier besonders wichtig das körperliche Klagen anzunehmen und als ein seelisches zu begreifen. Im Verlauf der Sitzungen rückten die Mitteilungen über das körperliche Geschehen stetig weiter in den Hintergrund.

Anhand dieser Darstellung interessierte, ob das bewusste Erleben von Resonanz durch andere Gruppenmitglieder und die Leitung in einer negativen Korrelation zur erlebten Beschwerdestärke stehen kann. Dafür wurde Hypothese 46 aufgestellt. Die Patienten schätzten ihre erlebte Resonanz sowie die erlebte Stärke der Beschwerden nach jeder Sitzung selbst ein. Hypothese 46 konnte anhand der Korrelationen statistisch nicht bestätigt werden.

Betrachtet man jedoch die Gesamtmittelwerte der erlebten Resonanz und der Beschwerdeintensität, wird sichtbar, dass die Beschwerdeintensität dort am geringsten ist wo die Resonanz im Gesamtmittelwert am größten ist. 
Zudem sinkt der Gesamtmittelwert der erlebten Beschwerdeintensität in den Stunden jedoch auch unabhängig von der erlebten Resonanz ab, so dass davon ausgegangen werden kann, dass Resonanz allein nicht der Wirkfaktor für das Absinken der erlebten Beschwerdestärke sein kann.

Die erlebte Resonanz kann vor allem als ein wichtiger Bruchteil im Prozess der Heilung angesehen werden. Ebenso wie in der Bindungsforschung von korrigierenden Erfahrungen im therapeutischen Setting gesprochen wird (Friedlmeier \& Holodynski, 1999), kann hier von korrigierenden Erfahrungen bezüglich des Resonanzerlebens auf körperliches und vor allem seelisches Klagen gesprochen werden.

Letztendlich wirkt sich fehlende Resonanz jedoch auch auf das Bindungsmuster aus. Deshalb ist anzunehmen, dass die Gruppenprozesse auch bindungskorrigierende Erfahrungen ermöglicht haben.

Für die innerhalb der tiefenpsychologischen Theorie postulierte fehlende Resonanz über Jahre hinweg, reicht ein Programm von acht Sitzungen nicht aus, um in Korrelation zum Beschwerdeerleben statistisch signifikante Veränderungsergebnisse hervorbringen zu können.

Es stellte sich jedoch heraus, dass die Patienten während den Gruppensitzungen signifikant weniger starke Beschwerden erlebten im Vergleich zu ihrer Alltagszeit während den Wochen zwischen den Sitzungen. Da sich hier zudem ein mittelgroßer Effekt von ,58 ergibt, ist davon auszugehen, dass die Erfahrungen in der Gruppe, wozu auch das permante Resonanzerleben zählt, für die therapeutische Praxis bedeutsam sind.

Das narzisstisch geprägte Bewältigungsverhalten, von dem Rudolf schreibt, verhindert, dass die Patienten zugänglich für die Wahrnehmung eigener Wünsche und Bedürfnisse werden. Daher wurde in Hypothese 48 erhoben, ob die Gruppenteilnehmer sich in der Interventionsgruppe weniger autonom und stark verhalten (können) als in ihrem Alltagsleben, wobei davon ausgegangen wurde, dass die erlebte Resonanz in der Gruppe dies ermöglichen kann.

Die Hypothese konnte sich mit ,02 als statistisch signifikant bestätigen. Auch hier kann die Effektstärke von ,56 die praktisch-therapeutische Bedeutsamkeit dieses Unterschieds untermauern. 
Wenn Patienten in der Gruppe beginnen können Teile des übermäßig benutzten Pseudoautonmie-Verhaltens abzulegen, wird dies nach einiger Zeit auch im Alltagsleben möglich werden.

Wichtig ist aber, wie auch Rudolf (2008a) empfiehlt, nicht die lange bewährten Bewältigungs- beziehungsweise Schutzmuster den Patienten gänzlich wegnehmen zu wollen.

Es macht wenig Sinn Menschen diesen Boden unter den Füssen wegzureissen. Statt dessen sollte an dieser Stelle eher dem "Was-Du-brauchst-Schema“ nach Hanne Seemann (1998) gefolgt werden, bei dem sie in der Arbeit mit psychosomatischen Patienten nicht das Ziel verfolgte, innen alle bisherigen Muster abzutrainieren oder gar schlecht zu sprechen, sondern vor allem darauf fokussiert war, was noch dazu kommen sollte, was auf der anderen Seite der Waage als Ausgleich fehlte.

Ein erstes Erleben eines solchen Ausgleichs sollte innerhalb der Gruppe ermöglicht werden. Dass die Probanden nach und nach dieses Erleben auch in ihren Alltag übertragen, ist zu hoffen.

Bezüglich der Interpretation des Ergebnisses über das narzisstische Verhalten in Gruppe und Alltag ist insgesamt allerdings Vorsicht zu wahren. Es basiert einzig auf zwei einzelnen Items, die zu beantworten waren. Standardisierte Fragebögen zu dem von Rudolf dargestellten narzisstisch-altruistischen Bewältigungsverhalten gibt es derzeit noch nicht.

Nach der Darstellung dieser Ergebnisse ist wichtig zu sagen, dass das Leben sicher nicht ewig aus dem Schutz der Gruppe bestehen wird. Das alltägliche Leben bietet weniger Rücksicht anderer und besteht aus einer wesentlich unreflektierteren Realität als diese, die in der Gruppe unter „Aufsicht“ erzeugt wird.

Wichtig ist aber zunächst überhaupt erst einmal eine erste, neue Erfahrung zu machen. Die Patienten sollen erfahren, dass ein neuer Umgang, eine sensiblere Art der Begegnung, die durch Wertschätzung, Mitfühlen und vor allem Ernsthaftigkeit geprägt ist, möglich ist. 


\section{Bedürfnisse im Symptom}

Das Ansprechen eines möglichen Symptomsinns erfordert viel Fingerspitzengefühl und vor allem volle Annahme und unbedingtes Ernstnehmen der körperlichen Symptomatik.

Vor dem Hintergrund des ständigen Wiederholens, dass die Symptomatik echt und wirklich vorhanden ist, war es zu späterem Zeitpunkt in allen Gruppen möglich, auch über Zusammenhänge im alltäglichen Leben zu sprechen.

Dabei traten vor allem die Wünsche des Bekümmert-, Gesehen- und Geliebtwerdens in den Vordergrund, welche viele der Betroffenen durch ihre eigene, eher altruistische Haltung wenig erfüllt bekamen. Es zeigte sich, dass viele nicht in der Lage waren, Bedürfnisse zu äußern oder überhaupt in Worten zu formulieren. Es bestand eine große Scham und ein deutliches Unbehagen darin, eigene Gefühle der Bedürftigkeit entstehen zu lassen und anzunehmen.

Viele der Patienten berichteten in diesem Zusammenhang auch von ihrem narzisstisch geprägten Verhalten und gestanden, teilweise unter Tränen oder ängstlicher Verzweiflung, dass sie so schwach und hilfebedürftig doch niemand kenne. Dabei drehte es sich nicht nur um Familie, Partner und Freunde. Vor allem erwies sich die Sicht der Patienten auf sich selbst als für sie fremd.

Es war faszinierend mitzuerleben, wie Patienten, die in der ersten Sitzung ausschließlich über ihre Symptomatik klagten am Ende des Programms an Themen wie dem Tod eines Kindes oder der großen Sehnsucht nach Liebe angelangt waren.

Sicher konnten acht Sitzungen nicht alles Notwendige leisten, aber das Ziel einer Sensibilisierung und des auf den „richtigen Weg Kommens" ist nach der Gruppenteilnahme sehr zufriedenstellend erreicht worden. 


\section{Ergebnisse im Vergleich zum gegenwärtigen Forschungsstand in der} Gruppenarbeit mit Somatisierungspatienten

In der bisherigen Diskussion wurden im Stand der Forschung referierte Angaben wie die Arzt-Patient-Beziehung, das Ärztehopping und die Psychotherapiebereitschaft bereits einbezogen.

Wie aber stellt sich nun abschließend das hier konzipierte und evaluierte Gruppenprogramm im Vergleich zu den bisher existierenden gruppenspezifischen Maßnahmen dar?

Wie unter Punkt 3.5 erläutert existieren im deutschsprachigen Raum bisher lediglich vier Arbeiten zur Gruppenarbeit mit Somatisierungspatienten, wobei drei dieser vier Gruppen nicht mit einer Kontrollgruppe gearbeitet haben.

Für das Gruppendesign von Nickle und Egle (1999) wurden keine statistischen Evaluationsergebnisse gefunden, wobei innerhalb des Berichtes der Gruppenarbeit auch lediglich von sieben Probanden gesprochen wird.

Drei der Programme grenzen sich durch den Fokus auf ein somatoformes Störungsbild für ihre Zielgruppe stark ein oder legen Kriterien fest, die nur schwer chronifizierte Patienten in psychosomatischen Kliniken erfüllen können.

Tschuschke und Weber (2007) kommen dem in dieser Studie dargestellten Programmkonzept am nächsten. Sie arbeiteten jedoch auch mit Patienten mit einer mittleren Krankheitsdauer von zehn Jahren und berichten nicht von einer Kontrollgruppe. Sie evaluierten nicht die Entwicklung von Krankheitsängsten, führten 20 anstatt 10 Sitzungen durch und stellten zudem keinen Kontakt zu den behandelnden Hausärzten her.

Das Kriterium, den Hausarzt so gut als möglich in die Intervention einzubeziehen, inn zu informieren, durch Datenschutzfreigabe einen Austausch über den Patienten zu ermöglichen und bestenfalls inn als Unterstützung und Ermutigung für den Betroffenen zu wissen, wurde in keiner der bisher veröffentlichen Studien berücksichtigt.

Da es keine echte Vergleichsstudie gibt, können direkte Parallelen nicht gezogen und Ergebnisvergleiche nicht durchgeführt werden. Umso interessanter wird die hoffentlich baldige Veröffentlichung der Heidelberger Forschungsgruppe „SpeziALL“ um Schäfert et al., deren Daten und Ergebnisse wohl am ehesten mit den Ergebnissen dieser Studie verglichen und diskutiert werden können. 
Zusammenfassend ergeben sich innerhalb dieses Gruppenkonzepts und auf die wissenschaftliche Arbeit bezogen folgende Neuheiten beziehungsweise Andersartigkeiten gegenüber den bisher veröffentlichen Gruppenstudien bei somatoformen Patienten:

- Versuchsgruppen und Kontrollgruppen-Design $\rightarrow$ versus nur Versuchsgruppe

- Kein Ausschluss einer speziellen somatoformen Störung für die Teilnahme am Programm $\rightarrow$ versus Eingrenzung auf ein spezielles somatoformes Störungsbild

- Einbindung des behandelnden Hausarztes $\rightarrow$ versus keine Kooperation

- Niedrigschwelliges psychosoziales Angebot, das unterhalb einer Psychotherapie anzuordnen ist und auch Betroffene ohne Chronifizierung anspricht $\rightarrow$ versus Programme, die Patienten erst in psychiatrischen Kliniken oder nach langem Chronifizierungsweg ansprechen.

- Das Programm ist relativ einfach zu wiederholen und kann von allen psychosozial gut ausgebildeten und mit theroretisch fundiertem Wissen ausgestatteten Kollegen und Kolleginnen durchgeführt werden. $\rightarrow$ versus unnötige Konkurrenzdiskussionen und Verkomplizierungen. 


\section{Das eigene „Idealobjekt“ werden? - Versteckte Bedürfnisse im Symptom} Über die Hypothesenüberprüfungen hinausgehende Überlegungen

Wenn jeder an sich selbst denkt, ist an jeden gedacht....oder: Wenn jeder sich selbst liebt und umsorgt, wird jeder geliebt und versorgt.

Ziel psychosozialer Arbeit ist es letztlich immer, dass die Betroffenen irgendwann lernen für sich selbst zu sorgen und Eigenverantwortung zu übernehmen. Gerade zu Beginn sind die Meisten jedoch auf ein Hilfs-Ich durch einen Helfer, wie es auch Rudolf (2008a) beschreibt, angewiesen. Das heißt, jemanden neben sich zu wissen, der stellvertretend Gefühle benennt, Bedürfnisse erkennt und sich stützend hinter und neben die Person stellt.

Vor allem die Arbeit in einer Gruppe ermöglicht, dass nicht nur der Leiter idealisiert und als einziges Hilfsobjekt herangezogen wird. Es können ebenso andere Mitglieder Stützen, Modelle und Ressourcen für die Betroffenen darstellen.

Ist es zu Beginn häufig noch notwendig, dass die Leitung Emotionen stellvertretend benennt und körperliches Erleben in einen Zusammenhang mit gegenwärtiger Lebensproblematik bringt, so haben dies innerhalb dieser Studie mit fortwährendem Prozess die Mitglieder in der Gruppe selbst füreinander übernommen.

Die alten Sehnsüchte nach liebenden, beschützenden und tröstenden Objekten dürfen und sollen gefühlt werden und Raum finden. 
Wenn die Trauer darüber gefühlt werden kann, ist es möglich in das „Jetzt“ zu schauen. Es gilt zu überlegen, ob heute fortwährend ein anderes Objekt in gleicher Massivität benötigt wird wie dies in der frühen Kindheit vonnöten war oder, ob die Patienten selbst beginnen können, diese schützenden und sorgenden Instanzen für sich zu sein.

Das bedeutet natürlich nicht, dass sie nicht mehr auf andere Menschen angewiesen sind oder getröstet, beschützt und geliebt werden möchten.

Es bedeutet lediglich, dass die Massivität dieses Wunsches, der letztlich völlig erfolglos, weil unklar kommuniziert, durch das Auftreten der Symptomatik gezeigt wird, nicht mehr das gesamte Alltags- und vor allem Beziehungsgeschehen bestimmt (Rudolf, 2008b).

Wenn die Betroffenen in sich selbst ihr behütendes und versorgendes Idealobjekt finden sollen, dann ist damit nicht eine neue Form der Großartigkeit und Pseudoautonomie gemeint. Es geht im Gegenteil darum, dass die Patienten auch als erwachsene Menschen noch emotionale Bedürfnisse haben und selbst dafür sorgen dürfen, dass diese möglichst gut befriedigt werden.

Dazu zählten in den Gruppensitzungen unter anderem Gespräche über Partnerschaftssituationen, in denen eine Scham bezüglich der Äußerung nach Wünschen für mehr Zärtlichkeit oder mehr gemeinsame Zeit miteinander bestanden. Die Symptomatik enthielt bei fast allen Betroffenen den Wunsch „Einfach in den Arm genommen zu werden“. Ein Satz, den jeder der Teilnehmer mindestens einmal äußerte.

Es liegt auf der Hand, dass ein eigentlich klares sich selbst Zugestehen dieses Bedürfnisses und ein Einfordern oder aktives Gestalten zwischenmenschlicher Zuneigung wesentlich angenehmer sein müsste als den Preis zu zahlen, mit permanenten Schmerzen durch das Leben zu gehen. Für Menschen mit somatoformen Störungen scheint es aber zunächst keinen anderen Weg der Kommunikation als über ihre Symptome zu geben.

In dieser Aussage liegt die Überzeugung, dass die körperliche Symptomatik die Botschaft enthält, an Fürsorge, Liebe und Zuneigung unterversorgt zu sein.

Dies deckt sich schliesslich mit dem von Rudolf (1998) dargestellten zugrundeliegenden depressiven Grundkonflikt. 
Davon abgesehen, dass die erlebten körperlichen Beschwerden sehr unangenehm sind, erzeugten die Symptome bei dem Großteil der Versuchsgruppenprobanden nach einer kurzen Zeit primär Ablehnung ihrer Partner und Freunde, weil diese davon genervt oder sich grenzenlos überfordert fühlten.

Der implizite einfache Wunsch nach beispielsweise mehr Zärtlichkeit oder Zuhören, blieb vielen Angehörigen aber auch den Patienten selbst vor der Gruppenteilnahme hinter den Symptomen verborgen.

Es wurde daher viel Wert darauf gelegt, einerseits eigene unterstützende Ressourcen zu finden und andererseits aktuelle Bedürfnisse zu benennen, vor allem wenn Patienten während den Sitzungen sagten, dass die Symptomatik gerade schlimmer geworden sei. Dies war immer eine gute Möglichkeit, im „Jetzt“ gemeinsam zu überlegen, was gerade nicht anders als über das Symptom kommunizierbar ist. 


\section{Diskussion nicht hypothesengebundener Ergebnisse}

Neben den hypothesengebundenen Ergebnissen wurden weitere nicht hypothesengebundene Daten erhoben.

Dazu zählen die Angaben der Probanden, ob sie bereits Psychotherapieerfahrung haben oder inzwischen eine Therapie begonnen haben oder beginnen möchten.

Von den insgesamt 20 Probanden der Versuchsgruppe möchten fünf keine Einzeltherapie beginnen, wobei vier dieser fünf weiterhin an der Gruppe teilnehmen und diese als ihre Therapie betrachten. Vier der 14 Probanden, die in die 14-tägige Gruppe kommen, gehen zudem zusätzlich in eine Einzeltherapie, was innen gut bekommt und sich mit der Gruppenarbeit gut vereinen lässt.

Innerhalb der Kontrollgruppe konnte oder wollte während der Studienteilnahme niemand eine Therapie beginnen, wobei drei Personen schon vor Studienteilnahme in einer laufenden Einzeltherapie waren, die noch nicht beendet ist. 11 der 20 Kontrollprobanden möchten keine Therapie (mehr) beginnen, wobei hier fraglich ist, ob diese lediglich aufgrund von Ablehnungen, langen Wartezeiten oder zu wenig Unterstützung den Mut verloren haben.

Außerdem wurde der Beschwerdeindex-Somatisierung ohne eine zuvor formulierte Hypothese erhoben. Dieser war nicht mit einer wesentlichen Veränderungserwartung behaftet. Wie bereits im Rahmen der Symptomentwicklung berichtet, erstaunen die Veränderungswerte bezogen auf die körperliche Symptomatik insgesamt. Auch für den Beschwerdeindex-Somatisierung haben sich sehr positve Veränderungen ergeben.

Erneut ist dies in beiden Gruppen zu beobachten, wobei die Versuchsgruppe im Durchschnitt beinah doppelt so viele Prozentrangwerte verlieren kann im Vergleich zu der Kontrollgruppe.

Die Pretestergebnisse liegen bei $M=58,85$ in der Versuchs- und $M=59,65$ in der Kontrollgruppe. Im Follow Up hat die Versuchsgruppe beinah 19 und die Kontrollgruppe ca. 10 Prozentrangpunkte verloren. Waren es in der Versuchsgruppe im Pretest nur 40,15\% einer psychosomatischen Vergleichsgruppe, die im BeschwerdeindexSomatisierung einen noch schlechteren Gesamtsomatisierungswert erreichten, sind es im Follow Up bereits 59,95\% deren Gesamtsomatisierungswert schlechter ausfällt als dieser, der Versuchsgruppe. 
Die fünf ausgewählten standardisierten Diagnostikinstrumente, wozu die Skalen zum Erleben von Emotionen (SEE), der Veränderungsfragebogen des Erleben und Verhaltens (VEV), der Gesundheitsfragebogen (PHQ-15), der Whiteley-Index (WI-7) und das Screenig für somatoforme Störungen (SOMS) zählten, erfüllten durchweg ihre dienende Funktion zur Überprüfung der Forschungshypothesen.

Hinzu kamen die zur Symptomstärke, Psychotherapiemotivation und eingeschätzten Fähigkeit der Emotionswahrnehmung gezielt eingesetzten Ratingskalen sowie diverse Einzelitems und offene Fragen, die durch standardisierte Fragebögen nicht abgedeckt werden konnten. Hier ist stets Achtsamkeit in der Interpretation der Ergebnisse bezüglich der Validität der Items zu bewahren. Es wurde in der Konstruktion jedoch bestmöglich auf Validität geachtet.

Die Entscheidung dafür, die verwendeten Rating-Skalen innerhalb der statistischen Berechnungen als Intervallskalen zu behandeln, konnte sich aufgrund der Kontrolle durch die nonparametrischen Verfahren als richtig bestätigen. Die Ergebnisse unterschieden sich dort nicht von denen des t-Tests.

Innerhalb der Diskussion soll sogar die These aufgeworfen werden, dass diese Skalen auch als Verhätlnisskalen gelten könnten. In allen Fällen handelte es sich um Skalen, die eine persönlich erlebte Einschätzung messen sollten, beispielsweise die erlebte Beschwerdestärke von 0-10. Die Skalen hatten also nicht den Anspruch eine objektive physiologische Eigenschaft zu erfassen, sondern lediglich das subjektive Empfinden. Es ist dabei davon auszugehen, dass Menschen diese Skalen mit einer „inneren Waage“ ansehen. Dabei ist den Einschätzenden bewusst, dass 10 der höchstmögliche und 0 der kleinstmögliche Wert ist. Aufgrund dieser Wahrnehmung, liegt es nahe, dass 5 den Wert exakt in der Mitte darstellt. Es ist anzunehmen, dass es vor dem Auge der „inneren Waage" logisch erscheint, dass das Ankreuzen des Wertes 10 eine doppelt so stark erlebte Beschwerdestärke beschreiben soll als ein Wert von 5 .

Selbstverständnlich ist die "innere Waage“ eines Menschen nicht geeicht und die Abstände sind nicht als linear nachzuweisen, jedoch stellt eine solche Einschätzungsskala für subjektives Erleben und Empfinden die wohl beste Möglichkeit der Messung dar.

Obwohl auch Schulnoten im streng mathematisch-statistischen Sinne nicht als Intervallskalen betrachtet werden können, da die Abstände zwischen einer 1 und einer 2 sicher nicht linear sind, so werden sie jedoch in der Praxis durchweg als solche behandelt. 
Innerhalb jeglicher Prüfungen, vom Abitur bis zur Promotion, werden die arithmetischen Mittel berechnet (numerus clausus) (Rost, 2005).

Für den Gesamtaufwand zur Beantwortung aller Fragen musste im Pretest, Posttest und Follow Up jeweils eine Zeit von ca. 45-60 Minuten beanschlagt werden.

Rückbilckend ist zu sagen, dass dies grenzwertig und wenig freundlich für die Kontrollprobanden konzipiert war. Durch die kostenlose Teilnahme am Gruppenprogramm galt es für die Versuchsprobanden als Teilnahmevorraussetzung die Bögen zu den drei angesetzten Zeitpunkten auszufüllen. Dies gestaltete sich demnach auch nicht als problematisch.

Hingegen der Versuchsgruppe erhielten die Kontrollprobanden jedoch keine Intervention. Als Anreiz für das vollständige und gewissenhafte Ausfüllen der Bögen diente lediglich eine nach Abschluss der Studie versprochene individuelle Einzelauswertung der Ergebnisse. Diese Einzelauswertungen für alle 20 Kontrollprobanden verursachten nach Studienabschluss einen, zuvor nicht erwarteten, hohen zusätzlichen zeitlichen Aufwand.

Die zeitliche Ökonomie zum Ausfüllen diagnostischer Fragebögen wäre in einer weiteren Studie deutlich zu verbessern, um Probanden nicht durch übermäßig lange Ausfüllzeiten zu demotivieren.

Bis auf den VEV waren alle Instrumente unmittelbar und leicht verstehbar. Der VEV bedurfte sehr genauem Lesen sowie absoluter Konzentration beim Ausfüllen. Es ergaben sich trotz der etwas komplizierten Aufmachung jedoch keine augenscheinlichen Verständnisprobleme bei den Patienten. Jeder VEV-Bogen wurde kontrollierend begutachtet und keiner gab Anlass zur Annahme, dass ein gravierendes Missverständnis beim ausfüllenden Patienten zu vermuten sei.

Der VEV erschien zunächst als kompliziertes Messintrument, lieferte dafür innerhalb dieser Studie jedoch hoch interessante Ergebnisse. Sein Einsatz war lohnenswert.

Im Rahmen der nicht hypothesengebundenen Ergebnisse wurden vor allem Aspekte über die Bewertung des Gruppenprogramms erfasst. Dies war deshalb von großem Interesse, da das Programm ggf. mit weiteren Patienten wiederholt wird und mit Hilfe der Angaben eine weitere Verbesserung vorgenommen werden kann.

Erfreulich ist, wie im Untersuchungsverlauf erwähnt, dass einzelne Hausärzte weiterhin Patienten in die bestehenden Gruppen verweisen. 
Die Evaluationen zur Bewertung des Gruppenprogramms fallen insgesamt sehr positiv aus und geben Anlass zur Annahme, dass auch andere Betroffene von diesem Angebot profitieren können.

Auf einer Skala von 0-10 ergibt sich ein Mittelwert von 9,4 als Befürwortung der Patienten dieses Programm als parallele Ergänzung zu ihrer hausärztlichen Behandlung durchzuführen.

Mit einem Mittelwert von 9,3 würden die Patienten das Programm auch anderen Betroffenen empfehlen.

Besonders wichtig ist die Angabe, dass die Probanden sich mit ihrer Symptomatik ernst genommen fühlten. Dies ist ein elementarer Baustein, um mit somatisierenden Patienten auch über psychische Anteile ins Gespräch kommen zu können.

Gerade in einer Gruppe können Betroffene sich gegenseitig Verständnis und Akzeptanz für ihre Symptomatiken entgegenbringen. Menschen, die selbst von einer somatoformen Störung betroffen sind, wissen, wie groß der Leidensdruck und die Angst ist, mit den Beschwerden zurückgewiesen, nicht ernst genommen oder als Simulant abgestempelt zu werden. Unter den Gruppenmitgliedern herrschte diesbezüglich große Wertschätzung und gegenseitige Annahme der körperlichen Klagen. Dies ermöglichte, dass nicht ausschliesslich die Leitung als helfendes Objekt idealisiert wurde.

Natürlich war Wertschätzung, Annahme der Klagen und des Leids auch die Grundhaltung der Leitung. Eine Person als Helfer kann aber sicher mehr Fehler begehen als eine ganze Gruppe an Menschen.

Ein einzelner Kommentar der Leitung konnte unerwartet treffend, verletzend und als zurückweisend empfunden werden. Die Tatsache, dass in solchen Momenten auf andere Gruppenmitglieder als Ressource zurückgegriffen werden konnte, war von besonderer Wichtigkeit dafür, dass die Patienten sich nicht schlagartig verschlossen oder aus dem Kontakt gingen. Sie konnten ein anderes Gruppenmitglied ansprechen und dort in der Beziehung neuen Halt und Sicherheit gewinnen.

Die quantitativen Ergebnisse zur Symptomentwicklung werden durch die Aussagen der Teilnehmer, bezüglich der Frage wie es innen körperlich nach der Teilnahme am Gruppenprogramm gehe, bestätigt. 
Niemand fühlt sich schlechter. 16 von 20 Patienten geht es körperlich besser oder sehr viel besser. Ein großer Erfolg.

Interessant ist, dass bei selbiger Frage bezogen auf das psychische Befinden nach der Gruppenteilnahme ein Patient mit „schlechter geworden“ geantwortet hat. Auch hier geben 17 Personen besser oder sehr viel besser an. Die einzelne Angabe „schlechter“ soll aber als bedeutsam markiert werden.

Dass ein Patient sich psychisch nach der Teilnahme schlechter fühlt als vor dem Programm ist sicher nicht wünschenswert, aber auch hier stellt sich die Frage, ob sich möglicherweise die psychischen Belange vor die zuvor so penetrant erlebten körperlichen Beschwerden „geschoben“ haben. Innerhalb der zwei-monatigen Intervention wurde darauf geachtet, dass keine besonders gravierenden oder traumatischen Erlebnisse aufgerissen wurden. Dennoch gibt es natürlich, trotz Achtsamkeit der Leitung, keine Garantie und absolute Steuerbarkeit innerpsychischer Prozesse, die in den Probanden ausgelöst werden.

Im besten Fall ist der Patient an für ihn schmerzliche, aber bedeutsame Punkte gestoßen, die mit seiner Somatisierungsstörung zusammenhängen und nun mehr an der Oberfläche sind, um sie bearbeiteten zu können.

Im schlechtesten Fall geht es dem Patienten nach der Intervention psychisch schlechter und körperlich nicht besser. Wichtig war deshalb, allen Teilnehmern zu raten und sie darin zu unterstützen, nach der Gruppenarbeit weiterhin psychosoziale Hilfe in Anspruch zu nehmen. Sie wurden ermutigt, dass eine Erstverschlimmerung als ein Zeichen bewertet werden kann, dass in ihnen Prozesse in Bewegung sind, die angesehen und bearbeitet werden können.

Für solche und ähnliche Problematiken war die Einverständniserklärung und Übernahme der Eigenverantwortung bezüglich der Gruppenteilnahme im Vorfeld sehr wichtig.

Für Patienten mit somatoformen Störungen kann es als ein Fortschritt angesehen werden, wenn es ihnen psychisch nach einer Intervention schlechter und körperlich besser geht. So können sie beginnen, nicht mehr ausschließlich über das Körpersymptom, sondern auch über das emotionale Erleben in den Kontakt zu treten.

Die Formulierung eines Alltagsziels zu Beginn der Intervention galt als wichtiger Motivationsfaktor konstant an allen angesetzten 8 Sitzungen teilzunehmen. 
Zu Beginn jeder Sitzung sprachen die Teilnehmer über den Stand der Zielerreichung. Dazu zählte das, was zum Erreichen des Ziels individuell aktuell beitragen konnte beziehungsweise noch dazu fehlte. Das Sprechen über ein Alltagsziel konnte fortwährend einen konkreten Bezug zum Alltag der Patienten aufrechterhalten.

Immerhin sind $\mathbf{1 6}$ der 20 Probanden ihrem Alltagsziel näher gekommen, als ihnen dies vor der Gruppenteilnahme möglich gewesen wäre.

Auffällig ist, dass 15 der 20 Teilnehmer die Dauer des Programms von zwei Monaten als zu kurz bewerten. Dieses Ergebnis erstaunt, da während der Konzeption bewusst auf eine möglichst überschaubare und kurze Zeit geachtet wurde, um potenzielle Patienten nicht durch unüberschaubare Endlosigkeit schon zuvor abzuschrecken und eine konstante Teilnahme wahrscheinlich zu machen.

Es wird vermutet, dass sich diese Erkenntnis in den Teilnehmern erst nach der Intervention entwickeln konnte. Eine wirklich psychotherapeutische Behandlung beansprucht mehr als zwei Monate oder acht Sitzungen, auch wenn dies Vertreter manch anderer Therapierichtungen anders einschätzen mögen.

Die Anzahl der Sitzungen sollte trotz der Einschätzung der Teilnehmer nicht erhöht werden, da dieses Gruppenprogramm tatsächlich als ein Sprungbrett zu weiteren psychosozialen Hilfsmaßnahmen dienen soll.

Patienten, die zuvor noch niemals Kontakt zu psychosozialen Hilfsmaßnahmen hatten, würden bei einer höheren Frequenz womöglich abgeschreckt werden. Das Ergebnis, dass die Versuchsgruppenprobanden im Nachhinein doch "mehr" wollten, ist ausschließlich als positiv zu bewerten.

Basierend auf diesem Ergebnis kann gesagt werden, dass die Intervention den Zweck erfüllte, für den sie konzipiert wurde: Eine Sensibilisierung für psychosoziale Hilfsmaßnahmen bei Patienten mit somatoformen Störungen.

Dass die Patienten die Maßnahme im Nachhinein als zu kurz bewerten, hat letztlich dazu geführt, dass sie sich selbst und aus eigenem Willen und eigener Kraft dafür eingesetzt haben, dass die Gruppen fortgeführt werden. 
Der Fokus liegt dabei darauf, dass die Teilnehmer Kräfte aus sich selbst heraus entwickeln, Ressourcen entdecken und die Leitung nur agiert, wenn es unbedingt notwendig ist. Die Menschen sollen ein Gefühl der Selbsthilfegruppe in sich tragen, um in keine all zu grossen Abhängigkeiten zu geraten.

Psychosoziale Maßnahmen können grundsätzlich nur hilfreich sein, wenn die Patienten aus eigenem Willen und eigener Motivation heraus daran teilnehmen.

Für Patienten, die zuvor überdurchschnittlich häufig den Hausarzt beanspruchten und einzig auf die somatischen Untersuchungen und Behandlungen fixiert waren, ist das eigene Engagement zur Fortsetzung psychosozialer Hilfe in der Gruppe ein sehr gutes und erfolgreiches Ergebnis.

Die im Ergebnisteil zuletzt aufgeführten Antworten zu den 13 offenen Fragen eröffnen durch die Beibehaltung der Originalwortlaute einen nahen und greifbaren Zugang zu den Studienteilnehmern. Ein Aspekt, der trotz oder gerade wegen der Anonymisierung innerhalb der Forschung wertvoll und wichtig ist. Letztlich sprechen wir seitenlang von „Probanden“, „Patienten“ oder „Personen“. Dies alles sind aber Individuen, die sich freiwillig zum Gegenstand einer Forschungsarbeit gemacht haben.

$\mathrm{Zu}$ verallgemeinernde Aussagen für Betroffene zu finden, ist sicher wichtig und sinnvoll. Innerhalb dieser Forschungsarbeit soll jedoch auch Wert auf die Individuen, die jeder für sich und in ihren Problematiken einzigartig sind, gelegt werden.

Ihren Aussagen auf die offenen Antworten kommt eine besondere Bedeutung zu, weil sie, neben den überwiegend quantitativ erhobenen Daten, neben Tabellen und Zahlen wieder den Mensch dahinter in den Mittelpunkt stellen. Sie zeigen, dass P1, P2, P3 etc. Menschen sind wie „Du und ich“ und erzeugen damit auch einen emotionalen Zugang zu den quantitativen Ergebnissen dieses Studienprojektes.

Forschung an seelischen Prozessen im Menschen wird letztlich ebenso in Zahlen, Tabellen und Kurven verarbeitet und dargestellt wie eine Arbeit mit Chemikalien im Labor.

Solch offene Antworten kommen dabei gerade recht, um zwischen chemischen Formeln und Menschen doch noch einen Unterschied aufzeigen zu können. 
Auch wenn der Mensch in der Biologie und Humanmedizin sicher in (chemischen) Formeln erklärt werden kann, so gibt es doch (hoffentlich) immer noch psychologische Phänomene, die nicht durch eine Formel endgültig dargestellt werden können. Sie bedürfen der individuellen gemeinsamen psychosozialen Arbeit.

Selbst wenn die Forschung sich um allgemeingültige Therapiekonzepte bemüht, bleibt jeder Mensch, auch in der Präsentation seines Störungsbildes, einzigartig. 


\subsection{Zusammenfassung der Ergebnisse und Schlussfolgerungen}

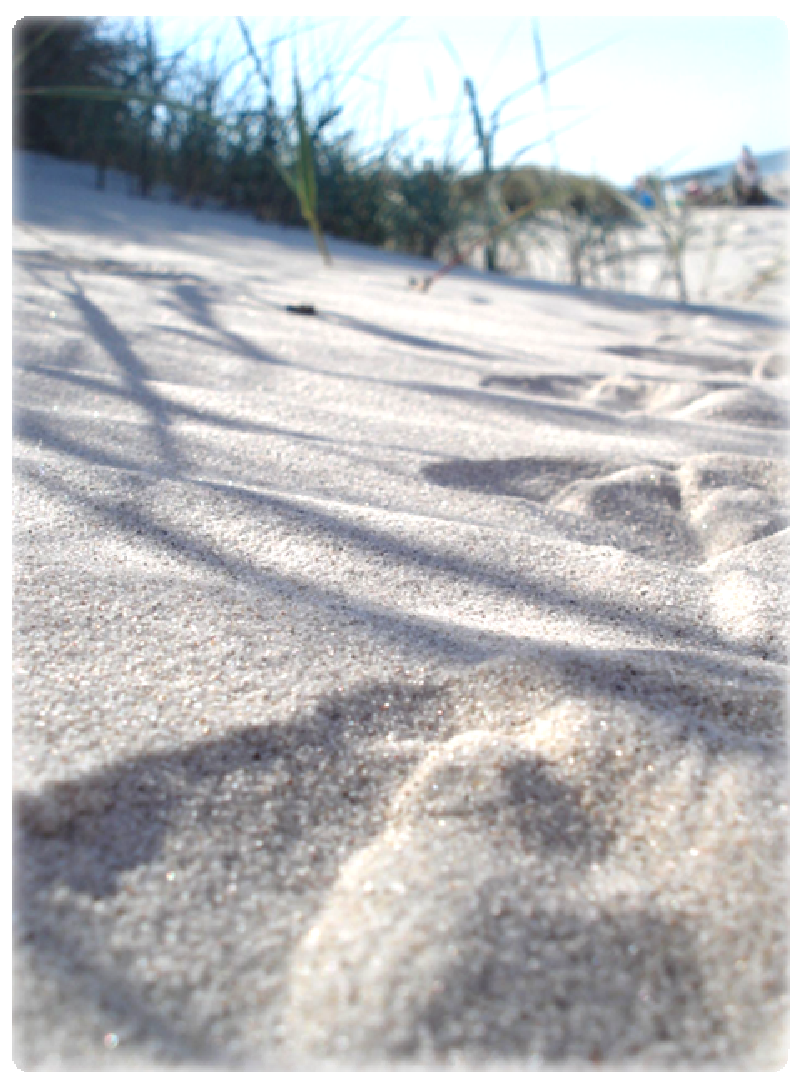

Wohin die Reise führte: Kurz gesagt...

Abschließend ist festzuhalten, dass sich das Gruppenprogramm für Patienten mit somatoformen Störungen als äußerst positiv dargestellt hat. Nach der Teilnahme konnte sich der Großteil aller Variablen zur körperlichen Symptomentwicklung innerhalb der Versuchsgruppe signifikant verbessern. Vor allem die großen Effektstärken lassen zu, dass die Umsetzung des Konzepts in die therapeutische Praxis vertretbar ist.

Die ebenfalls vorhandenen minimalen Verbesserungen in der Kontrollgruppe konnten demgegenüber überwiegend keine signifkanten Veränderungen ergeben. Bedeutsamer ist jedoch, dass sie zu keinem Zeitpunkt ähnlich hohe Effektstärken wie diese der Versuchsgruppe erreichen konnten.

Die Angaben der Ärzte, bezogen auf die erlebte Beziehungsqualität zu ihren Patienten, der Anzahl der Konsultationen sowie das Ausmaß an Forderungen weiterer medizinischer Untersuchungen von Patientenseite, konnten sich innerhalb der Versuchsgruppe statistisch signifikant verbessern. 
Die signifikante Verringerung der Krankheitsängste bei den Versuchgruppenprobanden wird in der Interpretation in Verbindung zur Verringerung der Anzahl an Arztkonsultationen sowie mit der Verbesserung der erlebten Beziehungsqualität zum behandelnden Arzt gebracht.

Angesichts der referierten Angaben über neunfach erhöhte Behandlungskosten bei Somatisierungspatienten (Smith et al., 1986) können die Verringerungen der Untersuchungsforderungen und der Anzahl an Arztkonsultationen mit großen Effektstärken von ,81 und 1,7 auch für gesundheitsökonomische Aspekte als bedeutsam hervorgehoben werden.

Somatoforme Störungen haben sich in dieser Untersuchung nicht als chronischveränderungsresistent im Sinne einer Persönlichkeitsstörung (Bass \& Murphy, 1995) gezeigt.

Der häufig chronische Verlauf dieser Erkrankung scheint eher in der Tatsache begründet, dass Somatisierungsstörungen von den behandelnden Hausärzten über viele Jahre hinweg unerkannt bleiben (Rief \& Hiller, 1998). Die Ergebnisse zur Ermittlung der Psychotherapiebereitschaft, zeigen in der Kontrollgruppe ein signifikantes Absinken von einer hohen Bereitschaft im Pretest zu einer niedrigen im Follow Up. Dies lässt die Interpretation zu, dass ein möglicher psychosozialer Handlungszeitpunkt überschritten wurde. Dies förderte die Chronifizierung der Beschwerden innerhalb dieser Gruppe.

Es stellt sich die Frage, ob betroffene Patienten von den Ärzten ausreichend auf psychosoziale Maßnahmen aufmerksam gemacht und in einer Behandlung unterstützt werden. Die fehlende Honorierung für Gespräche sowie die massive Zeitnot, unter der die Praktiker in ihrem Praxisalltag leiden, könnte eine mögliche Erklärung für diesen Missstand sein.

Auch weil Wartezeiten von einem Jahr für freie Psychotherapieplätze als normal gelten, soll das neu konzipierte Gruppenprogramm bis zu zehn Patienten eine gleichzeitige Teilnahme ermöglichen. Bestenfalls sollte in jede Hausarztpraxis ein psychosozialer Helfer vor Ort integriert werden, damit betroffene Patienten unmittelbare Hilfe erhalten und die oft leidvolle und kostenintensive Chronifizierung der somatoformen Störung vermieden werden kann. 
Dass in der Literatur erwähnte Ärztehopping-Verhalten (Henningsen et al., 2002b) konnte in dieser Untersuchung widerlegt werden. Keiner der Probanden hat im Verlauf von sechs Monaten einmal den Arzt gewechselt.

Die Drop-Out-Quote von 33,3\% lässt vermuten, dass es jedoch auch eine Patientengruppe gibt, die auf das Gruppenprogramm nicht anspricht.

Sie benötigen entweder eine längere Hinführung und Betreuung für die Teilnahme an einer psychosozialen Maßnahme oder sind für nicht-medizinische Angebote nicht erreichbar. Es wird allerdings eher vermutet, dass aufgrund von Angst vor Ablehnung und des narzisstisch geprägten Bewältigungsmusters sowie einer generellen Angst vor der Auseinandersetzung mit sich selbst, die hier ausgeschiedenen Patienten nicht erreicht werden konnten.

Besonders interessant stell sich die hohe Verbesserung der Symptomentwicklung gegenüber einer gering bedeutsamen Veränderung im emotionalen Erleben dar.

Erst im Posttest/Follow-Up-Vergleich konnte in der Versuchsgruppe eine signifikante Verbesserung der Akzeptanz eigener Emotionen erreicht werden. Da 14 der 20 Probanden im Anschluss an das Programm weiterhin an einer Gruppe teilnahmen, deutet dies darauf hin, dass erst eine längerfristige psychosoziale Begleitung emotionale Veränderungsprozesse in Gang bringen kann. Symptomverbesserungen sind hingegen innerhalb eines zwei-monatigen-Programms möglich.

Das Gruppenprogramm kann als "Therapie vor der Therapie“ benannt werden. Aus tiefenpsychologischer Sicht hat eine Verschiebung von der Symptom- auf die emotionaleEbene statt gefunden. Erst wenn die Symptome der Patienten genügend besprochen werden, sind sie in der Lage sich mit emotionalen Prozessen auseinanderzusetzen.

Neben der Verbesserung der körperlichen Symptome hat sich das Erleben und Verhalten der Versuchsgruppenpatienten im Vergleich zu diesen der Kontrollgruppe hoch signifikant zu mehr Entspannung, Gelassenheit und Optimismus verändert.

Bei Patienten der Kontrollgruppe verschlechterte sich das Erleben und Verhalten in Richtung Anspannung, Unsicherheit und Pessimismus signifikant. Die Ergebnisse der Verbesserung bzw. Verschlechterung des Erlebens und Verhaltens haben für die therapeutische Praxis aufgrund der hohen Effektstärken von 1,30 und 1,31 im Vergleich der Gruppen große Bedeutung. 
Für die innerhalb Rudolfs Theorie (1998) postulierte fehlende Resonanz über die Entwicklung der Patienten hinweg, konnte ein Programm von acht Sitzungen nicht ausreichen, um in Korrelation zum Beschwerdeerleben statistisch signifikante Veränderungeprozesse hervorzubringen. Dennoch zeigt sich eine Tendenz: Je höher die erlebte Resonanz ist, desto niedriger wird das Beschwerdeerleben eingeschätzt.

Patienten erlebten zudem signifikant weniger starke körperliche Beschwerden während der Sitzungen im Vergleich zu ihrer Alltagszeit in den Wochen zwischen den Stunden. Hierfür zeigte sich zudem eine mittelgroße Effektstärke von ,58.

Ähnliches gilt für das narzisstisch geprägte Verhalten der Probanden. Sie verhielten sich in den Gruppensitzungen signifikant weniger narzisstisch als in ihrem Altagsleben. Auch dieses Ergebnis konnte seine praktische Bedeutsamkeit durch eine mittelgroße Effektstärke von ,56 belegen.

Im Vergleich zum gegenwärtigen Forschungsstand in der Gruppenarbeit mit Somatisierungspatienten ist festzuhalten, dass diese Untersuchung, im Gegensatz zu bisher veröffentlichten Studien im deutschsprachigen Raum, über eine Kontrollgruppe verfügt. Lediglich eine der vier anderen Studien (Martin et al., 2007) referierten über eine Kontrollgruppe.

Das in dieser Arbeit angebotene Konzept schliesst im Gegensatz zu anderen Programmen kein somatoformes Störungsbild aus und ist niedrigschwellig konzepiert. Das Kriterium der Einbeziehung des Hausarztes wurde bisher in keiner gruppenspezifischen veröffentlichten Studie im deutschsprachigen Raum berücksichtigt.

Über die Hypothesenprüfungen hinausgehende Überlegungen enthalten die Empfehlung, mit Betroffenen darauf hinzuarbeiten, in sich selbst das behütende und versorgende Idealobjekt zu finden. Damit ist keine neue Form der Großartigkeit oder Pseudoautonomie gemeint. Es geht im Gegenteil darum, dass die Patienten auch als erwachsene Menschen emotionale Bedürfnisse haben dürfen und eine Art der Selbstfürsorge erlernen, die ihnen eine möglichst gute Befriedigung der Bedrüfnisse verschafft.

Im Rahmen der Programmdurchführung ergab sich der Eindruck, dass in jeder der somatoformen Symptoamtiken der Probanden die Botschaft an Fürsorge, Liebe und Zuneigung unterversorgt zu sein steckte. 
Im Rahmen der nicht hypothesengebundenen Ergebnisse ist die freiwillige Gruppenfortführung von 14 der 20 Versuchsgruppenprobanden als einer der wohl qualitativ größten Erfolge der Studie zu nennen.

Auch die durchweg sehr positive Bewertung des Programms lässt den Rückschluss zu, dass auch andere Somatisierungspatienten von einer Teilnahme profitieren können.

\section{Schlussfazit und Einordnung des Konzepts in die Praxis}

Die Bedeutung der Hausärzte für die richtige und sinnvolle Behandlung somatoformer Patienten ist zentral. Es ist daher unbedingt notwendig Hausärzte intensiver zu schulen und für direkte psychosoziale Kooperationsangebote zu sorgen.

Es sollte selbstverständlicher werden, dass neben somatischer Arbeit in einer Hausarztpraxis auch Möglichkeit zu psychosozialem Kontakt besteht. Ein Vorschlag, der als praktisches Fazit dieser Untersuchung betrachtet werden kann, ist die Integration eines psychosozialen Ansprechpartners direkt vor Ort in jeder Hausarztpraxis. Außerdem die Umsetzung des Konzepts sowohl als Möglichkeit der Frühintervention vor Eintritt einer Krankheitschronifizierung als auch als tertiärepräventives Interventionsangebot für Patienten mit somatoformen Störungen. Dabei dient das Programm überwiegend als „Therapie vor der (Psycho)Therapie“ und übernimmt eine Brückenfunktion zwischen medzinischer Behandlung und fundierter Psychotherapie.

Das größte Problem, was sich aus dieser Forderung ergeben mag betrifft die Kostendeckung.

Das Geld, was für unnötige wiederholende medizinische Maßnahmen ausgegeben wird, könnte langfristig für die psychosoziale Beratung und Begleitung der Patienten eingesetzt werden. Bedenkt man die hohen Prävalenzraten somatoformer Störungen von bis zu $40 \%$ in den allgemeinmedizinischen Praxen (Nanke und Rief, 2003), besteht kein Zweifel mehr, dass hier eine zusätzliche psychosoziale Betreuung unbedingt notwendig ist.

Die Vergütung für psychologische Interventionen in der Hausarztpraxis sollte an Gewichtung deutlich zunehmen. Wieder einmal ist die Frage, warum Verantwortliche im gesundheitspolitischen Bereich nicht erstrangig bereit sind, in Prävention oder Akutintervention zu investieren, als Menschen viele Jahre durch das System „zu jagen“ und auch „laufen zu lassen“. 
Die langen Odysseen und kostenintensiven Klinikaufenthalte sowie die durch zunehmende Chronifizierung häufig ein Leben lang anfallende Behandlungskosten gestalten sich im Endeffekt wohl wesentlich weniger ökonomisch und hilfreich als ein integrierter Helfer direkt vor Ort.

Die Bedeutung der Kosten verschwindet jedoch hinter dem immensen Leidensdruck der Betroffenen. Sie erfahren über Jahre hinweg keine angemessene Hilfe.

Dabei steht allerdings auch die Frage im Raum, ob diese Arbeit nicht die Ärzte selbst übernehmen können und wollen.

Dass sie dies teilweise gerne tun würden, ist anzunehmen. Es wird jedoch kritisch bemerkt, dass dies zum einen aufgrund der hohen Patientenzahlen überhaupt nicht realisierbar ist und zum anderen eine weitere, fundierte psychosoziale Ausbildung der Mediziner erfordern würde.

Viele der kooperierenden Ärzte waren froh, betroffene Patienten in die Gruppen abgeben zu können. Dies geschah mit dem Wissen, dass sie aber in somatischer Hinsicht weiterhin ihre vertrauten Behandler und Betreuer bleiben werden und mit der Gruppenleitung in Kooperation und Austausch gearbeitet wird. In diesem Sinne kann das Gruppenprogramm als paramedizinische Maßnahme, insbesondere als tertiäre Prävention betitelt werden.

Maßnahmen der Tertiärprävention werden nach der Manifestation einer Erkrankung durchgeführt, ihre Adressaten sind also Patienten. Die Ziele der Tertiärprävention bestehen einerseits in einer Reduktion der Progredienz (Voranschreiten) der Erkrankung und der Vermeidung von Rezidiven (Rückfall), andererseits in einer Reduktion von mit der Krankheit einhergehender Behinderung. Die Strategien der Tertiärprävention richten sich auf das Monitoring (Überwachen) von Patienten, die Frühbehandlung von Exazerbationen (Verschlimmerungen) und Rezidiven, die Modifikation von für die Krankheit bedeutsamen Risikofaktoren und schließlich die Rehabilitation (Wiederherstellung). Die rein kurativ orientierte Therapie zielt auf eine gänzliche Beseitigung einer Krankheit. Tertiärpräventive Maßnahmen richten sich in der Regel auf die Reduktion des Fortschreitens chronischer Erkrankungen oder auf Krankheiten, die drohen chronisch zu werden (König \& RiedelHeller, 2008). 
Die Rehabilitation richtet sich an Menschen mit vorliegender oder drohender chronischer Erkrankung oder Behinderung. Sie bezweckt mit ihren Maßnahmen, die „Behinderung abzuwenden, zu beseitigen, zu bessern, ihre Verschlimmerung zu verhüten oder ihre Folgen zu mindern“ (Haupt \& Delbrück, 1996). Rehabilitation wird der Tertiärprävention zugeordnet oder damit gleichgesetzt: „Gesundheitspolitisches Ziel von Tertiärprävention im Sinne von Rehabilitation ist es, bei vorliegender Erkrankung oder Behinderung die Leistungsfähigkeit soweit wie möglich wieder herzustellen und die Inzidenz bleibender Einbußen und Behinderungen abzusenken“ (VDR, 2000).

Nach SGB $\vee \S 11$ lautet der Auftrag für die gesetzliche Krankenversicherung durch Rehabilitation eine drohende Behinderung oder Pflegebedürftigkeit vorzubeugen, sie nach Eintritt zu beseitigen, zu bessern oder eine Verschlimmerung zu verhüten. Es gilt das Prinzip „Reha vor Pflege“.

Nach SGB VI §§ 9 und 10 lautet der Auftrag der gesetzlichen Rentenversicherung einer durch Krankheit oder Behinderung drohenden bzw. verursachten Beeinträchtigung der Erwerbsfähigkeit der Versicherten und einem damit verbundenen vorzeitigen Ausscheiden aus dem Erwerbsleben mittels Rehabilitation entgegenzuwirken. Es gilt das Prinzip „Reha vor Rente".

Der Rehabilitationsauftrag der genannten Sozialversicherungsträger dient letztlich der Vermeidung des Fälligwerdens anderer Sozialleistungen (Pflegekosten, Erwerbsminderungsrente) (Slesina, 2007).

Rehabilitation umfasst neben Maßnahmen zur Besserung oder Beseitigung von körperlichen, geistigen, seelischen und Funktionseinschränkungen immer Maßnahmen der tertiären Prävention (Verhütung von Krankheitsverschlechterung, Rückfällen).

In diesem Sinne sind tertiäre Präventionsmaßnahmen ein Bestandteil von häufig multiprofessioneller Rehabilitation für meist chronisch erkrankte Patienten.

Durch Maßnahmen der tertiären Prävention sollen neben der Verbesserung beeinträchtigter oder bedrohter Fähigkeiten und der Reduktion von Schmerzen insbesondere auch das Krankheitsverständnis und die Gesundheitsmotivation der Patienten gestärkt sowie Grundlagen für ein gesundheitsbewusstes, dem Gesundheitsproblem angemessenes Alltagsverhalten ermittelt werden (Slesina, 2007). 
Rehabilitation setzt unter normalen Bedingungen erst nach Abschluss einer angemessenen Behandlung einer Erkrankung ein. Patienten mit somatoformen Störungen wurden jedoch in der Regel häufig über Jahre hinweg nicht ausreichend oder falsch behandelt, so dass eine Störungschronifizierung meist bereist eingesetzt hat, ohne dass bereits wirklich effektiv, beispielsweise psychotherapeutisch, behandelt wurde. Daher müssen tertiär präventive Maßnahmen hier vor einer eigentlich fundierten anderweitig therapeutischen Arbeit angesetzt werden, um die Anschlussfähigkeit für eine notwendige psychotherapeutische Behandlung der Patienten überhaupt erst einmal zu erschaffen. Sollte ein psychosozialer Helfer direkt vor Ort in die hausärztlichen Praxen integriert werden, ist es jedoch auch gut möglich Patienten im Frühstadium einer somatoformen Störung mit einer Teilnahme am Gruppenkonzept zu erreichen und damit bestenfalls eine Störungschronifizierung zu vermeiden. Das Konzept stellt sich damit als ein integrativer Bestandteil der medizinischen Betreuung dar.

Betrachtet man die erfolgreichen Ergebnisse der Studie bzgl. der Reduktion der erlebten Symptomstärke der Patienten, ist eine weiterführende Psychotherapie für einen Großteil der Betroffenen möglicherweise aber auch nicht mehr gewünscht.

Dies wiederum kommt gesundheitsökonomischen Aspekten selbstverständlich entgegen. Das Gruppenprogramm ist demnach ein Angebot von psychosozialer Unterstützung.

Diese versucht der Psychotherapie zuvorzukommen. Wann eine Psychotherapie notwendig ist, entscheiden Ärzte und Psychologen. Psychosoziale Interventionsangebote hingegen sind häufig Maßnahmen der Prävention und vor allem „Hilfe zur Selbsthilfe“. (Härte \& Koch, 2000).

Bliebe das Gruppenangebot, so wie nach Beendigung des offiziellen Programms der Studie, bestehen und bliebe eine kostengünstige Teilnahmemöglichkeit erhalten, würde eine durch Arzt und psychosozialem Helfer multiprofessionelle Behandlung der überwiegend chronisch kranken Menschen, im Rahmen des Monitoring der Störungsentwicklung, bestehen bleiben.

Vertraute Ansprechpartner, die konstant und sicher verfügbar sind, können weitere Verschlechterungen der Krankheit so möglicherweise verhindern. 
Der Gruppenleiter sollte auch in Einzelgesprächen vor Ort in der Hausarztpraxis erreichbar sein, damit auch zwischen Hausarzt und psychosozialem Helfer ein ständiger Austausch möglich ist.

Wie auch durch die Aussagen anderer Forscher deutlich wird (Yates, 1994) kann Patienten mit somatoformen Störungen nur durch eine enge, direkt vor Ort gewährleistete, interdisziplinäre Kooperation zwischen unterschiedlichen Helfergruppen wirklich geholfen werden. 


\subsection{Eigene Stellungnahme zum Promotionsprojekt: Persönliche Erfahrungen und Entwicklungsprozesse}

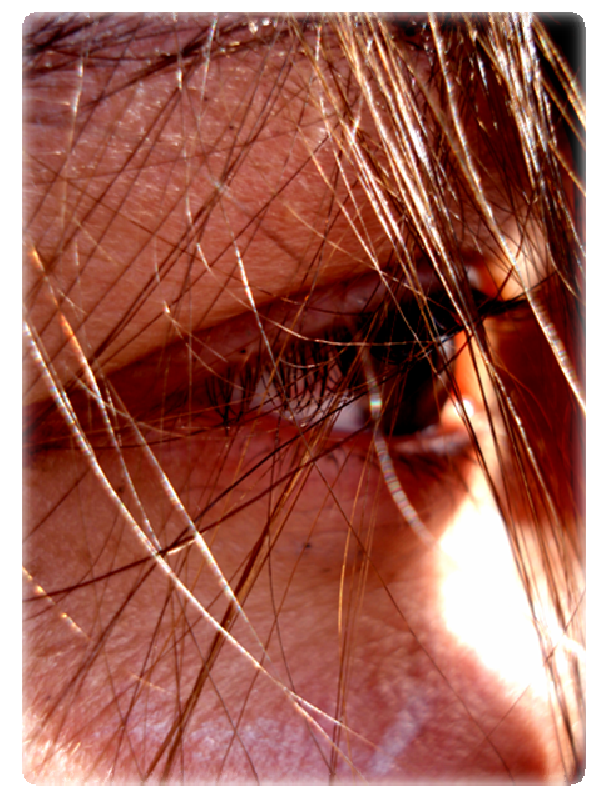

Zum Abschluss der Arbeit möchte ich mir dieses Kapitel gönnen, um hingegen aller "Gesetze des wissenschaftlichen Schreibens", gänzlich frei von jedem Ergebnis und jeder bisherigen Forschungsgrundlage, persönlich Stellung zu beziehen und über Erfahrungen und eigene Prozesse innerhalb des Studienprojekts berichten. So möge dies andere junge Forscher und Forscherinnen ermutigen, einen ähnlichen Weg zu wagen, an ihm zu wachsen, auf ihm zu fallen und wieder aufzustehen. Ich begann die Arbeit mit großen Visionen und Hoffnungen, die sich zum Teil erfüllten zu einem anderen Teil jedoch auch scheiterten, aber wie sagte Einstein:

\section{„Wenn eine Idee am Anfang nicht absurd klingt, gibt es für sie keine Hoffnung. “}




\section{Ängste und Sorgen vor Beginn der Studie und deren Entwicklung und (Auf)Lösung}

\section{Bin ich denn schon ,groß“ genug?}

Bei meinem Einstieg in die Promotion vor zwei Jahren war ich gerade 25 Jahre jung. Mein Studium konnte ich mit 1,0 mit Auszeichnung beenden. Ich war voller positiver Bestätigung, voller Mut, in meinem Fach „richtig“ zu sein und hatte nach diesem beflügelnden Erlebnis das Gefühl „alles“ erreichen und erforschen zu können, was sich in meinen jungen, idealistischen Phantasiewelten so tummelte.

Während meinen Diplomprüfungen nahm das Thema der somatoformen Störungen und der Psychosomatik bereits einen breiten Platz ein. Mich faszinierte das Zusammenspiel zwischen Physiologie und Psyche so sehr, dass ich begann meine hier verwirklichte Konzeptidee zu entwickeln. Nie werde ich vergessen, dass diese Idee wirklich an einem Abend gemeinsam mit meiner besten Studienfreundin Maria in meinem ersten eigenen Wohnzimmer entstand; meine Augen leuchtend, meine Motivation und mein Idealismus ungebrochen.

Ich wusste sicher zu diesem Zeitpunkt nicht, welche Berge der Arbeit und der Frustration ich überwinden muss, um bis zu dem heute erreichten Endpunkt zu gelangen. Gleichzeitig erahnte ich aber auch in keiner Weise, wie sehr ich praktisch an dieser Arbeit reifen und lernen würde und welche wunderbaren Konsequenzen sich durch das Fortbestehen der Gruppen für mich ergäben.

In mir lebte eine Mischung aus ungebändigter Lust und Motivation zur Verwirklichung dieses Projekts gepaart mit einer stillen, heimlichen Angst viel zu „klein“ und unerfahren zu sein.

Mein Äußeres hat einen eher jüngeren Charakter, so werde ich noch heute auf Anfang 20 geschätzt. Dazu besitze ich eine Stimme, die sich in den höheren Tonlagen zu Hause fühlt.

Zwar wusste ich um meine fachlichen Kompetenzen und auch der vielen Selbsterfahrung, die ich bis zu diesem Zeitpunkt bereits gesammelt hatte sowie meine praktische Phase innerhalb der Diplomarbeit und die positive Rückmeldung darin, aber nun war ich die Hauptverantwortliche und musste mich darin beweisen. 
Mir hat ein Satz, den mein geschätzter Doktorvater Jörg Fengler uns in seinen Vorlesungen und Seminaren stets mit gab, sehr geholfen. Er sagte: „Wenn Sie dann irgendwann Ihr Diplom in der Hand halten, dann haben Sie keine Angst, auch wenn Sie jung sind, am Anfang Ihrer beruflichen Karriere stehen, Sie dürfen sich dann den Mantel der Professionalität anziehen“.

Schön finde ich, dass dies eine Aussage ist, die er selbst von einem seiner Lehrmeister mitbekommen hatte als er jung war und welchen er mir nun erfolgreich als Mutmacher weitergeben konnte.

Meine Ängste, meine junge Stimme und mein Alter betreffend, bezogen sich darauf, nicht ernst genommen zu werden oder als Leitungsfigur nicht akzeptiert zu werden.

Dabei habe ich etwas Grundlegendes gelernt: Es kommt überhaupt nicht auf das Alter an, sondern darauf, wie sicher ich in meinem Tun, in meiner Planung und in meiner Kompetenz mir selbst gegenüber bin.

Das ist letztlich das, was ich den Menschen vermittele und, was sie an mir wahrnehmen. Diese stille Angst konnte sich sehr schnell auflösen. Zu keinem Zeitpunkt war mein Alter Thema für einen Gruppenteilnehmer. Ich wurde weder danach gefragt noch provokant darauf aufmerksam gemacht.

Diese Erfahrung war extrem wichtig für meine Selbsteinschätzung, Selbstwahrnehmung und das Finden von professioneller Sicherheit in meinem Handeln.

Aber nicht nur die Angst zu jung zu sein, sondern an der nun mal tatsächlich fehlenden Erfahrung zu scheitern, war vorhanden. Ich selbst hegte einen großen, vielleicht übergroßen und unrealistischen Anspruch an meine Fähigkeiten und an mein Wissen, das vorhanden sein musste.

Ich kam damit an einen Punkt, an dem ich mich selbst so unter Druck setzte, dass ich anfing an allem, was ich geleistet hatte, grundlegend zu zweifeln. Mir kam es vor als würde ich nun gar nichts mehr wirklich wissen und können.

In dieser Krise wurde mir bewusst, dass ich "nur" das sein kann, was ich bisher sein konnte: 25 Jahre alt, motiviert, interessiert, mit Wissen gut ausgestattet und bereit zu lernen und zu erfahren. Ich fing an zu akzeptieren, dass ich weder alles im Bereich Medizin, Psychologie und Therapie wusste, noch dass ich in der Lage war all das alleine zu leisten, was eigentlich ein Team von fünf oder zehn Mitarbeitern erfordert hätte. 
Mein Erleben ging dahin, dass es in Ordnung war, auch einmal etwas nicht zu wissen, eine Frage nicht beantworten zu können und ggf. Dinge, die mich überforderten an andere Menschen zu delegieren oder hinzunehmen, dass dies oder jenes eben nicht so wie geplant funktionieren konnte. Dieser Prozess ist bist heute sicher noch nicht abgeschlossen und ich arbeite weiter für mich daran, auch mit Unwissenheit bezüglich mancher Phänomene umgehen zu lernen.

Es verwundert nicht, dass ich bei Vorbildern, die vorwiegend in einem Alter von 60 Jahren sind, nur „weniger" sein kann. Ich bin nun allerdings dort angekommen, wo ich bemerke, dass "Ich" ganz eigen bin. Dass meine Art mit Menschen zu arbeiten "meine" ist und niemals diese oder jene Kopie eines Vorbilds sein wird und auch nicht sein soll. Ich bin dann „am besten“, wenn ich so arbeiten und handeln kann, wie es „ich“ ist.

Gerade in der Arbeit mit Menschen an tiefen, emotionalen Themen, funktioniert die Beziehung nicht, wenn von meiner Seite keine selektive Authentizität gelebt werden kann. Das habe ich schnell für mich herausgefunden.

Trotz, dass ich mir glücklicherweise eingestehen konnte, weder alles wissen noch alles direkt können zu müssen und durchaus auf die Hilfe anderer zurückgreifen zu dürfen, ist mir in einem solchen Projekt spürbar klar geworden, dass man doch am besten fährt, wenn man sich um möglichst viele organisatorische Aspekte selbst bemüht und kümmert. Verlasse dich nicht auf jemand anderen, wenn es DEIN Projekt ist. 


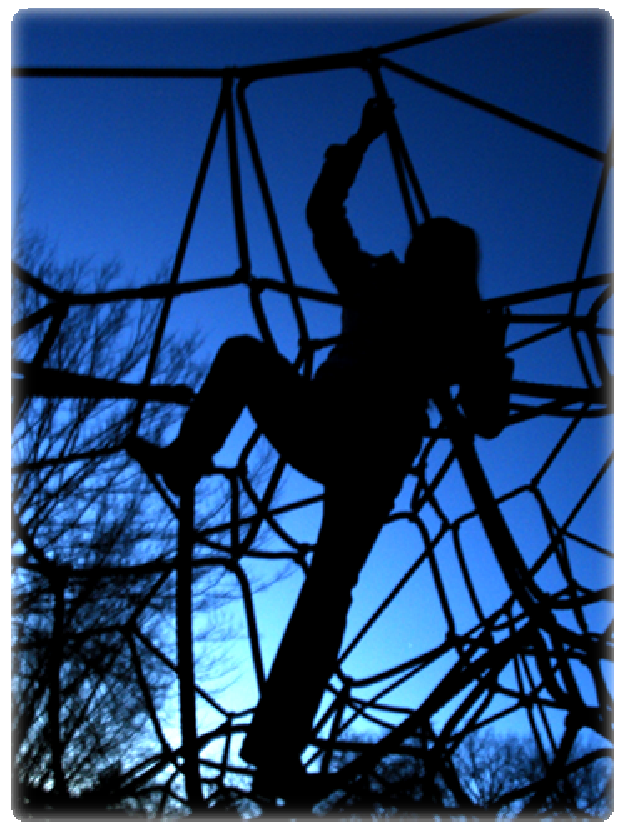

Es gibt immer etwas, das anders läuft als erwartet und was man in einstiger Ersmotivation im Angesicht des Antriebs, wie durch eine rosarote Brille übersah.

Ein Phänomen, das vermutlich jedem Doktoranden während seiner Arbeit begegnet ist die stetig erneute Konfrontation mit der Begrenzung.

Begrenzung bezogen auf das Thema, die Fragestellungen und dem, was überhaupt in einer Doktorarbeit zu leisten ist. Nach jedem erneuten Vorstellen im Doktorandenkolloquium war eine große Portion Frustrationstoleranz vonnöten, denn meist ging man dort völlig „neu“ und durchwühlt hinaus. Ideen und Vorhaben, die sich vorher fest in mir verankert hatten, mussten über Bord geworfen werden, weil sie entweder unlogisch oder einfach nicht realisierbar waren.

So wollte ich beispielsweise noch viel intensiver die Beziehungsdimensionen untersuchen und über die Bindungsmuster der Teilnehmer forschen. Ich wollte die Menschen in ihrem Altag befragen, um auch eine Fremdeinschätzung zu bekommen und in noch intensiveren Kontakt mit den Ärzten bezüglich der Beziehungsarbeit treten. 
All diese Aspekte erlebe ich nach wie vor als hochspannend und während ich sie aufschreibe, spüre ich meine Trauer, dass ich mich innerhalb dieser Arbeit von diesen verabschieden musste.

Immer wieder ging es darum herauszufinden: Was kann ich leisten? Wo übernehme ich mich? Was kann Bestandteil einer Dissertation sein und worauf kann und möchte ich mich letztlich fokussieren?

Neben dem immer währenden Thema der Begrenzung und Eingrenzung, des Minimierens und Fokussierens wurde mir sehr schnell deutlich, dass ich mir eine Projektidee erschaffen hatte, die nur in einer Vollzeitarbeit verwirklicht werden konnte.

Die persönliche Kontaktsuche zu den Ärzten und zu den Probanden sowie die gesamte Organisation der Gruppen, der Räumlichkeiten, die Durchführung des Programms und die Vor- und Nachbereitung beanspruchten meine gesamte Zeit, Kraft und meinen vollen Einsatz.

Dass dies schnell an meine finanziellen Grenzen führte, wurde von mir in anfänglicher Euphorie ebenfalls ausgeblendet. So begann ich, mich mit vollstem Engagement für ein Promotionsstipendium zu bewerben. Ein Prozess, den ich mir mit einem ausgezeichneten Diplomabschluss in Regelstudienzeit und zusätzlichem außeruniversitären sozialen Engagement sehr viel leichter vorgestellt hatte als er sich in der Realität darstellte.

Die Stiftungen verlangten nicht nur ein Zeugnis, die Bewerbungen selbst waren eine kleine Doktorarbeit, die in jeder Stiftung noch einmal anders aussehen musste. Nach einem Jahr Bewerbungsmarathon musste ich frustriert immer wieder Ablehnungen hinnehmen. Doch auch hier kam mir meine hartnäckige Verbissenheit letztlich zu Gute. Ich erhielt das Schmittmann-Wahlen-Stipendium, das jedes Jahr in einfacher Ausführung für einen ausgewählten Studenten an der Universität zu Köln vergeben wird. Über dieses wurde ich für zwei Jahre in meiner Promotionsarbeit finanziell unterstützt.

Ein Gefühl der Anerkennung meines Einsatzes und meiner Arbeit erfüllte mich wieder mit neuer Motivation.

Neben den Stipendienbewerbungen hatte ich mich außerdem bei diversen Krankenkassen nach Projektunterstützung erkundigt. Dies entsprang meiner Überzeugung, dass die Idee der Durchführung eines solchen Gruppenprogramms sich auch für die Krankenkassen wesentlich ökonomischer gestalten dürfte, als lange, chronfizierte und im Grunde unbehandelte Wege somatoformer Patienten. 
Leider stieß ich dort in keiner Weise auf eine Möglichkeit, finanziell mit dieser Idee unterstützt oder gefördert zu werden. Trotzdem möchte ich die AOK Bergisch-Gladbach dankend erwähnen, in der ich über einen sehr freundlichen und hilfsbereiten Kontakt einen Gruppenraum kostenlos zur Verfügung gestellt bekam.

\section{Die (ernüchternde) Erfahrung in der Kooperationsarbeit mit Hausärzten}

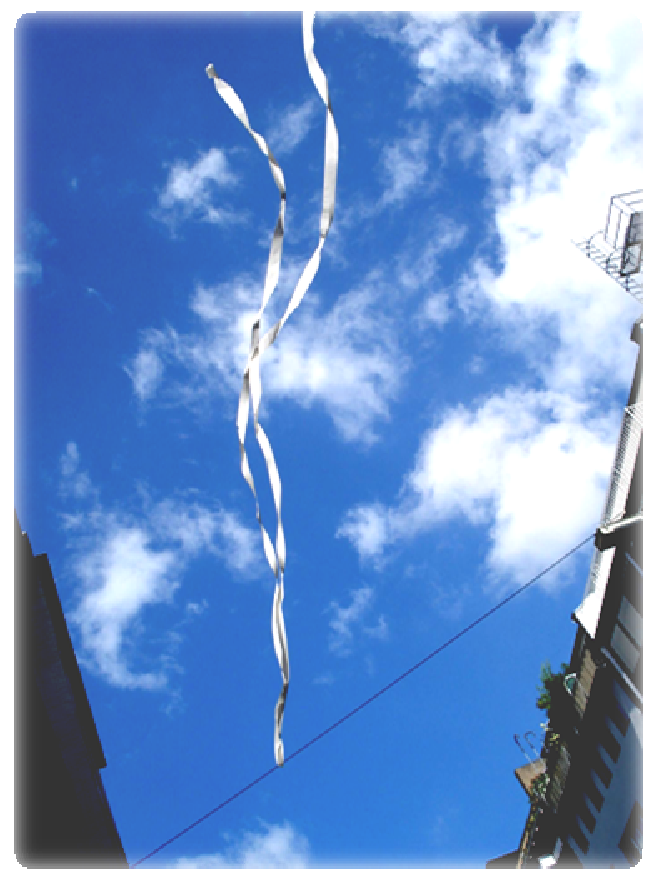

\section{Da kann ich Ihnen meine ganze Praxis schicken...}

Ich muss beinahe lächeln, wie optimistisch und euphorisch ich an die Idee der Kooperation mit behandelnden Hausärzten heranging. Innerhalb meiner Arbeit habe ich die Realität der Hausärzte erst richtig kennengelernt. Diese ist geprägt durch permanenten Zeitmangel, eigene Frustrationen über schlechte Vergütungen, eine viel zu hohe Patientenanzahl und wenig Raum für Gespräche.

Es ist nicht so als hätte ich kein Verständnis für das Handeln der Ärzte, denn bei den gegenwärtigen Bedingungen bleibt auch innen nicht mehr viel Spielraum. Weder für ausführliche Gespräche mit Patienten noch für eine Zusatzbelastung, die durch die Teilnahme an einer Studie wie dieser für sie entsteht, exisitert wirklich Zeit.

Die Praxen sind gekennzeichnet durch zunehmende Bürokratisierung, die mit einem hohen Mehraufwand an Schreibarbeit und Organisation verbunden ist. 
Die Ärzte können weniger denn je ihrem wirklichen Beruf nachgehen: Arzt zu sein und dies einfach und „nur“ im direkten Kontakt mit den Patienten.

Ich habe mit 70 Ärzten persönlich gesprochen und musste dafür zunächst die Barriere der Sprechstundenhilfen überqueren. Viele der Damen konnten mit den Begriffen meines Projekts wenig anfangen, so dass ich begann meine Ersterklärungen ständig weiter zu vereinfachen und zu minimalisieren, um überhaupt auf Anklang zu stoßen.

Schockierend musste ich feststellen, dass es unter den Ärzten tatsächlich Praktiker gab, die mit dem Begriff der somatoformen Störung zunächst nichts anzufangen wussten. Ein Skandal in Anbetracht der hohen Prävalenzraten in den Praxen.

Während meiner Werbung für mein Projekt kam ich mir selbst wie ein Vertreter vor, der Staubsauger verkaufen möchte. Eine Rolle, in der ich mich mehr als unwohl fühlte. Trotzdem gab es keinen anderen Weg als den unmittelbaren Kontakt zu suchen und dort im Gespräch alle Fragen direkt klären zu können.

Ich erlebte trotzdem von einem Großteil der Ärzte Aufmerksamkeit, wenn auch in nur sehr abgestecktem und hektischem Zeitrahmen. Das Interesse am und die Befürwortung eines solchen Projekts war beinah durchweg positiv. Ein Satz, den ein Hausarzt schmunzelnd zu mir sagte, war: „Na, da kann ich Ihnen ja meine ganze Praxis schicken." Leider habe ich von diesem Hausarzt jedoch nicht einen Patienten überwiesen bekommen.

Viele Ärzte befürworteten das Projekt, im Gespräch mit Betroffenen vergaßen sie aber doch wieder diese darauf aufmerksam zu machen, vielleicht auch, weil der Aufwand für sie in Anbetracht ihrer knapp bemessenen Zeit zu hoch war. Der Aufwand für die Ärzte wurde von mir jedoch auf einem wirklich geringen Niveau gehalten. Was sie zu tun hatten, war lediglich den Patienten einen Bogen zum Ausfüllen zu geben und zu den drei Messzeitpunkten drei Fragen zu diesem Patienten zu beantworten. Ein Minimalaufwand, der anscheinend für einen Großteil trotzdem noch zu hoch erschien.

Das Einholen der Arztbögen war zu allen Messzeitpunkten mit einem weiteren großen Energieaufwand meinerseits verbunden. Es erforderte häufiges freundliches Erinnern, Anrufen und Bitten um das Zurücksenden des Bogens, wobei selbstverständlich sowohl für die Ärzte als auch für die Patienten die Briefumschläge bereits frankiert und beschriftet waren. 
Obwohl über 300 Hausärzte im Umkreis praktizieren und alle durch ein Schreiben über die bevorstehende Studie informiert wurden, war das Finden von kooperativen Ärzten beschwerlich. Dafür waren diejenigen, die von sich aus Bereitschaft und Interesse zeigten, umso zuverlässiger und lieferten jeweils mehr als einen Patienten für das Gruppenprogramm.

Diese Ärzte sind es, die auch heute weiterhin betroffene Patienten in die Gruppen weiterleiten.

Eine Lösung für das Problem des Zeitmangels und der daraus resultierenden wenigen Zeit für Patienten mit somatoformen Störungen schwebt mir leider nicht vor.

Wieder ist mein Appell, dass es mehr direkte Kooperationen vor Ort zwischen Hausärzten und psychosozialen Helfern geben müsste, um diesem Problem angemessen begegnen zu können.

\section{Persönliche und professionelle Lernprozesse in der psychosozialen Gruppenarbeit}

\section{Angriffe, Grenzen und Gegenübertragung}

Das Wertvollste der ganzen Studienarbeit sind für mich persönlich die Dinge, die ich in der praktischen Tätigkeit mit und in der Gruppe gelernt habe.

Ich konnte mich erleben und erfahren im Umgang mit erwachsenen Patienten und kam dabei meinen eigenen Arbeitsmethoden stetig näher.

Als elementare Lernprozesse sind die „schwierigen“ Teilnehmer zu nennen. Etwas, das ich lernen musste und weiterhin lerne, sind negative Gegenübertragungen meinerseits zu bemerken und dabei zu versuchen, diese nicht unangemessen auszuagieren.

Unter den Teilnehmern gab es sehr fordernde Patienten, die mir permanent suggerierten: „Keiner kann mir helfen; Was Du machst, reicht auch nicht aus; Was soll mir das hier bringen?" Oder andere, die es sich zur Aufgabe gemacht hatten, mit mir in eine permanente Diskussion eintreten zu wollen, die es liebten, mir provokante Fragen zu stellen, sich mit mir auseinandersetzen und messen wollten.

Als letztes gab es diese, die nach permanenter Aufmerksamkeit rangen, durchweg reden wollten und anderen Mitgliedern kaum Raum ließen, um ebenfalls teilzunehmen. 
Diejenigen, die eher still und schweigsam waren galt es unter diesen nicht zu übersehen und nicht untergehen zu lassen.

Zu Beginn der Arbeit war ich natürlich sehr bemüht, es allen recht zu machen, wollte ich doch, dass bloß alle bleiben. Zudem wollte ich nicht „versagen“, war aufgeregt und sicher noch leicht aus der Bahn zu werfen. Dies gelang einzelnen recht gut, aber mit Hilfe von guter Supervision durch meinen Doktorvater und mit dem Doktorandenkolloquium hatte ich die Möglichkeit diese Prozesse zu reflektieren, für mich zu verstehen und anschliessend anders mit diesen Menschen umzugehen.

Latente Angriffe gegen mich, das Infragestellen der Sinnhaftigkeit des Projekts oder Vorwürfe, dass ich auch nicht helfen könne, begann ich weniger persönlich zu nehmen. Viel mehr machte ich diese Aussagen dann hin und wieder zum Gruppenthema, legte sie in den Raum und regte die anderen Teilnehmer an, sich dazu zu äußern oder ihre Empfindungen mitzuteilen. Eine direkte persönliche Auseinandersetzung mit Patienten ließ ich in der Gruppe nicht zu, nahm ihre Anmerkungen zur Kenntnis, reagierte darauf, aber stieg nicht auf das gleiche emotionale Level der Aggression, Provokation oder Wut und Enttäuschung ein. Dies funktionierte erstaunlich gut, vielleicht weil die Betroffenen so Sicherheit im Kontakt zu mir erleben konnten und erfuhren, dass ich trotz Provokationen konstant für sie erreichbar blieb.

Das Phänomen des Monologisierens einzelner, und deren häufige Fähigkeit, kurz vor Ende einer Sitzung nochmals ein "neues Fass“ zu öffnen, stellte sich ein wenig schwieriger für mich dar. Das zum Ende einer Stunde geöffnete „neue Fass“ konnte ich konkret benennen und die Frage mit auf den Weg geben, wozu und warum dieses Thema erst so spät angesprochen werden kann, wenn wissentlich keine Zeit mehr übrig ist, um es tiefergehend zu besprechen.

Teilnehmer, die allerdings zum monologisieren neigten, erlebte und erlebe ich auch heute noch für mich als schwierig handhabbar. Es besteht dabei immer ein schmaler Grad zwischen Verletzung desjenigen und Notwendigkeit, ihn auf sein Verhalten aufmerksam zu machen. Da die Gruppenmitglieder zuvor fast durchgehend noch keine Gruppenerfahrung gemacht hatten, war kaum zu erwarten, dass sie sich trauen würden, lange Monologe anderer von sich aus anzusprechen oder zu unterbrechen. 
Ich musste begrenzen, zum Schutz der anderen und um das Konzept nicht gänzlich in Gefahr zu bringen. Innerhalb des Projekts stieg diese Person im Anschluss an diese Stunde aus der Studie aus.

Natürlich verursachte dies in mir Zweifel und Unsicherheit, aber ich kam zu dem wichtigen Entschluss, dass nicht alle Menschen und auch nicht alle somatoformen Patienten für eine Gruppenarbeit geeignet sind und es dann auch meine Pflicht ist, die anderen Teilnehmer zu schützen und eine gute Gruppenarbeit nicht zu verhindern, sondern zu fördern.

Es war mir im Nachhinein dennoch wichtig, ein abschließendes Gespräch mit dieser Person zu führen, um sie mit gutem Gewissen aus der Studie „entlassen“ zu können.

Etwas, das ich ebenfalls mit „schwierigen Patienten“ lernen musste, ist, meine eigenen Ideale von Höflichkeit und Dankbarkeit beiseitezustellen, um nicht in Wut oder Enttäuschung über das Verhalten anderer zu versinken.

Dass die Teilnahme an diesem Programm kostenlos war, ich zu jeder Zeit für Getränke, permanente Präsenz und Freundlichkeit sorgte, schien für die überwiegende Anzahl der Teilnehmer normal zu sein. Diesbezüglich fiel es mir sehr schwer ihre Sicht einzunehmen, was mir letztlich aber doch gelang, wenn auch mit einem zurückbleibenden inneren Raunen und Grummeln.

Ein Punkt, den ich anfangs unterschätzte, war die große Bedeutung der Atmosphäre im Raum. Zwar habe ich mit Kerzen und einzelnen Lichtquellen dafür gesorgt, dass eine gewisse kühle Sachlichkeit eines Versicherungs-Raums an Gewichtung verliert, dennoch wurde diese von den Patienten immer wieder bemerkt und thematisiert. Inzwischen habe ich meinen eigenen Raum eingerichtet und sehr genau darauf geachtet, dass beim ersten Eintreten ein wohliges und wärmendes Gefühl entsteht. Eine Patientin sagte in der letzten Sitzung zu einer neuen Teilnehmerin: „Ich empfinde die Gruppe hier immer wie eine „Kuscheldecke“, wenn ich hier rausgehe, habe ich das Gefühl, in eine Decke eingewickelt und gewärmt zu sein." Ein wie ich finde, wunderbares Bild.

Hingegen anderer offener Gruppen, die über einen langen Zeitraum laufen, war es mir wichtig, auch schweigsame Personen von mir aus immer wieder einzubeziehen, herzlich und liebevoll anzusprechen, um sie nicht „aus dem Boot“ zu verlieren. Meine Erfahrung ist, dass es durchaus hilfreich war, wenn ich Patienten auch einmal direkt ansprach, wenn sie länger nichts mehr erzählt hatten. Oft waren sie erleichtert und brauchten hin und wieder diese Brücke, um zurück zur Gruppe finden zu können. 
Auch wenn dies in manchen Ansätzen weniger erwünscht ist, erlebte ich direktes Ansprechen als wichtig und notwendig, wie alle Interventionen jedoch selektiv und gut überlegt.

Das Thema der Art und Weise der Interventionen begleitete mich selbstverständlich von Anfang an in der praktischen Arbeit am aller meisten.

Immer wieder musste ich dabei an eine von meinem Doktorvater Jörg Fengler aufgestellte Dimension in der Intervention denken, die er „Leichtigkeit und Eleganz“ nennt.

Diese Qualität beschreibt, dass der Helfer Sorge tragen soll, dass sein Klient inn nicht schwerer, trauriger oder belasteter verlässt als er vor der Begegnung mit inm war.

Dies schließt Humor, eine gewisse Souveränität und Unangestrengtheit des Fachmanns ein. Ebenso wie ein professioneller Tänzer seine durchaus anstrengende Choreographie lange trainieren muss, bis sie leichtfüßig und flüssig aussieht, muss auch ein Helfer diese Qualität erst erlernen und erproben. Wesentliche Aspekte dieser Dimension sind die Ökonomie und Sparsamkeit in den Interventionen, das heißt: So viel wie nötig, so wenig wie möglich in den Gruppenprozess einzugreifen, Variabilität im Handlungsspielraum zu zeigen, aktuell gezeigte Ressourcen von Teilnehmern im Hier und Jetzt der Gruppensituation aufgreifen und nutzen zu können und Betroffenen bei einer gewissen Enthypnotisierung und Dekonstruktion bestimmter, festgefahrener Muster zu helfen und diese umzudeuten oder gemeinsam neue Formulierungen zu finden.

Eine lösungsorientierte Sichtweise erschafft ebenfalls eine gewisse Leichtigkeit in den Interventionen, während ungewollte, ungeschickte und unangenehme Deutungen von Seiten des Therapeuten häufig Ursache für einen Widerstand im Patienten sein können. Der Grund für Widerstände kann sehr vielseitig sein und liegt keinesfalls immer allein im Konflikt des Patienten begründet. Ein falscher Kontext, ungünstiger Zeitpunkt (Kairos) oder ein zu hohes Tempo können ebenso Ursache sein.

Fenglers Interventionstheorie war Bestandteil meines Diplomprüfungsthemas und hat sich durch ihre Übersichtlichkeit und Logik tief in mein praktisches Handeln eingebrannt. Seine vier beschriebenen Flügel mit dem Fuß der Beziehung als Grundlage erschaffen für jeden Helfer eine wichtige und hilfreiche Basis, um wirksame Interventionen kreieren und in einen guten Kontakt mit Patienten treten zu können. Als Literaturhinweis sei hierfür Fenglers Artikel „Triffst du nur das Zauberwort. Die Konstruktion wirksamer therapeutischer Interventionen“ (2003) genannt. 
Den Flügel der Leichtigkeit und Eleganz habe ich mir während meiner praktischen Arbeit immer wieder vor Augen geführt, um eine allzu große Verbissenheit und einem absoluten Perfektionsdrang angemessen begegnen zu können.

Gerade in der Gruppenarbeit habe ich nach und nach gelernt, wie oft und wann ich mich einbringen sollte und an welchen Punkten beispielsweise Schweigen für die Gruppe wichtig und förderlich war.

Etwas, das mich sehr bewegt hat, ist die Tatsache, dass es wirklich möglich ist, innerhalb so kurzer gemeinsamer Zeit intensiv miteinander zu arbeiten. Ich war beeindruckt und bewegt über den Verlauf der Sitzungen, die sich sehr vertraut und auch emotional gestalteten. Mein Eindruck war, dass auch ich als Leitung dazu einen großen Anteil beitragen konnte, indem ich der Gruppe meine ganze, volle und permanente Präsenz während den Sitzungszeiten schenkte.

Ein Leiter, der zwischendurch gänzlich abwesend ist, kann den Teilnehmern nicht das Gefühl von wirklichem Interesse geben. Die echte Präsenz führte allerdings auch dazu, dass ich von den Erzählungen und Emotionen der Teilnehmer ebenfalls ergriffen war und dabei Kräfte von mir investierte, deren Verlust ich nach den Stunden deutlich spürte. Während eines Selbsterfahrungsseminars über ein Wochenende zum Thema Gruppendynamik hatte mir mein Doktorvater einmal gespiegelt, dass er mich in den Interventionen so erlebt, dass ich mich beinah dort "hinein kniee“ und er sehen würde, wie sehr mir das auch körperlich zusetze, dass es mich Unmengen an Kraft koste und ich acht solcher Sitzungen am Tag so nicht auf Dauer durchstehen könne.

Das habe ich mir damals sehr zu Herzen genommen und bin diesbezüglich immer noch im Proze $ß$ an mir zu arbeiten. Für mich funktioniert diese intensive Arbeit mit anderen Menschen nicht, wenn ich nicht wirklich „dabei“ bin, allerdings gilt es wohl zu lernen, dass es auch Situationen und Tage geben darf, an denen $80 \%$ völlig ausreichen, um dennoch gute Arbeit zu leisten.

Ich halte diesen Lernprozess jedoch für sehr normal, wenn man am Anfang eines Helferberufes steht, in dem man gerade durch Gespräche und Präsenz in den Kontakt geht und nur um das Elementarste und den Kern eines Menschen kreist, bis man dann bestenfalls gemeinsam schließlich zu inm vordringt. Diese Arbeit erlebe ich als sehr erfüllend, bereichernd und absolut sinngebend. Gleichzeitig kostet sie viel eigene Kraft, die es gilt, haushalten zu lernen und sich einen Ausgleich dazu zu verschaffen. 
Eine Ausbildnerin meiner derzeitigen tanzpsychotherapeutischen Zusatzausbildung sagte auf die Frage der eigenen Psychohygiene hin, dass sie sich eine Art "Gegenwelt“ erschaffen hat, in der es überhaupt nicht um tiefe Kontakte geht.

Sie gräbt in ihrem Garten und ist fest auf dem Boden, schmutzig, mit der Natur in Kontakt und für sich alleine.

Das Bild einer "Gegenwelt“ finde ich sehr schön und kann es sehr gut auf mich und mein Leben übertragen, in dem es glücklicherweise viele Gegenwelten zu dieser, meiner Arbeit gibt.

Zuletzt möchte ich begründen, warum ich mich nur auf die deutschsprachigen Interventionen bezogen habe. Aus meiner Sicht haben Störungsbilder immer auch viel mit der Gegenwartsgesellschaft und den Bedingungen und Anforderungen darin zu tun. Ähnlich zu Freuds Zeiten, in denen das Thema Sexualität tabuisiert war und ggf. deshalb auch alle Störungsbilder auf verdrängte und nicht ausgelebte Sexualität zurückzuführen waren, erlebe ich es heute in Deutschland so, dass Emotionen und „Schwäche“, vor allem im Berufsleben, tabuisiert und verdrängt werden. Da ich selbst hier lebe und von einem Leben in den USA oder einem anderen Land zu wenig kenne und nicht wage, eine treffende Aussage zu formulieren, sehe ich die somatoforme Störung tatsächlich auch als eine „Krankheit unserer Zeit“. Eine Krankheit, die auf der Verleugnung von Abhängigkeiten, von emotionalen Bedürfnissen und der Überbetonung von narzisstischem Größenwahn und der Abwertung eigener Labilität beruht.

Die Alternative zum seelischen Klagen oder dem Äußern zwischenmenschlicher Bedürfnisse liegt in der Entwicklung körperlicher Symptome, die gesellschaftlich wesentlich anerkannter und akzeptierter sind als auszusprechen eine psychische Störung zu haben. Meines Erachtens wird auch der Begriff Depression durch die heutige Modediagnose Burnout (ICD-10:Z73.0) umgangen. Auch darin entwickeln die meisten Betroffenen körperliche Symptome, die zu besonders großem Leidensdruck führen.

Ich finde es alarmierend und sehr besorgniserregend, dass die Prävalenzraten somatoformer Störungen in den Allgemeinarztpraxen eine solche Höhe erreicht haben. Mir stellt sich die Frage, wo wir ankommen werden, wenn das Thema nicht endlich öffentlicher und selbstverständlicher werden wird. 
Dass eine somatoforme Störung in einer Hausarztpraxis weit häufiger diagnostiziert wird als eine Grippe sollte Grund genug sein, dieser Krankheit einen ebenso wichtigen Stellenwert zu geben und ebenso wirkungsvolle Behandlungsmaßnahmen zu erschaffen.

\section{„Wir wollen alle „nur“ geliebt werden“ (T.Franzen)}

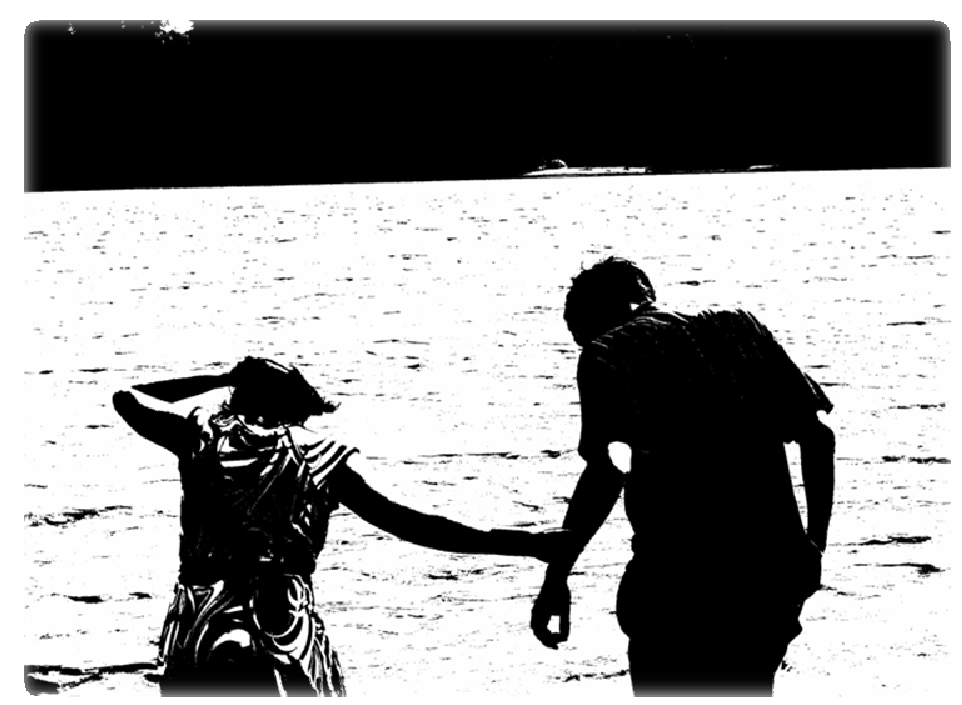

Mit diesem Zitat meines besten Freundes möchte ich die Arbeit schliessen. Er ist weder ein fachlicher Kollege, noch war inm bewusst, welch treffende Aussage er, auch für meine Arbeit, getroffen hat.

Neben all den vorhandenen komplexen psychodynamischen Erklärungsmodellen für die Entstehung somatoformer aber auch jeglicher anderer psychischer Störungen, liefert diese kurze und einfache Aussage aus meiner Sicht das Wesentliche:

Unser aller Sehnsucht nach Kontakt, Geborgenheit, konstanter Zuneigung, bedingungsloser Liebe, Sicherheit, Vertrauen und Verständnis.

Beobachtet man psychotherapeutische Behandlungen und jede andere Form der psychosozialen Hilfe, so hat schon Grawe (2000) herausgestellt, dass die Beziehung den wichtigsten Wirkfaktor für erfolgreiche psychosoziale Interventionen darstellt. 
Heute scheint die Sehnsucht nach solch tiefen, zuhörenden Beziehungen unendlich gross zu sein. In Kliniken und Therapien werden die vermissten Beziehungen gekauft, weil sie notwendig sind, um geheilt zu werden.

Natürlich ist dies auch meine Arbeit und alle Helfer sind auf sehnsüchtige Patienten angewiesen, um selbst überleben zu können.

Dennoch erschüttern mich der dramatische Anstieg psychischer Störungen und die damit einhergehende psychosoziale Unterversorgung vieler Betroffener sehr.

Warum verarmen wir anscheinend so massiv an echten, elterlichen und (be)schützenden Beziehungen?

Warum gehen so viele Menschen, insbesondere alte Leute, so häufig zu ihrem Hausarzt?

Wieso ist die Quote der erheblichen Inanspruchenahme medizinischer Dienste gerade in Deutschland so groß? In einer Fernsehdokumentation des ZDF wurde referiert, dass in keinem anderen Land der Welt die Menschen so häufig zum Arzt gehen wie in Deutschland.

Sind wir so hilfebedürftig?

Ich glaube: ja.

Menschen, die selbst wenig schützende Geborgenheit erfahren haben, sind nicht, oder nur mit Hilfe intensiver, langjähriger und reflektierender Arbeit an sich selbst, in der Lage solch ein Objekt für ihre Kinder darzustellen. Ein Teufelskreis, der eine Gesellschaft hervorbringen wird, in der immer mehr Menschen unsicher gebunden sein und an echtem Kontakt zu anderen und zu sich selbst verarmen werden.

Es mag ein wenig abstrakt klingen, aber ich bin der Überzeugung, dass gesunde Liebe heilt und wohl die beste Form der Prävention ist, die wir kostengünstig durchführen könnten.

Wie dies allerdings umzusetzen wäre, erscheint mir als ein gesellschaftliches Großprojekt, dem ich in dieser Dissertation wohl nicht mehr gerecht werden kann. 
Eine weiterführende Arbeit zum Thema: „Was fehlt Menschen mit psychischen Störungen eigentlich wirklich"?, der Frage nachzugehen, ob sich manch detaillierte Komplexität innerhalb der ätiologischen Ansätze nicht letztlich auf den einen Faktor, „Liebe“ herunterbrechen liesse, schwebt mir bereits als nächste Idee einer neuen herausfordernden und visionären Forschungsarbeit vor Augen. 


\section{$8 \quad$ Literatur}

Ahrens, S./Schneider, W. (2002). Lehrbuch der Psychotherapie und Psychosomatischen Medizin. 2.Auflage. Stuttgart: Schattauer.

Ainsworth, M.D.S./Blehar, M.C.Waters, E.Mall, S. (1978). Patterns of attachment. A psychological study of the strange. Hillshade, NJ: Erlbaum.

Albus, C./Herrmann-Lingen, C. (2007). Funktionelle Störungen in der Kardiologie. Psychosom Konsiliarpsychiatr; 1: 118-122.

American Psychiatric Association. (1994). Diagnostic and statistical Manual of Mental Disorder (4th edition). Washington,DC: APA.

Antor, G./Bleidick, U. (2001). Handlexikon der Behindertenpädagogik. Schlüsselbegriffe aus Theorie und Praxis. Stuttgart: Kohlhammer.

Arbeitskreis Klinische Psychologie in der Rehabilitation BDP. (1998). Somatoforme Störungen - Diagnostik und Therapie in der Rehabilitation. Beiträge zur 17. Jahrestagung des Arbeitskreises Klinische Psychologie in der Rehabilitation. Bonn: Deutscher Psychologen Verlag.

Bass, C/Murphy, M. (1995). Somatoform and personality disorders: Syndromal comorbidity and overlapping developmental pathways. J Psychosom Res; 39: 403-427.

Bauriedl, T. (1998). Ohne Abstinenz stirbt die Psychoanalyse. Über die Unvereinbarkeit von Psychoanalyse und Körpertherapie. Forum Psychoanal; 14: 325-341.

Behr, M./Becker, M. (2004). SEE Skalen zum Erleben von Emotionen. Manual. Göttingen: Hogrefe.

Bengel, J. (1998). Rehabilitation. In: Buddeberg, C. (Hrsg.) Lehrbuch Psychosoziale Medizin. 3. Auflage. Heidelberg. Springer.

Bengel, J./Strittmatter/R./Willmann, H. (1998). Was erhält den Menschen gesund? Antonovskys Modell der Salutogenese - Diskussionsstand und Stellenwert. Schriftenreihe der BZgA: Forschung und Praxis der Gesundheitsförderung. Band 6. Köln.

Bengel, J./Beutel, M./Broda, M./Haag, G./Härter, M./Lucius-Hoene, G./Muthny, FA./ Potreck-Rose, F./Stegie, R.Mels, J. (2003). Chronische Erkrankungen, psychische Belastungen und Krankheitsbewältigung. Herausforderungen für eine psychosoziale Versorgung in der Medizin. Psychother Psych Med; 53: 83-93.

Bortz, J., Döring, N. (2004). Statistik für Human- und Sozialwissenschaftler. 6. Aufl., Heidelberg: Springer.

Bortz, J., Döring, N. (2005). Forschungsmethoden und Evaluation für Humanund Sozialwissenschaftler. 4.Aufl., Heidelberg: Springer. 
Bowlby, J. (1984). Bindung. Frankfurt am Main: Fischer TB.

Bräutigam, W. /Christian, P./von Rad, M. (1997). Psychosomatische Medizin. Ein kurzgefasßtes Lehrbuch. 6.unveränderte Auflage. Stuttgart: Thieme.

Bredenkamp, J. (1969). Experiment und Feldexperiment. In: Grauman, C.F. (Hrsg). : Handbuch der Psychologie. Band 7/1: Sozialpsychologie. Göttingen: Hogrefe.

Brisch, K.H./Grossmann, K.E./Grossmann, K./Köhler, L. (Hrsg.) (2002). Bindung und seelische Entwicklungswege. Grundlagen, Prävention und klinische Praxis. Stuttgart: Klett-Cotta.

Brockmann, J./Schlüter, T./Eckert, J. (2003). Therapieziele, Zieländerungen und Zielerreichung im Verlauf psychoanalytisch orientierter und verhaltenstherapeutischer Langzeittherapie. Eine vergleichende Untersuchung aus der Praxis niedergelassener Psychotherapeuten. Psychother Psych Med; 53: 163-170.

Burger, S. (1996). Ökonomische Analyse der medizinischen Rehabilitation. Campus Verlag, Frankfurt, New York.

Campbell, D.T., Stanley, J.C. (1963). Experimental and quasieexperimental designs for research. Chicago: Rand McNally.

Caplan, G. (1963). Principles of preventive psychiatry. New York. Basic Books.

Comer, J.R/ (2001). Klinische Psychologie. Berling: Spektrum.

Conroy, R.M/Smith, O./Siriwardena, R./Fernandes, P. (1999). Health anxiety and characteristics of self-initiated general practioner consultations. J Psychosom Res; 46: 45-50.

Csef, H. (2001). Funktionelle (somatoforme) Störungen beim internistischen Hausbesuch. Der Internist; 11: 1476-1480.

Cuntz, U. (1998). Patients with Somatization Symptoms in General Practice - the Danger of Chronification or the Opportunity to Improve. In: Verhaltenstherapie, 8: 94-100.

Delbrück, E., Haupt, E. (Hrsg.). (1998). Rehabilitationsmedizin: ambulant, teilstationär, stationär. 2. Aufl. München. Urban \& Schwarzenberg.

Dilling, H./Mombour, W./Schmidt, M.H./Schulte-Markwort, E.(Hrsg.) (1994). Internationale Klassifikation psychischer Störungen. ICD-10 Kapitel V (F) Forschungskriterien. Bern: Verlag Hans Huber, Hogrefe AG.

Dilling, H./Mombour, W./Schmidt, M.H. (2010). Internationale Klassifikation psychischer Störungen. ICD-10 Kapitel V (F) Klnisch-diagnostische Leitlinien. 7., überarbeitete Auflage. Bern: Verlag Hans Huber, Hogrefe AG.

Ebel, H./Podoll, K. (1998). Komorbidität von somatoformen Störungen mit anderen psychischen Störungen. In: Somatoforme Störungen. Rudolf G., Henningsen P. (Hrsg.) Stuttgart: Schattauer. 
Eckert, J./Reimer, C./Strauß, B. (2007). Patientenziele in der Psychotherapie. Psychotherapeut; 52: 6-15.

Eckhardt-Henn, A./Tschan, R./Best, C./Dieterich, M. (2009). Somatoforme Schwindelsyndrome. Nervenarzt; 80: 909-917.

Egle, U.T./Hardt, J./Franz, M./Hoffmann, S.O. (2002a). Psychosoziale Belastungen in der Kindheit und Gesundheit im Erwachsenenalter. Möglichkeit der Prävention in der Psychosomatischen Medizin. Psychotherapeut; 47: 124127.

Egle, U.T./Hardt, J./Nickel, R./Kappis, B./Hoffmann, S.O. (2002b). Früher Stress und Langzeitfolgen für die Gesundheit - Wissenschaftlicher Erkenntnisstand und Forschungsdesiterate. Z. Psychosom. Med. Psychother; 48: 411-434.

Fäh, M./Fischer,G. Sinn und Unsinn in der Psychotherapieforschung . Eine kritische Auseinandersetzung mit Aussagen und Forschungsmethoden. (1998). Gießen: Psychosozial Verlag.

Felitti, V.J. (2002). Belastungen in der Kindheit und Gesundheit im Erwachsenenalter: Die Verwandlung von Gold in Blei. Z.Psychosom. Med. Psychother; 48: 359-369.

Fengler, J. (2003). Triffst Du nur das Zauberwort. Die Konstruktion wirksamer therapeutischer Interventionen. Report Psychologie; 28: 446-452.

Ferroni, B., Studer, HP. (2007). Komplementärmedizin in: Gesundheitswesen Schweiz 2007-2009. Bern. Hans Huber.

Fink, P./Sorensen, L./Engberg, M./Holm, M./Munk-Jorgensen, P. (1999). Somatization in primary care: prevalance, health care utilization, and general practioner recognition. Psychosomatics; 40: 115-130.

Fink, P./Rosendal, M./Toft, T. (2002). Assesment and Treatment of Functional Disorders in General Practice: The Extended Reattribution and Management Model - An Advanced Educational Program for Nonpsychiatric Doctors. Psychosomatics; 43: 93-131.

Fliegel, S./Kämmerer, A. (2006). Psycho-Therapeutische Schätze. 101 bewährte Übungen und Methoden für die Praxis. Tübingen: dgvt-Verlag.

Flückiger, C.Müsten, G. (2008). Ressourcenaktivierung. Ein Manual für die Praxis. Bern: Verlag Hans Huber, Hogrefe AG.

Franzkowiak, P. (2003). Prävention. In: BZgA (Hrsg). Leitbegriffe der Gesundheitsförderung. Schwabenheim a.d. Selz. Fachverlag Peter Sabo.

Friedlmeier, W./Holodynski, M. (1999). Emotionale Entwicklung. Funktion, Regulation und soziokultureller Kontext von Emotionen. Heidelberg, Berlin: Spektrum. 
Fritzsche, K./Larisch, A./Cierpka, M./Wirsching, M. (2004). Improving the biopsychosocial competence of german primary care physicians in diagnosing and treating somatoform disorders. Families, Systems \& Health; Vol 22, No3: 352364.

Fritzsche, K./Larisch, A./Schweickhardt, A. (2005). Psychotherapeutische Kurzintervention bei somatisierenden Patienten im Krankenhaus. Leitfaden für Psychotherapeuten im Rahmen des psychsomatischen Konsil- und Liaisondienstes. Psychotherapeut; 50: 404-414.

Fritzsche, K./Burghard, H.M/Schweickhardt, A./Wirsching, M. (2006). Was bewirken hausärztliche Interventionen bei Patienten mit psychischen Störungen? Eine systematische Literaturübersicht. Z Psychosom Med Psychother; 52: 4-22.

Froom, P., Benbassat, J. (2000). Inconsistencies in the Classification of Preventive Interventions. Preventive Medicine; 31: 153-158.

Fürstenau, P. (2007). Psychoanalytisch verstehen. Systemisch denken. Suggestiv intervenieren. Stuttgart: Pfeiffer bei Klett-Cotta.

Gerspach, M. (2009). Psychoanalytische Heilpädagogik. Ein systematischer Überblick. Stuttgart: Kohlhammer.

Grabe, H.J./Freyberger, J. (2003a). Körperliche Symptomatik ohne Ursache Somatisierungsstörung? In: Psychoneuro.; 29, 513-517.

Grabe, H.J/Meyer, C./Hapke, U./Rumpf, H.J./Freyberger, H.J./Dilling, H./John,U. (2003b). The Somatoform Pain Disorder in the Community. Psychotherapy and Psychosomatics; 72: 8-94.

Grabe, H.J./Meyer, C./Hapke, U./Rumpf, H.J./Freyberger, H.J./Dilling, H./John, U. (2003c). The "Specific Somatoform Disorder" in the General Population. Psychosomatics; 44: 304-311.

Gräfe, K/Quenter, A/Buchholz, C/Wild, B./Zipfel, S/Herzog, W/Löwe, B. (2001). Der Gesundheitsfragebogen für Patienten (PHQ-D) - Wie gut diagnostiziert ein Screeningfragebogen psychische Störungen? Nervenarzt; 20, 99.

Grawe, K. (2000). Psychologische Psychotherapie. Seattle: Hogrefe.

Grawe, K. /Donati, R./Bernauer, F. (2001). Psychotherapie im Wandel. Von der Konfession zur Profession. 5. unveränderte Auflage. Göttingen: Hogrefe.

Härter, M./Koch, U. (2000). Psychosoziale Dienste im Krankenhaus. Göttingen. Verlag für angewandte Psychologie.

Hargens, J./ Eberling, W. (2003). Einfach kurz und gut Teil 2. Ressourcen erkennen und nutzen. Dortmund: Verlag modernes lernen, Borgmann KG.

Haupt, E./Delbrück, H. (1996). Grundlagen der Rehabilitation. In: Delbrück, H., Haupt, E. (Hrsg) Rehabilitationsmedizin. München. Urban \& Schwarzenberg.

Hellerich, G. (2003). Selbsthilfe Psychiatrie-Erfahrener. Potenziale und Ressourcen. Bonn: Psychiatrie-Verlag GmbH. 
Henningsen, P. (1998). Somatisierung und Affektregulation: Elemente eines interpersonellen Modells. In: Rudolf, G/Henningsen, P (Hrsg.): Somatoforme Stöungen. Theoretisches Verständnis und therapeutische Praxis. Stuttgart: Schattauer.

Henningsen, P. (2000). Vom Gehirn lernen? Zur Neurobiologie von psychischer Struktur und innerer Repräsentanz. Forum Psychoanal; 16: 99-115.

Henningsen, P. (2002a). Körper und psychische Struktur: Anmerkungen zur Psychosomatik der Psyche. In: Rudolf, G./Grande, T./Henningsen, P. (Hrsg.) Die Struktur der Persönlichkeit. Vom theoretischen Verständnis zur psychotherapeutischen Anwendung des psychodynamischen Strukturkonzepts. Stuttgart: Schattauer.

Henningsen, P., Hartkamp, N., Loew, T., Sack, M., Scheidt, T., Rudolf, G. (2002b).Somatoforme Störungen. Leitlinien und Quellentext. Stuttgart: Schattauer.

Henningsen, P. (2007). Somatoforme Störungen: Patienten mit anhaltenden, organisch nicht ausreichend erklärbaren Körperbeschwerden. In: Rudolf, G., Henningsen, P. (Hrsg.) Psychotherapeutische Medizin und Psychosomatik, 6. Aufl. Stuttgart: Thieme.

Herzog, G. (2007). Klinische Interviews und psychologische Testverfahren in der Diagnostik somatoformer Störungen. Psychosom Konsiliarpsychiatr; 1: 97-105.

Hessel, A./Geyer, M./Gunzelmann, T./Schumacher, J./Brähler, E. (2003). Somatoforme Beschwerden bei über 60-Jährigen in Deutschland. Z.Gerontol Geriat; 36: 287-296.

Hiller, W./Rief, W. (1998). Therapiestudien zur Behandlung von Patienten mit somatoformen Störungen: Ein Literaturüberblick. In: Verhaltenstherapie; 8: 125136.

Hiller, W./Janca, A. (2003). Assesment of somatoform disorders: a review of strategies and instruments. Blackwell Munksgaard; 15: 167-179.

Hiller, W./ Rief, W. (2004a). Internationale Skalen für Hypochondrie. Deutschsprachige Adaption des Whiteley-Index (WI) und der Illness Attitude Scales (IAS). Bern: Hans Huber.

Hiller, W./Kroymann, R./Leibbrand, R./Cebulla, M./Korn, H.J./Rief, W./Fichter, M.M. (2004b). Wirksamkeit und Kosten-Nutzen-Effekte der stationären Therapie somatoformer Störungen. Fortschritte der Neurologie und Psychiatrie; 72: 136146.

Hofer, M.A. (1994). Early realtionships as regulators of infant physiology and behavior. Acta Paediatr Suppl; 397: 9-18.

Hotopf, M. (2004). Preventing somatization. Psychological Medicine. Cambridge University Press.; 34: 195-198. 
Hurrelmann, K./Klotz, T./Halsch, J. (2004). Lehrbuch Prävention und Gesundheitsförderung. Huber, Bern.

Isaac, M./Janca, A./Burke, K.C./Costa e Silva, J.A./Acuda, S.W./Altamura, A.C./Burke jr, J.D./Chandrashekar, C.R./Mirando, C.T-/Tacchini, G. (1995). Medically unexplainend somatic symptoms in different cultures. A preliminary report from phase I of the World Health Organization International Study of Somatoform Disorders. Psychother Psychosom; 64: 88-93.

Jacobi, F./Klose, M./Wittchen, H.U. (2004). Psychische Störungen in der deutschen Allgemeinbevölkerung: Inanspruchnahme von Gesundheitsleistungen und Ausfalltage. Bundesgesundheitsbl-Gesundheitsforsch-Gesundheitsschutz; 47: 736-744.

John, U., et al. Study of Health In Pomerania (SHIP). (2001). A health examination survey in an east German region: objectives and design. Sozial- und Präventivmedizin; 46: 186-94.

Kapfhammer, H.P. (2001a). Somatoforme Störungen. Historische Entwicklung und moderne diagnostische Konzeptualisierung. Nervenarzt; 27: 487-500.

Kapfhammer, H.P (2001b). Somatisierung - somatoforme Störungen Ätiopathogenetische Modelle. Fortschr Neurol Psychiat; 69: 58-77.

Kapfhammer, H.P (2007). Somatoforme Störungen. Konzept, Klinik, Ätiopathogenese und Therapie. Nervenarzt; 79: 99-117.

Katon, W./Korff, M. von/Lin, E./Lipscomb, P. et al. (1990). Distressed high utilizers of medical care_ DSM-III-R diagnoses and treatment needs. Gen Hosp Psychiatry; 12: 355-362.

Kholmogorova, A./Garanjan, N. (2001). Verknüpfung kognitiver und psychodynamischer Komponenten in der Psychotherapie somatoformer Erkrankungen. In: Psychother Psychosom med Psychol; 51, 212-218.

Klosterkötter, J./Maier, W. (2003). Früherkennung und Frühintervention bei psychischen Störungen: Ansätze zur Prävention und zur Vermeidung von Chronfizierungen. Deutsches Ärzteblatt; 100: 34-35.

Koch, U./Lucius-Hoene, G./Stegie, R. (Hrsg). (1988). Handbuch der Rehabilitationspsychologie. Heidelberg. Springer.

König, HH./Riedel-Heller, S. (2008). Prävention aus dem Blickwinkel der Gesundheitsökonomie. Internist; 49: 146-153.

Kroenke, K/Spitzer, R.L./Williams, J.B. (2001). The PHQ-9. Validity of a brief depression serverity measure. J Gen Intern Med; 16: 606-613.

Kroenke, K./Spitzer, R.L./Williams, J.B.W. (2002). The PHQ-15: Validity of a New Measure for Evaluating the Severity of Somatic Symptoms. Psychosomatic Medicine; 64: 258-266. 
Kruse, J./Heckrath, C../Tree, W. (1998). Die Diagnose psychogener Erkrankungen in der hausärztlichen Praxis. Problematik-AnalyseFortbildungskonzept. Psychotherapeut; 43: 164-170.

Kruse, J./Schmitz, N./Wöller, W./Heckrath, C./Tress, W. (2004): Warum übersieht der Hausarzt die psychischen Störungen seiner Patienten? In: Psychother Psych Med; 54: 45-51.

Kruse,J. (2008). Der Körper als Botschafter. Konzepte der psychodynamischen Psychotherapie. Aus der Praxis.PID; 3: 217-221.

Kurth, R.A./Pokorny, D./Körner, A./Geyer, M. (2002). Der Beziehungs-MusterFragebogen (BeMus): Validierung anhand einer repräsentativen Bevölkerungsstichprobe. Psychother Psych Med; 52: 179-188.

Kurth, R.A./Leweke, F./Milch, W./Brosig, B. (2007). Affektverarbeitung und Beziehungsgestaltung bei somatoformen Störungen. Eine Einzelfallstudie. Psychotherapeut; 52: 180-186.

Lamnek, S. (2005). Qualitative Sozialforschung. Lehrbuch. 4.Auflage. Weinheim, Basel: Beltz.

Larisch, A./Fisch, V./Fritzsche, K. (2005). Kosten-Nutzen-Aspekte psychosozialer Interventionen bei somatisierenden Patienten in der Hausarztpraxis. Zeitschrift für Klinische Psychologie und Psychotherapie; 34 (4): 282-290.

Leavall, HR./Clark, EG. (1965). Preventive Medicine for the doctor in his community. McGraw-Hill, New York.

Lotz, D. (1988). Heilpädagogik - Was ist das? In: BHP - Informationen: Heilpädagogik - was ist das?; 3: 1-8.

Löwe, B./Gräfe, K./Quenter, A./Zipfel, S./Herzog, W. (2002a). Diagnostik depressiver Störungen: Validität von Arztdiagnosen und drei Screeninginstrumenten im Vergleich. In: Balck F, Berth H, Dinkel A (Hrsg.) Medizinpsychologie.com. State of the Art der Medizinischen Psychologie. Lengerich: Pabst.; 52: 104-105.

Löwe, B./Gräfe, K/Quenter, A./Buchholz, C./Zipfel, S./Herzog, W. (2002b). Screening psychischer Störungen in der Primärmedizin: Validierung des "Gesundheitsfragebogens für Patienten (PHQ-D)“. Psychother Psych Med; 52: 104-105.

Martin, A./Korn, H.J/Cebulla, M./Saly, M./Fichter, M.M/Hiller, W. (2007). Kausalattributionen von körperlichen Beschwerden bei somatoformen Störungen. Zeitschrift für Psychiatrie, Psychologie und Psychotherapie; 55: 31-41.

Mertens, D. (1992). Das Andere in der Forschung. Nürnberg: DA Verlag. Das Andere $\mathrm{GmbH}$. 
Mertens, W. (2008). Einige Überlegungen zur randomisierten klinischen Studie („RTC). Zur Evidenzbasierung und zur Postulierung der Evidenzgrade. Department Psychologie. Klinische Psychologie und Psychotherapie. Abt. Psychoanalyse und psychodynamische Forschung. Ludwig-MaximilliansUniversität München.

Meyer, C. /Rumpf, H.J./Hapke, U./Dilling, H./John, U. (2000). Lebenszeitprävalenz psychischer Störungen in der erwachsenen Allgemeinbevölkerung. Ergebnisse der TACOS-Studie. Nervenarzt; 71: 535-542.

Michalak, J./Grosse Holtforth, M./Berking, M. (2007). Patientenziele in der Psychotherapie. In: Psychotherapeut; 52: 6-15.

Möhring, P. (1997). Körperlich Kranke in psychoanalytischer Psychotherapie. Zschr psychosom Med; 43: 57-79.

Morriss, R.K./Gask, L./Ronalds, C./Downes-Grainger, E./Thompson, H./Goldberg, D. (1999). Clinical and patient satisfaction outcomes of a new treatment for somatized mental disorder taught to general practioners. BR J Pract; 49: 263-267.

Morschitzky, H. (2002). Somatoforme Störungen. Diagnostik, Konzepte und Therapie bei Körpersymptomen ohne Organbefund. Wien: Springer.

Moser, T. (1999). Der Körper und die Psychoanalyse. Erwiderung auf Thea Bauriedl. Forum Psychoanal; 15: 167-174.

Motzkau, H., Rudolf, G. (1997). Biographie und Krankheit. Belastende Ereignisse und Faktoren pathogener Sozialisation bei psychisch und psychosomatisch Kranken. Kölner Zeitschrift für Soziologie und Sozialpsychologie; 49: 674-701.

Mück, H. (2005) Was ist „Strukturbezogene Psychotherapie“?. Köln. http://www.dr-mueck.de/HM FAQ/Strukturbezogene-Psychotherapie.htm (19.02.10)

Nanke, A./Rief, W. (2003). Zur Inanspruchnahme medizinischer Leistungen bei Patienten mit somatoformen Störungen. In: Psychotherapeut; 5- 2003: 83-91.

Nickel, R./Egle, UT. (2001 ). Manualisierte psychodynamisch-interaktionelle Gruppentherapie. Klinik für Psychosomatische Medizin und Psychotherapie der Johannes Gutenberg-Universität Mainz. Psychotherapeut; 48: 11-18.

Nissen, G. (2002). Psychosomatische Störungen. Ursachen - Erkennung Behandlung. Stuttgart, Berlin, Köln: Kohlhammer.

Oerter, R./Montada, L. (2002). Entwicklungspsychologie. 5. vollständig überarbeitete Auflage. Weinheim, Basel, Berlin: Beltz.

Pfrengle, A. (2004). Affektausdruck und Beziehungsregulation. Eine qualitativ empirische Studie über Patienten mit somatoformen Störungen. München: Martin Meidenbauer.

Pilkowsky, I. (1967). Dimensions of hypochondriasis. British Journal of Psychiatry; 113: 89-93. 
Potreck-Rose, F. Analysen zur Chronifizierung und Chronizität psychosomatischer Erkrankungen. (1995). Universität Freiburg/Br. Habilitationsschrift.

Potreck-Rose, F./Koch, U. (1994). Chronifizierungsprozesse bei psychosomatischen Patienten. Ergebnisse einer Expertise. Stuttgart. Schattauer.

Rief, W./Hiller, W. (1992). Somatoforme Störungen. Bern: Hans Huber.

Rief, W. (1995). Multiple somatoforme Symptome und Hypochondrie. Bern: Hans Huber.

Rief, W. (1998). Somatoforme Störungen - ein Überblick. In: Magraf, J./Neumer, S./Rief, W. (Hrsg.), Somatoforme Störungen. Ätiologie, Diagnose und Therapie. Berlin: Springer.

Rief, W./Fichter, MM. (1998). Somatoforme Störungen - Spiegel des modernen Gesundheitsverständnisses und Herausforderung für das Gesundheitswesen. Verhaltenstherapie; 8: 78-80.

Rief, W./Hessel, A./Braehler, E. (2001). Somatization symptoms and hypochondriacal features in the general population. Psychosom Med; 63: 595602.

Rief, W./Bleichhardt,G./Timmer, B. (2002). Gruppentherapie für somatoforme Störungen - Behandlungsleitfaden, Akzeptanz und Prozessqualität. Verhaltenstherapie; 12: 183-191.

Rief, W./Hiller, W. (2008). SOMS Screening für Somatoforme Störungen. Manual. 2., vollständig überarbeitete und neu normierte Auflage. Bern: Hans Huber, Hogrefe AG.

Rosenbrock, R./Kümpers, S. (2006). Zur Entwicklung von Konzepten und Methoden der Prävention. Psychotherapeut; 51: 412-420.

Rossa, B./Breull, A. (2004). Somatoforme Störungen in der Allgemeinmedizin. In: Z.allg.Med; 80: 282-288.

Rudolf, G. (1992). Körpersymptome als Schwierigkeit in der Psychotherapie. Praxis Psychother Psychosom; 37: 11-23.

Rudolf, G./Motzkau, H. (1997). Die Auswirkungen von biographischen Belastungen auf die Gesundheit von erwachsenen Frauen und Männern. Zeitschrift Psychomed, 43: 349-368.

Rudolf, G. (1998). Der Prozess der depressiven Somatisierung. In: Rudolf, G/Henningsen,P. (Hrsg). Somatoforme Störungen. Theoretisches Verständnis und therapeutische Praxis. Stuttgart: Schattauer.

Rudolf, G./Grande,T./Henningsen, P. (2002). Die Struktur der Persönlichkeit. Theoretische Grundlagen zur psychodynamischen Therapie struktureller Störungen. 
Rudolf, G., Henningsen, P. (2003) Psychotherapeutische Behandlung somatoformer Störungen. Z.Psychosom.Med.Psychother; 49: 3-19.

Rudolf, G. (2004). Individuelle und soziokulturelle Einflüsse auf die Entwicklung somatoformer Schmerzstörungen. In: Sandweg, R. (Hrsg.) Chronischer Schmerz und Zivilisation. Organstörungen, psychische Prozesse und gesellschaftliche Bedingtheiten. Göttingen: Vandenhoeck \& Rupprecht.

Rudolf, G. (2006a). Strukturbezogene Psychotherapie. Leitfaden zur psychodynamischen Therapie struktureller Störungen. Stuttgart: Schattauer.

Rudolf, G. (2006b). Somatoforme Störungen. In: Reimer, Ch., Rüger, U. Psychodynamische Psychotherapien, 3. Aufl. Berlin, Heidelberg: Springer.

Rudolf, G. (2007). Strukturbezogene Psychotherapie der BorderlinePersönlichkeitsstörung. In: Dammann/Janssen: Psychotherapie der BorderlineStörungen. Stuttgart: Thieme.

Rudolf, G. (2008a). Psychosomatik - konzeptuelle und psychotherapeutische Aspekte. Universität Heidelberg.

Rudolf, G. (2008b). Depressive Somatisierung. In: Rudolf, G., Henningsen, P. (Hrsg.) Psychotherapeutische Medizin und Psychosomatik, 6. Aufl. Stuttgart: Thieme.

Rudolf, G. (2008c). Psycho-Somatik: Erklärungsmodelle somatoformer Störungen. In: Rudolf, G./Henningsen, P. (Hrsg.) Psychotherapeutische Medizin und Psychosomatik, 6. Aufl. Stuttgart: Thieme.

Rudolf, G. (2008d) Unfallreaktive Somatisierungsstörungen. In: Rudolf, G./Henningsen, P. (Hrsg.) Psychotherapeutische Medizin und Psychosomatik, 6. Aufl. Stuttgart: Thieme.

Sachse, J./Strauß, B. (2002). Bindungscharakteristika und Behandlungserfolg nach stationärer psychodynamischer Gruppenpsychotherapie. Psychother Psych Med; 52: 134-140.

Sandweg, R. (2004). Chronischer Schmerz und Zivilisation. Organstörungen, psychische Prozesse und gesellschaftliche Bedingtheiten. Göttingen: Vandenhoeck \& Ruprecht.

Sauer, N./Eich, W. (2007). Somatoforme Störungen und Funktionsstörungen. Deutsches Ärzteblatt; 1-2007: 45-53.

Schäfert, R./Benedikt, G./Sauer, N./Wilke, S./Herzog, W./Szecscenyi, J./Zipfel, S./Henningsen, P. (2005). Früherkennung und Behandlung funktioneller/somatoformer Beschwerden in der Allgemeinarztpraxis. Notfall \& Hausarztmedizin 2005; 31.

Schäfert, R./Bölter, R./Faber, R./Kaufmann, C. (2008). Tangential, nicht frontal. Annäherung an eine schwierige Patientengruppe. PID;3: 1-8. 
Scheidt, C.E/Waller, E. (2005). Schmerz, Affekt und Bindung. Psychotherapie Forum; 13: 154-163.

Schneider, W. (2006). Gesundheitsverhalten und präventive Interventionen. Ausgewählte psychologische Aspekte. Psychotherapeut; 51: 421-432.

Schoepf, F. /Heun, R./Weiffenbach, O./Herrmann, S./Maier, W. (2003). 4Wochen-Prävalenz somatoformer Störungen und assoziierte psychosoziale Beeinträchtigung. Nervenarzt; 74: 245-251.

Schommer, N./Heilhammer, D. (2003). Psychobiologische Beiträge zum Verständnis stressbezogener Erkrankungen. In: Lehrbuch der Klinischen Psychologie und Psychotherapie: Modelle psychischer Störungen, Reinecker, H. Göttingen: Hogrefe.

Schoon, I. (2002). Die Langzeitwirkung sozial-ökonomischer Benachteiligung auf die psychosoziale Anpassung von Frauen. Z. Psychosom. Med. Psychother. ; 48: 381-395.

Schweickhardt, A./Larisch, A./Fritzsche, K. (2005). Differentiation of Somatizing Patients in Primary Care. Why the Effect of Treatment Are Always Moderate. The Journal of Nervous and Mental Disease; 193: 813-819.

Schweickhardt, A./Larisch, A./Wirsching, M./Fritzsche, K. (2007). Short-Term Psychotherapeutic Interventions for Somatizing Patiens in the General Hospital: A Randomized Controlled Study. Psychother Psychosom; 76: 339-346.

Seemann, H. (1998) Freundschaft mit dem eigenen Köper schließen. Über den Umgang mit psychosomatischen Schmerzen. München: Pfeiffer.

Slesina, W. (2007). Zur Bedeutung rehabilitativer Maßnahmen für die Prävention. Präv. Gesundheitsf; 2: 46-51.

Smith, GR./Monson, RA./ Ray, DC. (1986). Patients with multiples unexplained symptoms. Their characteristics, functional health, and health care utilization. Arch Intern Med; 146: 69-72.

Sonnenmoser, M. (2005). Behandlungserfolge nur interdisziplinär. In: Deutsches Ärzteblatt; 3: 122.

Spangler, G./Grossmann, K.E. (1993). Biobehavioral organization in securely and insecurely attached infants. In: Child Development; 64: 1439-1450.

Spangler, G./Zimmermann, P.(Hrsg.) (1995). Bindungstheorie. Grundlagen, Forschung und Anwendung. Stuttgart: Klett-Cotta.

Spangler, G. (1999a). Frühkindliche Bindungserfahrungen und Emotionsregulation. In: Friedlmeyer, W./Holodynsky, M. Emotionale Entwicklung. Funktion, Regulation und soziokultureller Kontext von Emotionen. Heidelberg, Berlin: Spektrum. 
Spangler, G./Zimmermann, P. (1999b). Die Bindungstheorie. Grundlagen, Forschung und Anwendung. 3. Auflage. Stuttgart: Klett-Cotta.

Speck, O. (1998). System Heilpädagogik. Eine ökologisch reflexive Grundlegung. 4.Aufl. München, Basel: Reinhardt.

Spitzer, R.L/Williams, J.B/ Kroenke, K./Linzer, M./deGruy, F.V,/Hahn, S.R. (1994). Utility of a new procedure for diagnosing mental disorders in primary care. The PRIME-MD 1000 study. JAMA; 272: 1749-1756.

Spitzer, R.L/Kroenke, K./Williams, J.B. (1999). Validation and utility of a selfreport version of PRIME-MD: the PHQ primary care study. JAMA; 282: 17371744.

Sroufe, L.A. (1996). Emotional development: the organization of emotional life in the early years. New York: Cambridge University Press.

Statstisches Bundesamt. (2002). Datenreport 2002. Zahlen und Fakten über die Bundesrepublik Deutschland. Bundeszentrale für politische Bildung, Bonn.

Steinhausen, O. (1997). Biographische Belastung und Bewältigungsstil bei Patienten mit chronifizierten somatoformen Störungen und Rententhematik. Dissertation an der Uniklinik Heidelberg.

Stern, D.N. (1992). Die Lebenserfahrung des Säuglings (Orginal erschienen 1986: The interpersonal world of the infant). Stuttgart: Klett-Cotta.

Strauß, B./Eckert, J./Tschuschke, V. (1996) Methoden der empirischen Gruppentherapieforschung. Ein Handbuch. Opladen: Westdeutscher Verlag $\mathrm{GmbH}$.

Tönnies, S./Breuer-Schneider, C./Schwieger, K. (1992). Psychologe und Arzt. Einstellung Hamburger Allgemeinmediziner und Klinischer Psychologen zu Gesundheitsversorgung und - politik, ihre berufliche Kooperation und gegenseitiges Berufsbild. Report Psychologie; 9: 31-30.

Trautner, H.M. (1978). Lehrbuch der Entwicklungspsychologie. Bd.1., Göttingen: Hogrefe.

Tschuschke, V. (2003). Kurzgruppenpsychotherapie. Theorie und Praxis. Wien: Springer.

Tschuschke, V. (2004). Gruppenpsychotherapie. In: Psychotherapeut; 49: 101109.

Tschuschke, V./Weber, R./Horn, E./Kiencke, P./Tress, W. (2007). Ambulante psychodynamische Kurzgruppenpsychotherapie bei Patienten mit somatoformen Störungen. In: Z.Psychiatr., Psychol.Psychother; 2: 87-95.

Tyrer, P./Fowler-Dixon, R./Ferguson, B./Keleman, A. (1990). A plea for the diagnosis of hypochondrial patients. Psychol Med; 23: 167-173.

Uexküll, von T./Adler, R.H/Herrmann, M./Köhle, K./Langewitz, W./Schonecke, O.W.Mesiack, W. (2003). Psychosomatische Medizin. Jena: Urban \& Fischer. 
US Preventive Services Task Force. (1996). Guide to clinical preventive services. Williams \& Wilkins, Baltimore.

VDR, Rehabilitationswissenschaftliche Abteilung (Hrsg). (2000). Aktiv Gesundheit fördern - Gesundheitsbildungsprogramm der Rentenversicherung für die medizinische Rehabilitation. Stuttgart. Schattauer.

Waller, E./Scheidt, C.E. (2002). Somatoforme Störungen und Bindungstheorie. Psychotherapeut; 47: 157-164.

Walter, U. (2003). Babylon im SGB? Eine Analyse der Begriffsvielfalt zur Prävention in den Sozialgesetzbüchern (http.dgvt.de)

Weber, R. (2004). Gruppenpsychotherapie bei Patienten mit somatoformen Störungen. In: Psychomed; 2: 83-91.

Werner, J. (1997). Lineare Statistik. Allgemeines lineares Modell. Weinheim: Beltz.

Wittchen, H./Sass, H./Zaudig, M./Kohler, K. (1989). Diagnostisches und Statistisches Manual psychischer Störungen (DSM-III-R). Weinheim: Beltz.

Wittchen, H.U. (2000). Bedarfsgerechte Versorgung psychischer Störungen. Abschätzungen aufgrund epidemiologischer, bevölkerungsbezogener Daten. Universität Dresden.

Wittchen, H.U/Jacobi, F. (2001). Die Versorgungssituation psychischer Störungen in Deutschland. Eine klinisch-epidemiologische Abschätzung anhand des Bundes-Gesundheitssurveys 1998. Bundesgesundheitsbl Gesundheitsforsch - Gesundheitsschutz; 44: 993-1000.

Wörterbuch der Sozialpolitik: http://www.socialinfo.ch/cgibin/discopossode/show.cfm?id=486

Wottawa, H./Thierau, H. (2003). Evaluation. 3. Auflage., Bern: Hans Huber.

Wottawa, H. (1996). Methoden der Evaluationsforschung. In: Erdfelder, E./Mausfeld, R./ Meiser, T./Rudinger, G. (Hrsg.). Handbuch quantitative Methoden. Weinheim: Beltz.

Yalom, I.D. (2005). Im Hier und Jetzt. Richtlinien der Gruppenpsychotherapie. Deutschland: Btb Verlag in der Verlagsgruppe Random House $\mathrm{GmbH}$.

Yates, B.J. (1994). Toward the incorporation of costs, costeffectiveness, analysis, and cost-benefit analysis into clinical research. J Consult Clin Psychol; 62: 729736.

Zielke, M./Kopf-Mehnert, C. (1978). VEV Veränderungsfragebogen des Erlebens und Verhaltens. Göttingen: Beltz Test GmbH. 
Zimmermann, T./Kaduszkiewicz, H. (2003). Funktionelle Körperbeschwerden in der Hausartzpraxis. In: Z.allg.Med; 79: 219-22.

Internetlink: Für die ICD-10: http://www.lumrix.de/icd.php?f=lumrixget $\& W=$ self $\& \mathrm{i}=0 \& \mathrm{r}=f \% 2 \mathrm{Ff} 48 . \mathrm{xml} \& \mathrm{t}=\mathrm{F} 48.0+$ Neurasthenie $\& \mathrm{~S}=\& \mathrm{~h}=1$ (13.02.10) 


\section{$9 \quad$ Tabellenverzeichnis}

Tabelle 1: $\quad$ Diagnostische Kriterien der Somatisierungsstörung 16 nach ICD-10

Tabelle 2: $\quad$ Diagnostische Kriterien der undifferenzierten

Somatisierungsstörung (UDS) nach ICD-10

Tabelle 3: $\quad$ Diagnostische Kriterien der hypochondrischen Störung (HS) nach ICD-10

Tabelle 4: $\quad$ Diagnostische Kriterien der somatoform autonomen Funktionsstörung (SAD) nach ICD-10

Tabelle 5: $\quad$ Diagnostische Kriterien der anhaltenden somatoformen Schmerzstörung (ASS) nach ICD-10

Tabelle 6: Diagnostische Kriterien sonstiger somatoformer Störungen nach ICD-10

Tabelle 7: $\quad$ Verwandte Störungen in der ICD-10

Tabelle 8: Strukturelle Aspekte der Somatisierung 25

Tabelle 9: Konflikt-Aspekte der Somatisierung 26

Tabelle 10: Psychodynamik des depressiven Grundkonflikts 27

Tabelle 11: Übersicht über bisherige gruppentherapeutische

Programme für Menschen mit somatoformen

Störungen im deutschsprachigen Raum

Tabelle 12: Hypothesen zur Entwicklung der körperlichen

Tabelle 13: Hypothesen zum Erleben und Verhalten

Tabelle 14: Hypothesen zum emotionalen Erleben

Tabelle 15: Hypothesen zur Arztbeziehung und dem Ärztehopping 65

Tabelle 16: Hypothesen zu den Einschätzungen und Angaben der 68 behandelnden Hausärzte

Tabelle 17: Hypothese zur Korrelation zwischen Resonanz- und

Beschwerdeerleben in den Gruppensitzungen

Tabelle 18: Hypothese zum Symptomerleben in Alltag und

Gruppe

Tabelle 19: Hypothesen zum narzisstischen Verhalten in Altag

und Gruppe

Tabelle 20: Hypothesen zur Psychotherapiebereitschaft 
Tabelle 21: Einschlusskriterien für die Teilnehmer der Studie 77 (nach der Einschätzung der behandelnden Hausärzte)

Tabelle 22: $\quad$ Gesamtübersicht deskriptive Daten der 78 Versuchsgruppe

Tabelle 23: Gesamtübersicht deskriptive Daten der Kontrollgruppe 79

Tabelle 24: Geschlechterverteilung in der Versuchsgruppe 79

Tabelle 25: Geschlechterverteilung in der Kontrollgruppe $\quad 79$

Tabelle 26: Beziehungsstatus: Häufigkeiten in der 79 Versuchsgruppe

Tabelle 27: Beziehungsstatus: Häufigkeiten in der Kontrollgruppe 80

Tabelle 28: Arbeitssituation: Häufigkeiten in der Versuchsgruppe 80

Tabelle 29: Arbeitssituation: Häufigkeiten in der Kontrollgruppe 80

Tabelle 30: Höchster Schulabschluss: Häufigkeiten in der 80 Versuchsgruppe

Tabelle 31: Höchster Schulabschluss: Häufigkeiten in der 80 Kontrollgruppe

Tabelle 32: $\quad$ Übersicht über Themen, Inhalte, Hauptziele und 82 allgemeine Ziele der Stunden

Tabelle 33: Gesamtübersicht über die geförderten Bereiche 83 innerhalb des gesamten Programms und den einzelnen Sitzungen

Tabelle 34: Übersicht der Bedeutungen hoher und niedriger Werte 96 in den Skalen der SEE

Tabelle 35: Auflistung der verwendeten statistischen Verfahren für 107 die Auswertung der Ergebnisse

Tabelle 36: Zeitlicher Verlauf der Untersuchung 113

Tabelle 37: Überprüfung der Vortest-Unterschiede zwischen 119 Versuchsgruppe und Kontrollgruppe nach den Ergebnissen des Pretests

Tabelle 38: Überprüfung der Normalverteilung der gesamten 120 Stichprobe im Pretest

Tabelle 39: Ergebnisse der Versuchsgruppe und der Kontrollgruppe in der erlebten Beeinträchtigung durch die Beschwerden (0-30)

Tabelle 40: $\quad$ Ergebnisse der Versuchsgruppe und der Kontrollgruppe in der erlebten Beschwerdeintensität (0-4)

Tabelle 41: Ergebnisse der Versuchsgruppe und der Kontrollgruppe für die Anzahl der erlebten Beschwerden 
Tabelle 42: Ergebnisse der Versuchsgruppe und der

Kontrollgruppe bezogen auf die Entwicklung der Krankheitsängste (0-7)

Tabelle 43: Ergebnisse der Versuchsgruppe und der

Kontrollgruppe bezogen auf die Entwicklung der erlebten Stärke der Beschwerden auf einer Skala von 0-10

Tabelle 44: Ergebnisse der Versuchsgruppe und der

Kontrollgruppe bezogen auf die Entwicklung der Akzeptanz eigener Emotionen (T-Wert 12-70)

Tabelle 45: Ergebnisse der Versuchsgruppe und der

Kontrollgruppe bezogen auf die Entwicklung des erlebten Mangels an Emotionen (T-Wert 28-91)

Tabelle 46: Ergebnisse der Versuchsgruppe und der

Kontrollgruppe bezogen auf die Entwicklung der Koppelung von körperlichen Beschwerden und psychischem Empfinden. (T-Wert 14-78).

Tabelle 47: Ergebnisse der Versuchsgruppe und der

Kontrollgruppe bezogen auf die Entwicklung der Selbsteinschätzung zur Wahrnehmung eigener Gefühle auf einer Skala von 0-10

Tabelle 48: Ergebnisse der Versuchsgruppe und der

Kontrollgruppe bezogen auf die Entwicklung des

Erlebens und Verhaltens im Alltag (42-294)

Tabelle 49: Korrelationsuntersuchung im Hinblick auf die erlebte Resonanz und der erlebten Beschwerdeintensität während den einzelnen Sitzungen

Tabelle 50: $\quad$ Ergebnisse der Versuchsgruppe der erlebten

Beschwerdestärke während einer ganzen Woche im Vergleich zur erlebten Beschwerdestärke während den Sitzungen auf einer Skala von 0-10

Tabelle 51: Ergebnisse der Versuchsgruppe und der

Kontrollgruppe bezogen auf die Entwicklung der Bewertung zu ihren behandelnden Hausärzten mit Schulnoten von 1-5

Tabelle 52: Ergebnisse der Versuchsgruppe und der Kontrollgruppe bezogen auf die Anzahl der Arztwechsel

Tabelle 53: Ergebnisse der Versuchsgruppe und der Kontrollgruppe zur Bewertung der Hausärzte über die Beziehung zu ihren Patienten mit Schulnoten von 1-5

Tabelle 54: Ergebnisse der Versuchsgruppe und der

Kontrollgruppe über die Anzahl der Arztkonsultationen 
Tabelle 55: Ergebnisse der Versuchsgruppe und der

Kontrollgruppe über das von den behandelnden Hausärzten angegebene Ausmaß an Forderungen nach weiteren medizinischen Untersuchungen auf einer Skala von 0-4

Tabelle 56: Ergebnisse der Versuchsgruppe und der

Kontrollgruppe bezogen auf die Entwicklung der

Psychotherapiebereitschaft auf einer Skala von 0-10

Tabelle 57: Ergebnisse der Versuchsgruppe für ein selbst

eingeschätztes narzisstisches Verhaltensmuster in den Gruppensitzungen im Vergleich zum Verhalten im Alltag auf einer Skala von 0-4

Tabelle 58: Ergebnisse der Versuchsgruppe und der

Kontrollgruppe im Beschwerdeindex Somatisierung nach dem SOMS

Tabelle 59: Häufigkeiten der Teilnahme an einer weiterführenden Gruppe nach Abschluss des offiziellen Programms der Patienten der Versuchsgruppe

Tabelle 60: Deskriptive Daten der Versuchsgruppe zur Durchführung einer Einzel-Psychotherapie

Tabelle 61: Deskriptive Daten der Kontrollgruppe zur Durchführung einer Einzel-Psychotherapie

Tabelle 62: Wie sehr empfehlen Probanden der Versuchsgruppe auf einer Skala von 0-10 anderen Menschen mit ähnlicher Problematik, die Teilnahme an diesem Programm

Tabelle 63: Wie sehr empfinden Probanden der Versuchsgruppe auf einer Skala von 0-10 die Teilnahme an diesem Programm als eine berechtigte und sinnvolle Ergänzung zu ihrer hausärztlichen Behandlung ihrer somatoformen Beschwerden?

Tabelle 64: Wie ernst genommen fühlen sich Probanden der Versuchsgruppe auf einer Skala von 0-10 von der Gruppenleitung?

Tabelle 65: Wie ernst genommen fühlen sich Probanden der Versuchsgruppe auf einer Skala von 0-10 von den anderen Gruppenmitgliedern?

Tabelle 66: Wie geht es den Probanden der Versuchsgruppe körperlich nach der Teilnahme am Gruppenprogramm im Vergleich zu vorher?

Tabelle 67: Wie geht es den Probanden der Versuchsgruppe psychisch nach der Teilnahme am Gruppenprogramm im Vergleich zu vorher? 
Tabelle 68: Wie nah sind die Probanden der Versuchsgruppe, ihrer persönlichen Einschätzung nach, ihrem vor dem Programm formulierten Alltagsziel gekommen?

Tabelle 69: Wie bewerten Probanden der Versuchsgruppe die 162 Dauer von zwei Monaten (acht Sitzungen) des Programms?

Tabelle 70: Wie bewerten Probanden der Versuchsgruppe die Dauer einer Sitzung a 90 Minuten?

Tabelle 71: Wie bewerten Probanden der Versuchsgruppe eine Gruppengröße von durchschnittlich acht Personen?

Tabelle 72: Als wie wichtig bewerten Proabnden der 163 Versuchsgruppe die Gestaltung und Atmosphäre des Gruppenraums, um sich öffnen zu können?

Tabelle 73: Wie bewerten Probanden der Versuchsgruppe das Verhältnis der Anteile von angeleiteten Übungen und freier Gruppensprechzeit? (Was wünschen sie sich?)

Tabelle 74: Offene Fragen und offene Antworten nach Beendigung des Programms 
Abbildung 1: Intrapsychische Aspekte der depressiven

Somatisierung

Abbildung 2: Interaktionelle Aspekte der depressiven

Somatisisierung

Abbildung 3: Psychophysiologische Aspekte der depressiven

Somatisierung

Abbildung 4: Nicht randomisierter Zwei-Gruppen-Plan mit Pretest, 86 Intervention, Posttest und Follow Up (quasiexperimentell)

Abbildung 5: Beispielabbildung zur Erfassung der erlebten

Veränderung des Erlebens und Verhaltens im Veränderungsfragebogen (VEV)

Abbildung 6: Graphische Darstellung: Mittelwertsvergleich zwischen Versuchs- und Kontrollgruppe bezogen auf die erlebte Anzahl der Beschwerden

Abbildung 7: Graphische Darstellung: Mittelwertsvergleich zwischen Versuchs- und Kontrollgruppe bezogen auf erlebten Krankheitsängste

Abbildung 8: Graphische Darstellung: Mittelwertsvergleich zwischen Versuchs- und Kontrollgruppe bezogen auf die erlebte Beschwerdestärke

Abbildung 9: Mittelwertsvergleich zwischen Versuchs- und

Kontrollgruppe bezogen auf die Entwicklung des

Erlebens und Verhaltens im Alltag (Richtung:

Entspannung/Gelassenheit/Optimismus oder Spannung/Unsicherheit/Pessimismus)

Abbildung 10: Graphische Darstellung: Mittelwertsvergleich zwischen Versuchs- und Kontrollgruppe bezogen auf die Entwicklung der Forderungen nach weiteren medizinischen Untersuchungen.

Abbildung 11: Graphische Darstellung: Mittelwertsvergleich zwischen Versuchs- und Kontrollgruppe bezogen auf die Entwicklung des BeschwerdeindexSomatisierung

Abbildung 12: Graphische Darstellung: Wie geht es den Probanden 161 der VG (A) körperlich nach der Intervention?

Abbildung 13: Graphische Darstellung: Wie geht es den Probanden 161 der VG (A) psychisch nach der Intervention? 
Abbildung 14: Graphische Darstellung: Wie nah sind die Probanden der VG $(A)$ ihrem vor der Itnervention formulierten Alltagsziel gekommen?

Abbildung 15: Graphische Darstellung der Bewertung der VG (A) 163 über den Anteil der angeleiteten Übungen und der freien Sprechzeit innerhalb des Gruppenprogramms. 


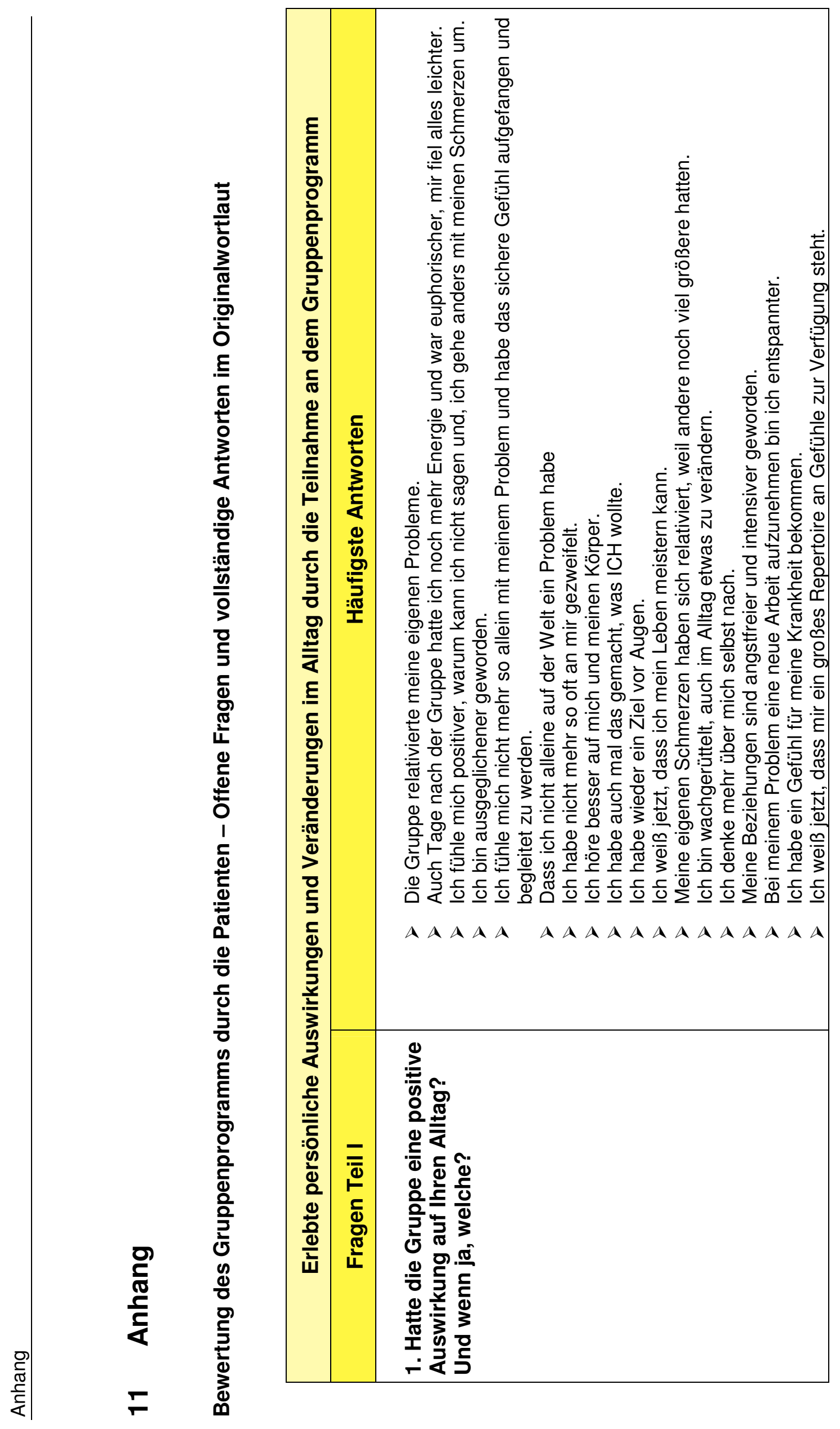

怘 


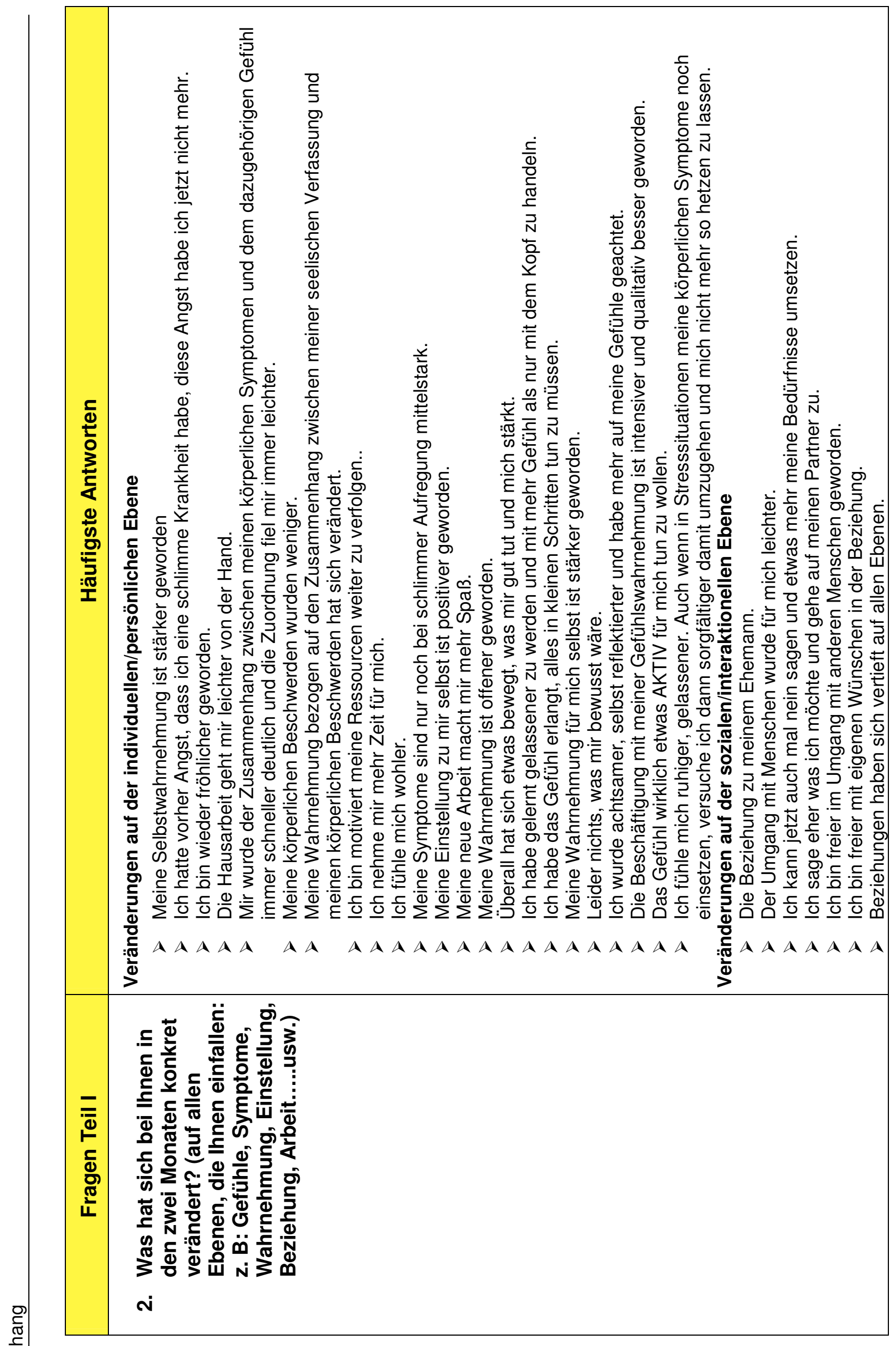

怘 


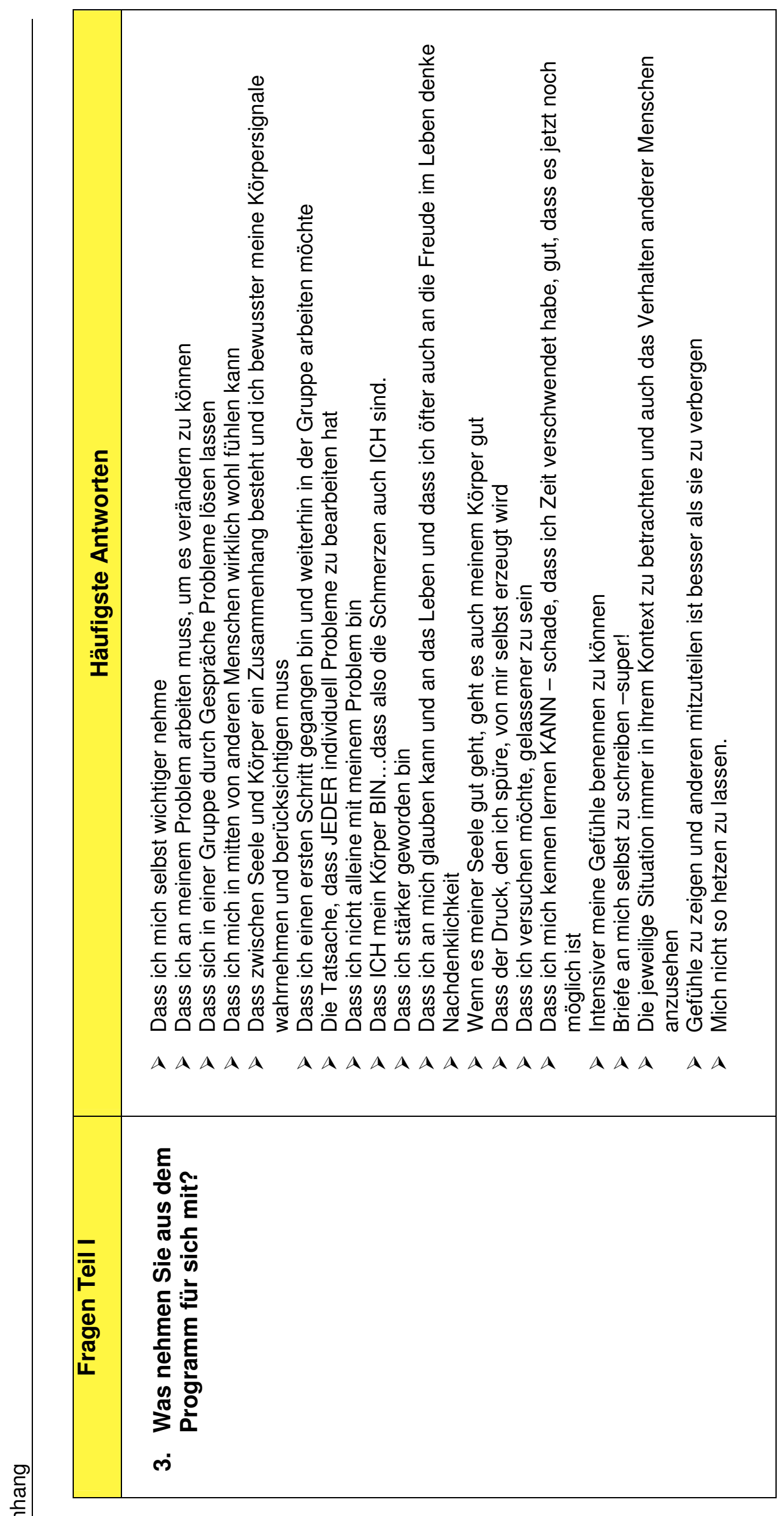

กิ 


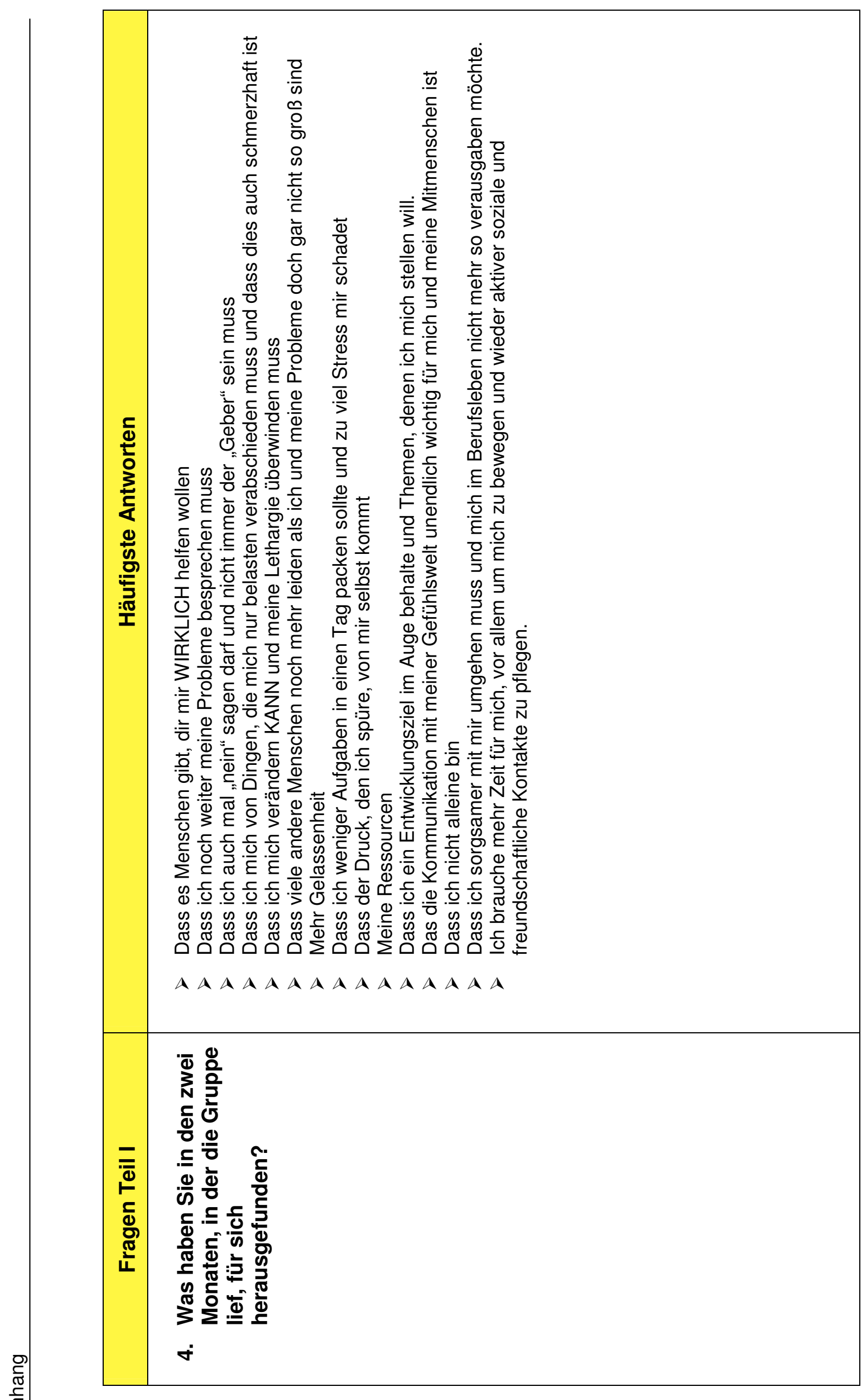

品 


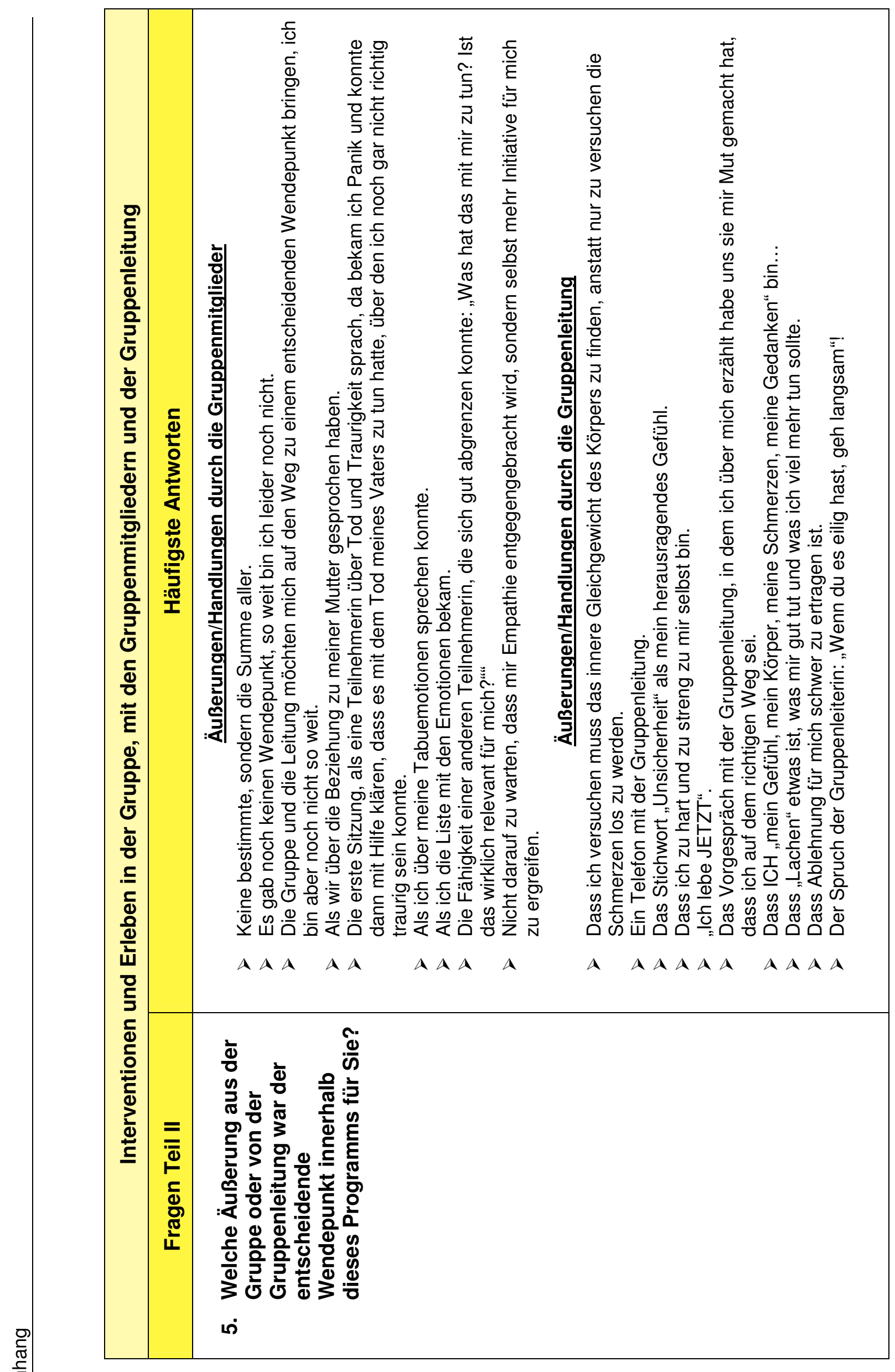

品 


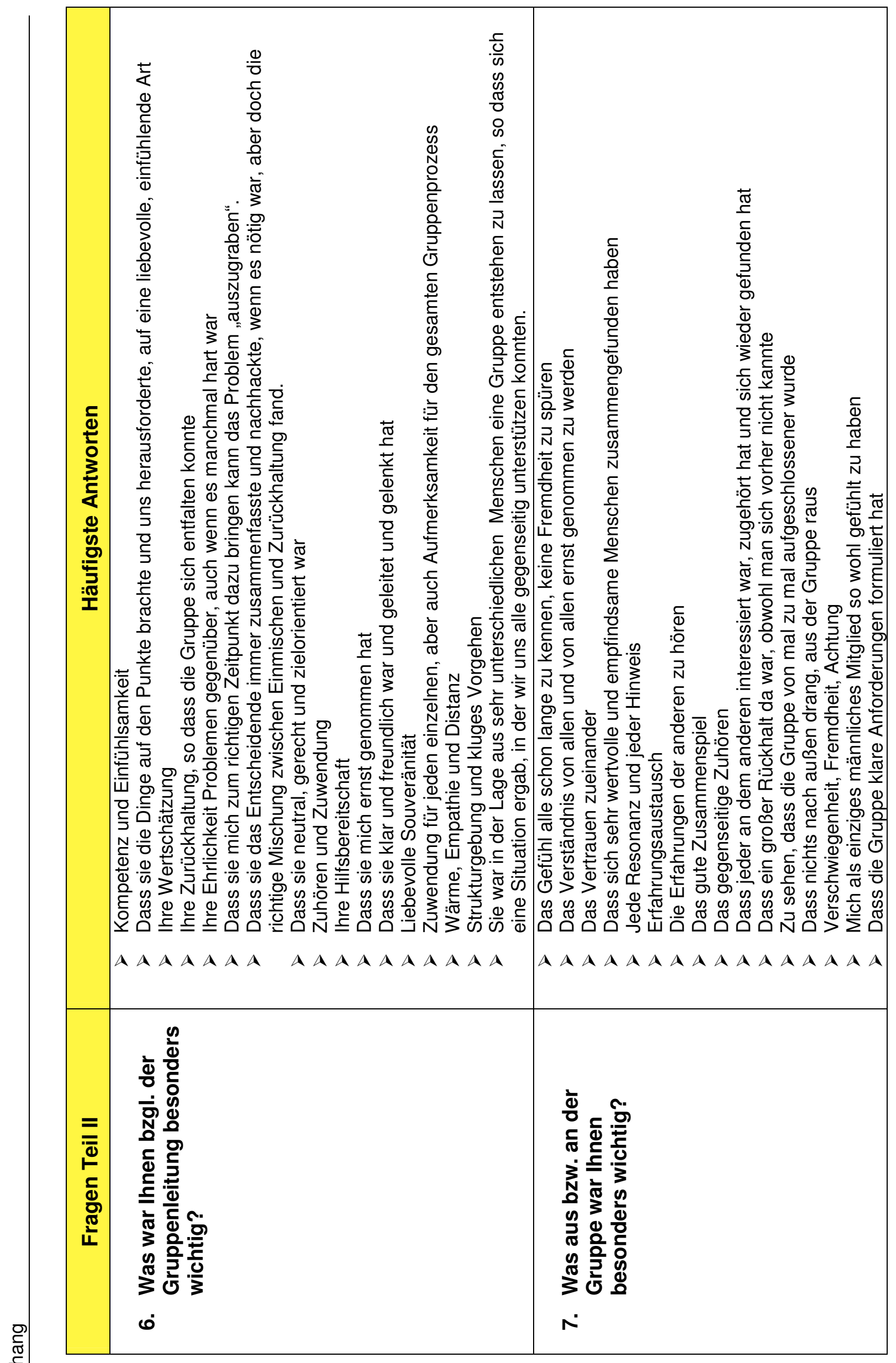

$\stackrel{\circ}{\circ}$ 


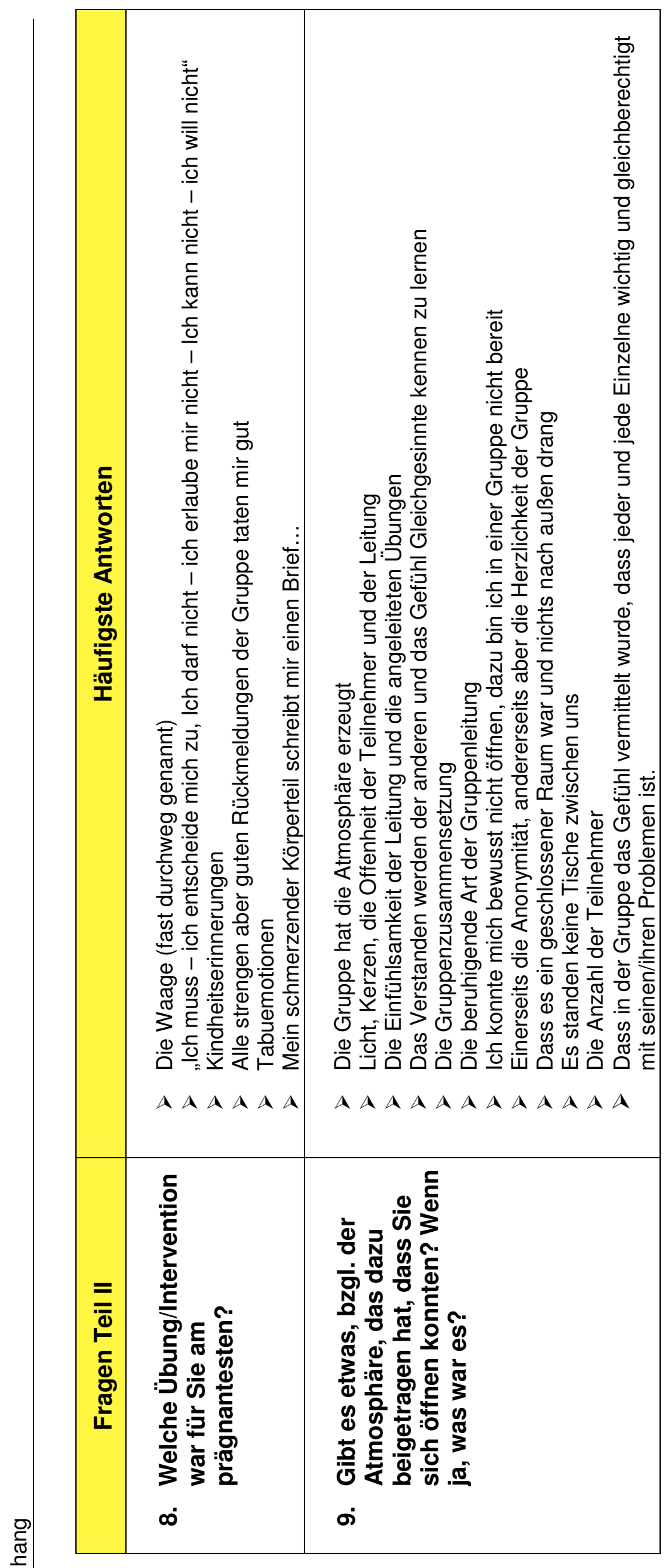

$\bar{\sigma}$ 


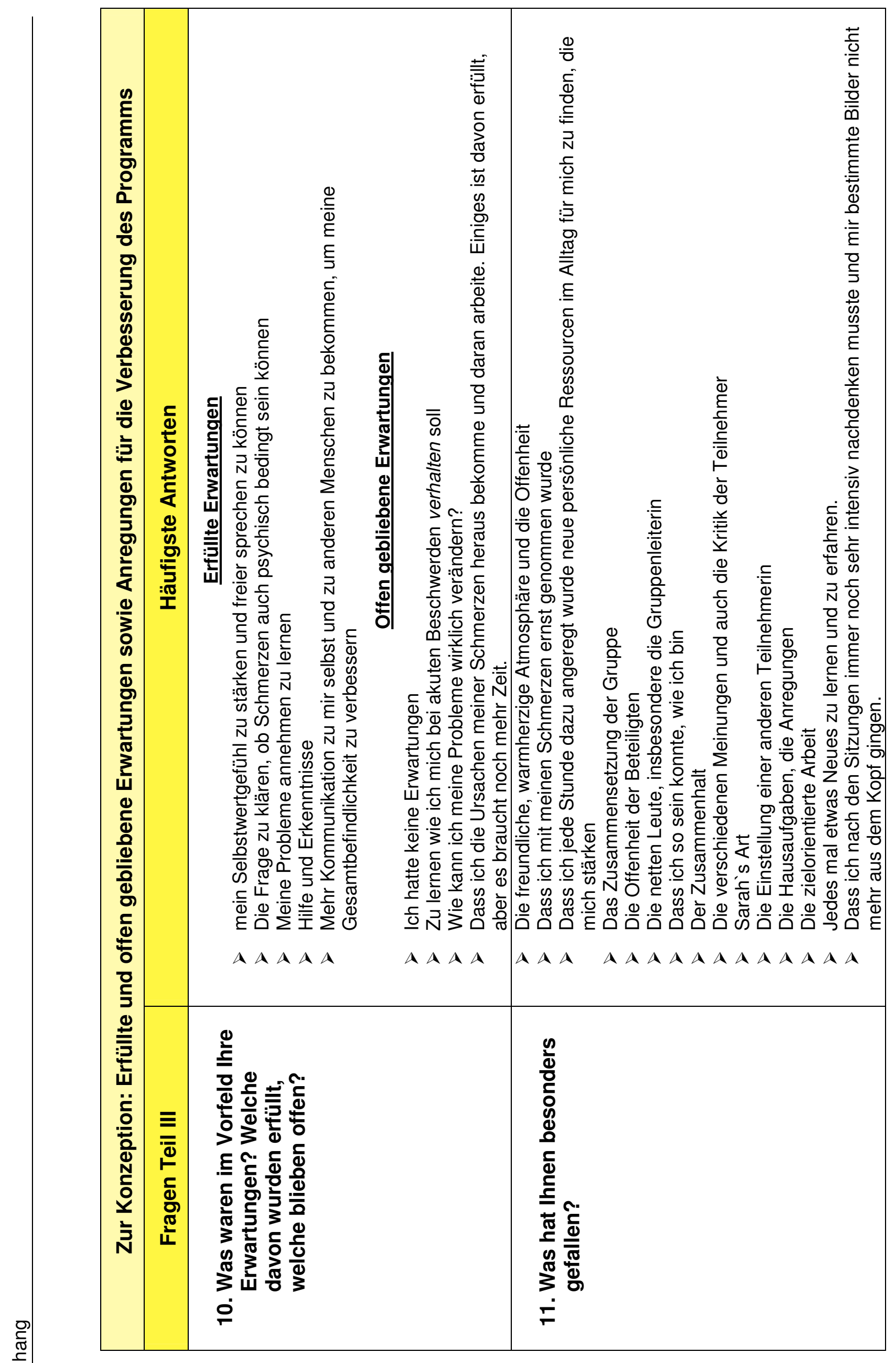

ญ 


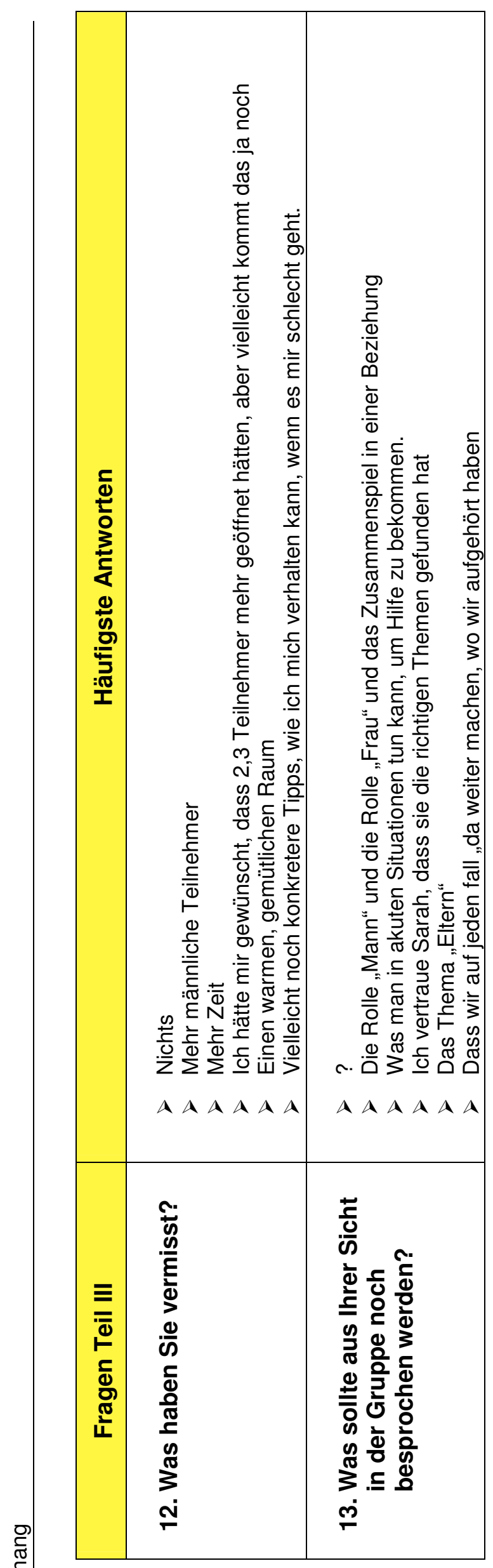




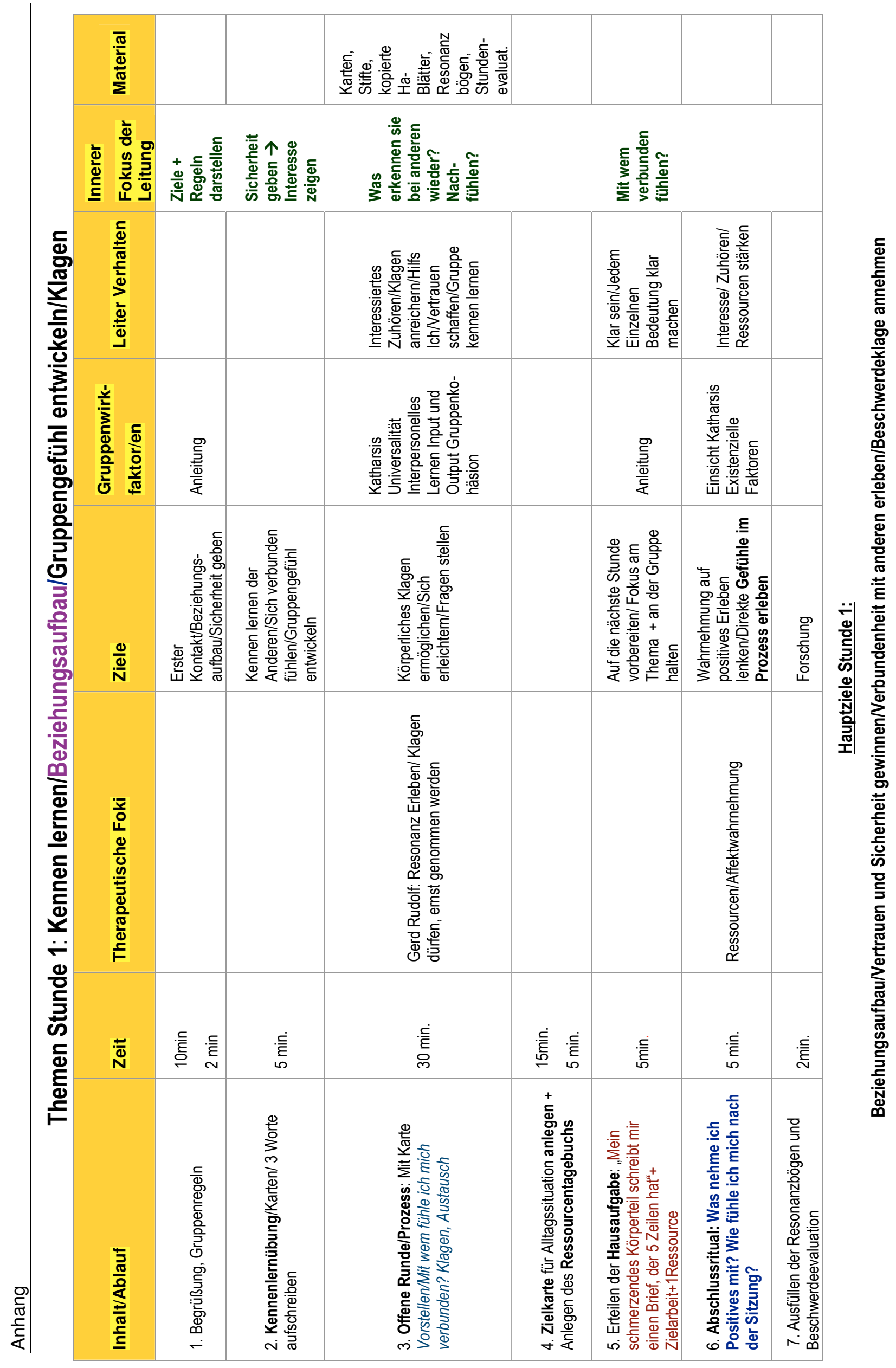




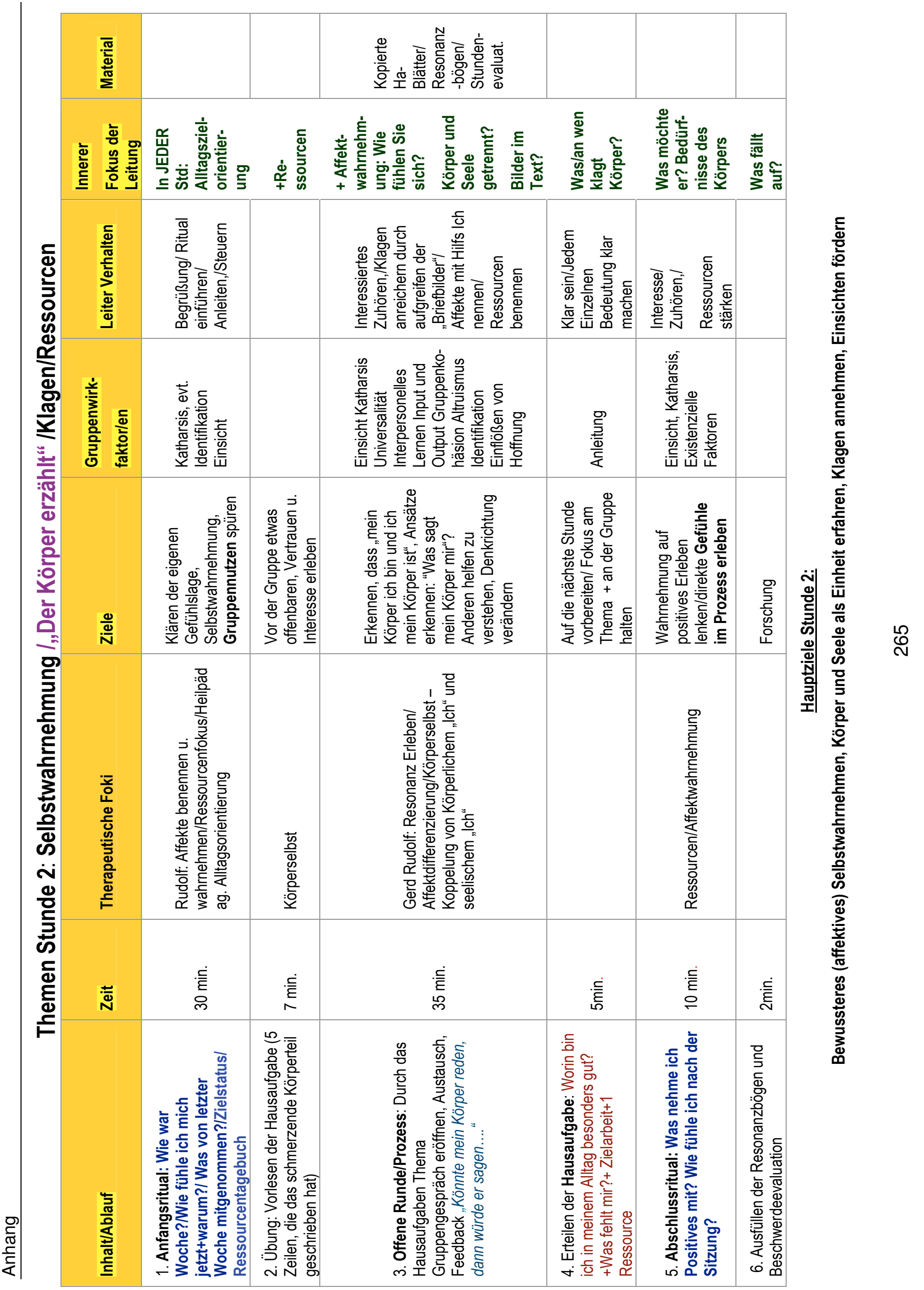




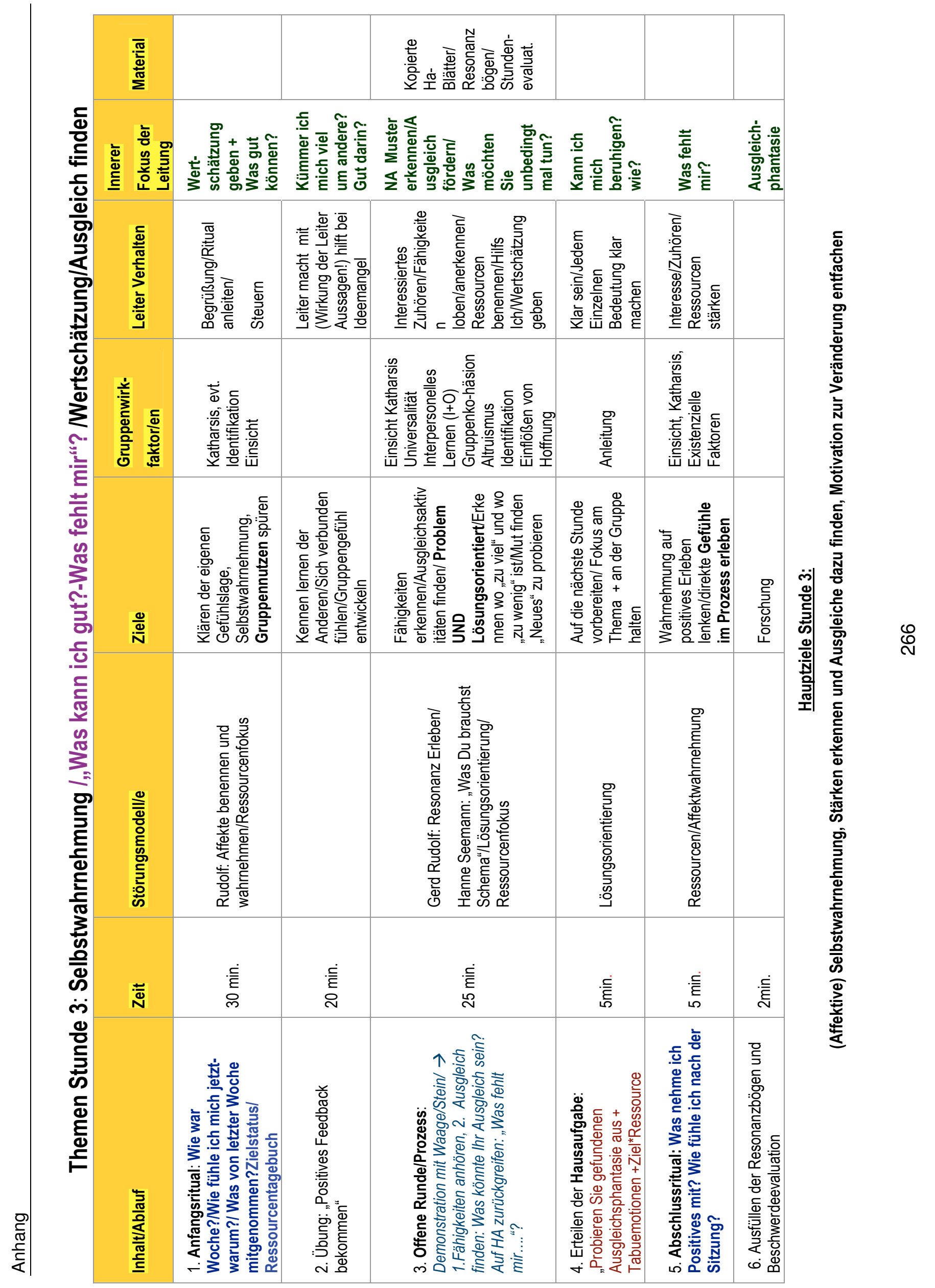




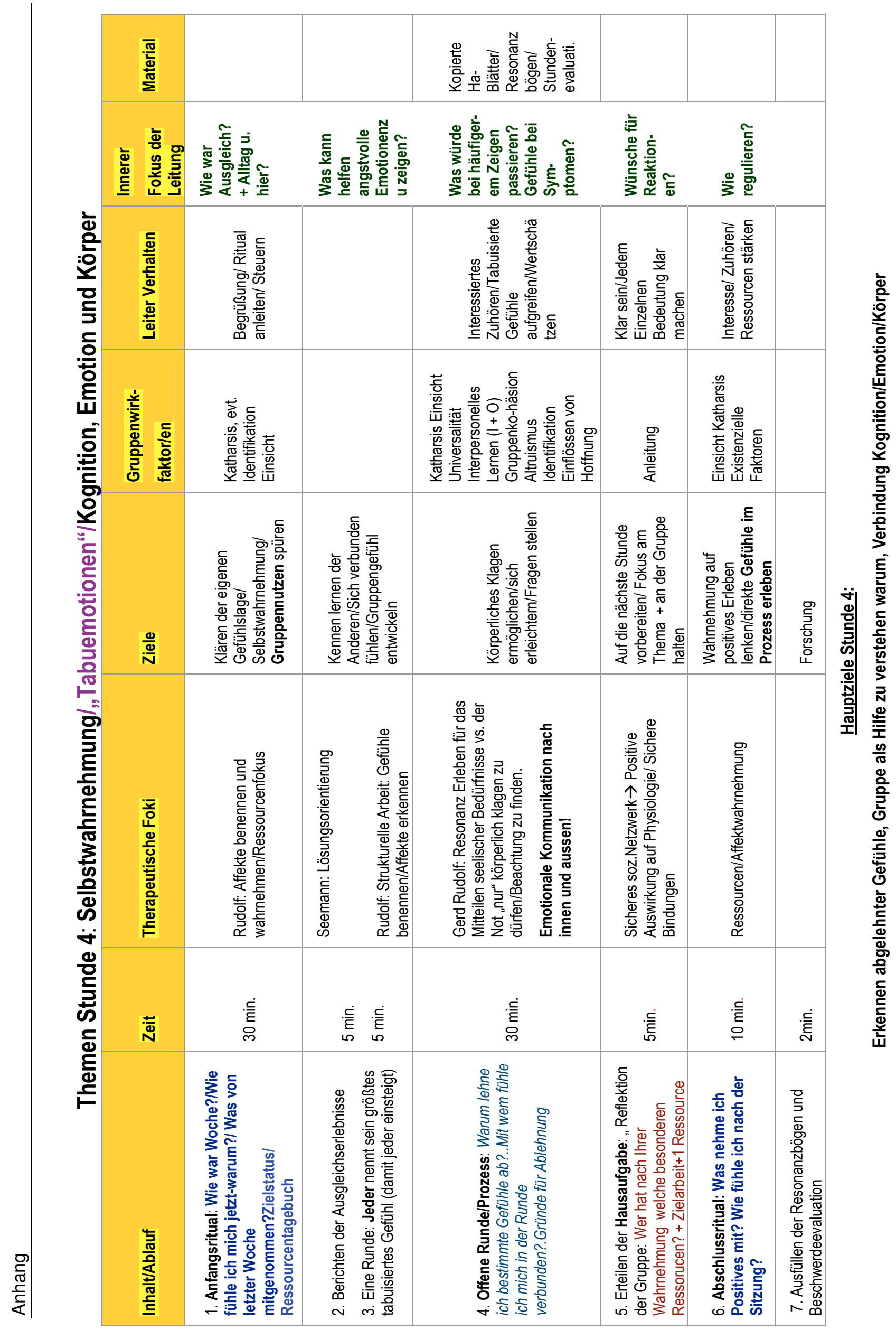

i̊ 


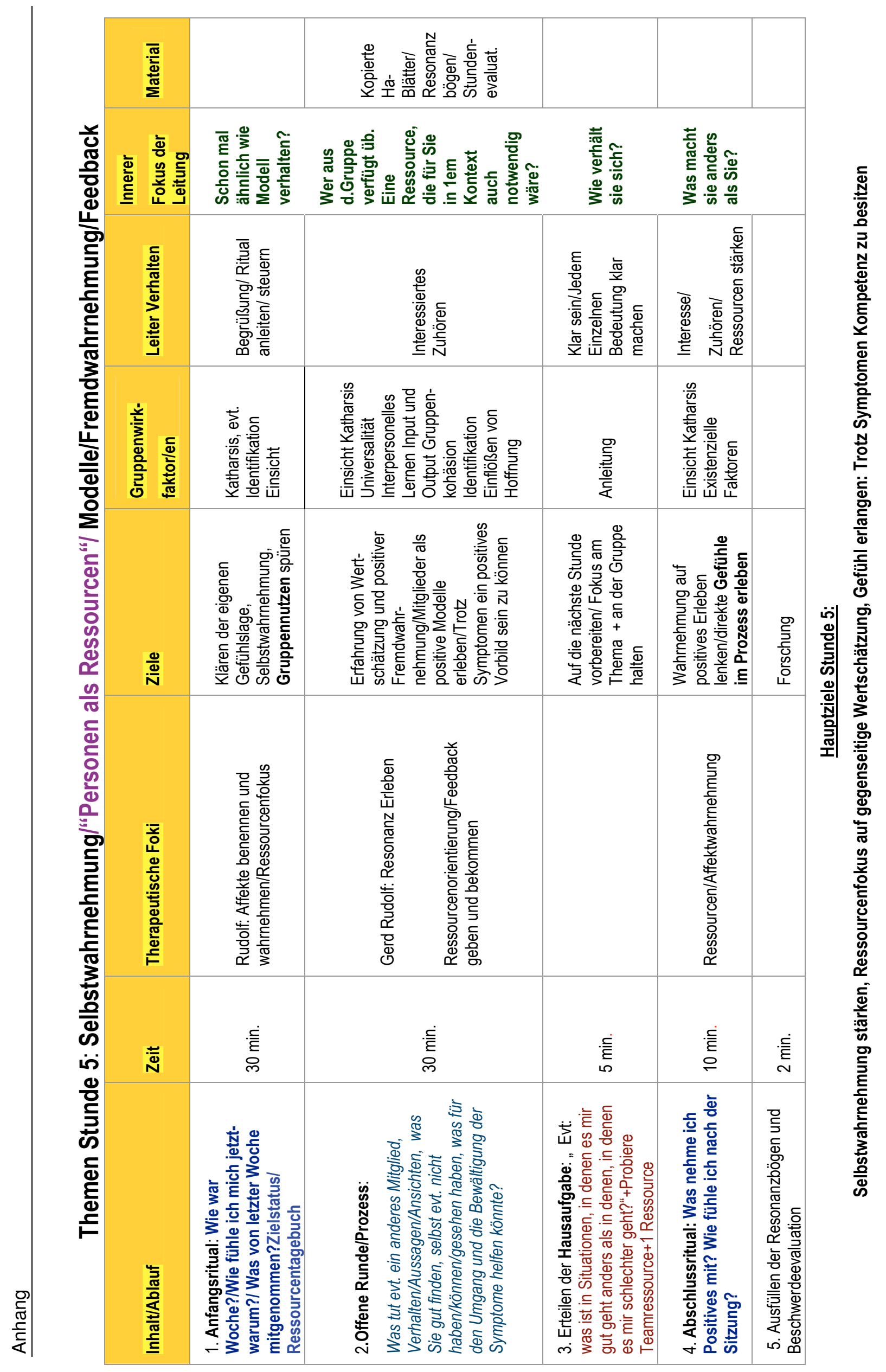

$\stackrel{\infty}{\sim}$ 


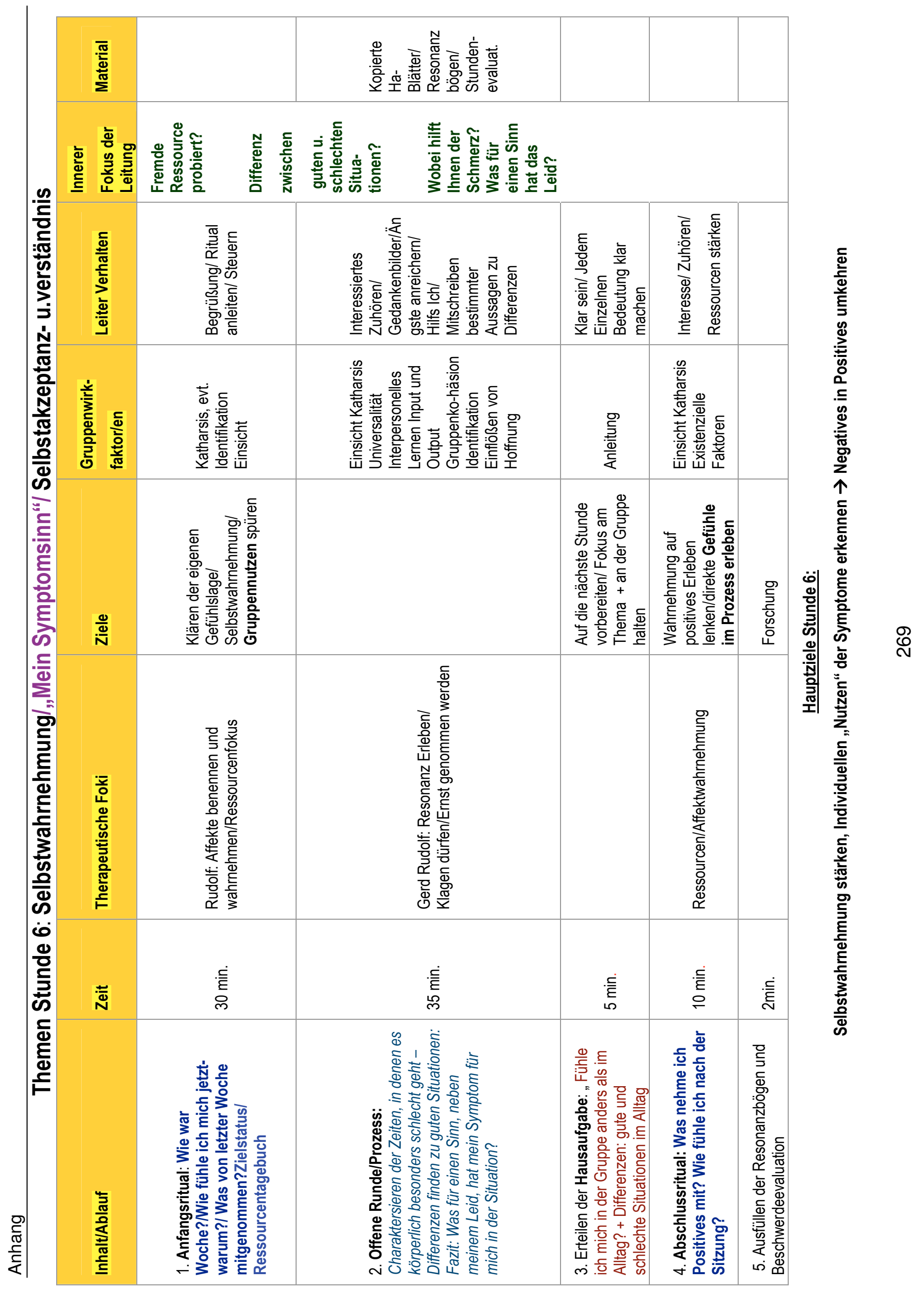




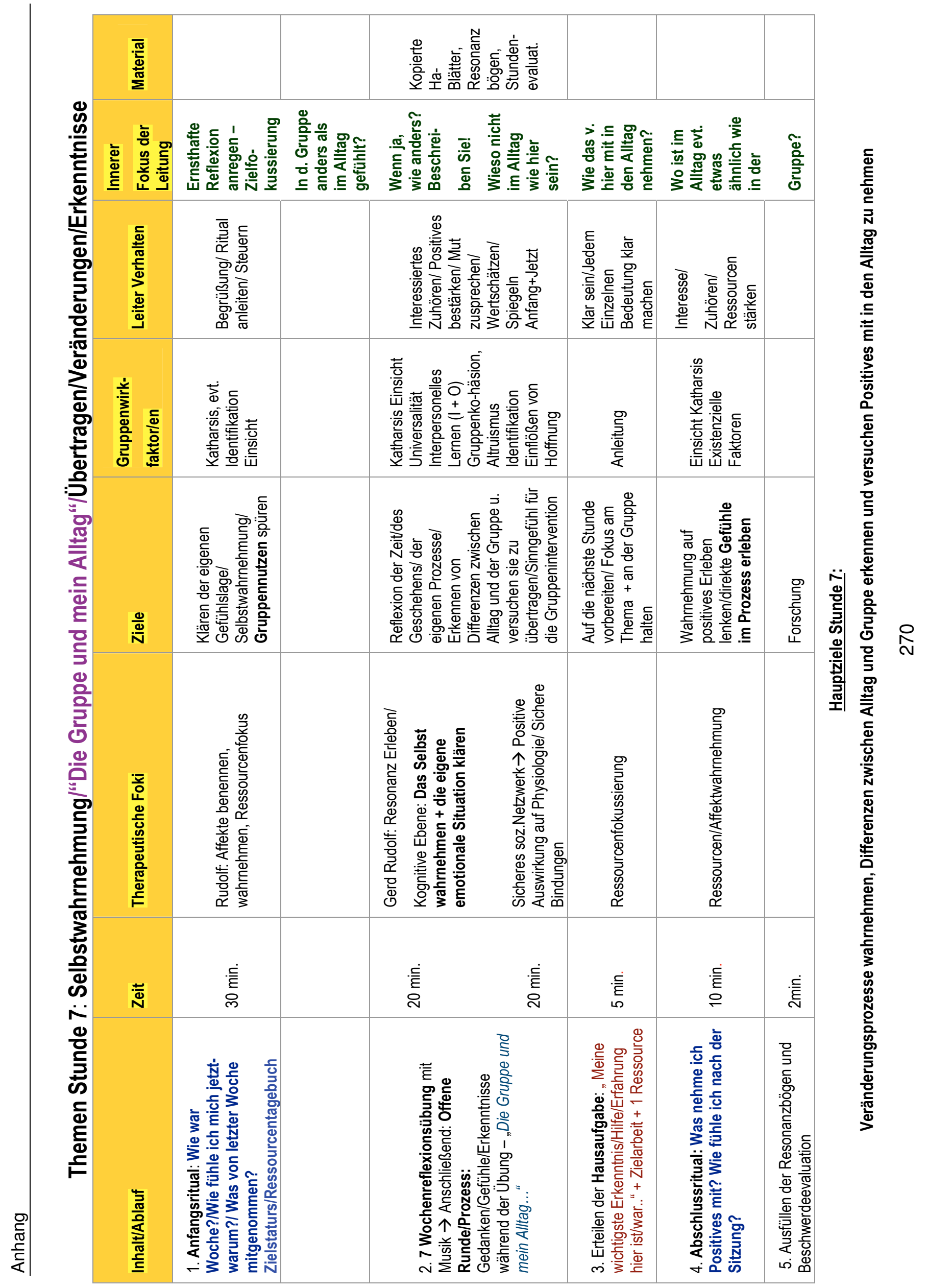




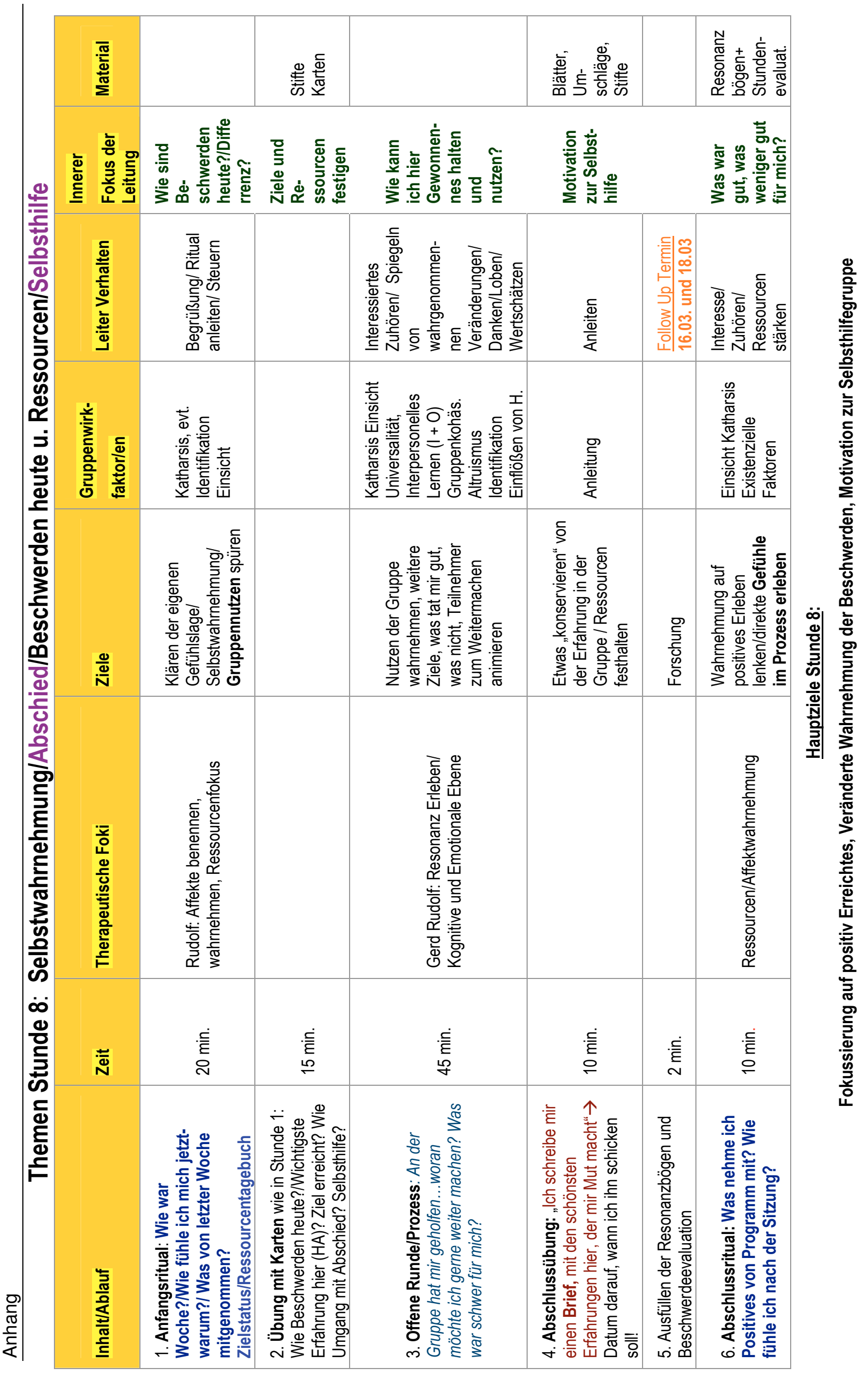

ล 

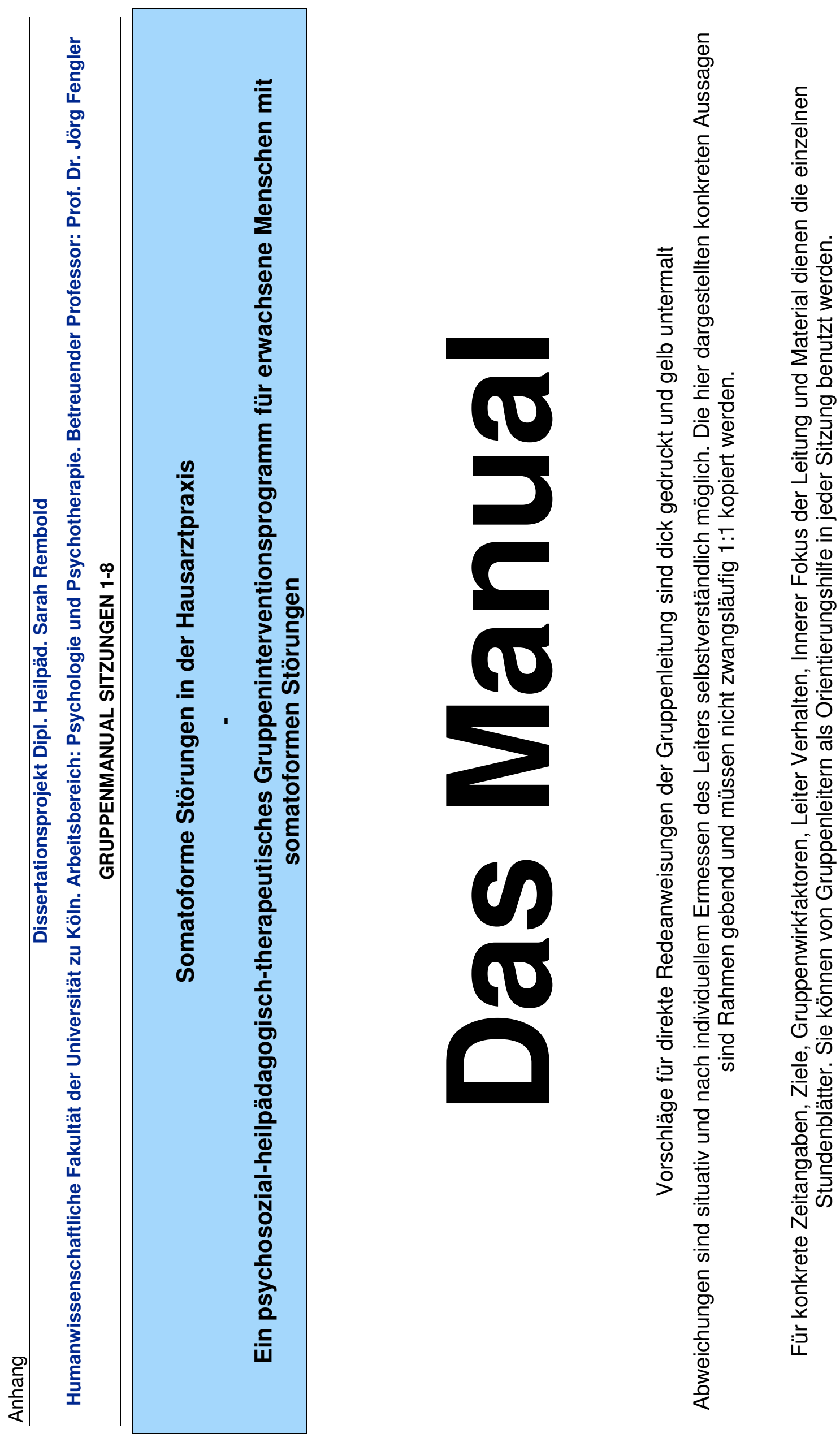


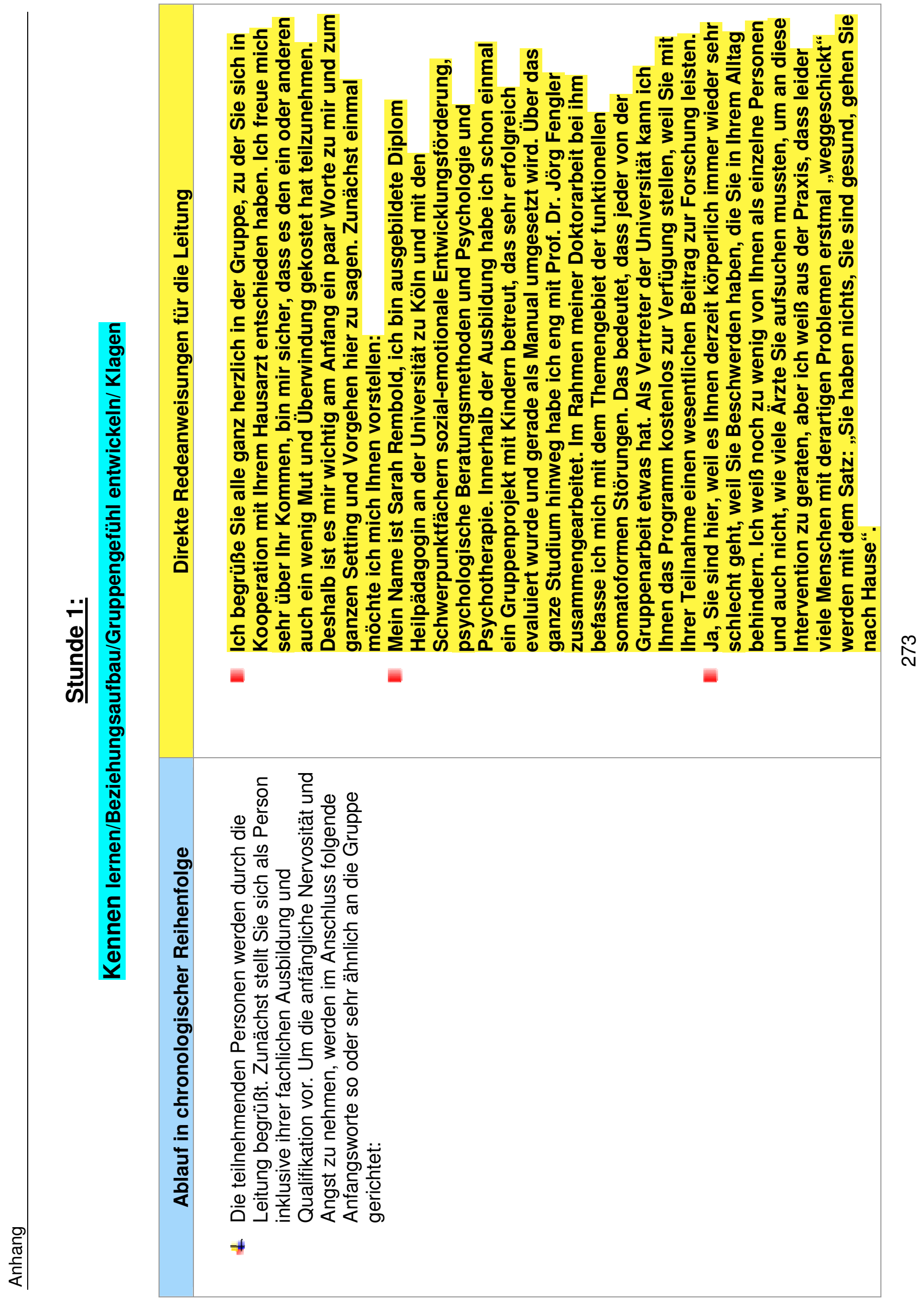




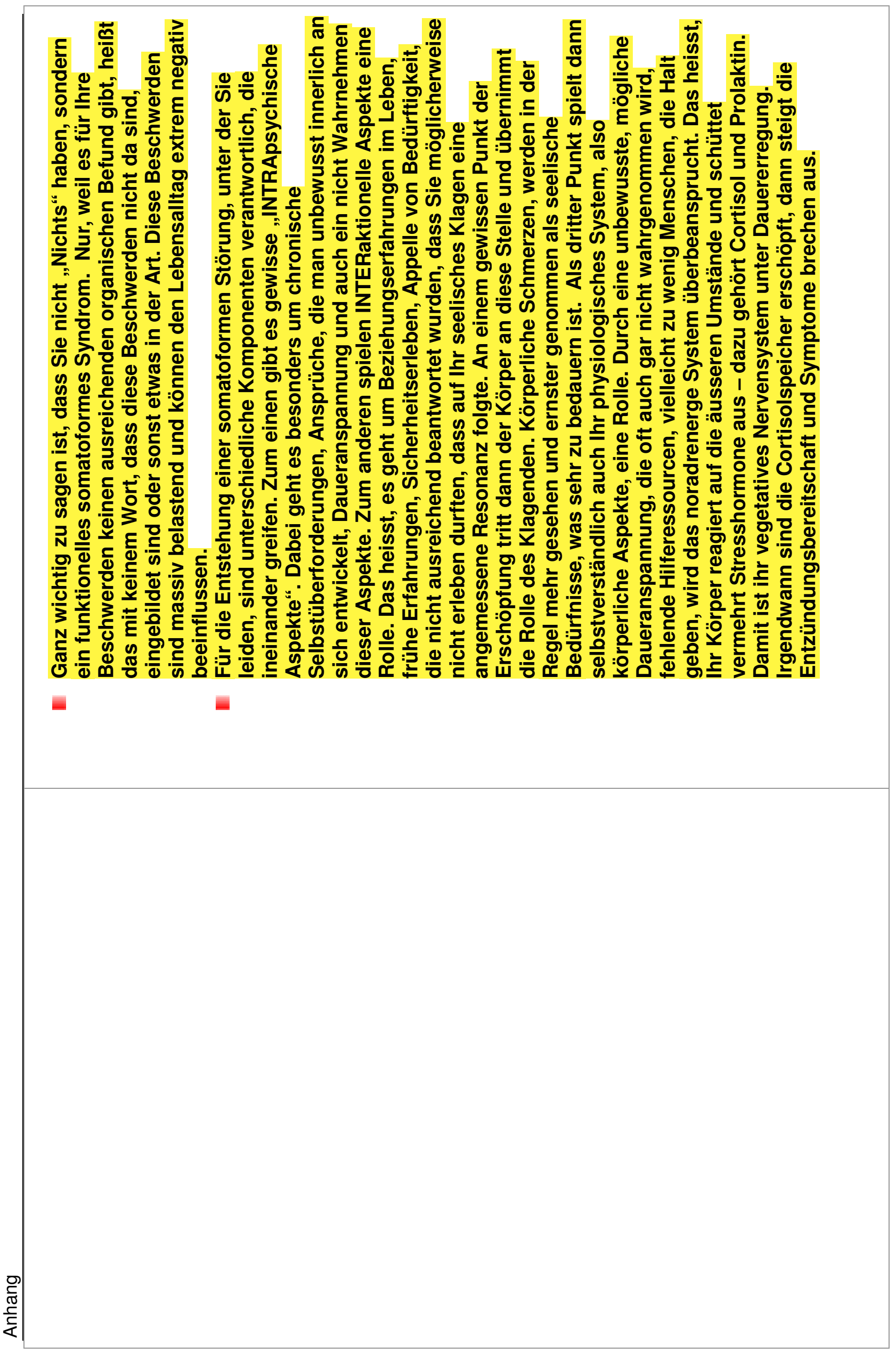




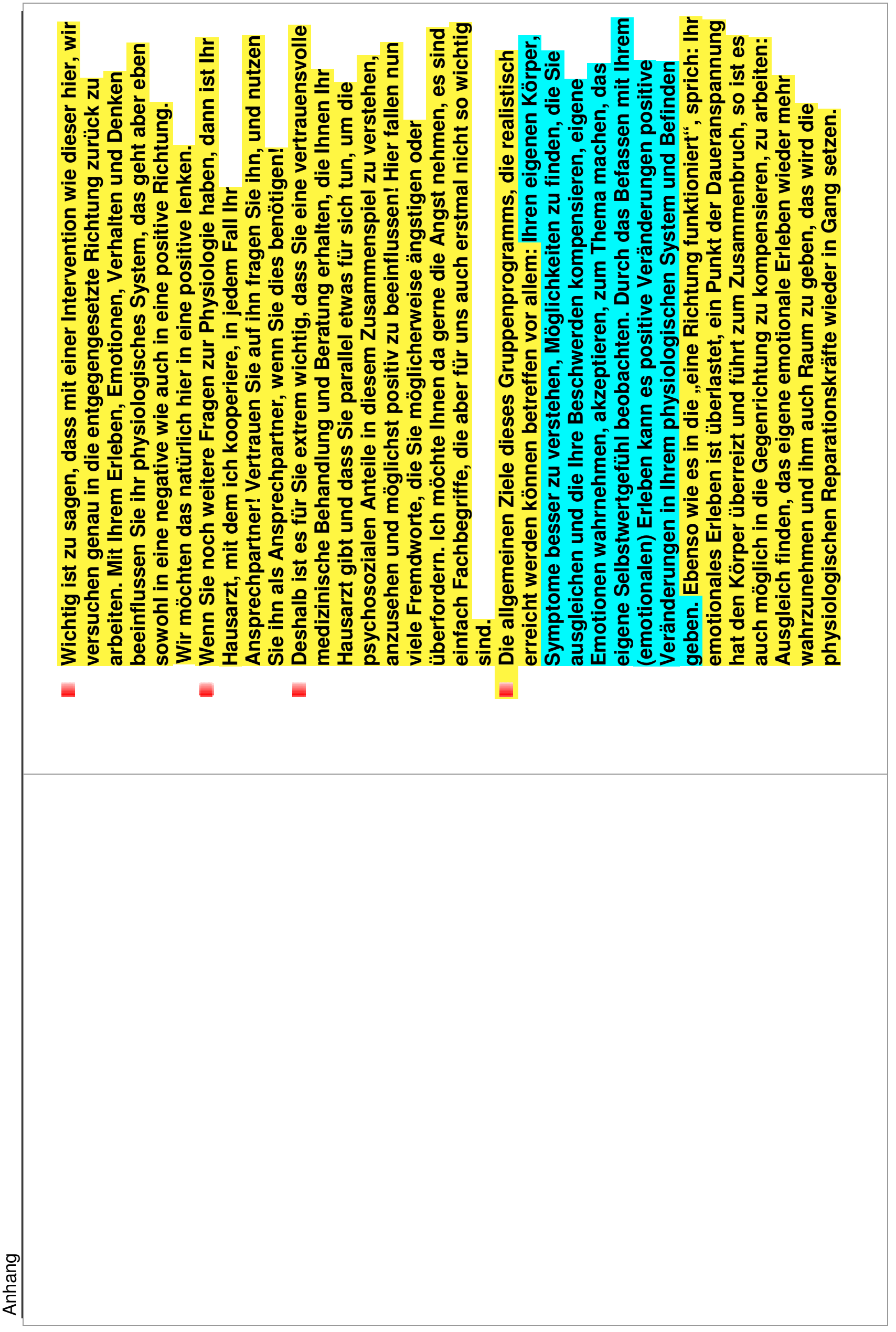




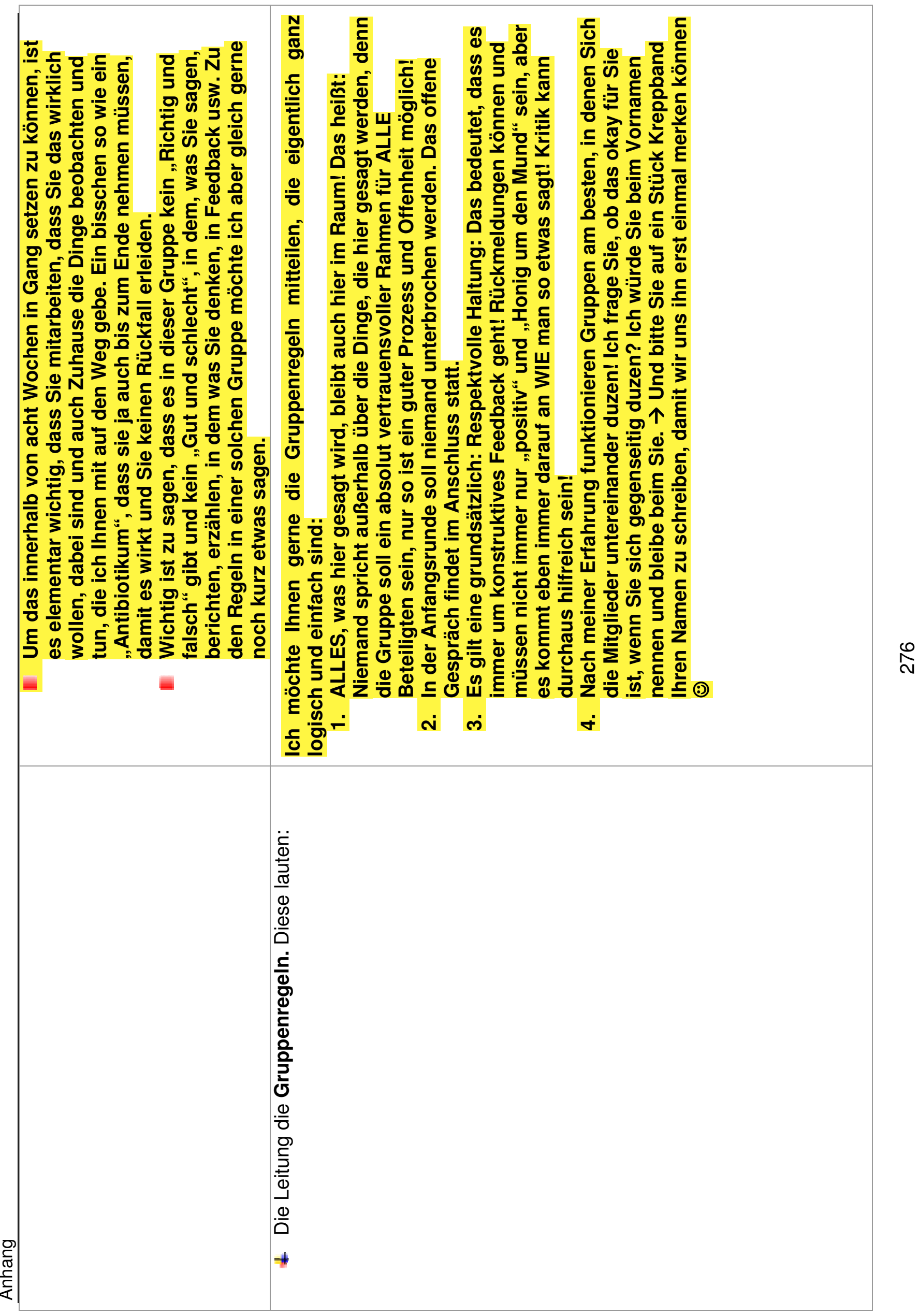




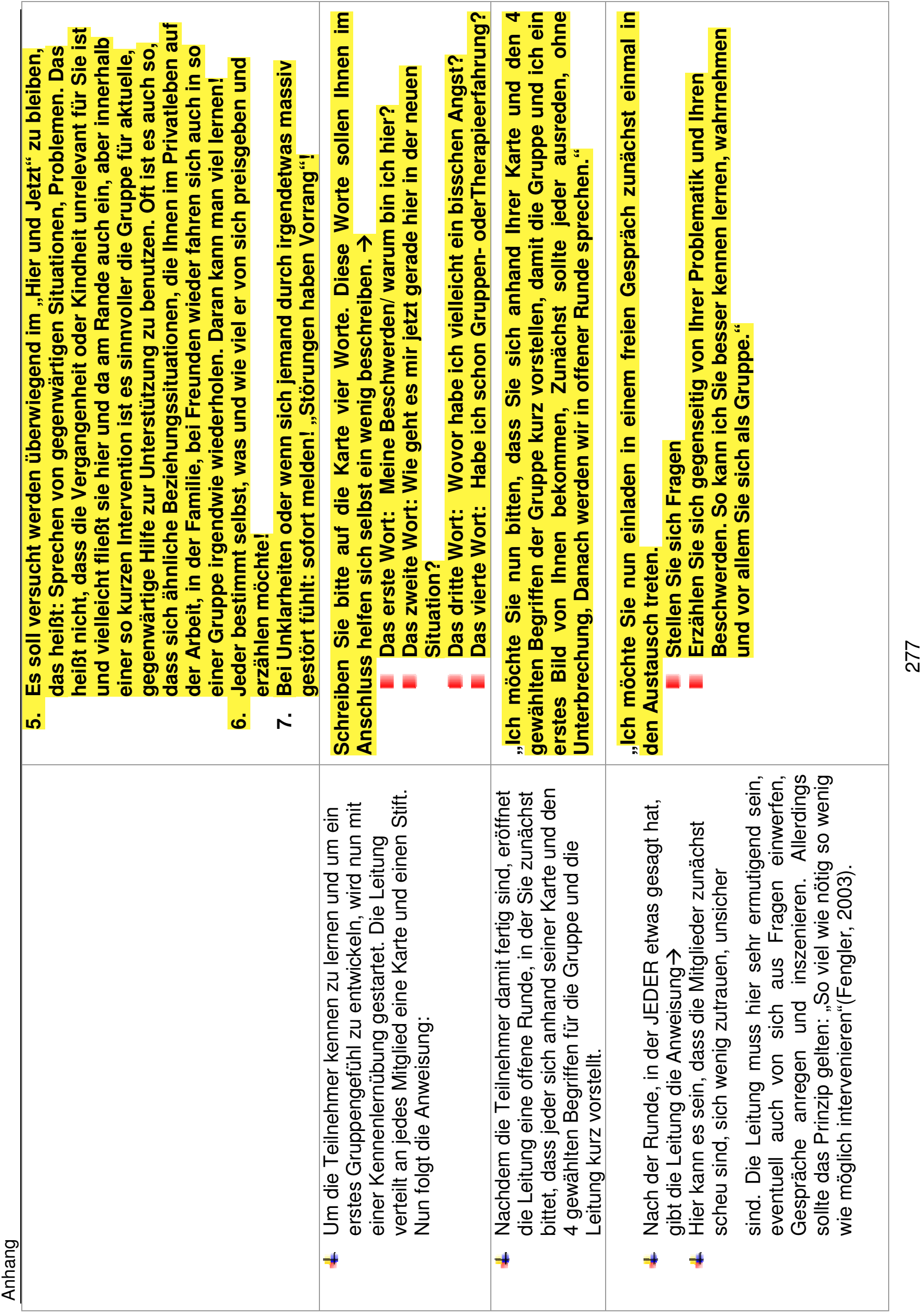




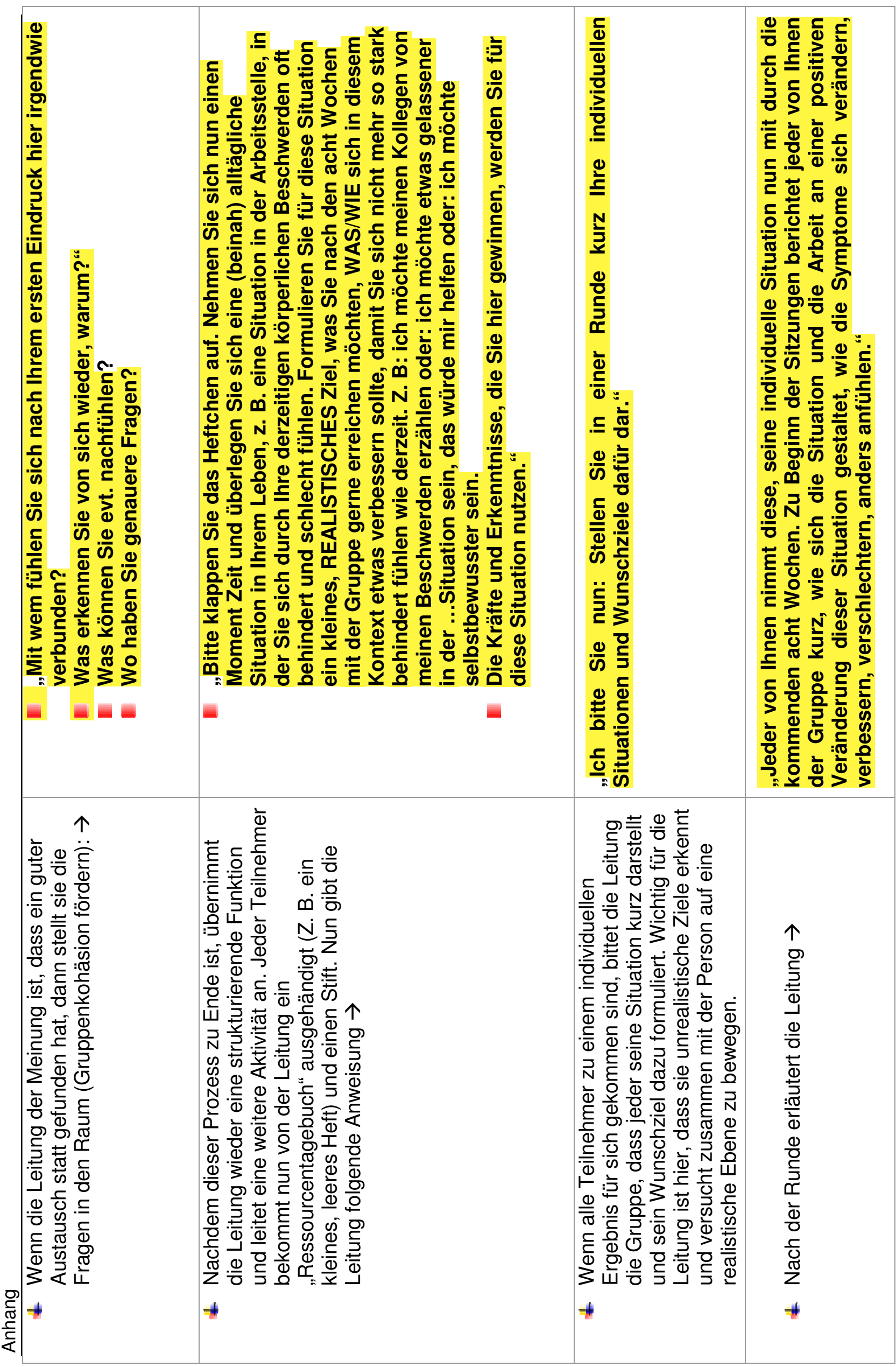

$\stackrel{\infty}{N}$ 


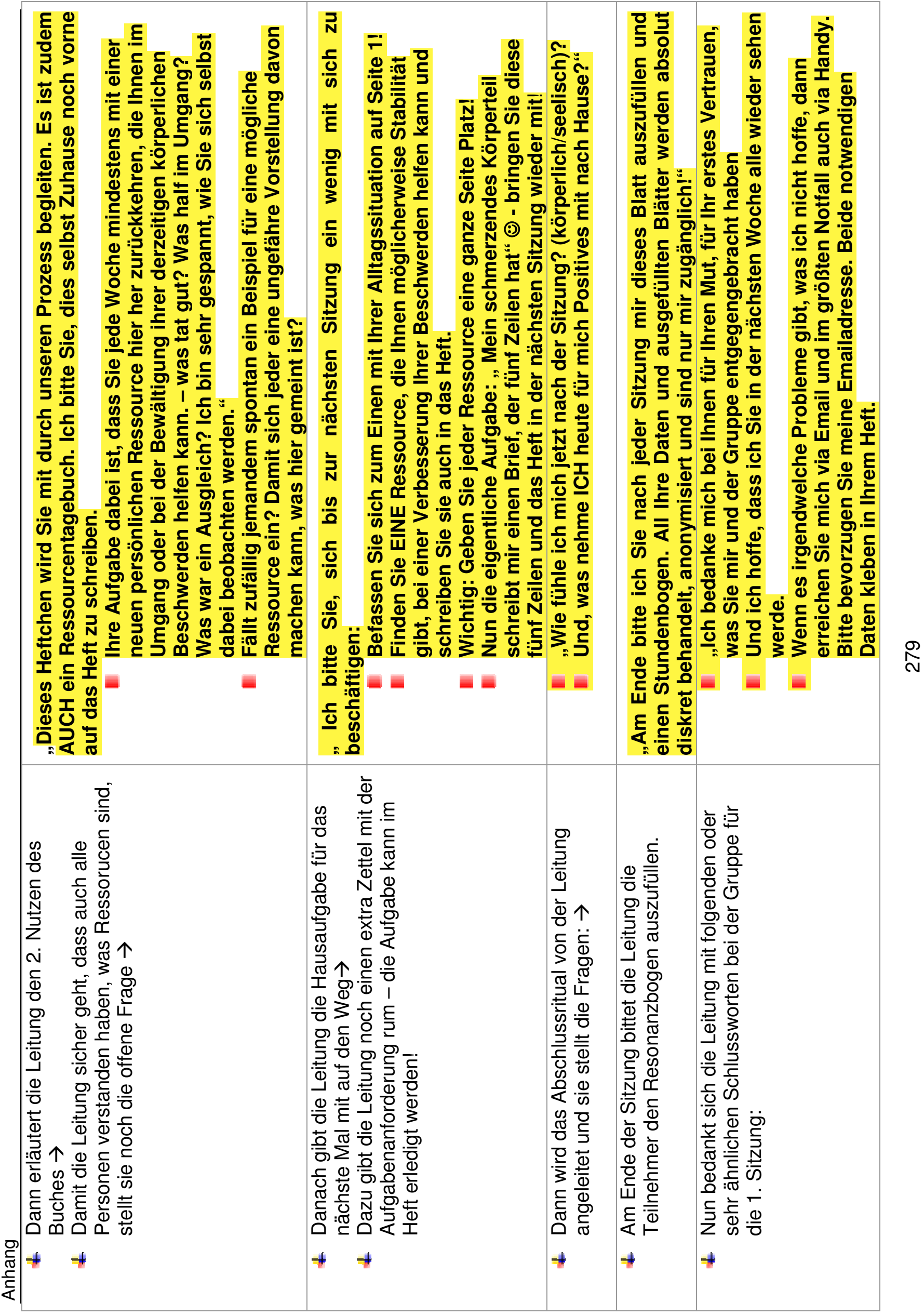




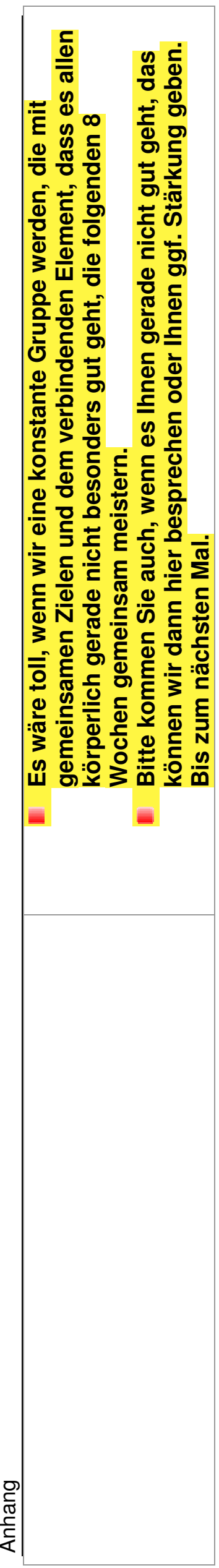




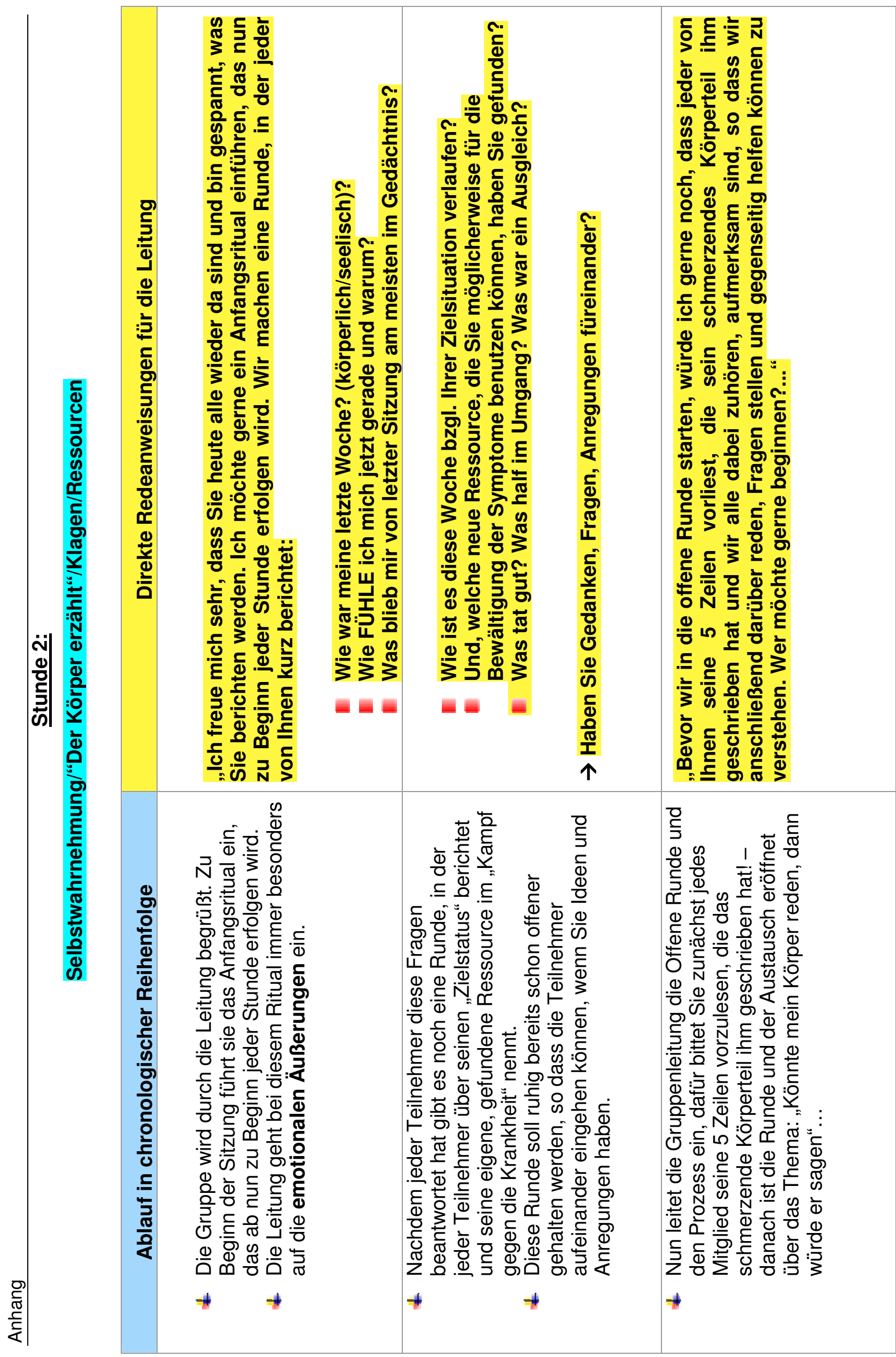




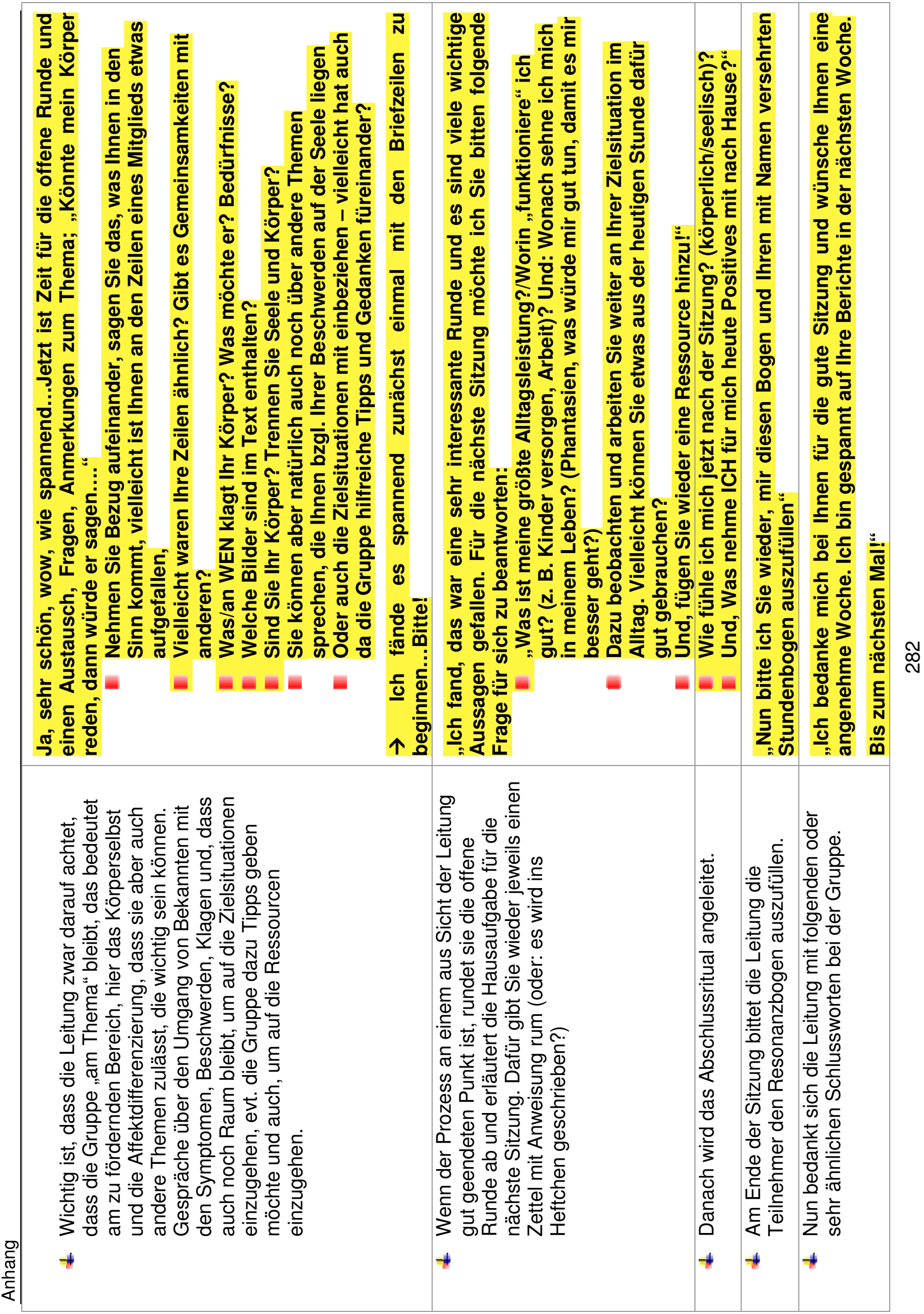




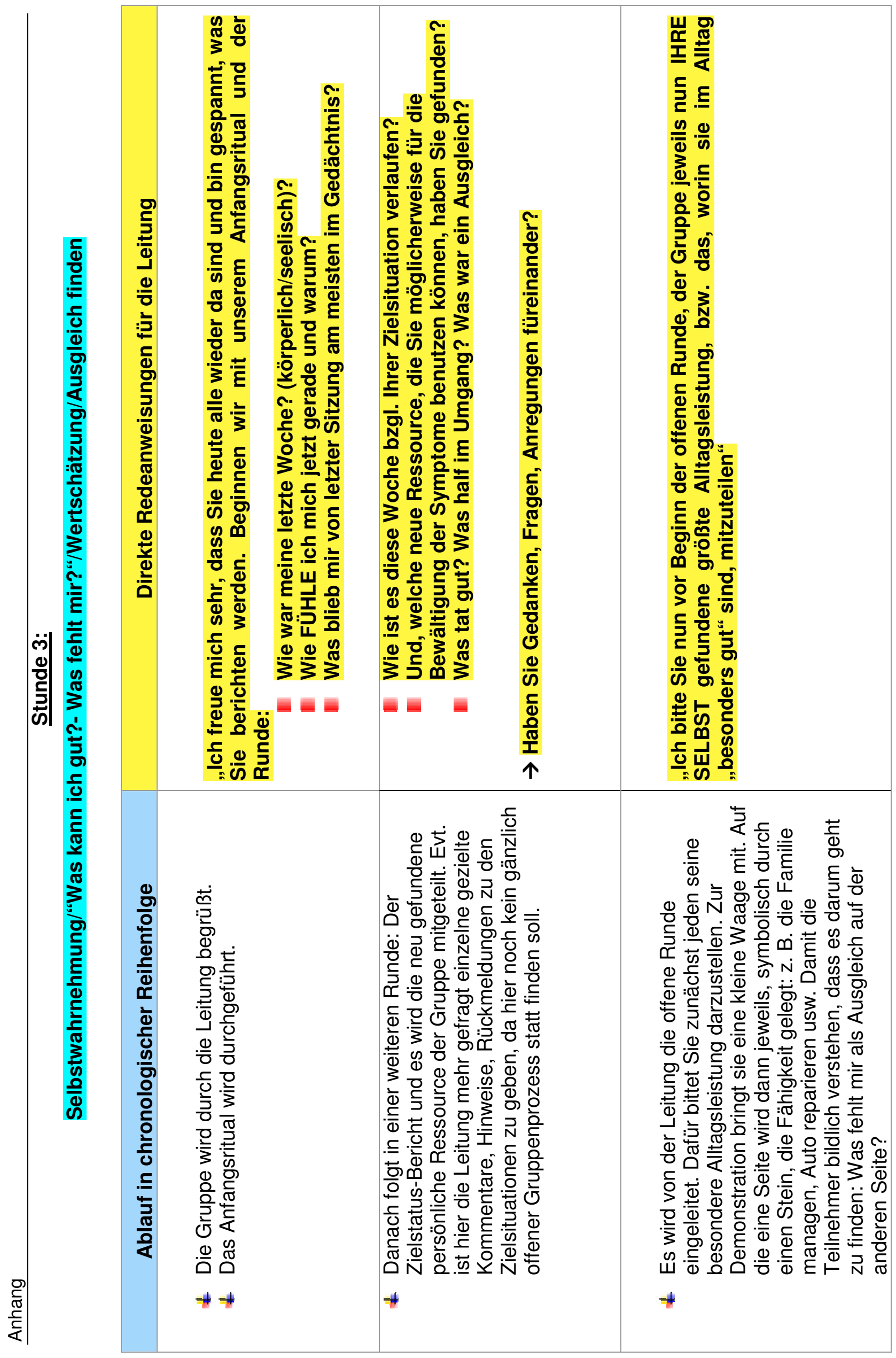

$\stackrel{\infty}{\infty}$ 


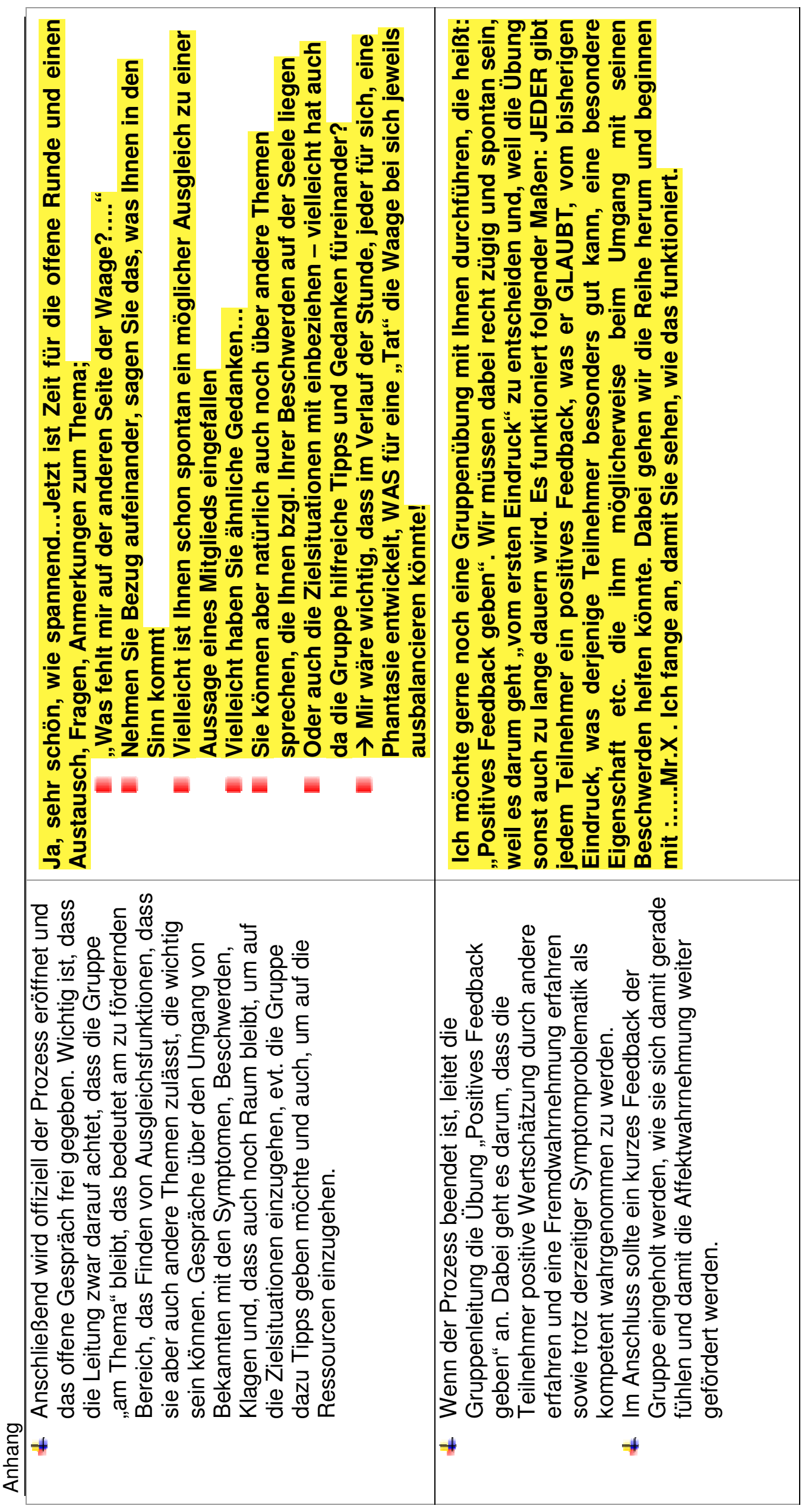




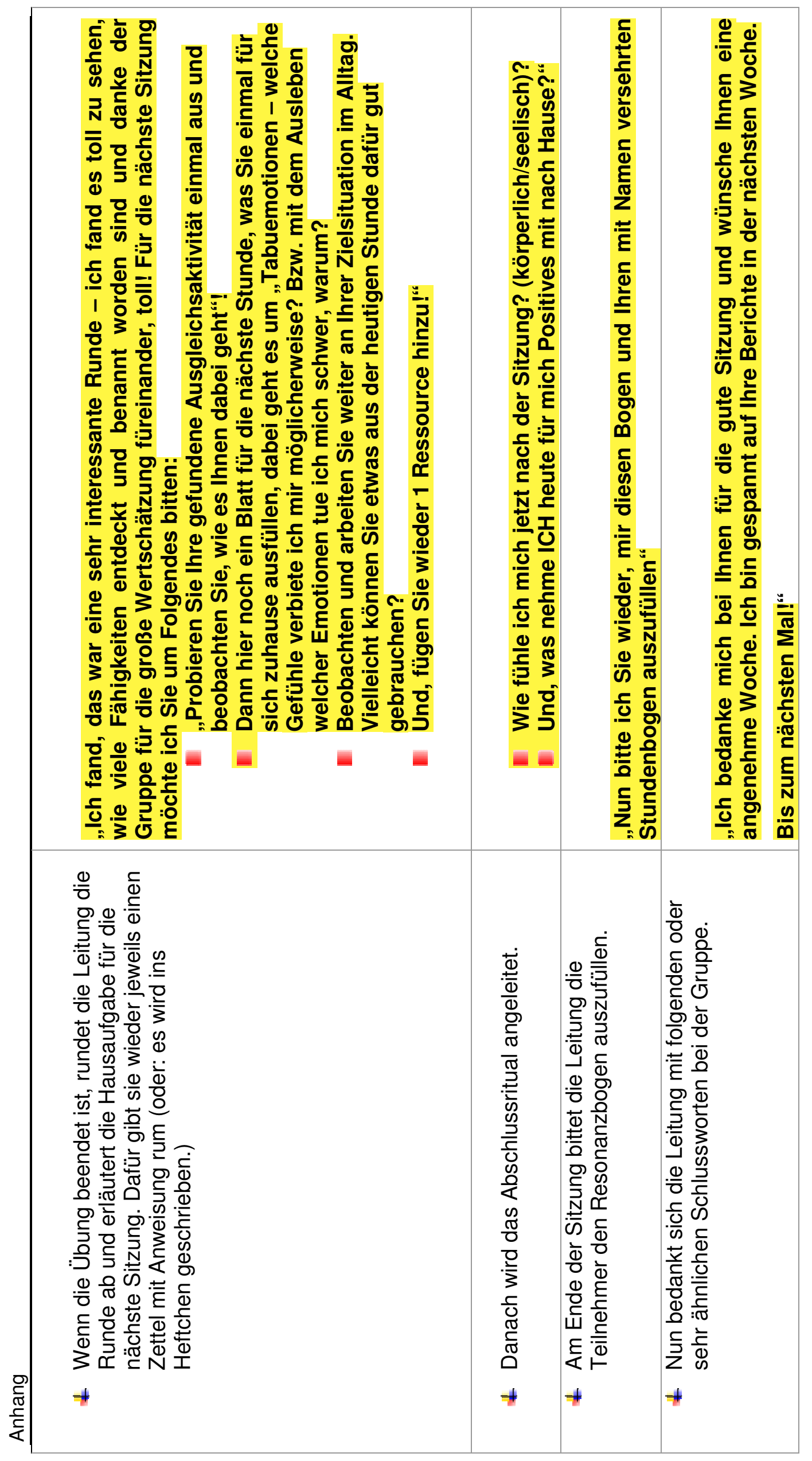




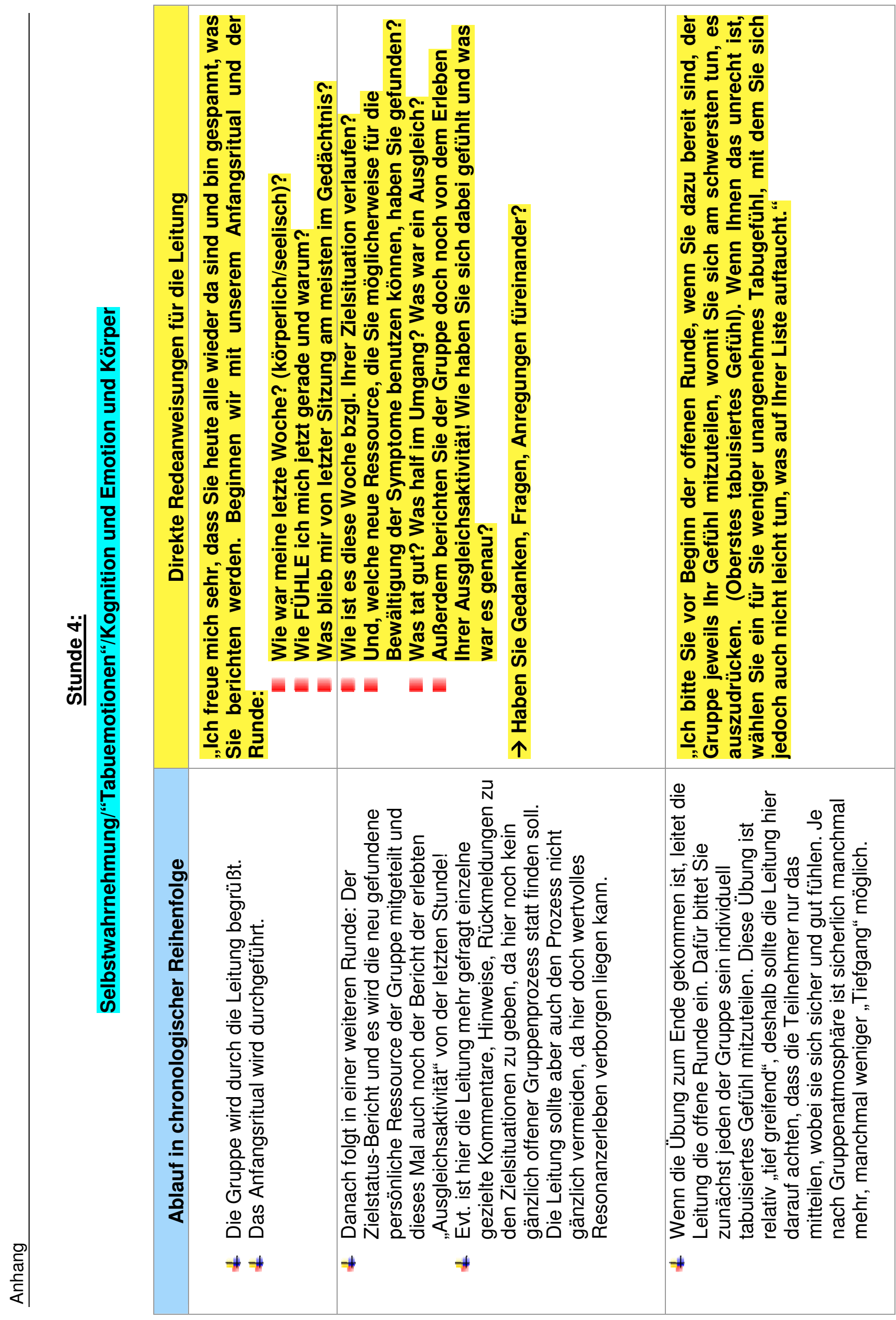

$\stackrel{\infty}{\sim}$ 


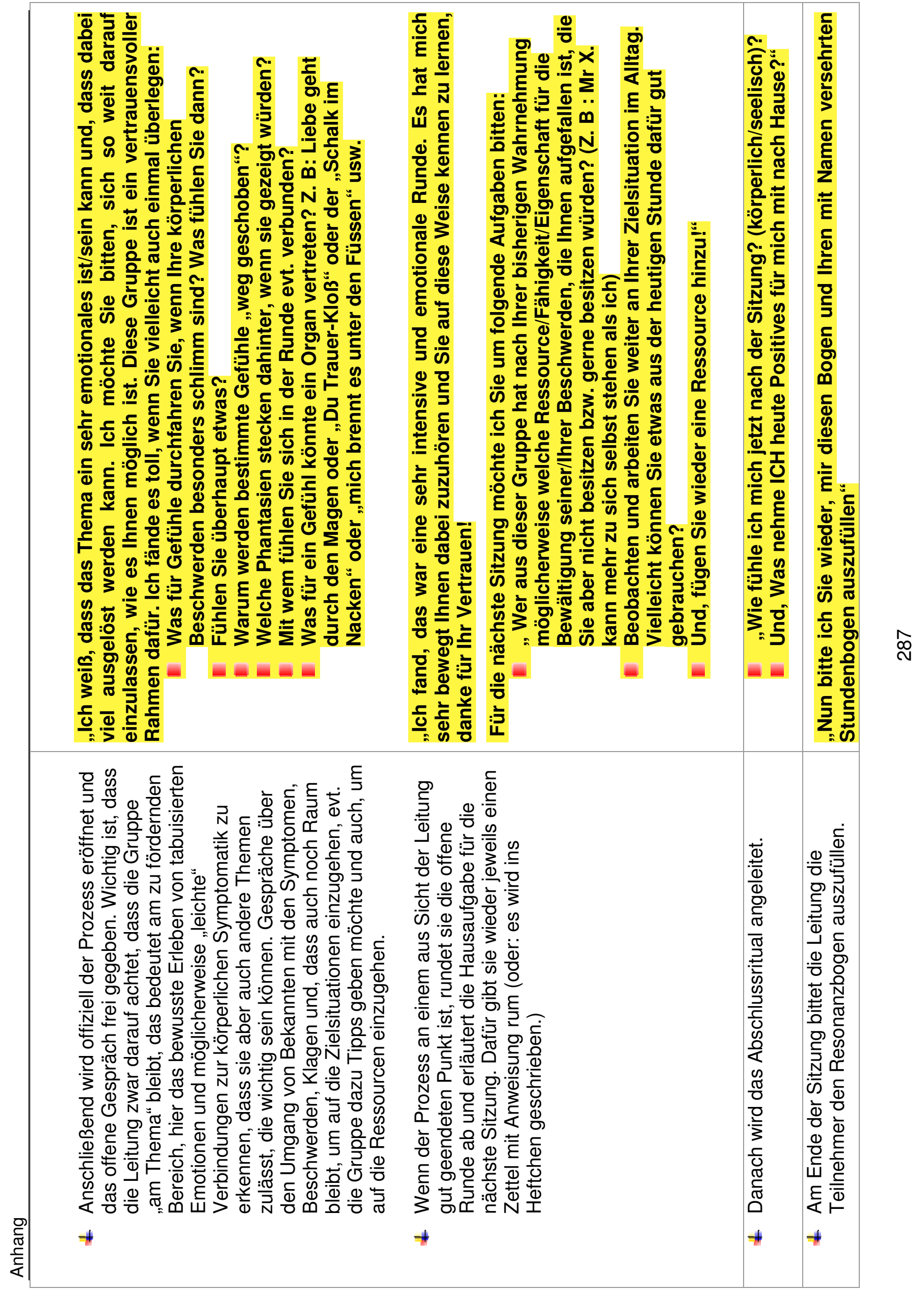




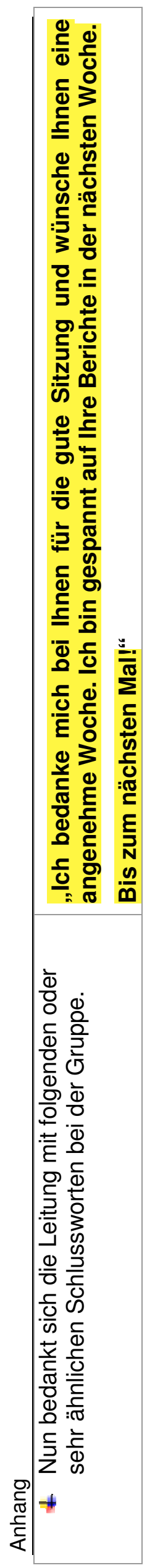




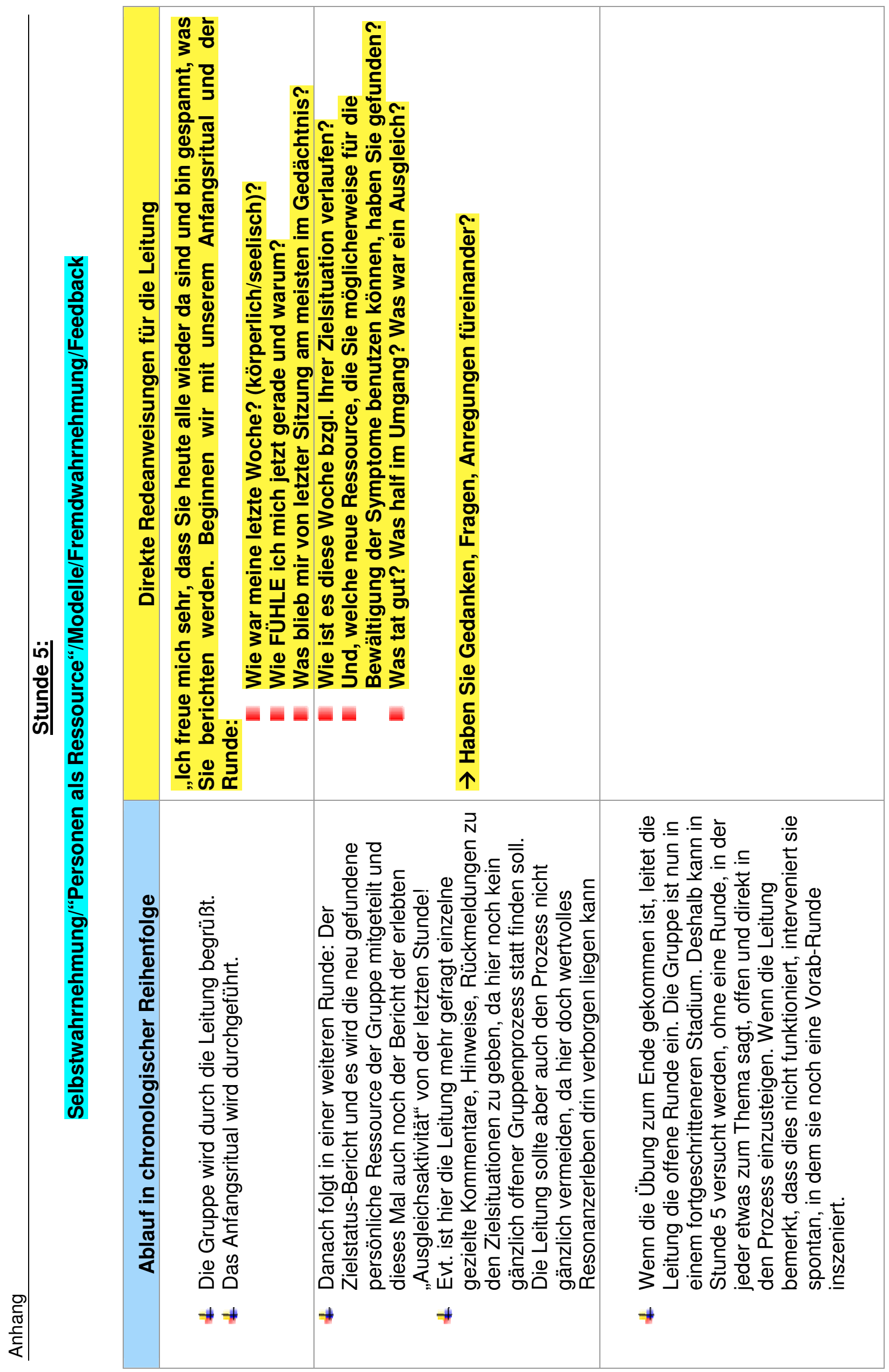

$\stackrel{8}{\sim}$ 


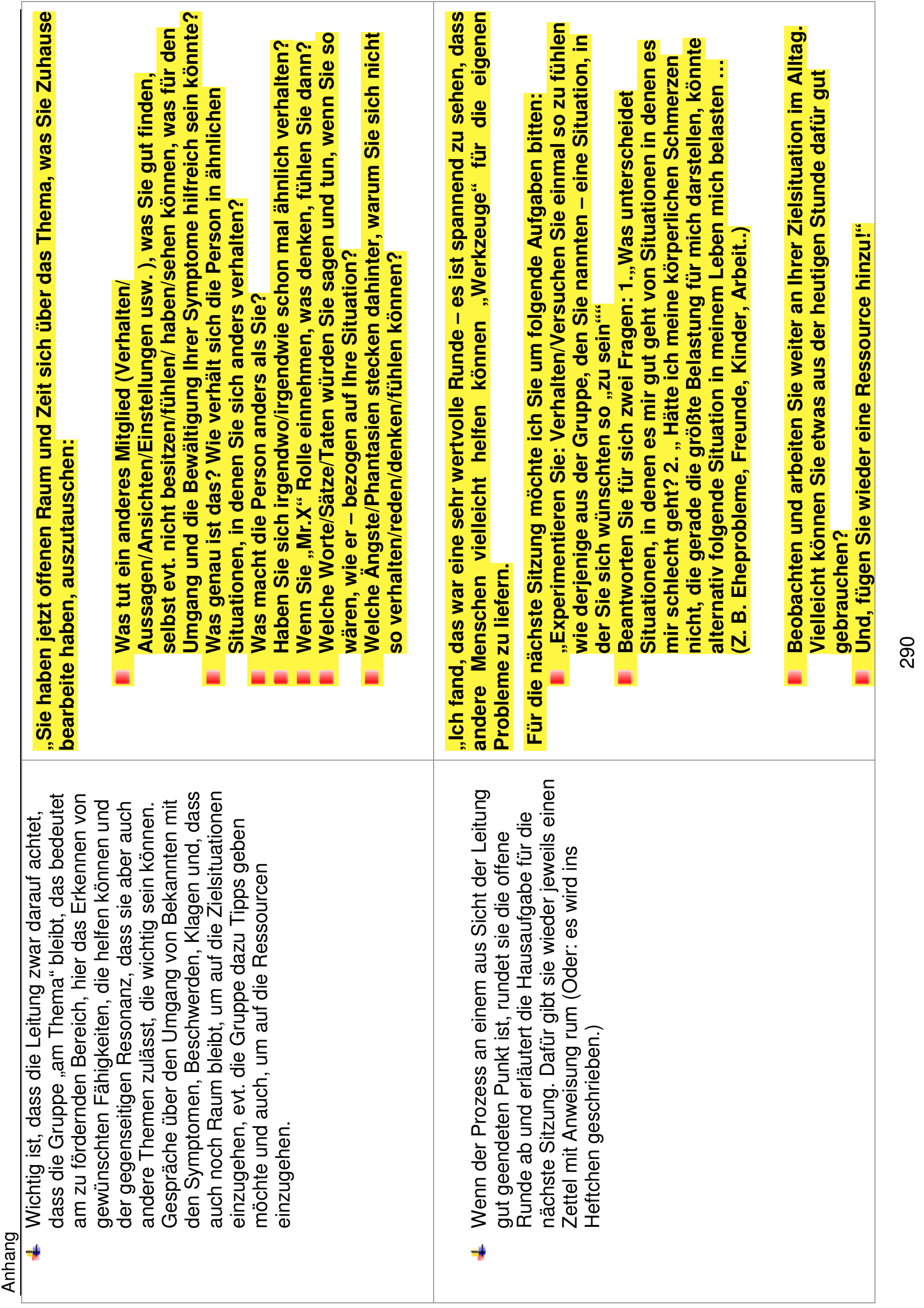




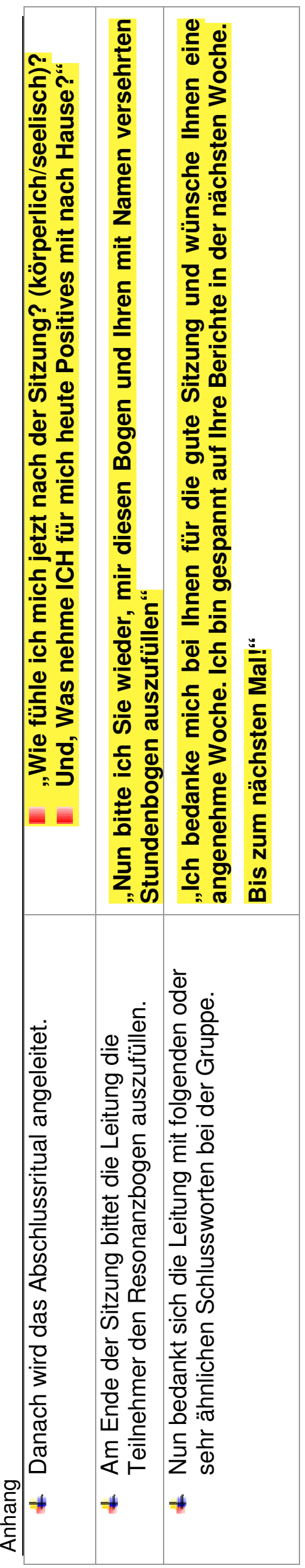




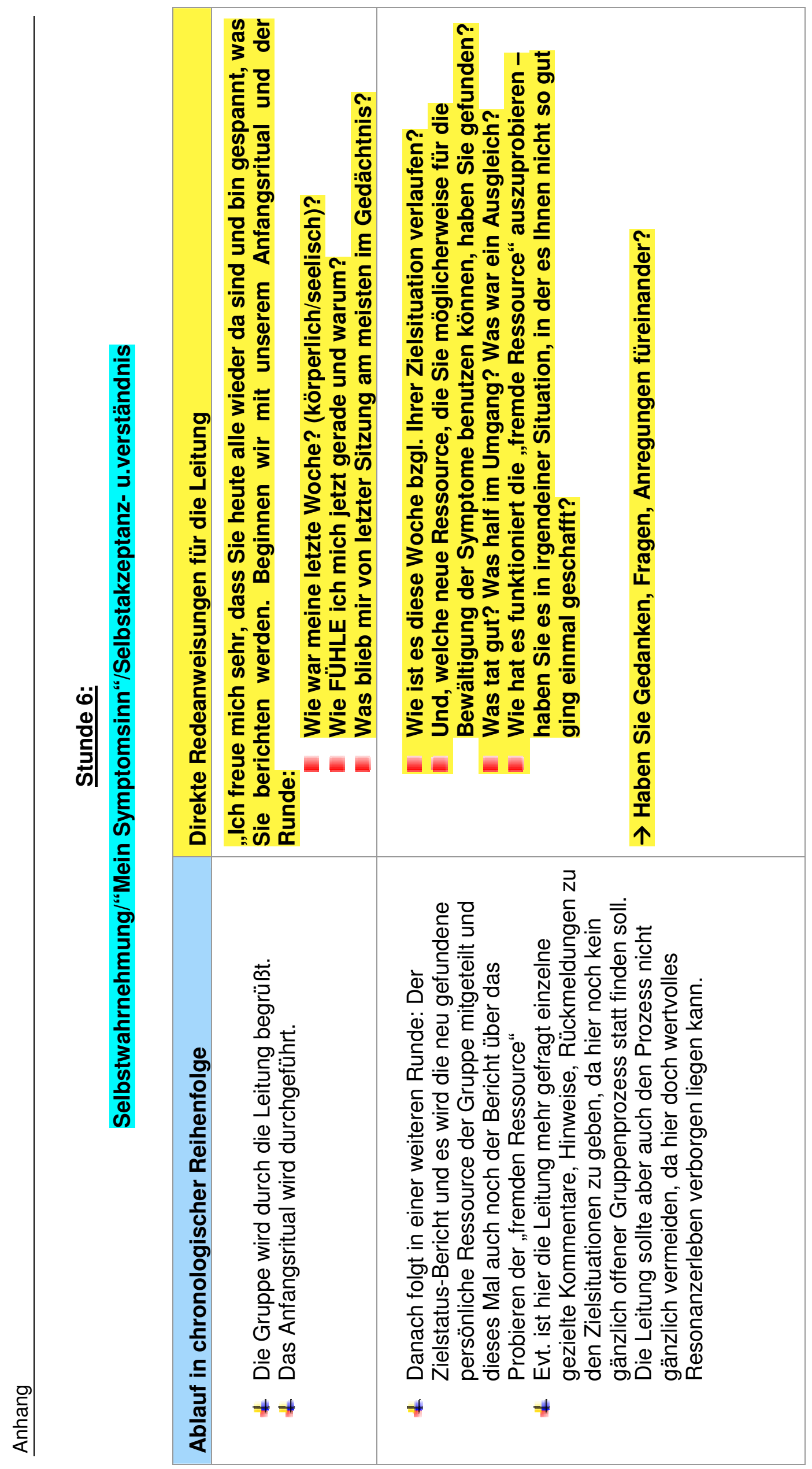

๙ 


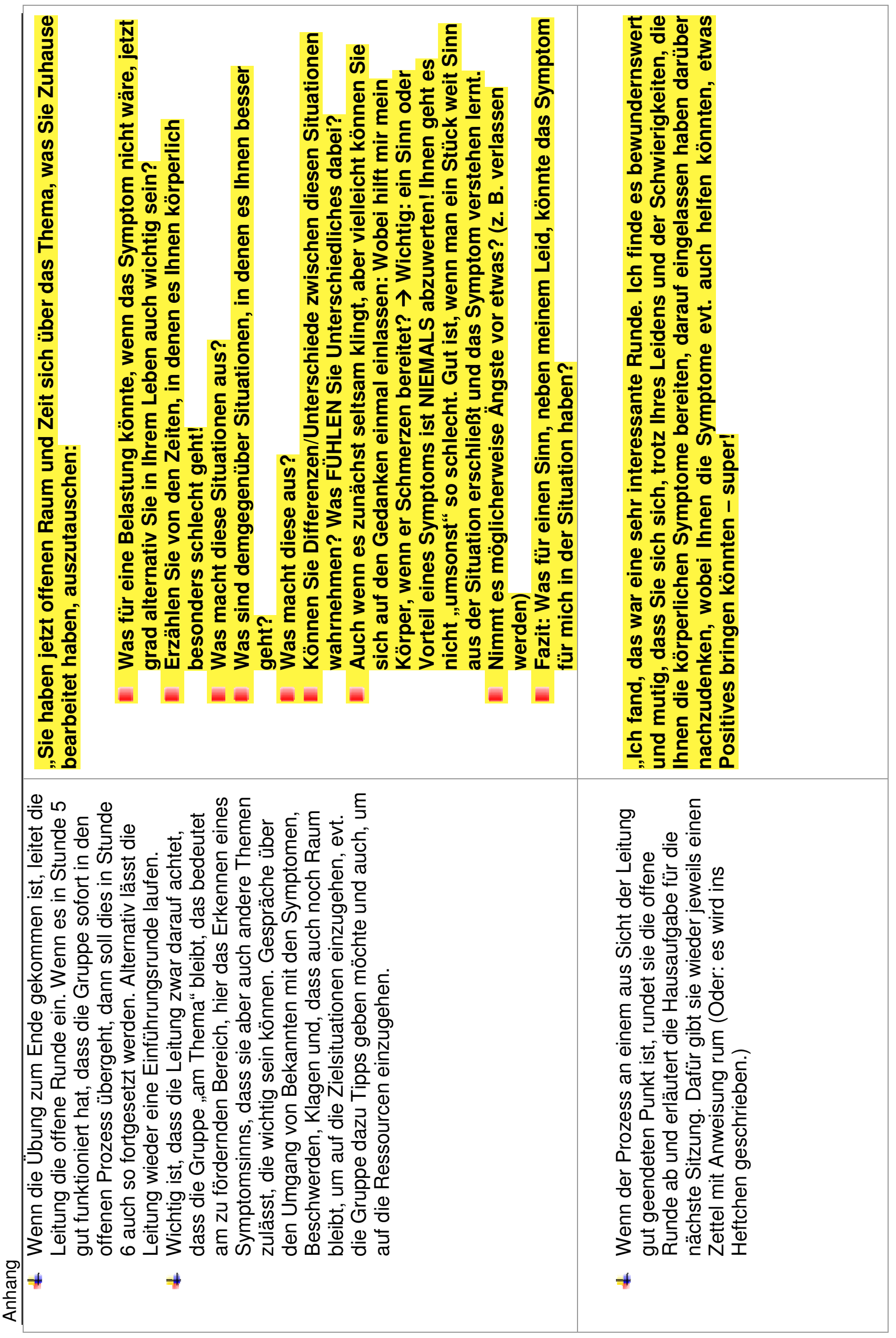

న్ 


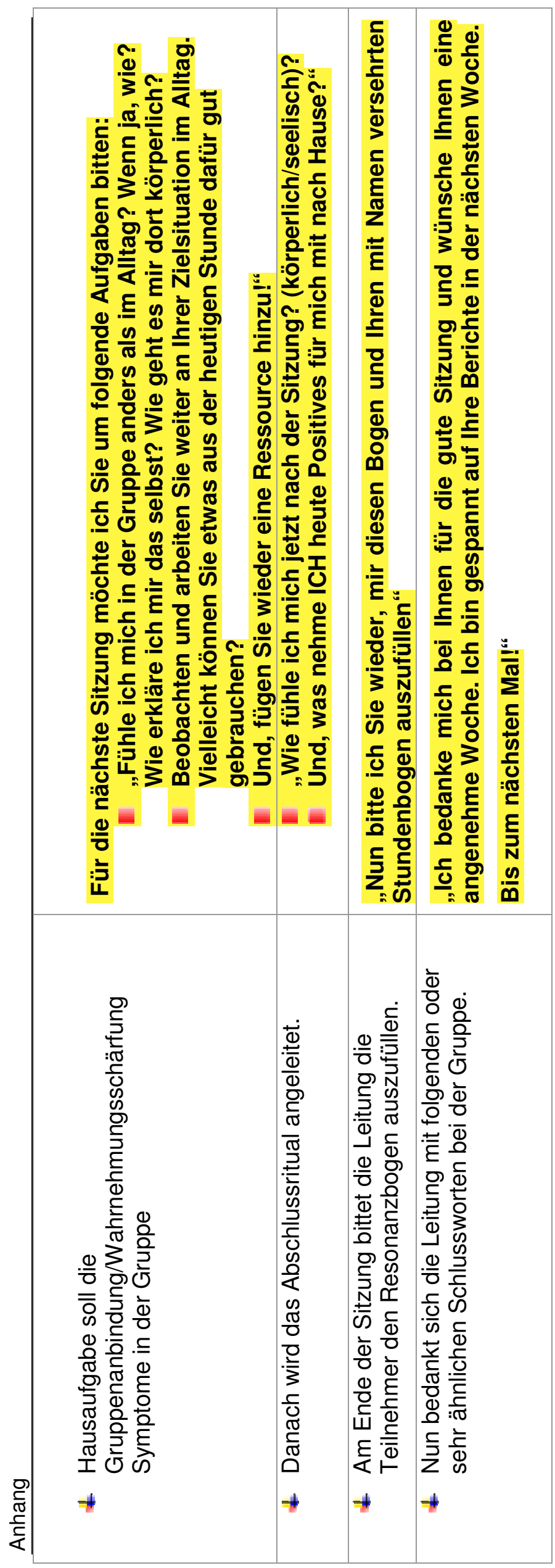




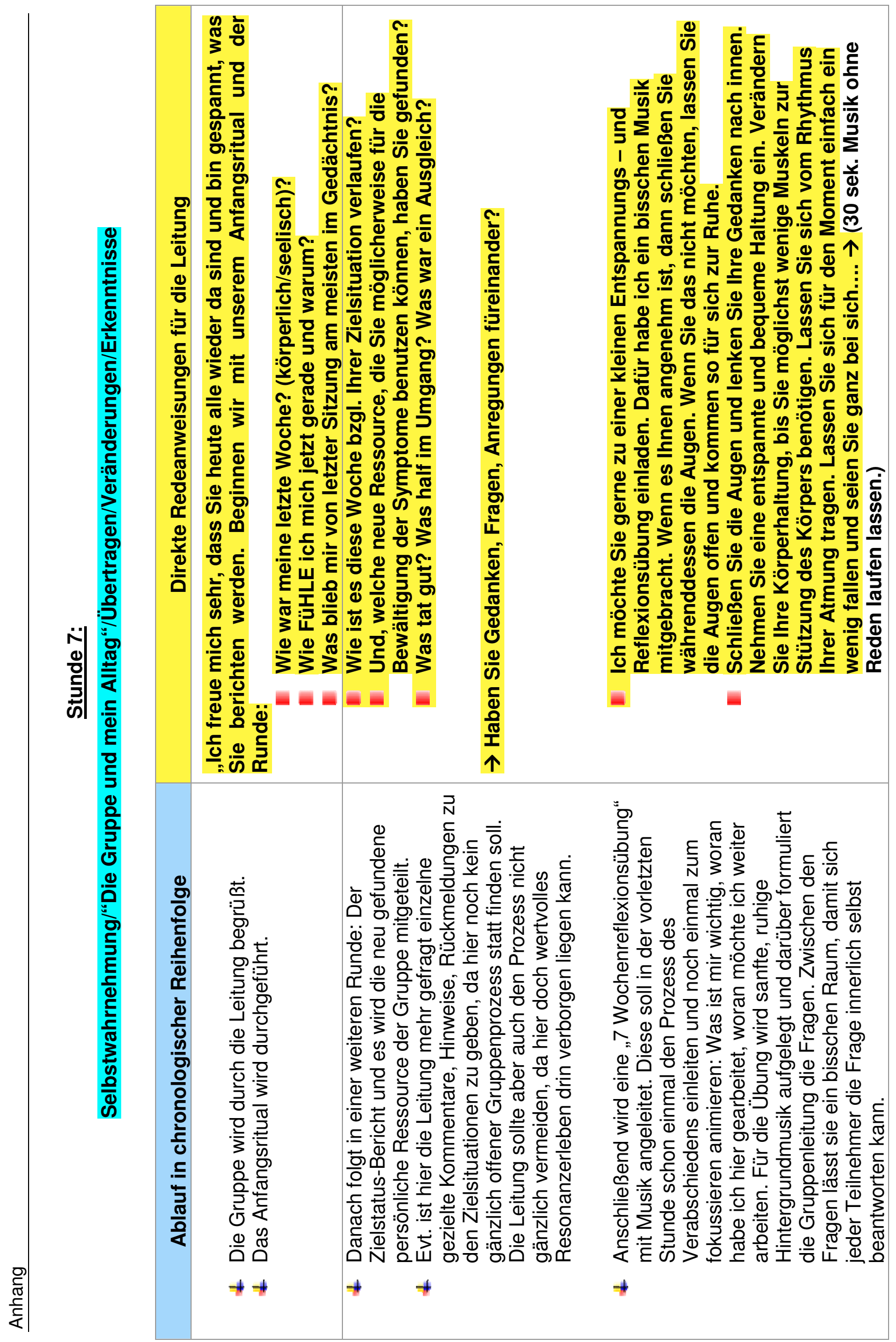




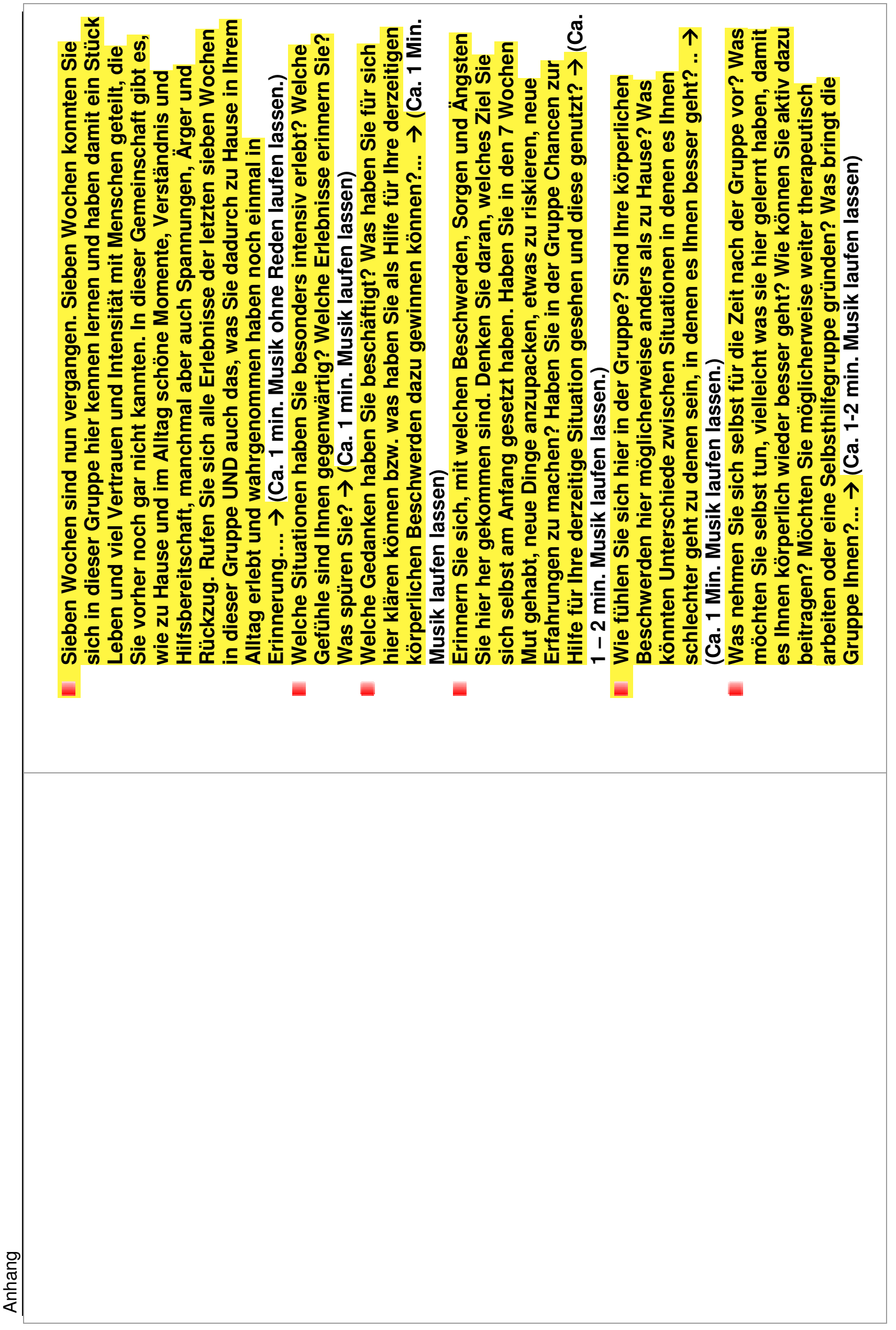




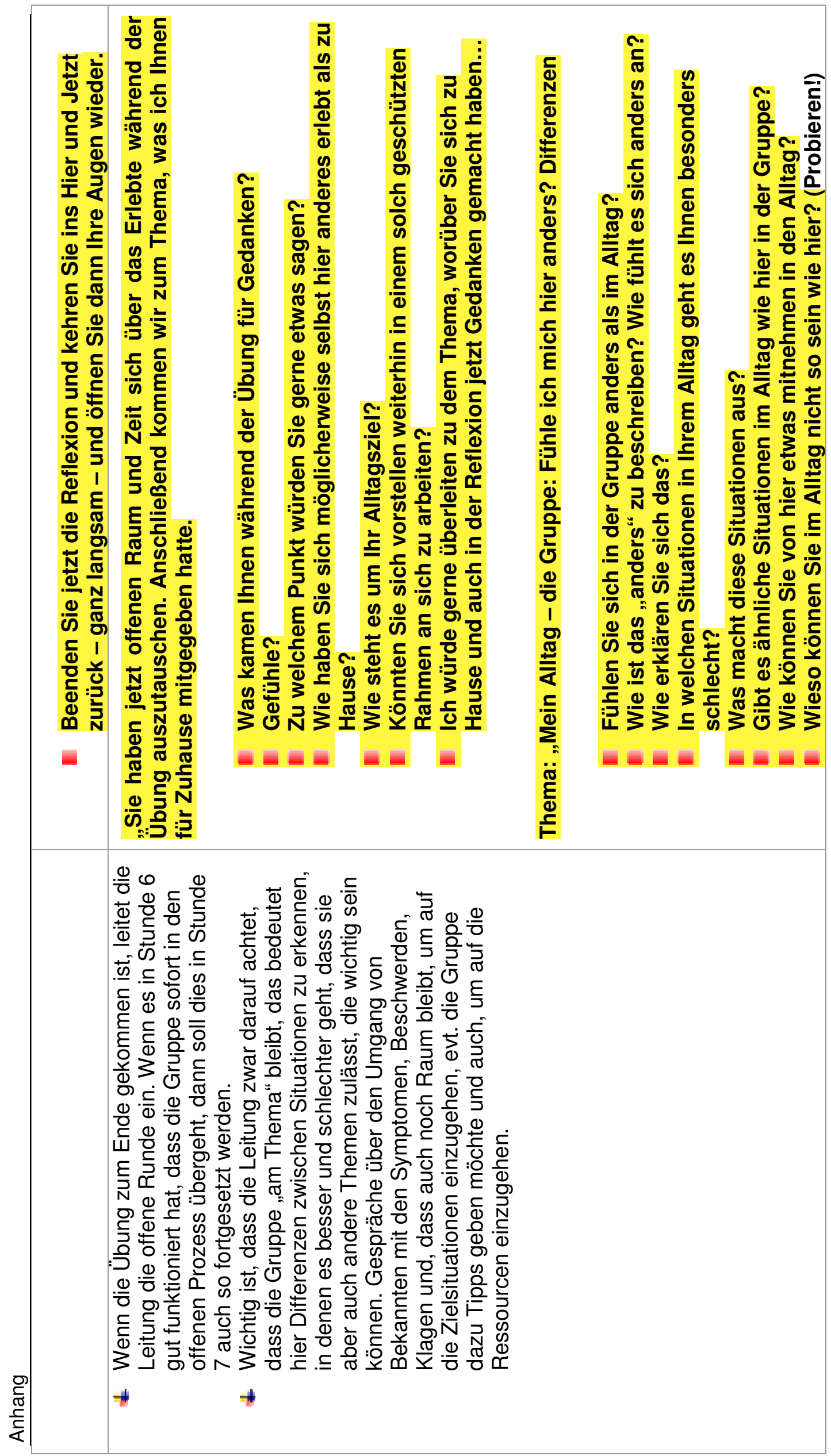




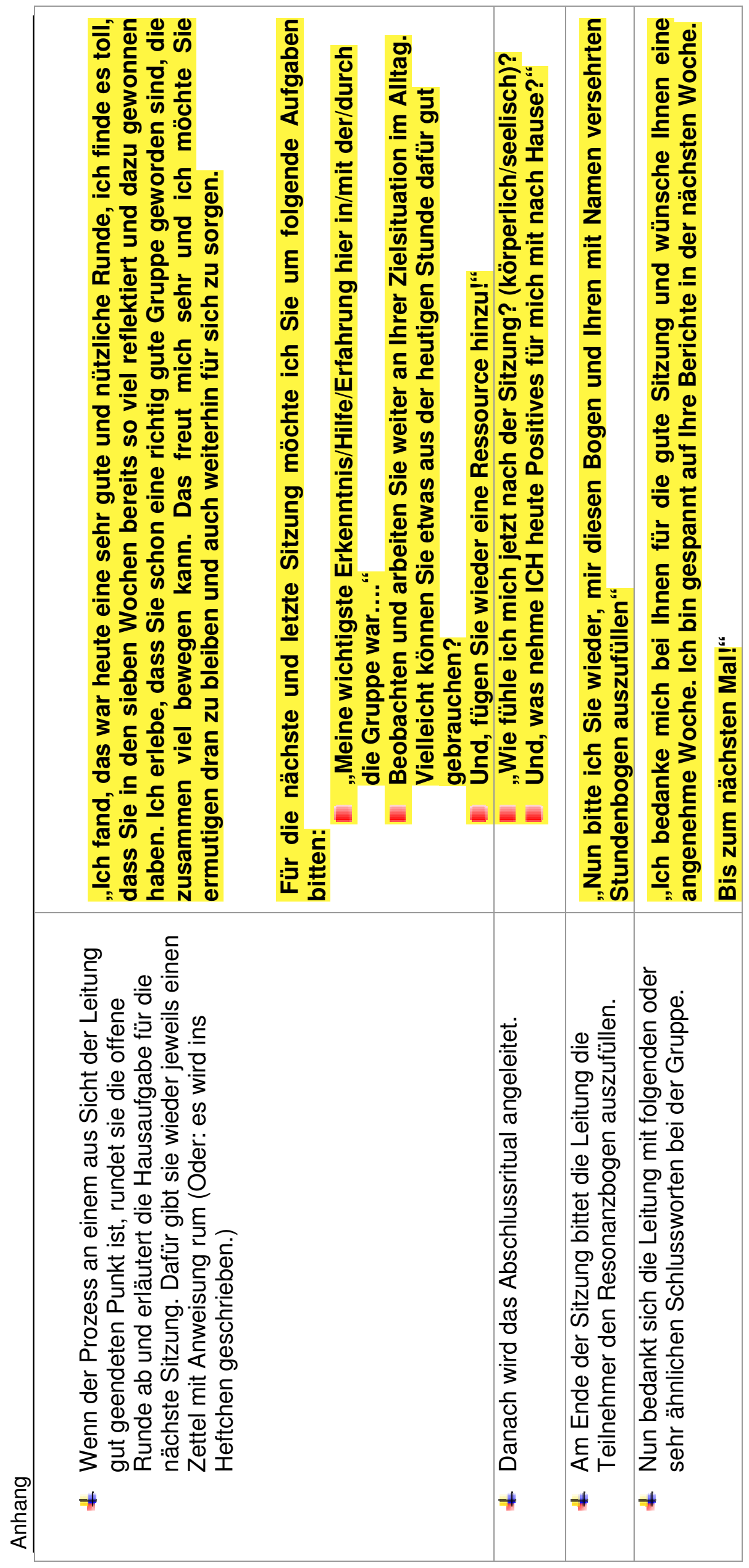

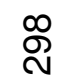




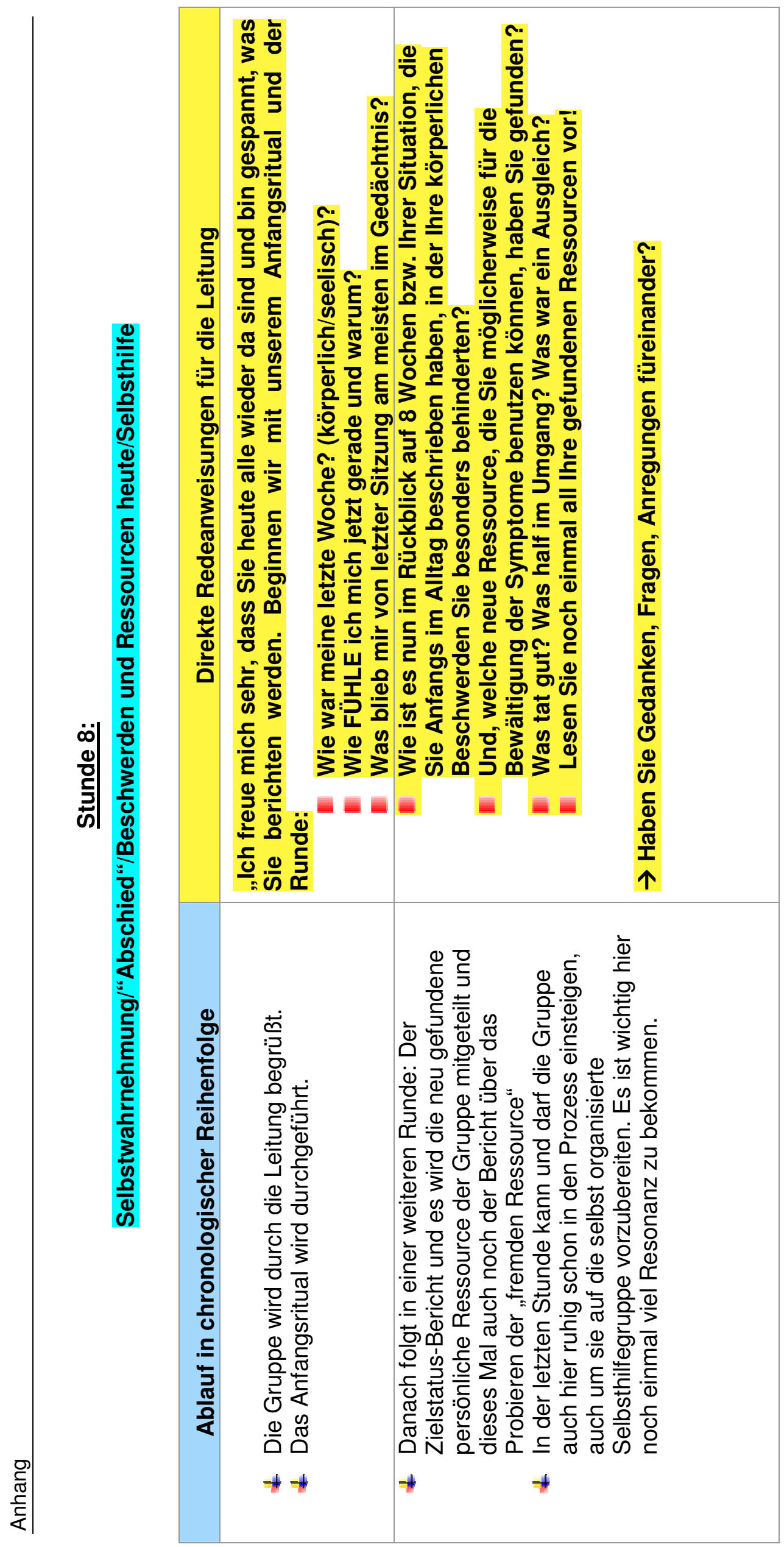




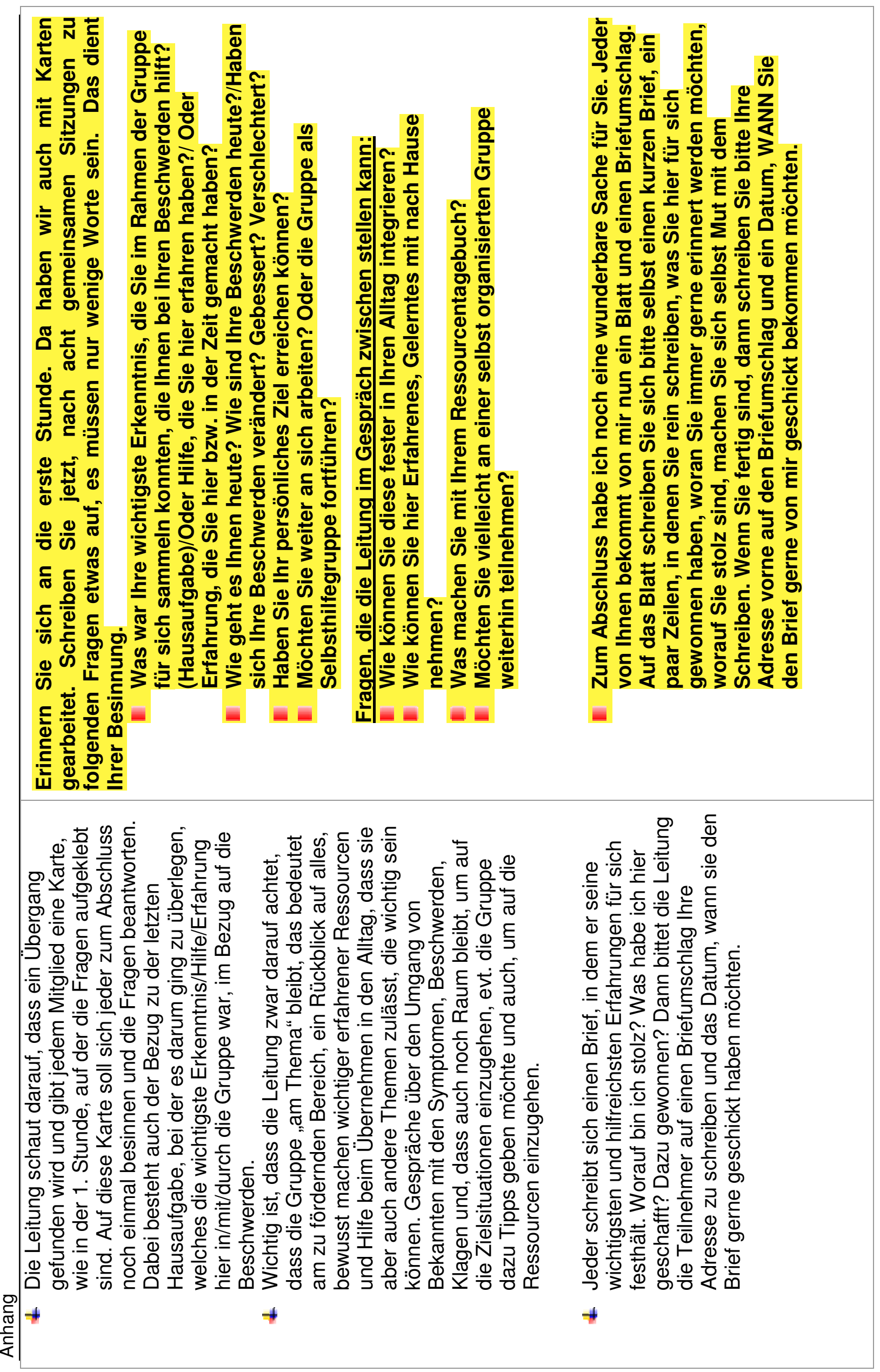




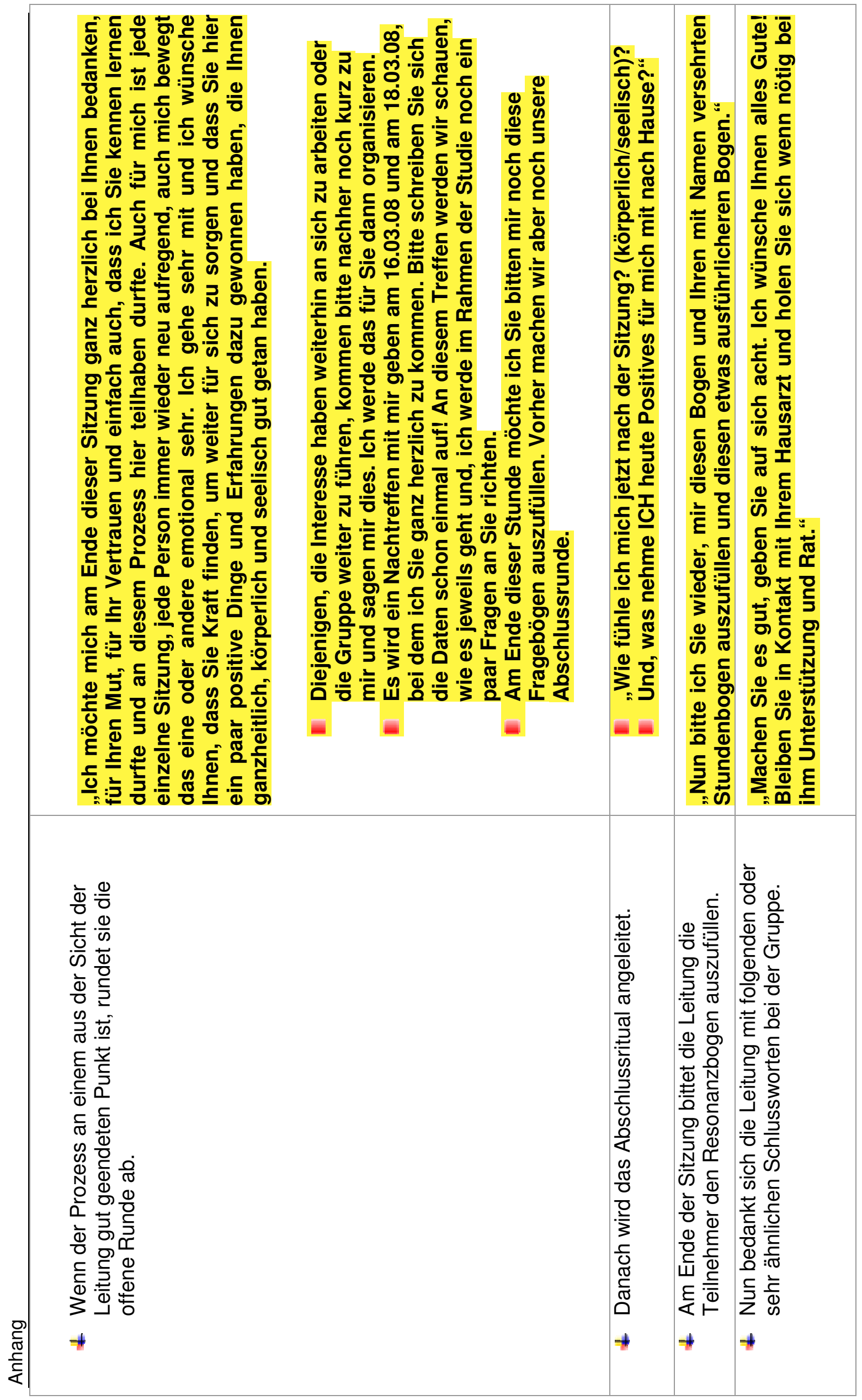

